\title{
Levels of lateral flange bending in straight, skewed and curved steel I -girder bridges during deck placement
}

\author{
Nohemy Y. Galindez \\ West Virginia University
}

Follow this and additional works at: https://researchrepository.wvu.edu/etd

\section{Recommended Citation}

Galindez, Nohemy Y., "Levels of lateral flange bending in straight, skewed and curved steel I -girder bridges during deck placement" (2009). Graduate Theses, Dissertations, and Problem Reports. 2897.

https://researchrepository.wvu.edu/etd/2897

This Dissertation is protected by copyright and/or related rights. It has been brought to you by the The Research Repository @ WVU with permission from the rights-holder(s). You are free to use this Dissertation in any way that is permitted by the copyright and related rights legislation that applies to your use. For other uses you must obtain permission from the rights-holder(s) directly, unless additional rights are indicated by a Creative Commons license in the record and/ or on the work itself. This Dissertation has been accepted for inclusion in WVU Graduate Theses, Dissertations, and Problem Reports collection by an authorized administrator of The Research Repository @ WVU.

For more information, please contact researchrepository@mail.wvu.edu. 
Levels of Lateral Flange Bending in Straight, Skewed and Curved Steel I-girder Bridges during Deck Placement

\author{
by \\ Nohemy Y. Galindez
Dissertation submitted to the College of Engineering and Mineral Resources at West Virginia University in partial fulfillment of the requirements for the degree of \\ Doctor of Philosophy \\ in \\ Civil Engineering
}

Approved by

Karl Barth, Ph.D., Chair Udaya Halabe, Ph.D. Bruce Kang, Ph.D. David Martinelli, Ph.D. John Zaniewski, Ph.D.

Department of Civil and Environmental Engineering

\author{
Morgantown, WV \\ 2009
}

Keywords: steel I-girder bridges, construction loads, lateral flange bending, cross-frame spacing, finite-element analysis

(C) 2009 Nohemy Y. Galindez 


\title{
Abstract \\ Levels of Lateral Flange Bending in Straight, Skewed and Curved Steel I-girder Bridges during Deck Placement
}

\author{
by Nohemy Y. Galindez
}

Exterior steel I-girders are required to withstand deck overhang loads during construction. This is partially accomplished by checking the flexural limit states for constructibility given by AASHTO. These limit states ensure that the maximum flange bending stresses produced during construction do not exceed the section flexural capacity of the girder.

For constructibility design, both the bending stresses and the flexural capacity of the flanges are affected by the loads corresponding to the deck placement sequence. Therefore, stiffness changes need to be considered during the various casting stages to compute the corresponding flange bending stresses and capacities. The specifications take into account this effect by defining separate limit states for discretely and continuously braced flanges. The limit states for discretely braced flanges involve not only the major-axis bending stresses produced by vertical loads but also the lateral flange bending (LFB) due to torsional responses or direct horizontal forces such as those produced by wind.

During construction, torsional effects are principally generated on exterior girders by deck overhang loads. In curved girders, it is also required to consider the significant torsional stresses introduced by the curvature, where the loads are eccentric with respect to the supports. Additionally, direct LFB may be induced in skewed bridges at crossframe locations caused by differential displacements or out-of-plane rotations.

Some simplified models have been proposed to estimate the LFB in exterior girders during deck placement conditions in straight bridges. However, the use of comprehensive models decreases the uncertainty in the lateral stiffness offered by structural elements such as the cross frames, the interior girders and the deck forms. In addition, the curvature and the skew angle effects have not been directly addressed in these simplified works.

AASHTO Specifications recommend approximate equations to estimate the torsional effects due to both deck overhang loads and curvature. For skewed bridges, the provisions recommend using $10 \mathrm{Ksi}$ as a conservative estimation of the unfactored LFB in bridges with discontinuous cross-frame lines and skew angles exceeding $20^{\circ}$. However, more precise approximations may be defined for each source of LFB if effects such as the continuity over the intermediate supports and the deck casting sequence are considered.

In this work, a comprehensive suite of finite element analyses is conducted on hypothetical three-span straight, skewed and curved bridges to assess the levels of flange bending during deck placement. The parameters varied include the span lengths, the cross-frame spacing, the skew angle and the radius of curvature. In addition, concentrated and distributed loading cases are considered to approximate the torsional effects due to eccentric overhang loading. A comprehensive formulation of the LFB 
effects due to curvature is also included for both loading cases. Numerical results were compared to current AASHTO Specifications and new approximations were proposed for predicting the LFB stresses. The flexural limit states for constructibility were also evaluated using the numerical stresses.

It was concluded that the curvature is the variable that most affects the limit states. Conversely, for the parameters exercised in this study, no significant effects were observed by varying the skew angle. The governing limit state of the casting sequence considered in this study corresponds to the ultimate strength for discretely braced flanges in compression. The yielding limit state controls in short span lengths while the web bend-buckling limit state becomes significant in the pier regions for long span lengths.

AASHTO does not include a specific recommendation for the spacing of cross frames in steel bridges. Therefore, the designer needs to either evaluate different configurations to select the most optimum spacing, or follow traditional practice that assures safe results. For that reason, a reliability analysis was proposed in this work to develop a practical method to select the cross-frame spacing for deck placement conditions considering the flexural limit states for constructibility that are affected by the cross-frame spacing. A Monte Carlo Simulation is performed for straight, skewed and curved steel I-girder bridges generating some fragility curves that allow identifying the maximum cross-frame spacing for deck-placement conditions according to the maximum tolerated level of risk. 


\section{Dedication}

To God, my lovely Daniel H. and my family. 


\section{Acknowledgements}

First, I would like to thank God for giving my husband and me the opportunity to accomplish this goal together.

Numerous individuals are also deserving of acknowledgment for their contributions to the successful completion of this dissertation. I would first like to express my deepest gratitude to my advisor, Dr. Karl E. Barth, for his valuable guidance and constant encouragement towards my professional realization.

Also, I would like to thank Drs. Udaya Halabe, Bruce Kang, David Martinelli and John Zaniewski for serving on my doctoral committee. Their contributions to this project are greatly appreciated. Dr. Ever Barbero and Dr. Julio Davalos are deserving of thanks as well for helping me to establish direct contact with Dr. Barth.

The contributions of Ms. Lauren E. Cullen at the beginning of this project are also acknowledged. I would like to thank my officemates as well, and especially my friends at West Virginia University for the countless good times spent during these years.

Financial support for this project was provided by the West Virginia Department of Transportation Division of Highways and is gratefully acknowledged.

Special thanks go to my family in Colombia for their unconditional support and love throughout my entire life. And lastly, I would like to express my deep appreciation to Daniel H. Cortes, my husband, for his encouragement to pursue my professional goals and for making the experience of living and studying abroad a very gratifying one. 


\section{Table of Contents}

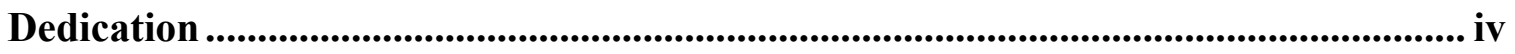

Acknowledgements ................................................................................................................... $\mathrm{V}$

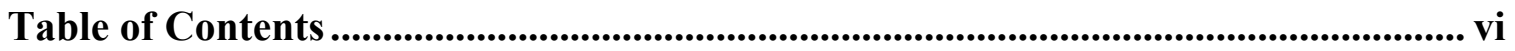

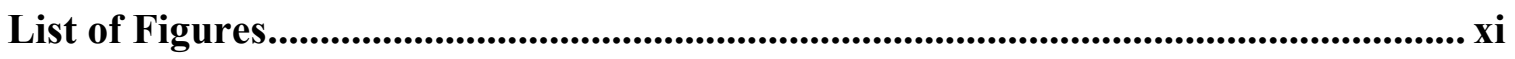

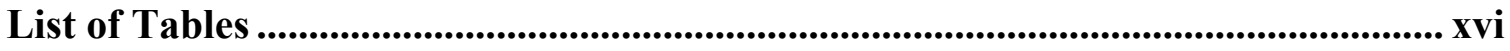

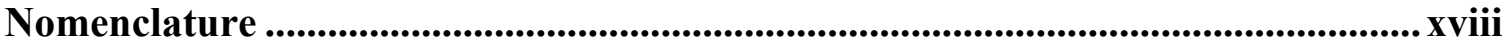

Chapter 1: Introduction ........................................................................ 1

1.1 PROBLEM, GOALS AND GENERAL OBJECTIVE .......................................... 1

1.2 Lateral Flange Bending In SteEl I-GiRder BRIDGES............................... 3

1.2.1 Lateral Flange Bending Fundamentals ................................................................ 3

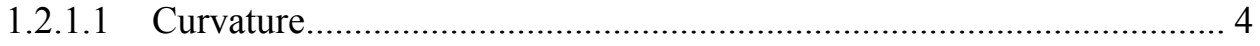

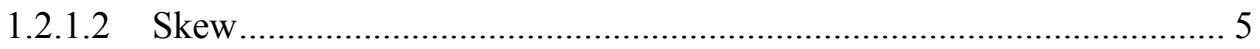

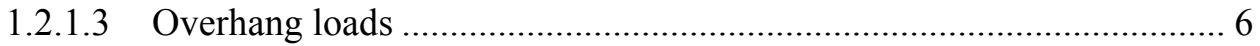

1.2.2 AASHTO approximate formulations for the $L F B$.............................................. 7

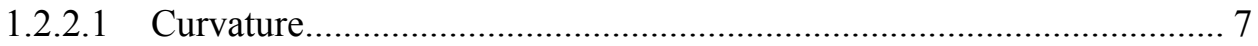

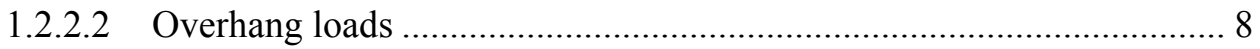

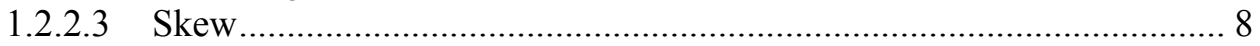

1.2.3 AASHTO Flexural Limit States for Constructibility ............................................... 9

1.2.3.1 Discretely braced flanges in compression.......................................... 9

1.2.3.2 Discretely braced flanges in tension .................................................. 10

1.2.3.3 Continuously braced flanges in tension or compression..................... 10

1.2.3.4 Maximum allowable LFB ............................................................... 10

1.3 RESEARCH OBJECTIVES, MOTIVATIONS AND METHODS ................................. 11

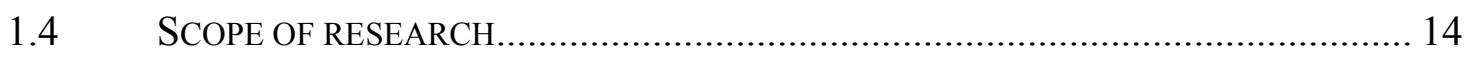

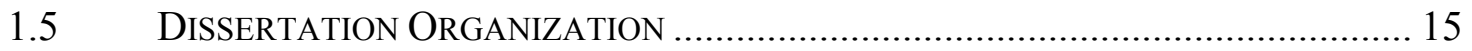

\section{Chapter 2: Literature Review......................................................................... 18}

2.1 CURVED STEEL I-GIRDER BRIDGES....................................................... 18

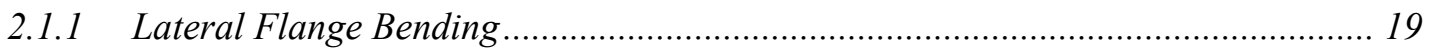

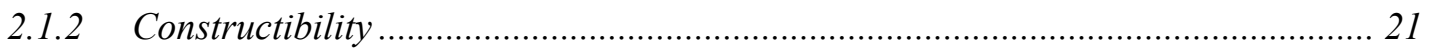




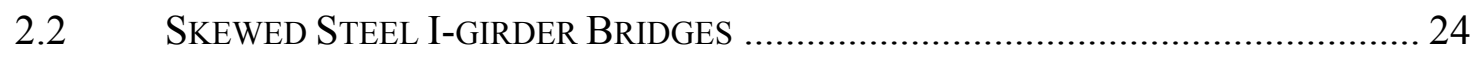

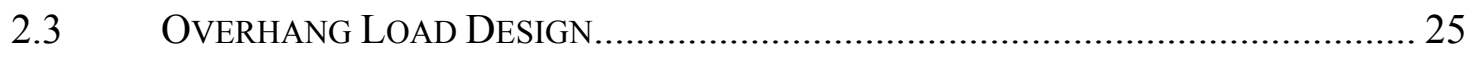

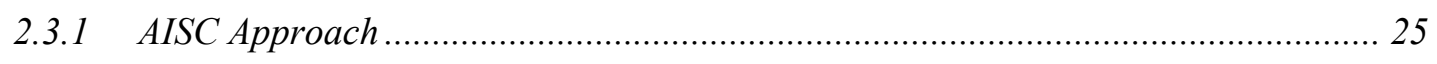

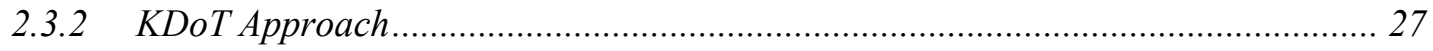

2.4 LATERAL FLANGE Bending DeSIGN ........................................................ 29

2.4.1 Standard Specifications for Highway Bridges - 15 ${ }^{\text {th }}$ Ed., AASHTO (1992): ........... 29

2.4.2 LRFD Bridge Design Specifications, SI Units $-I^{\text {st }}$ Ed., AASHTO (1994) ............... 30

2.4.3 LRFD Bridge Design Specifications, Customary U.S. Units $-2^{\text {nd }}$ Ed., AASHTO

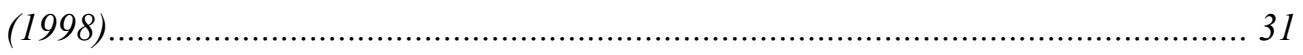

2.4.4 Horizontally Curved Steel Girder Highway Bridges, AASHTO (2003)................... 31

2.4.5 LRFD Bridge Design Specifications, Customary U.S. Units $-4^{\text {th }}$ Ed., AASHTO (2007)

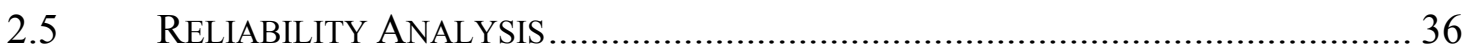

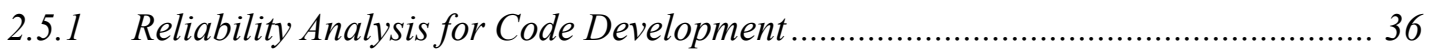

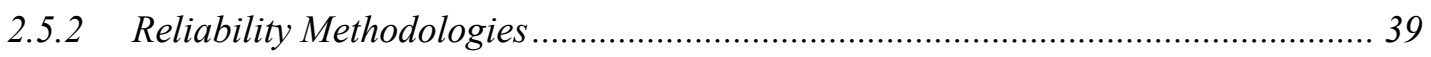

\section{Chapter 3: Overview of finite element modeling procedures................. 41}

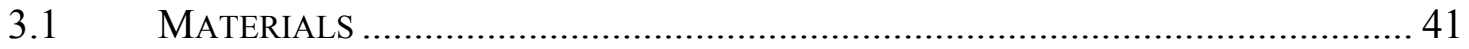

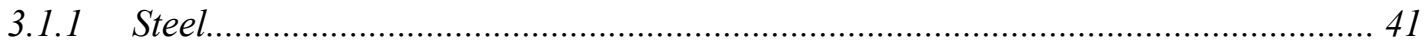

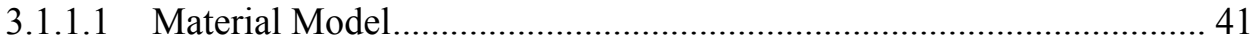

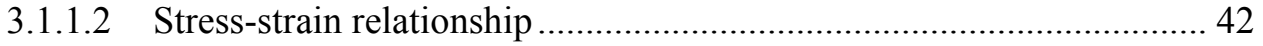

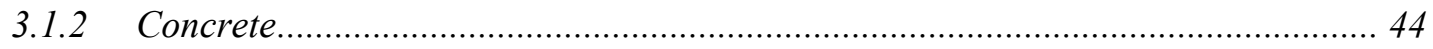

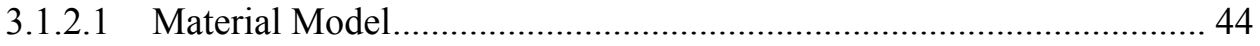

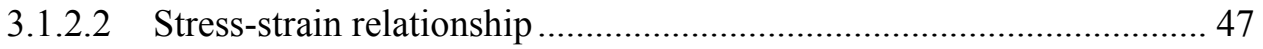

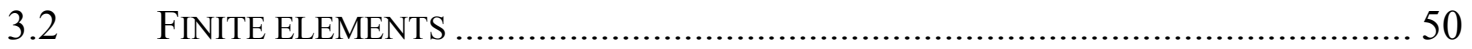

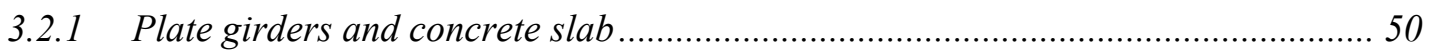

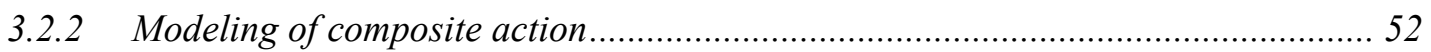

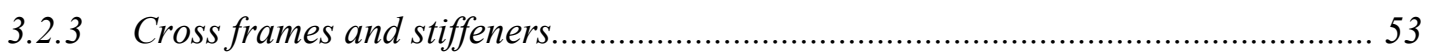

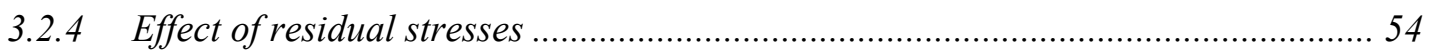




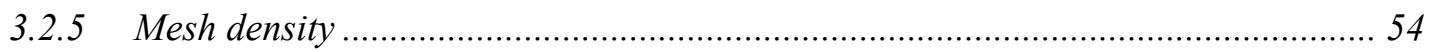

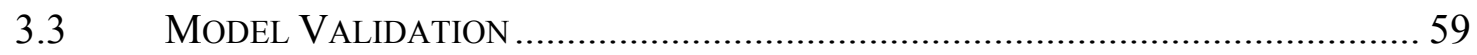

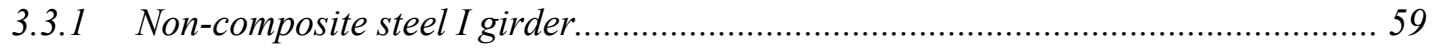

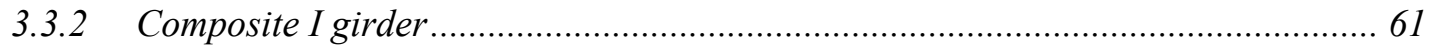

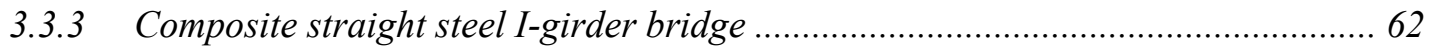

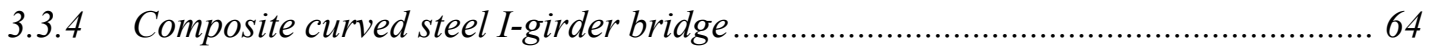

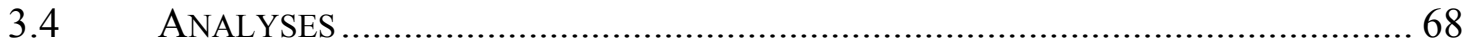

\section{Chapter 4: Parametric study of the lateral flange bending during} construction...................................................................................70

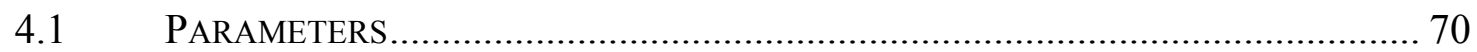

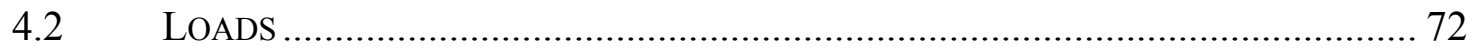

4.2.1 Deck placement sequence ........................................................................... 73

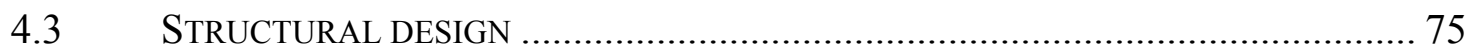

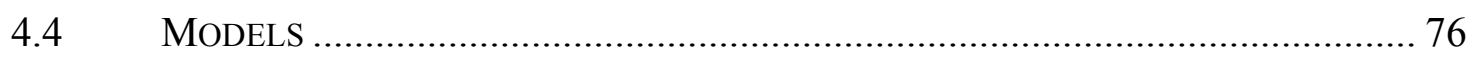

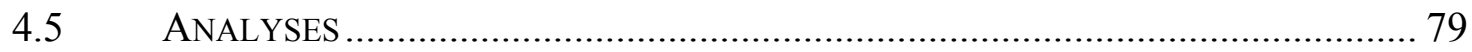

\section{Chapter 5: Approximation of the lateral flange bending in steel I-girder bridges ......................................................................................... 81}

5.1 DEFINITION OF THE BENDING STRESSES FROM FEA .................................... 81

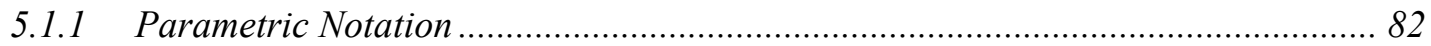

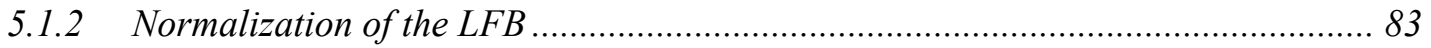

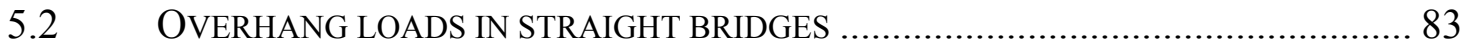

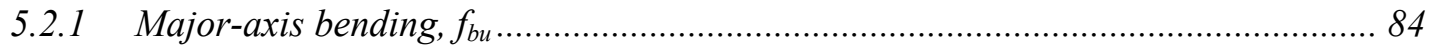

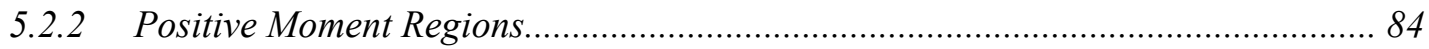

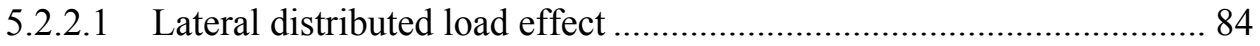

5.2.2.2 Lateral concentrated load effect ...................................................... 86

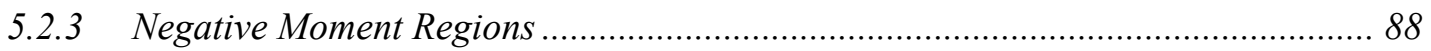

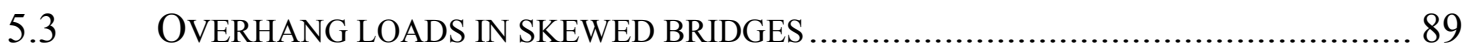

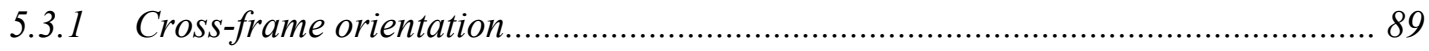




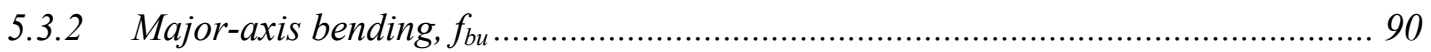

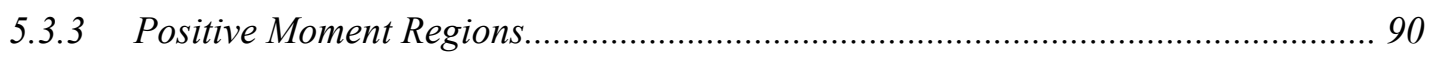

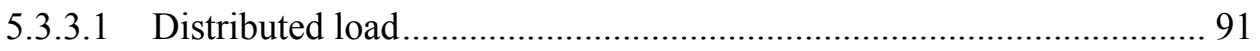

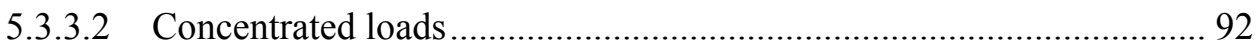

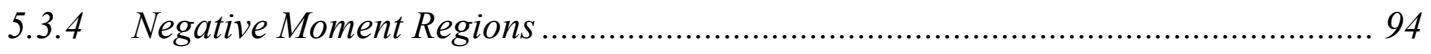

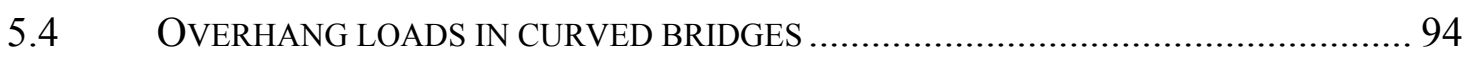

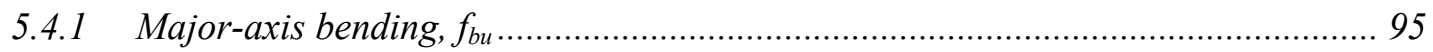

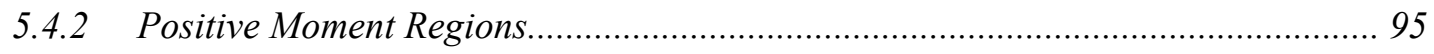

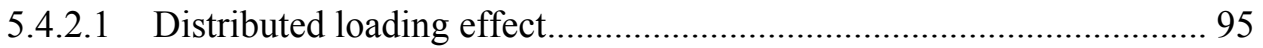

5.4.2.2 Concentrated loading effect .......................................................... 103

5.4.3 Negative Moment Regions .............................................................................. 109

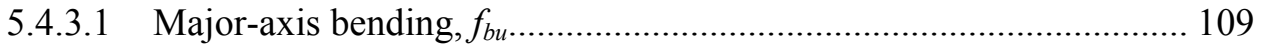

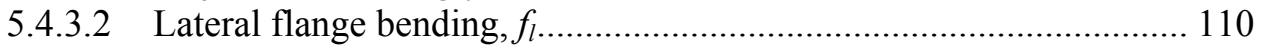

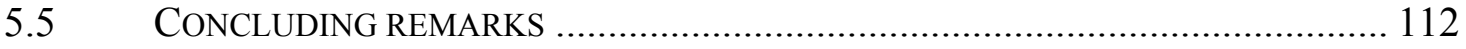

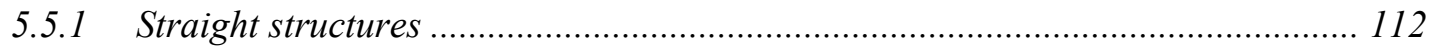

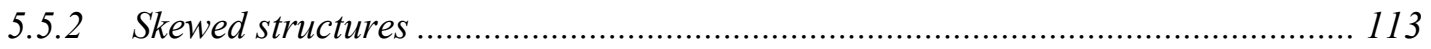

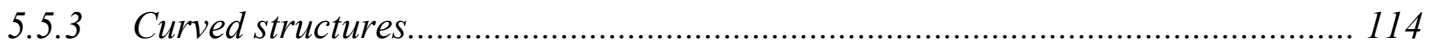

\section{Chapter 6: Evaluation of the flexural limit states for constructibility 117}

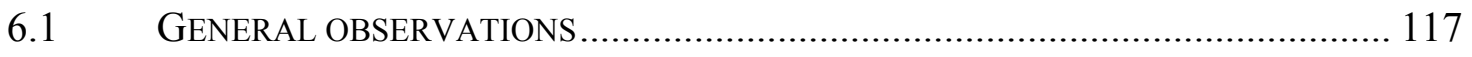

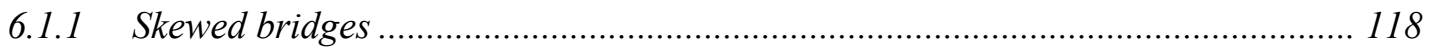

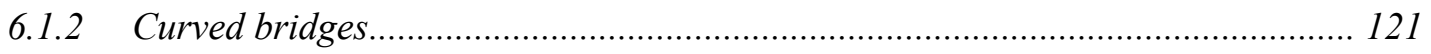

6.2 MAXIMUM ALLOWABLE SKEW ANGLE AND CURVATURE FOR STRAIGHT BRIDGES

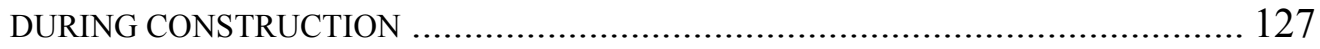

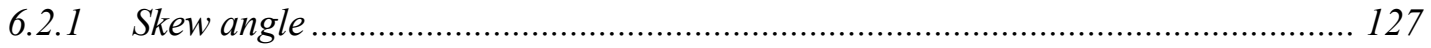

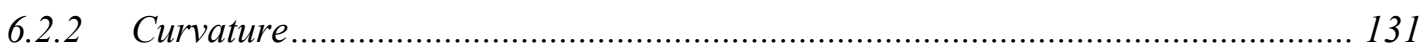

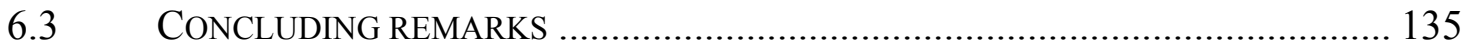

Chapter 7: Cross-frame spacing optimization ......................................... 138

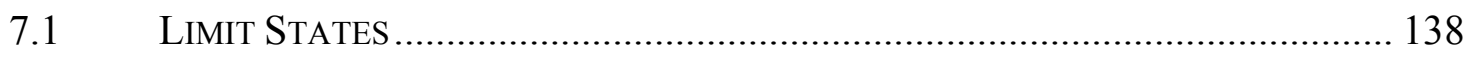

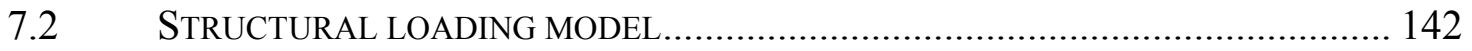




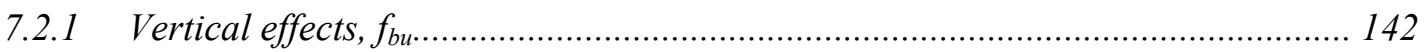

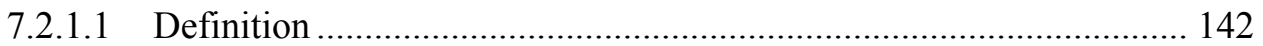

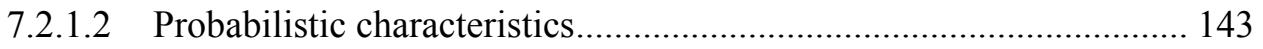

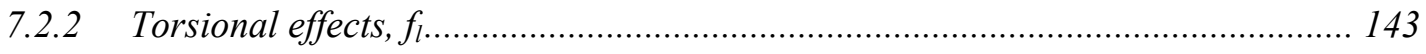

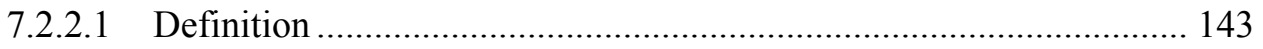

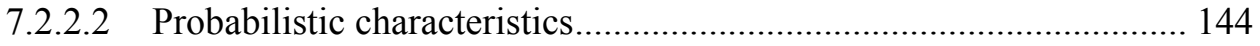

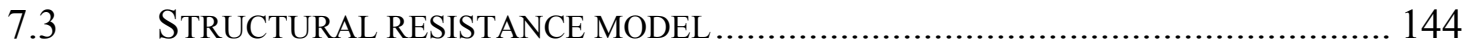

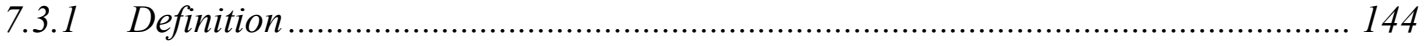

7.3.1.1 Lateral Torsional Buckling Resistance: .......................................... 144

7.3.1.2 Flange Local Buckling Resistance:................................................. 146

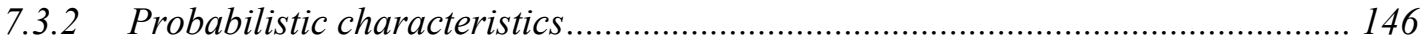

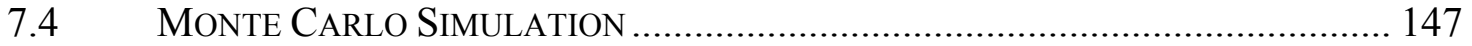

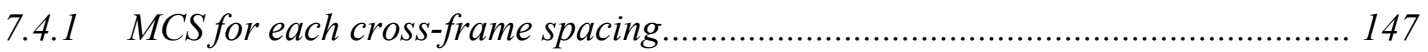

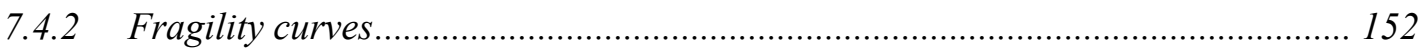

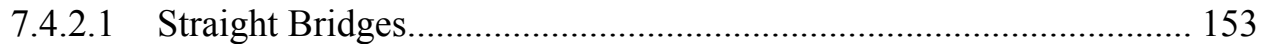

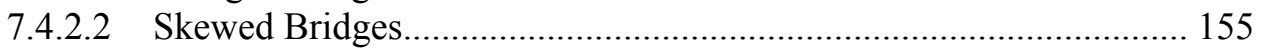

7.4.2.3 Curved Bridges .............................................................................. 158

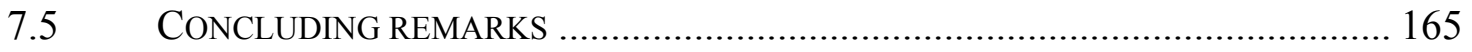

Chapter 8: Summary, conclusions and recommendations .................... 167

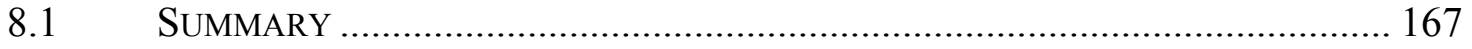

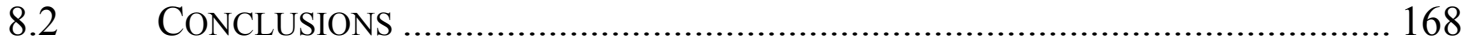

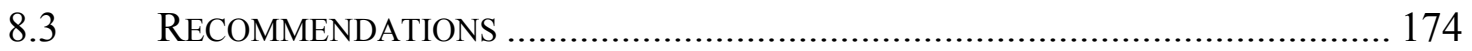

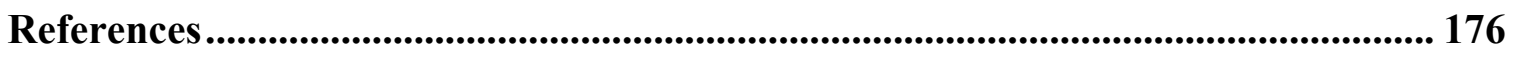

Appendix A: LFB in Skewed Bridges .......................................................................... 185

Appendix B: LFB in Curved Bridges............................................................................... 190

Appendix C: Limit States for constructibility in Skewed Bridges .............................. 200

Appendix D: Limit States for constructibility in Curved Bridges .............................. 212

Appendix E: Fragility Curves in Skewed Bridges ......................................................... 221

Appendix F： Fragility Curves in Curved Bridges......................................................... 225

Curriculum Vitae ....................................................................................................................... 248 


\section{List of Figures}

Figure 1-1. General state of stresses in an I-girder section (Cont.) ................................ 4

Figure 1-2. Torsional effects produced by curvature (Coletti \& Yadlosky, 2005)............ 5

Figure 1-3. Cross frames oriented perpendicular to the girders (Coletti \& Yadlosky, 2005)

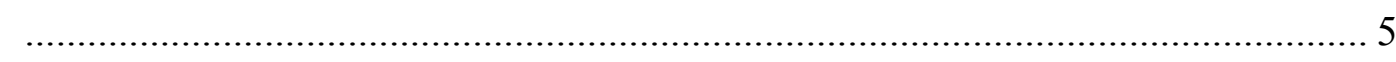

Figure 1-4. Cross frames oriented parallel to the skew (Coletti \& Yadlosky, 2005) ........ 6

Figure 1-5. Deck forming brackets on exterior girders............................................ 7

Figure 2-1. Plan view of bottom flange: a. Original b. Equivalent approximation. ......... 26

Figure 2-2. Idealized flange plastic stress distribution due to vertical and lateral bending.

Figure 2-3. Comparison of the complete and the approximate strength of a compact

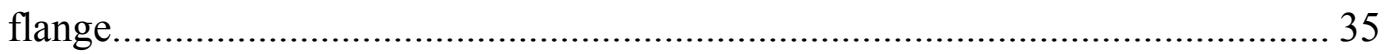

Figure 3-1. Engineering and true stress-strain relationships for the Grade-50 steel....... 42

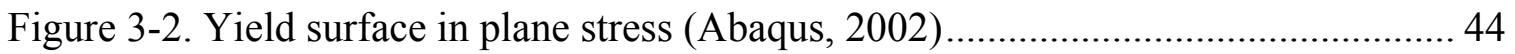

Figure 3-3. Concrete model response to uniaxial tension loading (Abaqus, 2002) ......... 45

Figure 3-4. Concrete model response to uniaxial compression loading (Abaqus, 2002) . 45

Figure 3-5. Effect of the compression stiffness recovery parameter $\mathrm{w}_{\mathrm{c}}$ (Abaqus, 2002) 47

Figure 3-6. Average compressive response of concrete (Jung, 2006) .......................... 48

Figure 3-7. Stress-strain relationship for the compressive behavior of concrete............. 48

Figure 3-8. Engineering stress-strain relationship for the tensile behavior of concrete ... 49

Figure 3-9. Change of shape of a fully integrated $a$. higher order $b$. linear element (Sun,

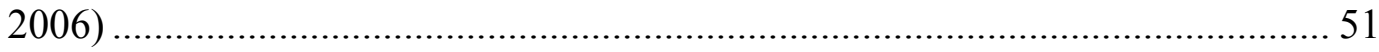

Figure 3-10. Change of shape of a reduced integration element (Sun, 2006) ................. 51

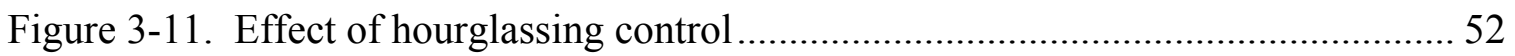

Figure 3-12. Effect of composite-action modeling (Jung, 2006) .................................. 53

Figure 3-13. Effect of residual stresses in the models (Jung, 2006) ............................. 54

Figure 3-14. Orientation of the finite elements in the simple supported beam model...... 55

Figure 3-15. Stress results for Case 1 of the mesh density study ................................ 56

Figure 3-16. Stress results for Case 2 of the mesh density study ................................. 57

Figure 3-17. Maximum LFB for a bridge configuration model with $\mathrm{Lm}=150 \mathrm{ft}$............ 58 
Figure 3-18. "D” girder specimen (Schilling \& Morcos, 1988) ................................... 59

Figure 3-19. Load-displacement response for a single steel I-shaped girder .................. 60

Figure 3-20. Geometry of Specimen POS1 (Mans, Yakel \& Azizinamini, 2001) ........... 61

Figure 3-21. Load-displacement response for a single composite steel I-shaped girder.. 61

Figure 3-22. Plan view of test bridge (Tiedeman, Albrecht \& Cayes, 1993) .................. 62

Figure 3-23. Cross section of test bridge (Tiedeman, Albrecht \& Cayes, 1993)............. 63

Figure 3-24. Comparison of measured and calculated bottom-flange stresses................ 63

Figure 3-25. Bridge test during the component strength study (Jung, 2006) .................. 64

Figure 3-26. Geometrical characterization of the bridge test (Jung, 2006) .................... 65

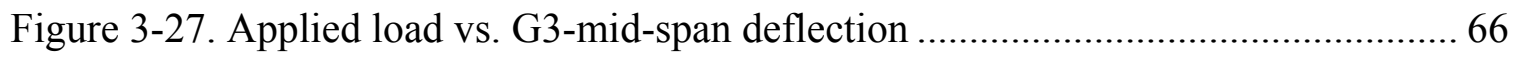

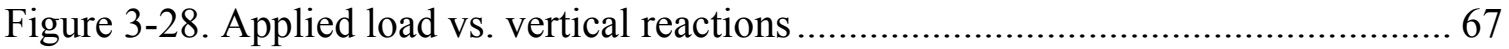

Figure 3-29. G3 bottom flange stresses at a load level of 570Kips (MAB: Major-axis

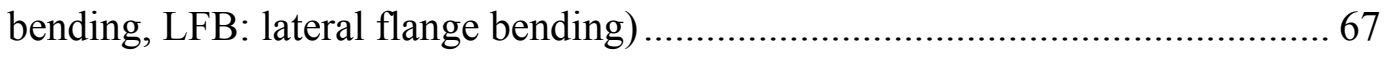

Figure 3-30. Load-displacement response of an unstable system (Abaqus, 2002).......... 68

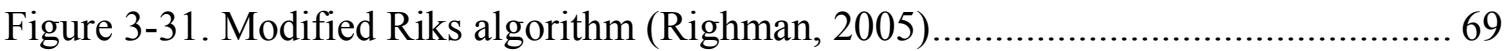

Figure 4-1. Visualization of the finishing machine on the exterior girder (KDT, 2005). 72

Figure 4-2. General view of a finishing machine (Bid-Well 4800) ............................. 73

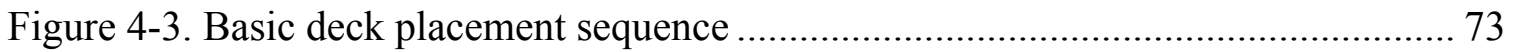

Figure 4-4. Detailed deck casting sequence............................................................ 74

Figure 4-5. Finite element model of a typical curved bridge configuration ..................... 76

Figure 4-6. Effect of the stay-in-place forms in the bending stresses of a curved bridge 78 Figure 4-7. Torsional effects on exterior girders produced by overhang loads ............... 79

Figure 4-8. Sequence of analysis for each bridge configuration ................................. 79

Figure 5-1. Identification of $f_{l}$ and $f_{b u}$ from the total flange bending response............... 81

Figure 5-2. Bending stresses on the exterior girder of a curved bridge model ................ 82

Figure 5-3. Effect of the cross-frame spacing in the major-axis bending ....................... 84

Figure 5-4. LFB due to distributed loads in the positive moment regions of straight

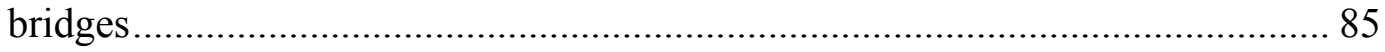

Figure 5-5. Effect of the concentrated load position in the LFB ................................. 86

Figure 5-6. LFB due to concentrated loads in straight bridges (Positive Moment).......... 87

Figure 5-7. LFB effects due to concentrated loads in straight bridges (Neg. Moment)... 88 
Figure 5-8. Effect of the skew angle in the LFB exhibited by the top flange................. 89

Figure 5-9. Effect of the cross-frame orientation in the LFB of skewed bridges ............ 90

Figure 5-10. Effect of the skew angle in the major-axis bending ................................. 91

Figure 5-11. LFB due to distributed loads in skewed bridges (Positive Moment).......... 92

Figure 5-12. LFB due to concentrated loads in skewed bridges (Positive Moment)....... 93

Figure 5-13. LFB effects due to distributed loads in skewed bridges (Neg. Moment)..... 94

Figure 5-14. Effect of curvature in the major-axis bending........................................ 95

Figure 5-15. Normalized $f_{b u}$ due to distributed loads in outer girders (Positive Moment)

Figure 5-16. Normalized $f_{b u}$ due to distributed loads in inner girders (Pos. Moment) .... 97

Figure 5-17. Hypothetical curved girder to approximate the curvature effects .............. 98

Figure 5-18. Visualization of the torsional effects due to curvature.............................. 99

Figure 5-19. Horizontal distributed force on the top flange ...................................... 99

Figure 5-20. Comparison of the overhang and curvature effects in the LFB ............... 101

Figure 5-21. LFB in the outer girder due to distributed loads ( $\mathrm{Lm}=150 \mathrm{ft}$, Pos. Moment)

Figure 5-22. LFB in the inner girder due to distributed loads $(\mathrm{Lm}=150 \mathrm{ft}$, Pos. Moment)

Figure 5-23. Normalized $f_{b u}$ due to concentrated loads in outer girders (Pos. Moment) 104

Figure 5-24. Normalized $f_{b u}$ due to concentrated loads in inner girders (Pos. Moment) 105

Figure 5-25. Overhang and curvature effects in the LFB due to concentrated loads .... 107

Figure 5-26. LFB in the outer girder due to concentrated loads ( $L m=150 \mathrm{ft}$, Pos. Moment) 108

Figure 5-27. LFB in the inner girder due to concentrated loads ( $L m=150 \mathrm{ft}$, Pos. Moment)

Figure 5-28. Normalized major-axis bending stresses in the negative moment regions 110

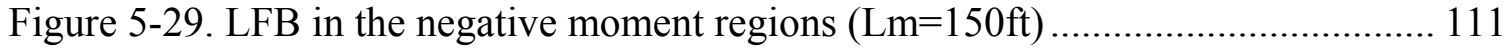

Figure 6-1. Variation of the limit state ratios along the length of skewed bridges......... 119

Figure 6-2. Effect of parametric variables in the limit states of skewed bridges (Cont.) 121

Figure 6-3. Variation of the limit state ratios along the length of curved bridges .......... 122

Figure 6-4. Variation of the LFB along the length of curved bridges 123 
Figure 6-5. Effect of parametric variables in the limit state ratios of Section S1

Figure 6-6. Effect of parametric variables in the limit state ratios of Section S2 ......... 125

Figure 6-7. Effect of parametric variables in the limit state ratios of Section S3 .......... 126

Figure 6-8. Identification of maximum skew angles for straight bridges with $\mathrm{Lm}=150 \mathrm{ft}$

Figure 6-9. Identification of maximum skew angles for straight bridges with $\mathrm{Lm}=240 \mathrm{ft}$

Figure 6-10. Identification of maximum skew angles for straight bridges with $\mathrm{Lm}=300 \mathrm{ft}$

Figure 6-11. Identification of maximum curvatures for straight bridges with $L_{m}=150 \mathrm{ft} 132$

Figure 6-12. Identification of maximum curvatures for straight bridges with $L_{m}=240 \mathrm{ft} 134$

Figure 6-13. Identification of maximum curvatures for straight bridges with $L_{m}=300 \mathrm{ft} 135$

Figure 7-1. Limit State function (Nowak \& Collins, 2000)

Figure 7-2. Probability functions of load and resistance (Nowak \& Collins, 2000) ...... 140

Figure 7-3. Reliability index in: $a$. general, and $b$. reduced coordinates (Melchers, 1999)

Figure 7-4. Graphical representation of the Reliability Index (Nowak \& Collins, 2000)

Figure 7-5. Inverse Transformation Technique Method (Haldar \& Mahadevan, 2000) 148

Figure 7-6. Histogram of the simulated values of $f_{b u}$ with $\mu=13.66 \mathrm{Ksi}$ and $\sigma=1.68 \mathrm{Ksi} 150$

Figure 7-7. Histogram of the simulated limit state LS2 with $\mu=35.18 \mathrm{Ksi}$ and $\sigma=4.88 \mathrm{Ksi}$

Figure 7-8. Simulated CDFs of LS1 and LS2 for different Lb values in a straight bridge

Figure 7-9. Fragility curves of LS2 using Monte Carlo Simulation .....

Figure 7-10. Fragility curves for the end-span sections in straight bridges with $L_{m}=150 \mathrm{ft}$

Figure 7-11. Fragility curves for the $L_{m}$ sections in straight bridges with $L_{m}=150 \mathrm{ft} \ldots . .155$

Figure 7-12. Fragility curves for the pier sections in straight bridges with $L_{m}=150 \mathrm{ft} \ldots 155$

Figure 7-13. Fragility curves for Section $\mathrm{S} 1$ in skewed bridges with $L_{m}=150 \mathrm{ft}$ and $\theta=30^{\circ}$ 
Figure 7-14. Fragility curves for Section S3 in skewed bridges with $L_{m}=150 \mathrm{ft}$ and $\theta=30^{\circ}$

Figure 7-15. Fragility curves for Section S2 in skewed bridges with $L_{m}=150 \mathrm{ft}$ and $\theta=30^{\circ}$

Figure 7-16. Fragility curves for section $\mathrm{S} 1$ in G4 with $L_{m}=150 \mathrm{ft}-\mathrm{L} / \mathrm{R}=0.30 \ldots \ldots \ldots \ldots . .160$

Figure 7-17. Fragility curves for Section S3 G4 with $L_{m}=150 \mathrm{ft}-\mathrm{L} / \mathrm{R}=0.30 \ldots \ldots \ldots \ldots \ldots . . .161$

Figure 7-18. Fragility curves for Section S2 in G4 with $L_{m}=150 \mathrm{ft}-\mathrm{L} / \mathrm{R}=0.30 \ldots \ldots \ldots \ldots . .162$

Figure 7-19. Fragility curves for Section $\mathrm{S} 1$ in G1 with $L_{m}=150 \mathrm{ft}-\mathrm{L} / \mathrm{R}=0.30 \ldots \ldots \ldots \ldots . .163$

Figure 7-20. Fragility curves for Section S3 in G1 with $L_{m}=150 \mathrm{ft}-\mathrm{L} / \mathrm{R}=0.30 \ldots \ldots \ldots \ldots . .164$

Figure 7-21. Fragility curves for Section S2 in G1 with $L_{m}=150 \mathrm{ft}-\mathrm{L} / \mathrm{R}=0.30 \ldots \ldots \ldots \ldots . .165$ 


\section{List of Tables}

Table 3-1. Approximations for a typical steel stress-strain behavior (Hartmann, 2005).. 43

Table 3-2. Average steel plate properties (Hartmann, 2005) ...................................... 43

Table 3-3. Mesh characteristics for Case 1 of the mesh density study ...........................55

Table 3-4. Mesh characteristics for Case 2 of the mesh density study ...........................57

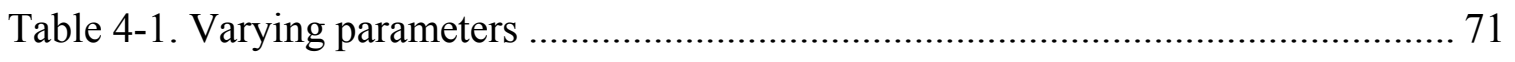

Table 4-2. Cross-frame spacing for positive moment regions ..................................... 71

Table 4-3. Cross-frame spacing for negative moment regions .................................... 71

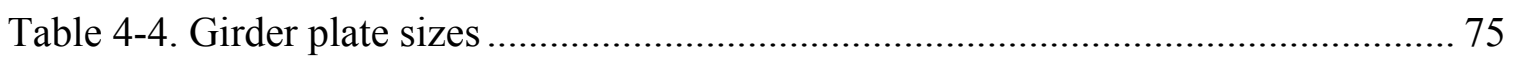

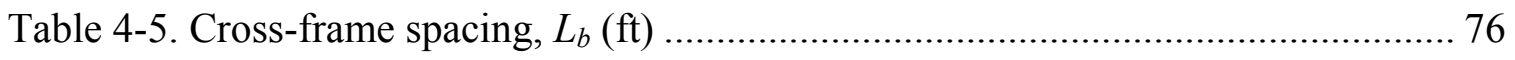

Table 5-1. Fitting parameters for distributed loads in straight bridges (Positive Moment).

Table 5-2. Fitting parameters for concentrated loads in straight bridges (Pos. Moment).

Table 5-3. Fitting parameters for distributed loads in straight bridges (Neg. Moment).. 88

Table 5-4. Fitting parameters for distributed loads in skewed bridges (Pos. Moment)... 92

Table 5-5. Fitting parameters for concentrated loads in skewed bridges (Pos. Moment).

Table 5-6. Fitting parameters for $f_{b u}$ due to distributed loads curved bridges (Pos.

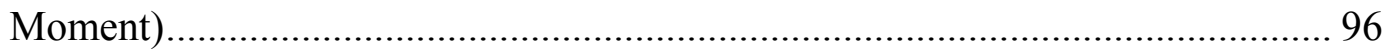

Table 5-7. Sign combination used to estimate $f_{b u}$ (Sign1, Sign2) (Pos. Moment) ........... 97

Table 5-8. Fitting parameters for distributed loads in the outer girders (Pos. Moment) 102

Table 5-9. Fitting parameters for distributed loads in inner girders (Pos. Moment) ..... 102

Table 5-10. Fitting parameters for fbu due to concentrated loads (Pos. Moment) ......... 104

Table 5-11. Fitting parameters for concentrated loads in the outer girders (Pos. Moment)

Table 5-12. Fitting parameters for concentrated loads in the inner girders (Pos. Moment) 108

Table 5-13. Fitting parameters for the negative moment regions .............................. 111

Table 7-1. General probabilistic characteristics of loads ........................................ 142 
Table 7-2. Probabilistic characteristics of distributed vertical loads in exterior girders 143 Table 7-3. Probabilistic characteristics of distributed lateral loads in exterior girders .. 144 Table 7-4. Probabilistic characteristics of resistance variables ...................................... 147 Table 7-5. Maximum $L_{b}(\mathrm{ft})$ corresponding to a code reliability level in straight bridges 153

Table 7-6. Maximum $L_{b}$ (ft) corresponding to a code reliability level in skewed bridges 156

Table 7-7. Maximum $L_{b}(\mathrm{ft})$ corresponding to a code reliability level in G4 ............... 158

Table 7-8. Maximum $L_{b}(\mathrm{ft})$ corresponding to a code reliability level in G1 ............... 158 


\section{Nomenclature}

$A_{s}: \quad$ Flange cross-sectional area (Eq. 2-4)

$b_{f}: \quad$ Flange width (Eq. 2-7, -8)

$B F$ : Bottom Flange

$b_{w}: \quad$ Reduced width at each edge of the bottom flange (Eq. 2-8)

$c$ : Width of each of the flange tips that correspond to the lateral flange moment (Eqs. 2-18, -19)

C1-C5: Specific stage from the deck-casting sequence

$C_{m}$ : $\quad$ Equivalent moment factor to account for the shape of the applied moment diagram (Eq. 2-4)

$C_{n}$ : $\quad$ Nominal construction load

$C V$ : $\quad$ Curved bridge

$D / C: \quad$ Demand-to-capacity ratio of limit states

D: $\quad$ Web depth (Eqs. 1-1, 2-11)

$d$ : $\quad$ Distance between flange centroids (Eq. 2-2)

$d_{\max }$ : $\quad$ Maximum eccentricity between the longitudinal axis in a curved girder and the straight line that connects the end supports

$D_{n}$ : Nominal dead load

$d_{t}, d_{c}$ : Damage variables for the concrete model in compression

E: $\quad$ Modulus of elasticity

$E_{0}$ : $\quad$ Initial elastic stiffness of concrete

$E_{s t}: \quad$ Strain hardening modulus of steel

F: $\quad$ Maximum induced stress in the bottom flange of the girder when the top flanges are continuously supported (Eq. 2-5)

Factored uniform lateral force (Eq. 2-16)

$f^{\prime} c$ : $\quad$ Maximum compressive strength of concrete

$F_{b s}: \quad$ Critical average flange stress of an equivalent straight girder flange (Eq. 214)

$f_{b u \text { max }}: \quad$ Maximum major-axis bending stress 
$f_{b u}: \quad$ Major-axis bending stress

$f_{\text {buo max }}$ : Maximum major-axis bending stress in a straight bridge

$F_{c b}$ : $\quad$ Flange bending stress produced by wind loading (Eq. 2-5)

$F_{c r}: \quad$ Flange critical buckling stress

Critical average flange stress for partially braced flanges (Eq. 2-14, -15)

$F_{c r w}: \quad \quad \quad \quad$ Nominal bend-buckling resistance for webs (Eq. 1-6)

FE: $\quad$ Finite Element

$F_{e}: \quad \quad$ Euler buckling stress of the flange in the plane of bending (Eq. 2-4)

FEA: $\quad$ Finete Element Analyses

$F_{l}$ : $\quad$ Statically equivalent uniformly distributed lateral force from the brackets due to the factored loads (Eq. 1-2)

$f_{l}$ : $\quad$ Lateral Flange Bending stress

$F_{n c}: \quad$ Nominal flexural resistance of a compression flange (Eq. 1-5)

$F_{Q}, F_{R}: \quad$ Cumulative Distribution Functions (CDF) of loads and resistance

$f_{Q}, f_{R}: \quad$ Probability Density Functions (PDF) of loads and resistance

$F_{r}: \quad$ Factored flexural resistance (Eq. 2-9)

$F_{u}$ : $\quad$ Flexural stress in the bottom flange due to the factored loads other than wind (Eq. 2-9)

$F_{w}$ : $\quad$ Flexural stress at the edges of the bottom flange due to the factored wind loading (Eq. 2-9)

$F_{y}: \quad$ Flange yield stress

$F_{y b}: \quad$ Specified minimum yield strength of the bottom flange (Eq. 2-8)

$F_{y c}$ : $\quad$ Specified minimum yield strength of a compression flange (Eq. 1-4)

$F_{y f}: \quad$ Specified minimum yield strength of the flange (Eq. 1-8)

$F_{y t}: \quad$ Specified minimum yield strength of a tension flange (Eq. 1-7)

g: $\quad$ Limit State designation

G1: $\quad$ Exterior girder in a straight and skewed bridge

Inner girder in a curved bridge

G4: $\quad$ Exterior girder in a straight and skewed bridge

Outer girder in a curved bridge

h: $\quad$ Web depth (Eq. 5-9) 
$K$ : $\quad$ Constant taken as 3 for compact flanges in compression and flanges in tension, and 1 for non-compact flanges in compression (Eq. 2-15)

$L / R: \quad$ Ratio of the middle span length to the radius of curvature

$l$ : $\quad$ Unbraced length (Eq. 1-1)

Unbraced arc length (Eq. 2-11)

L: $\quad$ Span length

Finite element length (Section 3.2.5)

$L_{b}: \quad$ Unbraced length in the girders

Cross-frame spacing

$l_{d}$ : Diaphragm spacing (Eq. 2-1)

$L_{e}: \quad$ End span length

$L_{m}$ : Middle span length

LS: $\quad$ Limit State

M: $\quad$ Major-axis bending moment (Eqs. 1-1, 2-11)

$M_{+}$: $\quad$ Maximum moment in-between the cross-frame spacing (Grubb, 1991)

$M_{f w}: \quad$ Maximum fixed-end moment at the cross-frame locations (Grubb, 1991)

$M_{l}$ : $\quad$ Lateral flange moment (Eq. 2-4)

$M_{\text {lat: }} \quad \quad$ Lateral flange bending moment (Eq. 1-1)

$M_{n b}: \quad$ Maximum normal bending moment (Eq. 2-2)

$M P C$ : Multi-point constraint

$M_{u}$ : $\quad$ Maximum moment capacity of the flange (Eq. 2-4)

$M u: \quad$ Factored major-axis bending

$M_{w}$ : $\quad$ Maximum lateral moment in the bottom flange due to factored wind loading (Eq. 2-8)

N: $\quad$ Constant taken as 10 or 12 (Eq. 1-1)

$P: \quad$ Concentrated lateral force at mid-panel (Eq. 2-16)

$P_{f:} \quad$ Probability of failure

$P_{l}$ : $\quad$ Statically equivalent concentrated lateral bracket force at the middle of the unbraced length due to the factored loads (Eq. 1-2)

Lateral concentrated load (Chapter 5)

$P_{u}: \quad \quad \quad$ Factored applied axial force in the flange (Eq. 2-4) 
$P u: \quad \quad \quad$ Vertical concentrated loads during deck placement

$P u_{l}: \quad \quad$ Lateral concentrated load acting on the flanges

Q: $\quad$ Loading variable

R: $\quad$ Radius of curvature

Factor that accounts for the effect of the bottom lateral bracing (Eqs. 2-5, -6)

Resistance variable (Chapter 7)

$R_{b}: \quad$ Web load-shedding factor (Eq. 7-11)

$R_{h}$ : $\quad$ Hybrid factor that accounts for the reduced contribution of the web to the nominal flexural resistance in sections with a higher-strength steel in the flanges (Eq. 1-4)

$R_{n}: \quad$ Nominal resistance

S1: Girder cross sections corresponding to the positive moment regions of the end spans

S2: $\quad$ Girder cross sections corresponding to the negative moment regions at the piers

S3: $\quad$ Girder cross sections corresponding to the positive moment regions of the middle span

$S_{d}$ : $\quad$ Diaphragm spacing (Eqs. 2-6, -7)

$S_{f}: \quad$ Flange section modulus

SK: $\quad$ Skewed bridge

ST: $\quad$ Straight bridge

$t_{f}: \quad$ Flange thickness (Eq. 2-7, -8)

TF: $\quad$ Top Flange

$T L_{n}: \quad$ Nominal total load

$V$ : Coefficient of variation of a random variable defined in general as the ratio of the standard deviation to the mean

w: $\quad$ Equivalent distributed lateral load (Eq. 2-1)

Finite element width (Section 3.2.5)

$W: \quad$ Wind loading along the exterior flange (Eq. 2-7)

wu: $\quad$ Vertical distributed loads during deck placement

$w u_{l}$ : $\quad$ Lateral distributed load acting on the flanges 
$w u_{\text {lmax }}$ : Maximum horizontal distributed force acting on the flanges of a curved girder

$x f 1, x f 2:: \quad$ Orientation of the cross frames: 1. perpendicular to the girders and 2. parallel to the skew

$\theta$ : $\quad$ Skew angle

Angle subtended by the span length in a curved girder (Eq. 5-8)

$\beta$ : $\quad$ Reliability index, also known as the safety index, is defined as the ratio of the mean value of the limit state to its standard deviation

$\mu$ : $\quad$ Mean value of a random variable

$\sigma: \quad$ Standard deviation of a random variable

$\sigma_{0.2 \%}: \quad$ Offset yield strength of steel

$\sigma_{\mathrm{co}}$ : Initial yield of concrete in compression

$\sigma_{\text {cu }} \quad \quad \quad$ Ultimate compressive stress in concrete

$\sigma_{\text {sy }}: \quad$ Static yield strength of steel

$\sigma_{t}, \sigma_{c}: \quad$ Tensile and compressive stress of concrete

$\sigma_{\text {to }}$ : Ultimate tensile stress in concrete

$\sigma_{\mathrm{u}}: \quad$ Tensile strength of steel

$\sigma_{y}: \quad$ Uniaxial yield strength of steel

$\varepsilon_{\mathrm{t}}^{\mathrm{pl}}, \varepsilon_{\mathrm{c}}{ }^{\mathrm{pl}}$ : Tensile and the compressive equivalent plastic strains for concrete

$\varepsilon_{s t}$ : Strain at the onset of strain hardening of steel

$\varepsilon_{u}$ : $\quad$ Strain at the tensile strength of steel

$\lambda$ : $\quad$ Bias factor defined as the mean-to-nominal ratio of a random variable

$\rho_{b}, \rho_{w}: \quad$ Strength reduction factors due to curvature (Eq. 2-14)

$\phi_{f}: \quad$ Resistance factor for flexure

$\Phi: \quad$ Cumulative Distribution Function of a standard normal variable 


\section{Chapter 1: Introduction}

\subsection{Problem, goals and general objective}

Horizontally curved and skewed steel I-girder bridges are frequently chosen as a practical solution for infrastructure projects that involve complicated interchanges or river crossings with specific site restrictions. In addition, these bridge configurations offer significant advantages related to economic and aesthetic aspects such as longer spans, which reduces the number of piers, and smooth transitions for urban environments with more uniform construction details. However, some inherent problems are exhibited during design and construction. In particular, the presence of curvature, skew angles or overhang construction loads produce additional bending effects which are counteracted by internal forces developed primarily in the flanges. The additional bending, known as lateral flange bending (LFB) is added to the major-axis bending produced by vertical loads, leading in some cases to premature strength and stability problems.

This dissertation is focused on the LFB exhibited by continuous straight, skewed and curved steel I-girder bridges during deck placement. AASHTO Load and Resistance Factor Design (LRFD) Specifications (2007) recommend some simplified equations to consider the LFB produced by curvature and overhang loads during construction. No specific equations are given to include the skew effect. The existing approximations included in the specifications are based on simplified models of the girder flanges that neglect effects such as the continuity over the supports, the deck-casting sequence and the interaction of the whole bridge superstructure. The inclusion of these effects by comprehensive models allows estimating the LFB more accurately. Therefore, the primary objective of this work is to develop improved equations to estimate the LFB of continuous steel I-girder bridges during deck placement. A parametric study based on finite element analyses (FEA) is employed to accomplish this objective.

Furthermore, the numerical bending results from the parametric study are used to evaluate the flexural limit states for constructibility. This evaluation allows the parametric variables that most affect the limit states, the governing limit states, and the 
critical stages during the deck-placement sequence considered in this study to be identified. In addition, the numerical major-axis bending stresses together with the improved LFB equations are used to define the maximum permissible skew angles and curvatures to meet the flexural limits for constructibility of bridges designed originally as straight. The definition of these limits will simplify the design process for constructibility of more complex bridges based on their straight girder counterparts.

Lastly, AASHTO (2007) does not include a specific recommendation for the spacing between cross frames. Consequently, the designer needs to evaluate different cross-frame configurations to select the most appropriate spacing that assures safe conditions especially during construction when the girders act in a non-composite state. For that reason, the final aim of this study is to optimize the cross-frame spacing during deck placement conditions. This is achieved by conducting reliability analyses of the flexural limit states for constructibility that are directly affected by the cross-frame spacing.

Therefore, the contribution of this research work to practice is to improve the estimation of the LFB in continuous steel I-girder bridges during deck placement. In addition, practical simplified checking procedures for constructibility are derived from the achievement of the primary goal combined with the corresponding flexural limit states: the definition of the maximum permitted skew angle and curvature for a bridge designed as straight and the selection of the optimum cross-frame spacing. As a result of these efforts, both the design and rating processes of steel I-girder bridges for constructibility will be improved.

Section 1.2 includes the definition of the LFB and its causes in steel I-girder bridges followed by the corresponding AASHTO simplified approximations. The flexural limit states for constructibility are also presented, since they constitute the criteria used in this project to evaluate the structural performance of the steel I-girder bridges. 


\subsection{Lateral Flange Bending in Steel I-girder Bridges}

An overview of the mechanical behavior of I-shaped girders is initially presented for a better understanding of the physical concept of LFB, along with a description of the principal sources of these additional bending effects in steel I-girder bridges.

\subsubsection{Lateral Flange Bending Fundamentals}

General cross sections resist torsion in the form of pure torsion and restrained warping (Seaburg \& Carter, 1997). The pure torsion resistance is obtained by means of shear stresses and if the warping is restrained, additional shear and normal stresses are incorporated to the original state of stresses. Warping becomes the primary mean to resist torsion in I-shaped girders since the St. Venant torsional stiffness for open cross sections is low. Therefore, the additional torsional effects are added to the initial axial and bending stresses produced by the gravity loads, as shown in Figure 1-1.

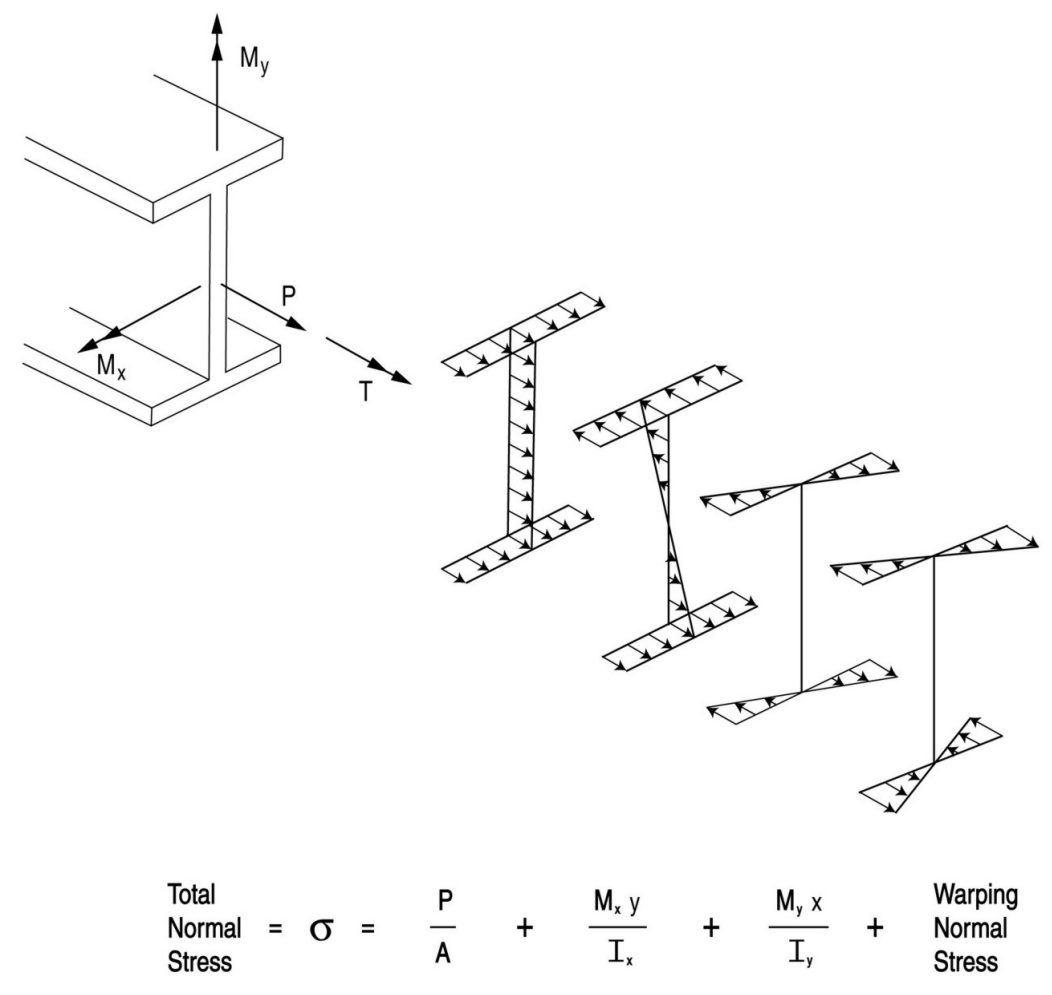

Figure 1-1. General state of stresses in an I-girder section (Coletti \& Yadlosky, 2005) 


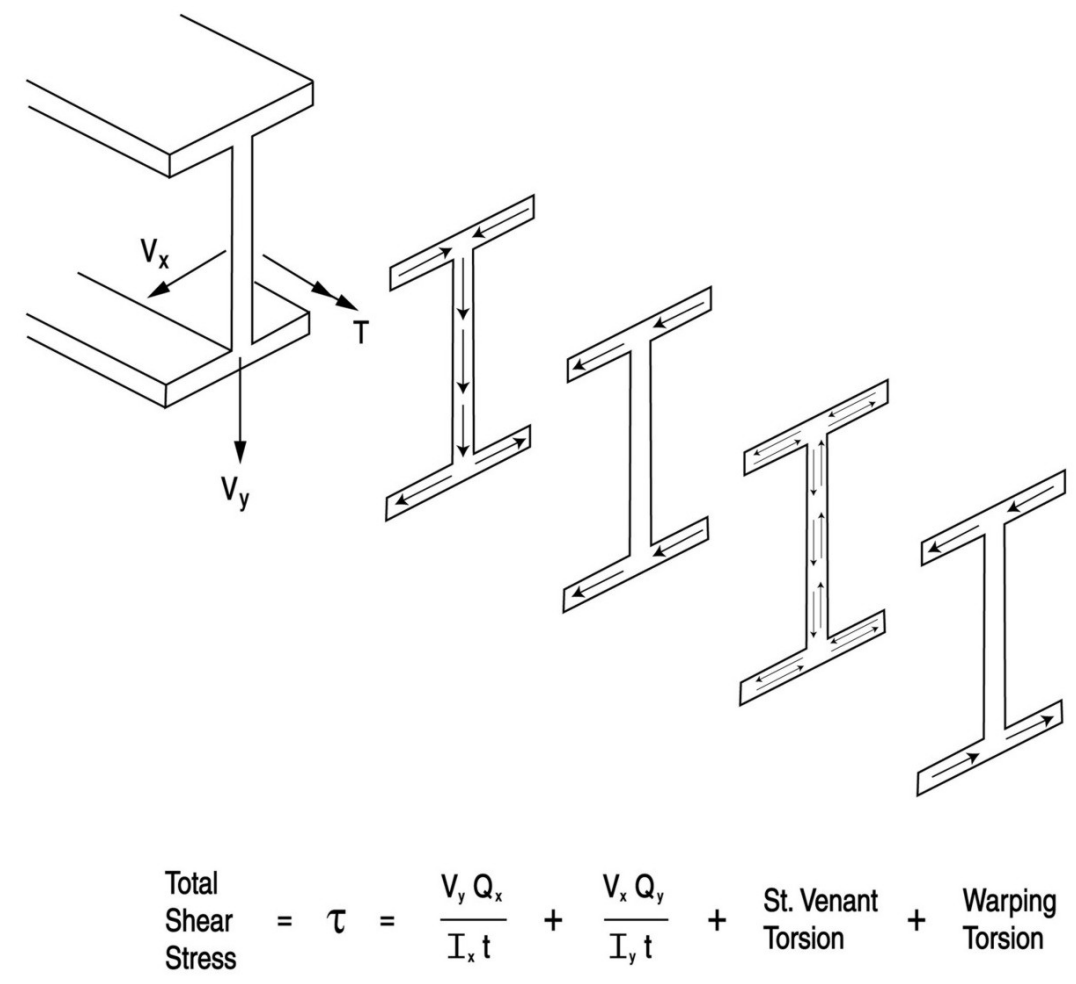

Figure 1-1. General state of stresses in an I-girder section (Cont.)

The warping normal stresses are basically carried by the girder flanges in the form of bending stresses and represent one of the factors introducing LFB. The curvature of the girders and the overhang load brackets in exterior girders during construction are some examples of structural configurations where the LFB is caused by torsional effects. Another source of LFB is given in skewed bridges where the cross frames induce additional lateral forces in the girder flanges. Further details about the mechanisms of these LFB sources are given in subsequent sections.

\subsubsection{Curvature}

The bending stresses in the girders of horizontally curved steel I-girder bridges are affected considerably by the geometry. The curvature introduces significant torsional stresses due to the eccentricity of the supports with respect to the loads, as shown in Figure 1-2. This curvature effect leads to a combined state of bending and torsional stresses that may cause potential strength or stability related problems. Cross frames are used to reduce these adverse effects since they increase the torsional stiffness of the 
bridge and offer lateral flange support, becoming part of the primary structural system of the bridge.

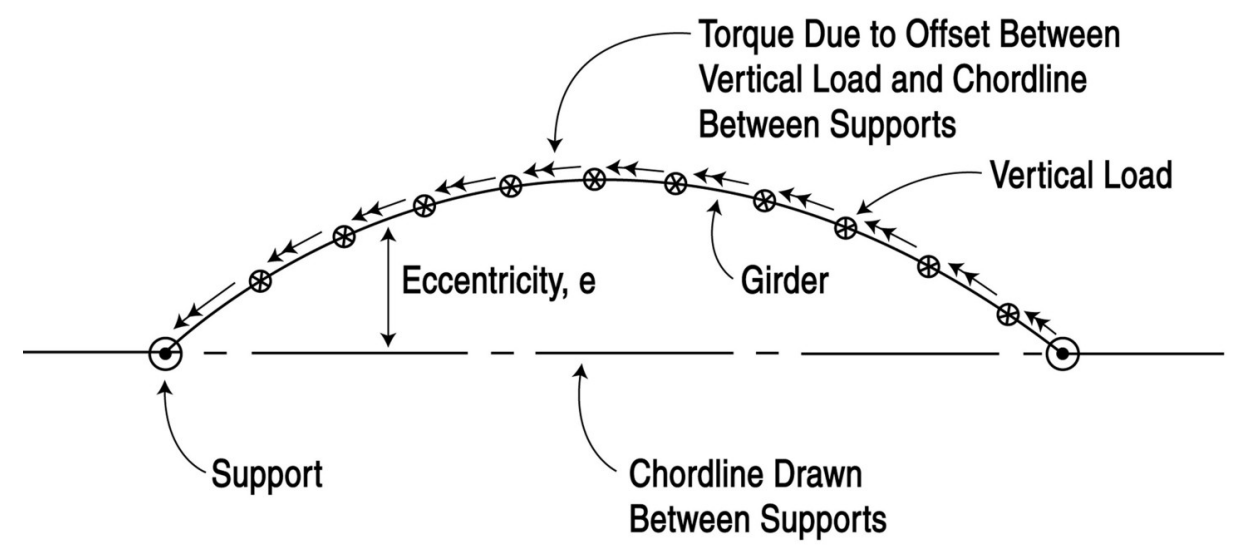

Figure 1-2. Torsional effects produced by curvature (Coletti \& Yadlosky, 2005)

\subsubsection{Skew}

Skewed bridges also exhibit significant levels of LFB at intermediate and end cross-frame locations. For example, Figure 1-3 shows intermediate cross frames oriented perpendicular to the bridge centerline. The cross frames connect adjacent girders with different levels of displacement at the connection points. As a consequence of this differential displacement, internal forces are generated in the cross frames that induce LFB in the girders (Coletti \& Yadlosky, 2005).

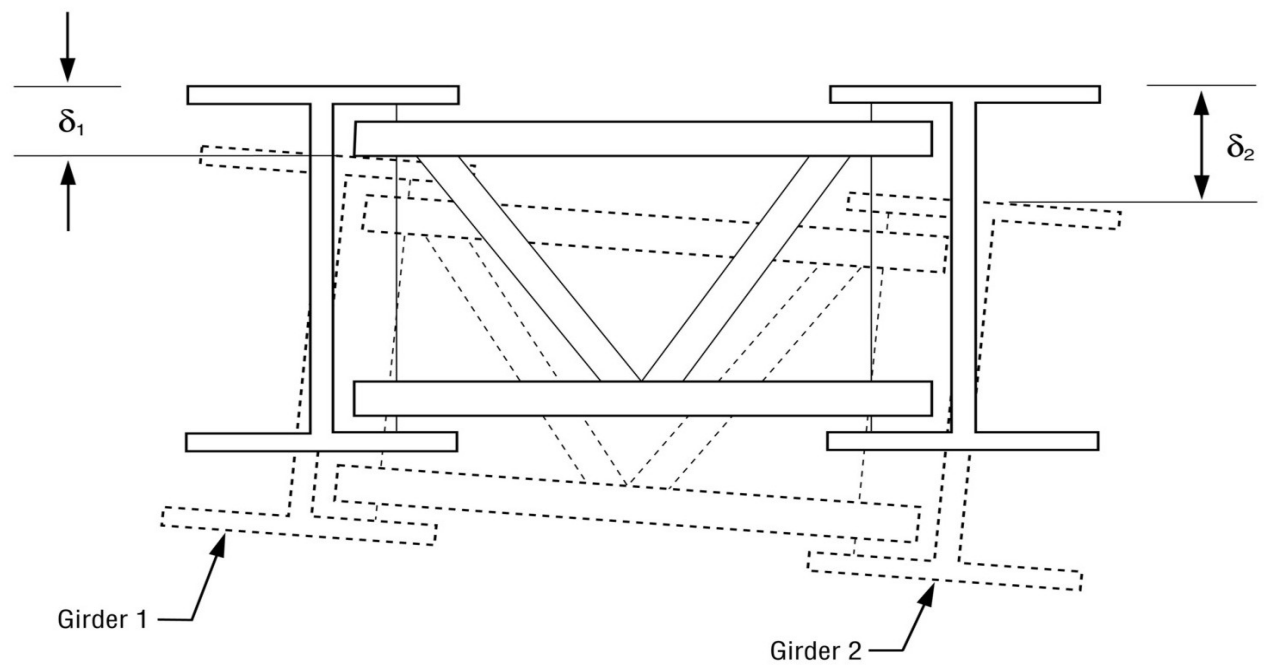

Figure 1-3. Cross frames oriented perpendicular to the girders (Coletti \& Yadlosky, 2005) 

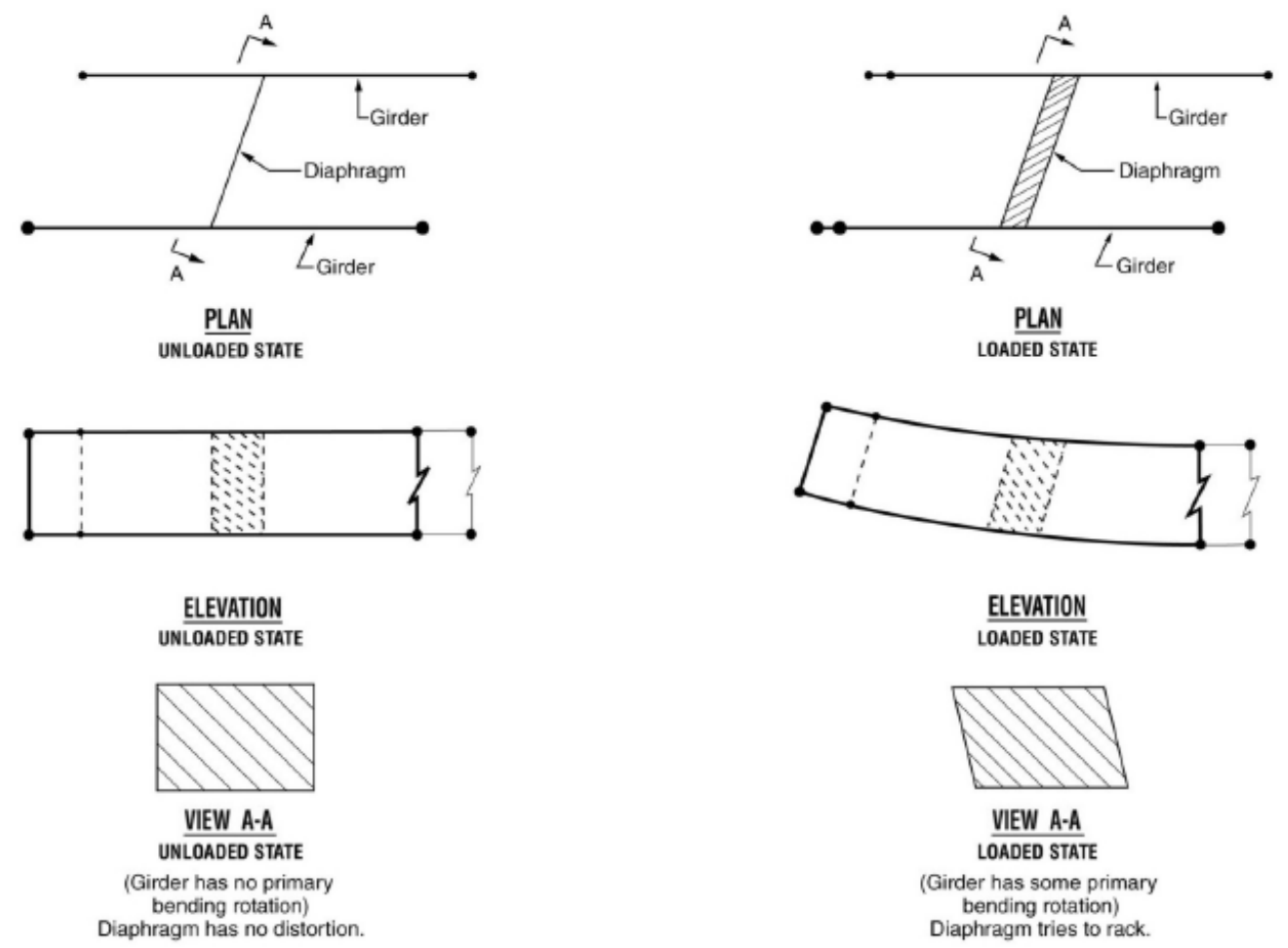

$\frac{\text { ELEVATION }}{\text { LOADED STATE }}$

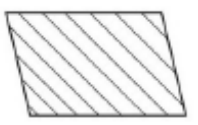

VIEW A-A LOADED STATE

(Girder has some primary bending rotation) Diaphragm tries to rack.

Figure 1-4. Cross frames oriented parallel to the skew (Coletti \& Yadlosky, 2005)

Cross frames oriented parallel to the skew angle also produce LFB since the girders at the cross-frame locations tend to rotate about an axis parallel to the skew (Beckmann \& Medlock, 2005). This rotation and additional deflection produce a lateral displacement between the flanges that distorts the original shape of the cross frames generating additional LFB as shown in Figure 1-4.

\subsubsection{Overhang loads}

Exterior girders are most affected during deck placement by overhang brackets loads. These loads are applied along the girders by deck forming brackets placed every three to four feet, as indicated in Figure 1-5. The overhang loads include the weight of the concrete over the deck overhang length, the overhang deck forms, the concrete finishing machine and its corresponding railing accessories, and a live load component representing the construction workers. Therefore, the exterior girders are subjected to torsional loading effects that produce LFB and web deformations that need to be considered during the design process. 


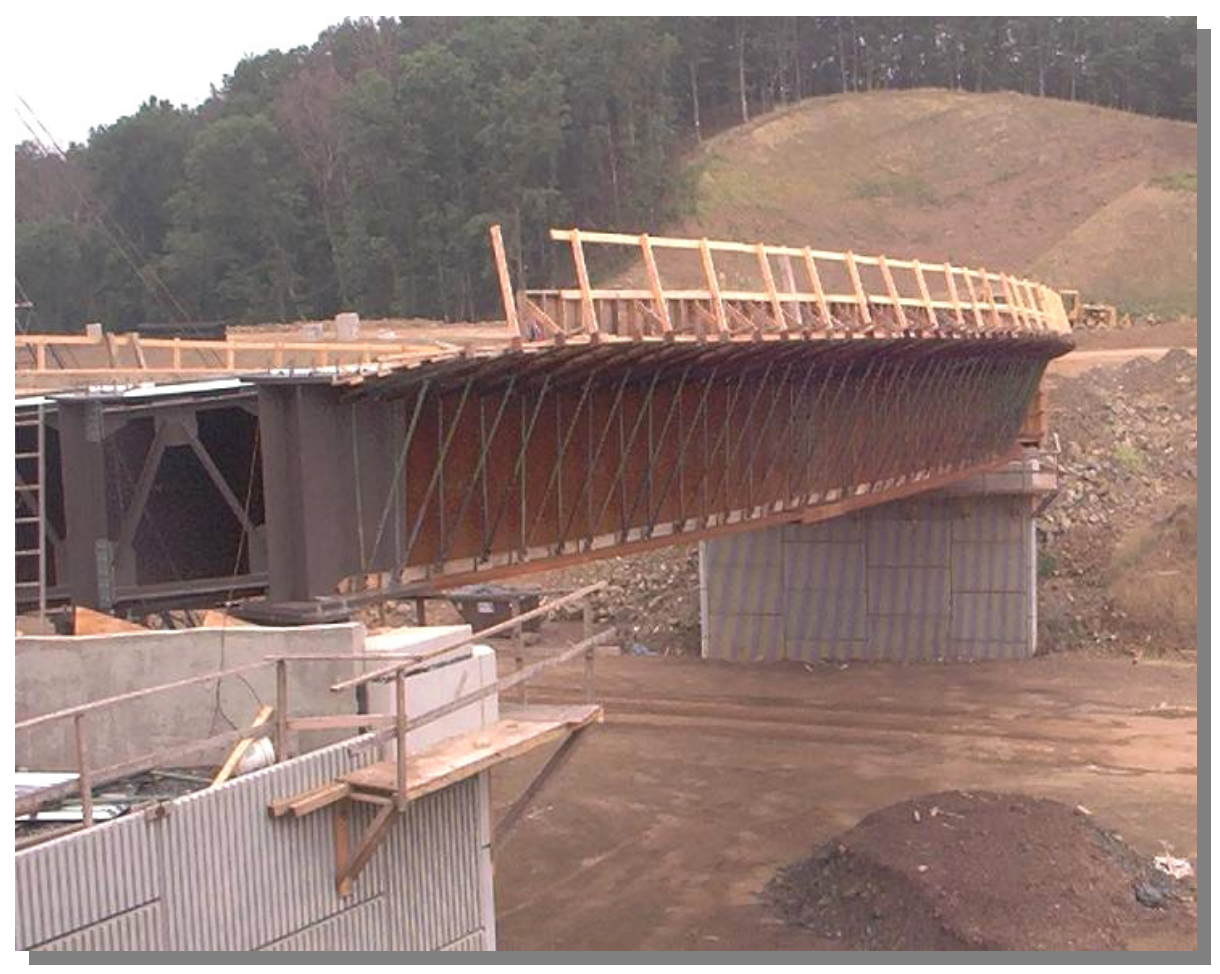

Figure 1-5. Deck forming brackets on exterior girders

\subsubsection{AASHTO approximate formulations for the LFB}

This section includes a description of the simplified approximations given by AASHTO (2007) to estimate the LFB due to the curvature, the overhang loads and the skew in the design of steel I-girder bridges.

\subsubsection{Curvature}

AASHTO (2007) states that curved girders meeting the following requirements can be analyzed as straight girders with the span length equal to the arc span length. The effects of curvature can also be ignored for major-axis bending moments and bending shears in these cases:

- Concentric girders

- Maximum skew angle of bearing lines is $10^{\circ}$

- Similar stiffness of the girders

- The angle subtended by any span is less than 0.06 radians 
However, the effect of curvature must always be considered on the torsional behavior of the girders regardless of the amount of curvature. Therefore, an approximate equation for the lateral flange bending moment due to curvature is recommended in lieu of a refined analysis:

$$
M_{l a t}=\frac{M l^{2}}{N R D}
$$

where $M$ is the major-axis moment, $l$ is the unbraced length, $R$ is the girder radius, $D$ is the web depth and $N$ is a constant taken as either 10 or 12 depending on the desired level of conservatism.

\subsubsection{Overhang loads}

The code provisions require considering the torsional effects due to construction loads on the strength and the stability of girders and cross frames. The corresponding commentary includes some approximate equations to compute the lateral flange moments due to eccentric loads applied on the overhang deck as follows:

$$
\begin{aligned}
& M_{\text {lat }}=\frac{F_{l} L_{b}^{2}}{12} \\
& M_{\text {lat }}=\frac{P_{l} L_{b}}{8}
\end{aligned}
$$

where $F_{l}$ is the statically equivalent uniformly distributed lateral force from the brackets due to the factored loads, $L_{b}$ is the unbraced arc length, and $P_{l}$ is the statically equivalent concentrated lateral bracket force at the middle of the unbraced length.

\subsubsection{Skew}

AASHTO (2007) does not include an approximate equation to account for the skew effect. However, the code provisions recommend using $10 \mathrm{Ksi}$ as a conservative estimation of the total unfactored LFB in bridges with discontinuous cross-frame lines and skew angles exceeding $20^{\circ}$ in lieu of a refined analysis. The total unfactored LFB is distributed between the load types in the same proportion as the unfactored major-axis stresses. 


\subsubsection{AASHTO Flexural Limit States for Constructibility}

After the sources of LFB during the deck-placement sequence are identified, the combined effect of the resulting LFB and the major-axis bending stresses, $f_{l}$ and $f_{b u}$, are evaluated using the flexural limit states for constructibility. These limit states are classified according to the state of stress at the flange and its bracing condition, as follows:

\subsubsection{Discretely braced flanges in compression}

During some phases of the deck placement, the girders work in a non-composite state. Moreover, the most critical condition is exhibited by the top flanges of the positive moment regions which are laterally supported by the cross frames. These compression flanges are usually smaller than the bottom flanges since they are designed for the service loads as composite sections continuously braced by the deck.

The bottom flanges in the negative moment regions are also compression flanges discretely braced by the cross frames. However, this condition is exhibited not only during construction but also during the service life of the bridge. Consequently, an adequate flange size is provided during design.

The limit states that govern the behavior of discretely braced flanges in compression are yielding, ultimate strength and web-bend buckling:

- Yielding limit state: This limit state shall not be checked for sections with slender webs and LFB equal to zero.

$$
f_{b u}+f_{l} \leq \phi_{f} R_{h} F_{y c}
$$

- Ultimate strength: considering lateral torsional buckling (LTB) and flange local buckling (FLB) based limit states.

$$
f_{b u}+\frac{1}{3} f_{l} \leq \phi_{f} F_{n c}
$$

- Web bend-buckling limit state: This limit state shall not be checked for sections with compact or noncompact webs.

$$
f_{b u} \leq \phi_{f} F_{c r w}
$$

where $f_{b u}$ is the flange stress calculated without consideration of LFB, $f_{l}$ is the LFB stress, $\phi_{f}$ is the resistance factor for flexure, $R_{h}$ is the hybrid factor that accounts for the reduced 
contribution of the web to the nominal flexural resistance in sections with a higherstrength steel in the flanges, $F_{y c}$ is the specified minimum yield strength of a compression flange, $F_{n c}$ is the nominal flexural resistance of a compression flange, and $F_{c r w}$ is the nominal bend-buckling resistance for webs.

\subsubsection{Discretely braced flanges in tension}

During construction, the bottom flanges in the positive moment regions and the top flanges in the negative moment regions are examples of tension flanges discretely braced by the cross frames. In the positive moment regions, this bracing condition remains during the service life of the bridge, but it changes in the negative moment regions when the girder starts to act as a composite section.

In tension flanges, only the yielding limit state is considered since stability is not an issue.

- Yielding limit state:

$$
f_{b u}+f_{l} \leq \phi_{f} R_{h} F_{y t}
$$

where $F_{y t}$ is the specified minimum yield strength of a tension flange.

\subsubsection{Continuously braced flanges in tension or compression}

This situation corresponds to the final phase of the deck placement when the girders are composite sections. The continuously braced condition is provided by the deck to the top flanges in compression and tension of the positive and negative moment regions, respectively.

This bracing condition prevents the presence of LFB in the flange. Therefore, the only limit state that needs to be checked is yielding.

- Yielding limit state:

$$
f_{b u} \leq \phi_{f} R_{h} F_{y f}
$$

where $F_{y f}$ is the specified minimum yield strength of the flange.

\subsubsection{Maximum allowable LFB}

In addition to the limit states that govern the interaction between $f_{b u}$ and $f_{l}$, the specifications define a limit for LFB up to where the limit states are satisfactorily valid. 
According to AASHTO (2007), $f_{l}$ corresponds to the largest value of stress due to lateral bending throughout the unbraced length in the flange under consideration. These stresses are calculated based on factored loads and should be taken as positive values in all resistance equations. All flanges are required to meet the following restriction to control the maximum levels of LFB:

$$
f_{l} \leq 0.6 F_{y f}
$$

Furthermore, amplifications factors for $\mathrm{f}_{l}$ are specified in cases where secondorder effects are required to be considered.

Section 1.3 describes the specific goals of the present work along with their motivation and the methods employed to carry them out.

\subsection{Research objectives, motivations and methods}

The general purpose of this research project is to evaluate the levels of LFB in steel I-girder bridges during deck placement in order to state practical, accurate and reliable design recommendations for constructibility. Consequently, this effort comprises basically three primary goals to be accomplished considering only the loading conditions exhibited during the deck placement, as follows:

1. Develop improved equations to estimate the LFB of continuous steel I-girder bridges.

Rationale: As discussed in Section 1.2, skewed and curved steel I-girder bridges exhibit significant levels of LFB due to their geometrical configurations that may cause potential strength and stability problems in both flanges and webs. Particularly, the structure is more susceptible to these problems during the deck placement when the girders act in a non-composite state.

AASHTO Specifications recommend some approximate equations to estimate the torsional effects due to deck-overhang loads and curvature that produce LFB. These approximations are based on simplified models where the unbraced segment of the flange is taken as a fixed-end beam. For skewed bridges, the provisions recommend using $10 \mathrm{Ksi}$ as a conservative estimation of the unfactored LFB in bridges with discontinuous 
cross-frame lines and skew angles exceeding $20^{\circ}$. However, more precise approximations may be defined for each source of LFB if effects such as the continuity over the intermediate supports, the deck-casting sequence and the participation of the whole superstructure are considered in the response.

Previous research efforts (Grubb 1991 \& Roddis et. al. 1999) have been conducted to approximate the LFB in exterior girders during deck placement conditions in straight bridges. Although these works add more complexities to the models, the approximations are still conservative compared to the results obtained in more comprehensive models. In addition, the curvature and the skew effects were not directly addressed in these simplified approximations.

Methods: A parametric study based on the FEA of continuous three-span steel Igirder bridges is employed to accomplish this objective. The varying parameters include the middle span length, the cross-frame spacing, the skew angle, and the angle subtended by the middle span in curved bridges. Additionally, the loading conditions and stiffness vary in the structural analyses according to the deck-placement sequence, since it is assumed that all preceding deck casts are composite for the casts that follow.

2. Identify the parametric variables that most affect the flexural limit states for constructibility, and define the maximum permissible skew angles and curvatures for bridges designed originally as straight.

Rationale: The identification of aspects such as the effect of the curvature and skew in the limit states, the critical stages during the deck-placement sequence, the critical girder sections and the governing limit states allow the designer to gain insight into how the bridge responds structurally to the conditions imposed during the deck placement. Moreover, this information may be also used for preliminary calculations in the planning phase of projects to define the most important design checks to be considered and the sections where they are most critical.

On the other hand, the definition of the maximum permitted skew angles and curvatures for bridges designed originally as straight is intended to simplify the constructibility design process in certain situations. The definition of these limits will allow the engineer to design for constructibility curved or skewed bridges based on their 
straight girder counterparts. Furthermore, no additional constructibility designs or checks would be necessary in case that a bridge designed as straight requires a geometrical modification within the limits established.

Methods: The numerical bending results from the parametric study are used to evaluate the demand-to-capacity ratios of the flexural limit states for constructibility. The variation of these ratios is presented as a function of the girder length to identify the critical sections along the bridge. In addition, the maximum ratios of the positive and negative moment regions are presented in terms of the cross-frame spacing, the deckcasting stage and the governing limit state to facilitate the identification of the relationships among the variables.

To define the skew and curvature limits for a straight bridge, the flexural limit states for constructibility are initially stated using: $i$. the maximum numerical major-axis bending stresses obtained during the parametric study, and ii. the proposed LFB equations that depend directly on the variables required. Then, the maximum skew and curvatures are solved from the governing limit state equation for different cross-frame distances.

3. Optimize the distance between cross frames.

Rationale: During construction, the lateral support of the flanges is only provided by the cross frames, where their spacing represents the unbraced length $(\mathrm{Lb})$ used to compute the bending capacity of the compression flanges. In addition, the LFB depends directly on $\mathrm{Lb}$ since the flanges act as continuous beams supported by the cross frames. Therefore, the selection of the appropriate cross-frame spacing will assure that the structure meets satisfactorily the performance limit states during deck placement using an optimum number of cross frames. However, AASHTO (2007) does not include a specific recommendation for the spacing between cross frames. Consequently, the designer needs to evaluate different cross-frame configurations to select the most appropriate spacing that assures safe conditions especially during construction when the girders act in a non-composite state. Therefore, the achievement of this objective by defining the maximum allowable cross-frame spacing that meets satisfactorily the flexural limit states for constructibility will simplify considerably the design process. 
Methods: This goal is accomplished by conducting reliability analyses of the flexural limit states for constructibility that are directly affected by the cross-frame spacing. This structural reliability problem was solved using a Monte Carlo Simulation which is a simulation technique that numerically simulates the behavior of the random variables and limit states involved in the problem. The probabilistic characteristics of the random variables were adopted from the research works carried out to calibrate the current AASHTO Specifications. The cross-frame spacing is presented in an appropriate format based on the maximum tolerated probability of failure of the considered limit states.

\subsection{Scope of research}

The focus of this research is to evaluate the levels of LFB in steel I-girder bridges during deck placement and the scope of this project consists of four major components: definition of the parametric study, approximation of the LFB effects, evaluation of the flexural limit states for constructibility and optimization of the cross-frame spacing.

A comprehensive parametric study is conducted using finite element (FE) models of steel I-girder bridges. Some characteristics are set as fixed such as the number of girders, the number of spans, the girder spacing, the overhang length, the concrete deck thickness, the material specifications and the ratio of the end-span to the middle-span lengths. The varying parameters include the middle span length, the cross-frame spacing, the skew angle, and the angle subtended by the middle span in curved bridges. A deckplacement sequence is defined for all models and the corresponding changes in the structural stiffness during the various stages are considered. For that reason, it is assumed in the analyses that all preceding deck casts are composite for the casts that follow. The LFB effects due to overhang loads, skew and curvature are approximated based on the numerical bending stresses obtained from this parametric study. Comparisons with the approximations recommended by AASHTO are also established.

The flexural limit states for constructibility constitute the criteria used to evaluate the structural performance of the bridges considered in this work. These limit states are computed using the numerical bending results obtained from the parametric study to 
identify the impact of the parametric variables in the design. The limit states and the proposed LFB equations are also used to determine the maximum available skew and curvatures of bridges originally designed using a straight girder formulation.

The final component of this work is the optimization of the distance between cross frames which simplifies the design process of steel I-girder bridges. A reliability analysis is performed using a Monte Carlo Simulation to generate the probabilistic distribution of the random variables and to evaluate the reliability of the flexural limit states for constructibility. The parameters required to define the load and resistance structural models that describe this reliability problem were adopted from previous research efforts intended to calibrate the AASHTO Specifications. The optimum crossframe spacing is selected from a curve in terms of the probability of failure or reliability index of the considered limit states.

\subsection{Dissertation Organization}

The body of this dissertation consists of eight chapters. This first chapter, Introduction, provides general background information of the research work, discusses the need for this project, highlights the main research objectives and describes the methods employed to accomplish them.

A literature review of the research efforts related to the present work is included in Chapter 2. This chapter is organized into five sections as follows: (1) a description of the studies considering LFB, constructibility issues and code specifications in curved steel I-girder bridges; (2) an overview of work addressing the behavior of skewed steel Igirder bridges; (3) a presentation of the most important works carried out to develop design guides for LFB in exterior girders due to deck overhang loads during construction; (4) an overview of the development of the bridge specifications regarding the LFB effects

and constructibility issues; and (5) a description of some relevant studies related to structural reliability and code development procedures.

Chapter 3 discusses the principal modeling procedures employed to conduct FEA in this project. A description of the material models for steel and concrete and their corresponding stress-strain relationships is initially presented. Then, the finite elements 
and techniques required to model the bridge behavior during deck placement conditions are described. Benchmarking of these techniques is also presented. Finally, a description of the analysis methodology employed in the FE models is given.

The parametric study used to investigate the effects of the deck-placement process on the LFB of steel I-girder bridges is described in Chapter 4. The parametric study is defined by initially selecting the parameters considered to be most significant; describing the loads that represent the conditions during the casting sequence; performing the structural design of the bridge configurations obtained from the parametric evaluation, and finally defining the FE models and the corresponding analyses for each parametric configuration that represent properly the deck casting sequence in the bridges.

Chapter 5 presents the methods used to approximate the LFB in straight, skewed and curved steel I-girder bridges due to construction loads during deck placement based on the results obtained from the parametric study described in Chapter 4. The major-axis bending stresses are also analyzed, where the principal contribution is made for curved bridges since it is shown that the torsional effects do not affect the vertical bending response in straight and skewed bridges.

In Chapter 6, the flexural limit states for constructibility are evaluated according to AASHTO Specifications for the parametric bridges analyzed in this work. First, the major-axis bending and the LFB stresses obtained from FEA are used to evaluate the demand-to-capacity ratios of the flexural limit states for constructibility. This evaluation allows identifying the critical sections along the bridge and the effects of the cross-frame spacing and the deck-placement sequence in the governing limit states. Second, the maximum allowable skews and curvatures are computed for bridges designed originally as straight. The flexural limit states for constructibility constitute the criteria used to achieve this goal. The major-axis bending stresses are taken directly from FEA and the LFB stresses are estimated using the approximate equations proposed in Chapter 5.

A reliability analysis was proposed in this work and performed in Chapter 7 to develop a practical method to select the cross-frame spacing for deck placement conditions considering the flexural limit states for constructibility that are affected by the cross-frame spacing. Initially, a general description of a structural reliability study is given along with the limit states considered. Then, the adopted structural loading and 
resistance models are described and their corresponding probabilistic characteristics are presented. Finally, a Monte Carlo Simulation is performed for straight, skewed and curved steel I-girder bridges generating fragility curves that allow identification of the maximum cross-frame spacing for deck-placement conditions according to the maximum tolerated level of risk.

Chapter 8, Summary, Conclusions, and Recommendations, summarizes the results of this work and provides recommendations for future research in this area.

Finally, a list of cited references is included along with six Appendices of figures. 


\section{Chapter 2: $\quad$ Literature Review}

As discussed in Chapter 1, the primary objective of the present research work is to study the levels of LFB in steel I-girder bridges during deck placement. Therefore, a literature review of the research efforts related to this objective is included in this chapter. The literature review is organized into five sections: Section 2.1, Curved Steel I-girder Bridges, describes the studies considering LFB, constructibility issues and code specifications; Section 2.2, Skewed Steel I-girder Bridges, reviews the works studying the effect of the skew on the bridge response; Section 2.3, Overhang Load Design, presents the most important works carried out to develop design guides for the LFB in exterior girders subjected to deck overhang loads during construction; Section 2.4, Lateral Flange Bending Design, describes the LFB and constructibility approaches according to the design specifications; and Section 2.5, Reliability Analysis, cites some relevant studies related to structural reliability and code development procedures.

\subsection{Curved Steel I-girder Bridges}

Since the 1960s, several studies have been conducted to investigate different typical aspects of the structural behavior on curved girders such as: the strength capacity under vertical loads (Jung et. al., 2005; Davidson, Balance \& Yoo, 2000; Thevendran et. al., 2000; Thevendran et. al., 1999; Shanmugan, 1995); modeling strategies (Chang et. al., 2005); analysis methods (Nevling, Laman \& Linzell, 2006; Zureick \& Naqib, 1999); load distribution (Samaan, Sennah \& Kennedy, 2005; Zhang, Huang \& Wang, 2005; DePolo \& Linzell, 2005; McElwain \& Laman, 2000; Brokenbrough, 1986; Heins \& Jin, 1984); dynamic response (Tilley, Barton \& Gomez, 2006; Maneetes \& Linzell, 2003); stability (Davidson, Balance \& Yoo, 1999; Davidson \& Yoo, 1999); etc. However, the present work deals directly with the LFB and constructibility issues, in particular during deck placement. Therefore, the studies closely related to the objectives of this work will be presented in more detail in the subsequent sections: Section 2.1.1 contains the research

work related to the LFB on curved girders, Section 2.1.2 describes studies addressing 
constructibility issues, and Section 2.1.3 includes an overview of the specifications for curved girders since detailed design aspects are considered in Section 2.4.

\subsubsection{Lateral Flange Bending}

The New York State Department of Transportation published a series of research reports (Beal, 1978; Kissane and Beal, 1972; Beal and Kissane, 1971) based on a research study started in 1967 to evaluate the behavior of horizontally curved girder bridges and to establish appropriate design procedures. The project involved field testing of four existing bridges and theoretical analyses using planar grid models based on the stiffness method. In particular, Kissane (1978) published a report where experimental and analytical results from two small scale models were used to establish an empirical formulation of the LFB moments in simply supported and uniformly loaded curved girders. As a result of this effort, an empirical equation for the LFB moment on curved girders was proposed:

$$
\begin{gathered}
M_{\text {lat }}=\frac{w l_{d}^{2}}{12} \\
w=\frac{M_{n b}}{R d}
\end{gathered}
$$

where $l_{d}$ is the diaphragm spacing, $w$ is the equivalent distributed lateral load, $M_{n b}$ is the maximum normal bending moment, $R$ is the radius of curvature, and $d$ is the distance between flange centroids.

The factor 1/12 in Equation 2-1 was taken as 1/10 in the specifications valid at the time of the research project. This value was selected as a compromise between the extreme factors given for fixed (1/12) and simply-supported beams (1/8). However, the research results showed that the LFB behavior is similar to the main bending stresses exhibited by continuous girders. Currently, the specifications still recommend this approximation to take into account the curvature but using a divisor factor between 10 and 12, depending on the desired level of conservatism.

Schilling (1996) presented a series of yield-interaction relationships for compact, compact-flange, and noncompact sections under combined vertical and lateral moments to define the bending capacity of curved girders. The sections had to satisfy web 
slenderness and compression-flange bracing limits. The proposed interaction equations allow defining the vertical bending capacity based on a steel section with reduced flanges, where the reduced flange widths depend on the lateral moment. This philosophy is the basis of the "one-third rule" equations which are the format of the flexural state limits in current design specifications, as described later in Section 2.4.5.

Davidson and Yoo (1996) numerically studied the effect of curvature on the local buckling of curved girder compression flanges and proposed a practical reduction factor of the buckling strength in straight girders as a function of the warping-to-bending stress ratio. Additionally, based on the numerical results, a design equation was derived for the maximum width-thickness ratio of compression flanges.

Davidson, Keller and Yoo (1996) carried out a parametric study using detailed FE models of curved steel bridges connected by cross frames. The results were compared to straight girder systems concluding that parameters such as curvature level, span length and flange width were the most significant factors affecting the structural behavior of curved girders. Approximate equations to estimate the adequate cross-frame spacing and predict the warping-to-bending stress ratio were developed based on a nonlinear statistical regression. Davidson and Yoo (2000 \& 2003) verified the accuracy of these approximations using detailed finite-element models of the curved three-girder test frame which was constructed under the Curved Steel Bridge Research Project experimental phase (Zureick et. al., 2000; Duwadi et. al., 2000) to evaluate the curvature effects on the bending strength of curved I-girders.

Yoo and Davidson (1997) presented a complement of the work of Schilling (1996) developing yield-interaction equations for singly symmetric noncomposite and composite sections in both positive and negative bending regions. Complete plastification for compact sections, partial yield penetration for compact-flange sections and initial yield at the flange tip for noncompact sections were considered as the limit states for the study. Analysis of the results indicated that the denominator of the lateral moment equation (see Eq. 2-1) should be taken as 14 instead of 12 or 10.

White, Zureick and Jung (2001) developed a unified approach for the flexural design of both straight and curved I-girder bridges that was implemented in AASHTO (2004) Specifications. The flexural design equations, also called the "one-third rule" 
equations, exhibit a favorable characteristic with respect to past approximations, since they handle in a practical manner combined vertical and lateral bending from any load source regardless of the girder type: curved or straight (see Section 2.4.5).

\subsubsection{Constructibility}

Grubb, Yadlosky and Duwadi (1996) described different issues that arise during the construction of horizontally curved steel bridges. In fact, according to the authors, most of the problems exhibited by curved girders are related to the fabrication and assembly procedures, since the designer does not account for the deformations presented during construction which are more complex than those experienced by straight girders. In particular, during deck placement, the non-composite girders are subjected to significant demands from dead loads that include: member self weight, weight of the wet concrete slab, miscellaneous steel, and construction equipment. In this state, it is common to have more than one-half of the web depth in compression since the top flanges are usually smaller than the bottom flanges in positive-moment regions generating potential stability problems. The deck-casting sequence is also described as an important aspect to consider during construction since stiffness changes affect the deflection profile during and after the deck casting. It is recommended to cast the deck in positive-moment zones first to minimize the slab cracking and maintain the casting sequence as symmetrical as possible to avoid unbalanced loading and differential deflections.

Galambos et. al. (2000) studied the behavior of the steel superstructure of a curved steel I-girder bridge during all phases of construction. Comparisons of the fieldmeasured and elastic numerical stresses yielded good correlation for the main bending stresses and deflections. However, the warping and minor-axis bending effects exhibited less predictable behavior. During deck placement, the stress correlation improved as the casting progressed since local fit-up stresses dissipated under additional loading. Finally, the authors recognized the importance of considering the composite behavior on negative-moment regions to simulate the live load stresses.

Linzell, Leon and Zureick (2004) assessed the ability of numerical procedures for predicting response during erection. The work is based on experimental results of a full- 
scale curved steel bridge structure that was part of the Federal Highway Administration project intended to develop rational design guidelines. The comparisons showed good predictions by the finite-element models, where the main differences were attributed to construction effects. The test results indicated that providing minimal radial restraint for curved girders during construction has beneficial effects on the structural behavior.

Domalik, Shura and Linzell (2005) presented the issues raised during the design and construction of a two-span curved steel I-girder bridge. A global twisting of the superstructure was produced due to the unbalanced distribution load of the unequal spans. Additionally, the authors recommend a technique to include into the girder design the additional lateral bending moments produced by the out-of-plumb effects. This technique computes the rotation of the girder based on the girder depth and deflection; then, the vertical bending moment is resolved into a lateral and an aligned bending component with respect to the out-of-plumb web. Shura and Linzell (2006) published additional results about the field measurements during the superstructure erection and the deck placement. These results indicated that two-dimensional grillage models were not able to reproduce flange stresses and warping of the girders during erection. The authors proposed an exterior-to-interior single girder erection sequence to reduce dead load stresses and deflections.

Chavel and Earls (2006a \& 2006b) evaluated the erection problems encountered in a curved steel I-girder bridge using finite-element analyses. Most of the erection difficulties were attributed to an inconsistency in the detailing and fabrication of the structure, since the girders and cross frames were detailed for a no-load condition different than the one exhibited by the bridge during construction. As a consequence, elements like the cross frames may be too short or too long which generates additional stresses and deformations into the girders and increases the costs. The authors recommend the use of an appropriate temporary support system to resemble the no-load condition assumed in the design process.

Bell and Linzell (2007) studied a curved six-span steel I-girder bridge which was monitored during corrective procedures intended to counteract some erection problems. The results indicated that the erection of paired girders reduces radial and vertical deformations. Additionally, providing shoring towers at span quarters and the use of 
lateral bracing in exterior girder spaces were proven to be effective means of reducing levels of deflection.

Howell and Earls (2007) evaluated the effect of the web plumbness during the erection of the steel superstructure. Finite-element models were used to study the effect that the web out-of-plumbness has on flange tip stresses, vertical and lateral deflections, cross-sectional distortion and cross-frame demands. The results showed that the flange tip stresses are significantly influenced by the web plumbness exhibiting stress changes up to $20 \%$ with respect to calculations that ignore this effect. The vertical deflections were not altered by the web plumbness effects. However, the lateral deflections show increases as much as $250 \%$ at the midspan of the outer girder. Finally, the cross-frame forces were also sensitive to increasing out of plumbness where a proportional effect is typically exhibited.

\subsubsection{Specifications - background}

The CURT project (Consortium of University Research Teams) in 1969 was the first major work led to create some design provisions for curved girder bridges based on experimental and analytical research (Linzell et. al., 2004). As a result of this effort, specifications based on the allowable-stress-design format (ASD) were proposed in 1976. However, the load factor design (LFD) was adopted by AASHTO in 1980 and added to the previous ASD provisions, becoming the first version of the AASHTO Guide Specifications for Horizontally Curved Highway Bridges. A second edition was issued in 1993. However, the 1980-original provisions were generally kept unchanged in its primary content. Therefore, as a result of the National Cooperative Highway Research Program (NCHRP) 12-38 Project, an updated version was published in 2003 using the LFD format (AASHTO, 2003). These specifications made significant improvements to the design guidelines of the 1993 Guide Specs, since they identified the need to correlate the LFB with the vertical bending (see Section 2.4.4).

Finally, AASHTO issued the LRFD Bridge Design Specifications in 2004 (see Section 2.4.5) as part of the work done during the NCHRP 12-52 project, intended to develop provisions for curved bridges in the AASHTO load and resistance factor design format (LRFD). The primary objective of this work was to incorporate the design 
provisions for curved bridges into the then-existing specifications for straight bridges (White \& Grubb, 2004). These provisions were initially published in 2004 using statistically calibrated data for straight girders (AASHTO, 2004). However, the provisions corresponding to curved girders were published in the 2006 interim to the AASHTO LRFD specifications. The NCHRP 12-52 project concluded from the statistical calibration of the load and resistance factors, that the LRFD factors for straight girders were still valid for curved girders (NCHRP, 2006b). The important achievement of these specifications lays on the fact of handling a combined solicitation of LFB and major-axis bending, independent of the load source and the type of girder (curved or straight).

\subsection{Skewed Steel I-girder Bridges}

It is a common practice to ignore the skew effect in the structural behavior of skewed bridges which are designed mostly as general tangent structures. As a consequence, there are far less studies addressing the skew effect than works focused on the curvature effect. Some of these research efforts deal with both effects simultaneously (Ozgur \& White, 2007; Coletti \& Yadlosky, 2005), and some others evaluate specific aspects of skewed bridges such as load distribution (Huang, Shenton \& Chajes, 2004; Khaloo \& Mirzabozorg, 2003), cross-frame systems (Herman et. al., 2005), etc. Construction issues are addressed in some specific works described below.

Norton, Linzell and Laman (2003) compared the field response of a skewed bridge during deck placement to analytical model predictions. Specifically, the study evaluated the effect of pouring the concrete both perpendicular to the centerline of the bridge and parallel to the abutments. The authors concluded that the first deck placement procedure exhibits high support reactions and displacements during intermediate construction stages, while the second technique presents a more homogeneous response.

Beckmann and Medlock (2005) described rotation and deflection issues that may affect the structural behavior of highly skewed steel bridges. In particular, they mention the rotation normal to the skewed supports that the girders exhibit when the noncomposite loads are applied, which increases the demand on the cross frames. This 
rotation displaces the top flange transversely from the bottom flange and causes the outof-plumb effect on the web. On the other hand, the principal issue for intermediate cross frames oriented perpendicular to the girders is related to the differential deflections. These problems require an accurate detailing work of the cross frames to meet the noload fit, steel dead-load fit or the full dead-load fit, and avoid over demands of stresses and deformations.

Choo et. al. (2005) investigated the response of a continuous skewed bridge with High Performance Steel (HPS) under the effects of the deck placement during construction. Finite-element models were calibrated using field measurements that were highly sensitive to the temperature changes. The authors also evaluated the effects of placing the concrete both perpendicular to the girders and parallel to the skew. The results indicated that placing the concrete parallel to the supports reduces the differential deflections and stresses across the bridge superstructure. This beneficial effect is more evident in simply supported girders than in girders under continuous support conditions.

\subsection{Overhang Load Design}

Some simplified procedures have been suggested to design exterior girders for the effects caused by the concrete deck overhang loads: LFB and web distortion. The most significant design guides were developed by the American Institute of the Steel Construction (AISC) (Grubb, 1991) and the Kansas Department of Transportation (KDoT) (Roddis, Kriesten \& Liu, 1999).

\subsubsection{AISC Approach}

In this procedure, it is assumed that the cross frames act as torsionally rigid supports that prevent out-of-plane warping. Therefore, exterior-girder flanges, which are the resister elements of the torsion imposed by the overhang loads, are taken as a laterally loaded fixed-end beam with a span length equal to the distance between the cross frames, as shown in Figure 2-1 for the bottom flange.

The design guide includes a simplified analysis where tabulated coefficients in terms of the overhang length and the girder height are multiplied by the square of the 
cross-frame spacing to obtain the maximum fixed-end moment at the cross-frame locations $\left(M_{f w}\right)$. The maximum moment in-between the cross-frame spacing $\left(M_{+}\right)$is calculated multiplying the corresponding $M_{f w}$ by 0.53 and 0.60 for the uniform overhang loads (slab, overhang form and walkway live load) and the finishing machine loads, respectively.

For the top flanges on exterior girders, the guide recommends to use rebar ties attached to the stud shear connectors at the third points of the cross-frame spacing. This configuration reduces the lateral moment and increases the buckling strength of the top flange. Therefore, top flanges that meet this requirement and are part of cross frames where their distances do not exceed 25 feet, are assumed to control permanent deformations caused by yielding and ensure adequate ultimate strength without requiring an explicit checking procedure.
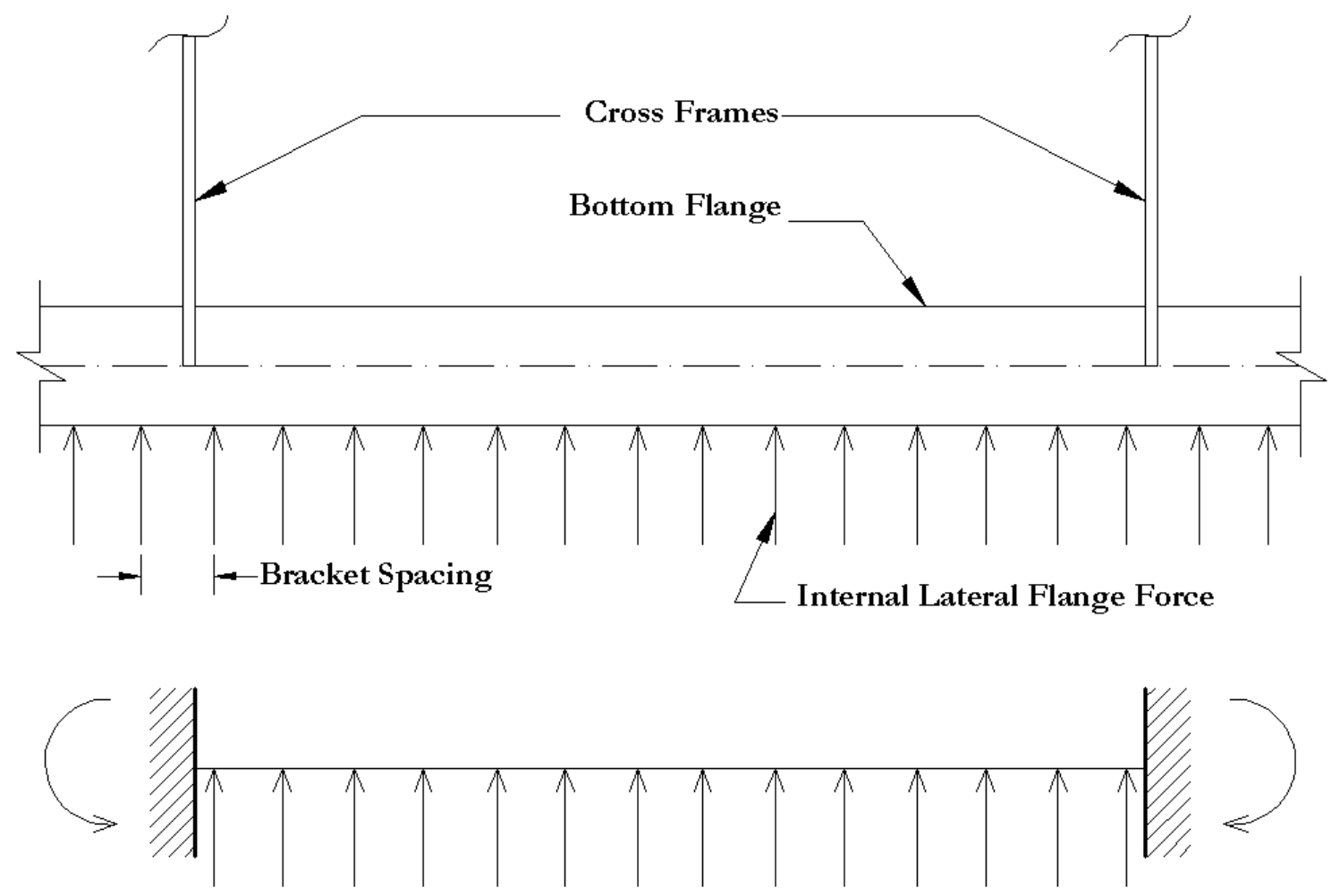

Figure 2-1. Plan view of bottom flange: a. Original b. Equivalent approximation. 
On the other hand, the following limit states are defined for the bottom flanges:

- Strength:

1. Yielding Limit State: To control permanent deformations of tension and compression flanges at and between cross frames.

$$
f_{b u}+f_{l} \leq F_{y}
$$

where $f_{b u}$ is the maximum factored normal bending stresses, $f_{l}$ is the LFB stresses, and $F_{y}$ is the flange yield stress.

2. Ultimate strength: Interaction equation of axial and bending effects for compression flanges in between the cross frames.

$\frac{P_{u}}{0.85 A_{s} F_{c r}}+\frac{M_{l} C_{m}}{M_{u}\left(1-\frac{P_{u}}{A_{s} F_{e}}\right)} \leq 1.0$

where $P_{u}$ is the factored applied axial force in the flange, $A_{s}$ is the flange cross-sectional area, $F_{c r}$ is the flange critical buckling stress, $M_{l}$ is the applied lateral flange moment, $C_{m}$ is the equivalent moment factor to account for the shape of the applied moment diagram, $M_{u}$ is the maximum moment capacity of the flange, and $F_{e}$ is the Euler buckling stress of the flange in the plane of bending.

- Stability:

1. Web distortion: To control potential web instabilities the guide suggests that the cantilever brackets should be supported within a maximum of six inches from the bottom flange. This requirement is intended to prevent a direct contact of the brackets and the web in a compression zone.

\subsubsection{KDoT Approach}

The University of Kansas and the KDoT developed a software program called “Torsional Analysis for Exterior Girders - TAEG" based on a research project including field tests and numerical analyses. This approximation overcomes some inaccurate assumptions of the AISC approach such as the totally fixed-support condition given by the cross frames and the worst case midspan-to-fixed-end-moment ratios ( 0.53 for distributed loads and 0.60 for screed loads). 
The program has a step-by-step procedure to design overhang and cross-frame dimensions, cross-frame distances and to check falsework schemes. The following basic assumptions were adopted in the KDoT approach according to the results obtained in the research work:

- The flange flexure analogy is valid to represent the torsional effects.

- A simplified flange model with three continuous spans with fixed ends is sufficient to achieve good accuracy compared to the AISC simple-span assumption.

- The lateral support in the bottom flange needs to be considered and varies with the type of support: cross frames or diaphragms.

- The effect of temporary supports needs to be considered.

- The dynamic effects due to the movement of the motor carriage are negligible.

- Impact loads during the deck placement are also considered insignificant.

Three basic load schemes are considered along the three-span beam to define the maximum demands: $i$. dead load, live construction load and concrete for the initial span of the beam; $i$. dead load, live construction load, concrete and the finishing machine for the middle span; and iii. dead load and live construction load for the remaining span. The position of the wheel loads in $i i$ is varied within the second span of the continuous girder to identify the critical location that generates the maximum effects. All the loads are simulated as distributed including the wheel loads applied over the width of the finishing machine stand. The cross frames and diaphragms are modeled as pinned supports for the top flange. For the bottom flange, the cross frames are also considered as pinned supports while the diaphragms as well as the temporary supports are modeled with equivalent springs.

The principal calculations that the program performs based on the three-span continuous beam model and the stiffness method are:

- Maximum stresses in the flanges

- Ultimate strength check for the top flange

- Deflection of the flanges

- Rotation and deflection of the girder at the screed rail

- Internal forces of the overhang brackets

- Support reactions 
- Stresses in the diaphragms

- The bolt load and critical bolt load in case of bolted connections between the girder and diaphragms

In comparison to the AISC approach, the stress results obtained with the KDoT program are approximately $20 \%$ higher for the positive moment regions and $20 \%$ lower for the negative moment zones. Thus, an economical benefit is obtained using the program since usually the negative moments govern the design.

\subsection{Lateral Flange Bending Design}

Section 1.2, Lateral Flange Bending in Steel I-girder Bridges, described the physical conditions that produce LFB in curved and skewed bridges and the interaction of these stresses with the normal bending effects produced by vertical loads. Given that the LFB behavior has been defined, it is necessary to establish how the design specifications have addressed this particular issue for steel I-girder sections.

\subsubsection{Standard Specifications for Highway Bridges - $15^{\text {th }}$ Ed., AASHTO (1992):}

These specifications consider lateral bending effects due to wind loading in order to only evaluate the maximum forces generated in diaphragms and cross frames. The maximum induced stress $(F)$ in the bottom flange of the girder when the top flanges are continuously supported is defined as follows:

$$
\begin{aligned}
& F=R F_{c b} \\
& R=(0.2272 L-11) S_{d}^{-2 / 3} \text { BLB } \\
& R=(0.059 L-0.64) S_{d}^{-1 / 2} \text { No BLB } \\
& F_{c b}=\frac{72 M_{c b}}{t_{f} b_{f}^{2}}(\mathrm{psi}) ; \quad M_{c b}=\frac{W S_{d}^{2}}{12}(\mathrm{lb}-\mathrm{ft})
\end{aligned}
$$

where $R$ is the factor to account for the effect of the bottom lateral bracing (BLB), $F_{c b}$ is the flange bending stress produced by wind loading, $W$ is the wind loading along the 
exterior flange $(\mathrm{lb} / \mathrm{ft}), S_{d}$ is the diaphragm spacing $(\mathrm{ft}), \quad L$ is the span length $(\mathrm{ft}), t_{f}$ is the flange thickness (in), and $b_{f}$ is the flange width (in).

In cases when the top flanges are not continuously supported, the code recommends performing an explicit analysis of the structural elements to identify the lateral bending stresses.

\subsubsection{LRFD Bridge Design Specifications, SI Units - $1^{\text {st }}$ Ed., AASHTO} (1994)

Wind effects on girder flanges are considered in these specifications for composite sections classified either as compact or non-compact. For compact sections, these lateral effects are assumed to be carried by a reduced width at each edge of the bottom flange (Schilling, 1996), defined as:

$$
b_{w}=\frac{b_{f}-\sqrt{b_{f}^{2}-\frac{4 M_{w}}{t_{f} F_{y b}}}}{2} \leq \frac{b_{f}}{2}
$$

where $b_{f}$ is the bottom flange width $(\mathrm{mm}), t_{f}$ is the bottom flange thickness $(\mathrm{mm}), F_{y b}$ is the specified minimum yield strength of the bottom flange (MPa), and $M_{w}$ is the maximum lateral moment in the bottom flange due to factored wind loading (N-mm). The remaining flange width is used to compute the composite girder capacity for the vertical loads involved in the corresponding load combination.

In the case of non-compact sections, an interaction of stresses has to be checked for the bottom flange as follows:

$$
\begin{aligned}
& \left(F_{u}+F_{w}\right) \leq F_{r} \\
& F_{w}=\frac{6 M_{w}}{t_{f} b_{f}^{2}}
\end{aligned}
$$

where $F_{u}$ is the flexural stress in the bottom flange due to the factored loads other than wind $(\mathrm{MPa}), F_{w}$ is the flexural stress at the edges of the bottom flange due to the factored wind loading $(\mathrm{MPa}), F_{r}$ is the factored flexural resistance according to the these specifications ( $\mathrm{MPa})$, and $M_{w}$ is the maximum lateral moment in the bottom flange due to factored wind loading (N-mm). 
The construction issues are addressed by selecting the appropriate construction loads to compute the strength and the stability during construction. In particular, it is recommended to consider changes in loads, stiffness and bracing during the deck pouring sequence. The corresponding commentary gives particular attention to the strength and stability problems produced by the cantilever forming brackets. The nominal flexural resistance is defined by the yield moment capacity of the non-composite section, when it meets certain slenderness requirements. Otherwise, the lateral-torsional buckling capacity for non-composite and non-compact sections is applied. However, there is a lack of explicit bending limit states during construction that permit the addition of the LFB effects in a practical approach, such as that required for a design code format.

\subsubsection{LRFD Bridge Design Specifications, Customary U.S. Units $-2^{\text {nd }}$ Ed., AASHTO (1998)}

In these specifications, the design guidelines given for wind loading conditions remain similar as those described in Section 2.4.2. The constructibility incorporates a new requirement to limit the maximum compressive flexural stress in the web, resulting from the various stages of the deck placement sequence, to the theoretical elastic bendbuckling stress of the web. Nevertheless, as explained in Section 2.4.2, there is still not an explicit definition of the flexural limit states with specific restrictions on the LFB effects.

\subsubsection{Horizontally Curved Steel Girder Highway Bridges, AASHTO (2003)}

AASHTO (2003) introduces formally the definition and the notation of the LFB as follows:

- Lateral Flange Bending (LFB) - Flexural action in the plane of the flange with respect to the vertical axis through the flange. LFB may be due to lateral loads applied to the flange and/or nonuniform torsion in the member. In these provisions LFB moments refer to those at brace points.

- $f_{i}$ : calculated total factored LFB stress at the section under consideration.

- $M_{\text {lat: }}$ LFB moment. 
The code allows the use of a simplified line girder analysis, when the plan configuration of the bridge meets certain geometrical restrictions. In that case, the lateral bending moment in I-girder flanges due to curvature is defined as:

$$
M_{\text {lat }}=\frac{6 M l^{2}}{5 R D}
$$

where $M$ is the vertical bending moment (K-ft), $l$ is the unbraced arc length ( $\mathrm{ft}), R$ is the girder radius ( $\mathrm{ft}$ ), and $D$ is the web depth (in).

Additionally, these specifications limit the maximum values of $f_{l}$, recognizing the adverse impact that these stresses may have on the general flange bending behavior of curved girders, according to the following equations:

$$
\begin{aligned}
& f_{l} \leq 0.5 F_{y} \\
& \left|f_{l} / f_{b}\right| \leq 0.5
\end{aligned}
$$

where $F_{y}$ is the specified minimum yield stress (Ksi) and $f_{b}$ is the calculated factored average flange stress at the section under consideration (Ksi). Equation 2-13 is valid when $f_{b}$ is greater than or equal to the smaller of $0.33 F_{y}$ or $17 \mathrm{ksi}$.

The critical average flange stress for partially braced flanges, $F_{c r}$, is taken as the smaller of $F_{c r l}$ and $F_{c r 2}$ :

$$
\begin{aligned}
& F_{c r 1}=F_{b s} \rho_{b} \rho_{w} \\
& F_{c r 2}=F_{y}-\frac{\left|f_{l}\right|}{K}
\end{aligned}
$$

where $F_{b s}$ is the critical average flange stress of an equivalent straight girder flange (Ksi); $\rho_{b}$ and $\rho_{w}$ are the strength reduction factors due to curvature effects expressed in terms of the $l / R$ ratio, $\rho_{w}$ is also function of the stress ratio $f_{l} / f_{b u} ; K$ is a constant taken as 3 for compact flanges in compression and flanges in tension, and 1 for non-compact flanges in compression; $l$ is the unbraced arc length; and $R$ is the girder radius.

These flange strength definitions include directly the reduction in the flexural capacity of the flange due to the lateral stress effects, contrary to previous code versions, where the LFB is not explicitly taken into account in either the strength formulations or limit states equations. This format allows the engineer to evaluate the effects produced by any type of load combination and limit state. 
Finally, a complete section of the specifications is dedicated to the constructibility, giving practical recommendations for deck placement and overhang brackets. The corresponding commentary includes some approximate equations (similar to those presented in AASHTO 2007) to compute lateral flange moments depending on how the lateral load is assumed applied to the top flange:

$$
M_{\text {lat }}=\frac{F l^{2}}{12} ; \quad M_{\text {lat }}=\frac{P l}{8}
$$

where $F$ is the factored uniform lateral force $(\mathrm{K} / \mathrm{ft}), l$ is the unbraced arc length (ft), and $P$ is the concentrated lateral force at mid-panel (Kip).

\subsubsection{LRFD Bridge Design Specifications, Customary U.S. Units $-4^{\text {th }}$ Ed., AASHTO (2007)}

AASHTO (2007) corresponds to the present time specifications which were described in detail at Sections 1.2.2, AASHTO approximate formulations for the LFB, and 1.2.3, AASHTO Flexural Limit States for Constructibility. Approximate equations for considering curvature and overhang loading were explained along with the flexural limit states that govern the construction stage. In particular, the second limit state equation for discretely braced compression flanges (see Eq. 1-5), called the "one-third rule equation" (White \& Grubb, 2005; White, Zureick \& Jung, 2001), is intended to assure that the member has sufficient capacity for the lateral torsional and flange local buckling limit states. This equation is basically an interaction expression similar to those given for beam-column elements, where $\mathrm{f}_{\mathrm{bu}}$ represents the axial load and $\mathrm{f}_{l}$ the bending moment. The one-third factor corresponds to a linear approximation of the equivalent beam-column resistance for levels of LFB below the $60 \%$ of the nominal flange yield strength. The theoretical derivation of the one-third rule equation according to White et. al. (2001) is presented below:

- Derivation of the "one-third rule" equation:

Consider an I-girder flange subjected to combined vertical and lateral bending effects. The flange lateral moment produced by an elastically behaved lateral bending stress, $f_{l}$, is defined by Equation 2-17. However, if the flange is compact, a fully plastic stress distribution can be developed as shown in Figure 2-2, where the flange tips of 
width $c$ correspond to the flange force related to the lateral moment, given by Equation 218. Therefore, the remaining width of the flange is required to take the flange force associated with the vertical bending (see Eq. 2-19), which corresponds to a uniform stress distribution, $\mathrm{f}_{\mathrm{bu}}$, over the complete flange width, $\mathrm{b}_{\mathrm{f}}$, in absence of the lateral effects.

$$
\begin{aligned}
& M_{l}=\frac{f_{l} b_{f}^{2} t_{f}}{6} \\
& M_{l}=F_{y} c t_{f}\left(b_{f}-c\right) \\
& f_{b u}=F_{y} \frac{b_{f}-2 c}{b_{f}}
\end{aligned}
$$

where $t_{f}$ is the flange thickness and $F_{y}$ is the yield strength of the flange.

The width $c$ can be obtained by equating Equations 2-17 and 2-18, and then placed in Equation 2-19 to obtain $f_{b u}$ as a function of $f_{l}$ and the flange fully plastic strength, $F_{y}$, as indicated below:

$$
f_{b u}=F_{y} \sqrt{1-\frac{2}{3} \frac{f_{l}}{F_{y}}}
$$

For practical purposes where $f_{l}$ is below $60 \%$ of $\mathrm{F}_{\mathrm{y}}$, Equation $2-20$ is approximated accurately by the following linear relationship, as shown in Figure 2-3:

$$
f_{b u}=F_{y}-\frac{1}{3} f_{l}
$$

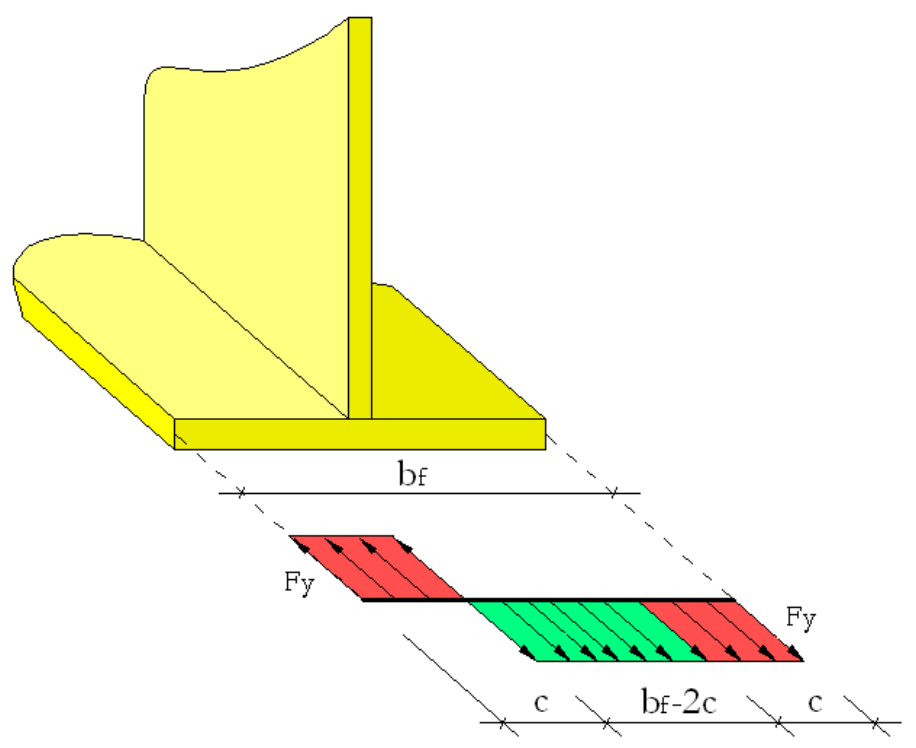

Figure 2-2. Idealized flange plastic stress distribution due to vertical and lateral bending. 


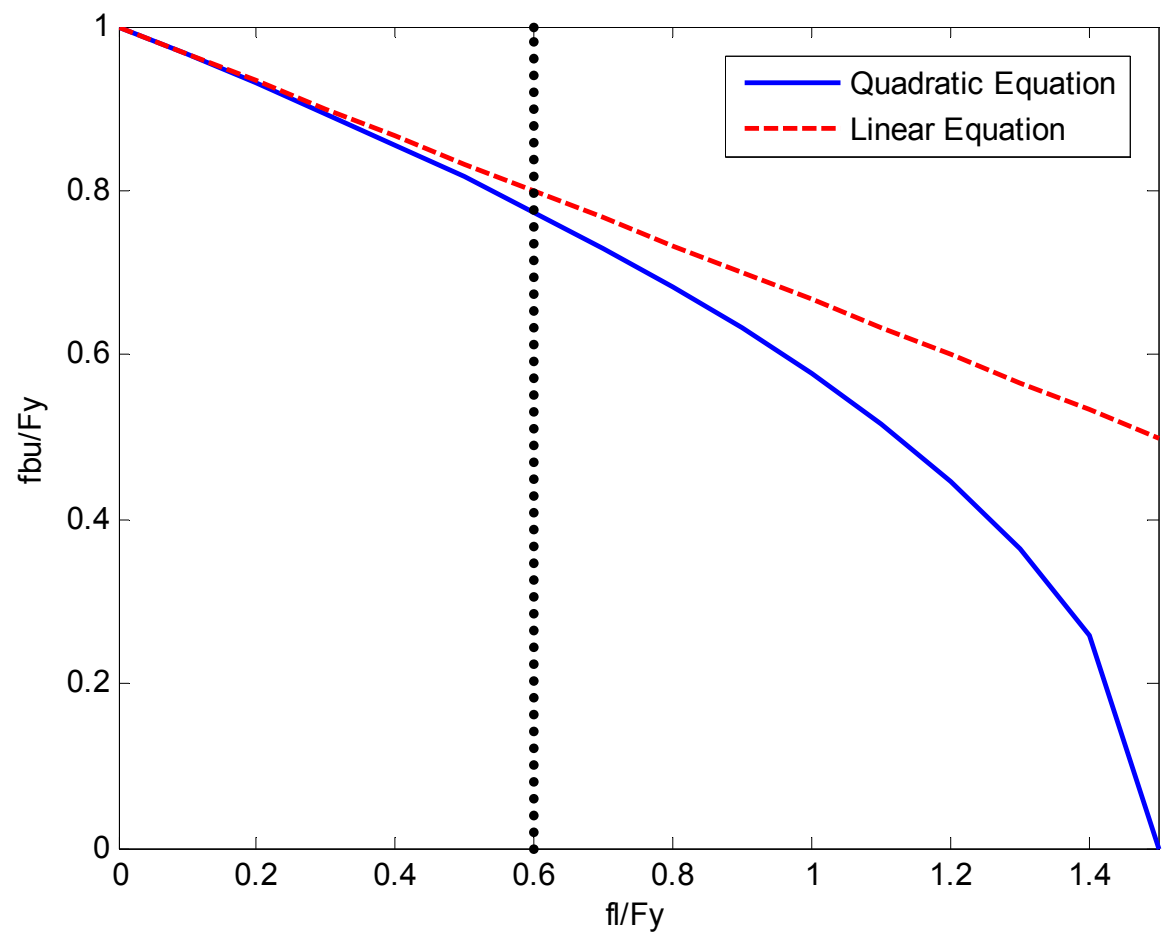

Figure 2-3. Comparison of the complete and the approximate strength of a compact flange

Equation 2-21 does not account for the reduction in the flexural capacity due to local flange or lateral-torsional buckling in compression flanges. Therefore, in this case, the yield strength of the flange is replaced by the design flexural resistance of the flange, $\phi_{f} F_{n c}$, computed according to the specifications.

The one-third rule equation has the advantage of being valid either for straight or curved girders, since it does not depend directly on the radius of curvature as the $\rho$ factors in Equation 2-14. Therefore, the equation does not exhibit any anomaly when the radius of curvature goes to infinity. Additionally, this formulation allows computing independently the load demands from the strength capacities of the flanges, which improves the practical application of the provisions. Finally, the one-third rule applies not only for the constructibility condition but also for the strength limit state including compact and non-compact sections, and flanges in either tension or compression states. 


\subsection{Reliability Analysis}

The prediction of the structural performance of any system is based on the combination of approximate analysis methodologies to determine loading effects and experimental tests to establish material properties or member behaviors. However, there are always inherent uncertainties related to these processes that distort the subsequent predicted results. Variables such as the material properties, approximations in the analyses, simplification of the models, randomness of the loads, etc., affect the demand and capacity estimations of the members in the structural system. Therefore, the reliability analyses are intended to account for these uncertainties to estimate the level of confidence exhibited by the structure to achieve the desired structural performance.

An overview of research efforts conducted in reliability is presented as follows: first, a description of the reliability analysis for code development, where a synopsis of the most important performance-based codes and specifications based on structural reliability is presented; and, a presentation of some reliability methodologies, including specific research works on the methods and procedures to compute the reliability of a structural member or system.

\subsubsection{Reliability Analysis for Code Development}

In the 1960s, the philosophy of the code specifications was based on the allowable stress principles (ASD, Allowable Stress Design). The structure was supposed to behave elastically, and the uncertainties were taken into account by a safety factor which divided the maximum stress according to the limit state considered. However, since these safety factors were selected subjectively, the risk of failure associated with that decision was unknown. Therefore, this practice has become impractical from the economical and safety point of view.

During the 1960s and 1970s, different natural disasters took place around the world causing extensive loss of human lives and economical damage. The evidence of deficiencies in design specifications revealed the need for the elaboration of codes based in a different design philosophy. More rational code definitions were defined identifying the basic limit states that any structure should achieve: safety under high load scenarios 
and comfort during normal load conditions. This approach became the basis of most of the structural design specifications even for current codes.

In 1978, Ellingwood, Galambos, MacGregor and Cornell developed a set of design specifications using advanced reliability analysis methods and statistical data (Ellingwood, 1985). The fundamental concept of this design procedure is the basis of the Partial Factor Method, which is considered a "Simplified Probabilistic Design" method (Vrounwenvelder, 2001). In this method, a structural failure occurs if the load effects are larger than the resistance capacity of the element or system, and since both of these variables are considered random, a probability-based criterion may be applied. The issue consisted in defining the target probabilities considered as "safe" for design, in order to find the appropriate threshold between safety and economy. Several current structural design standards such as the AISC's LRFD Specifications for Steel Structures, ASCE Standard 16 on LRFD for Engineered Wood Construction, American Concrete Institute Standard 318, and the International Building Code 2000, are based on these principles.

Initially, the partial factors that account for uncertainties in loads and resistances were defined based only on past experiences and the observed behavior of the structures. However, current systems demand more accurate methods to determine these factors, since high uncertainties may carry catastrophic consequences which are unacceptable in today's practice. The probabilistic analyses satisfy this requirement and enhance the confidence level of the structures.

Some new code specifications address the structural design problem from the performance-based point of view, which is the final target of the evolution of the design codes. Vision 2000, FEMA 356 (FEMA, 2000a), FEMA 350 (FEMA, 2000b), ATC-40 (ATC, 1996) and NEHRP (FEMA, 2003) are some examples of this new trend in the code developments, principally for seismic design. The designer needs to define a structural performance objective which consists of different intensity load scenarios that control the design of structural and no-structural members. The definition of the loads and the capacities are based on probabilistic analyses according to real conditions.

Specific research works on the calibration of code specifications for steel bridges have been done by several authors (Nowak, 1995; Nowak, 2004; Barker \& Zacher, 1997; Czarnecki \& Nowak, 2006). In these studies, the probabilistic definitions of loads and 
capacities have been addressed during the service stage of the bridge. In particular, Nowak et. al. (2006) calibrated the resistance factors for steel curved bridges including construction stages using three representative structures. The authors concluded that the resistance factors used for straight bridges are valid for curved bridges and that the construction stage is very important for curved bridges because of the significant variation of stresses during this phase.

Eamon et. al. (2000) presented a reliability-based criterion for wood bridges in the Load and Resistance Factor Design (LRFD) format. Load and resistance models were developed based on statistical analyses of test results. The limit state considered was the flexural capacity where the failure is limited by the moment of rupture. A wide range of reliability indexes was exhibited principally due to significant differences between codepredicted stresses and analytical results. The authors concluded that more accurate design approximations based on experimental and analytical results are required to improve the reliability for a wood bridge code.

Galambos (2004) determined the theoretical reliability of steel beams, columns and beam-columns designed according to the projected 2005 AISC specifications. The reliability indices were evaluated based on contemporary material properties and recent experimental strength data. It was concluded that the notional reliability of the proposed specification is identical to the one exhibited by the LRFD Specifications of 1986.

White and Jung (2008) evaluated the lateral-torsional and flange local buckling (LTB \& FLB) predictions from 2004 AASHTO and 2005 AISC provisions versus uniform bending experimental test results. The reliability indices were estimated where the corresponding values for FLB were shown to be larger than those for LTB. These specifications were also evaluated using the results obtained from moment gradient experimental tests (White \& Kim, 2008). In this work, the estimated reliability indices for FLB of end-loaded segments were found to be similar to those determined by White and Jung (2008) in most cases. For LTB, the reliability indices for end-loaded segments with moderate to large unbraced lengths were slightly larger than the values estimated from the uniform bending tests. 


\subsubsection{Reliability Methodologies}

Some specific research works are presented below to describe the importance of the selection of the reliability methods according to the available resources and intended goals.

Gayton et. al. (2004) described the advantages and disadvantages of the existing calibration methods for the partial factors of reliability-based design codes, in order to evaluate their validity and improve their efficiency. The global optimization methods are highly accurate but time consuming; hence, the approximate methods become more popular and practical with a consequent accuracy cost. Four numerical examples are presented to illustrate the application and the differences of the global and approximate methods. The authors give recommendations to select the appropriate method according to result quality and computation time.

Zhao and Ono (2001) studied five methods to approximate the probability of failure of a structural system based on the higher order moments of a random variable. Compared to the most popular first-order reliability methods (FORM), these procedures are simpler and do not require iterations or derivative computations, which makes them attractive for structural reliability analysis.

Foschi, Li and Zhang (2002) described an approach to integrate structural response calculations with procedures for the calculation of reliability (forward problem) or calculate design parameters for specified target reliabilities (inverse problem). The inverse problem is usually more time consuming since it requires an iterative optimization process of the design parameters. However, the efficiency is improved if a reliability database is constructed in terms of the design parameters, where interpolation algorithms are applied to obtain the appropriate factor values. These performance-based procedures allow designing structures with specific demands or particular characteristics that are out of the standard-specifications scope.

$\mathrm{Li}$ and $\mathrm{Li}$ (2004) performed a reliability-based integrated design (RID) of steel portal frames with tapered members. The authors address the reliability concept of the structural system rather than their individual components, thus the structural redundancy and ductility are involved in the limit states. The authors employ a nonlinear integrated analysis model to reduce the uncertainty of the results. The final design using this 
procedure consumes less steel than the LRFD method and produces a satisfactory reliability index. Cheng, Xu and Jiang (2006) also presented a method to compute the approximate reliability using a linear Taylor approximation of the accurate reliability that reduces computational effort. The method comprises a reliability analysis and optimization of design variables. 


\section{Chapter 3: Overview of finite element modeling procedures}

This chapter discusses the principal modeling procedures employed to conduct FEA in this research. First, the material models for steel and concrete and their corresponding stress-strain relationships are described. Then, the finite elements and techniques required to model the bridge behavior during deck placement conditions are presented. A validation of the modeling procedures is also included using the results from experimental tests with different levels of complexity, starting from one steel Ishaped girder and finishing with the Federal Highway Administration's full-scale curved bridge structure. Finally, a description of the analysis methodology employed in the FE models is given.

\subsection{Materials}

This section includes a description of the material models and the experimental data from where the stress-strain relationships were derived.

\subsubsection{Steel}

\subsubsection{Material Model}

A plasticity metal model with Mises yield surfaces, associate plastic flow and isotropic hardening was used in this study. This model is considered appropriate to simulate rate-independent behavior of a metal under a relatively monotonic loading where creep effects may be neglected (Righman, 2005).

The Mises yield criterion is a rate-independent plasticity model for isotropic materials, i.e. isotropic yielding. This criterion states that yielding begins when the strain energy of distortion reaches a critical value in terms of the uniaxial yield strength of the material $\left(\sigma_{\mathrm{y}}\right)$. The associated plastic flow considers that the inelastic deformation rate is in the direction of the normal yield surface; consequently, the plastic deformation is volume invariant. Finally, the isotropic hardening defines the change of the yield-surface 
size which is uniform in all directions and varies according to the plastic strain (Abaqus, 2002).

\subsubsection{Stress-strain relationship}

The engineering stress-strain relationship used in this study consists of a multilinear approximation (Hartmann, 2005), which is summarized in Table 3-1. This approximation is based on the average properties of selected plates (see Table 3-2) of a full-scale curved bridge structure that is part of the Federal Highway Administration's Curved Steel Bridge Research Project. As a result, the curve indicated in Figure 3-1 was used to characterize the mechanical behavior of the Grade-50 steel. A representative value for the modulus of elasticity ( $E=29600 \mathrm{Ksi}$ ) was specified to construct the curve. Finally, the engineering stress-strain relationship is converted to the true stress-strain curve, as required for input into Abaqus ${ }^{\circledR}$.

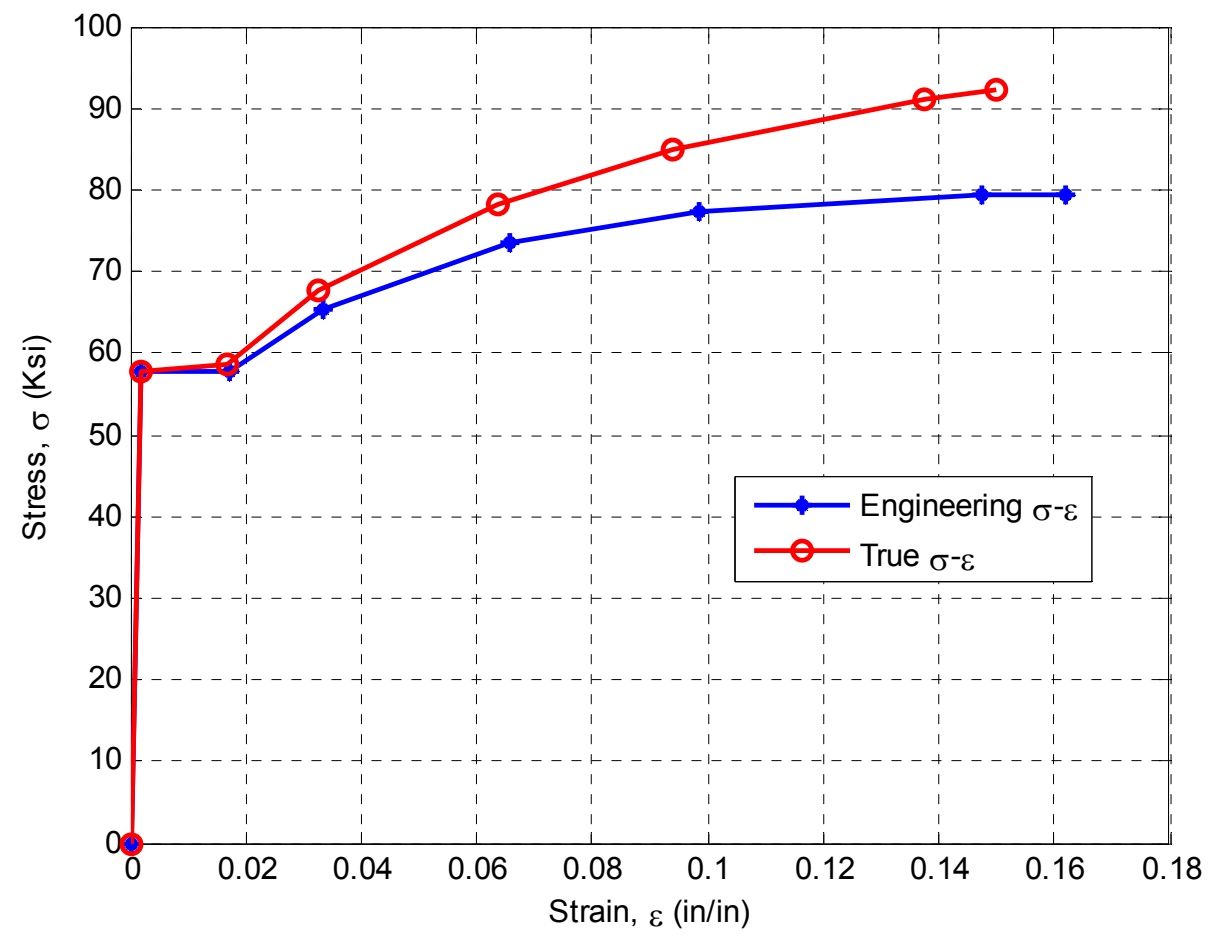

Figure 3-1. Engineering and true stress-strain relationships for the Grade-50 steel 
Table 3-1. Approximations for a typical steel stress-strain behavior (Hartmann, 2005)

\begin{tabular}{ccc}
\hline Point & Strain & Stress \\
\hline 1 & $\frac{\sigma_{s y}}{E}$ & $\sigma_{s y}$ \\
\hline 2 & $\frac{\left(\varepsilon_{u}-\varepsilon_{s t}\right)}{10}+\varepsilon_{s t}$ & $\sigma_{s y}$ \\
\hline 3 & $\frac{2\left(\varepsilon_{6}-\varepsilon_{3}\right)}{7}+\varepsilon_{3}$ & $\frac{E_{s t}\left(\varepsilon_{u}-\varepsilon_{s t}\right)}{10}+\sigma_{s y}$ \\
\hline 5 & $\left.\frac{2\left(\varepsilon_{6}-\varepsilon_{3}\right)}{7}+\varepsilon_{4}\right)$ \\
\hline 6 & $\frac{2\left(\sigma_{6}-\sigma_{3}\right)}{7}+\sigma_{4}$ \\
\hline 7 & $\frac{\sigma_{s y}}{\varepsilon_{u}-\frac{\sigma_{u}}{10}}\left(\varepsilon_{u}-\frac{100\left(\varepsilon_{u}-\varepsilon_{s t}\right)}{\sigma_{s t}}\right.$ \\
\hline
\end{tabular}

Table 3-2. Average steel plate properties (Hartmann, 2005)

\begin{tabular}{lc}
\hline \multicolumn{1}{c}{ Property } & Average Value \\
\hline Static yield strength, $\sigma_{s y}(\mathrm{Ksi})$ & 57.65 \\
\hline Offset yield strength, $\sigma_{0.2 \%}(\mathrm{Ksi})$ & 59.42 \\
\hline Strain at the onset of strain hardening, $\varepsilon_{s t}(\%)$ & 1.71 \\
\hline Strain hardening modulus, $E_{s t}(\mathrm{Ksi})$ & 537.37 \\
\hline Tensile strength, $\sigma_{u}(\mathrm{Ksi})$ & 81.88 \\
\hline Strain at the tensile strength, $\varepsilon_{u}(\%)$ & 16.18 \\
\hline
\end{tabular}




\subsubsection{Concrete}

\subsubsection{Material Model}

The concrete damaged plasticity model proposed by Lee and Fenves (1998) is selected as the constitutive model to represent the concrete behavior in the FEA. In this model, it is assumed that the main two failure mechanisms are tensile cracking and compressive crushing of the concrete. This is a plastic-damage model based on the concepts of fracture-energy damage and stiffness degradation from the continuum damage mechanics.

The concrete damaged plasticity model assumes non-associated potential plastic flow. This flow potential, which is continuous and smooth, ensures that the flow direction is always uniquely defined. The model makes use of the modified yield function proposed by Lee and Fenves (1998) to account for different evolution of strength under tension and compression. The evolution of the yield (or failure) surface is controlled by two hardening variables, the tensile $\left(\varepsilon_{\mathrm{t}}^{\mathrm{pl}}\right)$ and the compressive $\left(\varepsilon_{\mathrm{c}}{ }^{\mathrm{pl}}\right)$ equivalent plastic strains, which are associated to their corresponding failure mechanisms. Figure 3-2 illustrates the initial shape of the yield surface, in the principal stress space for plane stress.

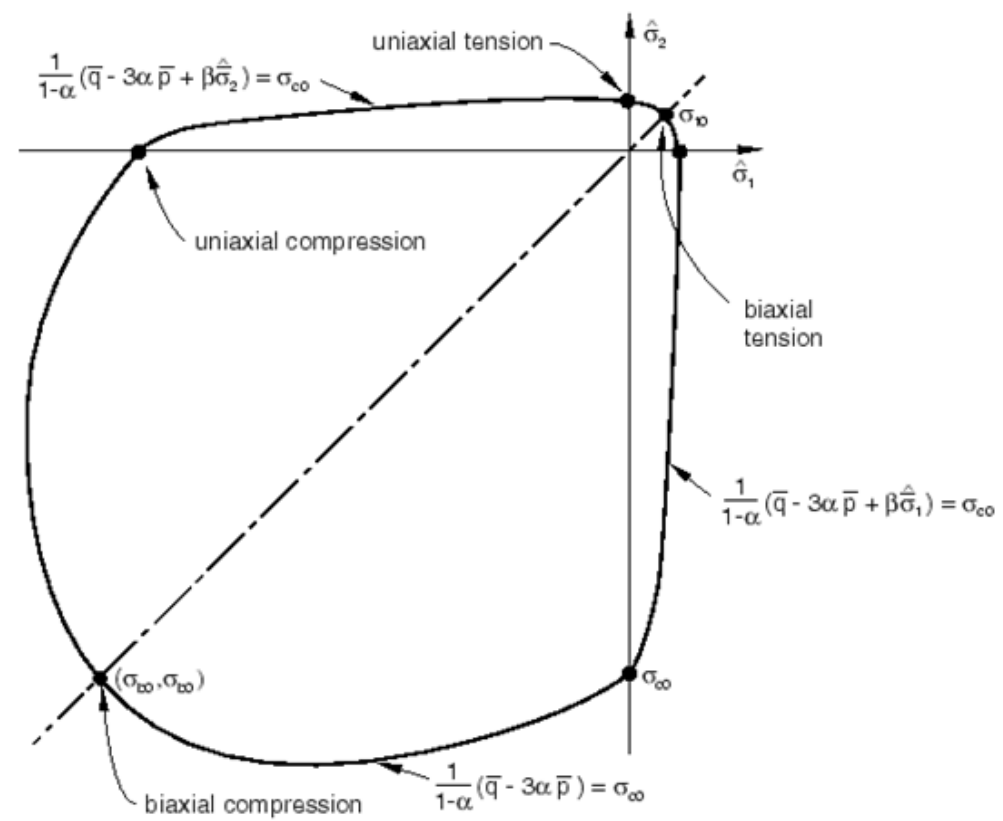

Figure 3-2. Yield surface in plane stress (Abaqus, 2002) 
- Uniaxial tension behavior:

Under uniaxial tension the stress-strain response follows a linear elastic relationship, as shown in Figure 3-3, until the value of the failure stress $\left(\sigma_{\mathrm{to}}\right)$ is reached. The failure stress is related to the onset of micro-cracking in the concrete material. Then, the formation of micro-cracks is represented macroscopically with a softening stressstrain response, which induces strain localization in the concrete structure (Abaqus, 2002).

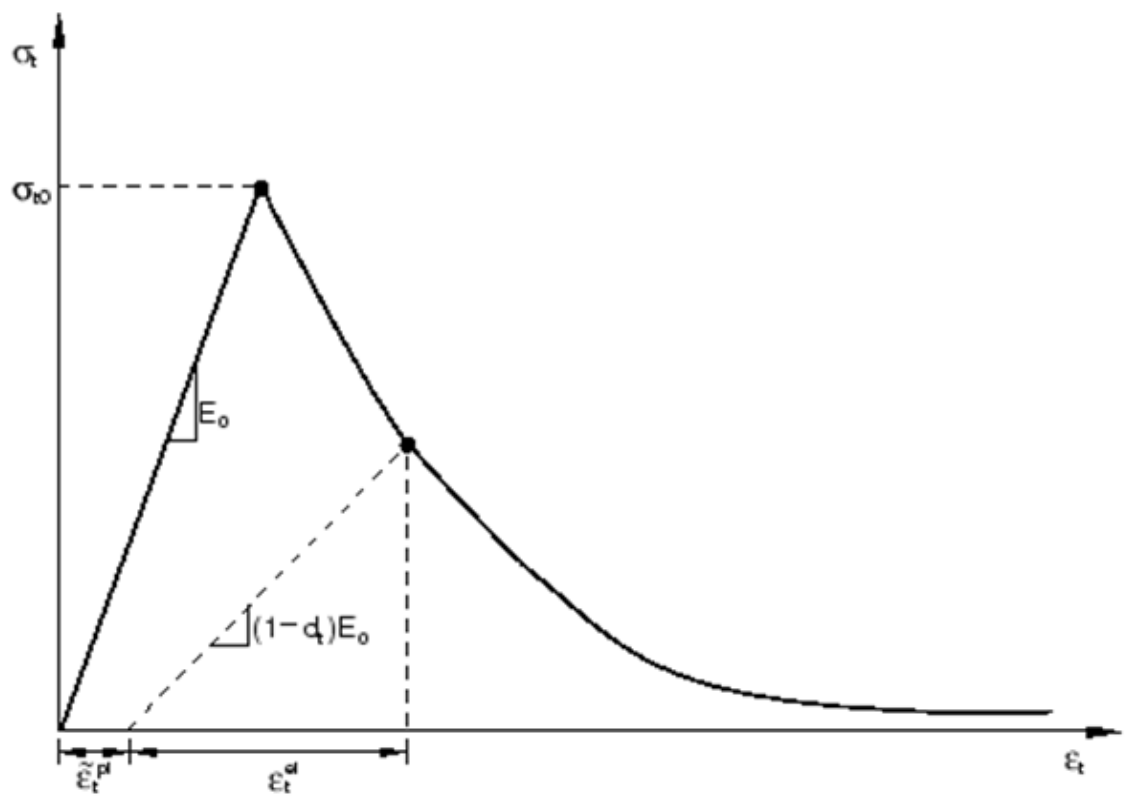

Figure 3-3. Concrete model response to uniaxial tension loading (Abaqus, 2002)

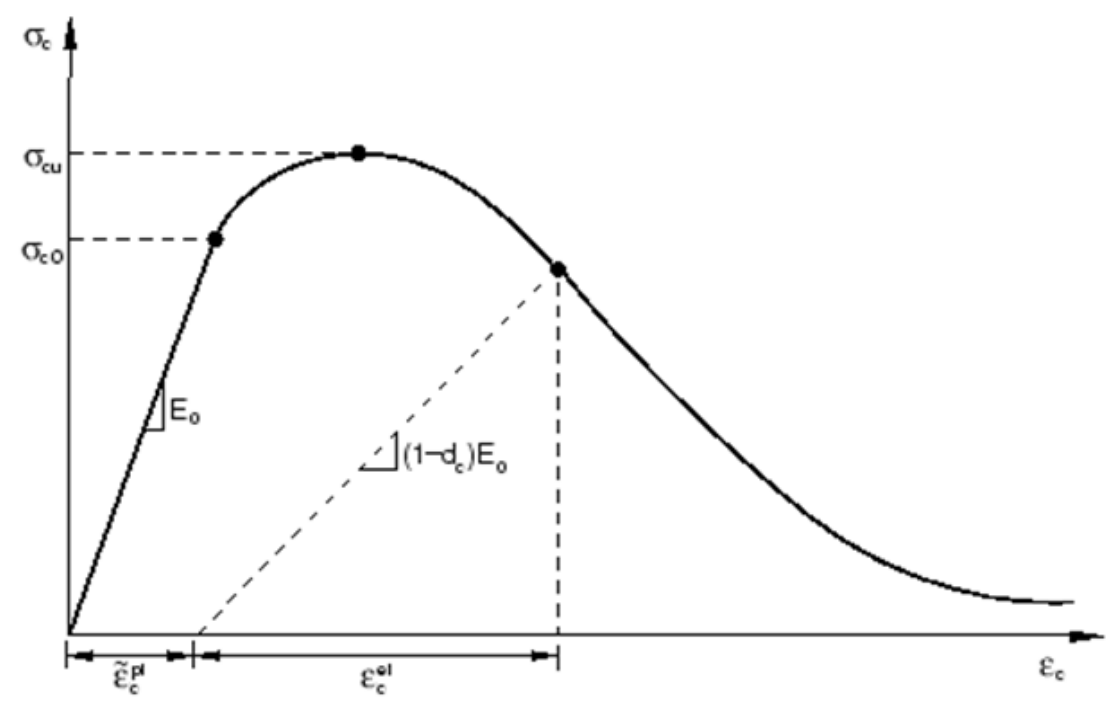

Figure 3-4. Concrete model response to uniaxial compression loading (Abaqus, 2002) 
- Uniaxial compression behavior:

Under uniaxial compression the response is linear until the value of initial yield $\left(\sigma_{\mathrm{co}}\right)$, as shown in Figure 3-4. The plastic response is typically characterized by stress hardening followed by strain softening beyond the ultimate stress $\left(\sigma_{\mathrm{cu}}\right)$. This simplified representation satisfactorily reproduces the main features of the response of concrete in compression (Abaqus, 2002).

According to Figure 3-3 and Figure 3-4, when the concrete is unloaded from any point on the strain softening branch, the unloading response is weakened, i.e. the elastic stiffness of the material is degraded. The degradation of the elastic stiffness is characterized by two damage variables, $d_{t}$ and $d_{c}$, which are assumed to be functions of the equivalent plastic strains. These damage variables can take values from zero, representing the undamaged material, to one, which represents total loss of strength. If $E_{0}$ is the initial elastic stiffness of the material, the stress-strain relationships under uniaxial tension and compression loading are defined by:

$$
\begin{aligned}
& \sigma_{t}=\left(1-d_{t}\right) E_{0}\left(\varepsilon_{t}-\widetilde{\varepsilon}_{t}^{p l}\right), \\
& \sigma_{c}=\left(1-d_{c}\right) E_{0}\left(\varepsilon_{c}-\widetilde{\varepsilon}_{c}^{p l}\right)
\end{aligned}
$$

The effect of some recovery of the elastic stiffness when the load changes sign is also considered for the uniaxial cyclic behavior of this concrete model. The concrete damaged plasticity model assumes that the general reduction of the elastic modulus depends on a scalar degradation variable $d$, as follows:

$$
E=(1-d) E_{0}
$$

where $d$ is a function of the stress state and the uniaxial damage variables, $\mathrm{d}_{\mathrm{t}}$ and $\mathrm{d}_{\mathrm{c}}$. In particular, Abaqus uses the following expression for the uniaxial cyclic conditions:

$$
(1-d)=\left(1-s_{t} d_{c}\right)\left(1-s_{c} d_{t}\right)
$$

where $s_{t}$ and $s_{c}$ are functions of the stress state, which are intended to model stiffness recovery effects associated with stress reversals:

$$
\begin{aligned}
& s_{t}=1-w_{t} r^{*}\left(\sigma_{11}\right) ; \quad 0 \leq w_{t} \leq 1, \\
& s_{c}=1-w_{c}\left(1-r^{*}\left(\sigma_{11}\right)\right) ; \quad 0 \leq w_{c} \leq 1 \\
& r^{*}\left(\sigma_{11}\right)=\left\{\begin{array}{lll}
1 & \text { if } & \sigma_{11}>0 \\
0 & \text { if } & \sigma_{11}<0
\end{array}\right.
\end{aligned}
$$


where $w_{t}$ and $w_{c}$ are the weight factors that control the recovery of the tensile and compressive stiffness upon load reversal as shown in Figure 3-5.

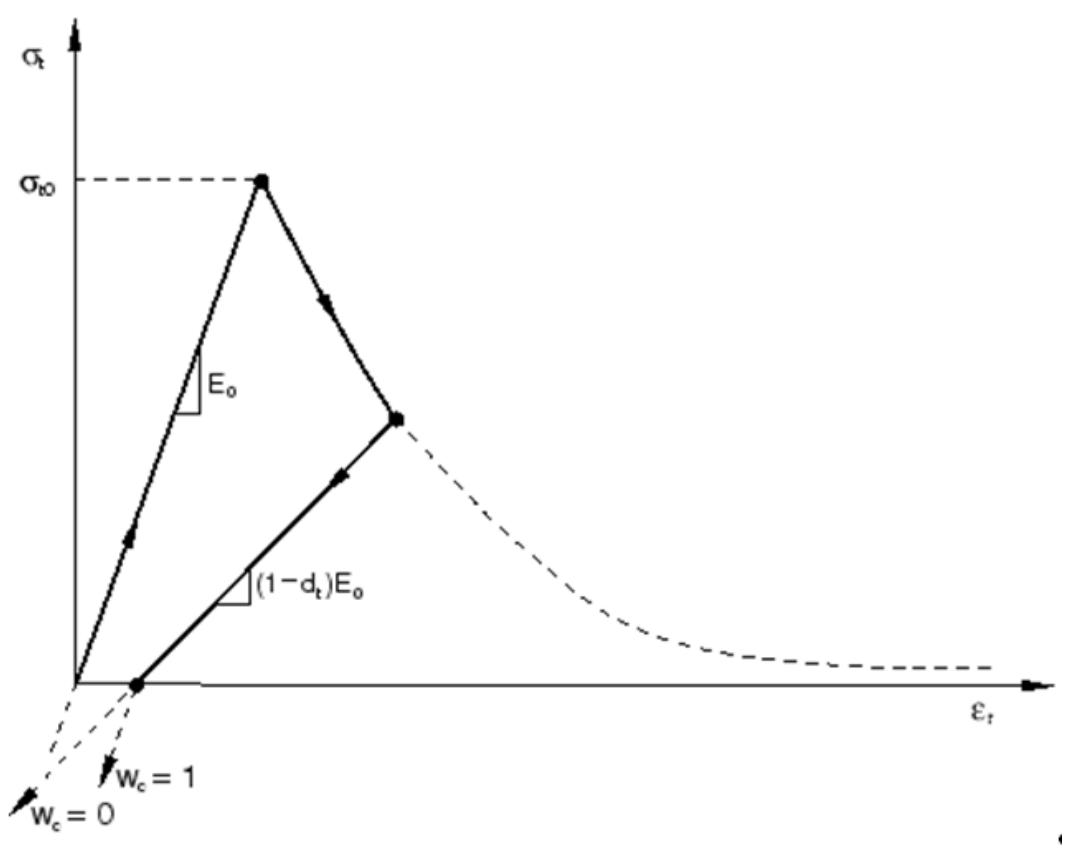

Figure 3-5. Effect of the compression stiffness recovery parameter $\mathrm{w}_{\mathrm{c}}$ (Abaqus, 2002)

\subsubsection{Stress-strain relationship}

The stress-strain curves used in this work to represent the concrete behavior are also based on the experimental data obtained from the Federal Highway Administration's Curved Steel Bridge Research Project. These results were reported in detail by Jung (2006).

- Compressive strength:

The approximate compressive stress-strain relationship is based on measured stress-strain curves of six 298-day cylinder tests, where the average compressive stressstrain response is presented in Figure 3-6.

The average maximum strength was $4.87 \mathrm{ksi}\left(f^{\prime} c\right)$, which was used to compute the approximate initial yield strength of the curve as $0.45 \mathrm{f}^{\prime} \mathrm{c}=2.19 \mathrm{ksi}$ (ACI-318, 2002). Then a multi-linear approximation consisting of eight additional points is followed as shown in Figure 3-7. 


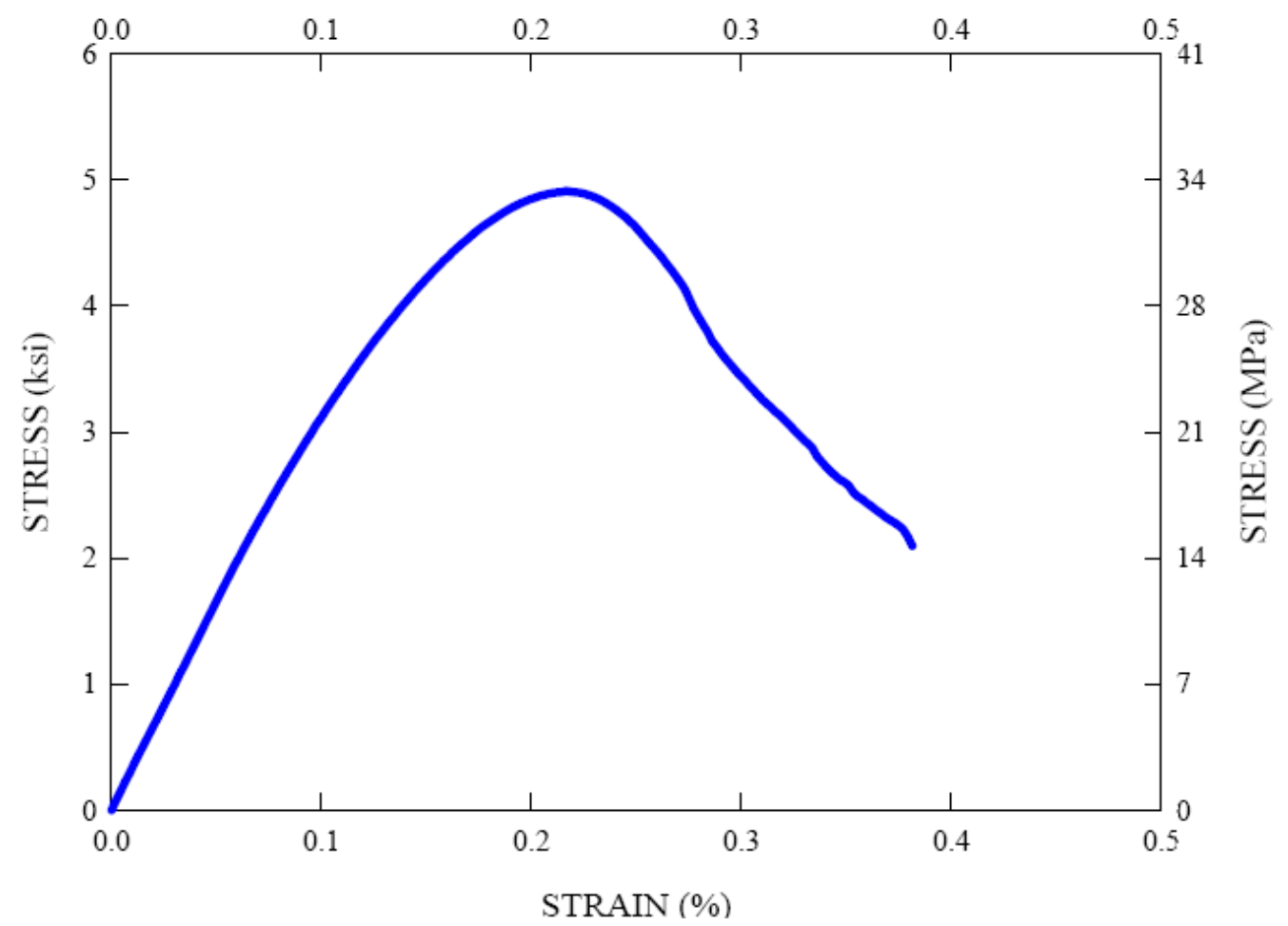

Figure 3-6. Average compressive response of concrete (Jung, 2006)

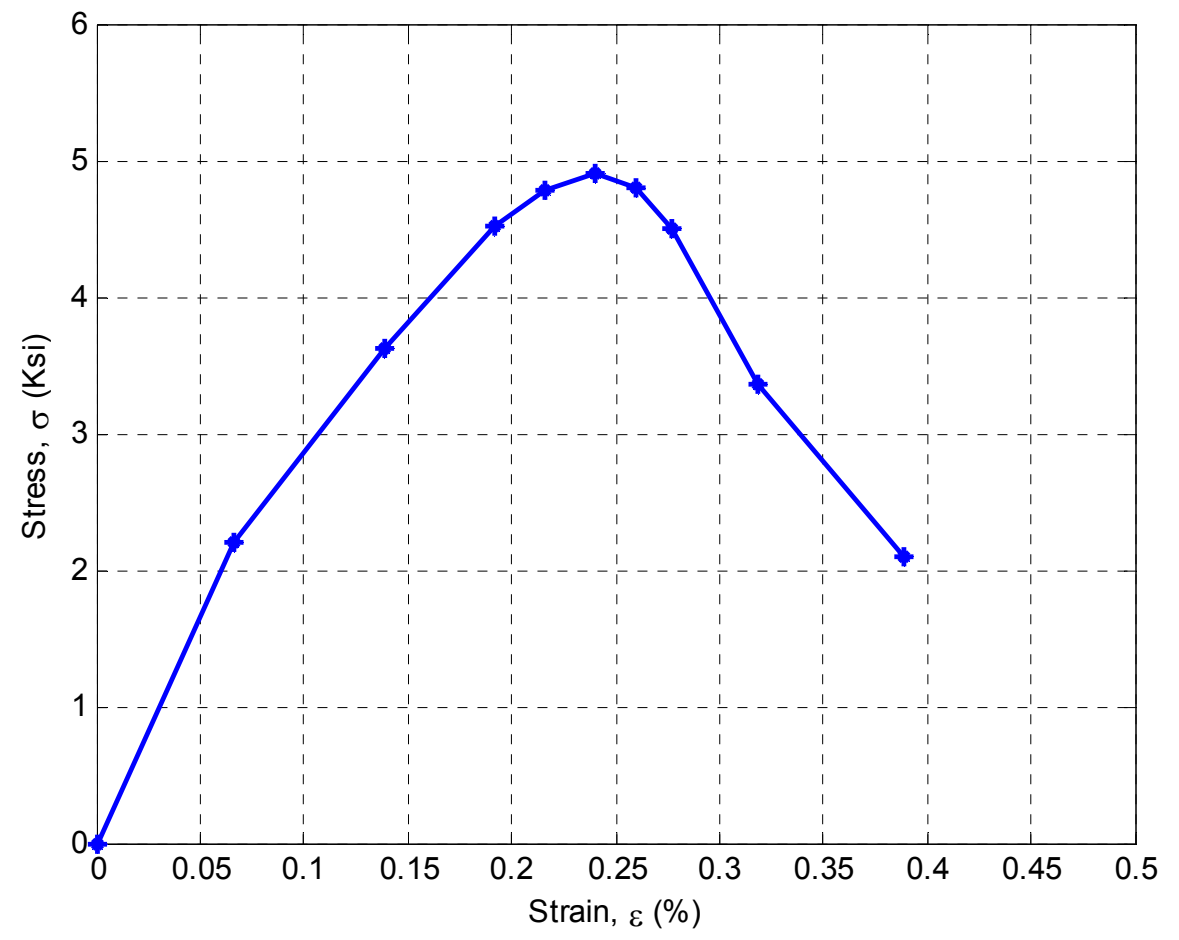

Figure 3-7. Stress-strain relationship for the compressive behavior of concrete 
- Tensile strength:

The tensile stress-strain curve is based on the average maximum tensile splitting strength (0.50ksi) of six 298-day concrete cylinders. The descending branch of the curve is computed based on the elastic stiffness degradation (damage value) obtained from cyclic tension tests.

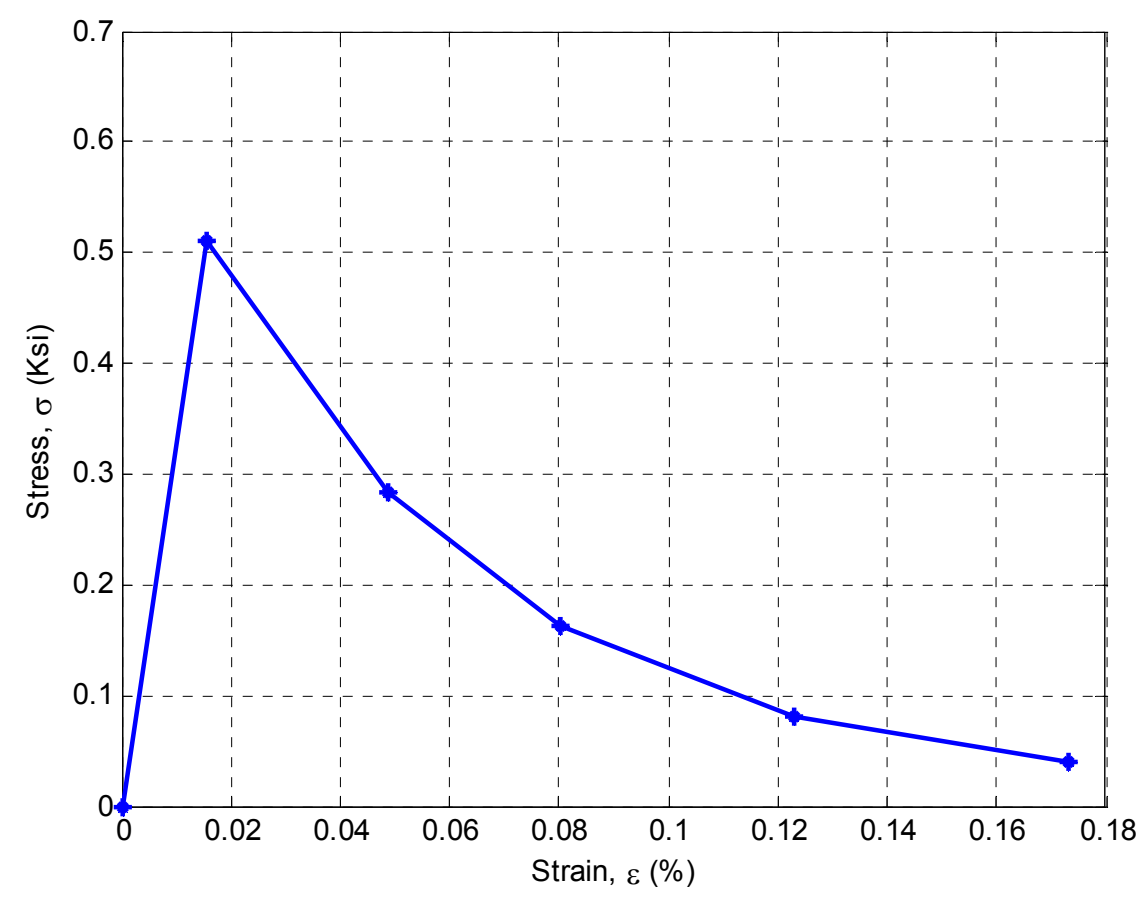

Figure 3-8. Engineering stress-strain relationship for the tensile behavior of concrete

The interaction between the reinforcement and the concrete tends to reduce the mesh sensitivity of the numerical solution, provided that a reasonable amount of tension stiffening is introduced in the concrete model to simulate this interaction (Abaqus, 2002). Therefore, higher tensile strengths were considered for some FE models that exhibited convergence problems, based on the assumption that the steel reinforcement increases the tension capacity of the concrete.

- Elastic stiffness degradation:

The measured elastic stiffness degradations for compression and tension $\left(\widetilde{d}_{c}, \widetilde{d}_{t}\right)$ reported by Jung (2006) were used in this study. These values were computed following 
the procedure described by Lee and Fenves (1998) for the corresponding stress-strain relationships described previously.

\subsection{Finite elements}

In this section, a description of the finite elements used to model the different members of the bridges is included. Additionally, modeling procedures performed to define the effects of the residual stresses and the mesh density are also presented.

\subsubsection{Plate girders and concrete slab}

Four-node (or linear) shell elements with reduced integration and enhanced hourglass control (S4R) were used to model the plate girders and the concrete slab. The "reduced integration" accounts for the ability of the S4R element to use one integration point instead of four, as in the case of the fully integrated and general-purpose shell element (S4). Therefore, S4R is computationally more efficient for comprehensive analyses since it significantly reduces running times. The reduced elements also address the typical shear locking effect produced by fully integrated linear elements. For example, under pure bending conditions, the linear elements are not able to bend to curves causing an artificial shear stress, as shown in Figure 3-9b. Then, the linear fully integrated element becomes locked or overly stiff under bending moment producing an incorrect response. However, this problem is avoided using reduced integration elements which permit shape distortions.

Nevertheless, the reduced first order elements exhibit a numerical problem called hourglassing due to their excessive flexibility. Figure 3-10 shows that the lines corresponding to the integration point do not deform under bending moment. Therefore, the stresses are zero and no strain energy is generated at the integration point. This is a non-physical response that may produce meaningless results. Therefore, an appropriate hourglassing control command is introduced in the definition of the S4R element in Abaqus to overcome this problem. 

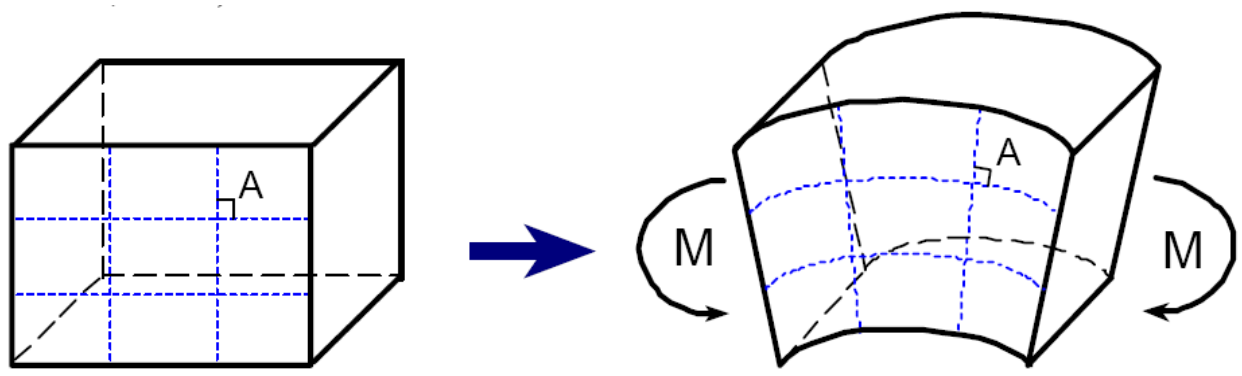

$a$.
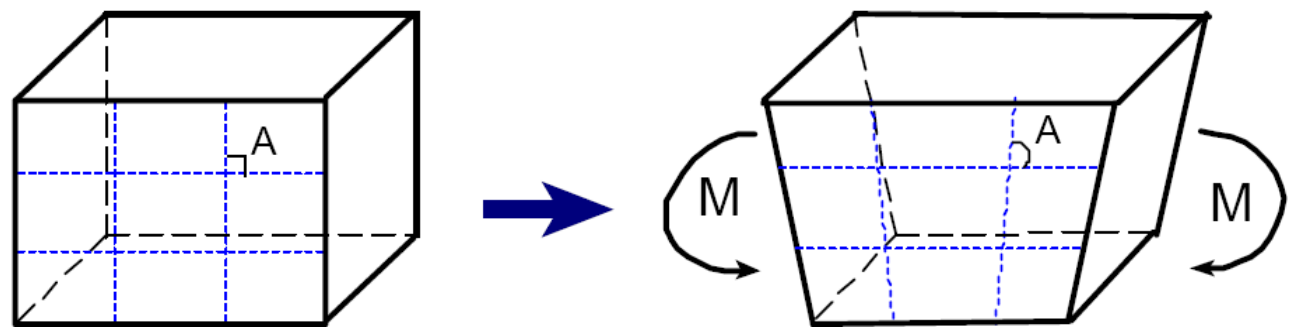

$b$.

Figure 3-9. Change of shape of a fully integrated $a$. higher order $b$. linear element (Sun, 2006)
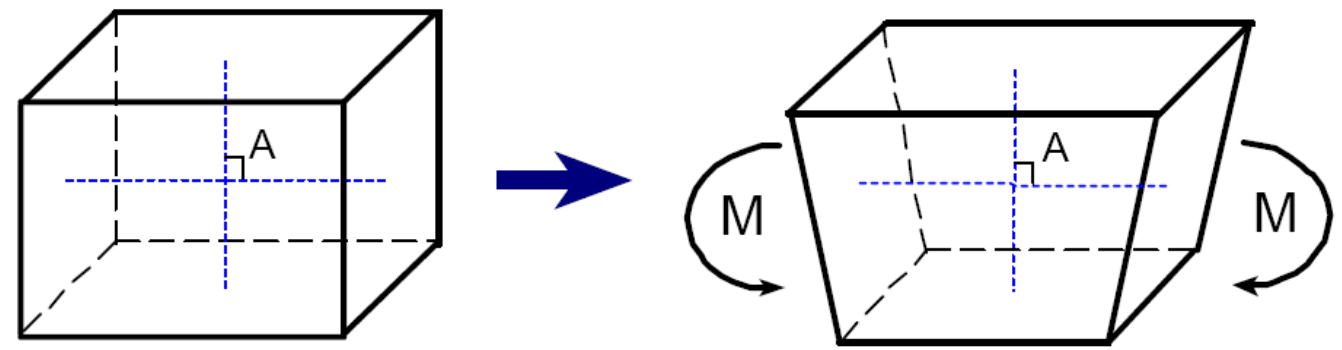

Figure 3-10. Change of shape of a reduced integration element (Sun, 2006)

The hourglassing control effect was analyzed using a simply supported beam subjected to a uniform load of $0.1 \mathrm{kip} / \mathrm{in}$. The beam is $20 \mathrm{ft}$ long with a rectangular cross section of 1 in $x 12 \mathrm{in}$. The FE model uses three shell elements throughout the height of the web. Therefore, a minimum stress value of $-20 \mathrm{Ksi}$ is expected at the integration point of the upper elements. According to Figure 3-11, only the models using fully integrated linear elements (S4) and reduced first order elements (S4R HC) with hourglassing control exhibit a stress level close to the theoretical value. 


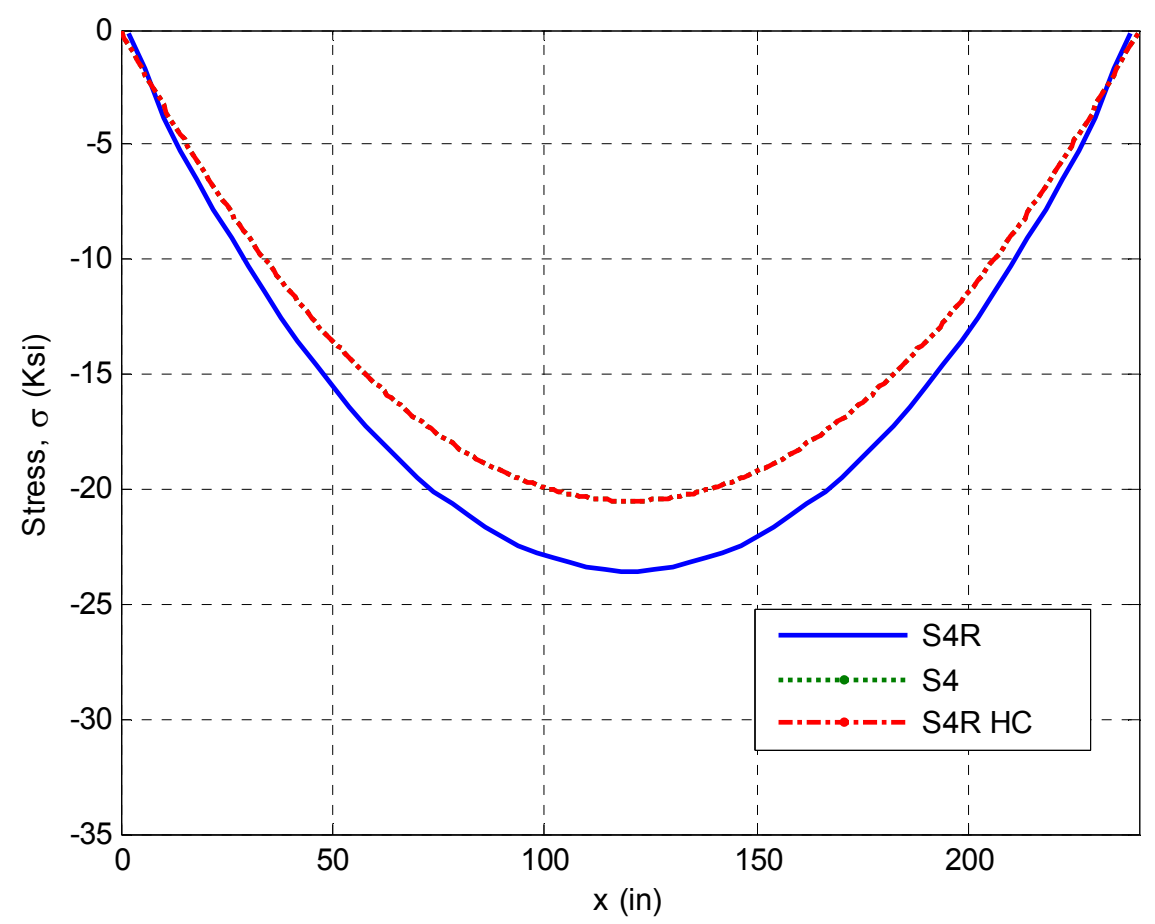

Figure 3-11. Effect of hourglassing control

\subsubsection{Modeling of composite action}

Jung (2006) used two modeling approaches to consider the composite action between the concrete slab and the steel I girders. The first approach employs discrete nonlinear spring elements to represent the shear connector response as a function of the relative slip that occurs at the concrete-steel interface. In the second modeling approach, it is assumed that there is no relative slip in the concrete-steel interface region and a rigid beam-type multi-point constraint (MPC) is used to connect the top flange nodes of the steel girders to the bridge slab nodes. Load-deflection responses of two models using these approaches were compared as shown Figure 3-12, from where it is observed that both modeling techniques produce similar results. Consequently, the modeling approach using beam MPC was adopted in this work to simulate the deck composite action. 
VERTICAL DEFLECTION (mm)

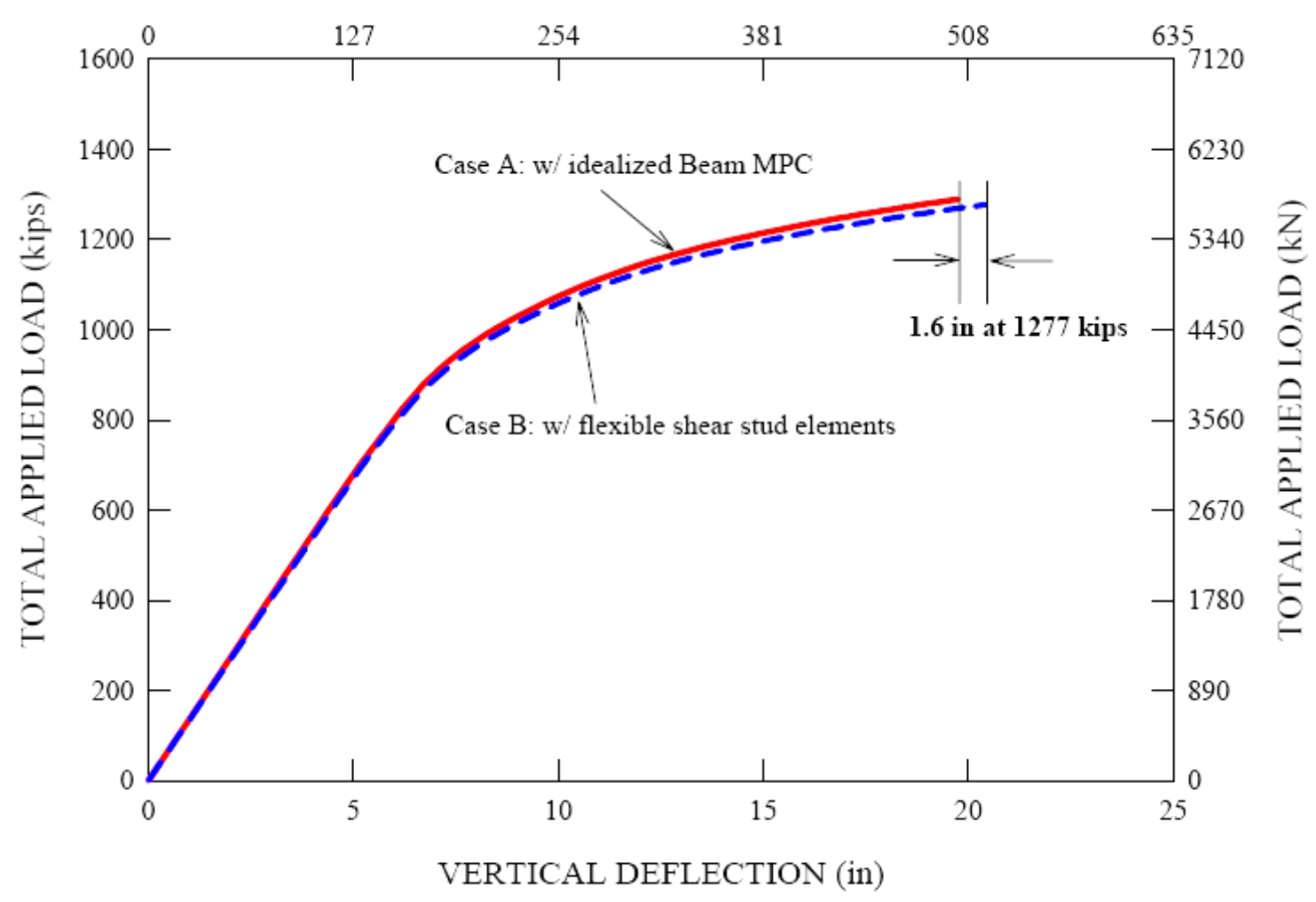

Figure 3-12. Effect of composite-action modeling (Jung, 2006)

\subsubsection{Cross frames and stiffeners}

The cross frames and the stiffeners were modeled using slender beam elements (B33) based on the Euler-Bernoulli formulation. The Euler-Bernoulli formulation uses cubic interpolation functions to increase their accuracy in case of distributed loads. These elements are intended for small strain and large rotation analyses. Additionally, beam elements do not allow for transverse shear deformation, i.e. plane sections initially normal to the beam's axis remain plane (if there is no warping) and normal to the beam axis. B33 elements should only be used to model slender beams, which are characterized by having small cross-sectional dimensions compared to typical distances along its axis. For beams made of uniform material and typical dimensions in the cross-section less than about $1 / 15$ of typical axial distances, the transverse shear flexibility may be negligible. It was considered a linear section behavior assuming that the internal forces remain in the elastic range (Abaqus, 2002). 


\subsubsection{Effect of residual stresses}

The effect of the residual stresses was ignored in the modeling process of this work based on some FE model evaluations performed by Jung (2006). The evaluations comprised three FE models of the Federal Highway Administration's curved bridge employing different elements to represent the girder flanges. Two models use shell elements for the girder flanges (Model B), but only one of them includes the residual stresses, while the third model (Model A) utilizes beam elements for the girder flanges without considering the residual stresses. The load-deflection response indicated in Figure 3-13 of one of the girders suggests that this effect may be ignored for the global structural response independent of the modeling complexity of the flanges.

\section{G3 MID-SPAN VERTICAL DEFLECTION (mm)}

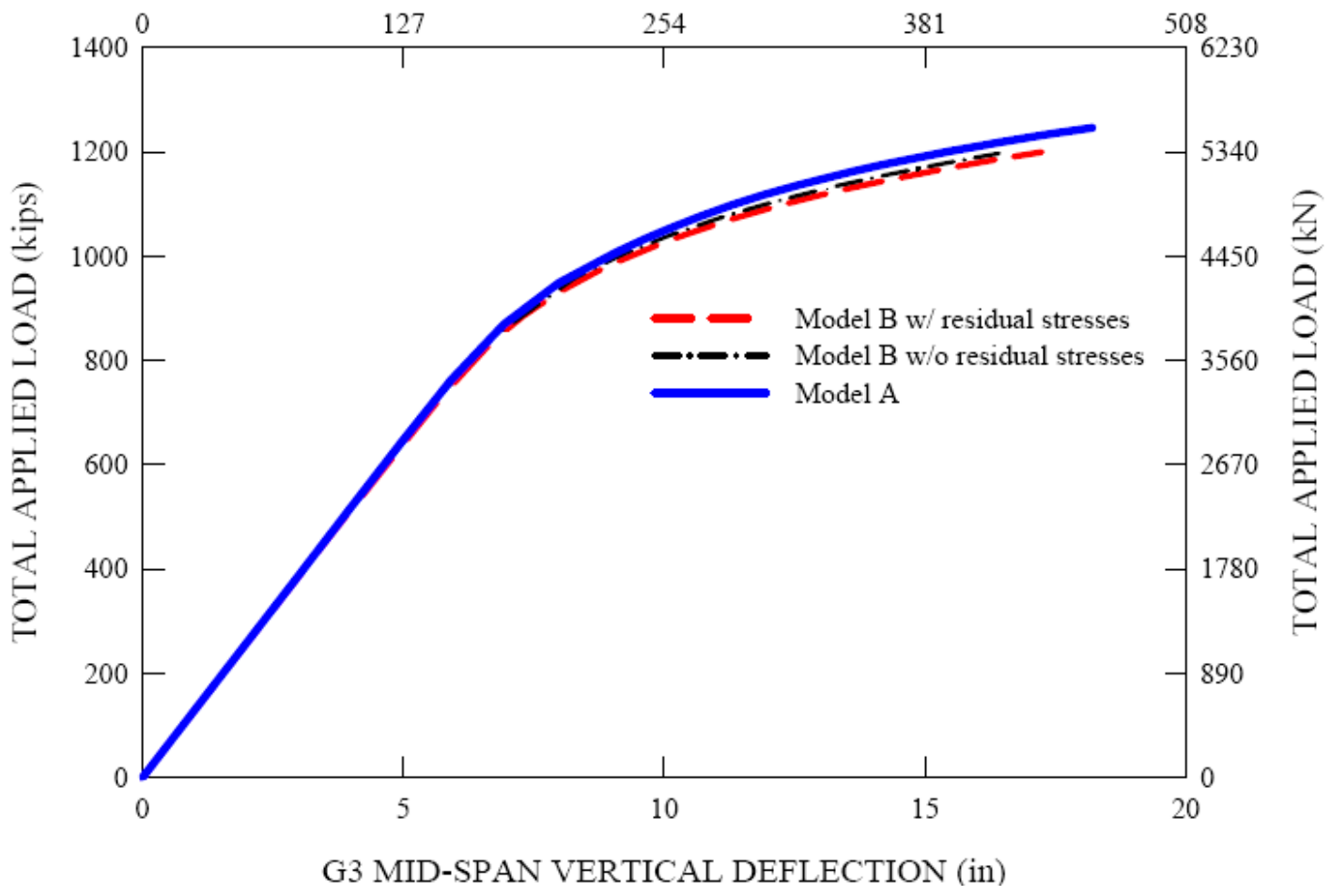

Figure 3-13. Effect of residual stresses in the models (Jung, 2006)

\subsubsection{Mesh density}

The simply supported beam model used to investigate the effect of the hourglassing control was also employed to determine the appropriate mesh density of the 
plate girders for this study. Two cases were considered to analyze the mesh density effect: $i$. when the length (L) of the FEs is varied and their width (w) is fixed; and $i i$. the opposite situation when $\mathrm{w}$ is varied and $\mathrm{L}$ is fixed. The length of the FEs is always measured along the longitudinal direction of the beam as indicated in Figure 3-14.

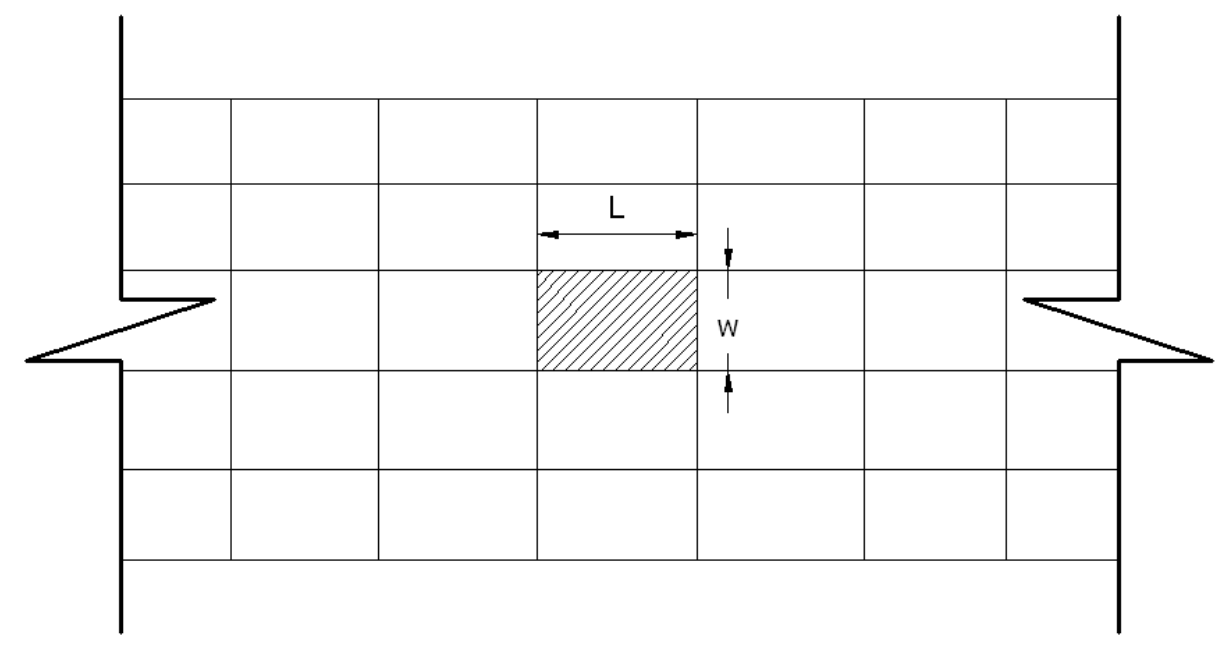

Figure 3-14. Orientation of the finite elements in the simple supported beam model

- Case 1: Varying $L$ and fixed $w$

Several FE models were constructed assuming six different aspect ratios (L/w). A fixed $\mathrm{w}$ of 4 in is considered for this particular case, hence the number of transversal divisions $(\mathrm{NW}=3)$ remains constant. The length of the elements and the number of finite divisions obtained longitudinally (NL) are indicated in Table 3-3.

Table 3-3. Mesh characteristics for Case 1 of the mesh density study

\begin{tabular}{ccc}
\hline $\mathbf{L} / \mathbf{w}$ & $\mathbf{L}$ (in) & $\mathbf{N L}$ \\
\hline 1 & 4 & 60 \\
\hline 1.2 & 4.8 & 50 \\
\hline 1.5 & 6 & 40 \\
\hline 2 & 8 & 30 \\
\hline 3 & 12 & 20 \\
\hline 5 & 20 & 12 \\
\hline
\end{tabular}


A theoretical stress value of $-30 \mathrm{ksi}$ is expected on the superior fiber at the cross section corresponding to the maximum moment. However, Figure 3-15 shows that the models exhibit a minimum stress close to -20ksi due to the low number of elements used throughout the depth of the beam. It is also observed that the fact of increasing NL (i.e., low $\mathrm{L} / \mathrm{w}$ values) does not improve the accuracy of the response, since the stresses are computed at the integration point of the elements and the position of the integration point depends on NW. Therefore, the accuracy of the bending stresses in a long structure is affected by the number of elements used transversally rather than the number of elements used longitudinally.

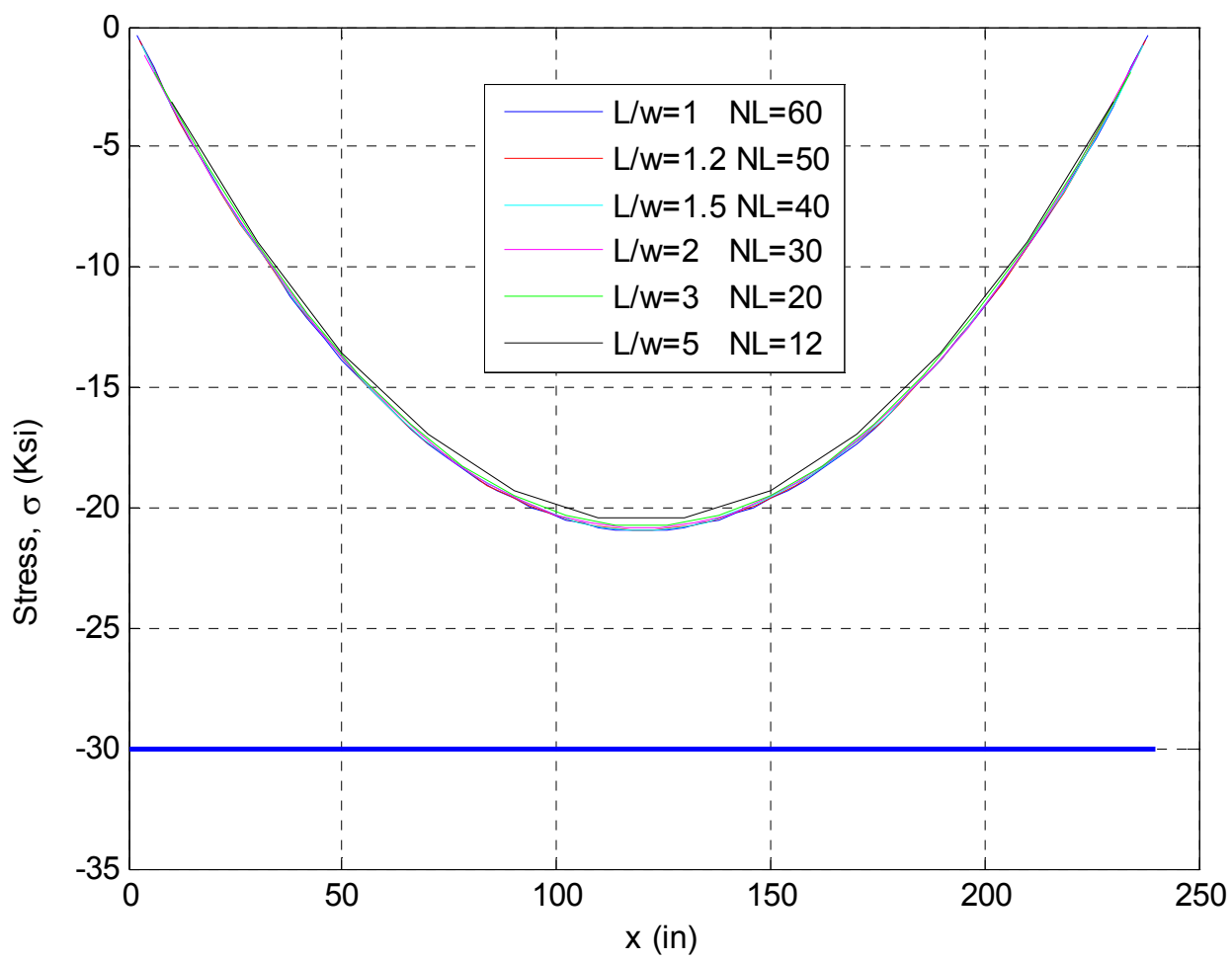

Figure 3-15. Stress results for Case 1 of the mesh density study

- Case 2: Varying w and fixed L

In this case, the FE models were constructed assuming a fixed $\mathrm{L}$ of 6 in for the elements. Therefore, a total of 40 longitudinal divisions (NL) were generated. The values for $\mathrm{w}, \mathrm{NW}$ and $\mathrm{L} / \mathrm{w}$ are presented in Table 3-4. 
Figure 3-16 shows that high aspect ratios may be used in order to improve the accuracy of the results and decrease the number of elements. However, the selection of the appropriate NW depends on the stress gradient throughout beam depth, which is defined as the bending moment over the moment of inertia (M/I). For example, the use of 16 elements across the beam height would produce a reasonable level of accuracy for this particular example according to Figure 3-16.

Table 3-4. Mesh characteristics for Case 2 of the mesh density study

\begin{tabular}{ccc}
\hline $\mathbf{L} / \mathbf{w}$ & $\mathbf{w}$ (in) & $\mathbf{N W}$ \\
\hline 1 & 6 & 2 \\
\hline 1.5 & 4 & 3 \\
\hline 2 & 3 & 4 \\
\hline 3 & 2 & 6 \\
\hline 4 & 1.5 & 8 \\
\hline 8 & 0.75 & 16 \\
\hline 16 & 0.375 & 32 \\
\hline
\end{tabular}

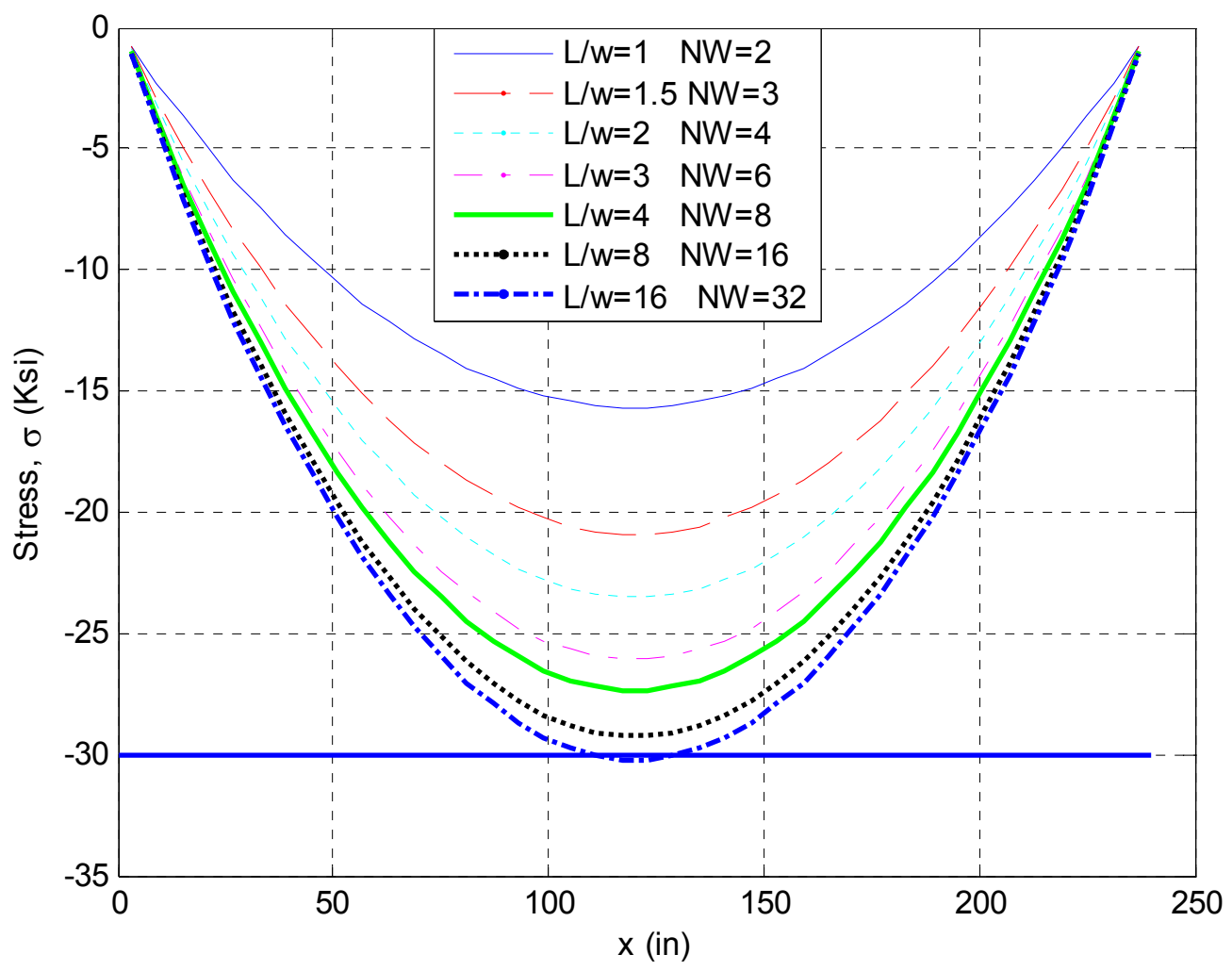

Figure 3-16. Stress results for Case 2 of the mesh density study 
Conversely, the stress gradient produced by LFB is much lower than the one corresponding to a major-axis bending, as in the previous example. Therefore, a lower NW may be required for the LFB in order to achieve a reasonable level of accuracy. For that reason, a curved bridge model from the parametric study explained in Chapter 4 was analyzed using different NWs as shown in Figure 3-17, where FEL $=$ NW and $A R=(L / w)$. From the figure, a mesh configuration using six elements across the flange width with aspect ratios close to four was selected as the appropriate to produce significant results.

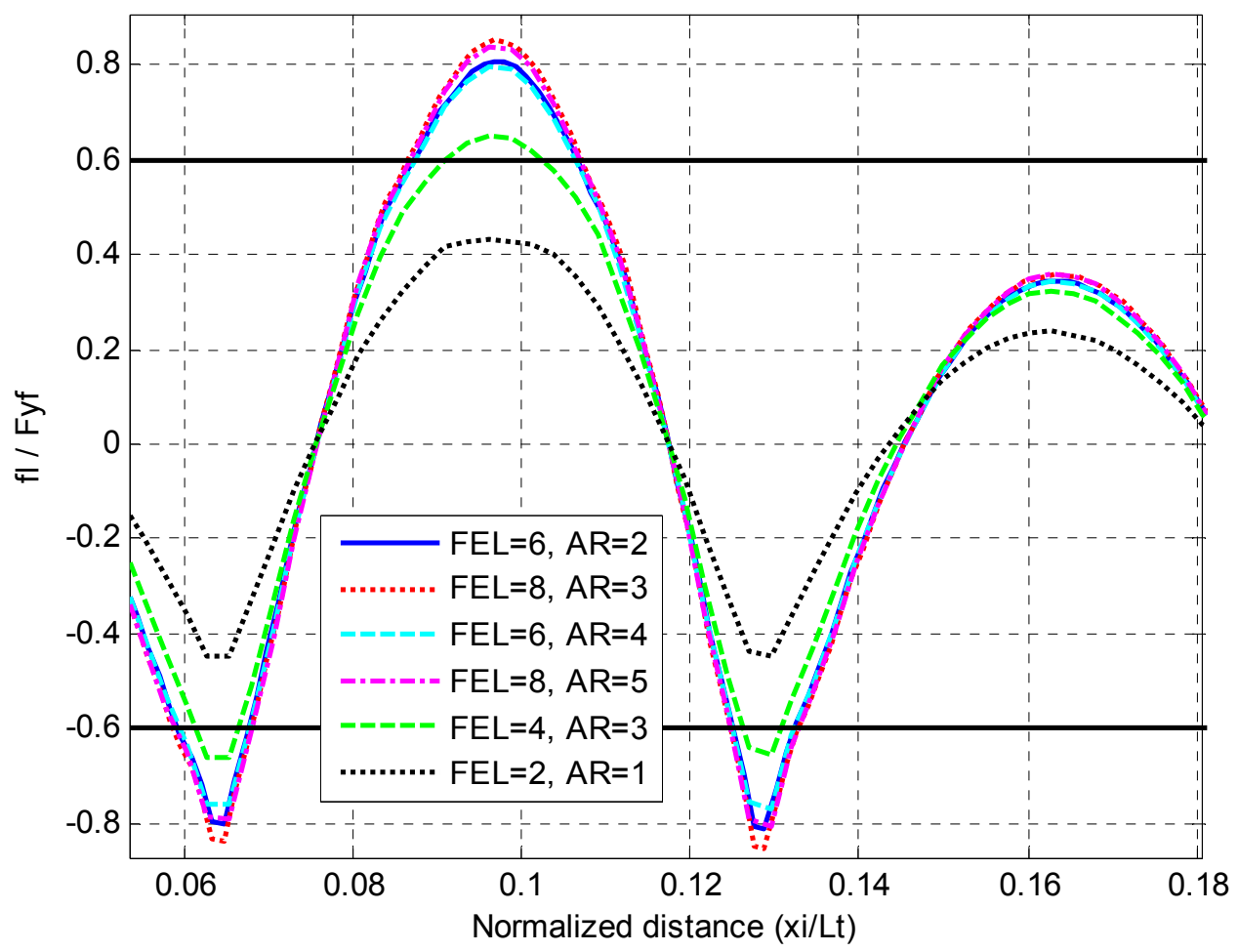

Figure 3-17. Maximum LFB for a bridge configuration model with $\mathrm{Lm}=150 \mathrm{ft}$

All shell elements in the girders of straight and skewed bridges are 12-inch long, while for curved bridges this length corresponds to the central radius of the bridge, i.e. the outer-girder elements are longer than those corresponding to the inner girder. On the other hand, the number of elements used throughout the web depth depends on its height, since an aspect ratio close to one was selected for all web elements. Finally, the shell elements of the concrete slab were set as squares with side lengths equal to 24-in for 
straight bridge configurations, with some slight modifications in the shape for skewed and curved bridge models.

\subsection{Model Validation}

This section is focused on the correlation of FEA predictions using the modeling procedures described previously with results from representative experimental tests. The tests described below exhibit different levels of complexity, starting from one steel Ishaped girder and finishing with the Federal Highway Administration's full-scale curved bridge structure.

\subsubsection{Non-composite steel I girder}

Three steel I-girder specimens, labeled as "S" (Shallow), "M" (Medium) and "D" (Deep) were reported by Schilling and Morcos (1988). The girders were designed with ultra-compact flanges and the slenderness of the webs was varied from a value that was nearly compact for specimen " $\mathrm{S}$ " to a value that was at the upper limit for classification as non-compact for specimen "D". Specimen "M" had an intermediate web slenderness between those corresponding to specimens " $S$ " and " $D$ ". However, only specimen " $D$ " was selected for the verification process since its web slenderness is more representative of the girders assessed in this study (see Figure 3-18).
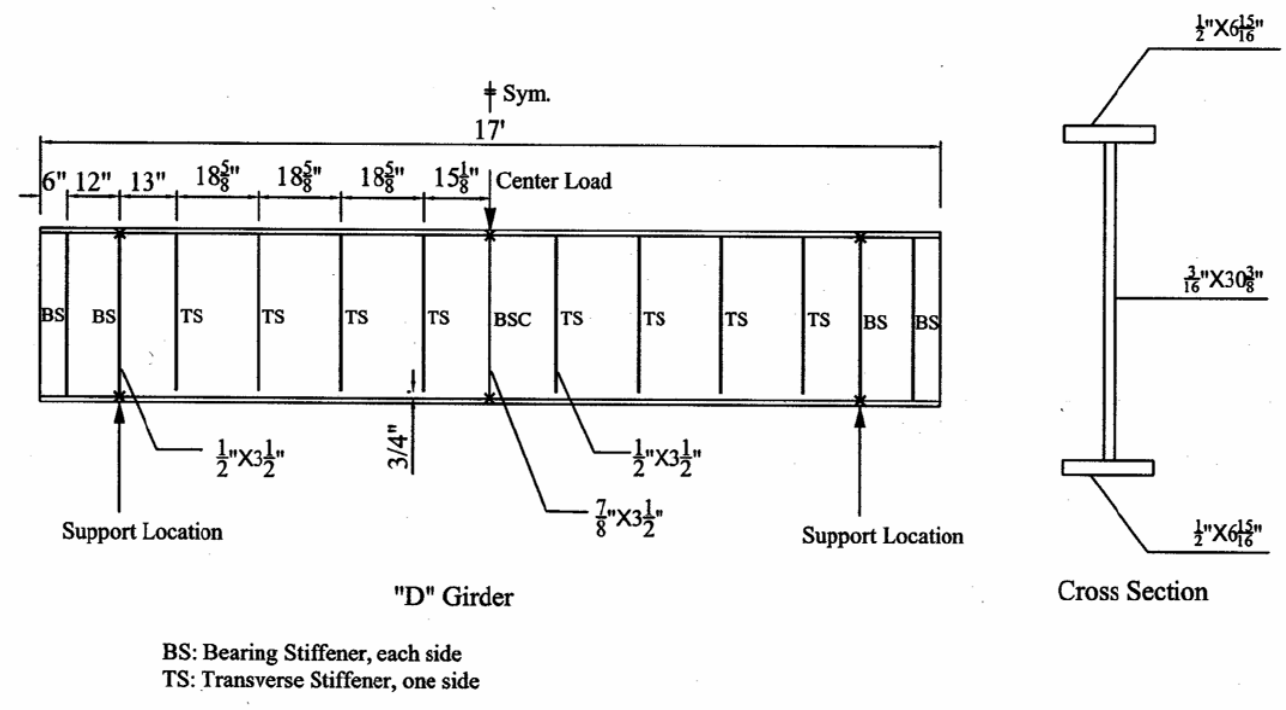

Figure 3-18. "D” girder specimen (Schilling \& Morcos, 1988) 
All three girders were fabricated using ASTM A572 Grade 50 steel. Each specimen was tested as a simply supported beam with a single load at midspan to simulate the conditions of an interior support in a continuous-span bridge. However, the specimen was inverted with respect to the normal bridge position to simplify the testing setup. Therefore, the center load in the test simulated the reaction at the interior support of the bridge, while the simply supports in the test simulated the adjacent inflection points in the bridge. Figure 3-19 shows a comparison between the numerical and experimental load-displacement responses. A good agreement is observed for the elastic range but the model fails to reach the maximum load obtained during the test. This is probably due to the geometrical imperfections added to the model that are intended to account for physical misalignments of the specimen. The presence of geometrical imperfections in the model forces some degree of buckling to occur before the critical load is reached.

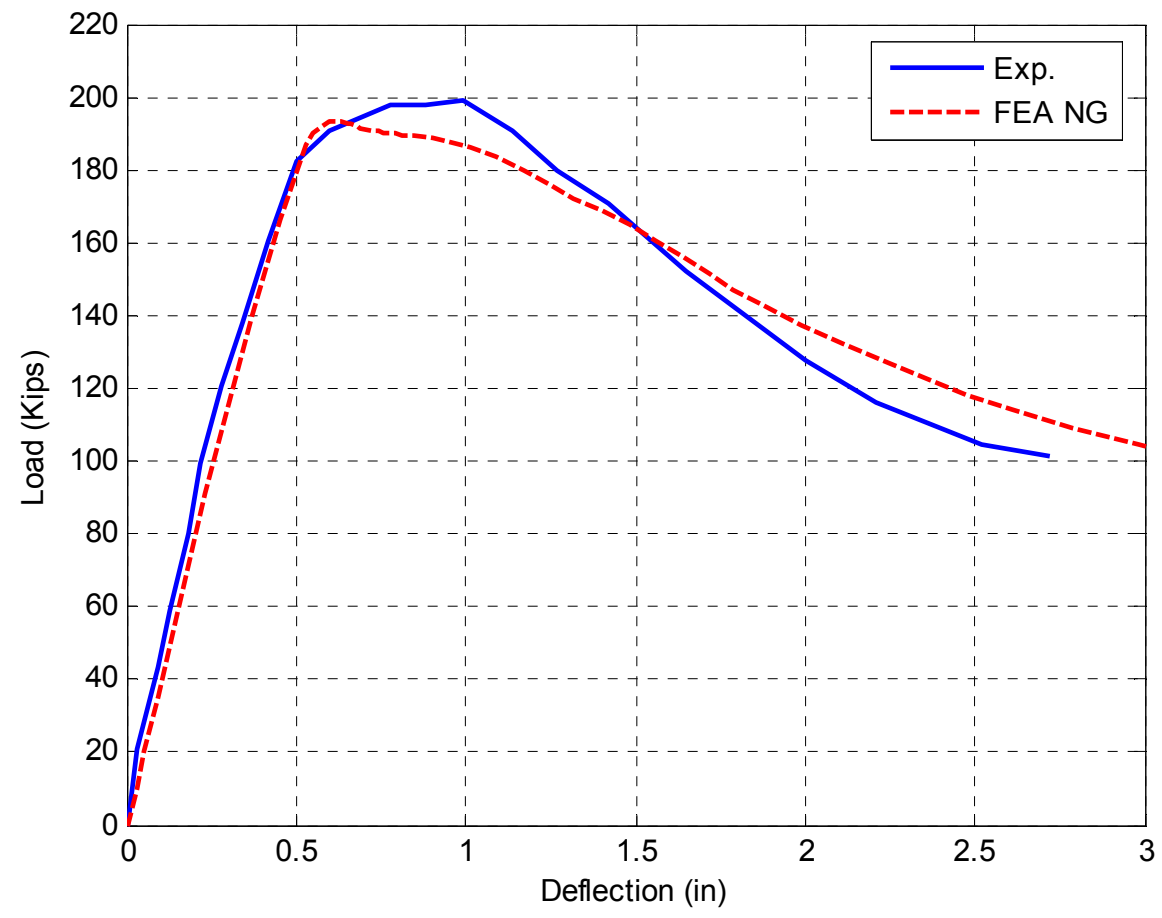

Figure 3-19. Load-displacement response for a single steel I-shaped girder 


\subsubsection{Composite I girder}

Mans, Yakel and Azizinamini (2001) investigated experimentally the applicability of the positive bending ductility requirements to composite girders constructed of HPS$70 \mathrm{~W}$. The first specimen, POS1, was designed to reach the plastic moment capacity and achieve a high level of ductility. Intermediate stiffeners were placed to give a very conservative shear strength controlled by elastic buckling. The flanges and the web were made of HPS-70W steel and the stiffeners were fabricated from steel with the minimum specified yield strength of 50Ksi. The slab is $60 \mathrm{in}$ in width and $7.25 \mathrm{in}$ in depth.

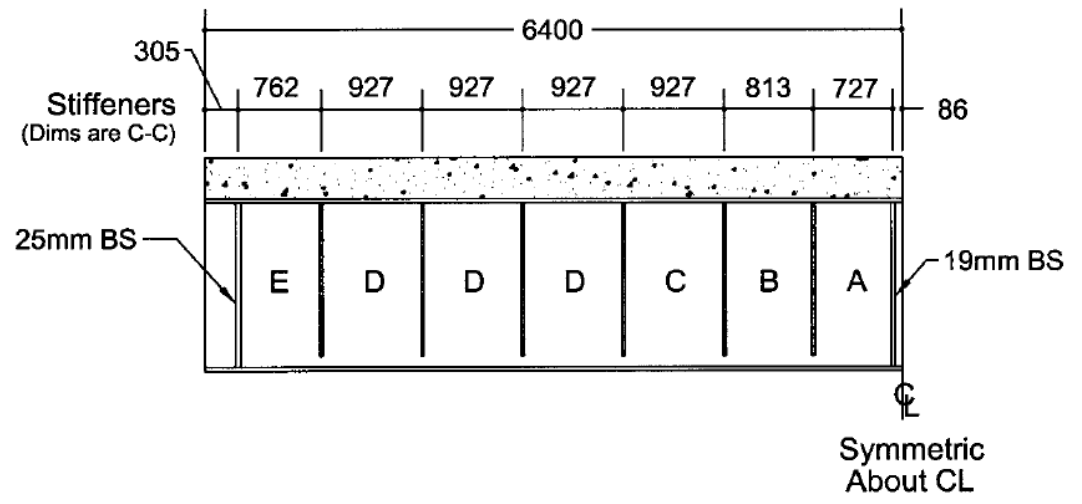

Figure 3-20. Geometry of Specimen POS1 (Mans, Yakel \& Azizinamini, 2001)

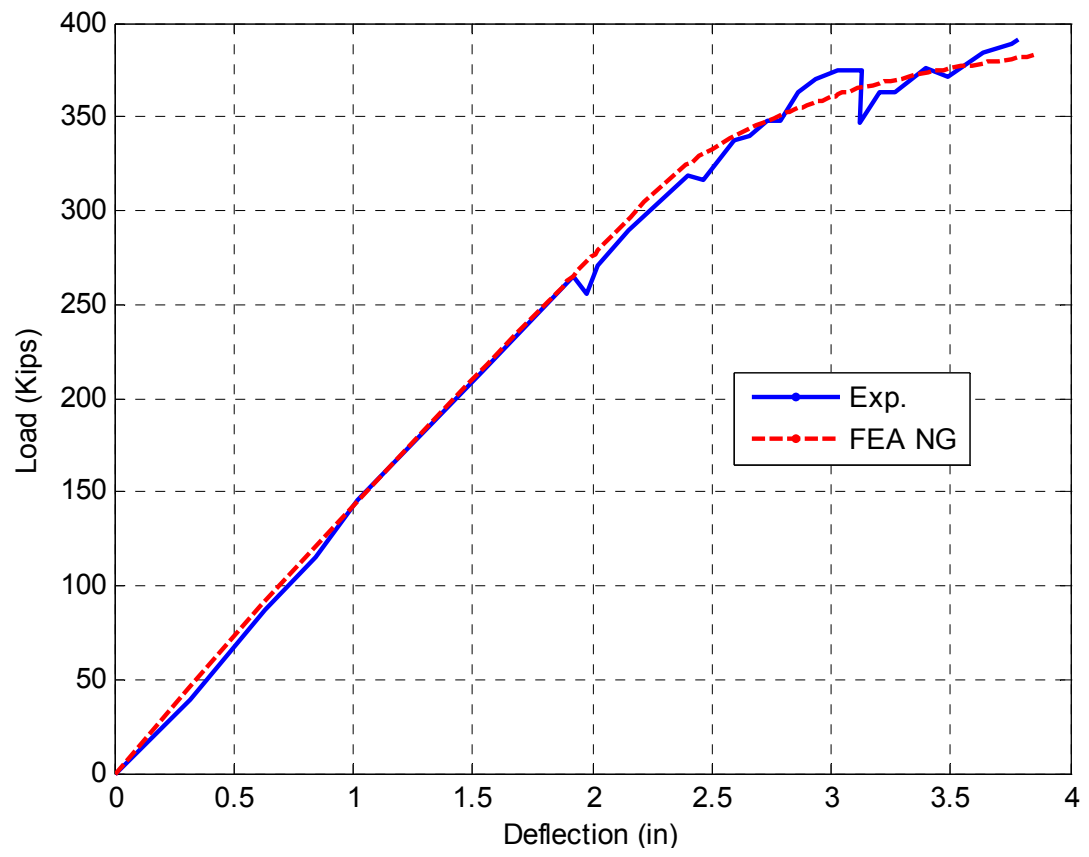

Figure 3-21. Load-displacement response for a single composite steel I-shaped girder 
Figure 3-20 shows the overall dimensions and locations of intermediate and bearing stiffeners for the specimen. Good agreement is observed between the experimental and numerical global response of the specimen according to Figure 3-21.

\subsubsection{Composite straight steel I-girder bridge}

Tiedeman, Albrecht and Cayes (1993) compared the measured reactions, stresses, moments, displacements and rotations of a bridge tested in the Federal Highway Administration's laboratory. The bridge was a 0.4-scale model of a prototype bridge. The prototype bridge was designed by the autostress design method for the AASHTO HS20 truck loading and alternate military loading specified for bridges on the interstate system. The test bridge consisted of two symmetrical $56 \mathrm{ft}$ spans and three girders spaced $6.8 \mathrm{ft}$ on centers as shown in Figure 3-22. The modular deck was composed of 35 precast concrete panels. Diaphragms were located over each pier, and every $10 \mathrm{ft}$ from the end piers per AASHTO requirements. The diaphragms consisted of rolled members (WT $2 \mathrm{x}$ 6.5) arranged in a V-type cross bracing as shown in Figure 3-23.

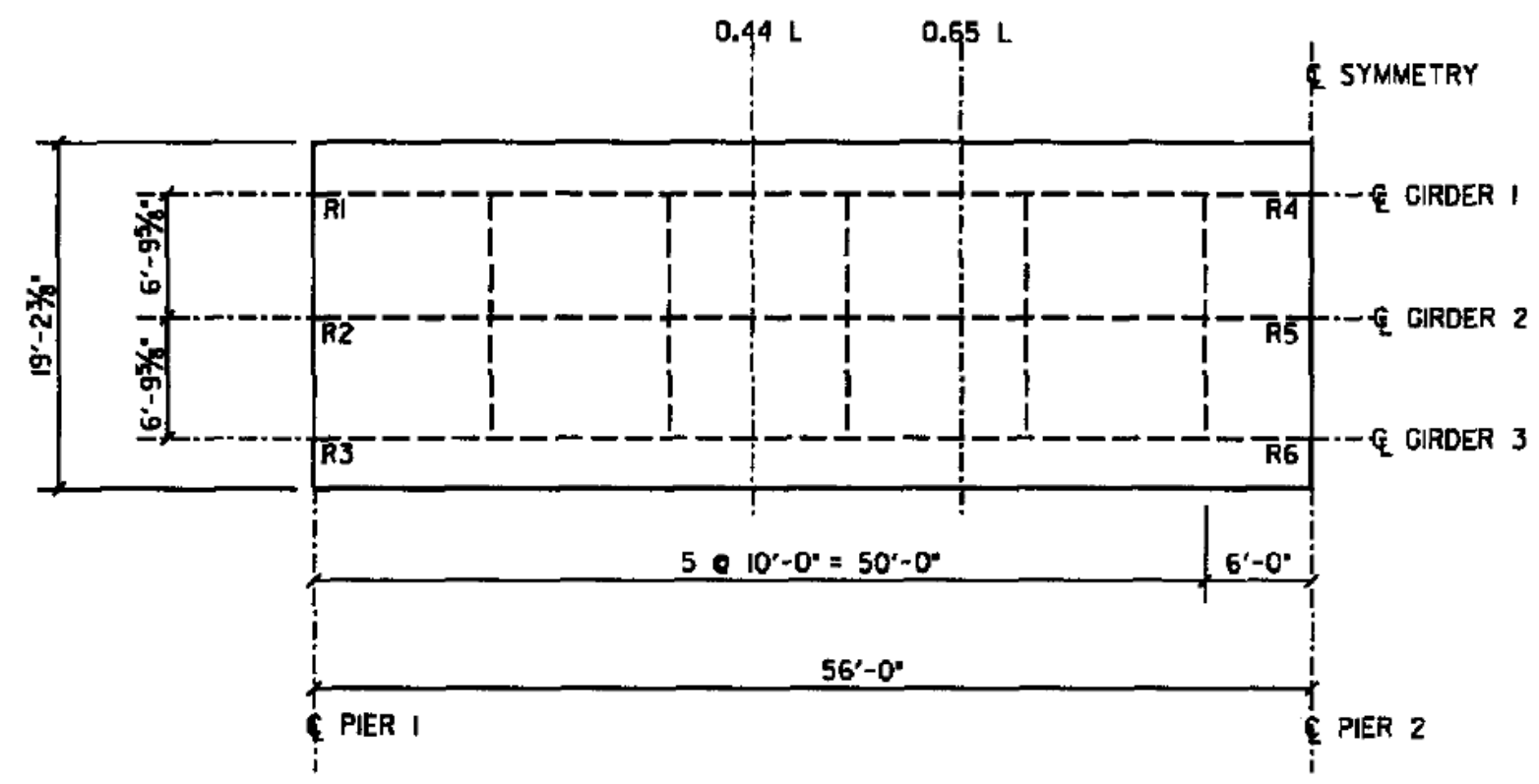

Figure 3-22. Plan view of test bridge (Tiedeman, Albrecht \& Cayes, 1993) 


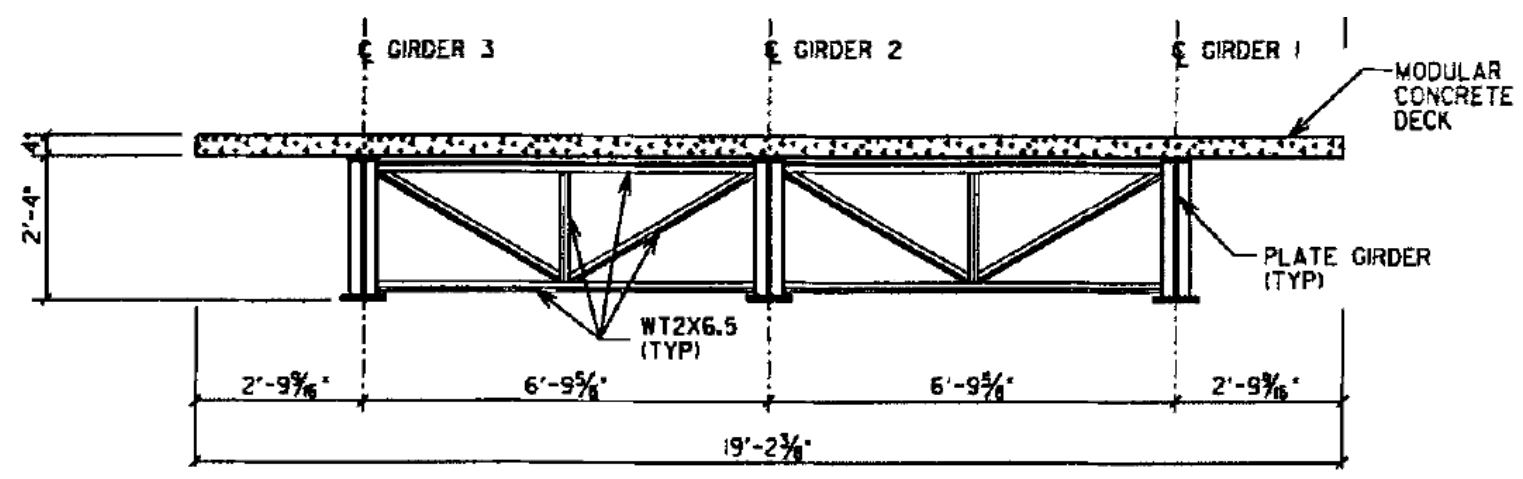

Figure 3-23. Cross section of test bridge (Tiedeman, Albrecht \& Cayes, 1993)

A single axle of an AASHTO HS20 truck was simulated with a pair of concentrated loads that were applied by a hydraulic jacking system. The two 7Kip loads of each axle were applied transversely on the west span only. The spacing of the loads corresponds to the scaled spacing of the wheels of the truck in a single lane or in multiple lanes. The loads are placed longitudinally at $0.44 \mathrm{~L}$ and $0.65 \mathrm{~L}$ to produce the largest approximate positive and negative moment at $0.4 \mathrm{~L}$ and $1.0 \mathrm{~L}$ (the interior support), respectively. In both cases, the loads were applied on one span only.

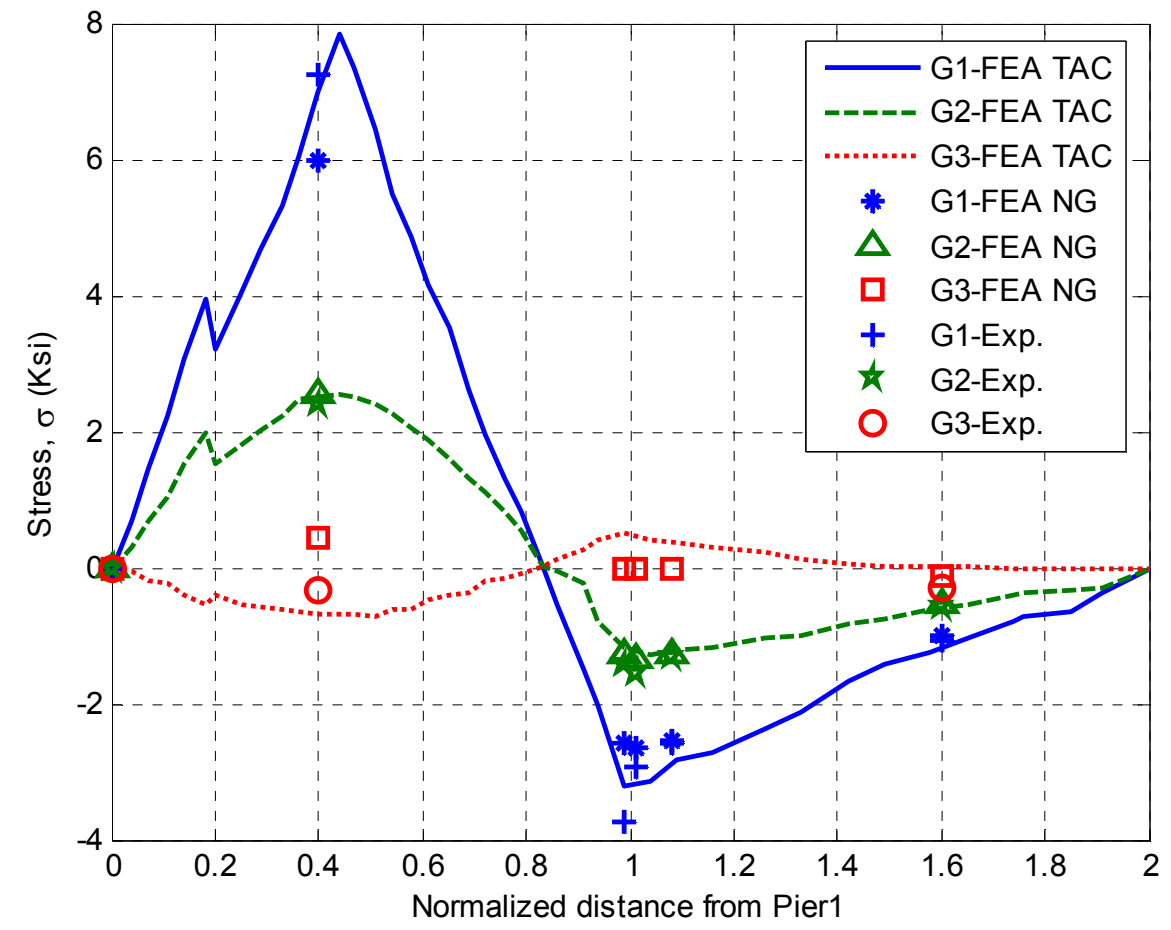

Figure 3-24. Comparison of measured and calculated bottom-flange stresses 
Figure 3-24 shows a comparison of the measured and the FEA bottom-flange stresses when the axles of a single line are placed as close as possible to Girder 1 to maximize the moments on that exterior girder at $0.44 \mathrm{~L}$. The numerical results predict satisfactorily the experimental measurements, principally for those girders closer to the loads (G1 and G2).

\subsubsection{Composite curved steel I-girder bridge}

In 1992, the Federal Highway Administration developed the Curved Steel Bridge Research Project to investigate the behavior of curved steel bridges and their components in order to establish more rational design specifications. The study comprised three phases: an erection study, a component strength study (see Figure 3-25) and a composite bridge study. Jung et. al. (2005) reported some experimental results from the third phase, which are used in this work for the validation process.

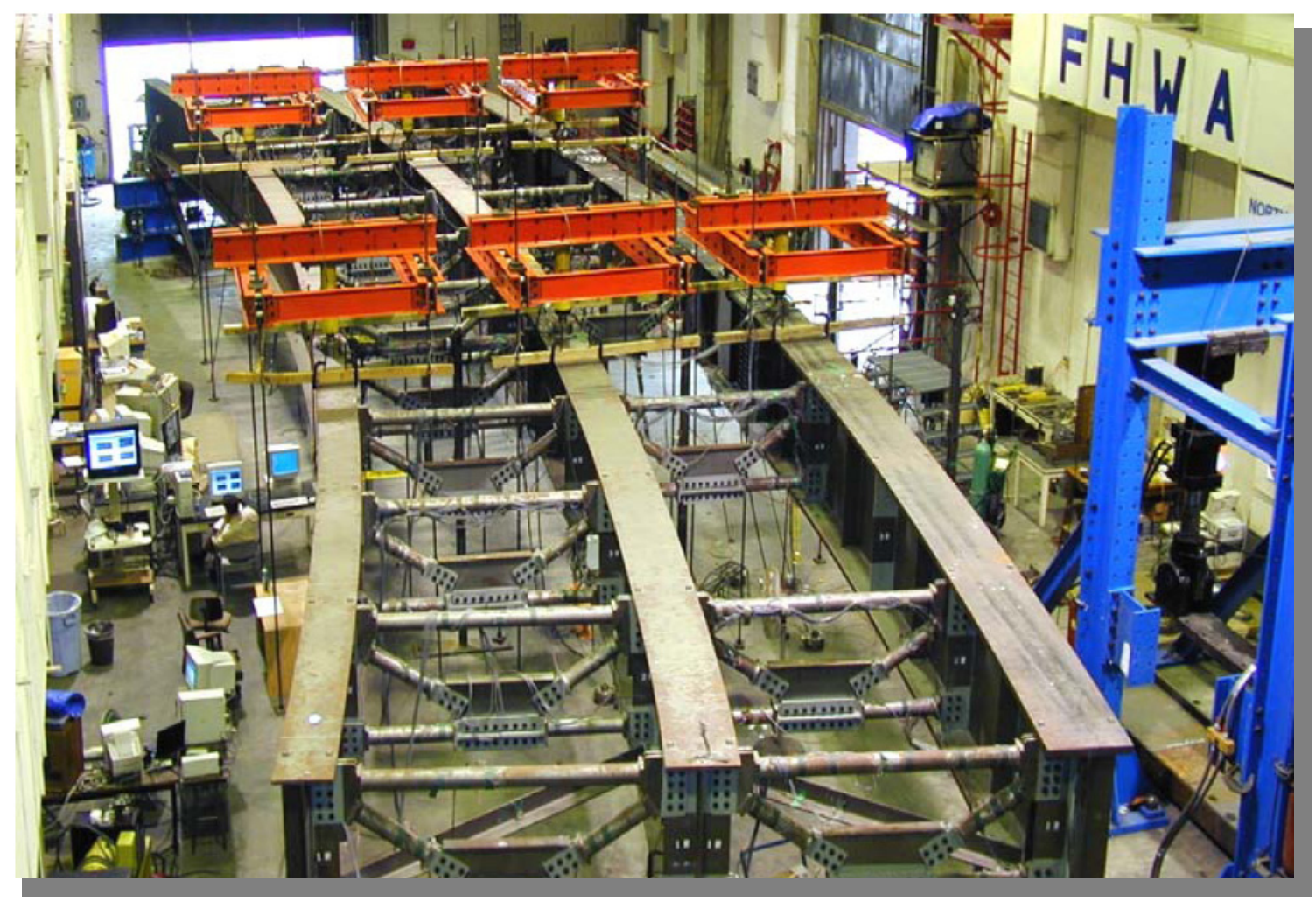

Figure 3-25. Bridge test during the component strength study (Jung, 2006) 
Figure 3-26 illustrates the cross section and plan view of the test bridge. The cross section of the bridge consists of three prismatic I-girders spaced at $8.75 \mathrm{ft}$ and the bridge span is $90 \mathrm{ft}$ measured along its centerline. The radii of curvature are $191.25 \mathrm{ft}$, $200 \mathrm{ft}$ and $208.75 \mathrm{ft}$ for the inside (G1), middle (G2) and outside (G3) girders, respectively. All of the steel plates used A709 Gr.50 steel with the exception of the bottom flange of G3 which used HPS 70W. The cast-in-place concrete slab is 8in thick with 3 in haunches and $3 \mathrm{ft}$ overhangs. The composite action between the slab and the plate girders is given by studs (6in $\mathrm{x}$ 3/4in) spaced at 6in both longitudinally and transversely. The bridge has five equally spaced K-shaped cross frames, which are strong enough to develop the ultimate capacity of the composite bridge system without any cross-frame member failure.
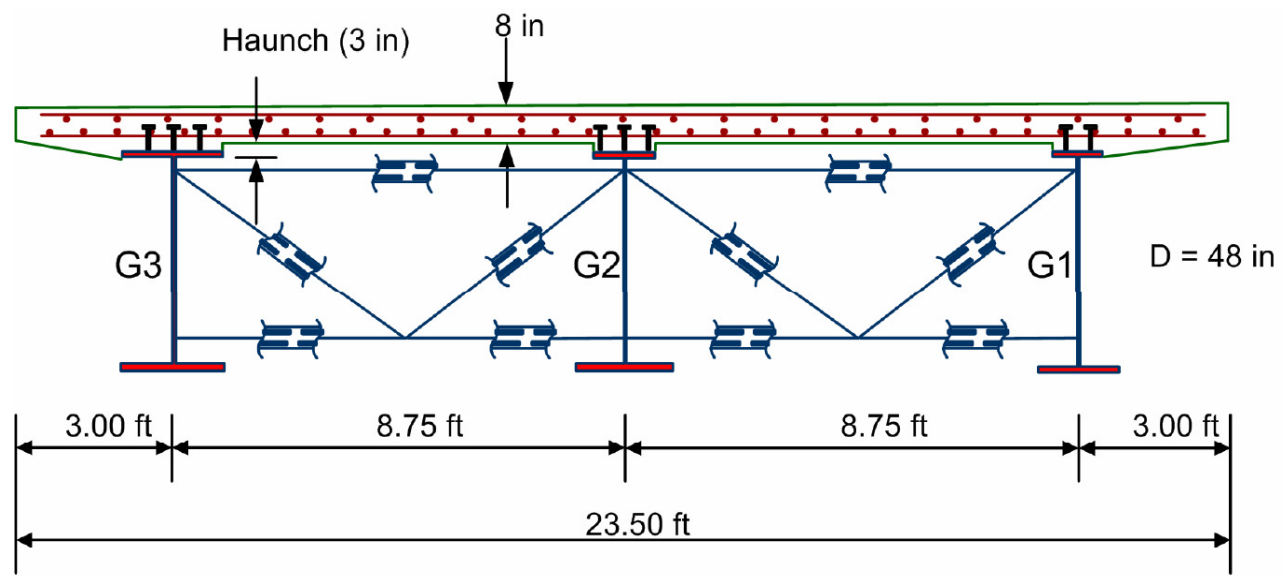

a. Cross section of the test bridge

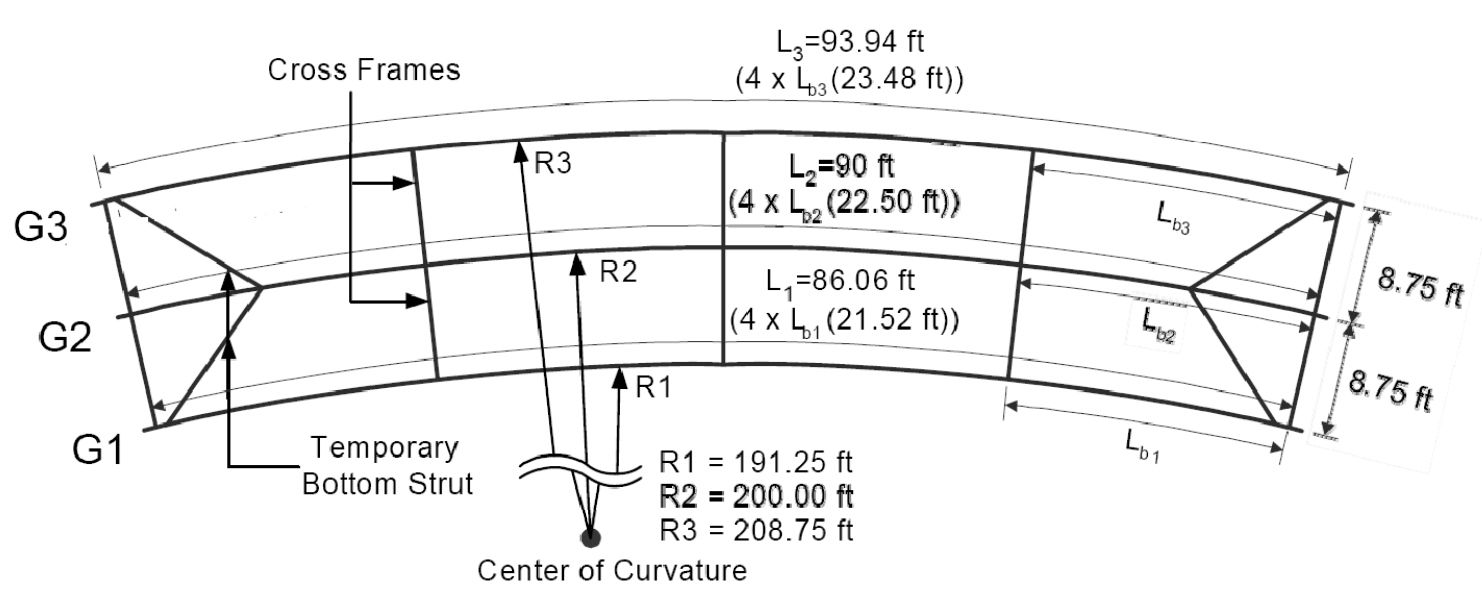

b. Plan view of the test bridge

Figure 3-26. Geometrical characterization of the bridge test (Jung, 2006) 
The experimental results shown in Figure 3-27, Figure 3-28 and Figure 3-29 correspond to a testing loading that simulates the effects of two design trucks aligned side-by-side at the middle of the bridge plus two lanes. This loading condition governs the flexural design of G3. In general, a satisfactory agreement is observed between the numerical and experimental results for the global response of the test bridge. Although the model fails to predict the maximum positive LFB stresses, the maximum negative LFB stresses (which are larger than the positive ones) seem to be adequately calculated.

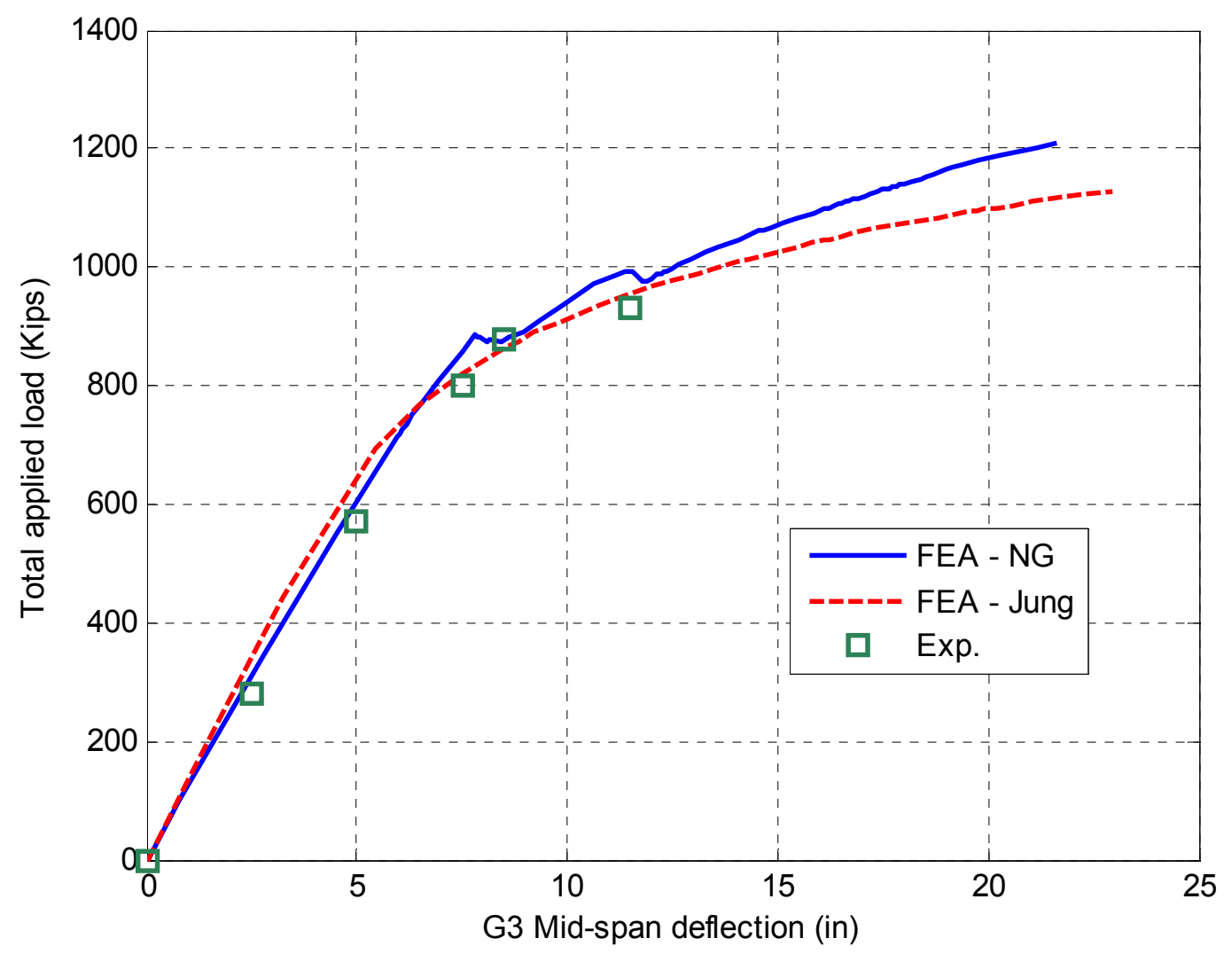

Figure 3-27. Applied load vs. G3-mid-span deflection 


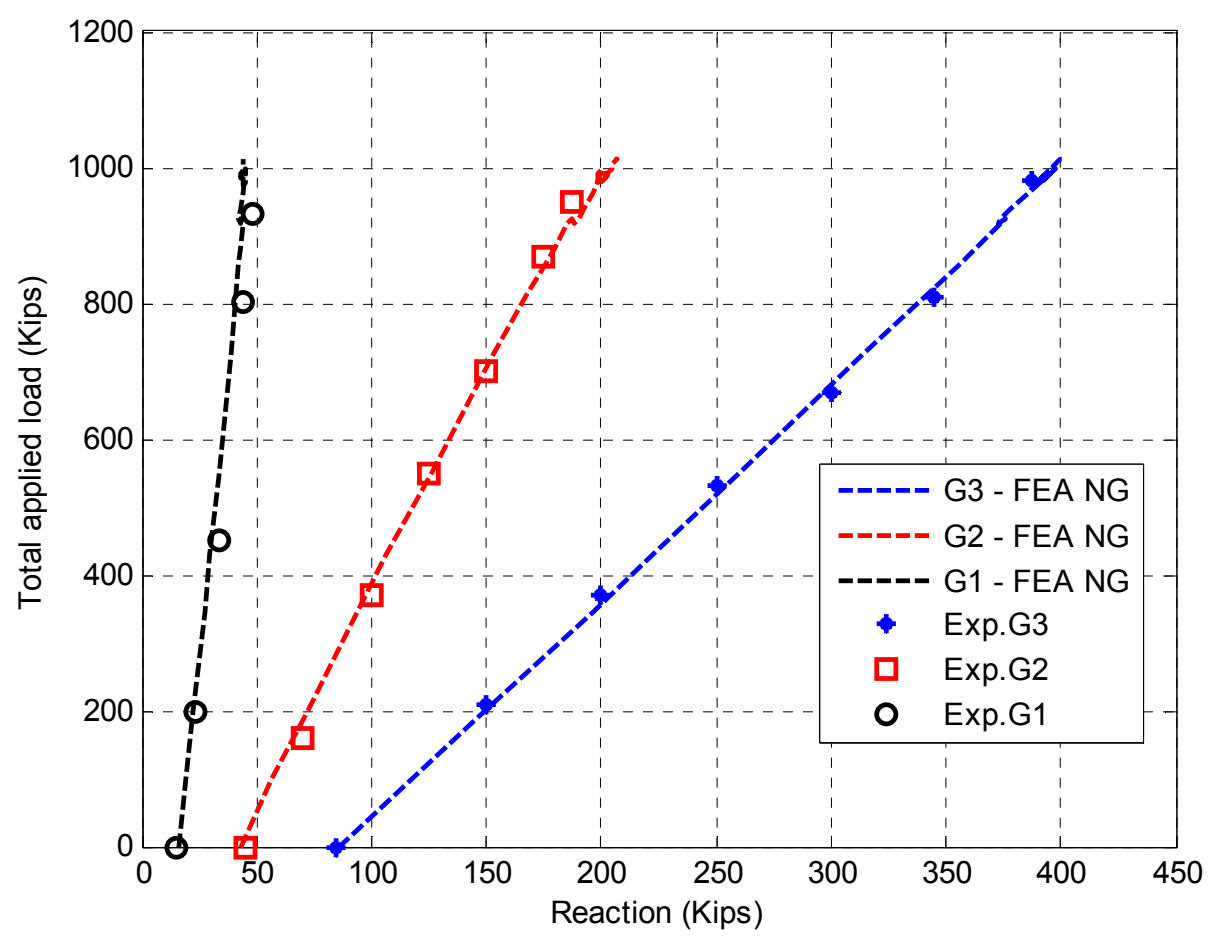

Figure 3-28. Applied load vs. vertical reactions

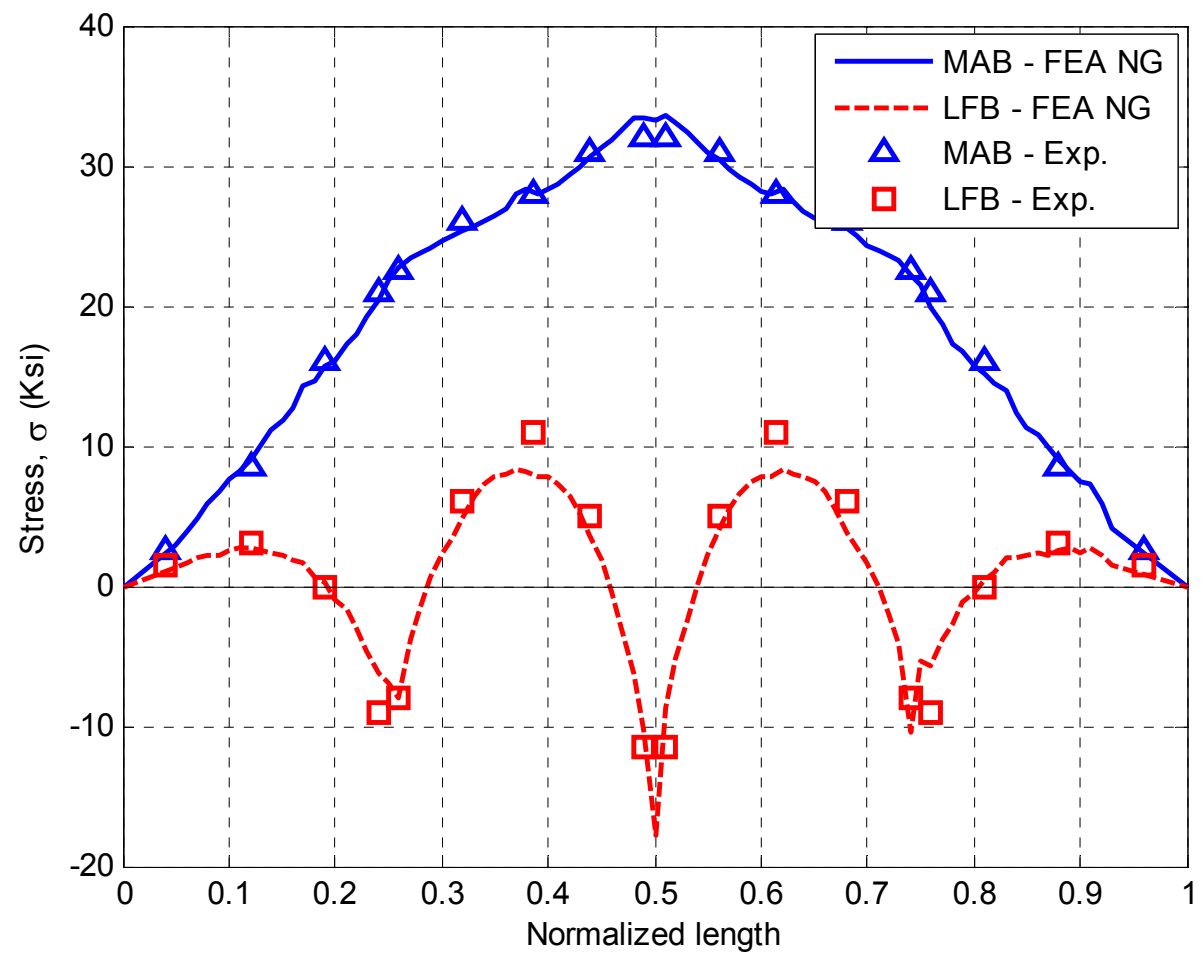

Figure 3-29. G3 bottom flange stresses at a load level of 570Kips (MAB: Major-axis bending, LFB: lateral flange bending) 


\subsection{Analyses}

A user-defined sub-routine in Matlab ${ }^{\circledR}$ was used to create the input file for each FE model that was analyzed by ABAQUS® Version 6.7.1, a commercially available FEA software program. The initial loads such as the steel weight and the distributed construction loads were applied using linear static stress analyses, since the structure behaves within the elastic ranges without the presence of instabilities at this load level. However, the concrete deck placement was simulated using a nonlinear static analysis to consider probable material nonlinearity or geometrically nonlinear behavior causing buckling or local instabilities.

The nonlinear analysis is based on the modified Riks algorithm available in Abaqus ${ }^{\circledR}$. This method has the ability to pass beyond the limit point and trace the unloading portion of the nonlinear equilibrium path, as shown in Figure 3-30. The Riks method uses the load magnitude as an additional unknown; it solves simultaneously for loads and displacements. Therefore, another quantity must be used to measure the progress of the solution. ABAQUS/Standard uses the "arc length," l, along the static equilibrium path in load-displacement space. This approach provides solutions regardless of whether the response is stable or unstable (Abaqus, 2002).

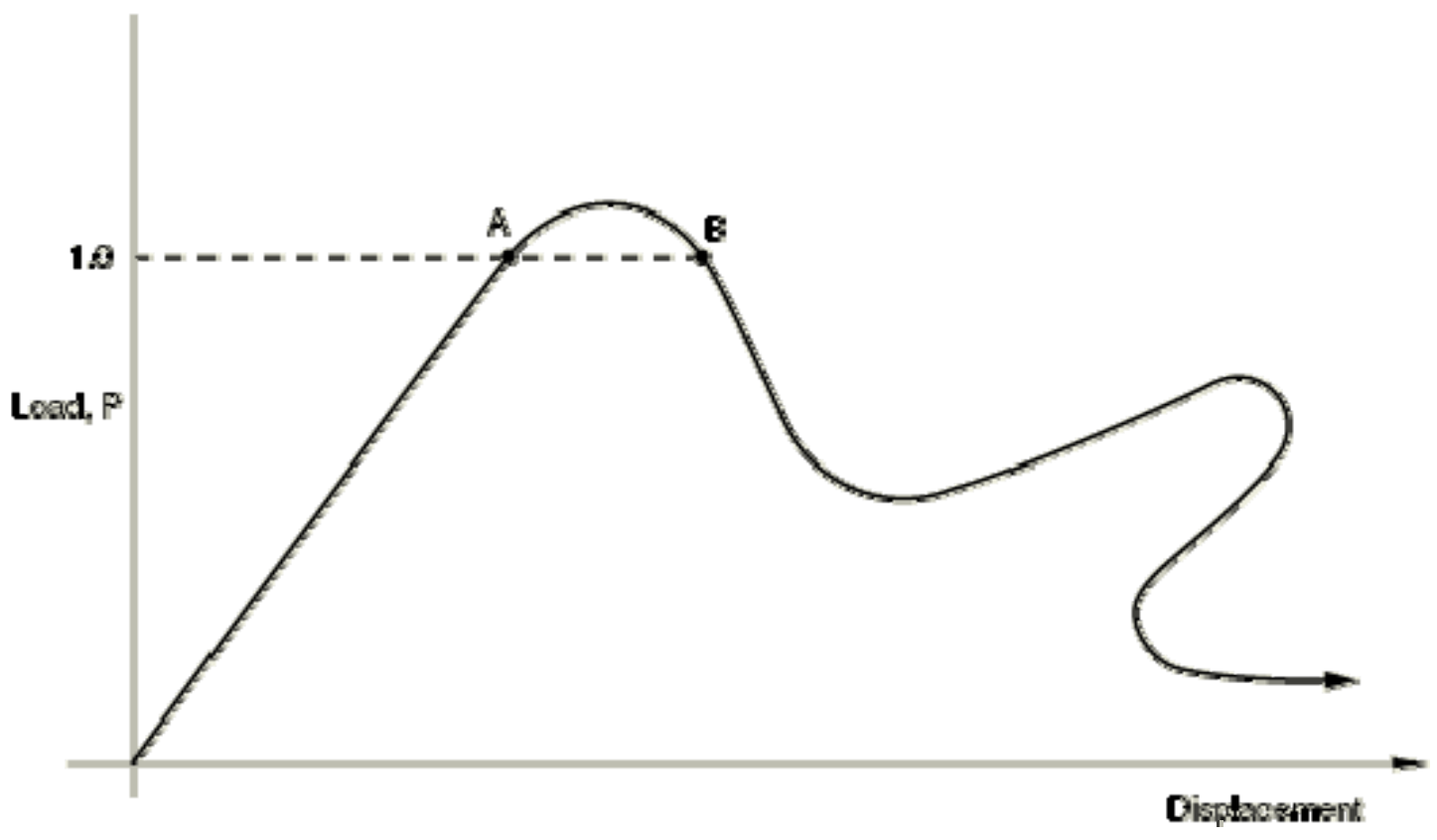

Figure 3-30. Load-displacement response of an unstable system (Abaqus, 2002) 
This method basically finds the solution of a single equilibrium path in a space that is defined by the nodal variables and the loading parameter. The solution during each increment is found by moving a given distance along the tangent line to the current solution point and then searching for equilibrium in the plane that not only passes through the point thus obtained, but also is orthogonal to the same tangent line. Once this plane is determined, the Newton's method is used to solve the equilibrium equations. It is assumed that the response is reasonably smooth, i.e. sudden bifurcations do not occur. The algorithm of the modified Riks method is shown in Figure 3-31, (Righman, 2005).

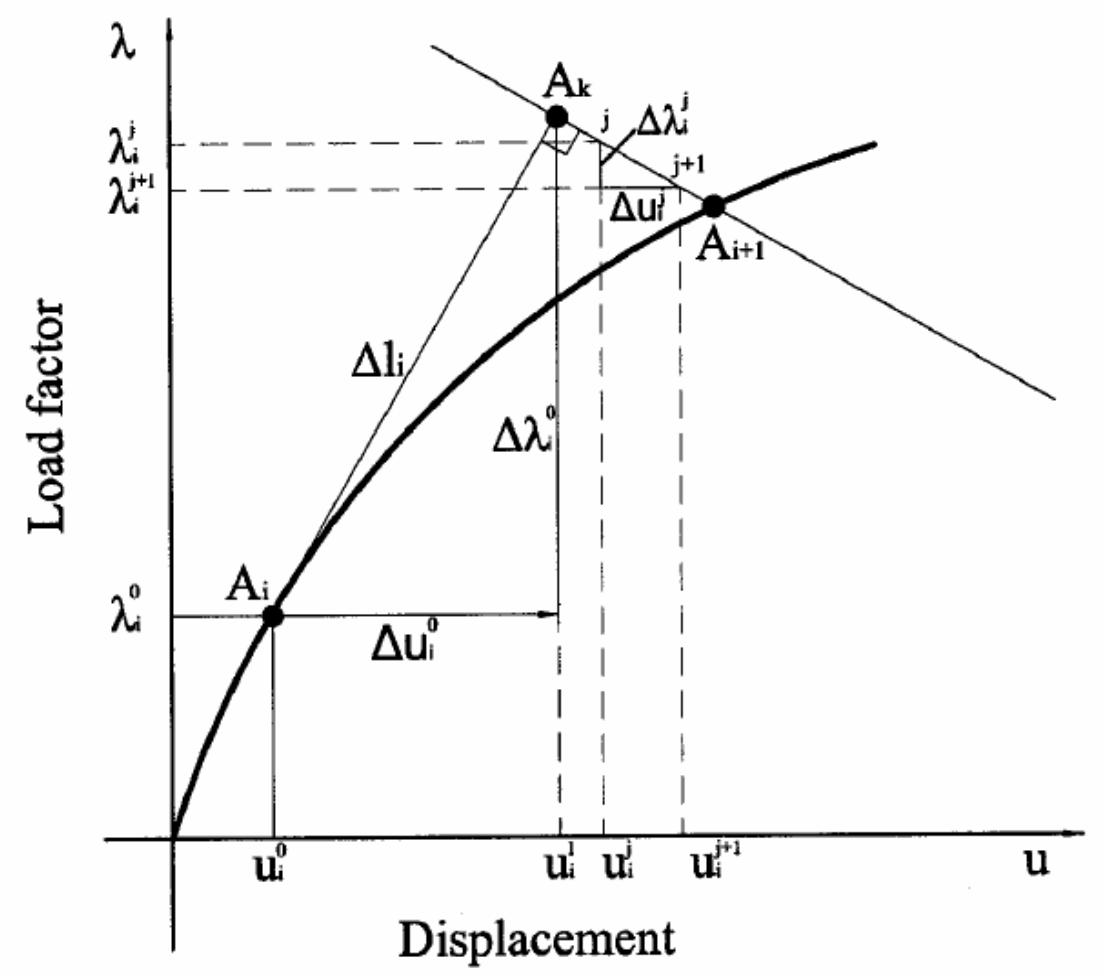

Figure 3-31. Modified Riks algorithm (Righman, 2005) 


\section{Chapter 4: Parametric study of the lateral flange bending during construction}

A parametric study is used to investigate the effects of the deck-placement process on the LFB of straight, skewed and curved steel I-girder bridges. The parametric study is defined by: $i$. the parameters considered as significant in the response of the structure; ii. the loads that represent the real conditions during the deck casting sequence; iii. the design according to the code provisions of the parametric configurations; $i v$. the models used to represent the physical behavior of the bridges; and $v$. the analyses defined for each bridge configuration to represent the deck casting sequence.

\subsection{Parameters}

The selection of the variables in this work is intended to cover a wide range of variation in the parameters that govern the practical design of skewed and curved steel bridges. All bridge configurations have three spans arranged such that the end spans $\left(L_{e}\right)$ are $80 \%$ of the middle span $\left(L_{m}\right)$. This span configuration helps to assure an optimum and homogeneous depth of the girders in all spans (NSBA, 2002). The cross sections consist of four girders spaced at $12 \mathrm{ft}$ centers with $3.6 \mathrm{ft}$ deck overhangs. Structural steel having a specified minimum yield stress of 50Ksi is used throughout. The deck is conventional cast-in-place concrete with a specified minimum 28-day compressive strength of $4 \mathrm{Ksi}$. The total deck thickness is $9.5 \mathrm{in}$, including a one-half inch integral wearing surface. The concrete deck haunch is assumed to be 3.5in deep measured from the top of the web to the bottom of the concrete deck. A future wearing surface of $25 \mathrm{psf}$ is specified for design. Permanent steel deck forms of $15 \mathrm{psf}$ are also assumed to be used between girders.

Table 4-1 presents the varying parameters considered in this project and their corresponding values. Straight, skewed and curved bridge configurations (ST, SK and $\mathrm{CV}$ ) were generated for each middle span length $\left(L_{m}\right)$ considered in the study, resulting in a total of 21 hypothetical bridges without including the additional configurations 
generated for each cross-frame spacing. Curved bridges with skewed supports were not considered in this work.

Table 4-1. Varying parameters

\begin{tabular}{lc}
\hline \multicolumn{1}{c}{ Parameter } & Value \\
\hline Middle span length $\left(L_{m}\right)$ & $150 \mathrm{ft}-240 \mathrm{ft}-300 \mathrm{ft}$ \\
\hline Ratio of $L_{m}$ to radius of curvature $\left(L_{m} / R\right)$ & $0.30-0.45-0.60$ \\
\hline Skew angle $(\theta)$ & $0^{\circ}-30^{\circ}-45^{\circ}-60^{\circ}$ \\
\hline Range of cross-frame spacing $\left(L_{b}\right)$ & $22 \mathrm{ft}-42 \mathrm{ft}$ \\
\hline
\end{tabular}

Bridge configurations using different cross-frame distances $\left(L_{b}\right)$ were constructed as indicated in Table 4-2 and Table 4-3 to analyze the variation of the LFB effects which depend directly on this parameter.

Table 4-2. Cross-frame spacing for positive moment regions

\begin{tabular}{ccccc} 
& & \multicolumn{3}{c}{$\boldsymbol{L}_{\boldsymbol{b}}(\mathbf{f t})$} \\
\cline { 3 - 5 } Bridge type & End span, $\boldsymbol{L}_{\boldsymbol{e}}(\mathbf{f t})$ & $\mathbf{1 2 0}$ & $\mathbf{1 9 2}$ & $\mathbf{2 4 0}$ \\
\hline ST, SK, CV & Model Lb1 & 25 & 25 & 22 \\
\hline ST, SK, CV & Model Lb2 & 27 & 28 & 27 \\
\hline ST, SK, CV & Model Lb3 & 32 & 33 & 35 \\
\hline ST, SK, CV & Model Lb4 & 37 & 39 & 37 \\
\hline ST & Model Lb5 & 42 & 42 & 42 \\
\hline ST, SK, CV & Middle span, $\boldsymbol{L}_{\boldsymbol{m}}(\mathbf{f t})$ & $\mathbf{1 5 0}$ & $\mathbf{2 4 0}$ & $\mathbf{3 0 0}$ \\
\hline ST, SK, CV & Model Lb1 & 22 & 25 & 22 \\
\hline ST, SK, CV & Model Lb2 & 27 & 27 & 28 \\
\hline ST, SK, CV & Model Lb3 & 32 & 35 & 34 \\
\hline ST & Model Lb4 & 40 & 37 & 38 \\
\hline
\end{tabular}

Table 4-3. Cross-frame spacing for negative moment regions

\begin{tabular}{ccccc} 
& & \multicolumn{3}{c}{$\boldsymbol{L}_{\boldsymbol{b}}(\mathbf{f t})$} \\
\hline Bridge type & Middle span, $\boldsymbol{L}_{\boldsymbol{m}}(\mathbf{f t})$ & $\mathbf{1 5 0}$ & $\mathbf{2 4 0}$ & $\mathbf{3 0 0}$ \\
\hline ST, SK, CV & Model Lb1 & 20 & 20 & 20 \\
\hline ST, SK, CV & Model Lb2 & 21 & 26 & 24 \\
\hline ST, SK, CV & Model Lb3 & 27 & 33 & 31 \\
\hline ST, SK, CV & Model Lb4 & 35 & 36 & 36 \\
\hline ST & Model Lb5 & 36 & 36 & 40 \\
\hline
\end{tabular}




\subsection{Loads}

The loading condition corresponds to the loads acting during the deck casting process: permanent dead loads and construction loads. Permanent dead loads include the self weight of the structural members and construction loads comprise the following elements (NSBA, 2002; KDoT, 2005):

- Overhang form brackets: $50 \mathrm{lb}$ each, spaced every $3 \mathrm{ft}$

- Formworks: $10 \mathrm{lb} / \mathrm{ft}^{2}$

- Screed rail: $85 \mathrm{lb} / \mathrm{ft}$

- Railing: $25 \mathrm{lb} / \mathrm{ft}$

- Walkway: $50 \mathrm{lb} / \mathrm{ft}^{2}$

- Finishing machine: $813 \mathrm{lb} /$ wheel for a total of 4 wheels per exterior girder (see Figure 4-1 and Figure 4-2)

The factored loads correspond to the Strength Load Combination I of the Specifications, which recommend that the load factors shall not be taken less than 1.25 and 1.5 for the dead and the construction loads, respectively (AASHTO, 2007). Therefore, these recommended factors were used for the ultimate loads presented in this work.

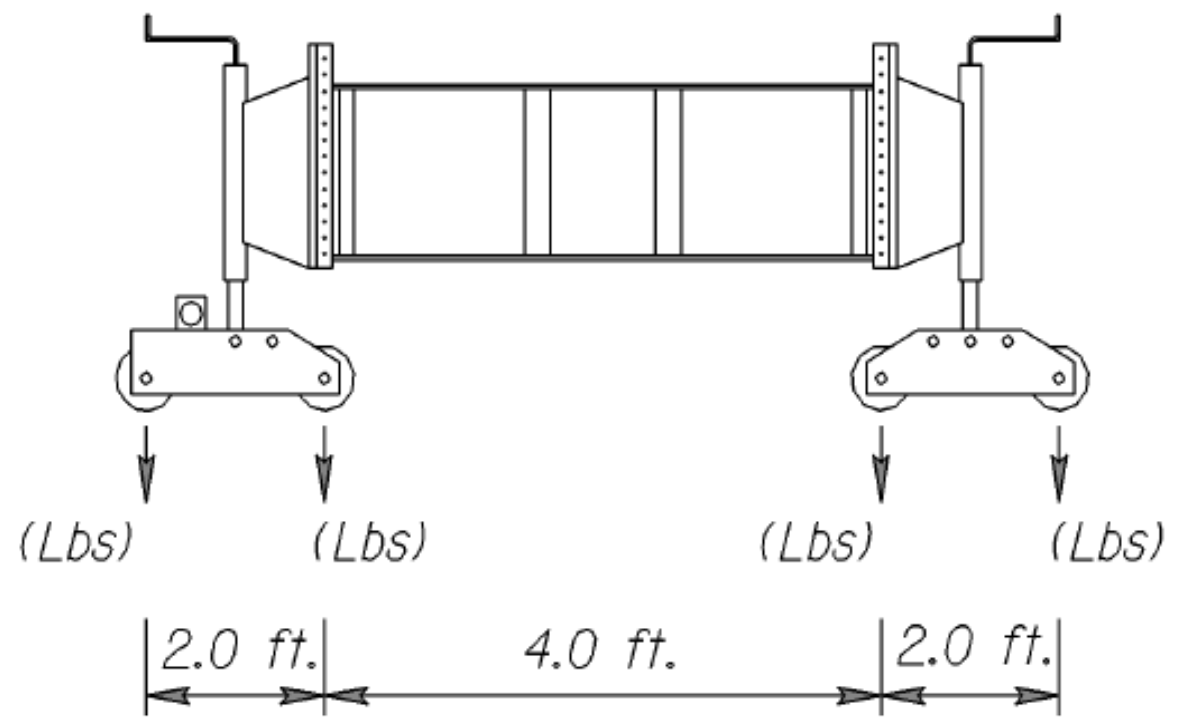

Figure 4-1. Visualization of the finishing machine on the exterior girder (KDT, 2005) 


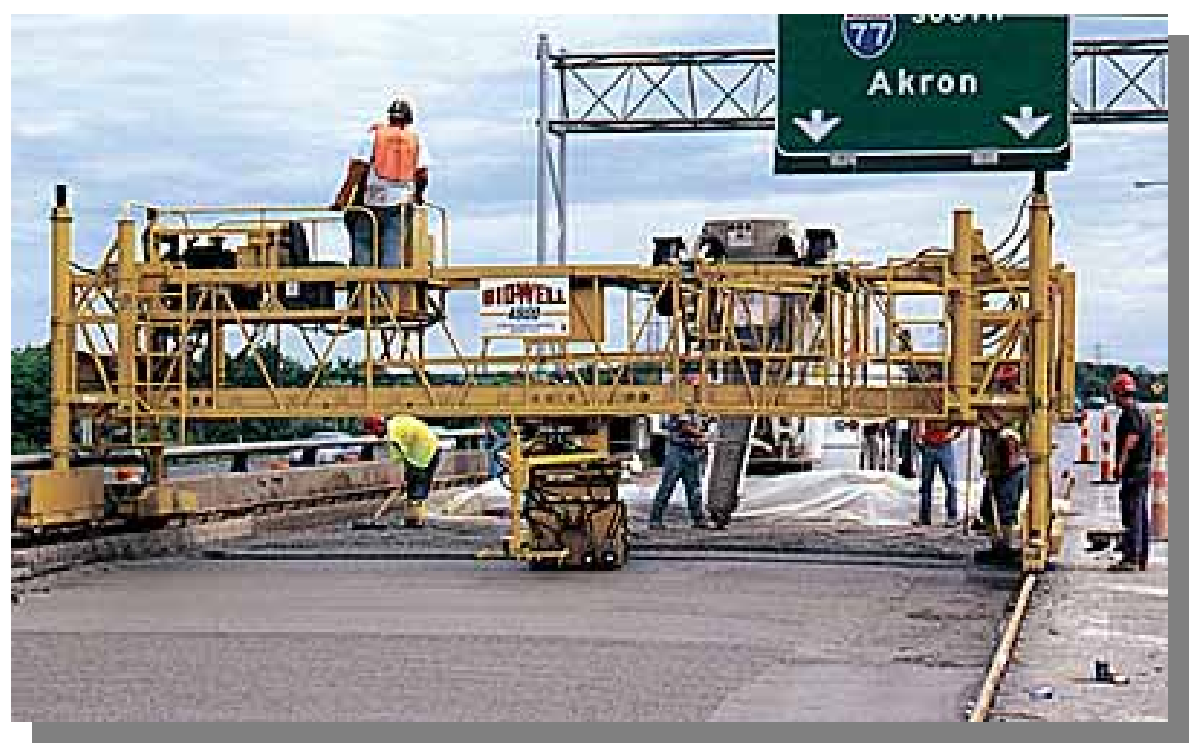

Figure 4-2. General view of a finishing machine (Bid-Well 4800)

\subsubsection{Deck placement sequence}

A symmetric deck placement sequence composed of three basic consecutive stages was considered as shown in Figure 4-3, where the concrete is poured first in the positive moment region of the end spans followed by that corresponding to the middle span (NSBA, 2002). The sequence is completed by placing the concrete in the negative moment regions over the pier zones. This sequence scheme is intended to minimize cracking of the concrete slab primarily in the negative moment regions. Additional intermediate stages are incorporated into the basic three-step sequence to consider the effect of the finishing-machine wheels in the positive moment regions as shown in Figure 4-4.

द

$\varepsilon$

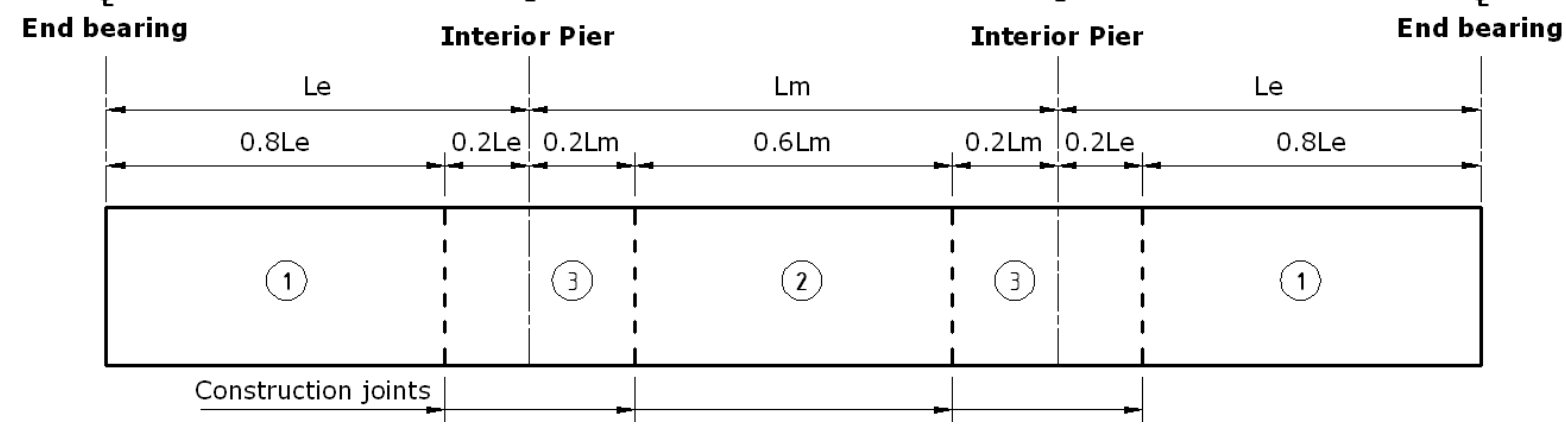

Figure 4-3. Basic deck placement sequence 
The LFB effects due to distributed $(w u)$ and concentrated $(P u)$ loads were evaluated separately in the positive moment regions. First, the effects of the distributed loads are assessed from the casting stages that consider only the weight of the fresh concrete, i.e. Castings 2 and 4 in Figure 4-4 for the end and middle spans, respectively. Then, the concentrated load effects are analyzed from the casting stages that include the finishing-machine wheels combined with the fresh concrete, i.e. Castings 1 and 3.

The negative moment regions are mostly controlled by the final stage from the sequence shown in Figure 4-4. However, the maximum bending results in these regions may be obtained at intermediate phases. Therefore, the complete casting sequence is considered for the pier regions independent of the casting stage.

Casting 1: Concrete and finishing machine - End spans

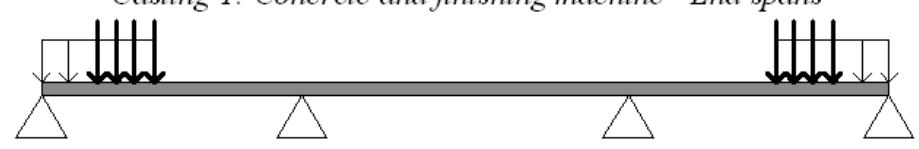

Casting 2: Concrete - End spans

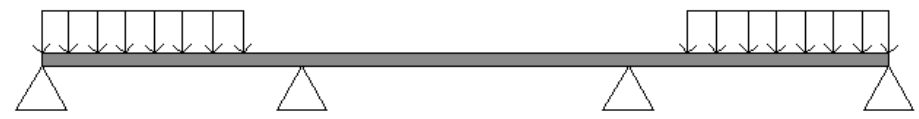

Casting 3: Concrete and finishing machine - Middle span

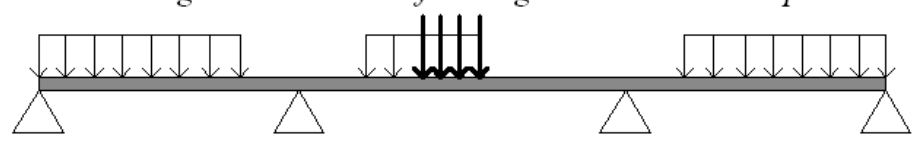

Casting 4: Concrete - Middle span

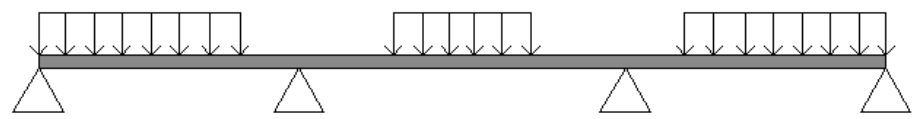

Casting 5: Concrete and finishing machine - Pier regions

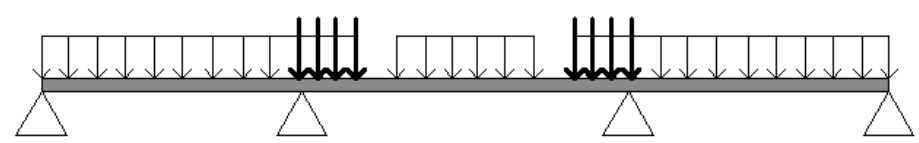

Figure 4-4. Detailed deck casting sequence 
For skewed bridges, the deck placement was considered parallel to the supports. The construction joints for curved and skewed bridges are radial and parallel to the skew angle, respectively.

\subsection{Structural design}

Girders, cross frames and stiffeners of the straight bridge configurations were sized according to 2007 AASHTO Specifications, based on the parameters and loads defined previously. Changes of section that coincide with the construction joints are assumed in regions close to the piers, as shown in Figure 4-3. Table 4-4 includes the girder plate sizes for the different cross sections employed at the straight bridges. The same sections were used for the skewed and curved bridges analyzed in this work to achieve one of the research objectives consisting of finding the maximum available skew angle and curvature for bridges originally designed as straight.

Table 4-4. Girder plate sizes

\begin{tabular}{|c|c|c|c|}
\hline$L_{m}(\mathbf{f t})$ & $\begin{array}{c}\text { End Span } \\
\text { S1 }\end{array}$ & $\begin{array}{l}\text { Pier Zone } \\
\text { S2 }\end{array}$ & $\begin{array}{c}\text { Middle Span } \\
\text { S3 }\end{array}$ \\
\hline \multirow{4}{*}{150} & TF: 20"x1" & TF: 18”x2” & TF: $16 "{ }^{7}{ }^{7} / 8 "$ \\
\hline & BF: 20"x1" & BF: 20"x2" & BF: 18"x1" \\
\hline & $\mathrm{W}: 60 " \times 1 / 2 "$ & $\mathrm{~W}: 60 " \mathrm{x}$ 1/2" & W: : 60"x 1/2" \\
\hline & $\mathrm{L}_{\mathrm{S}}: 96 \mathrm{ft}$ & $\mathrm{L}_{\mathrm{S}}: 54 \mathrm{ft}$ & $\mathrm{L}_{\mathrm{S}}: 90 \mathrm{ft}$ \\
\hline \multirow{4}{*}{240} & TF: 24"x11/2" & TF: 28"x23/4" & TF: 20"x11/4" \\
\hline & BF: 24"x11/4" & BF: $28 " x 23 / 4 "$ & BF: 24"x11/4" \\
\hline & $W: 80 " x^{5} / 8 "$ & W: $80 " x^{5} / 8 "$ & $W: 80 " x^{5} / 8 "$ \\
\hline & $\mathrm{L}_{\mathrm{S}}: 154 \mathrm{ft}$ & $\mathrm{L}_{\mathrm{S}}: 86 \mathrm{ft}$ & $\mathrm{L}_{\mathrm{S}}: 144 \mathrm{ft}$ \\
\hline \multirow{4}{*}{300} & TF: 28"x11/4" & TF: 32"x23/4" & TF: 26"x11/4" \\
\hline & BF: 28"x11/4" & BF: $32 " x 23 / 4 "$ & BF: $26 " x 11 \frac{1}{4} "$ \\
\hline & W: 105"x/4" & W: $105 " x^{3} / 4 "$ & W: $105 " x^{3} / 4 "$ \\
\hline & $\mathrm{L}_{\mathrm{S}}: 192 \mathrm{ft}$ & $\mathrm{L}_{\mathrm{S}}: 108 \mathrm{ft}$ & $\mathrm{L}_{\mathrm{S}}: 180 \mathrm{ft}$ \\
\hline
\end{tabular}

TF: Top Flange; BF: Bottom Flange; W: Web; $\mathrm{L}_{\mathrm{S}}$ : Length of the section

For design purposes, the cross frames of each straight bridge were arranged such that their maximum spacing $\left(L_{b}\right)$ in the positive-moment regions was close to the 
traditional spacing used in practice, $25 \mathrm{ft}$. However, in the pier regions the cross frames were set at shorter distances (17ft - 20ft), as indicated in Table 4-5.

Table 4-5. Cross-frame spacing, $L_{b}(\mathrm{ft})$

\begin{tabular}{ccccc}
\cline { 2 - 5 } & \multicolumn{2}{c}{ End Span } & \multicolumn{2}{c}{ Middle Span } \\
\hline Lm (ft) & M+ & M- & M- & M+ \\
\hline 150 & 25 & 20 & 20 & 22 \\
\hline 240 & 25 & 17 & 20 & 25 \\
\hline 300 & 22 & 20 & 18 & 22 \\
\hline
\end{tabular}

\subsection{Models}

A FE model was developed for each straight, skewed and curved bridge configuration using a MATLAB ${ }^{\circledR}$ code that generates the input files which are subsequently processed by Abaqus ${ }^{\circledR}$. Figure 4-5 shows a typical FE model of a curved bridge configuration at the end of Casting 2 .

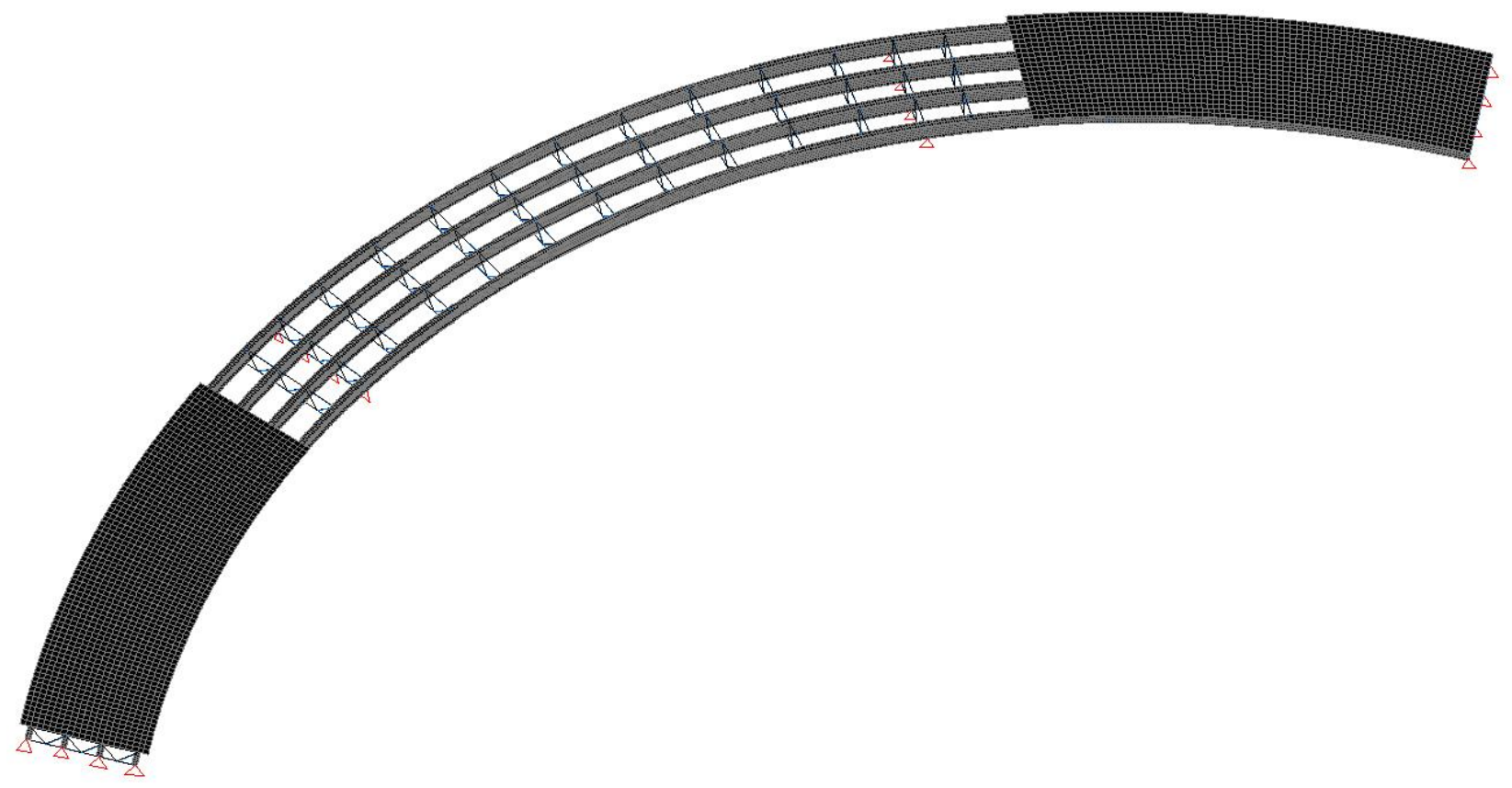

Figure 4-5. Finite element model of a typical curved bridge configuration 
As discussed in Chapter 3, four-node shell elements with reduced integration and enhanced hourglass control were used to model the plate girders and the concrete slab. The deck composite action was simulated using rigid elements that connect the top flanges of the girders to the slab. The cross frames and the stiffeners were modeled using slender beam elements based on the Euler-Bernoulli formulation, assuming a linear section behavior. The mesh configuration in the flanges employs six elements across the flange width with aspect ratios close to four. An aspect ratio close to one was selected for the webs. All shell elements in the girders are approximately 12-inch long.

In addition, the stay-in-place (SIP) forms were included in the modeling process of the bridges when the girders act in a non-composite state. This inclusion was required to identify the position of the shell elements that represent the concrete deck after the non-composite girders have deformed during the placement of the fresh concrete. The deck formwork was modeled by shell elements with the same coordinates as those elements used to model the concrete deck, but with a lower stiffness represented by the thickness $(t=2 \mathrm{in})$ and the material $(E=33.2 \mathrm{ksi})$. Figure $4-6$ shows the bending stresses in the top flange of a curved bridge model with and without including the stay-in-place forms. It is observed that the fbu is practically not affected by the inclusion of the forms, while the LFB decreases up to $20 \%$ in regions of maximum moment in a curved bridge model. In addition, the forms provide stability to the inner girder of curved bridges, since the LFB varies regularly about the zero stress instead of exhibiting a global buckling mode. The decreasing effect on the LFB due to the inclusion of the deck formwork is also exhibited by the skewed and straight bridges, but to a much lesser degree.

The continuity over the intermediate supports also contributes to reduce the levels of LFB due to the increase of the structural stiffness. In addition, the LFB of a noncomposite segment due to fresh concrete is relieved when previous casts are considered as composite in the analyses.

The vertical loads other than the self weight of the steel members are considered in the models using concentrated forces applied directly on the girder nodes. The self weight of steel was included as an element-based body load due to gravity. On the other hand, the torsional effects produced by the overhang loads are represented on the exterior girders by horizontal forces as shown in Figure 4-7. A maximum depth of 70in is used 
for the overhang brackets. However, this distance varies for each bridge configuration according to the web depth and the FE dimensions.
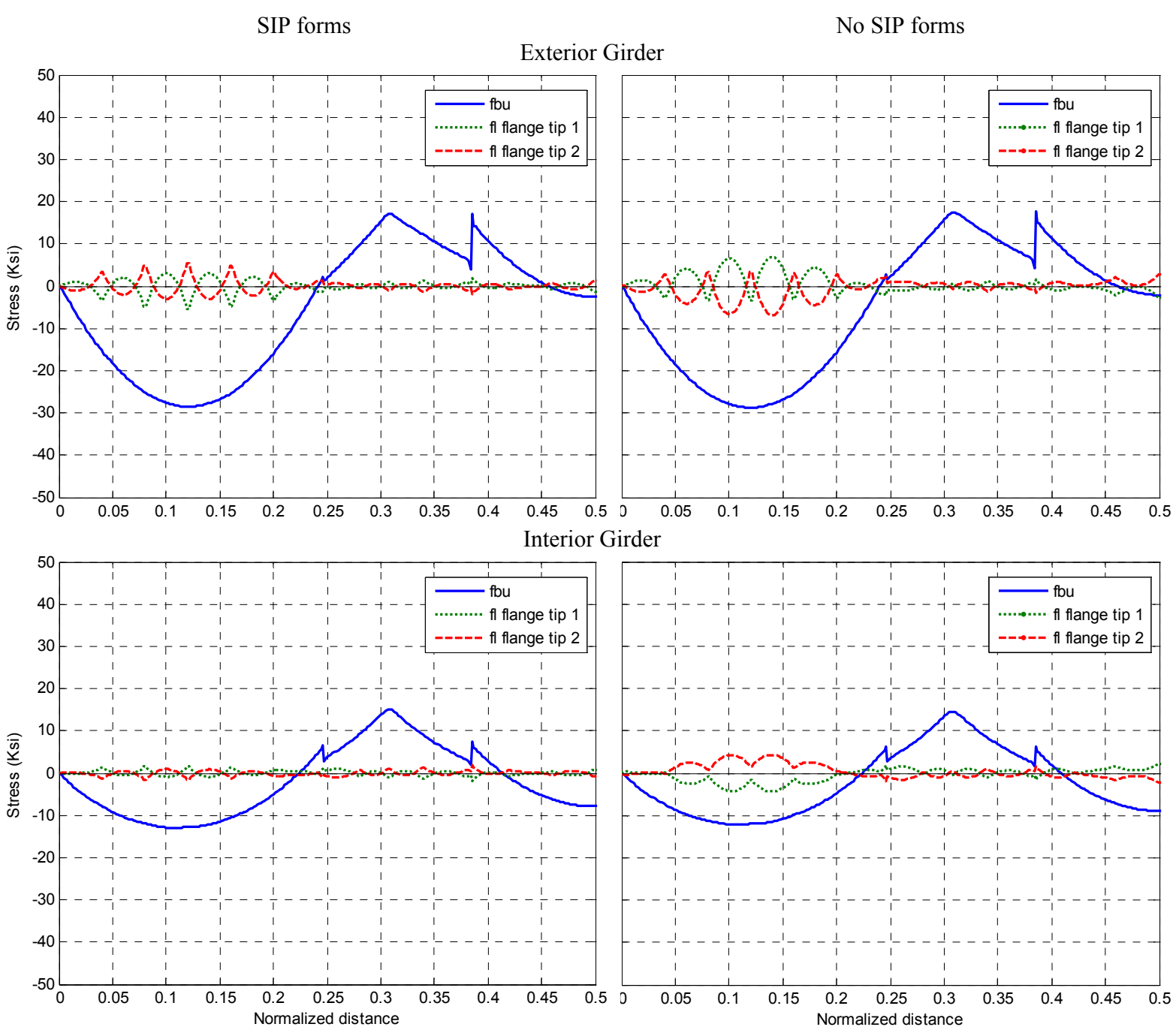

Figure 4-6. Effect of the stay-in-place forms in the bending stresses of a curved bridge 

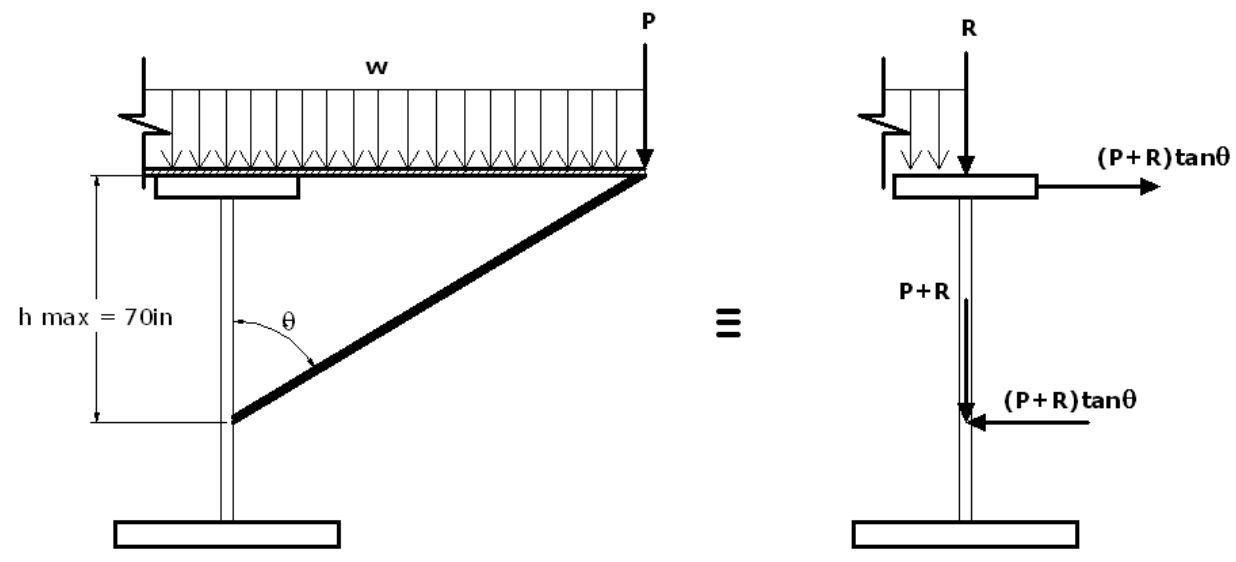

Figure 4-7. Torsional effects on exterior girders produced by overhang loads

\subsection{Analyses}

Five static stress analyses are required for each bridge configuration to simulate the deck placement, as shown in Figure 4-8. The arrows in the figure point to the previous analysis from where the initial conditions are taken. As mentioned before, the weight of the wet concrete slab is considered in the corresponding cast as explicit concentrated loads applied directly on the non-composite girders. However, in the analyses, earlier concrete casts are made composite for each subsequent cast.

End spans

$\underline{\text { Middle Span }}$

$\underline{\text { Pier regions }}$

Casting 1:Pu and $w u$

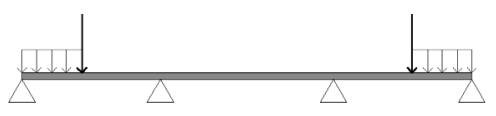

Casting 2: $w u$

Casting 3: $P u$ and $w u$

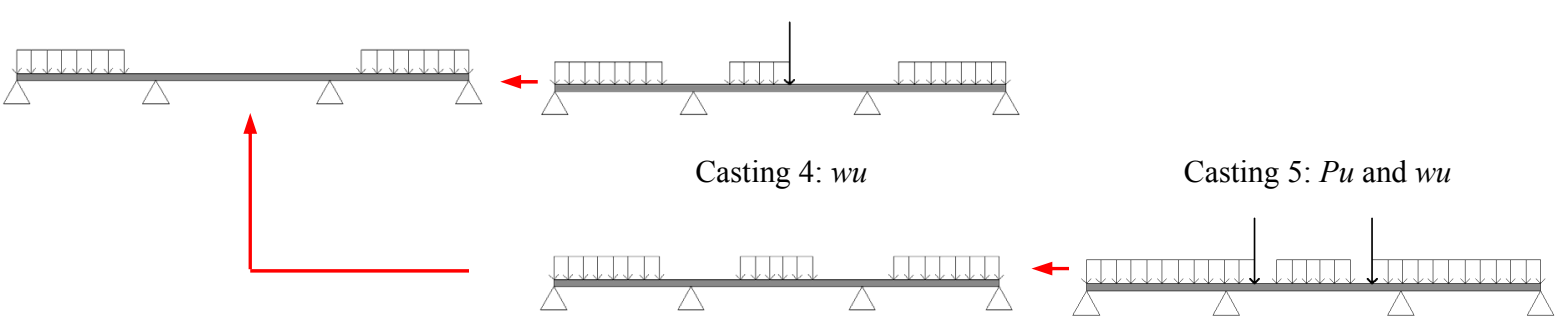

Figure 4-8. Sequence of analysis for each bridge configuration 
A detailed explanation of each one of the casting stages and the corresponding models from Figure 4-8 is given below:

- End spans:

Casting 1: The initial conditions are zero for this cast. The loads applied sequentially consist of: $i$. the weight of the steel superstructure, $i i$. the construction loads without considering the finishing machine, and iii. the weight of the fresh concrete corresponding to one-half of the positive moment regions at the end spans along with the finishing machine.

Casting 2: In this cast, the initial conditions are also assumed as zero since the fresh concrete of the positive moment regions at the end spans is applied at once. Therefore, the load sequence is similar to the one described for Casting 1. However, in this case, the fresh concrete is applied over the complete positive moment regions of the end spans without including the finishing machine effects.

- Middle span:

Casting 3: The initial conditions correspond to the final stresses and deformations obtained in Casting 2. In this stage, the shell elements of the concrete deck corresponding to Casting 1 need to be initially activated. Then, the weight of the fresh concrete is applied over one-half of the positive moment region of the middle span, including the finishing machine.

Casting 4: The difference between Castings 3 and 4 consists in that the weight of the fresh concrete in Casting 4 is applied over the complete positive moment region of the middle span, and the finishing machine effects are neglected.

- Pier regions:

Casting 5: The initial conditions are taken from the final response of Casting 4. The shell elements corresponding to the concrete slab placed in Casting 2 are activated to simulate the composite action in this zone. In this cast, the weight of the fresh concrete is extended from the beginning of the negative moment regions at the end spans to the midpoint between the pier and the end of the negative moment regions at the middle span. The finishing machine effects are included as well. 


\section{Chapter 5: Approximation of the lateral flange bending in steel I-girder bridges}

This chapter presents the methods used to approximate the LFB in straight, skewed and curved steel I-girder bridges due to construction loads during deck placement based on the results obtained from the parametric study described in Chapter 4. The major-axis bending stresses are analyzed as well. However, the principal contribution to estimate $f_{b u}$ is made for curved bridges since it is shown that the torsional effects do not affect the vertical bending response in straight and skewed bridges.

\subsection{Definition of the bending stresses from FEA}

The approximations proposed in this work to estimate the bending effects in steel I-girder bridges during deck placement are based on the results obtained from the FEA of the parametric bridge configurations. However, the direct response obtained from the Abaqus ${ }^{\circledR}$ output corresponds to the total bending stresses at each flange tip $\left(f_{1}\right.$ and $\left.f_{2}\right)$, as shown in Figure 5-1. Therefore, a Matlab ${ }^{\circledR}$ code was developed to compute the majoraxis bending $\left(f_{b u}\right)$ and LFB $\left(f_{l}\right)$ stresses from $f_{1}$ and $f_{2}$ assuming a linear response, as follows:

$$
\begin{aligned}
& f_{b u}=\frac{f_{1}+f_{2}}{2} \\
& f_{l}=f_{\text {total }}-f_{b u}
\end{aligned}
$$

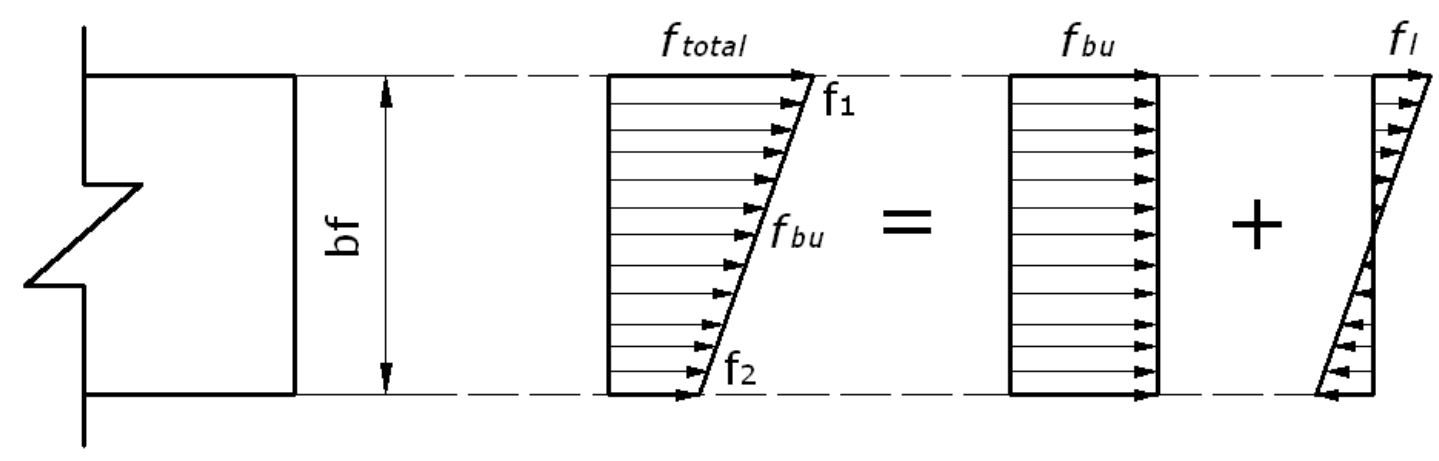

Figure 5-1. Identification of $f_{l}$ and $f_{b u}$ from the total flange bending response 
For example, the resulting bending stresses computed in Matlab ${ }^{\circledR}$ for the top and bottom flanges of the exterior girder in a curved bridge model are shown in Figure 5-2.
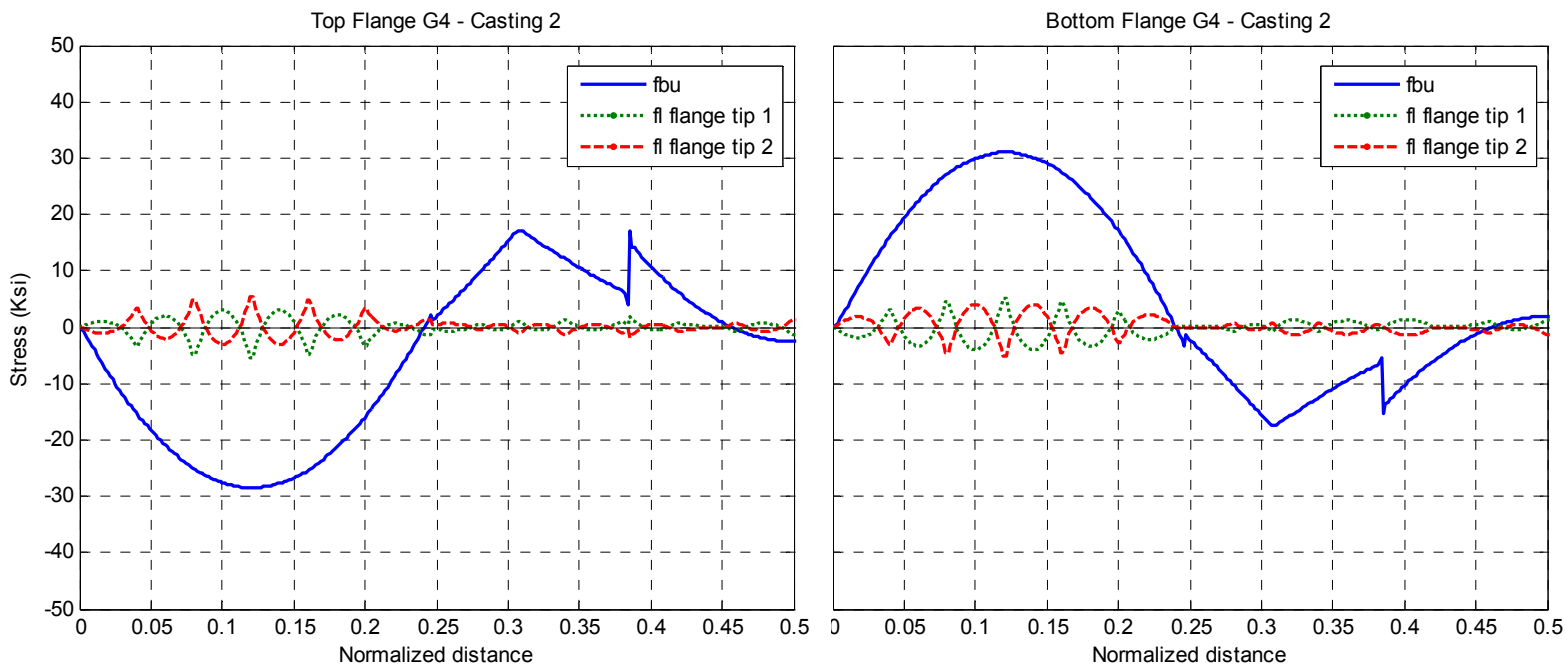

Figure 5-2. Bending stresses on the exterior girder of a curved bridge model

\subsubsection{Parametric Notation}

The effects on $f_{l}$ and $f_{b u}$ of the deck placement loads are evaluated in this project using the following parametric notation:

- Span lengths: The span lengths of the bridge models are identified by referencing their middle span length $\left(L_{m}=150 \mathrm{ft}, 240 \mathrm{ft}\right.$ or $\left.300 \mathrm{ft}\right)$ only, since the end span lengths depend on $L_{m}\left(L_{e}=80 \% \mathrm{Lm}\right)$.

- Flange position: Top flange (TF) or bottom flange (BF).

- Girder section: S1 for the section corresponding to the positive moment regions of $L_{e}, \mathrm{~S} 2$ for the negative moment regions over the piers, and S3 for the positive moment region of $\mathrm{Lm}$.

- Casting sequence and load type: These variables are related to each other since the effects of the distributed loads $(w u)$ are evaluated from Castings 2 and 4 for S1 and $\mathrm{S} 3$, respectively. The concentrated load effects $(P u)$ are analyzed from Castings 1 and 3 for $\mathrm{S} 1$ and $\mathrm{S} 3$, since these casts include the finishing-machine wheels combined with the fresh concrete. For S2, the complete casting sequence is considered. 
- Girder position: This parameter is particularly important for curved bridges to indicate the position of the girder with respect to the curvature (outside G4 and inside G1).

- Skew angle $(\theta): 30^{\circ}, 45^{\circ}$ or $60^{\circ}$.

- Curvature angle $\left(L_{m} / R\right): 0.30,0.45$ or 0.60

- Bridge type: Straight bridges (ST-0), skewed bridges with $\theta=30^{\circ}, 45^{\circ}$ or $60^{\circ}$ (SK30, SK45 or SK60), and curved bridges with $L / R=0.30,0.45$ or 0.60 (CV30, CV45 or CV60).

- Cross-frame orientation in skewed bridges: Perpendicular to the girders (xfl) and parallel to the abutments (xf2).

\subsubsection{Normalization of the $L F B$}

The LFB stresses obtained from FEA are normalized in order to establish fitting models independent of the cross section properties. Therefore, the numerical stresses, $f_{l}$, are multiplied by the flange section modulus, $S_{f}$, to obtain the lateral flange moment, $M_{\text {lat }}$. Then, the lateral moment is divided by the corresponding lateral load which depends directly on the overhang bracket depth for construction loads, or the web depth for curvature effects. Consequently, the following expressions were used to present the LFB effects in this work:

$$
\begin{gathered}
\frac{f_{l} S_{f}}{F_{l}}=\frac{M_{l a t}}{F_{l}} \\
\frac{f_{l} S_{f}}{P_{l}}=\frac{M_{l a t}}{P_{l}}
\end{gathered}
$$

where $F_{l}$ and $P_{l}$ are the lateral distributed and concentrated loads that represent the torsional effects.

\subsection{Overhang loads in straight bridges}

The LFB effects produced by distributed and concentrated loads are studied initially from the straight bridge configurations where the curvature and skew effects do not take place. 


\subsubsection{Major-axis bending, $f_{b u}$}

Figure 5-3 shows that the major-axis bending exhibited by the exterior girders of straight bridges is independent of the cross-frame spacing. Therefore, $f_{b u}$ may be taken directly from FEA using any cross-frame distance.
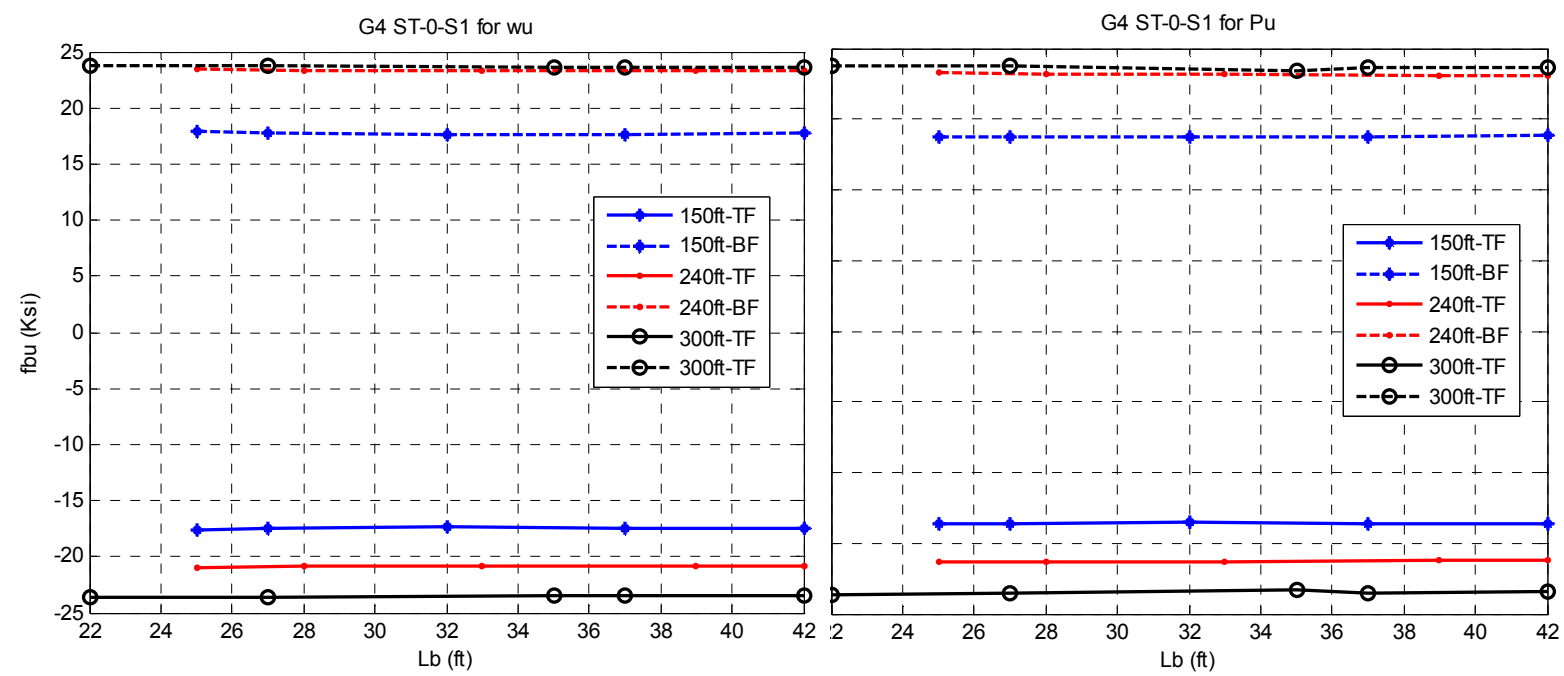

Figure 5-3. Effect of the cross-frame spacing in the major-axis bending

\subsubsection{Positive Moment Regions}

\subsubsection{Lateral distributed load effect}

For distributed loads in the positive moment regions, the LFB stresses were approximated using the following fitting model:

$$
\frac{M_{l a t}}{w u_{l}}=\frac{L_{b}^{a}}{A}
$$

where $w u_{l}$ is the lateral distributed load due to fresh concrete and $L_{b}$ is the cross-frame spacing. The variables $a$ and $A$ are defined in Table 5-1 for both top and bottom flanges. Figure 5-4 compares the results obtained using the proposed equation (Eq. 5-5) with the effects given by FEA and the code approximate equation (Eq. 1-2). It is observed that the code equation is highly conservative in all cases, principally for large cross-frame distances. However, the proposed equation predicts satisfactorily the LFB at the exterior 
girders of the end span over the entire range of cross-frame distances. Although the LFB exhibited in the middle span is slightly lower, the same equations are proposed for $L_{m}$ to simplify the recommended approximations for straight bridges.

Table 5-1. Fitting parameters for distributed loads in straight bridges (Positive Moment).

\begin{tabular}{ccc}
\hline & $a$ & $\begin{array}{c}\mathbf{A} \\
\left(\mathbf{f t}^{a-2}\right)\end{array}$ \\
\hline $\mathrm{TF}$ & 1.22 & 2.46 \\
\hline $\mathrm{BF}$ & 1.92 & 29.24 \\
\hline
\end{tabular}
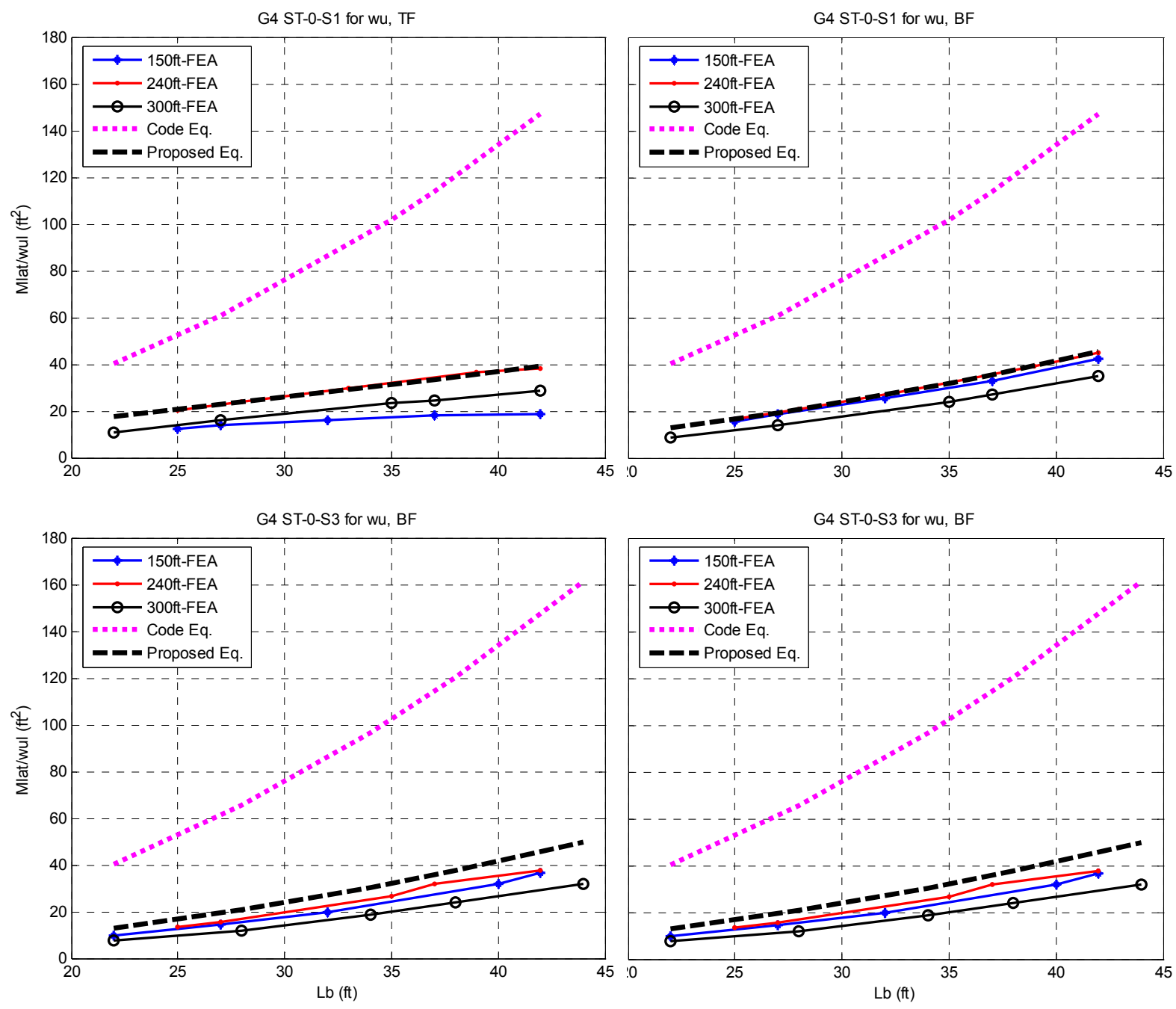

Figure 5-4. LFB due to distributed loads in the positive moment regions of straight bridges 


\subsubsection{Lateral concentrated load effect}

The evaluation of the concentrated load effects on LFB is more complex than for distributed loads due to several reasons:

- Both distributed and concentrated loads act simultaneously in Castings 1 and 3.

- The position of concentrated loads at the corresponding cross-frame spacing depends on the cross-frame distribution, i.e. the wheel loads may be located over one crossframe location or anywhere within two consecutive cross frames. For example, a simply supported straight bridge was analyzed separately to identify the effect of the concentrated load position on the LFB. Figure 5-5 shows that a linear trend parallel to the code equation is followed by models where the load is placed at the middle of a cross-frame spacing, being the critical location. However, other intermediate positions decrease the LFB effect.

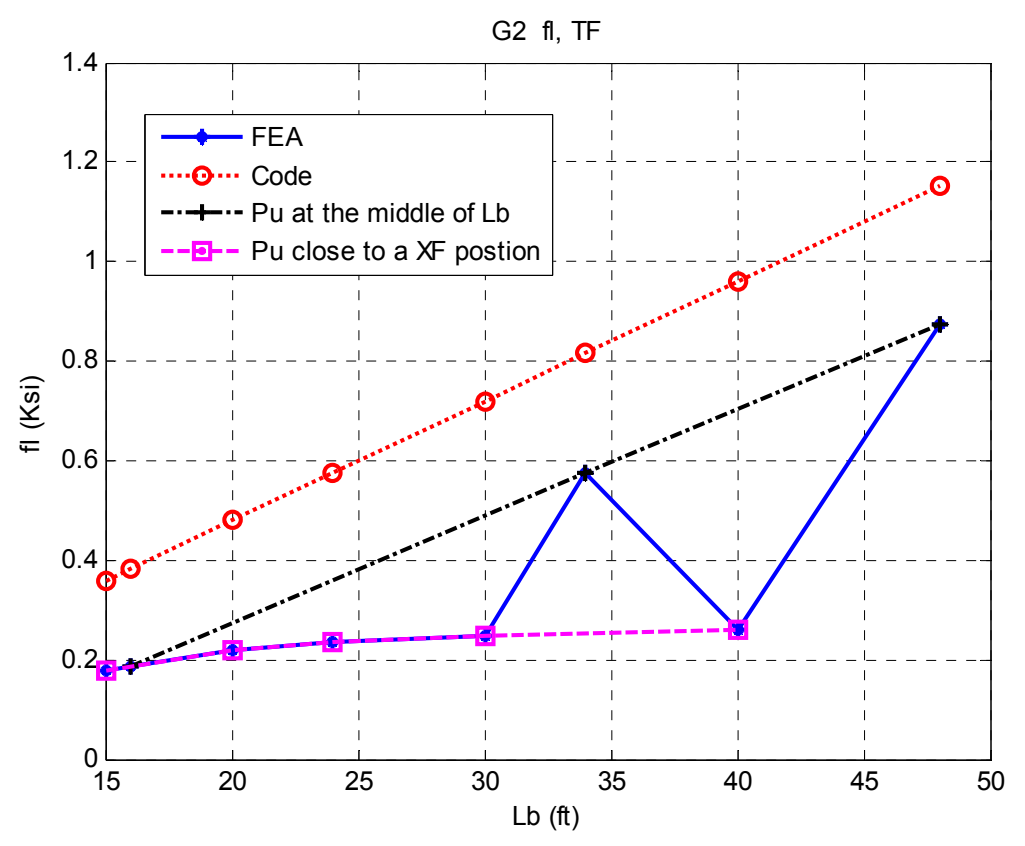

Figure 5-5. Effect of the concentrated load position in the LFB

- A similar situation occurs for distributed loads which extend up to the position of the leading wheel in the finishing machine.

- The finishing machine effect on an exterior girder consists of four wheels acting within a distance of $8 \mathrm{ft}$. However, in the approximated equations, it is conservatively assumed that the forces are all applied at the same point. 

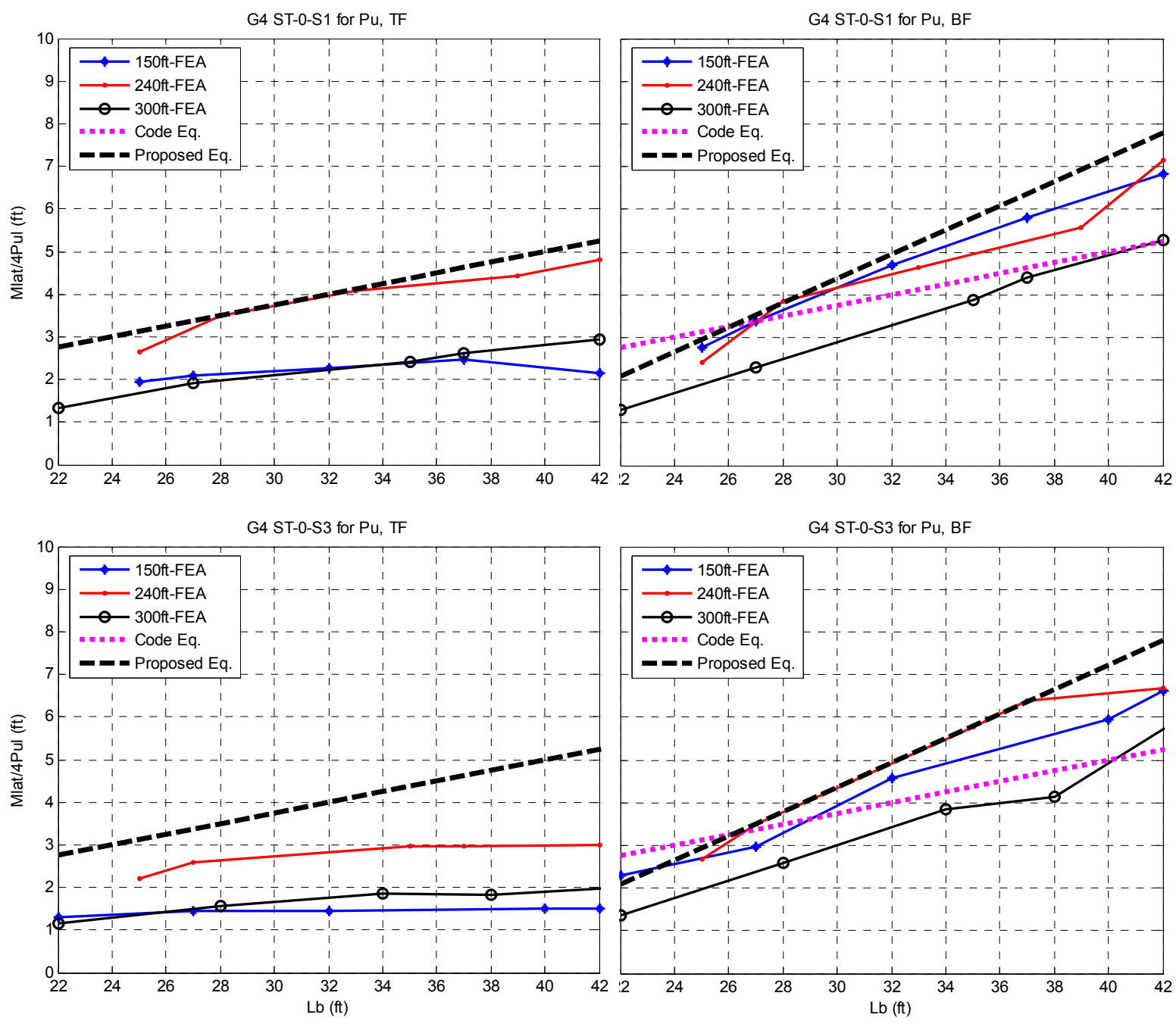

Figure 5-6. LFB due to concentrated loads in straight bridges (Positive Moment).

Consequently, a linear model similar to the code equation was adopted in this work to describe the combined effect of the lateral concentrated load, $P u_{l}$, representing a single wheel with the distributed load due to fresh concrete. However, the model only considers explicitly the concentrated loads while the concrete effect is considered by the combination of the variables $B$ and $C$, as follows:

$$
\frac{M_{\text {lat }}}{4 P u_{l}}=\frac{L_{b}}{B}+C
$$

The results of the models are shown in Table 5-2 and Figure 5-6. It is observed that the linear regression considers the most external points from the critical curves, since these points correspond to models where the concentrated loads are placed at the middle 
of a cross-frame spacing. It is also noticed that the proposed equation for the bottom flange governs over the code equation for cross-frame distances larger than $26 \mathrm{ft}$. However, both the code and the proposed approximations coincide for the top flange.

Table 5-2. Fitting parameters for concentrated loads in straight bridges (Pos. Moment).

\begin{tabular}{ccc}
\hline & B & $\begin{array}{c}\text { C } \\
(\mathbf{f t})\end{array}$ \\
\hline $\mathrm{TF}$ & 8 & 0 \\
\hline $\mathrm{BF}$ & 3.5 & -4.2 \\
\hline
\end{tabular}

\subsubsection{Negative Moment Regions}

The LFB effect on the negative moment regions was approximated considering only the distributed loading case since the contribution from the concentrated loads is negligible. Therefore, Equation 5-5 is taken as a valid model with the corresponding fitting parameters contained in Table 5-3. Figure 5-7 shows that the proposed equation satisfactorily predicts the LFB in both flanges.

Table 5-3. Fitting parameters for distributed loads in straight bridges (Neg. Moment)

\begin{tabular}{ccc}
\hline & $a$ & $\begin{array}{c}\mathbf{A} \\
\left(\mathbf{f t}^{a-2}\right)\end{array}$ \\
\hline $\mathrm{TF}$ & 1.37 & 4.42 \\
\hline $\mathrm{BF}$ & 2.02 & 22.45
\end{tabular}
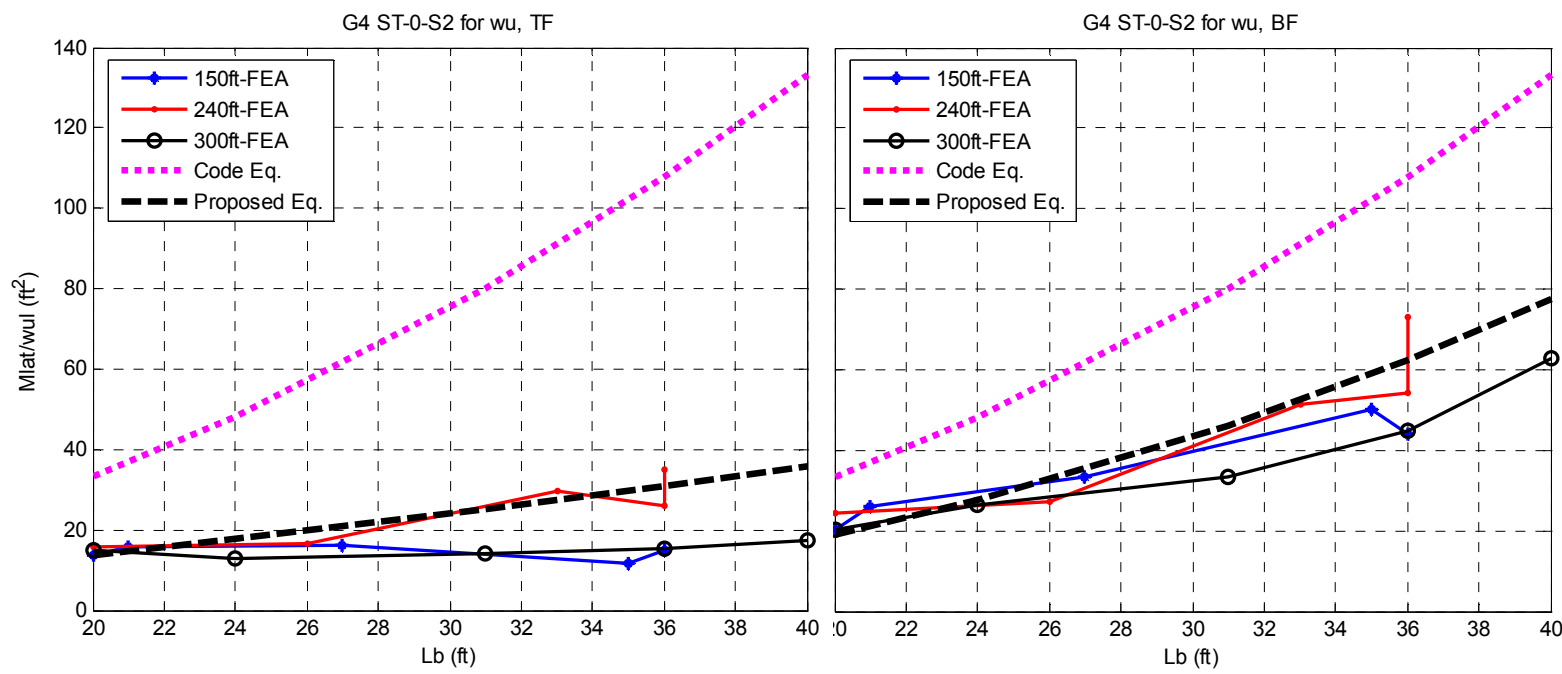

Figure 5-7. LFB effects due to concentrated loads in straight bridges (Neg. Moment). 


\subsection{Overhang loads in skewed bridges}

In all skewed bridges, the concrete is placed parallel to the skew. An evaluation of the cross-frame orientation was initially performed using bridge configurations with $L_{m}=150 \mathrm{ft}$. Then, the bending effects in skewed bridges are analyzed employing models with the recommended cross-frame orientation.

\subsubsection{Cross-frame orientation}

Figure 5-8 compares the bending results exhibited by the top flange of an exterior girder in a straight and skewed bridge subjected to vertical loads, e.g. steel weight. The results indicate that skewed bridges exhibit LFB even when torsional loads are not applied. However, in the presence of torsional effects, the LFB effect is slightly more pronounced when the cross frames are oriented parallel to the supports as shown in Figure 5-9. Therefore, a perpendicular orientation of the cross frames is recommended for general skewed bridges to increase the lateral stiffness of the structure, hence it is used in the models described next to evaluate the bending effects.
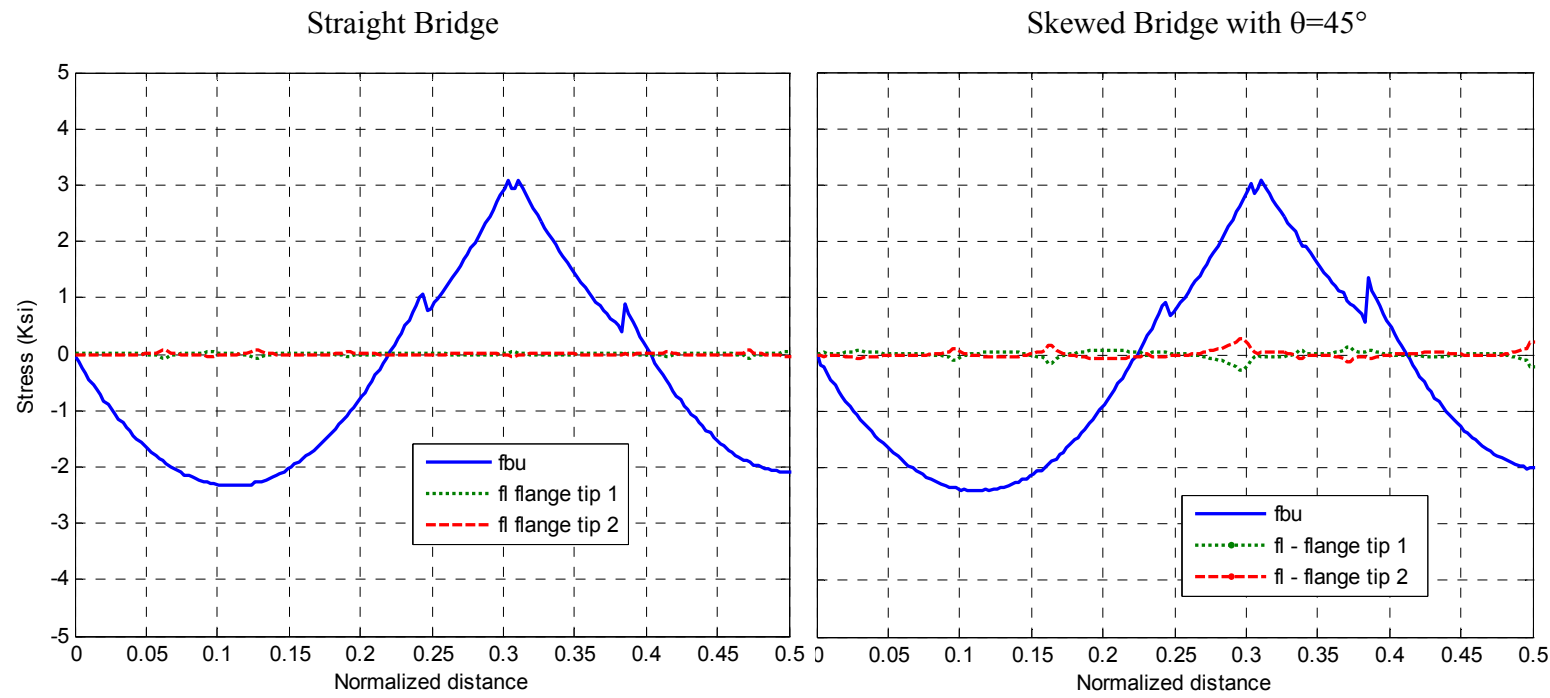

Figure 5-8. Effect of the skew angle in the LFB exhibited by the top flange 

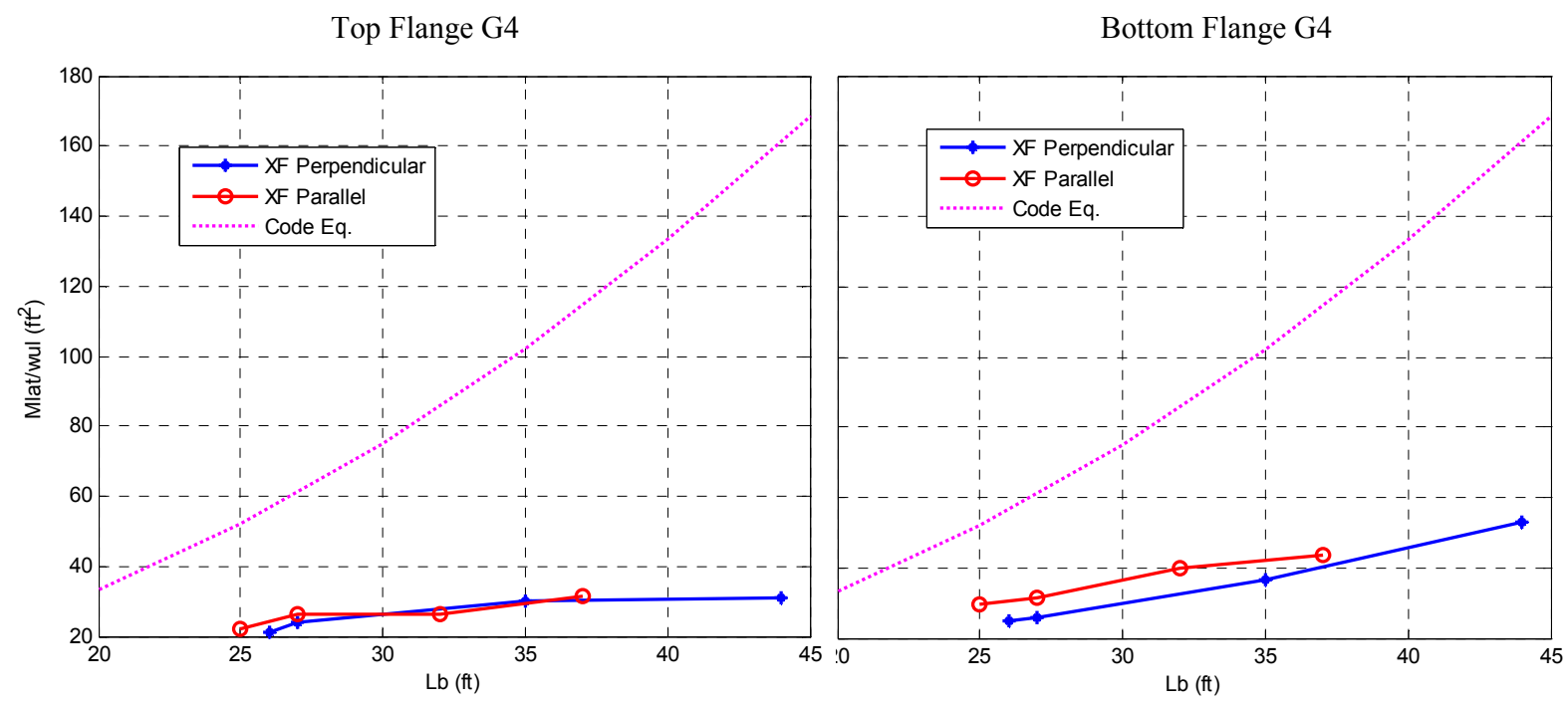

Figure 5-9. Effect of the cross-frame orientation in the LFB of skewed bridges

\subsubsection{Major-axis bending, $f_{b u}$}

Figure 5-10 shows that the major-axis bending in skewed bridges is not only independent of the cross-frame spacing but also of the skew angle. Therefore, $f_{b u}$ may be taken from FEA performed for skewed bridges with any cross-frame spacing and skew angle, including their straight counterpart.

\subsubsection{Positive Moment Regions}

An approach analogous to the procedure described for straight bridges was followed to approximate the LFB due to distributed and concentrated loads in the positive moment regions of skewed bridges. 

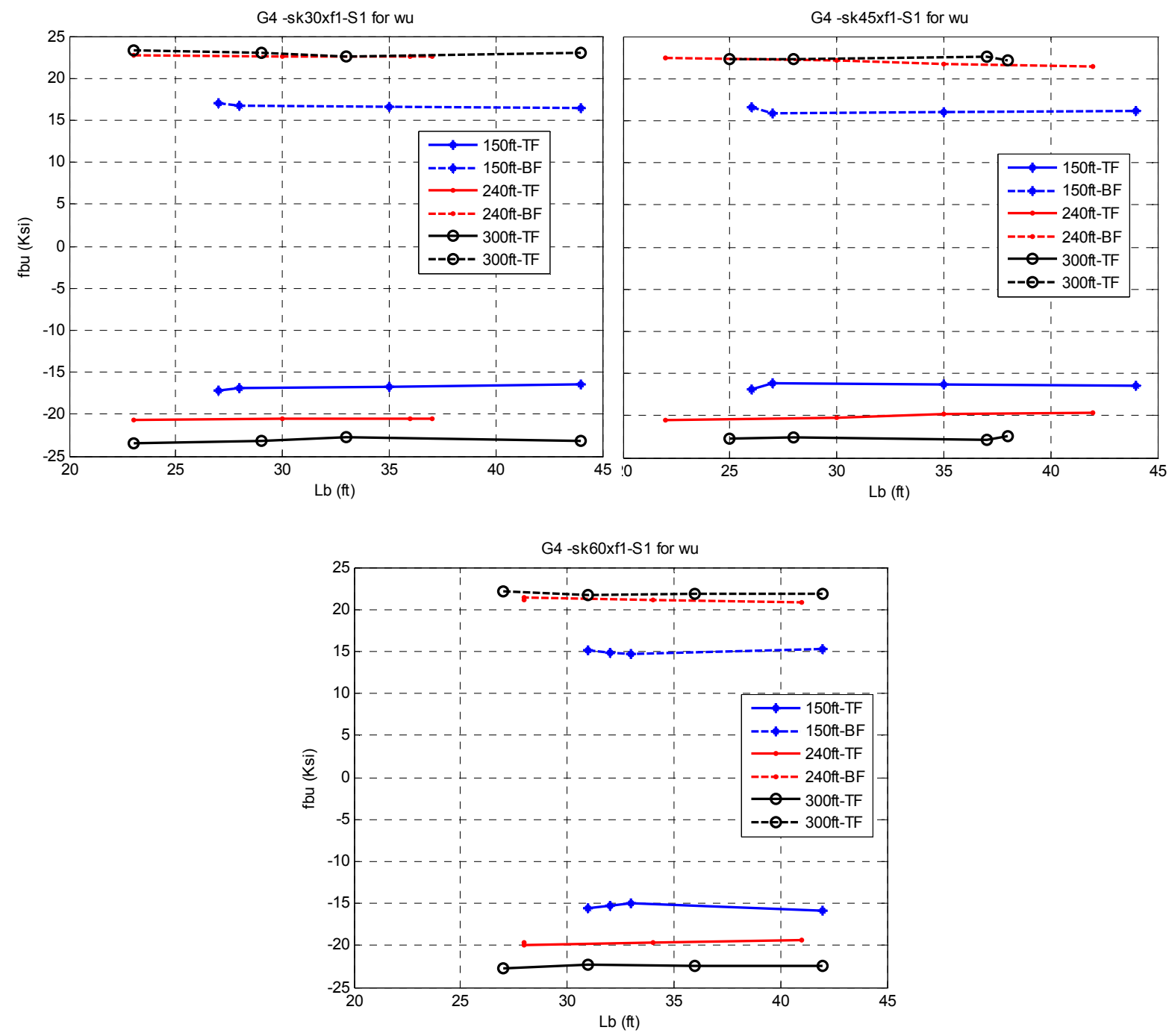

Figure 5-10. Effect of the skew angle in the major-axis bending

\subsubsection{Distributed load}

The corresponding fitting parameters are indicated in Table 5-4. Figure 5-11 shows the normalized LFB for bridges with $\theta=30^{\circ}$ (see Appendix A for $\theta=45^{\circ}$ and $60^{\circ}$ ). It is observed that the code recommended equation (Eq. 1-2) significantly overestimates the LFB principally for long cross-frame distances. However, the code fails to predict the LFB exhibited by the bottom flange of bridges with low $L_{b}$ values, especially for high skew angles. For that reason, a lower limit equal to 30,45 and $70 \mathrm{ft}^{2}$ for $\theta=30^{\circ}, 45^{\circ}$ and $60^{\circ}$, respectively, was introduced in the proposed equation for the normalized LFB at the bottom flange. 
Table 5-4. Fitting parameters for distributed loads in skewed bridges (Pos. Moment).

\begin{tabular}{ccccccc}
\hline & \multicolumn{3}{c}{$a$} & & \multicolumn{3}{c}{$\begin{array}{c}\mathbf{A} \\
\left(\mathbf{f t}^{a-2}\right)\end{array}$} \\
\hline $\boldsymbol{\theta}$ & $\mathbf{3 0}^{\circ}$ & $\mathbf{4 5}^{\circ}$ & $\mathbf{6 0}^{\circ}$ & $\mathbf{3 0}^{\circ}$ & $\mathbf{4 5}^{\circ}$ & $\mathbf{6 0}^{\circ}$ \\
\hline $\mathrm{TF}$ & 1.44 & 1.26 & 1.41 & 3.59 & 1.73 & 2.31 \\
\hline $\mathrm{BF}$ & 1.29 & 1.13 & 1.26 & 2.19 & 1.00 & 1.42 \\
\hline
\end{tabular}
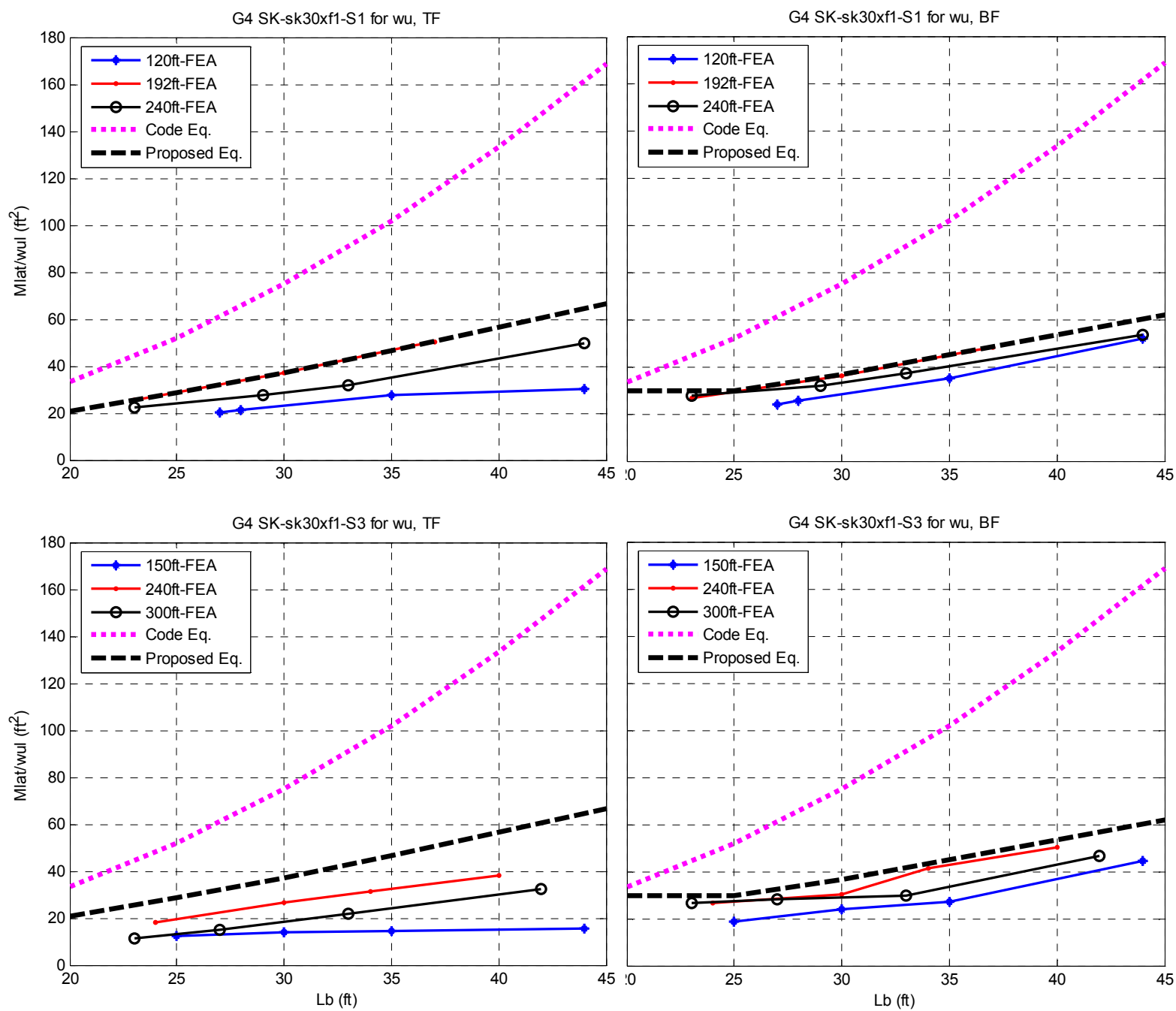

Figure 5-11. LFB due to distributed loads in skewed bridges (Positive Moment).

\subsubsection{Concentrated loads}

Table 5-5 contains the definition of the variables $\mathrm{B}$ and $\mathrm{C}$ from the fitting model for concentrated loads according to the numerical stresses obtained in the parametric 
study. Figure 5-12 (including the figures from Appendix A) shows that the code equation fails to predict satisfactorily the LFB in most of the cases, principally for the bottom flange.

Table 5-5. Fitting parameters for concentrated loads in skewed bridges (Pos. Moment).

\begin{tabular}{ccccccc}
\hline & & $\mathbf{B}$ & & \multicolumn{3}{c}{$\mathbf{C}(\mathbf{f t})$} \\
\hline $\boldsymbol{\theta}$ & $\mathbf{3 0}^{\circ}$ & $\mathbf{4 5}^{\circ}$ & $\mathbf{6 0}^{\circ}$ & $\mathbf{3 0}^{\circ}$ & $\mathbf{4 5}^{\circ}$ & $\mathbf{6 0}^{\circ}$ \\
\hline $\mathrm{TF}$ & 8 & 7.4 & 7.4 & 0 & 0 & 1.5 \\
\hline $\mathrm{BF}$ & 4.5 & 5.0 & 14 & -2.0 & 0 & 6.0 \\
\hline
\end{tabular}
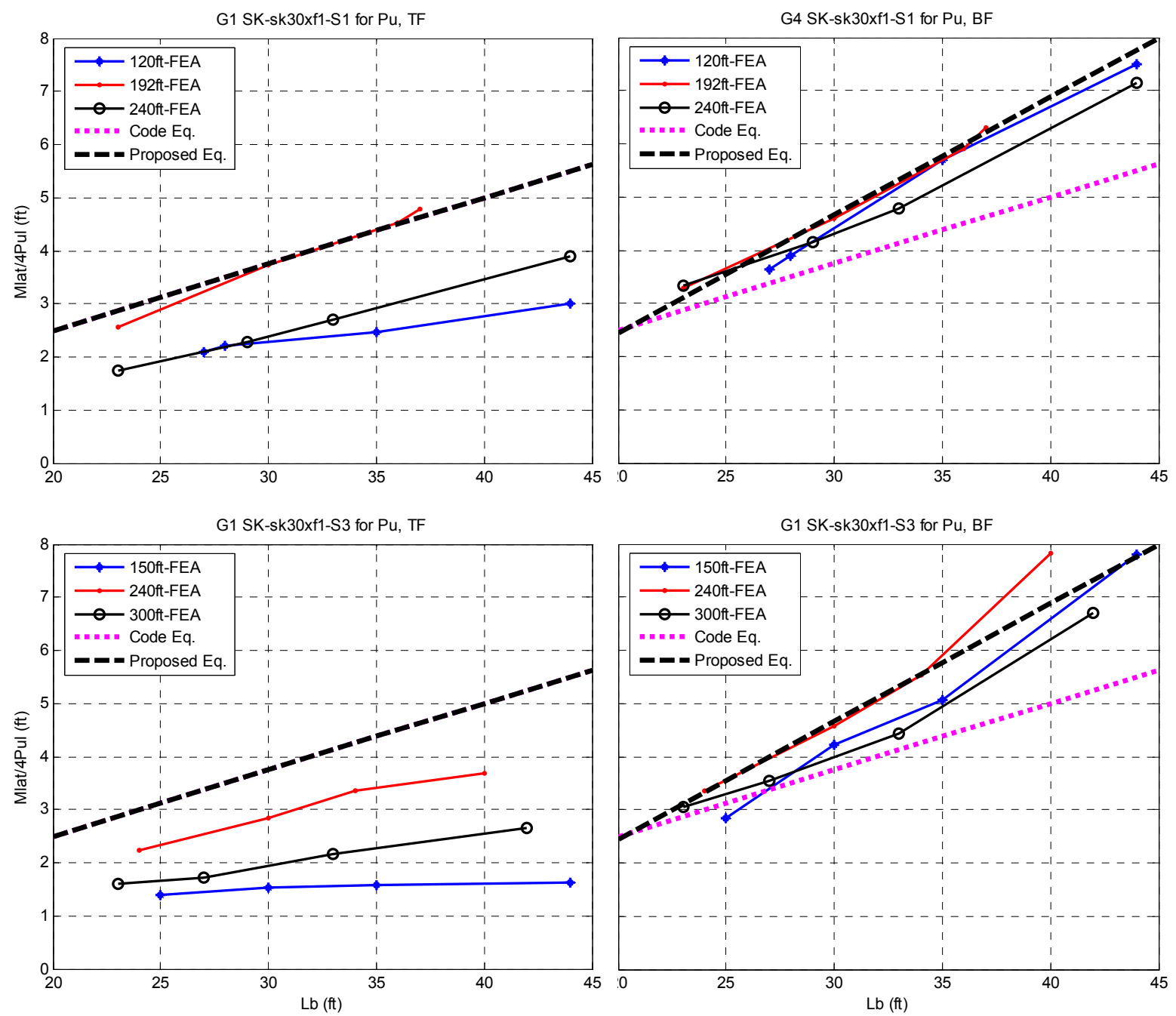

Figure 5-12. LFB due to concentrated loads in skewed bridges (Positive Moment) 


\subsubsection{Negative Moment Regions}

The LFB in negative moment regions was approximated by a constant term that depends on the skew angle. This term was defined according to the numerical results as 4, 6 and $8 \mathrm{Ksi}$ for $\theta=30^{\circ}, 45^{\circ}$ and $60^{\circ}$, respectively. The constant approximation is proposed to conservatively estimate the stresses which do not exhibit a consistent trend. Figure 5-13 presents the LFB stresses for bridge configurations with $\theta=30^{\circ}$, the remaining figures corresponding to $\theta=45^{\circ}$ and $60^{\circ}$ are contained in Appendix A. The results indicate that the code recommendation of using $10 \mathrm{Ksi}$ for the unfactored LFB stresses in skewed bridges is conservative even compared to factored stresses.
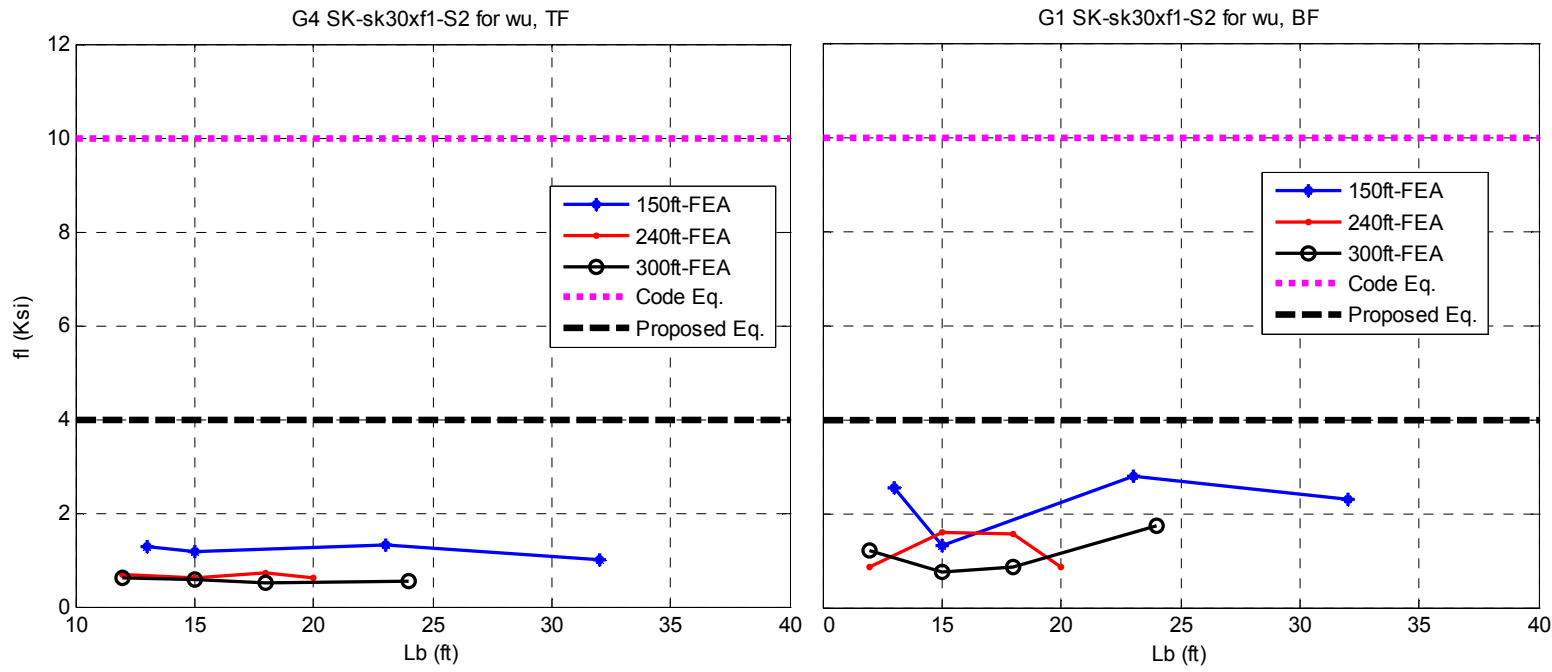

Figure 5-13. LFB effects due to distributed loads in skewed bridges (Neg. Moment).

\subsection{Overhang loads in curved bridges}

The evaluation of the bending stresses in curved bridges indicates that $f_{b u}$ and $f_{l}$ are deeply affected by the curvature. In addition, the results showed that the participation of the overhang loading term in the LFB effects is low compared to the curvature effects. 


\subsubsection{Major-axis bending, $f_{b u}$}

Figure 5-14 shows that the $f_{b u}$ in curved bridges is independent of $L_{b}$ but is affected by the curvature and the girder position. These relationships will be described in detail below.
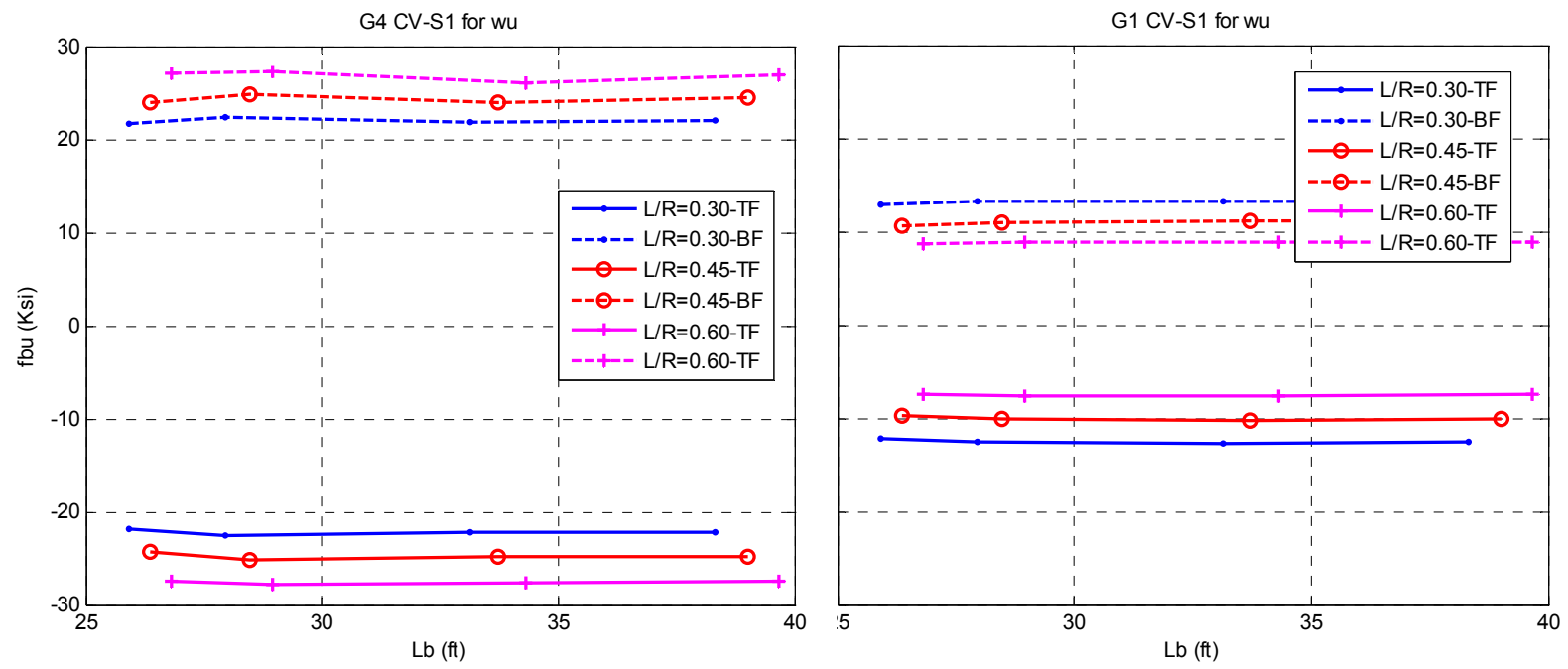

Figure 5-14. Effect of curvature in the major-axis bending

\subsubsection{Positive Moment Regions}

\subsubsection{Distributed loading effect}

\subsection{Major-axis bending, fbu}

The proposed linear model intended to estimate $f_{b u}$ in curved bridges during construction is given by:

$$
\frac{f_{\text {bu } \max }}{\left|f_{\text {buo } \max }\right|}=(\operatorname{sign} 1) b\left(\frac{L}{R}\right)+(\operatorname{sign} 2) c
$$

where $f_{b u \text { max }}$ is the maximum major-axis bending stress and $f_{b u \text { max }}$ is the maximum major-axis bending stress of the straight counterpart $(L / R=0)$. Table 5-6 and Table 5-7 contain the variables $b$ and $c$, and the sign combinations (sign1, sign2) for the bridge models evaluated in this project. Figure 5-15 and Figure 5-16 show that the majorbending stresses increase linearly with the curvature at the end spans. Conversely, a 
constant term is proposed for the middle span since $f_{b u}$ is not significantly affected by the curvature over this zone.

Table 5-6. Fitting parameters for $f_{b u}$ due to distributed loads curved bridges (Pos. Moment)

\begin{tabular}{cccccccc}
\cline { 2 - 7 } & \multicolumn{2}{c}{$\boldsymbol{L}_{\boldsymbol{m}}=\mathbf{1 5 0 f t}$} & \multicolumn{2}{c}{$\boldsymbol{L}_{\boldsymbol{m}}=\mathbf{2 4 0 f t}$} & \multicolumn{2}{c}{$\boldsymbol{L}_{\boldsymbol{m}}=\mathbf{3 0 0 f t}$} \\
\hline Outer Girder, G4: End span, $L_{e}$ & 0.95 & 1 & 1.3 & 1 & 1.5 & 1 \\
\hline Outer Girder, G4: Middle span, $L_{m}$ & 0 & 1 & 0 & 1 & 0 & 1.1 \\
\hline Inner Girder, G1: End span, $L_{e}$ & 0.85 & 1 & 1.06 & 1 & 1.2 & 1 \\
\hline Inner Girder, G1: Middle span, $L_{m}$ & 0 & 1 & 0 & 1 & 0 & 1.1 \\
\hline
\end{tabular}
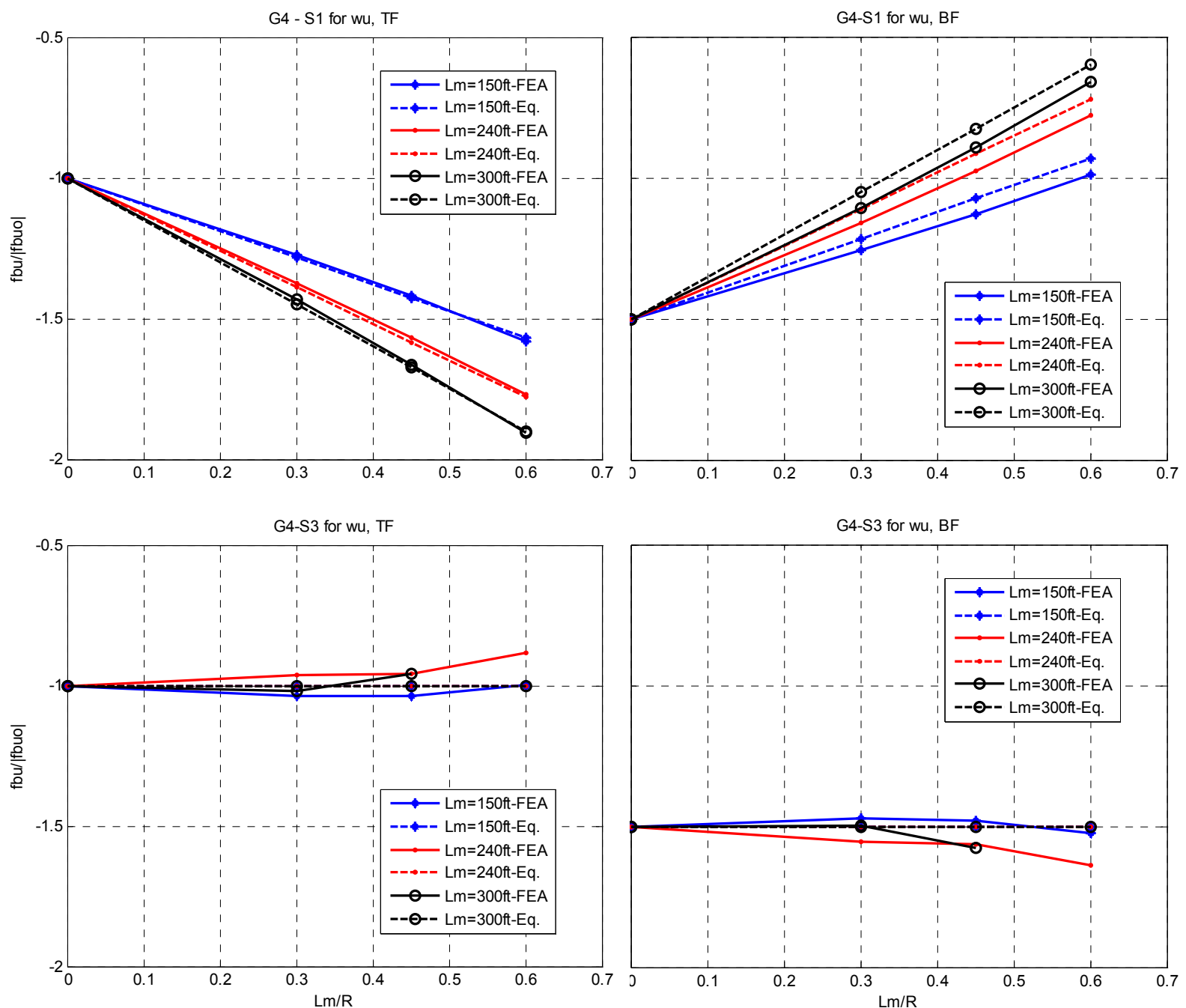

Figure 5-15. Normalized $f_{b u}$ due to distributed loads in outer girders (Positive Moment) 
Table 5-7. Sign combination used to estimate $f_{b u}$ (Sign1, Sign2) (Pos. Moment)

\begin{tabular}{ccc}
\hline & Top flange, TF & Bottom Flange, BF \\
\hline G4 &,-- &,++ \\
\hline G1 &,+- &,-+ \\
\hline
\end{tabular}
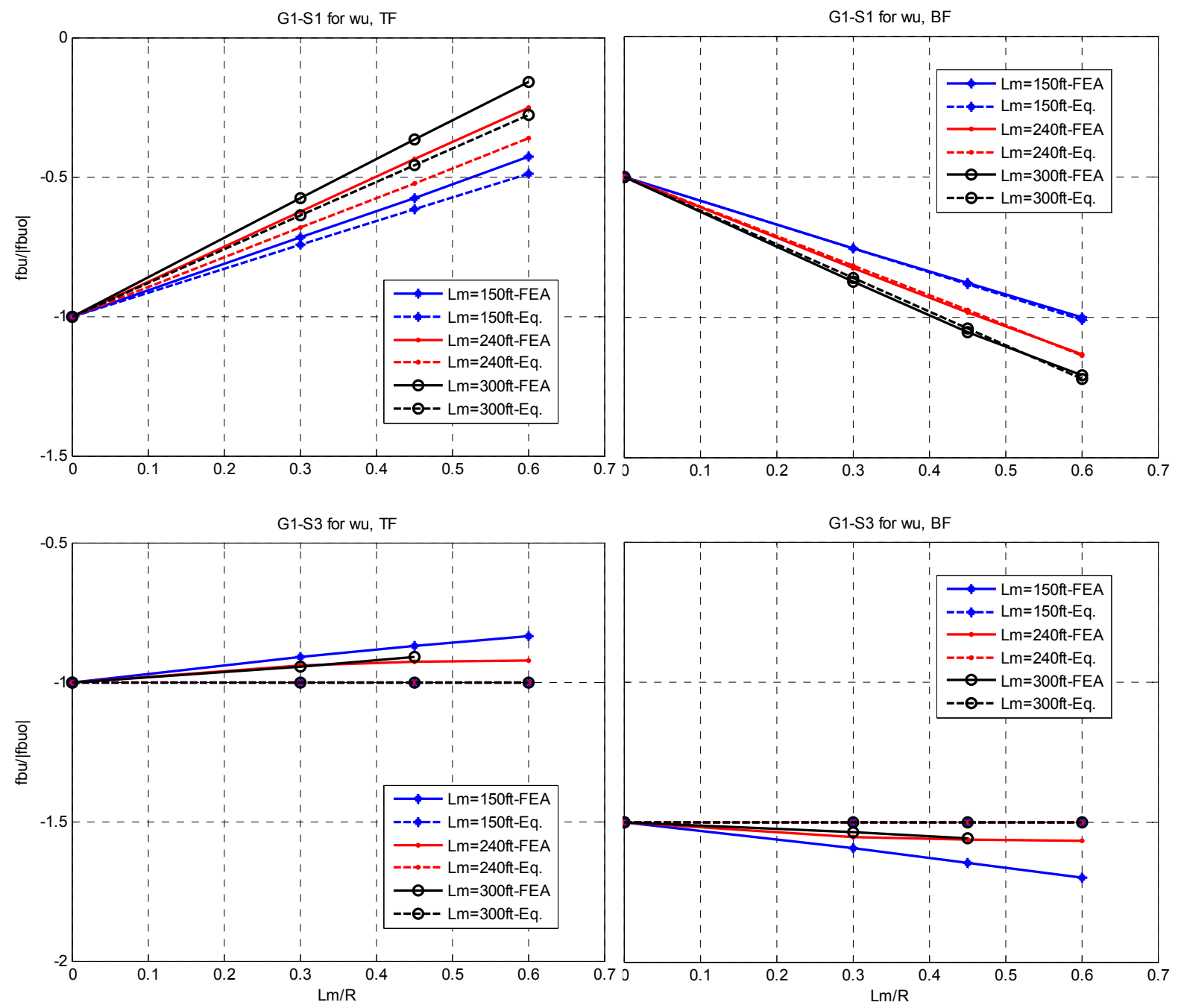

Figure 5-16. Normalized $f_{b u}$ due to distributed loads in inner girders (Pos. Moment)

\subsection{Lateral flange bending, $f_{l}$}

The LFB stresses were approximated considering a hypothetical curved I girder subjected to a uniform vertical distributed load, $w u$. The girder has a radius of curvature, $R$, and a span length, $L$, that subtends an angle $\theta$, as shown in Figure 5-17. The 
eccentricity, $d$, between the vertical load and the straight line that connects the end supports is a function of the longitudinal position. The maximum eccentricity, $d_{\max }$, is at the middle of the span length (Eq. 5-8). As a result of this eccentricity, a distributed torsional moment, $T$, is generated along the girder which is equivalent to a couple of horizontal distributed forces acting on the flanges, $w u_{l}$, as shown in Figure 5-18. The magnitude of $w u_{l}$ varies from zero at the end supports to the maximum value at the middle of the span, $w u_{\operatorname{lmax}}$ (Eq. 5-9), as indicated by Figure 5-19. Therefore, the maximum LFB stress, $f_{l}$, will correspond to the cross-frame spacing, $L_{b}$, closer to the span midpoint. The variation of $w u_{l}$ along the central cross-frame spacing is assumed to be small and consequently may be considered uniformly distributed. Finally, $f_{l}$ is approximated by Equation 5-10 where a curve fitting process is required to define the order of the approximation, $d$, and the denominator, $D$.

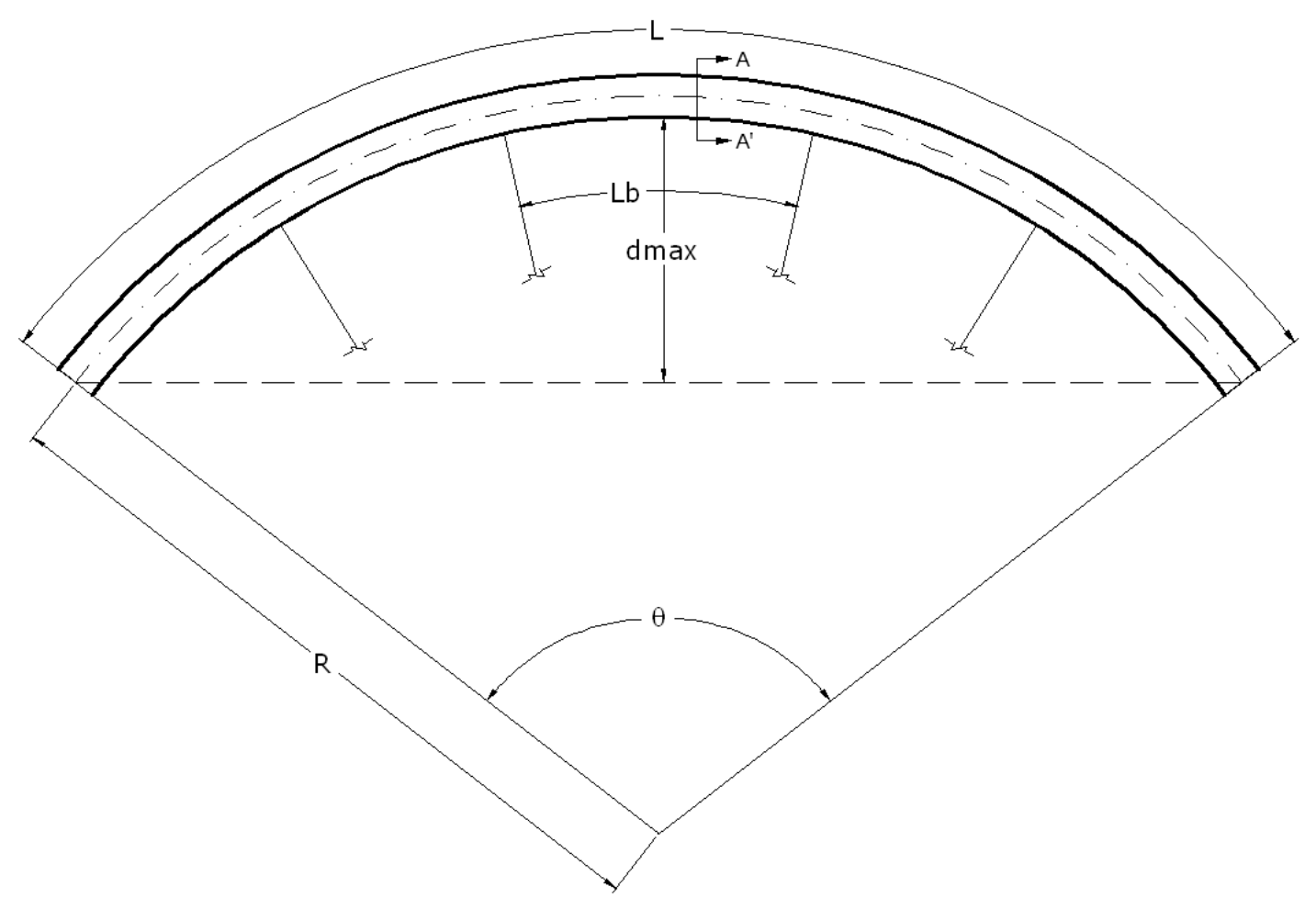

Figure 5-17. Hypothetical curved girder to approximate the curvature effects 

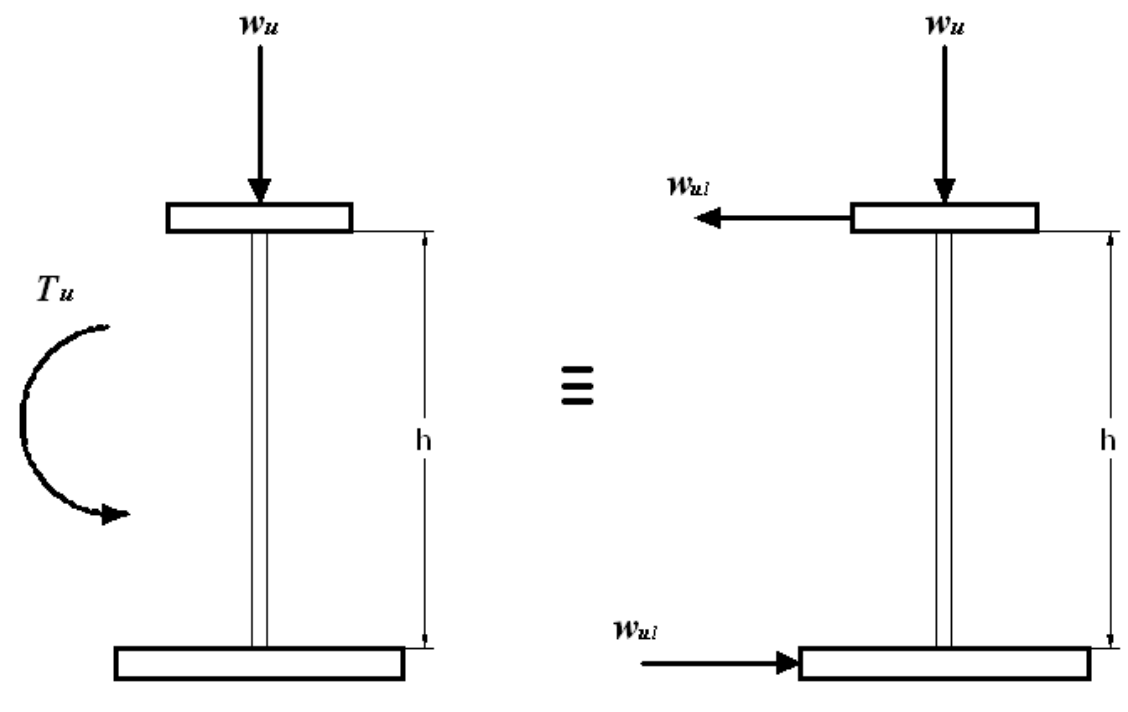

$A-A^{\prime}$

Figure 5-18. Visualization of the torsional effects due to curvature

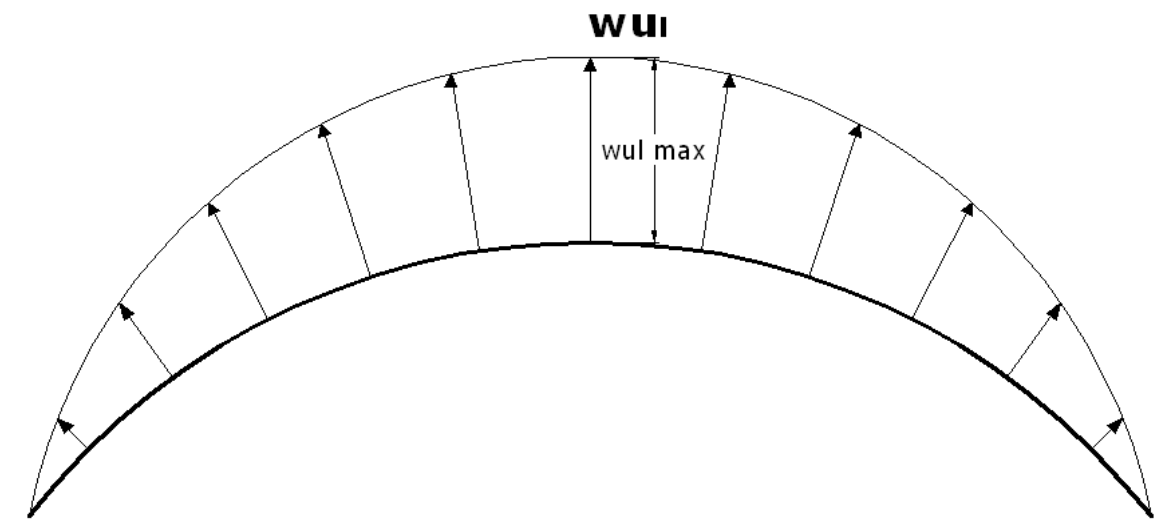

Figure 5-19. Horizontal distributed force on the top flange

$$
\begin{aligned}
& d_{\text {max }}=R\left(1-\cos \frac{\theta}{2}\right) \\
& w_{u l \text { max }}=\frac{w_{u} d_{\text {max }}}{h} \\
& f_{l}=\frac{w_{u l \text { max }} L_{b}^{d}}{D S_{f}}
\end{aligned}
$$


The LFB for curved girders is caused by two different sources: the curvature and the overhang loads during construction. The curvature and the overhang torsional effects have the same direction in the outer girder while they counteract at the inner girder, as follows:

$$
\begin{array}{ll}
M_{\text {lat }}=\left(M_{\text {lat }}\right)_{\text {curv. }}+\left(M_{\text {lat }}\right)_{o v .} & \text { for } \mathrm{G} 4 \\
M_{\text {lat }}=\left(M_{\text {lat }}\right)_{\text {curv. }}-\left(M_{\text {lat }}\right)_{o v .} & \text { for G1 } \\
M_{\text {lat }}=\frac{w_{u l \max } L_{b}^{d}}{D} \pm \frac{w u_{l} L_{b}^{a}}{A} &
\end{array}
$$

However, the participation of the overhang loading in the LFB is low compared to the curvature effects as shown in Figure 5-20. In fact, the overhang-to-curvature effect ratio reduces as the length of the span and the curvature increase. Therefore, the total LFB is estimated using the curvature term only. However, the torsional effects due to overhang loads are implicitly considered since the curve fitting process to estimate the parameters $d$ and $D$ in Equation 5-10 is applied to the total LFB obtained from the parametric study.

The critical case between the top and bottom flanges was selected to define the proposed equations for curved bridges. The corresponding variables from the curve fitting process are contained in Table 5-8 and Table 5-9. It is observed from Figure 5-21 that the LFB effects of the end spans are practically unaffected by the variation of the curvature, while a slight effect is observed in the middle spans. The proposed equation (Eq. 5-10) works adequately in both exterior girders. Significant reductions were found in most of the cases compared to the estimations given by the AASHTO approximation (Eq. 1-1). The major-axis bending moment, $M$, in the code equation was computed using both the numerical and the estimated (Eq. 5-7) major-axis bending stresses, obtaining similar results. This is a practical observation since the LFB in a curved bridge can be approximated using the code equation (Eq. 1-1) together with the $f_{b u}$ computed from the information of the straight counterpart. However, the figures contained in Appendix B for $L_{m}=240 \mathrm{ft}$ and $300 \mathrm{ft}$ indicate that the code equation fails to predict the LFB at the inner girder of highly curved bridges with long spans. Therefore, it is recommended to use the equation proposed in this work which predicts satisfactorily the LFB in all cases. 

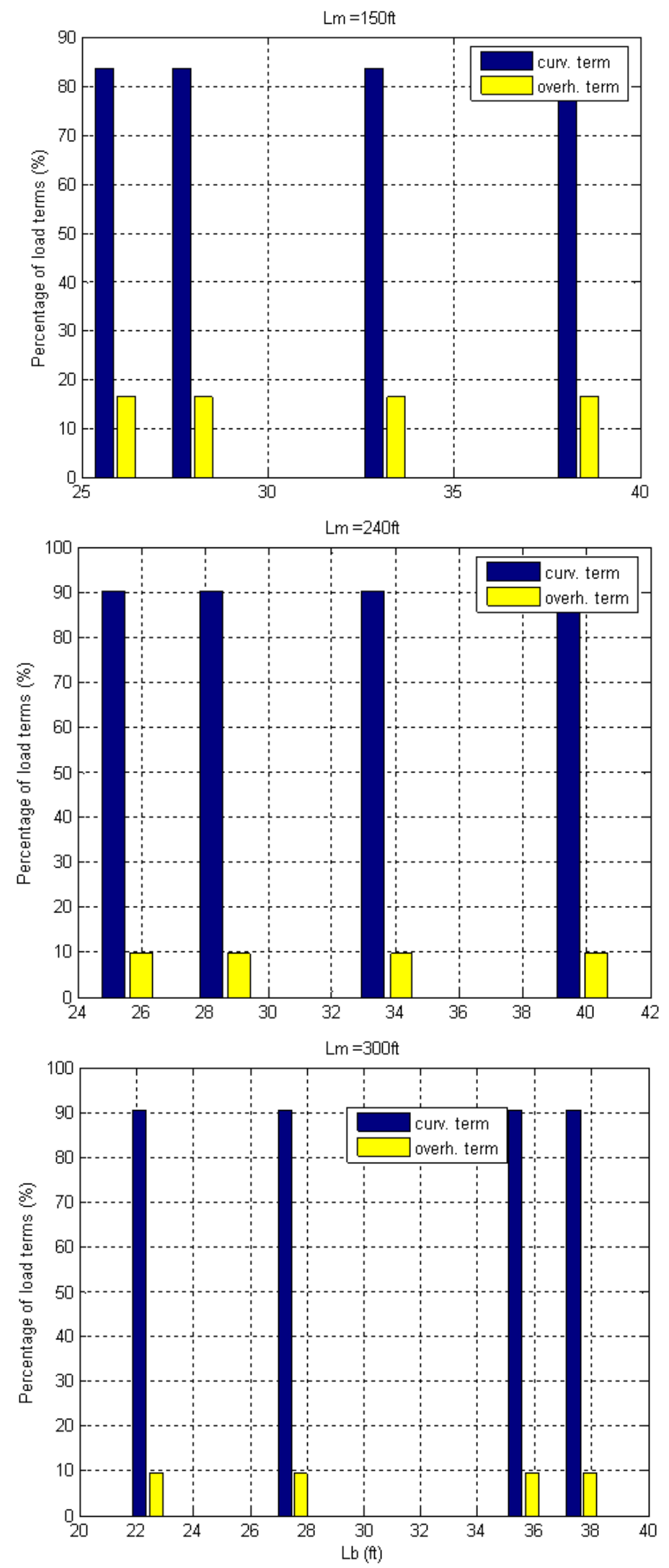

Figure 5-20. Comparison of the overhang and curvature effects in the LFB 
Table 5-8. Fitting parameters for distributed loads in the outer girders (Pos. Moment)

\begin{tabular}{ccccccc}
\hline Section & \multicolumn{2}{c}{ 150ft } & \multicolumn{2}{c}{ 240ft } & \multicolumn{2}{c}{ 300ft } \\
\hline & $\mathrm{d}$ & $\begin{array}{c}\mathrm{D} \\
\left(\mathrm{ft}^{\mathrm{d}-2}\right)\end{array}$ & $\mathrm{d}$ & $\begin{array}{c}\mathrm{D} \\
\left(\mathrm{ft}^{\mathrm{d}-2}\right)\end{array}$ & $\mathrm{d}$ & $\begin{array}{c}\mathrm{D} \\
\left(\mathrm{ft}^{\mathrm{d}-2}\right)\end{array}$ \\
\hline $\mathrm{S} 1$ & 1.99 & 31.14 & 1.32 & 2.49 & 1.72 & 9.55 \\
\hline $\mathrm{S} 3$ & 1.99 & 89.42 & 1.81 & 55.6 & - & - \\
\hline
\end{tabular}

Table 5-9. Fitting parameters for distributed loads in inner girders (Pos. Moment)

\begin{tabular}{ccccccc}
\hline Section & \multicolumn{2}{c}{ 150ft } & \multicolumn{2}{c}{ 240ft } & \multicolumn{2}{c}{ 300ft } \\
\hline & $\mathrm{d}$ & $\begin{array}{c}\mathrm{D} \\
\left(\mathrm{ft}^{\mathrm{d}-2}\right)\end{array}$ & $\mathrm{d}$ & $\begin{array}{c}\mathrm{D} \\
\left(\mathrm{ft}^{\mathrm{d}-2}\right)\end{array}$ & $\mathrm{d}$ & $\begin{array}{c}\mathrm{D} \\
\left(\mathrm{ft}^{\mathrm{d}-2}\right)\end{array}$ \\
\hline $\mathrm{S} 1$ & 2.52 & 396.4 & 2.36 & 262.4 & 0.81 & 1.06 \\
\hline $\mathrm{S} 3$ & 2.31 & 443.2 & 2.51 & 839.7 & - & - \\
\hline
\end{tabular}

G4 CV-S1 for wu, TF

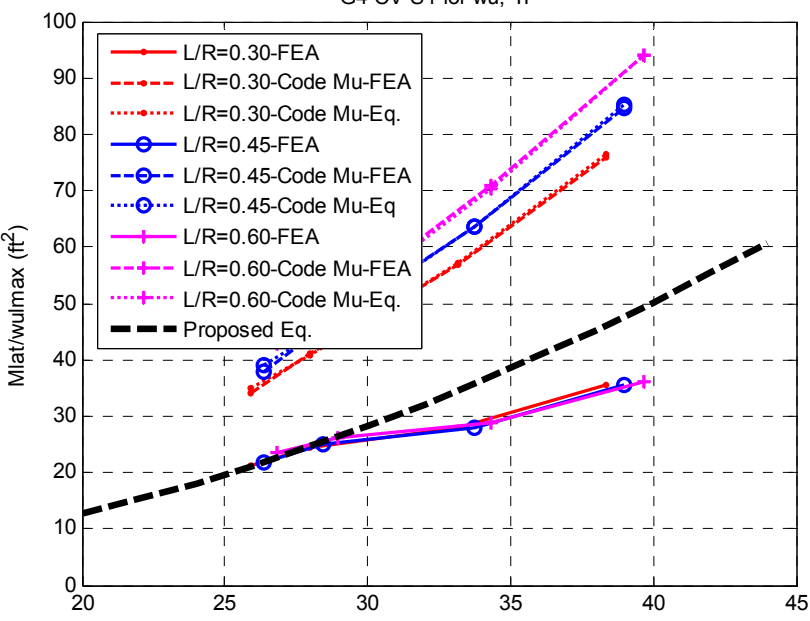

G4 CV-S3 for wu, TF

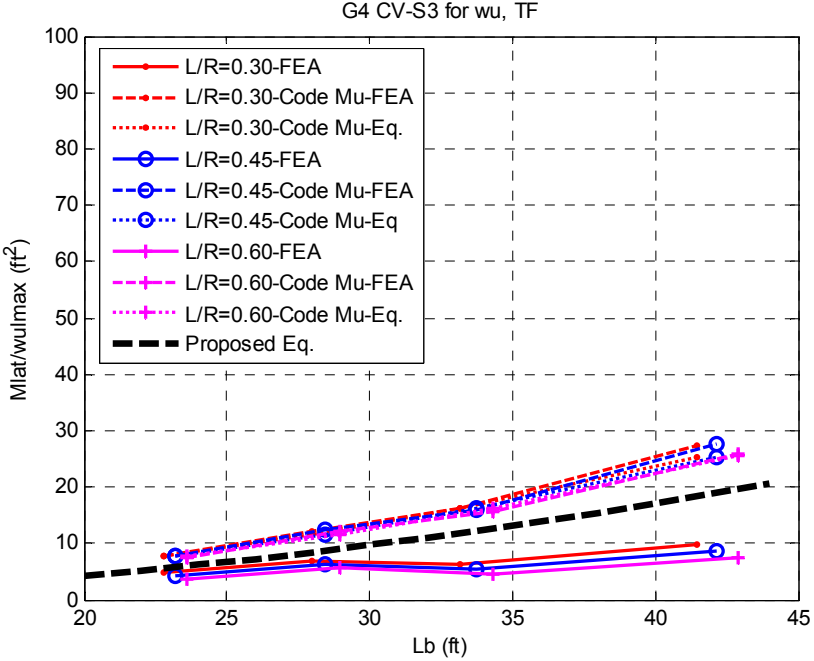

G4 CV-S1 for wu, BF

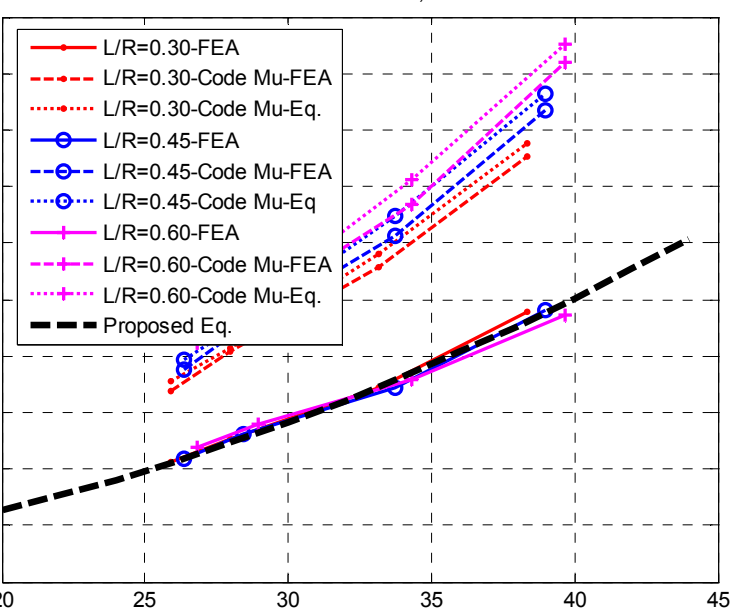

G4 CV-S3 for wu, BF

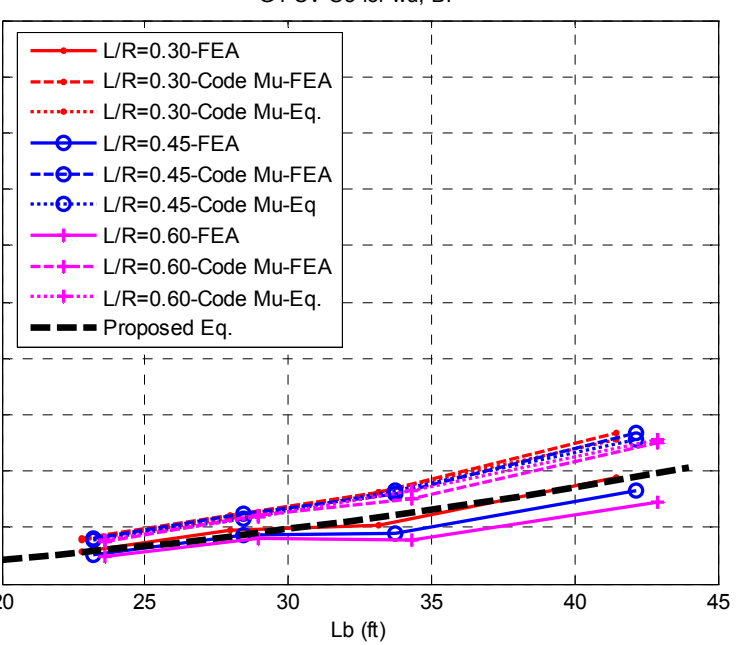

Figure 5-21. LFB in the outer girder due to distributed loads ( $\mathrm{Lm}=150 \mathrm{ft}$, Pos. Moment) 
G1 CV-S1 for wu, TF

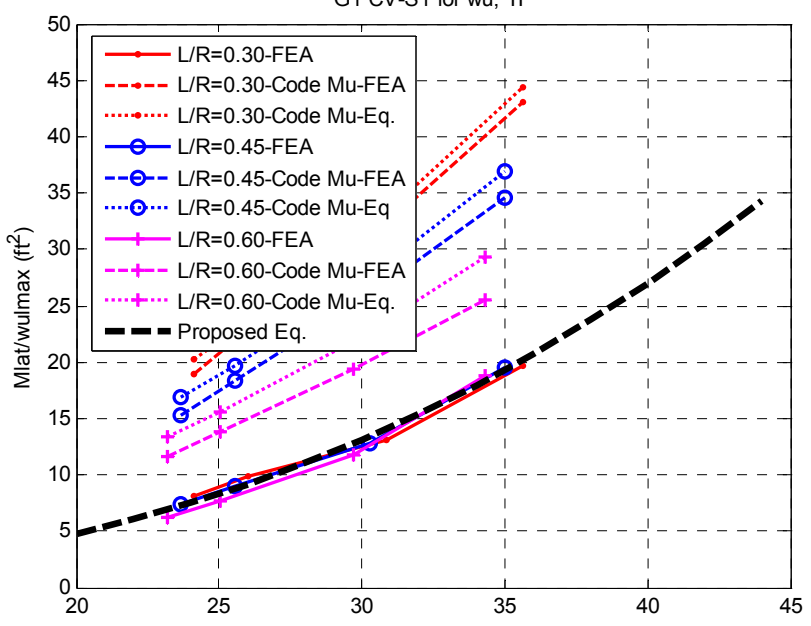

G1 CV-S3 for wu, TF

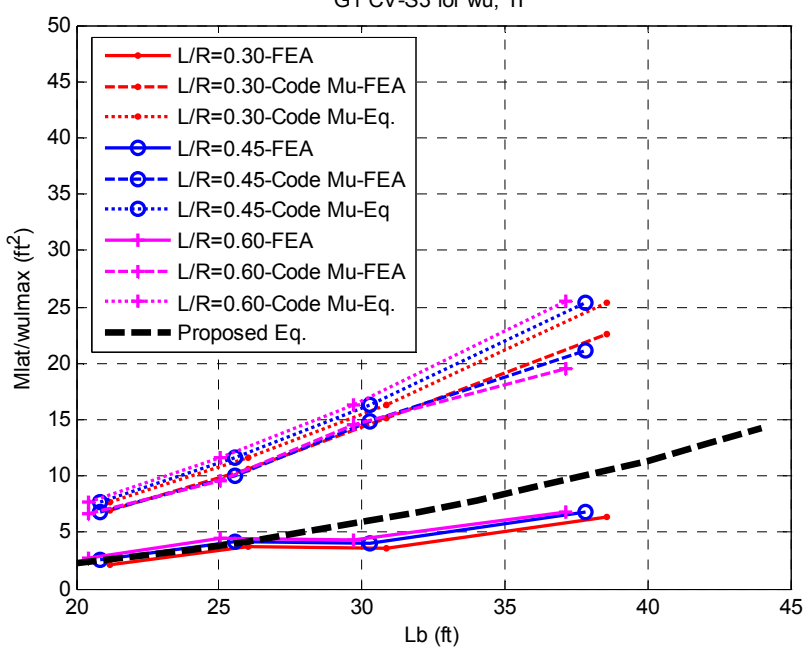

G1 CV-S1 for wu, BF

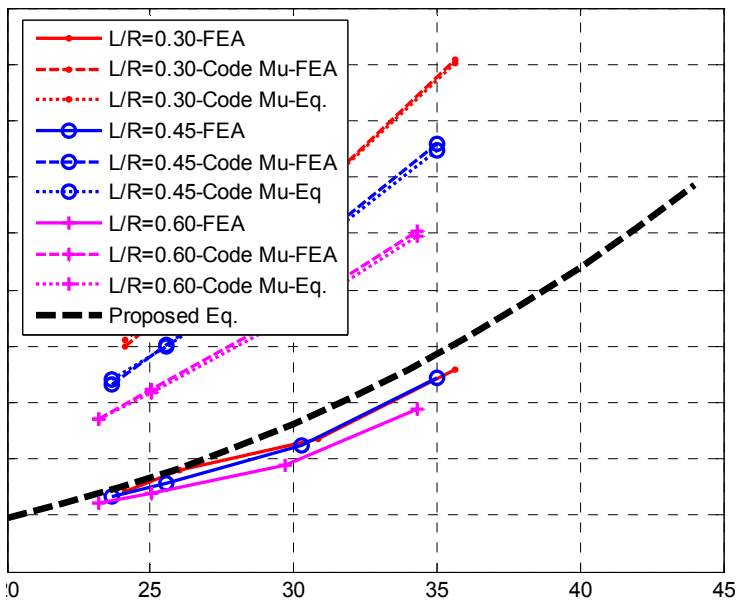

G1 CV-S3 for wu, BF

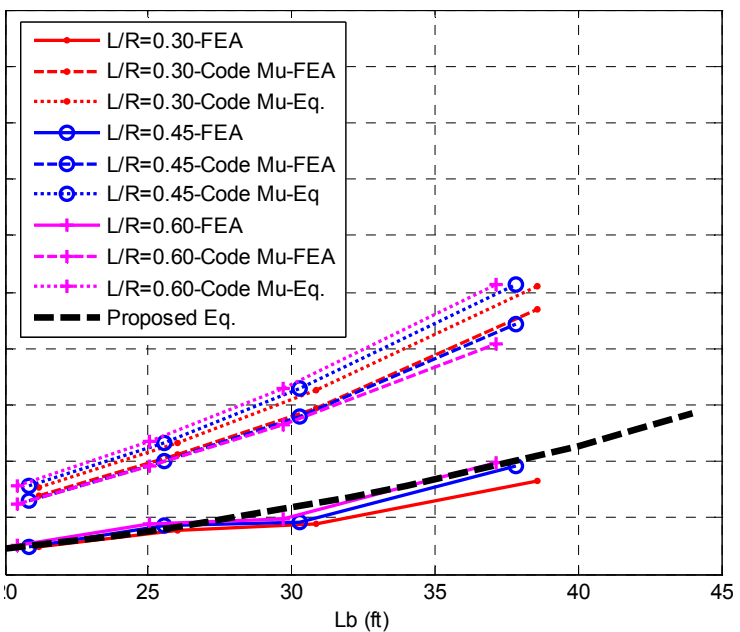

Figure 5-22. LFB in the inner girder due to distributed loads ( $\mathrm{Lm}=150 \mathrm{ft}$, Pos. Moment)

\subsubsection{Concentrated loading effect}

An approximation similar to the one described in the previous section is proposed to estimate the bending effects in curved bridges due to concentrated loads.

\subsection{Major-axis bending, $f_{b u}$}

The proposed model to estimate $f_{b u}$ given by Equation 5-7 is still valid in this case. The difference lies on the specific values of $b$ and $c$ contained in Table 5-10. Figure 5-23 and Figure 5-24 compares the numerical stresses with those estimated using Equation 5-7. 
Table 5-10. Fitting parameters for fbu due to concentrated loads (Pos. Moment)

\begin{tabular}{ccccccc}
\cline { 2 - 6 } & \multicolumn{2}{c}{$\boldsymbol{L}_{\boldsymbol{m}}=\mathbf{1 5 0 f t}$} & $\boldsymbol{L}_{\boldsymbol{m}}=\mathbf{2 4 0 f t}$ & $\boldsymbol{L}_{\boldsymbol{m}}=\mathbf{3 0 0 f t}$ \\
\hline Outer Girder, G4: End length, $L_{e}$ & 0.8 & 1 & $\mathbf{b}$ & $\mathbf{c}$ & $\mathbf{b}$ & $\mathbf{c}$ \\
\hline Outer Girder, G4: Middle length, $L_{m}$ & 0 & 1 & 0 & 1 & 0 & 1.25 \\
\hline Inner Girder, G1: End length, $L_{e}$ & 0.75 & 1 & 0.9 & 1 & 1 & 1 \\
\hline Inner Girder, G1: Middle length, $L_{m}$ & 0 & 1 & 0.45 & 1 & 0.45 & 1 \\
\hline
\end{tabular}
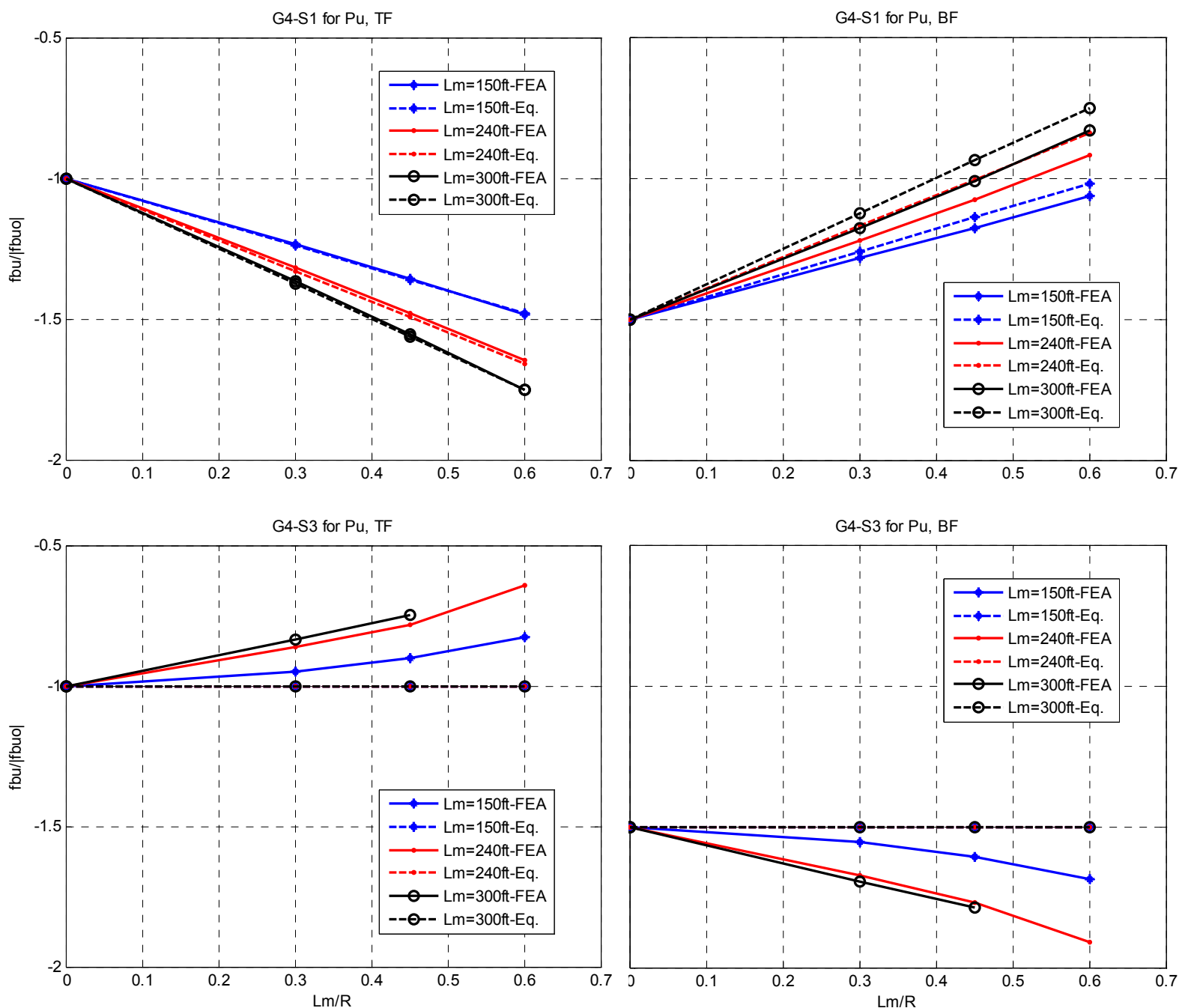

Figure 5-23. Normalized $f_{b u}$ due to concentrated loads in outer girders (Pos. Moment) 

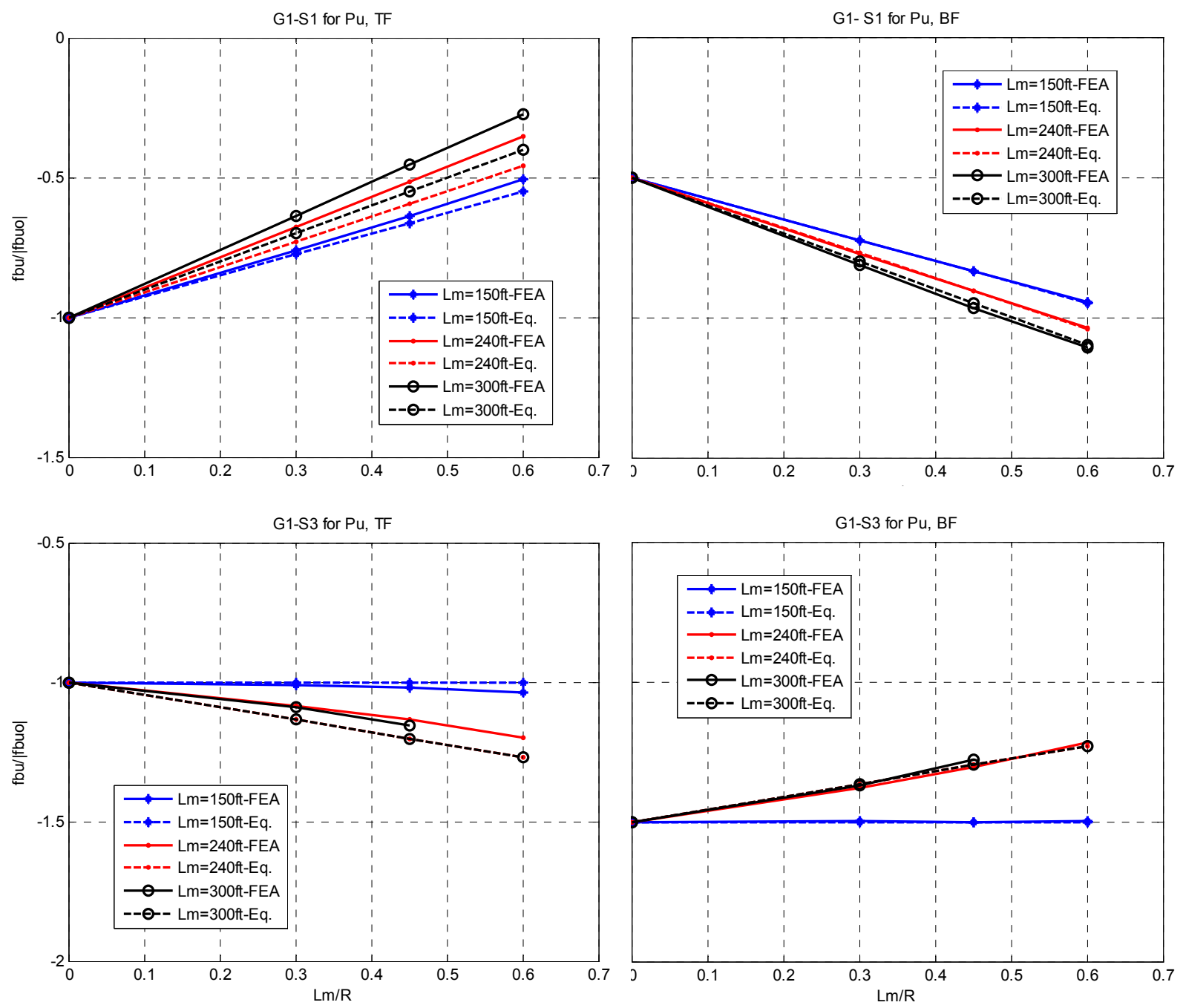

Figure 5-24. Normalized $f_{b u}$ due to concentrated loads in inner girders (Pos. Moment)

\subsection{Lateral flange bending, $f_{l}$}

In this case, the definition of $f_{l}$ is modified to allow the inclusion of the lateral concentrated load, $P_{l}$, as follows:

$$
\begin{gathered}
P_{l}=\frac{P_{u} d_{\text {max }}}{h} \\
f_{l}=\frac{P_{l} L_{b}^{f}}{F S_{f}}
\end{gathered}
$$

where $f$ and $F$ are the exponent and denominator, respectively, that define the corresponding lateral flange moment. 
Equation 5-14 shows the proposed equation to estimate the LFB due to concentrated loads considering both the curvature and overhang torsional effects. However, the participation of the overhang terms is also shown to be low compared to the curvature effects (Figure 5-25). Therefore, the overhang loading effects are neglected in this case as well.

$$
\begin{aligned}
& M_{\text {lat }}=\left(M_{\text {lat }}\right)_{\text {curv. }} \pm\left(M_{\text {lat }}\right)_{\text {ov. }} \\
& M_{\text {lat }}=\left[\frac{w_{u l \max } L_{b}^{d}}{D}+\frac{\left(4 P_{l}\right) L_{b}^{f}}{F}\right] \pm\left[\frac{w u_{l} L_{b}^{a}}{A}+\frac{\left(4 P u_{l}\right) L_{b}}{B}+\left(4 P u_{l}\right) C\right]
\end{aligned}
$$

Table 5-11 and Table 5-12 contain the values of the fitting variables $d, D, f$ and $F$ for the outer and inner girders, respectively. Figure 5-26 and Figure 5-27 (including the figures contained in Appendix B) show that the proposed equations satisfactorily predict the numerical results and reductions compared to AASHTO estimations in most of the cases. It is also observed that the bottom flange exhibits the most critical LFB effects compared to the top flange, especially for short span lengths. A variation in the curvature is also considered negligible in the LFB, especially for the end spans.

\begin{tabular}{|c|c|c|c|c|c|c|}
\hline & \multicolumn{2}{|c|}{$150 \mathrm{ft}$} & \multicolumn{2}{|c|}{$240 \mathrm{ft}$} & \multicolumn{2}{|c|}{$300 f t$} \\
\hline & d & $\begin{array}{c}D \\
\left(\mathbf{f t}^{\mathrm{d}-2}\right)\end{array}$ & d & $\begin{array}{c}D \\
\left(\mathrm{ft}^{\mathrm{d}-2}\right)\end{array}$ & d & $\begin{array}{c}D \\
\left(\mathrm{ft}^{\mathrm{d}-2}\right)\end{array}$ \\
\hline Positive moment region: End span & 2.05 & 41.59 & 2.04 & 42.06 & 1.79 & 23.02 \\
\hline \multirow[t]{2}{*}{ Positive moment region: Middle span } & 0 & 0 & 0 & 0 & - & - \\
\hline & f & $\begin{array}{c}F \\
\left(\mathbf{f t}^{\mathrm{f}-1}\right)\end{array}$ & f & $\begin{array}{c}F \\
\left(\mathrm{ft}^{\mathrm{f}-1}\right)\end{array}$ & f & $\begin{array}{c}F \\
\left(\mathbf{f t}^{\mathrm{f}-1}\right)\end{array}$ \\
\hline Positive moment region: End span & 0 & 0 & 0 & 0 & 1.35 & 14.38 \\
\hline Positive moment region: Middle span & 1.68 & 57.0 & 1.96 & 167.39 & - & - \\
\hline
\end{tabular}

Table 5-11. Fitting parameters for concentrated loads in the outer girders (Pos. Moment) 

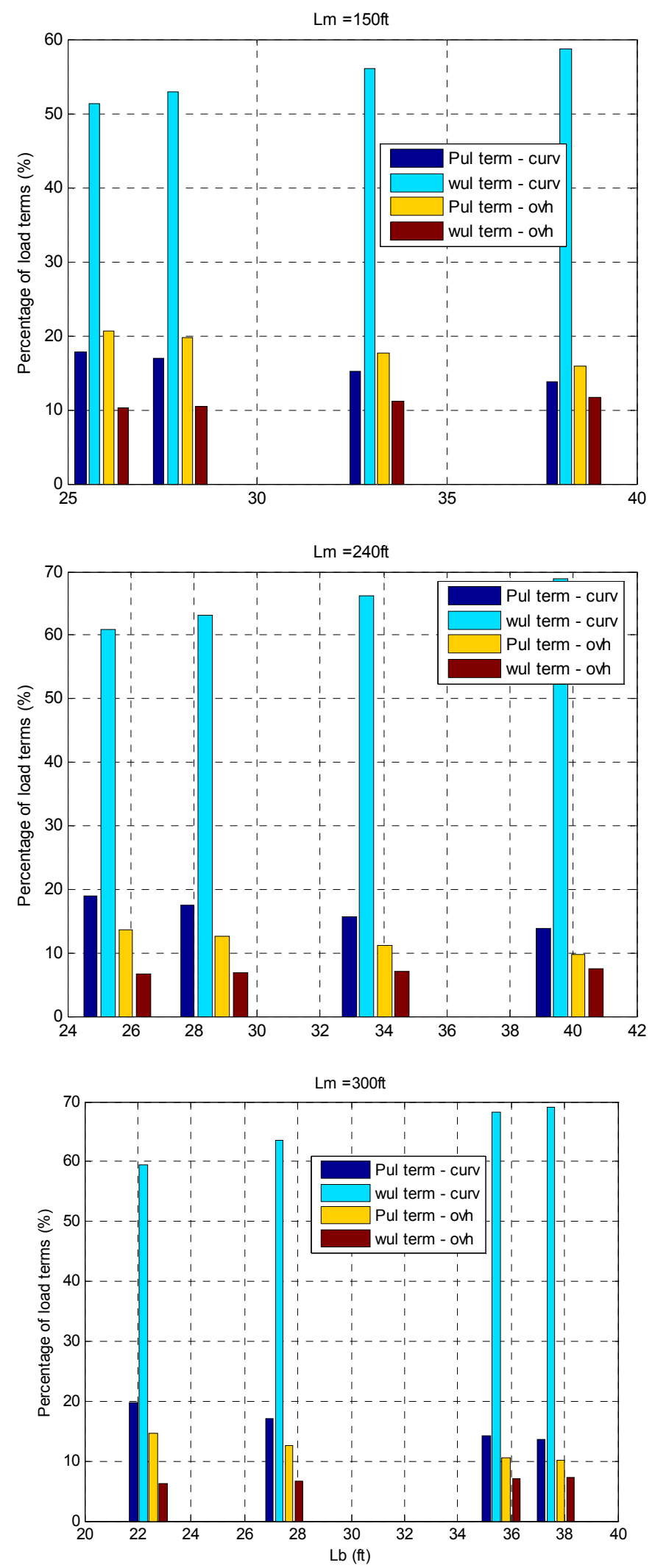

Figure 5-25. Overhang and curvature effects in the LFB due to concentrated loads 
Table 5-12. Fitting parameters for concentrated loads in the inner girders (Pos. Moment)

\begin{tabular}{|c|c|c|c|c|c|c|}
\hline & \multicolumn{2}{|c|}{$150 \mathrm{ft}$} & \multicolumn{2}{|c|}{$240 \mathrm{ft}$} & \multicolumn{2}{|c|}{$300 \mathrm{ft}$} \\
\hline & d & $\begin{array}{c}D \\
\left(\mathrm{ft}^{\mathrm{d}-2}\right)\end{array}$ & d & $\begin{array}{c}D \\
\left(\mathbf{f t}^{\mathrm{d}-2}\right)\end{array}$ & d & $\begin{array}{c}D \\
\left(\mathbf{f t}^{\mathrm{d}-2}\right)\end{array}$ \\
\hline Positive moment region: End span & 2.88 & 1855.8 & 2.40 & 443.73 & $\begin{array}{l}(2.36, \\
1.57)^{*}\end{array}$ & $\begin{array}{l}(359.0, \\
58.44)^{*}\end{array}$ \\
\hline \multirow[t]{2}{*}{ Positive moment region: Middle span } & 0 & 0 & 0 & 0 & - & - \\
\hline & f & $\begin{array}{c}F \\
\left(\mathbf{f t}^{\mathrm{f}-1}\right)\end{array}$ & f & $\begin{array}{c}F \\
\left(\mathbf{f t}^{\mathrm{f}-1}\right)\end{array}$ & f & $\begin{array}{c}F \\
\left(\mathbf{f t}^{\mathrm{f}-\mathbf{1}}\right)\end{array}$ \\
\hline Positive moment region: End span & 0 & 0 & 0.77 & 20.80 & $\begin{array}{l}(0.99 \\
0.82)^{*}\end{array}$ & $\begin{array}{l}(42.45, \\
3.48)^{*}\end{array}$ \\
\hline Positive moment region: Middle span & 2.45 & 1557.1 & 3.06 & 9744.3 & - & - \\
\hline
\end{tabular}

*(TF, BF)

G4 CV-S1 for Pu, TF

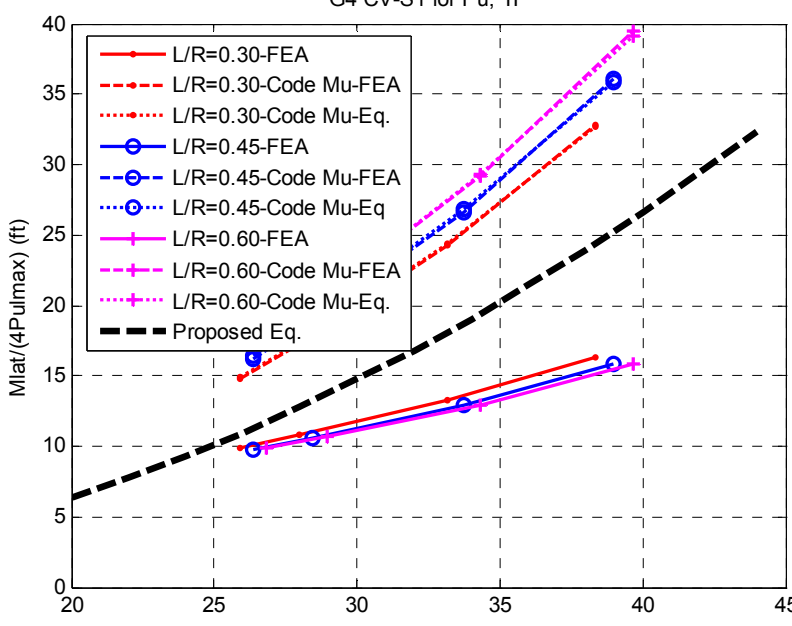

G4 CV-S3 for Pu, TF

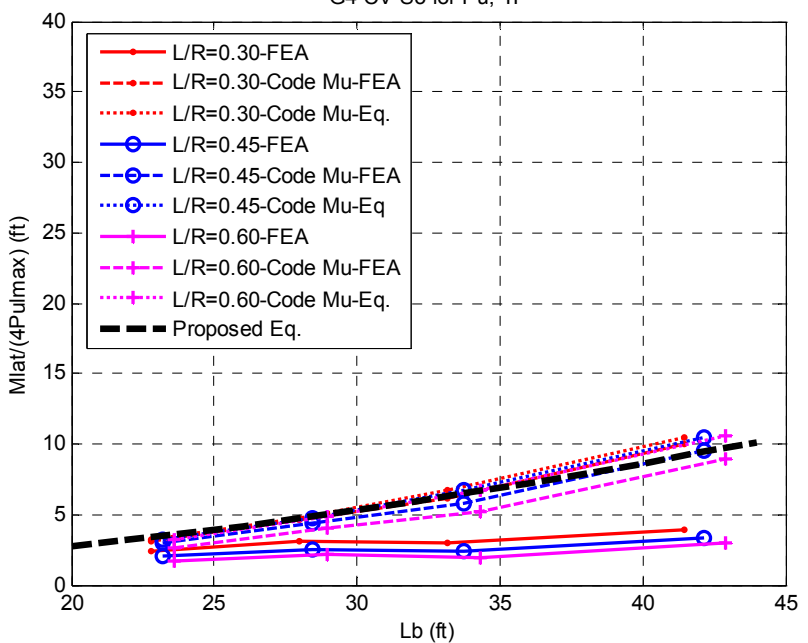

G4 CV-S1 for Pu, BF

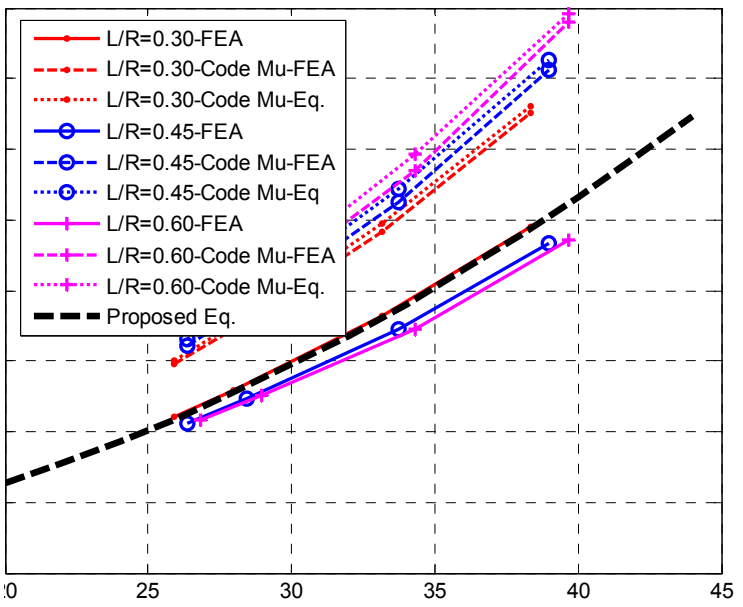

G4 CV-S3 for Pu, BF

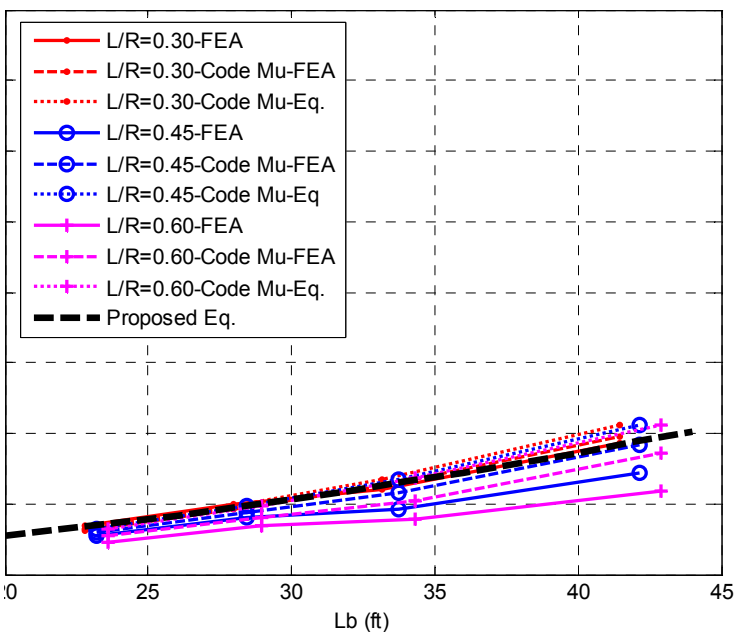

Figure 5-26. LFB in the outer girder due to concentrated loads ( $L m=150 \mathrm{ft}$, Pos. Moment) 

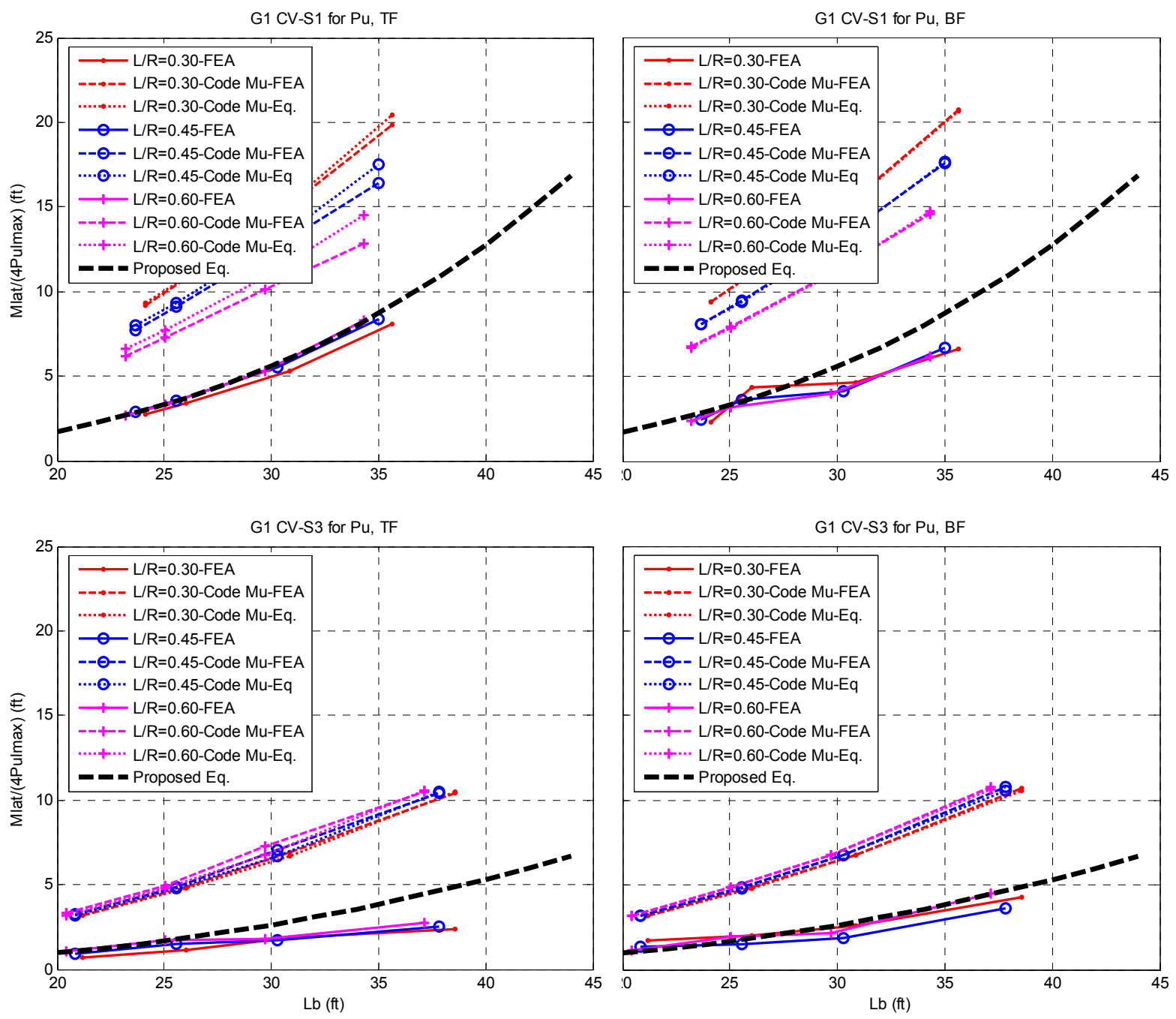

Figure 5-27. LFB in the inner girder due to concentrated loads ( $L m=150 \mathrm{ft}$, Pos. Moment)

\subsubsection{Negative Moment Regions}

Although the curvature does not directly affect the pier zones since there is no eccentricity between the loads and the supports, the results show that the torsional restriction offered by the pier regions affects the bending stresses in these zones.

\subsubsection{Major-axis bending, $f_{b u}$}

The major-bending stresses exhibit a linear trend independent of the span length as shown in Figure 5-28. As a result, the definition of $b$ in Equation 5-7 reduces to 0.43 and 0.30 for the outer and inner girders, respectively. The variable $c$ is always taken as 
one. Additionally, the sign combinations indicated by Table 4 are reversed to account for the change in the stress state.
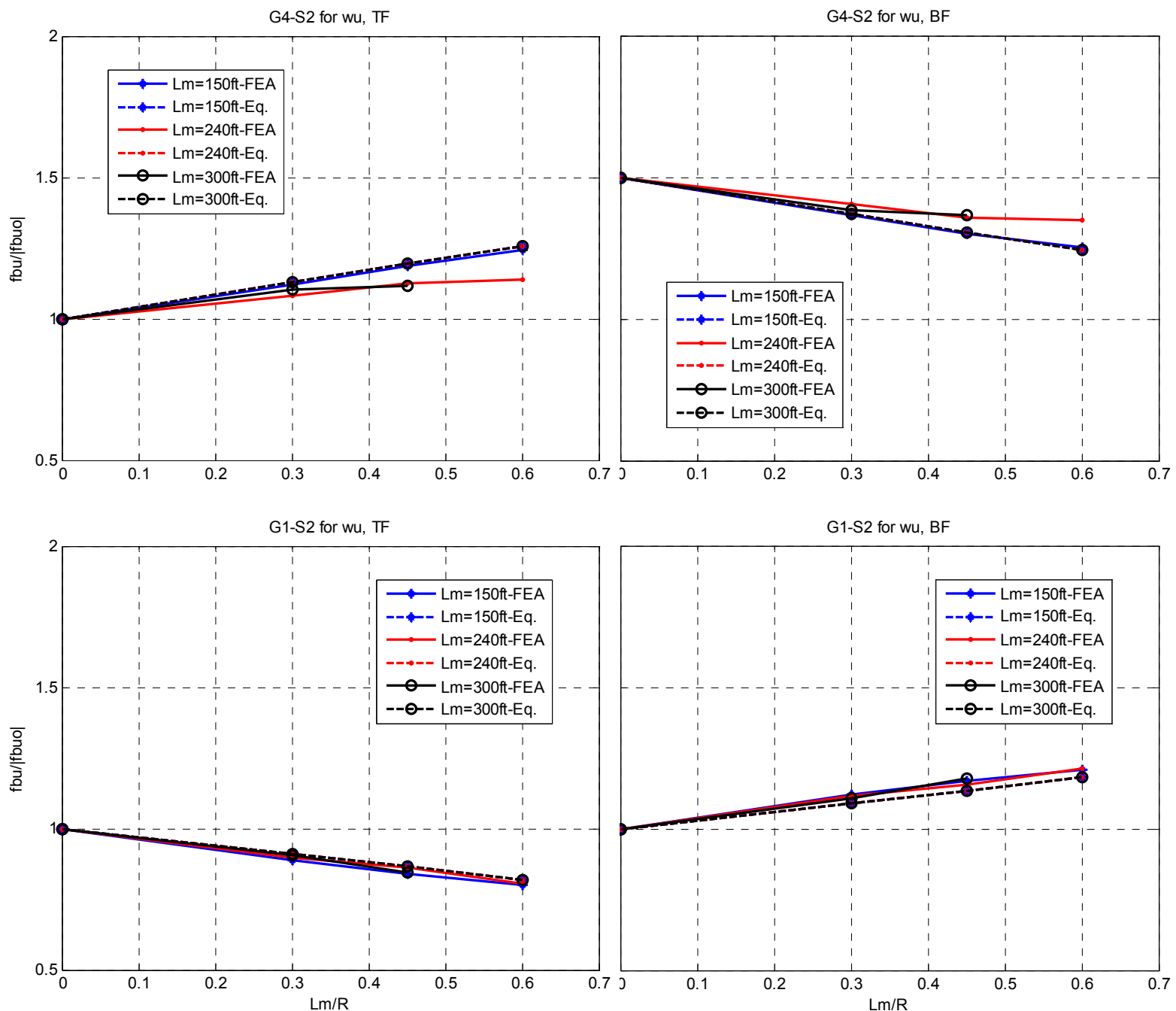

Figure 5-28. Normalized major-axis bending stresses in the negative moment regions

\subsubsection{Lateral flange bending, $f_{l}$}

The LFB is satisfactorily predicted using Equation 5-10 with the parameters contained in Table 5-13. However, the variable $w_{u l \max }$ is computed as the average of the values corresponding to the end and middle spans, since the maximum eccentricity $\left(d_{\max }\right)$ depends on the span length. 
Table 5-13. Fitting parameters for the negative moment regions

\begin{tabular}{ccccccc}
\hline & \multicolumn{2}{c}{ 150ft } & \multicolumn{2}{c}{ 240ft } & \multicolumn{2}{c}{ 300ft } \\
\hline & $\mathrm{d}$ & $\begin{array}{c}\mathrm{D} \\
\left(\mathrm{ft}^{\mathrm{d}-2}\right)\end{array}$ & $\mathrm{d}$ & $\begin{array}{c}\mathrm{D} \\
\left(\mathrm{ft}^{\mathrm{d}-2}\right)\end{array}$ & $\mathrm{d}$ & $\begin{array}{c}\mathrm{D} \\
\left(\mathrm{ft}^{\mathrm{d}-2}\right)\end{array}$ \\
\hline $\mathrm{G} 4$ & 1.84 & 21.52 & 1.63 & 10.17 & 1.52 & 5.73 \\
\hline $\mathrm{G} 1$ & 1.72 & 12.93 & 1.69 & 12.18 & 1.36 & 3.76 \\
\hline
\end{tabular}

Figure 5-29 (including the Figures in Appendix B) shows that the proposed equations predict satisfactorily the bending effects in both exterior girders, while the code equations are over conservative for most cases. It is also observed that the LFB in these regions is practically unaffected by the curvature.
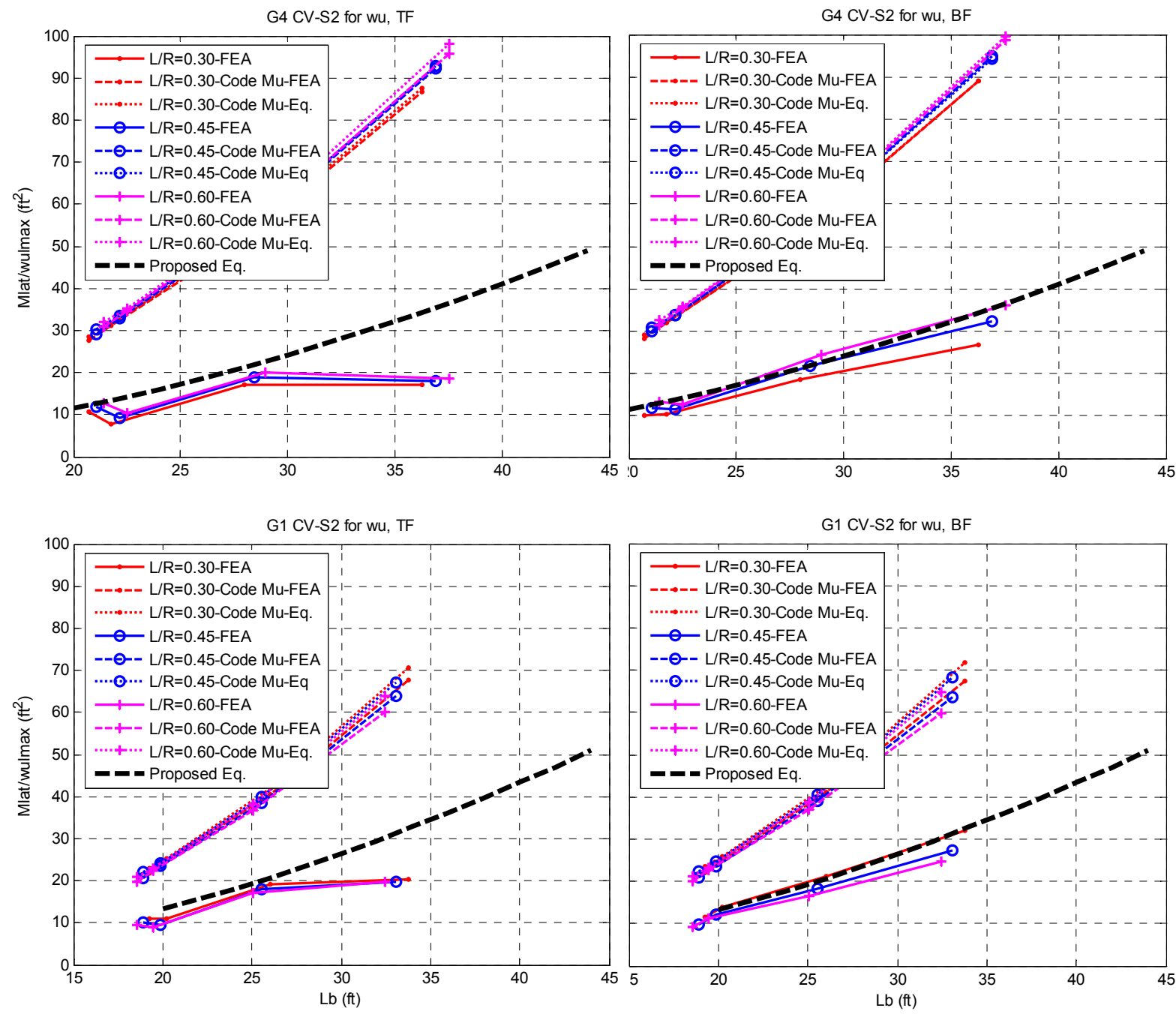

Figure 5-29. LFB in the negative moment regions $(\mathrm{Lm}=150 \mathrm{ft})$ 


\subsection{Concluding remarks}

The bending stresses during the deck placement in straight, skewed and curved steel I-girder bridges were evaluated using the results obtained from the parametric study described in Chapter 4. The LFB stresses caused by distributed and concentrated overhang loads were estimated finding the curves that have the best fit to the critical stresses obtained from the parametric study. According to this evaluation, the following major observations were made.

\subsubsection{Straight structures}

The major-axis bending stresses exhibited by the exterior girders of straight bridges are independent of the cross-frame spacing. Therefore, these stresses may be obtained from the structural analysis of either a simplified model that does not consider explicitly the cross frames or a more detailed model involving any cross-frame distance.

For the positive moment regions, the LFB due to distributed loads was satisfactorily predicted by the equation proposed in this work over the entire range of cross-frame distances, principally at the end spans. Although the LFB obtained in the middle span is lower, the same equations were proposed to simplify the approximations. Comparisons with the code estimations showed that the Specifications are highly conservative in all cases, especially for large cross-frame distances.

For concentrated loads, a linear regression considering the most external points from the critical curves was performed to adjust the numerical stresses. These external points correspond to models where the concentrated loads are placed at the middle of a cross-frame spacing which is the critical location. The proposed equation for the bottom flange governs over the code equation for cross-frame distances larger than $26 \mathrm{ft}$. However, both the code and the proposed approximations coincide for the top flange.

The LFB effect on the negative moment regions was approximated considering only the distributed loading case since the contribution from the concentrated loads is negligible. The results showed that the proposed equation satisfactorily predicts the LFB in both flanges. 


\subsubsection{Skewed structures}

The results showed that skewed bridges exhibit LFB even when torsional loads are not applied. However, in the presence of torsional effects, the LFB effect is slightly more pronounced when the cross frames are oriented parallel to the supports. Therefore, a perpendicular orientation of the cross frames is recommended for general skewed bridges to increase the lateral stiffness of the structure. This perpendicular configuration was adopted in this work to evaluate the LFB effects.

The results indicated that the major-axis bending in skewed bridges is not only independent of the cross-frame spacing but also of the skew angle. Therefore, these stresses may be taken from structural analyses performed for skewed bridges with any cross-frame spacing and skew angle, including their simplified straight counterpart.

The LFB in the positive moment regions was approximated using curve fitting models similar to the ones used for straight bridges. For distributed loads, a lower limit was introduced in the proposed equation for the bottom flange since this flange exhibits LFB even for closely spaced cross frames. The minimum LFB stress considered in the bottom flange is proportional to the skew angle. The results were compared to the code equation for overhang loads and significant overestimates of the LFB were found principally for long cross-frame distances.

On the other hand, for concentrated loads, the results showed that the code equation for overhang loads fails to predict satisfactorily the LFB in most of the cases, principally in the bottom flange.

The LFB in the negative moment regions was approximated in this work by a constant term that depends on the skew angle. This approximation was adopted to conservatively estimate the stresses which do not exhibit a consistent trend in these regions. The results also indicated that the code recommendation of using 10Ksi for the unfactored LFB stresses in skewed bridges is conservative even compared to factored stresses. 


\subsubsection{Curved structures}

The evaluation of the bending stresses in curved bridges indicated that $f_{b u}$ and $f_{l}$ are deeply affected by the curvature and the position of the girder, i.e. outer or inner girder.

The analyses showed that the major-bending stresses in curved bridges are independent of the cross-frame spacing. However, they increase linearly with the curvature in the positive moment regions of the end spans. Conversely, these stresses at the middle span of the outer girder are not significantly affected by the curvature. Therefore, a linear model was proposed to estimate the major-bending effects in the positive moment regions of both exterior girders. This model computes the major-axis bending in curved bridges based on the major-axis bending stresses exhibited by their straight counterpart. Therefore, the proposed equation allows estimating $f_{b u}$ in curved bridges from structural analyses of simplified straight bridges. In the negative moment regions, the major-bending stresses also exhibit a linear trend but it is independent of the span length.

The assumption of computing $f_{b u}$ based on a simplified model with the real arc length of the curved girder does not help to represent the curvature effects on $f_{b u}$. In fact, the AASHTO recommendation of ignoring the curvature effects for $f_{b u}$ when $L / R$ is lower than 0.06 introduces an error of approximately $10 \%$ in long span bridges.

The LFB in curved girders is caused by both the curvature and the overhang loads. However, it was shown that the participation of the overhang loads in the LFB is low compared to the curvature effects. In fact, the overhang-to-curvature effect ratio reduces as the length of the span and the curvature increase. Therefore, the overhang term was dropped from the final expression proposed for the LFB which simplifies to the curvature term only. However, the torsional effects due to overhang loads are implicitly considered since the curve fitting process is applied to the total LFB obtained from the parametric study. A comprehensive formulation to estimate the LFB effects due to curvature was developed for distributed and concentrated loads, respectively. The critical case between the top and bottom flanges was selected to define the equations proposed in this work to estimate the LFB in curved bridges. 
The results indicated that the LFB is practically unaffected by the variation of the curvature, a slight effect was only observed in the positive moment regions of the middle span. This observation indicates that these effects need to be considered even in bridges with large curvature radii.

The equations proposed in this project to estimate the LFB in curved bridges work adequately in both exterior girders. Significant reductions were found in most of the cases compared to the estimations given by the AASHTO Specifications.

The major-axis bending effects in the AASHTO equation were computed using both the numerical and the estimated major-axis bending stresses, obtaining similar results. The estimated major-axis bending stresses correspond to the stresses computed using the equation proposed in this work to estimate $f_{b u}$ in curved bridges from the results obtained in their straight counterpart. Therefore, the LFB in a curved bridge can be conservatively approximated using the code equation together with the major-axis bending from the corresponding straight bridge. The advantage of the proposed equations over the code approximation is that it is not required to know in advance the major-bending effects to compute the LFB. However, the principal disadvantage is that different expressions are required to define the effects of distributed and concentrated loads, while the code approximation consists of one single equation that applies for all load cases independent of the girder location and flange position.

However, the results from the parametric study indicated that the code equation fails to predict the LFB due to distributed loads at the inner girder of highly curved bridges with long spans. Therefore, it is recommended to use the equation proposed in this work which predicts satisfactorily the LFB in all cases. It was also observed that the bottom flange under concentrated loads exhibits the most critical LFB effects compared to the top flange, especially for short span lengths.

The results indicated that the outer girder exhibits the most critical combined bending effects. On the contrary, the curvature decreases the magnitude of the majorbending stresses in the inner girder resulting in a combined bending action much lower than that corresponding to the outer girder. Therefore, the design of both exterior girders shall be based on the evaluation of the outer girder, unless an optimization of the inner 
girder is pursued. In that case, the effects of the girder stiffness in the behavior of the whole cross section of the deck shall be investigated.

In general, it is recommended to distribute the cross frames such that a cross frame is placed at the maximum vertical bending moment to decrease the combined effect of $f_{l}$ and $f_{b u}$ during construction. 


\section{Chapter 6: Evaluation of the flexural limit states for constructibility}

In this chapter, the flexural limit states for constructibility are evaluated according to AASHTO Specifications for the parametric bridges analyzed in this work.

First, the major-axis bending and the LFB stresses obtained from FEA are used to evaluate the demand-to-capacity ratios of the flexural limit states for constructibility. This evaluation allows identifying the critical sections along the bridge and the effects of the cross-frame spacing and the deck-placement sequence in the governing limit states.

Second, the maximum allowable skews and curvatures are computed for bridges designed originally as straight. The flexural limit states for constructibility constitute the criteria used to achieve this goal. The major-axis bending stresses are taken directly from FEA and the LFB stresses are estimated using the approximate equations proposed in Chapter 5.

\subsection{General observations}

The bending results from FEA were evaluated using the flexural limit state equations for constructibility. These limit states are applied in a normalized form (demand-over-capacity) as follows:

- Discretely braced flanges in compression:

Yielding: $\quad \frac{f_{b u}+f_{l}}{\phi_{f} R_{h} F_{y c}} \leq 1$

Ultimate strength: $\quad \frac{f_{b u}+\frac{1}{3} f_{l}}{\phi_{f} F_{n c}} \leq 1$

Web bend-buckling: $\quad \frac{f_{b u}}{\phi_{f} F_{c r w}} \leq 1$

- Discretely braced flanges in tension:

$$
\text { Yielding: } \quad \frac{f_{b u}+f_{l}}{\phi_{f} R_{h} F_{y t}} \leq 1
$$


- Continuously braced flanges in tension or compression:

Yielding:

$$
\frac{f_{b u}}{\phi_{f} R_{h} F_{y f}} \leq 1
$$

Limit for LFB: $\quad \frac{f_{l}}{0.6 F_{y f}} \leq 1$

In the following sections, the limit state ratios of the skewed and the curved bridges are compared to the results obtained from their straight counterparts.

\subsubsection{Skewed bridges}

For the skewed bridge configurations analyzed in this work, it is observed that the effect on the limit state ratios of the girder position, the cross-frame orientation and the skew angle is almost negligible as shown in Figure 6-1. However, the cross-frame spacing has a significant effect on the limit state ratios of discretely braced flanges in compression in the positive moment regions, since the LFB depends on $L_{b}$ and the capacity of the flange is reduced as $L_{b}$ increases.

Although the limit state ratios are not visibly affected by the skew, the proposed equations for the LFB presented in Chapter 5 are intended to represent the maximum possible variations on $f_{l}$ caused by the skewed geometry of the structure.

Figure 6-2 (see other figures in Appendix C) also shows that sections S1 and S3 are controlled by the second and fourth castings, respectively. The corresponding flanges in compression in both sections are governed by the ultimate strength limit state.

The negative moment regions are controlled by the fifth casting stage. The flanges in compression are governed by the yielding limit state when the span lengths are short. However, the web bend-buckling limit state governs the compression flanges for long span lengths. 

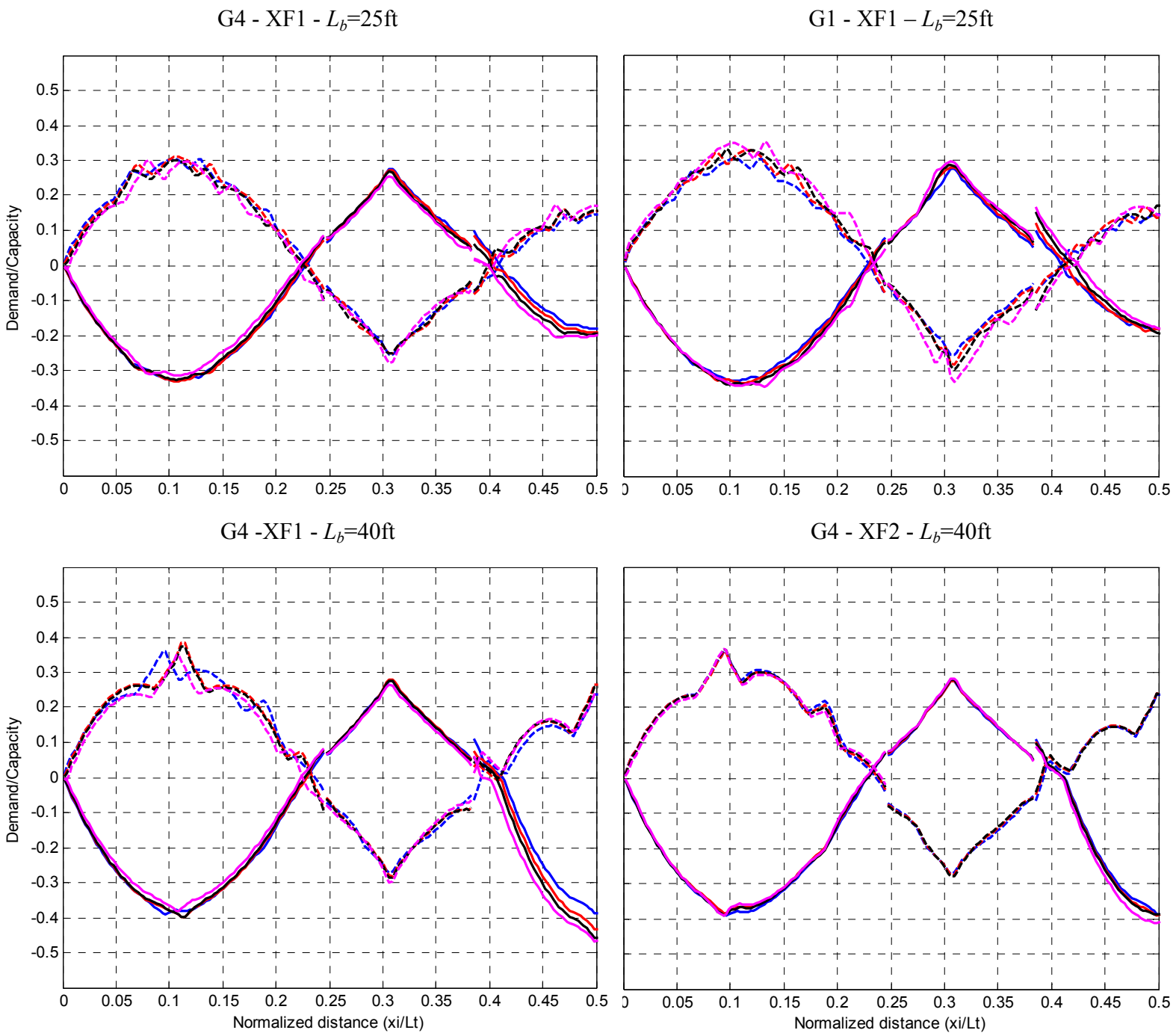

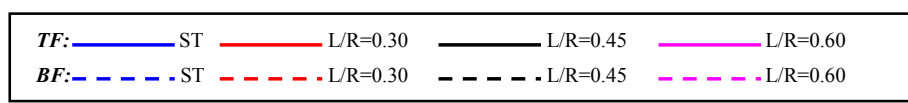

Figure 6-1. Variation of the limit state ratios along the length of skewed bridges 

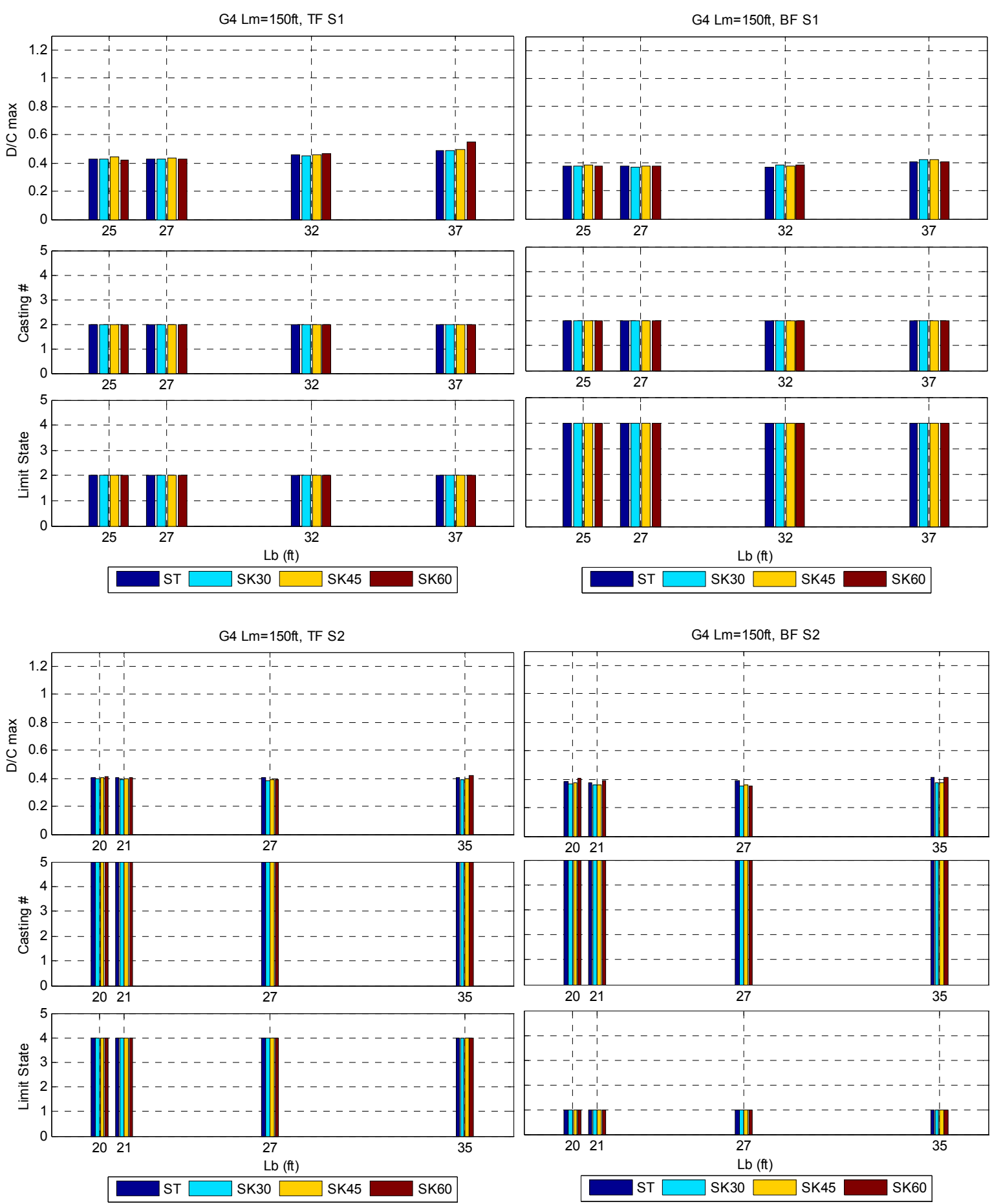

Figure 6-2. Effect of parametric variables in the limit states of skewed bridges 

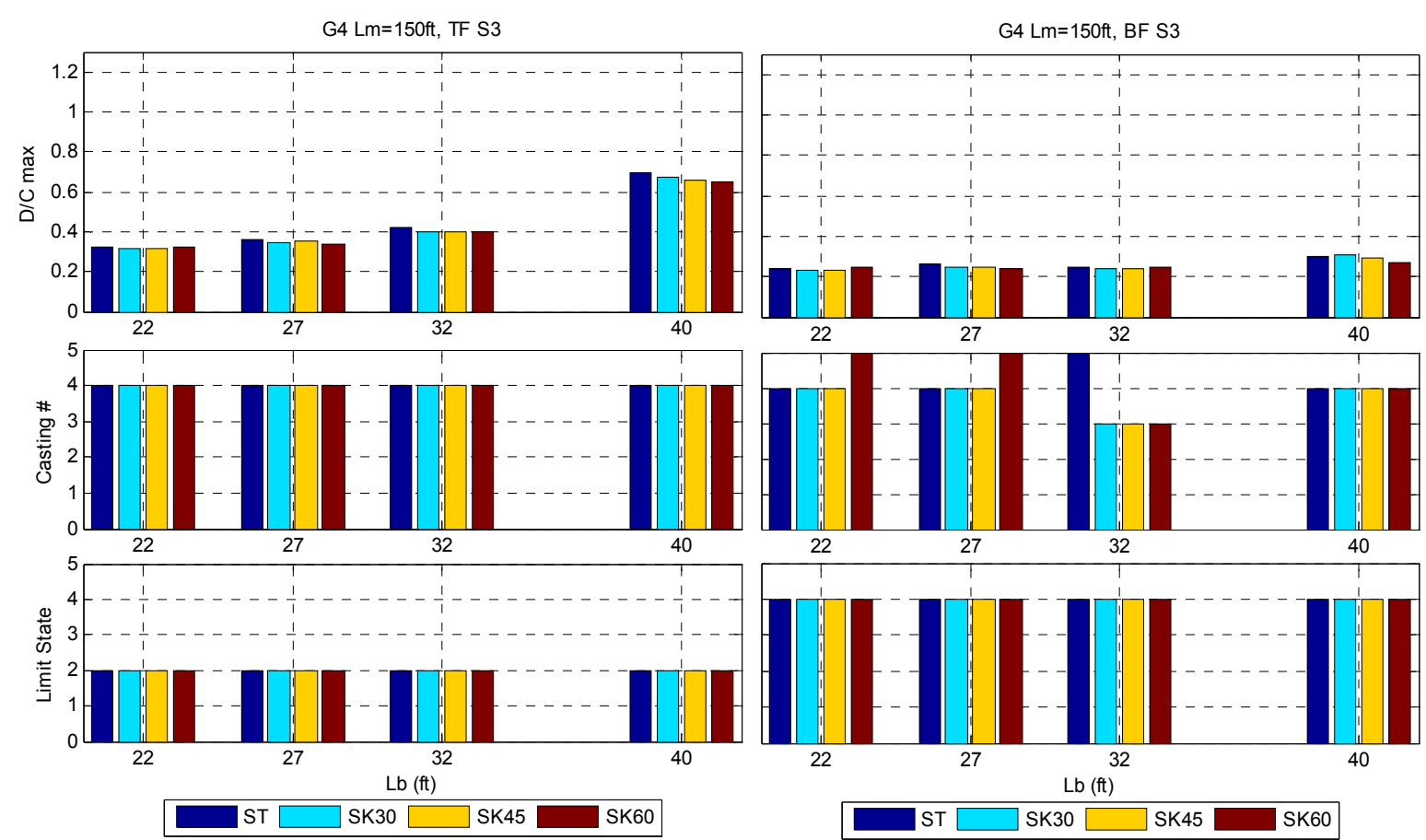

Figure 6-2. Effect of parametric variables in the limit states of skewed bridges (Cont.)

\subsubsection{Curved bridges}

Figure 6-3 illustrates that both the curvature and the cross-frame spacing highly affect the limit state ratios in the outer girder principally in the positive moment regions, while a stress relief is observed in the inner girder. This is caused by the length adjustments due to curvature since interior girders shorten as the curvature increases generating lower major-axis bending stresses. The opposite situation occurs in the outer girders where the vertical stresses increase. Additionally, the LFB exhibited by the inner girder is relieved since its eccentricity with respect to the supports is smaller.

In fact, the LFB undergoes a reversal effect in the inner girder compared to its straight counterpart, as shown in Figure 6-4. This is due to the curvature and overhang loading torsional effects counteracting each other in the inner girder. However, the curvature effects govern over the torsion produced by the overhang loads. 


\section{$L_{b}=25 \mathrm{ft}$}
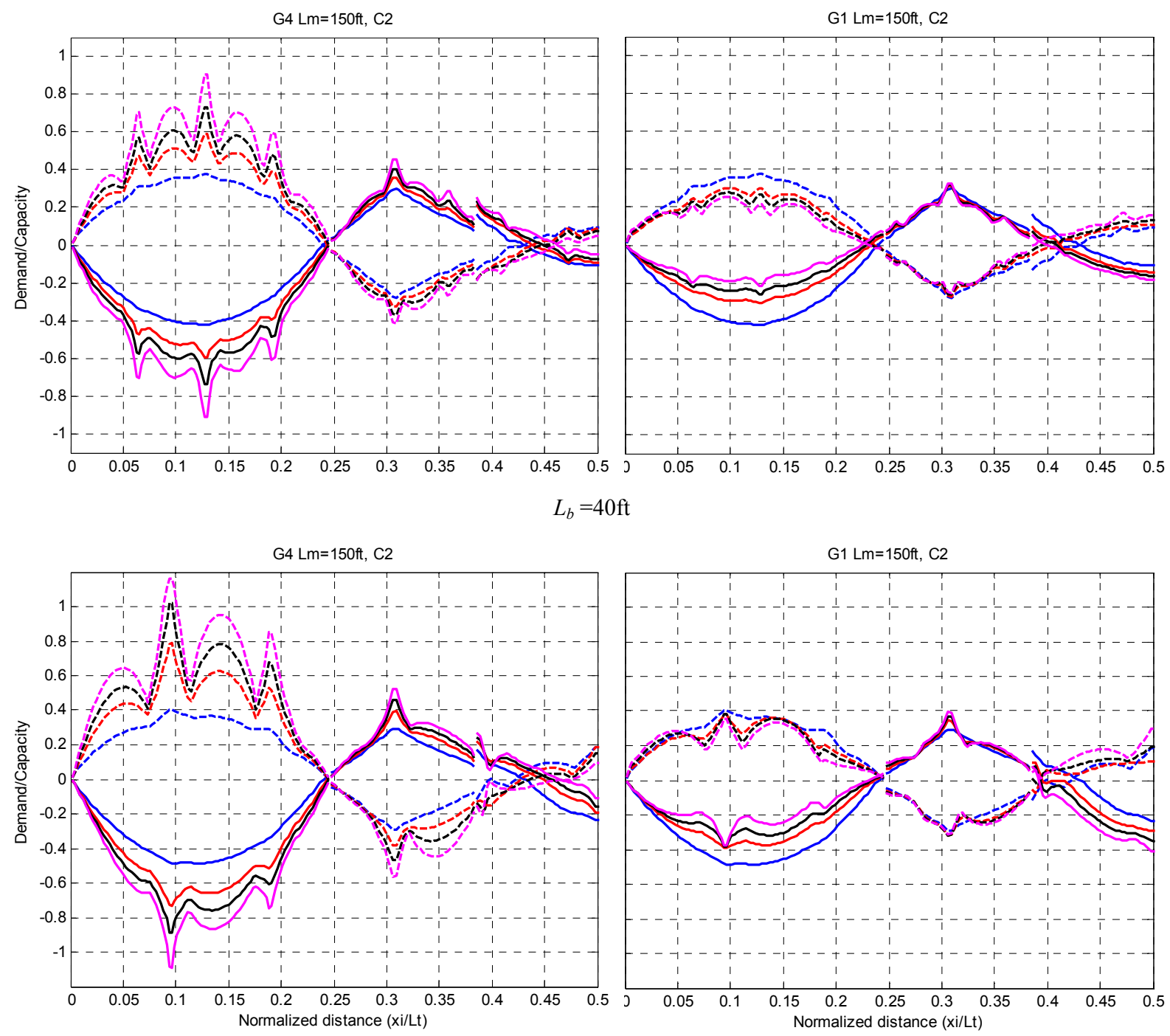

TF: $\mathrm{ST} \longrightarrow \mathrm{L} / \mathrm{R}=0.30$

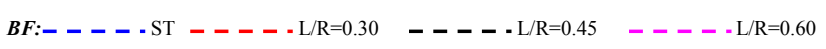

Figure 6-3. Variation of the limit state ratios along the length of curved bridges 

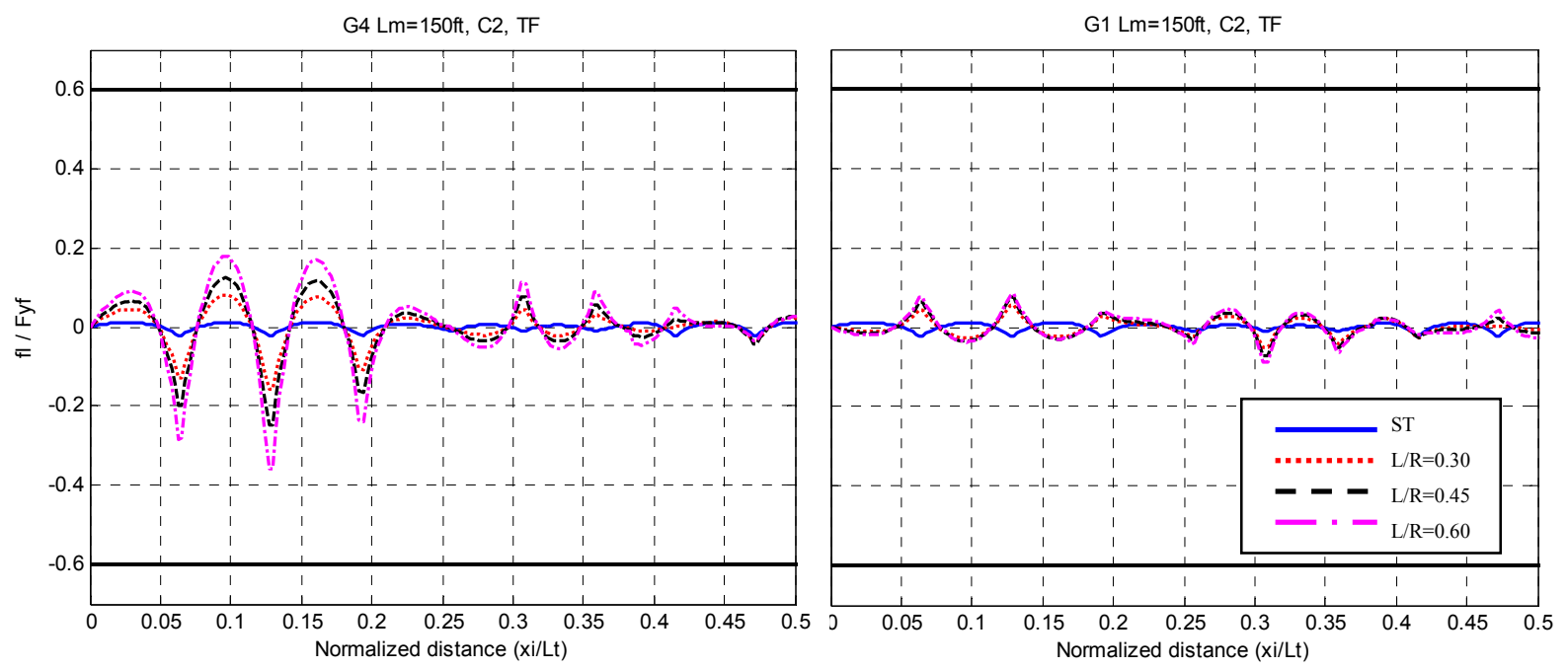

Figure 6-4. Variation of the LFB along the length of curved bridges

Figure 6-5, Figure 6-6 and Figure 6-7 show that the cross-frame spacing affects the limit state ratios in the outer girders as well. It is also observed that second and fourth castings from the deck-placement sequence control sections S1 and S3, respectively. Compression flanges in these sections are governed by the ultimate strength limit state. However, the yielding limit state may control in short span lengths with high curvatures.

As for skewed bridges, the negative moment regions are controlled by the fifth casting stage. The yielding limit state governs the compression flanges of bridges with short spans and the web bend-buckling limit state becomes significant in the pier regions for long span bridges (see Figures in Appendix D). 

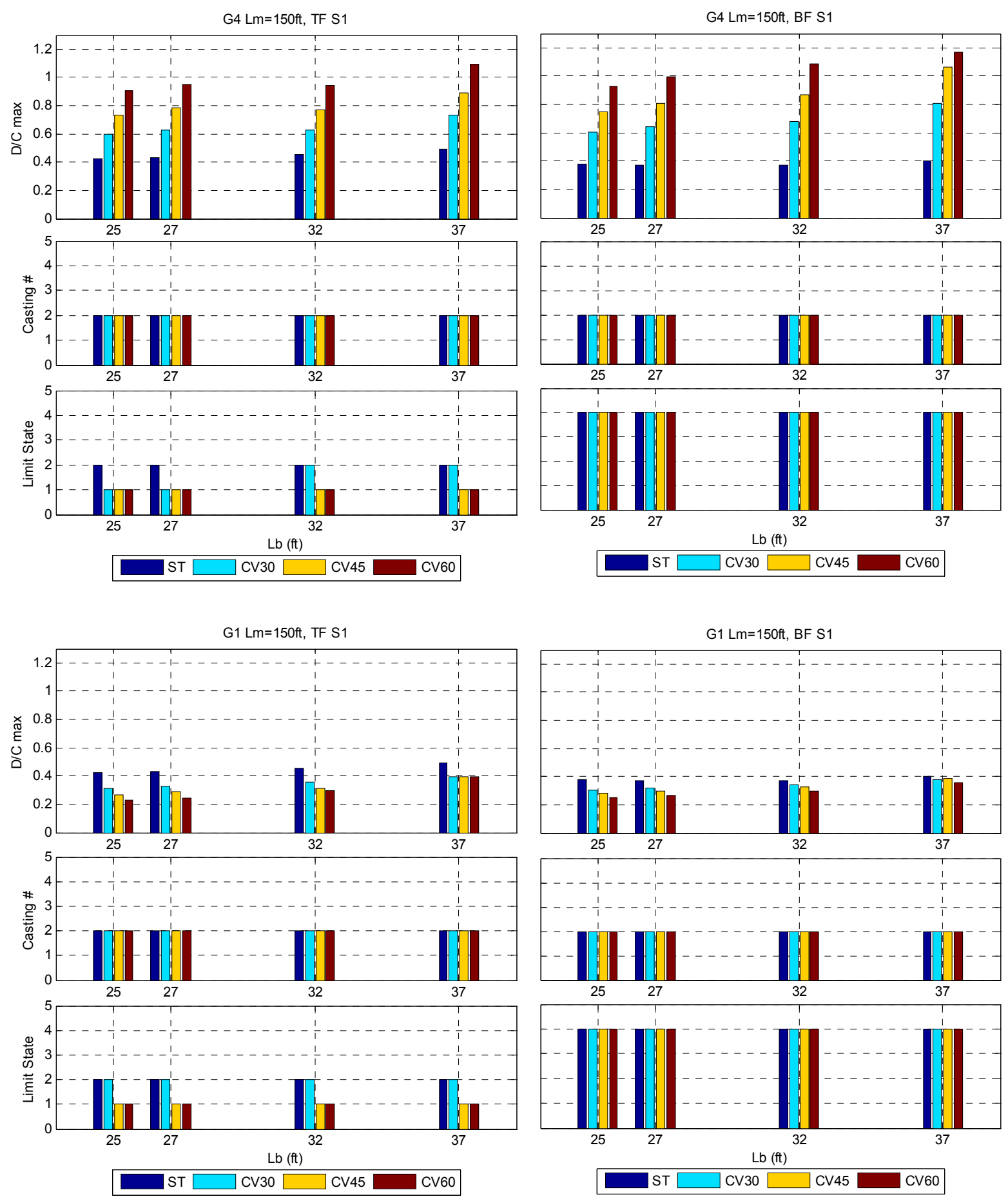

Figure 6-5. Effect of parametric variables in the limit state ratios of Section S1 

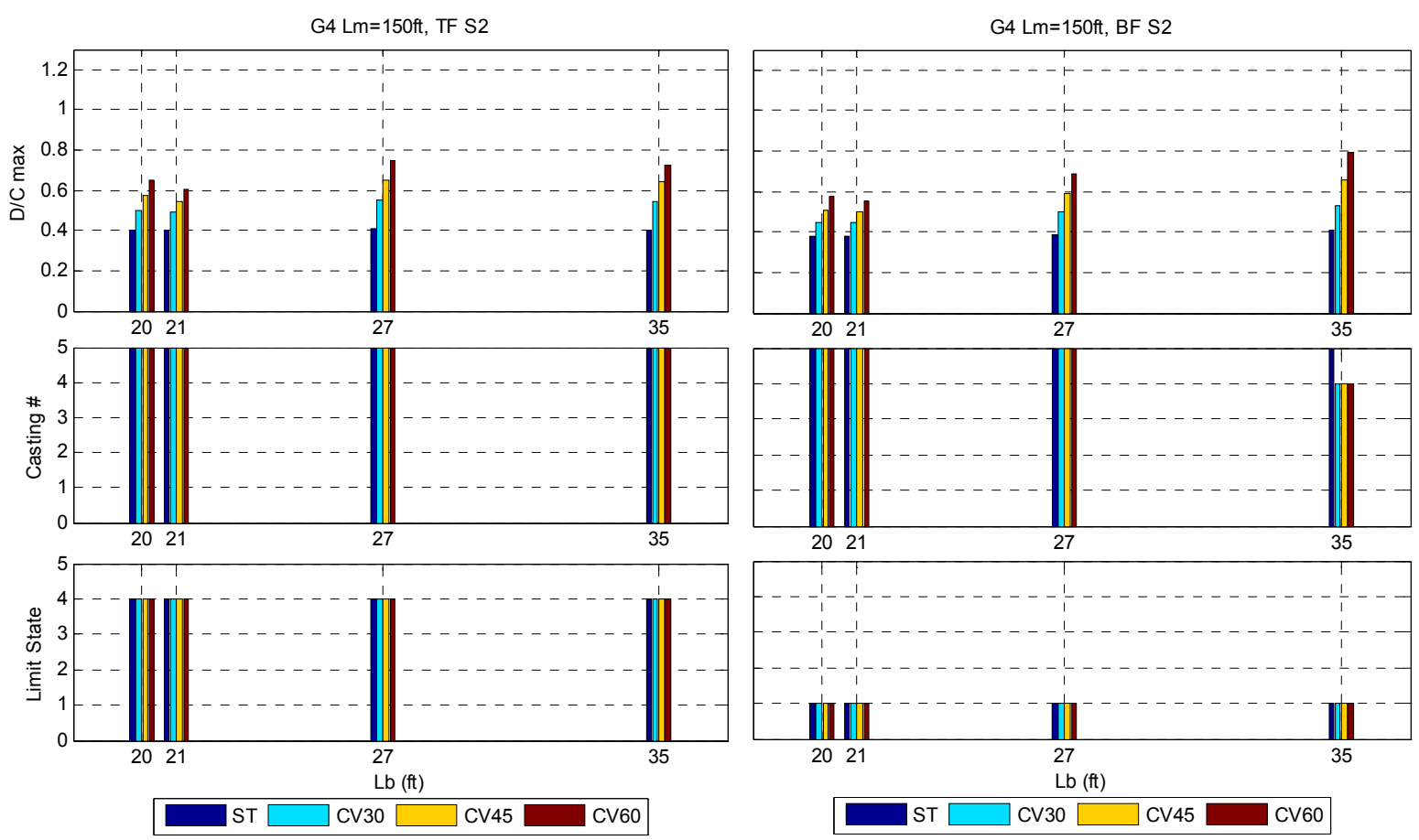

$\mathrm{G} 1 \mathrm{Lm}=150 \mathrm{ft}$, TF S2
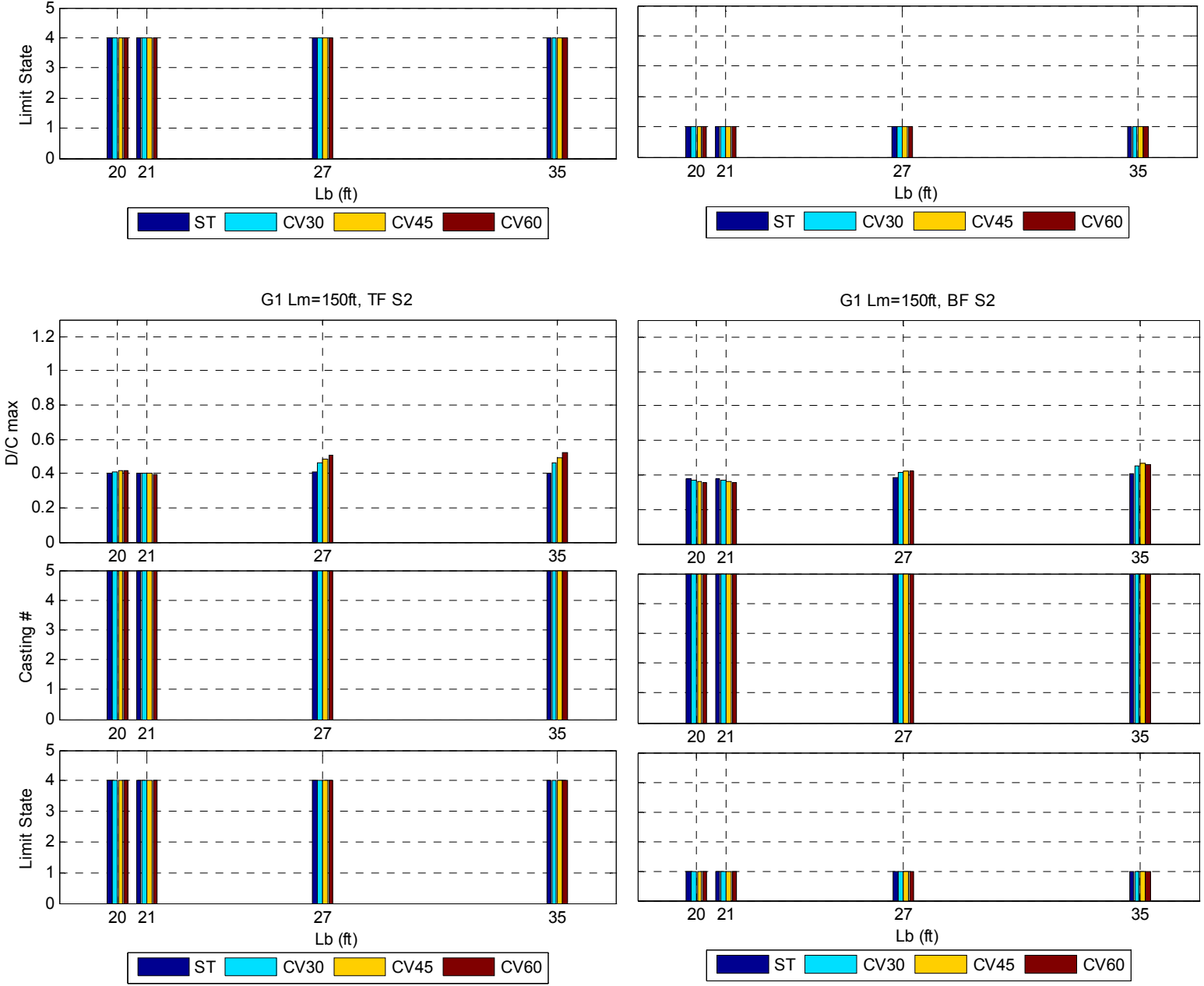

Figure 6-6. Effect of parametric variables in the limit state ratios of Section S2 

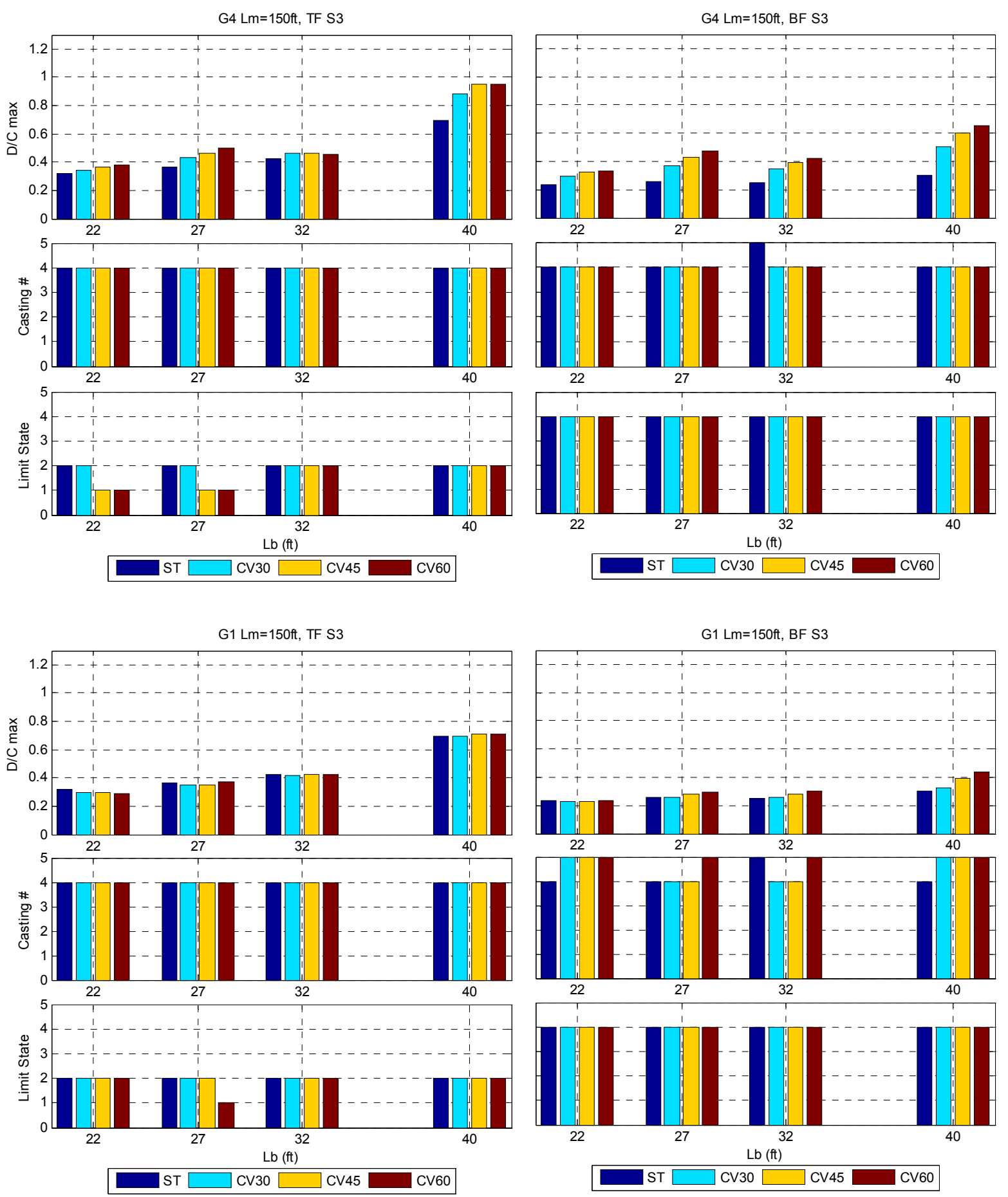

Figure 6-7. Effect of parametric variables in the limit state ratios of Section S3 


\subsection{Maximum allowable skew angle and curvature for straight bridges during construction}

In this section, the straight bridge models are evaluated using the flexural limit states for constructibility in order to define the maximum allowable skew angles and curvatures for the loading conditions given during deck placement. This evaluation will provide the engineer with design information for curved and skewed bridges based on their straight girder counterparts. Furthermore, no additional constructibility designs or checks would be necessary in case that a bridge designed as straight requires a geometrical modification within the limits established.

To achieve this goal, the maximum cross-frame spacing $\left(L_{b \max }\right)$ was computed for each straight bridge considered in this work from the limit state equations for constructibility that directly depend on $L_{b}$ (Eqns. 6-1, 6-2, 6-4 and 6-6). In the limit state equations, the major-axis bending effects were taken from FEA and the LFB stresses were expressed in terms of $L_{b}$ using the approximate equations recommended in Chapter 5 for straight bridges. After finding $L_{b \max }$ by solving the critical limit state equation, the maximum skew angle and curvature are computed for different $L_{b}$ values based on the limit state equations for constructibility as well.

\subsubsection{Skew angle}

The maximum skew was computed by solving the limit state equations for each $L_{b}$ value. However, the LFB in these equations are expressed in terms of $L_{b}{ }^{a} / A$ or $L_{b} / B+C$ for distributed and concentrated loads in the positive moment regions, respectively. Therefore, these terms are solved from the limit state equations and then are compared to the corresponding values defined in Chapter 5 for $\theta=30^{\circ}, 45^{\circ}$ and $60^{\circ}$ as shown in Figure 6-8, Figure 6-9 and Figure 6-10 for $L_{m}=150 \mathrm{ft}, 240 \mathrm{ft}$ and 300ft, respectively. For the negative moment regions, the comparisons are established in terms of stresses.

It is observed that the maximum cross-frame spacing in skewed bridges is controlled by the ultimate strength limit state in the compression flange (Eq. 6-2). Conversely, the tension flange seems to be unaffected by the skew angle. 

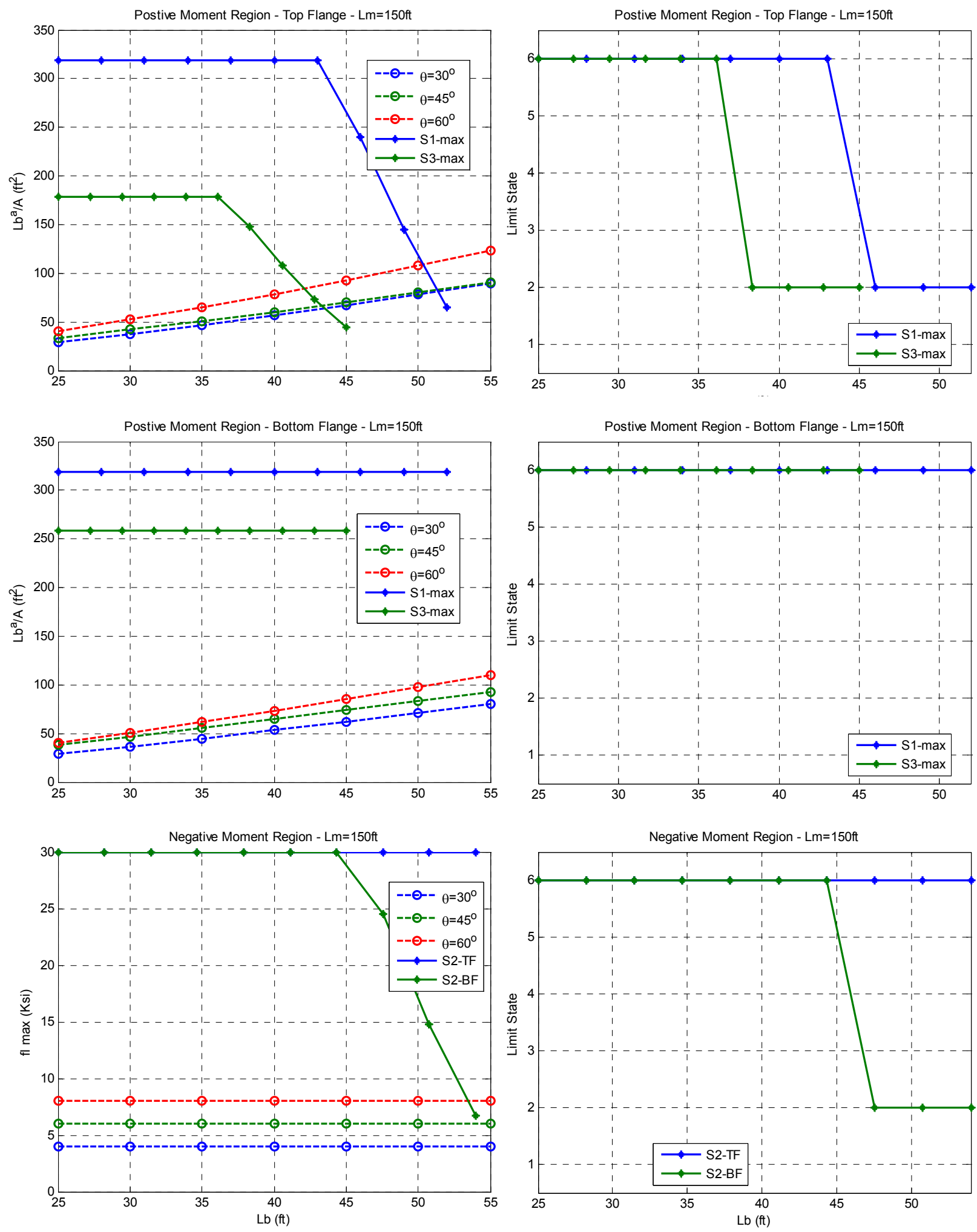

Figure 6-8. Identification of maximum skew angles for straight bridges with $\mathrm{Lm}=150 \mathrm{ft}$ 

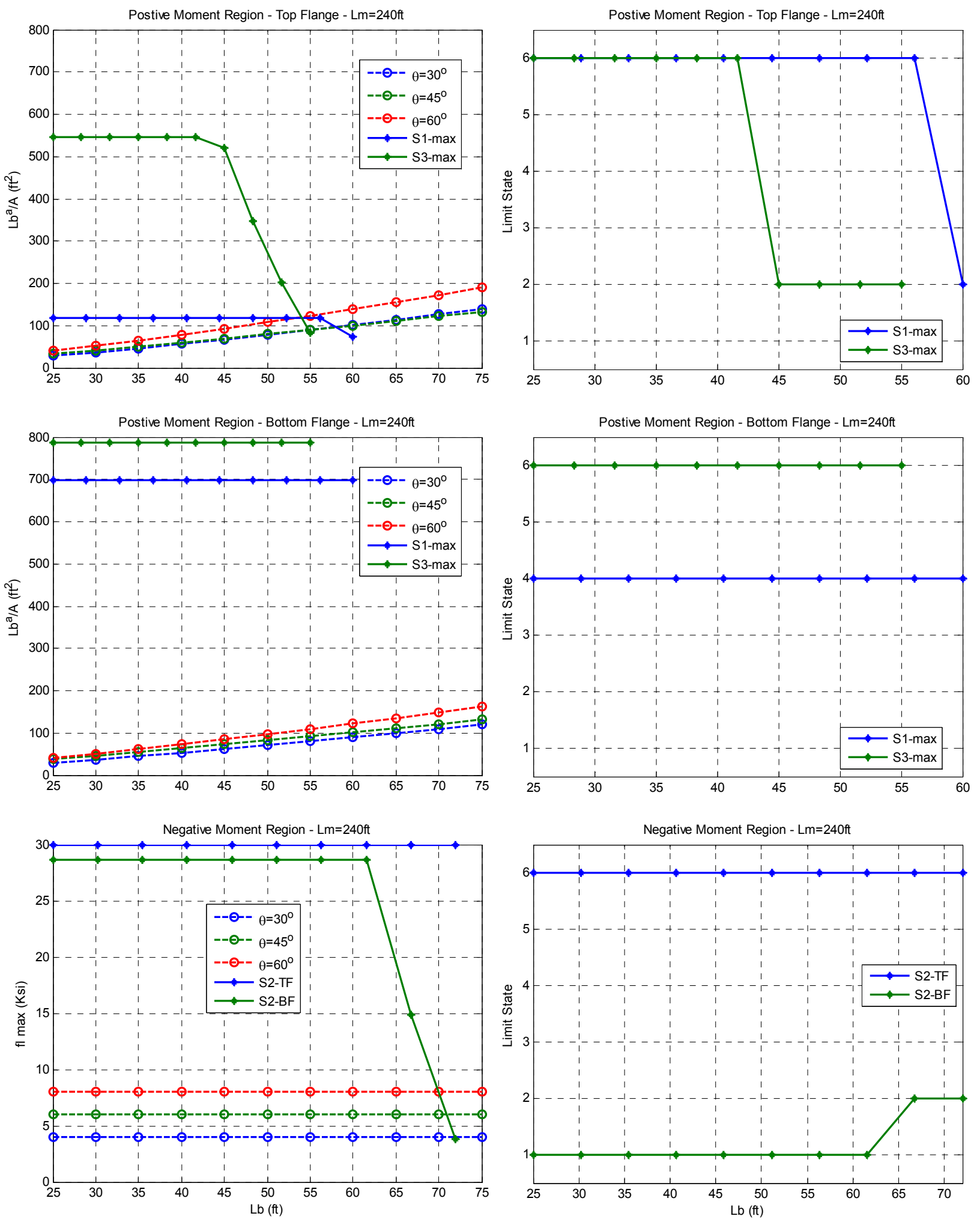

Figure 6-9. Identification of maximum skew angles for straight bridges with $\mathrm{Lm}=240 \mathrm{ft}$ 

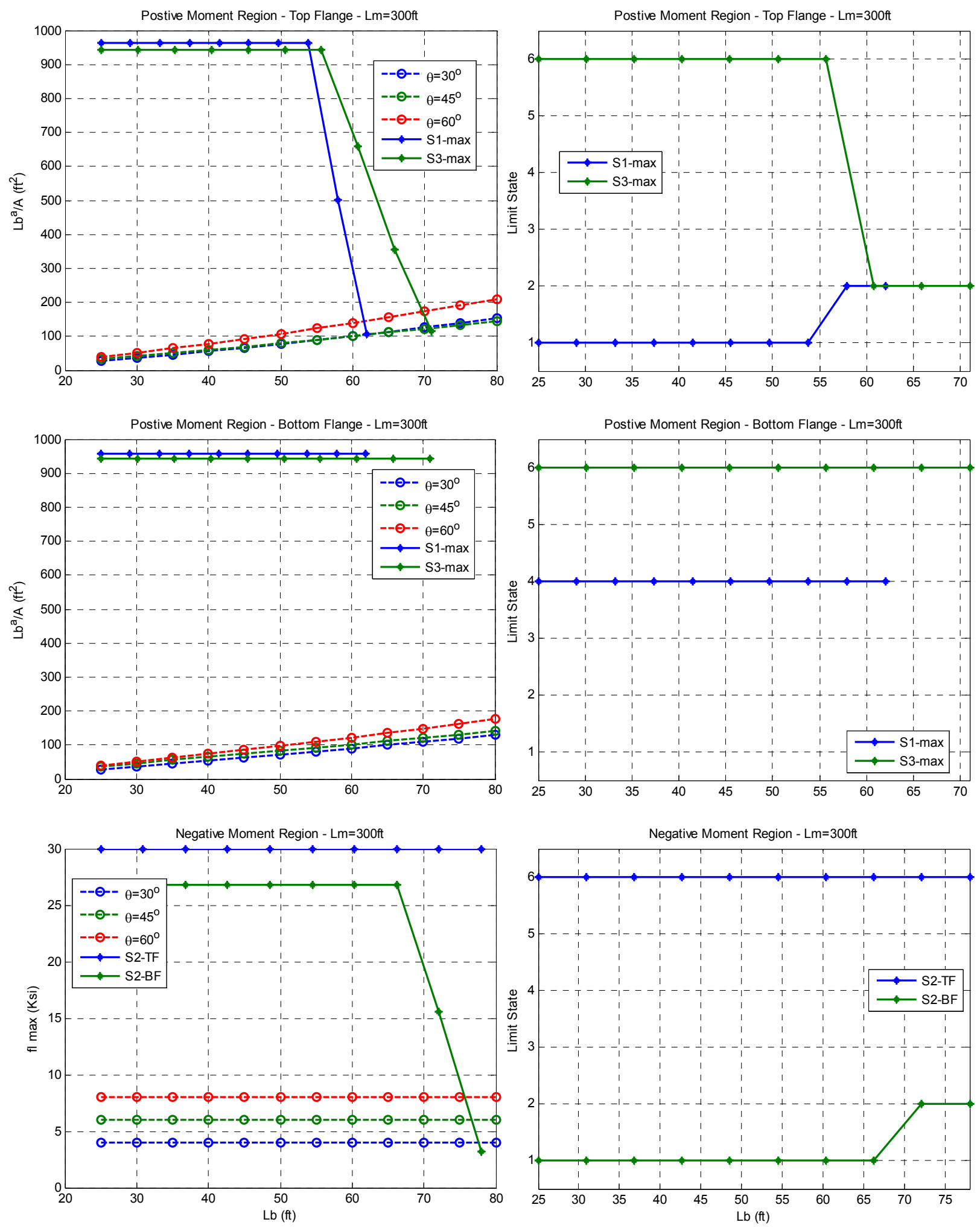

Figure 6-10. Identification of maximum skew angles for straight bridges with $\mathrm{Lm}=300 \mathrm{ft}$ 


\subsubsection{Curvature}

For curved bridges, both $f_{b u}$ and $f_{l}$ depend on the curvature as indicated by the approximate equations proposed in Chapter 5. The maximum curvature $L / R$ is solved from the limit state equations for different $L_{b}$ values as shown in Figure 6-11, Figure 6-12 and Figure 6-13 for $L_{m}=150 \mathrm{ft}, 240 \mathrm{ft}$ and $300 \mathrm{ft}$, respectively. It is noticed that the maximum curvature is controlled by the end-span cross section in the outer girder. In addition, it is also shown that the ultimate strength limit state of the compression flange controls the maximum allowable curvature for high $L_{b}$ values.

The code specifies a maximum cross frame spacing for curved bridges as follows:

$$
\begin{aligned}
& L_{b \max }=0.1 R=0.1 \frac{L}{L / R} \\
& \therefore(L / R)_{\max }=0.1 \frac{L}{L_{b}}
\end{aligned}
$$

where $R$ is the radius of curvature. Comparisons with the numerical results show that the code recommendation of $L / R$ is adequate in short span bridges (150ft) when $L_{b}$ is less than 40ft approximately. However, long span bridges present an allowable curvature lower than the one recommended in the code when the cross sections are designed from a straight girder formulation.
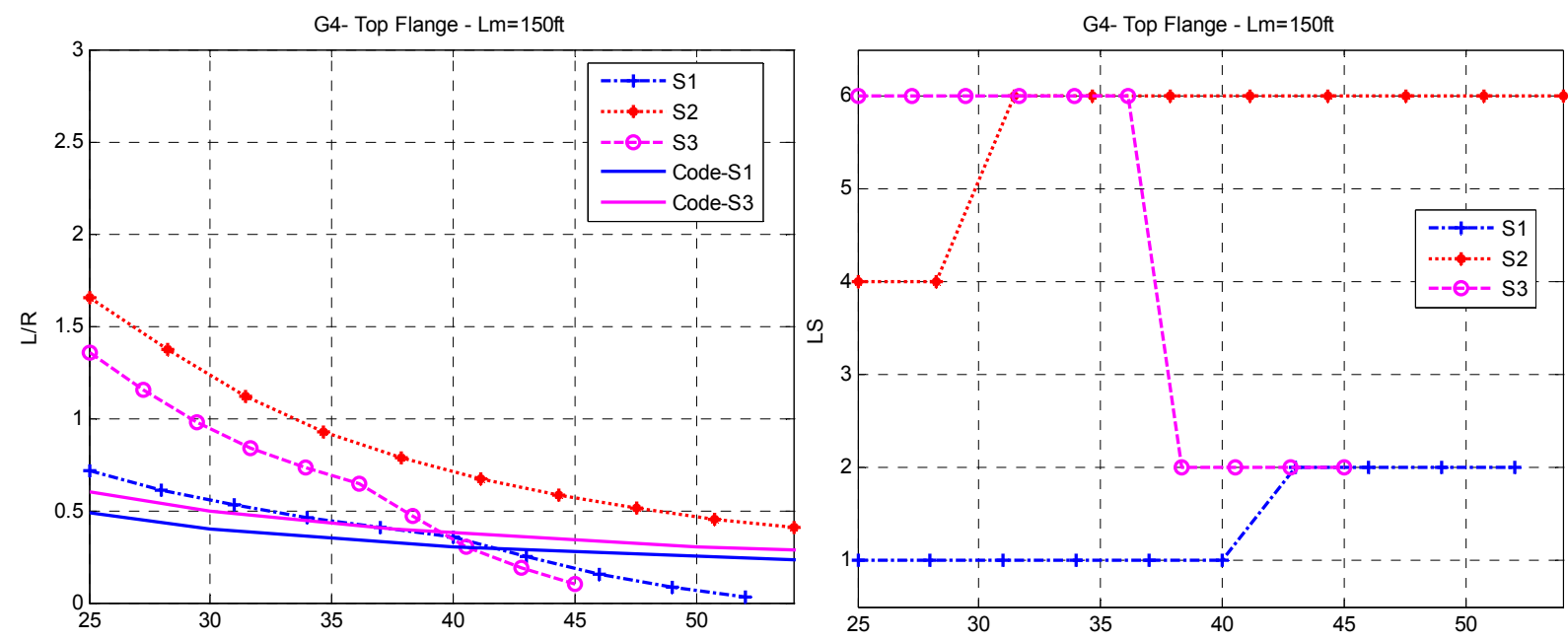

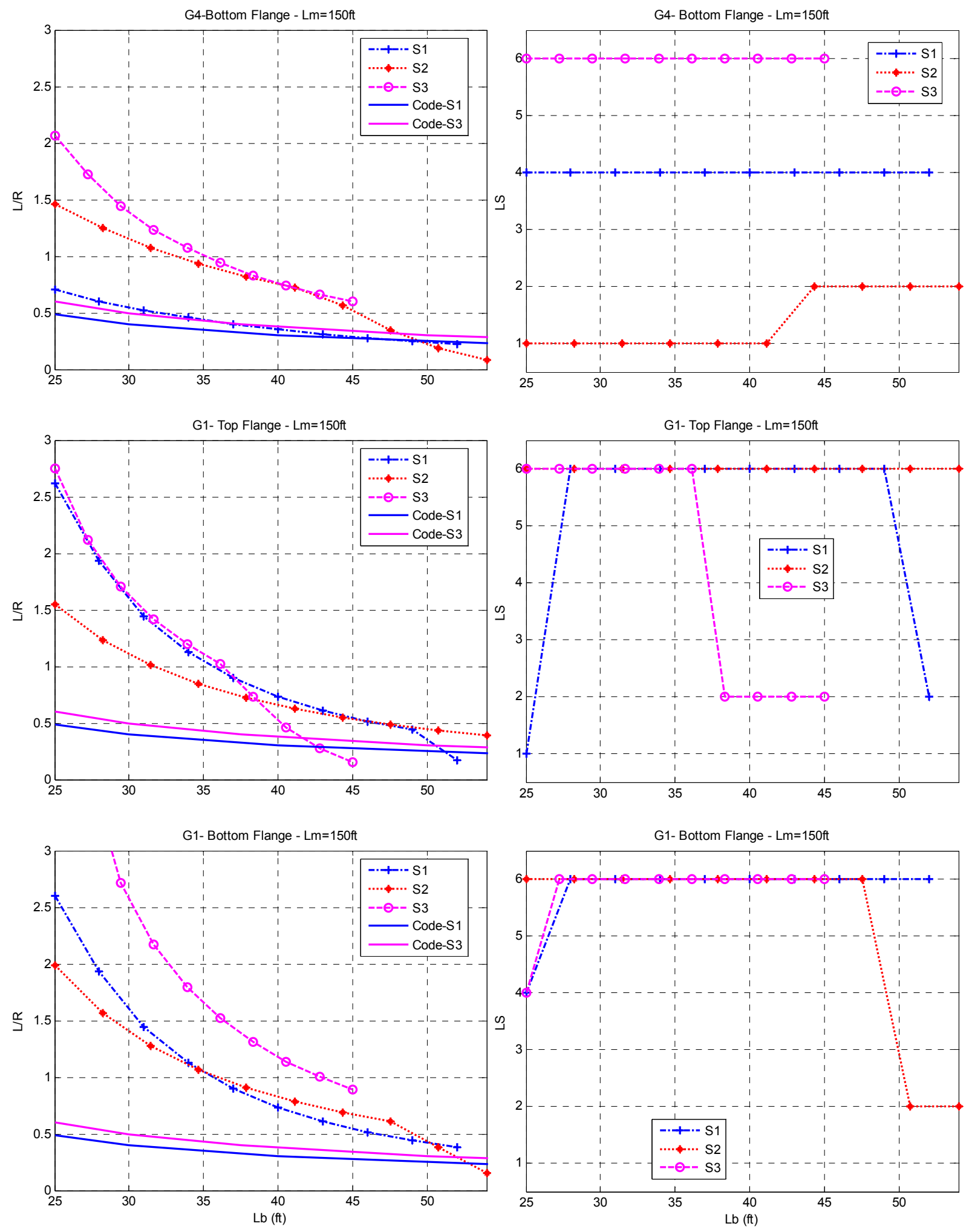

Figure 6-11. Identification of maximum curvatures for straight bridges with $L_{m}=150 \mathrm{ft}$ 

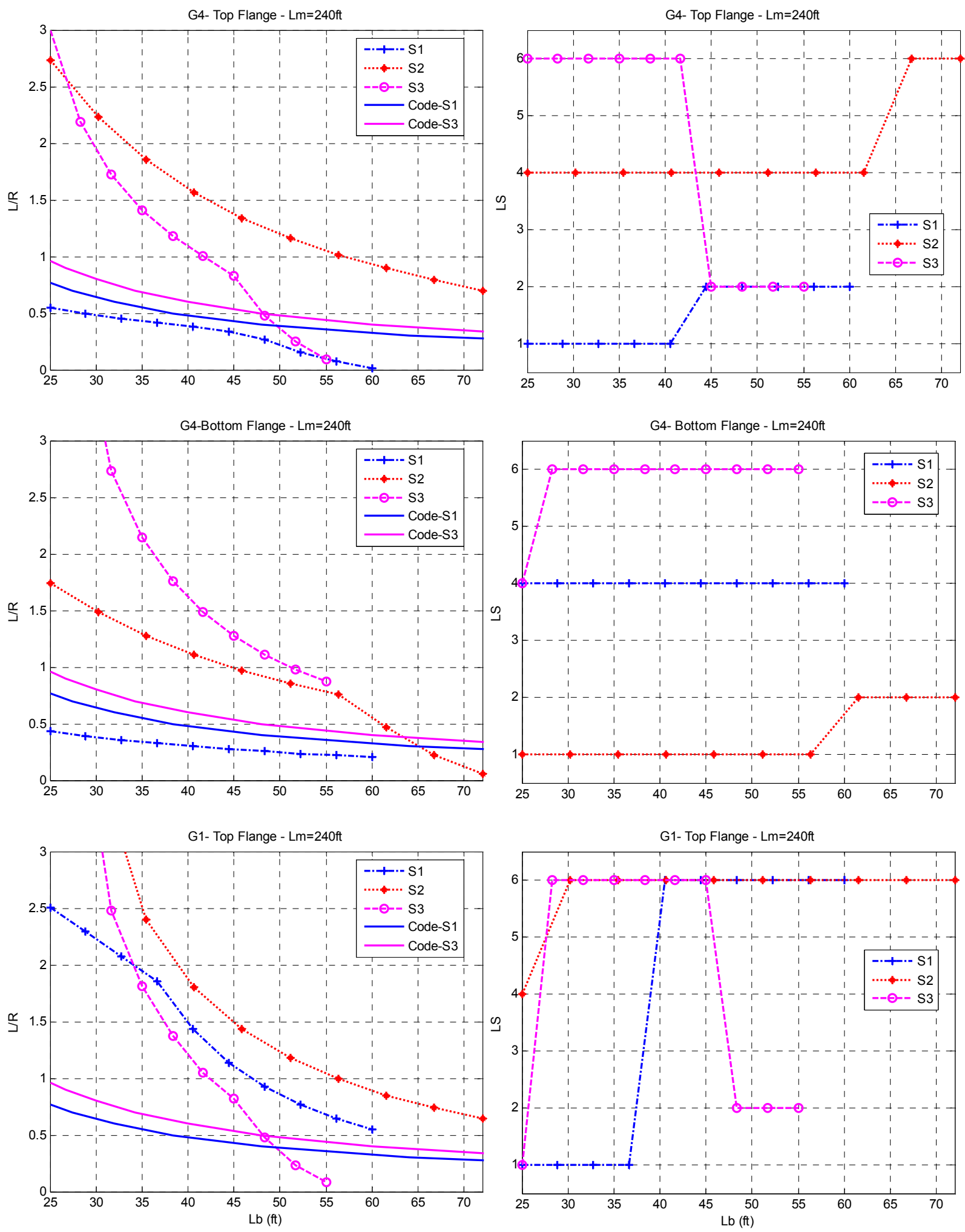

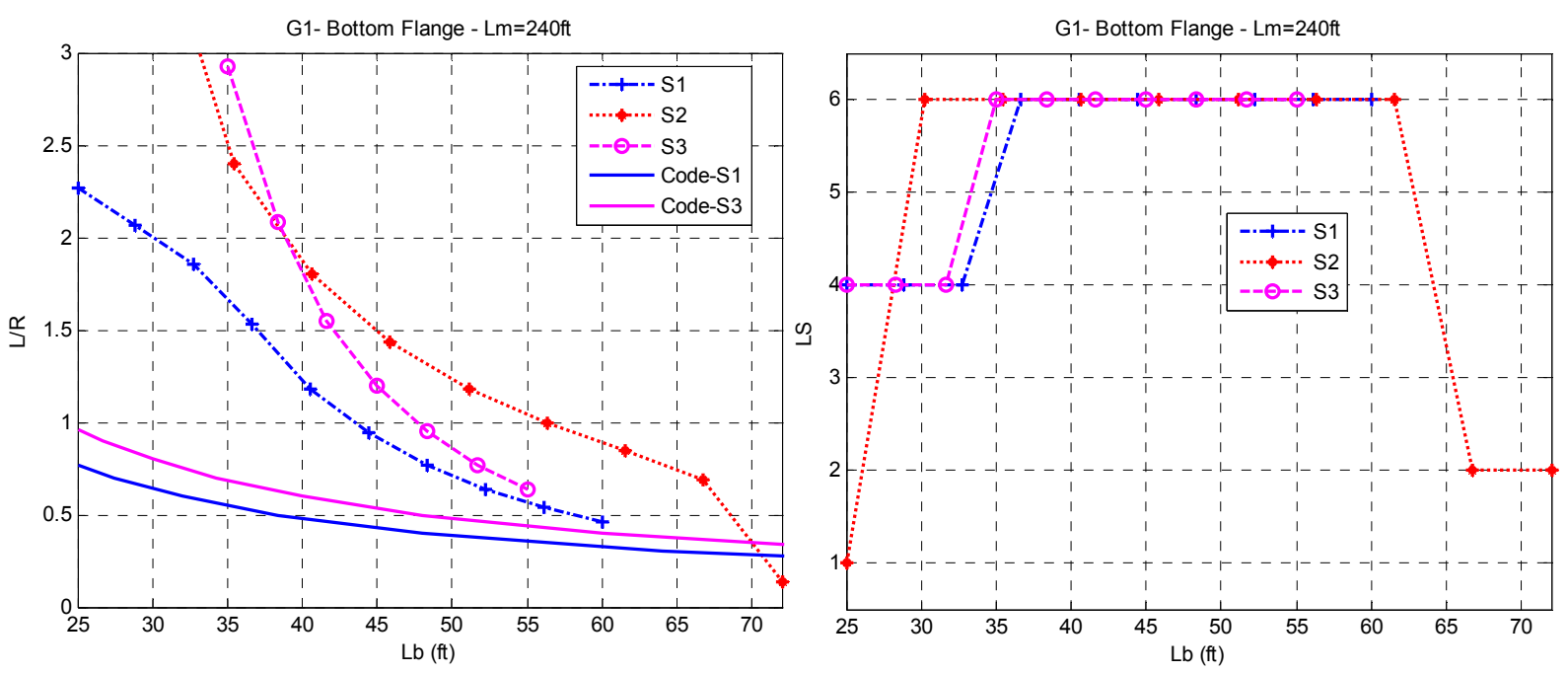

Figure 6-12. Identification of maximum curvatures for straight bridges with $L_{m}=240 \mathrm{ft}$
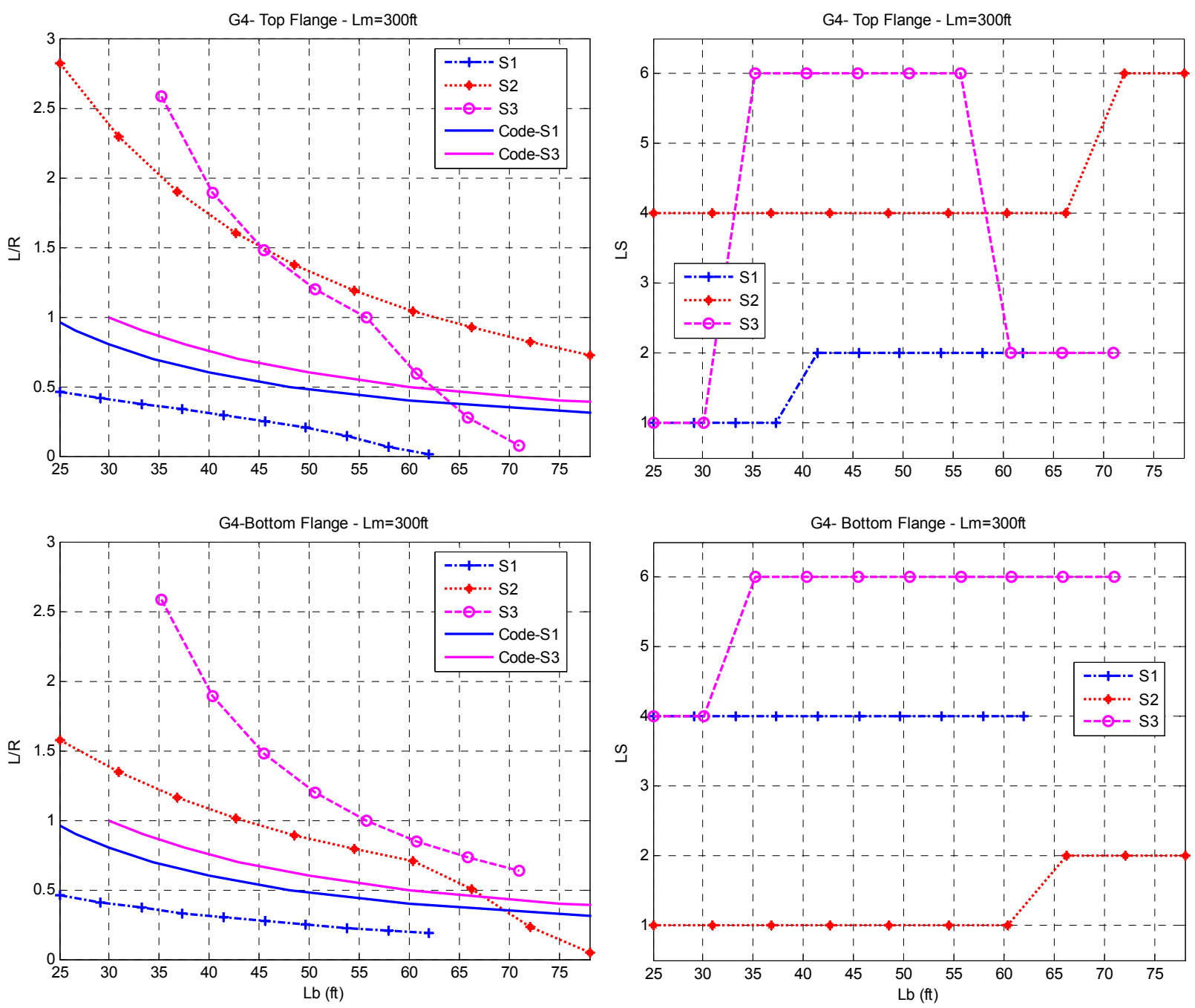

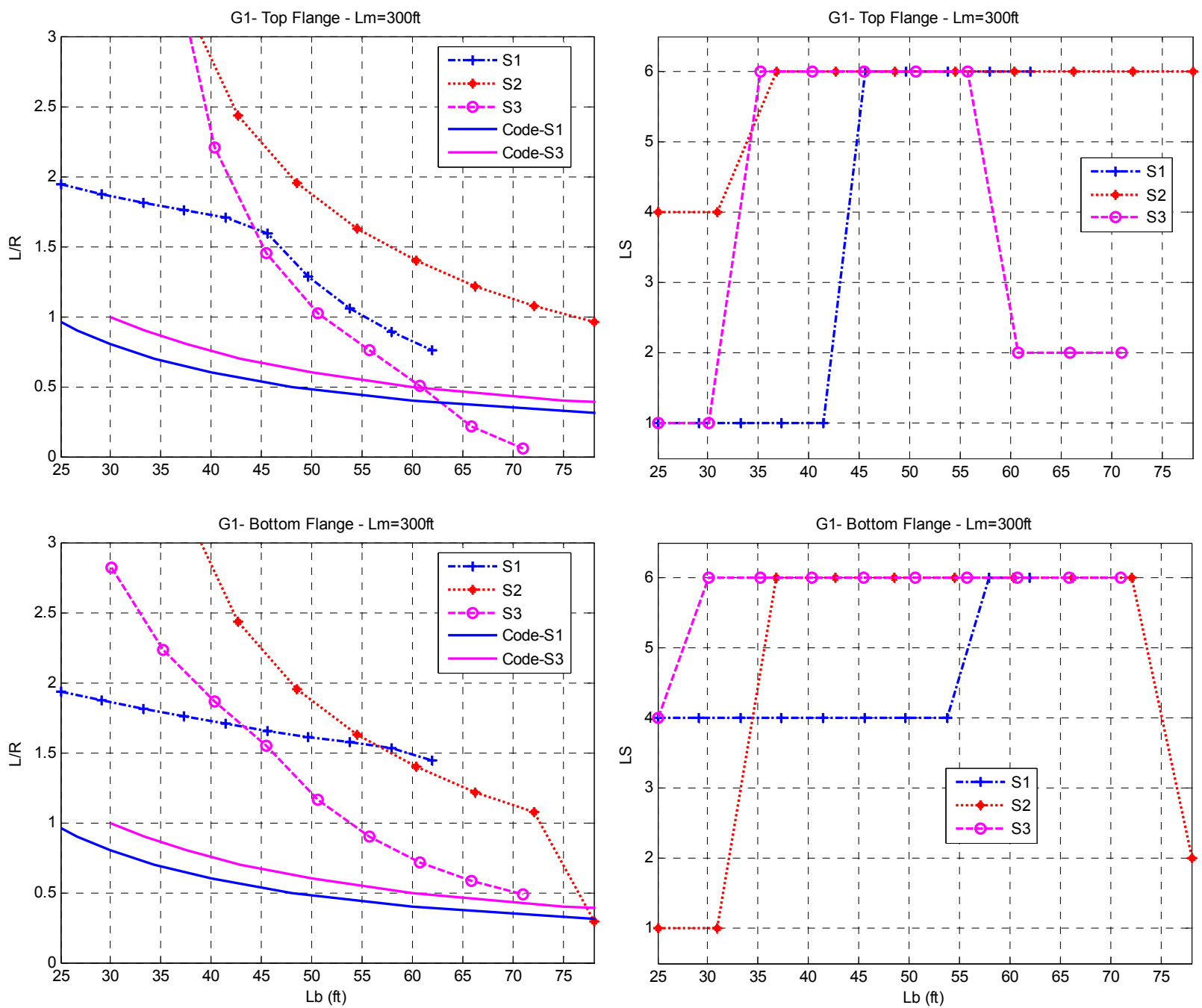

Figure 6-13. Identification of maximum curvatures for straight bridges with $L_{m}=300 \mathrm{ft}$

\subsection{Concluding remarks}

In the first part of this chapter, the behavior of the limit states for constructibility were evaluated based on the FEA results obtained from the parametric study. The following major conclusions were made.

For the skewed bridges analyzed in this work, it was observed that the effect on the limit states of the girder position, the cross-frame orientation and the skew angle is almost negligible. However, the cross-frame spacing has a significant effect on the limit state of discretely braced flanges in compression principally in the positive moment 
regions, since the LFB depends on the cross-frame distance and the capacity of the flange reduces as this parameter increases.

It was also observed that the cross sections at the positive moment regions of the end and middle spans are controlled by the second and fourth castings, respectively. The corresponding flanges in compression in both sections are governed by the ultimate strength limit state. The negative moment regions are controlled by the fifth casting stage. The flanges in compression are governed by the yielding limit state for short span lengths bridges. However, the web bend-buckling limit state governs the compression flanges for longer spans.

In curved bridges, the results showed that both the curvature and the cross-frame spacing highly affect the limit state ratios in the positive moment regions of the outer girder. However, a stress relief was observed in the inner girder caused by the length adjustments due to curvature. Additionally, the LFB exhibited by the inner girder is relieved since its eccentricity with respect to the supports is smaller. In fact, the LFB undergoes a reversing effect in the inner girder compared to its straight counterpart. This is caused from the torsional effects that are controlled by the curvature counteracting the overhang load effects in the inner girder.

It was observed that the variables that most affect the limit states are the curvature followed by the cross-frame spacing. The second and fourth castings from the deckplacement sequence control the sections at the positive moment regions of the end and middle spans, respectively. The ultimate strength is the limit state that principally governs the compression flanges in these sections. However, the yielding limit state may control in short span lengths with high curvatures.

As for skewed bridges, the negative moment regions are controlled by the fifth casting stage. The yielding limit state governs the compression flanges of bridges with short spans and the web bend-buckling limit state becomes significant in the pier regions for long span bridges.

None of the critical limit state values resulted from a casting stage where the finishing machine is considered. 
In the second part of Chapter 6, the maximum allowed skews and curvatures were computed for straight bridges based on the limit states for constructibility and the LFB equations proposed in Chapter 5. The following principal observations were established.

For skewed bridges, it was observed that the maximum cross-frame spacing in skewed bridges is controlled by the ultimate strength limit state in the compression flange. Conversely, the tension flange seems to be unaffected by the skew angle.

On the other hand, the maximum allowed curvature is controlled in most of the cases by the end-span cross section in the outer girder. It was also shown that the ultimate strength limit state of the compression flange controls the maximum allowable curvature for large cross-frame distances. Comparisons with the code recommendation about the maximum cross-frame spacing in curved bridges showed that this specification is adequate in short span bridges when the distance between cross frames is less than approximately $40 \mathrm{ft}$. However, long span bridges present an allowable curvature lower than the one recommended in the code when the cross sections are designed from a straight girder formulation. 


\section{Chapter 7: Cross-frame spacing optimization}

AASHTO (2007) does not include a specific recommendation for the spacing of cross frames in steel bridges. Therefore, the designer needs to either evaluate different configurations to select the optimum spacing, or follow traditional practice that assures safe results. For that reason, a reliability analysis was proposed in this work and performed in Chapter 7 to develop a practical method to select the cross-frame spacing for deck placement conditions considering the flexural limit states for constructibility that are affected by the cross-frame spacing. Initially, a general description of a structural reliability study is given along with the limit states considered in this work. Then, the adopted structural loading and resistance models are described and their corresponding probabilistic characteristics are presented. Finally, a Monte Carlo Simulation is performed for straight, skewed and curved steel I-girder bridges generating some fragility curves that allow the maximum cross-frame spacing for deck-placement conditions according to the maximum tolerated level of risk to be identified.

\subsection{Limit States}

According to Nowak and Collins (2000), a limit state is the boundary between the desired and undesired performance of a structure. The definition of a limit state is based on the identification of a potential structural failure that makes the structure unable to perform as intended originally in the design, e.g. excessive deflections, exceeding the moment capacity, buckling. The structural limit states are generally classified as ultimate, serviceability or fatigue limit states depending on the failure type prevented. Thus, ultimate limit states correspond to the loss of load-carrying capacity, the serviceability limit states are related with the user's comfort and the fatigue limit states control the loss of strength due to cyclic loads.

Mathematically, the limit states are represented by a performance function, defined as:

$$
g(R, Q)=R-Q
$$


where, $R$ represents the "capacity or resistance" and $Q$ the "demand". The limit state is obtained specifically when $g=0$, which is the border between the safe and unsafe zones as shown in Figure 7-1. If $g \geq 0$, the structure is safe, otherwise, an undesired structural performance is exhibited. The basic variables $R$ and $Q$ are functions of other specific variables such as load components, influence factors, resistance parameters, material properties, dimensions, etc. Some of these variables may be of random nature. Therefore, the failure of the system needs to be addressed probabilistically.

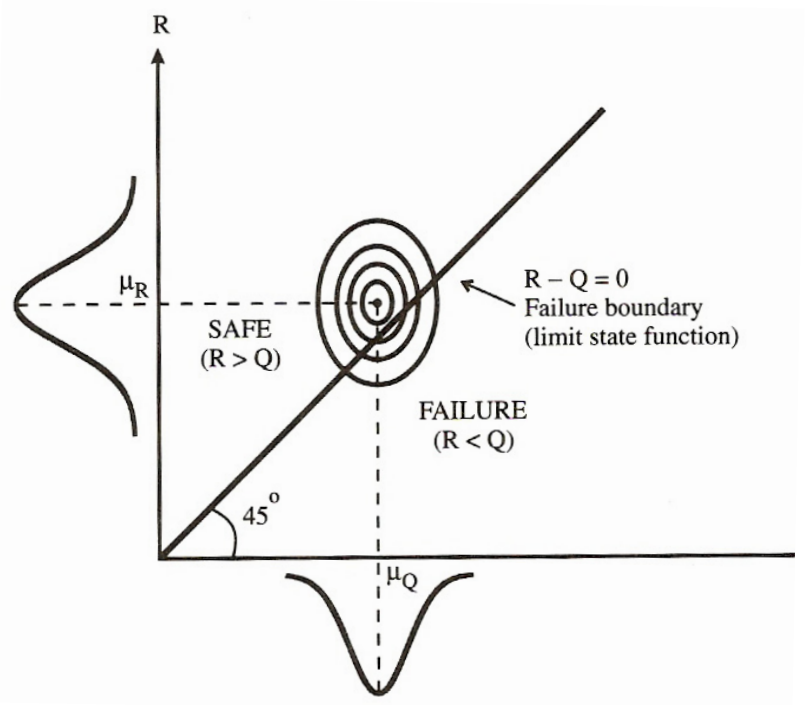

Figure 7-1. Limit State function (Nowak \& Collins, 2000)

Random variables are characterized by their probability density function (PDF), as shown in Figure 7-2 for $R$ and $Q$. Moreover, $R-Q$ is also a random quantity in which the probability of failure is represented by the shaded area where the demand exceeds the capacity. The analytical expression of the probability of failure is given by (Nowak \& Collins, 2000):

$$
P_{f}=1-\int_{-\infty}^{+\infty} F_{Q}\left(r_{i}\right) f_{R}\left(r_{i}\right) d r_{i}=\int_{-\infty}^{+\infty} F_{R}\left(q_{i}\right) f_{Q}\left(q_{i}\right) d q_{i}
$$

where $F_{Q}$ and $F_{R}$ are the Cumulative Distribution Functions (CDF) of $Q$ and $R, f_{Q}$ and $f_{R}$ are the Probability Density Functions (PDF) of $Q$ and $R$, and $q_{i}$ and $r_{i}$ are specific values of $Q$ and $R$. Since Eq. 7-2 is difficult to evaluate analytically, alternative procedures are used to indirectly compute the probability of failure based on the concept of the reliability index. 


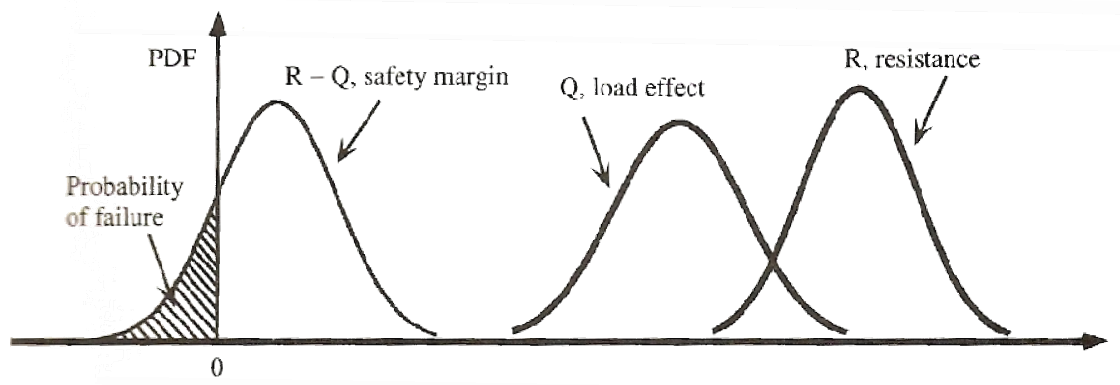

Figure 7-2. Probability functions of load and resistance (Nowak \& Collins, 2000)

The reliability index, also known as the safety index, is the ratio of the mean value of the limit state to its standard deviation, as shown in Figure 7-3. However, a more common definition is given when the limit state is expressed in terms of the reduced variables, which are a nondimensional form of the original random variables $\mathrm{R}$ and $\mathrm{Q}$ as follows:

$$
\begin{aligned}
& Z_{R}=\frac{R-\mu_{R}}{\sigma_{R}} \\
& Z_{Q}=\frac{Q-\mu_{Q}}{\sigma_{Q}}
\end{aligned}
$$

where $Z, \mu$ and $\sigma$ are the reduced variable, the mean value and the standard deviation, respectively. If $R$ and $Q$ are represented graphically in a space of reduced variables as shown in Figure 7-4, the reliability index corresponds to the shortest distance from the origin to the line representing the limit state $(g=0)$. Using equation 7-3 and geometry, the reliability index is defined as:

$$
\beta=\frac{\mu_{R}-\mu_{Q}}{\sqrt{\sigma_{R}^{2}+\sigma_{Q}^{2}}}
$$

Additionally, if $R$ and $Q$ are normally distributed random variables, the reliability index is related to the probability of failure by:

$$
\beta=-\Phi^{-1}\left(P_{f}\right) \quad \text { or } \quad P_{f}=\Phi(-\beta)
$$

where $\Phi$ is the CDF of a standard normal variable. For random variables different than normally distributed and uncorrelated variables, equation 7-5 only gives an approximate definition of $\beta$. 

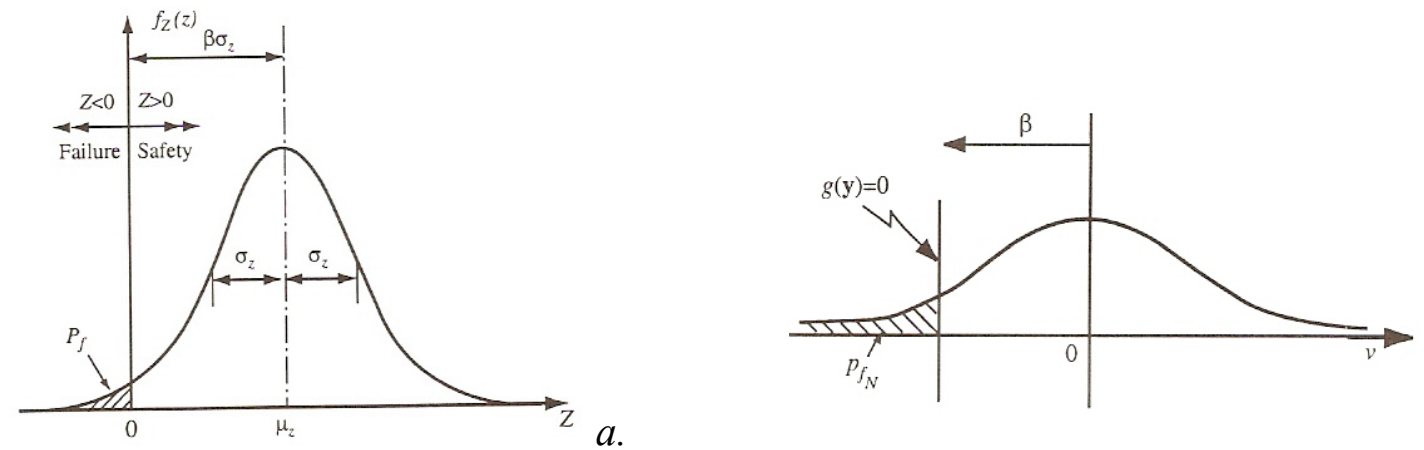

$b$.

Figure 7-3. Reliability index in: $a$. general, and $b$. reduced coordinates (Melchers, 1999)

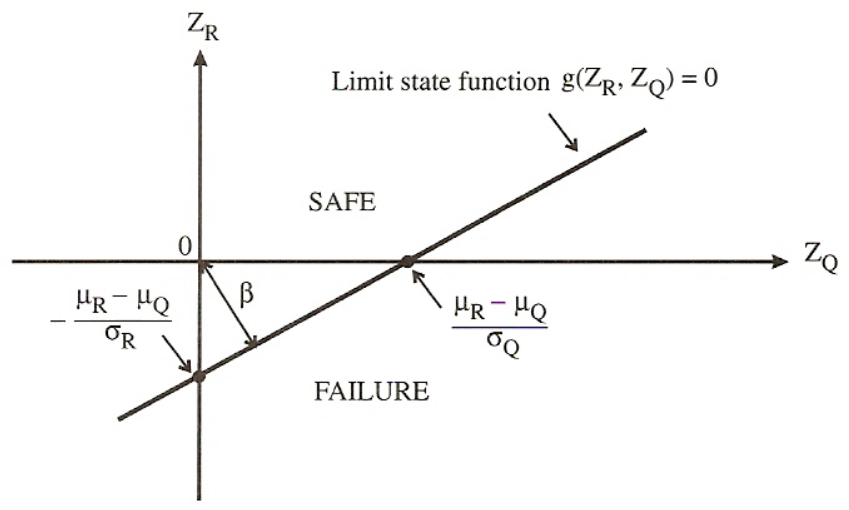

Figure 7-4. Graphical representation of the Reliability Index (Nowak \& Collins, 2000)

The present work evaluates the reliability of the flexural limit states for constructibility that are directly affected by the cross-frame spacing, i.e. limit states that consider LFB, as follows:

- Discretely braced flanges in compression:

Yielding:

$$
f_{b u}+f_{l} \leq \phi_{f} R_{h} F_{y c}
$$

Ultimate strength: $\quad f_{b u}+\frac{1}{3} f_{l} \leq \phi_{f} F_{n c}$

- Discretely braced flanges in tension:

Yielding:

$$
f_{b u}+f_{l} \leq \phi_{f} R_{h} F_{y t}
$$

Limit for LFB:

$$
f_{l} \leq 0.6 F_{y f}
$$

In order to evaluate the reliability of the limit state equations, it is necessary to define the probabilistic characteristics of the loading and capacity variables that are 
involved in the equations. These definitions are called structural loading and resistance models and are described next.

\subsection{Structural loading model}

In this work, the demand $\left(f_{b u}, f_{l}\right)$ comes from factored loads corresponding to the Strength-I load combination for construction, where the load factors are taken as 1.25 and 1.5 for the dead and the construction loads, respectively (AASHTO, 2007).

All loading variables are treated as normal random variables. The probabilistic properties of the dead and construction loads are taken from the work developed by Nowak et. al. (2006) for the calibration of the LRFD design specifications for steel curved girder bridges. The bias factors $(\lambda)$ and coefficients of variation $(\mathrm{V})$ indicated in Table 7-1 are based on field measurements made by the University of Minnesota.

Table 7-1. General probabilistic characteristics of loads

\begin{tabular}{ccc}
\hline & $\begin{array}{c}\text { Bias Factor } \\
(\lambda)\end{array}$ & $\begin{array}{c}\text { Coefficient of Variation } \\
(\mathbf{V})\end{array}$ \\
\hline Dead Loads (D) & 1.0 & 0.150 \\
\hline Construction Loads (C) & 1.1 & 0.215 \\
\hline
\end{tabular}

\subsubsection{Vertical effects, $f_{b u}$}

\subsubsection{Definition}

Direct vertical loads produce the major-axis bending stress, $f_{b u}$, in the girder flanges. In this study, $f_{b u}$ corresponds to the maximum values obtained in the FEA of the parametric study described in Chapter 4. Because the influence of the cross-frame spacing in $f_{b u}$ is almost negligible as shown in Chapter $5, f_{b u}$ is taken only from the FE models with $L_{b}=25 \mathrm{ft}$. 


\subsubsection{Probabilistic characteristics}

Because $f_{b u}$ is a total factored stress resulting from the considered load combination, it is necessary to define $\lambda$ and $V$ for the total vertical load (TL), as follows:

$$
\begin{aligned}
& \lambda_{f b u}=\frac{\overline{T L}}{T L_{n}} \\
& V_{f b u}=\frac{\sqrt{\left(D_{n} \lambda_{D} V_{D}\right)^{2}+\left(C_{n} \lambda_{C} V_{C}\right)^{2}}}{\overline{T L}} \\
& \overline{T L}=\lambda_{D} D_{n}+\lambda_{C} C_{n} \\
& T L_{n}=1.25 D_{n}+1.5 C_{n}
\end{aligned}
$$

where $X_{n}$ is the nominal or design value recommended in the specifications for the $X$ load and $\bar{X}$ is the corresponding mean value. Table 7-2 contains the values computed for the exterior girders subjected to distributed vertical loads. The same properties are considered for concentrated loads, since in this case the construction-to-dead load ratios are smaller.

Table 7-2. Probabilistic characteristics of distributed vertical loads in exterior girders

\begin{tabular}{ccccccc}
\hline $\boldsymbol{L}_{\boldsymbol{m}}(\mathbf{f t})$ & $\boldsymbol{D}_{\boldsymbol{n}}(\mathbf{K i p} / \mathbf{f t})$ & $\boldsymbol{C}_{\boldsymbol{n}}(\mathbf{K i p} / \mathbf{f t})$ & $\mathbf{T L}_{\mathbf{n}}$ & $\overline{T L}$ & $\lambda f_{b u}$ & $\mathbf{V} f_{b u}$ \\
\hline 150 & 1.45 & 0.64 & 2.77 & 2.16 & 0.777 & 0.123 \\
\hline 240 & 1.67 & 0.64 & 3.06 & 2.38 & 0.779 & 0.123 \\
\hline 300 & 1.81 & 0.64 & 3.23 & 2.52 & 0.780 & 0.123 \\
\hline
\end{tabular}

\subsubsection{Torsional effects, $f_{l}$}

\subsubsection{Definition}

Torsional effects generate indirect LFB. In this work, the LFB effects are computed according to the approximated equations recommended in Chapter 5. Although these stresses depend directly on the cross-frame spacing, $L_{b}$ is considered as a deterministic variable in the reliability analyses and is varied from $15 \mathrm{ft}$ to $100 \mathrm{ft}$ using $5 \mathrm{ft}$ increments. 


\subsubsection{Probabilistic characteristics}

An approach similar to the one described for vertical loads is followed to define the probabilistic characteristics of the lateral factored loads due to eccentric overhang loading, as shown in Table 7-3. However, the same values contained in Table 7-2 are used for the curvature effects since the proposed LFB equations for curvature depend on the vertical loads only.

Table 7-3. Probabilistic characteristics of distributed lateral loads in exterior girders

\begin{tabular}{ccccccc}
\hline $\boldsymbol{L}_{\boldsymbol{m}}(\mathbf{f t})$ & $\boldsymbol{D}_{\boldsymbol{n}}(\mathbf{K i p} / \mathbf{f t})$ & $\boldsymbol{C}_{\boldsymbol{n}}(\mathbf{K i p} / \mathbf{f t})$ & $\mathbf{T L}_{\mathbf{n}}$ & $\overline{T L}$ & $\lambda \boldsymbol{f}_{\boldsymbol{l}}$ & $\mathbf{V} \boldsymbol{f}_{\boldsymbol{l}}$ \\
\hline 150 & 0.17 & 0.20 & 0.52 & 0.40 & 0.761 & 0.137 \\
\hline 240 & 0.13 & 0.15 & 0.38 & 0.29 & 0.761 & 0.137 \\
\hline 300 & 0.12 & 0.14 & 0.37 & 0.28 & 0.761 & 0.137 \\
\hline
\end{tabular}

\subsection{Structural resistance model}

\subsubsection{Definition}

In the limit states described in Section 7.1, the resistance $R$ corresponds to the right side of the inequalities given by Equations 7-3, 7-4, 7-5 and 7-6. The resistance factor, $\phi_{f}$, is taken as 1.0 for flexure and $R_{h}$ is 1.0 for homogenous girders (AASHTO, 2007). The resistances $F_{y c}, F_{y t}$ and $F_{y f}$ correspond to the specified minimum yield strength of a compression, tension or any-stress-state flange, respectively. According to the material properties, these resistances are equal to $50 \mathrm{ksi}$.

On the other hand, $F_{n c}$ is the nominal flexural resistance of a compression flange. This resistance is governed by the most critical failure mode between the lateral torsional buckling (LTB) and the flange local buckling (FLB). The definition of these resistances is given below according to AASHTO (2007).

\subsubsection{Lateral Torsional Buckling Resistance:}

The lateral torsional buckling resistance of the compression flange is defined as:

- If $L_{b} \leq L_{p}$, then: 


$$
F_{n c}=R_{b} R_{h} F_{y c}=F_{y c}
$$

since the web load-shedding factor, $R_{b}$, is taken as 1.0 for constructibility (AASHTO, 2007).

- If $L_{p}<L_{b} \leq L_{r}$, then:

$$
\begin{aligned}
& F_{n c}=C_{b}\left[1-\left(1-\frac{F_{y r}}{R_{h} F_{y c}}\right)\left(\frac{L_{b}-L_{p}}{L_{r}-L_{p}}\right)\right] R_{b} R_{h} F_{y c} \leq R_{b} R_{h} F_{y c} \\
& L_{p}=1.0 r_{t} \sqrt{\frac{E}{F_{y c}}} \\
& r_{t}=\frac{b_{f c}}{\sqrt{12\left(1+\frac{D_{c} t_{w}}{3 b_{f c} t_{f c}}\right)}} \\
& L_{r}=\pi r_{t} \sqrt{\frac{E}{F_{y r}}}
\end{aligned}
$$

where $C_{b}$ is the moment gradient modifier, conservatively taken as $1.0 ; L_{b}$ is the unbraced length; $L_{p}$ is limiting unbraced length to achieve the nominal flexural resistance under uniform bending; $L_{r}$ is limiting unbraced length to achieve the onset of nominal yielding in either flange under uniform bending with consideration of compression flange residual stress effects; $F_{y r}$ is the smaller of $0.7 F_{y c}$ and $F_{y w}$, but not less than $0.5 F_{y c} ; D_{c}$ is the depth of the web in compression; $b_{f c}$ and $t_{f c}$ are the width and thickness of the flange in compression; $t_{w}$ is the web thickness; and $E$ is the steel modulus of elasticity taken as 29000 Ksi.

- If $L_{b}>L_{r}$, then:

$$
\begin{aligned}
& F_{n c}=F_{c r} \leq R_{b} R_{h} F_{y c} \\
& F_{c r}=\frac{C_{b} R_{b} \pi^{2} E}{\left(\frac{L_{b}}{r_{t}}\right)^{2}}
\end{aligned}
$$

where $F_{c r}$ is the elastic lateral torsional buckling stress. 


\subsubsection{Flange Local Buckling Resistance:}

The local buckling resistance of a compression flange is taken as:

- If $\lambda_{\mathrm{f}} \leq \lambda_{\mathrm{pf}}$, then:

$$
F_{n c}=R_{b} R_{h} F_{y c}=F_{y c}
$$

- Otherwise:

$$
\begin{aligned}
& F_{n c}=C_{b}\left[1-\left(1-\frac{F_{y r}}{R_{h} F_{y c}}\right)\left(\frac{\lambda_{f}-\lambda_{p f}}{\lambda_{r f}-\lambda_{p f}}\right)\right] R_{b} R_{h} F_{y c} \leq R_{b} R_{h} F_{y c} \\
& \lambda_{f}=\frac{b_{f c}}{2 t_{f c}} \\
& \lambda_{p f}=0.38 \sqrt{\frac{E}{F_{y c}}} \\
& \lambda_{r f}=0.56 \sqrt{\frac{E}{F_{y r}}}
\end{aligned}
$$

where $\lambda_{\mathrm{f}}$ is the slenderness ratio for the compression flange, $\lambda_{\mathrm{pf}}$ is the limiting slenderness ratio for a compact flange, and $\lambda_{\mathrm{rf}}$ is the limiting slenderness ratio for a noncompact flange.

\subsubsection{Probabilistic characteristics}

In reliability analysis, the resistance $R$ is taken as the product of the nominal resistance $\left(R_{n}\right)$ used in design and three factors that account for the uncertainties due to material properties, fabrication and analysis (Nowak \& Collins, 2000):

$$
R=R_{n} M F P
$$

where $M$ is the ratio of the actual to nominal material properties, $F$ corresponds to the ratio of the actual to nominal cross-sectional properties and $P$ is the professional factor defined as the ratio of the test to predicted capacity. The mean value of the resistance model presented in Equation 7-24 and its corresponding $\lambda_{R}$ and $\mathrm{V}_{R}$ are defined as:

$$
\bar{R}=R_{n} \bar{M} \bar{F} \bar{P}
$$




$$
\begin{aligned}
\lambda_{R} & =\bar{M} \bar{F} \bar{P} \\
V_{R} & =\sqrt{V_{M}^{2}+V_{F}^{2}+V_{P}^{2}}
\end{aligned}
$$

In this study, the resistance is taken as a lognormal variable. Table 7-4 contains the probabilistic characteristics of the resistance parameters (Nowak et. al. 2006; Galambos, 2004; White et. al. 2008):

Table 7-4. Probabilistic characteristics of resistance variables

\begin{tabular}{ccc}
\hline Variable & $\lambda$ & $\mathbf{V}$ \\
\hline Material $(M)$ & 1.06 & 0.06 \\
\hline Fabrication $(F)$ & 1.00 & 0.05 \\
\hline Professional factor $(P)$ & 1.10 & 0.05 \\
\hline Resistance $(R)$ & 1.166 & 0.093 \\
\hline
\end{tabular}

\subsection{Monte Carlo Simulation}

In this work, the reliability analysis is applied to the factored loads and resistances instead of the corresponding nominal values. This is because the load and resistance factors have already been calibrated in the Specifications to meet a target reliability index, $\beta_{\mathrm{T}}=3.5$. However, the reliability analysis is performed to study the effect of the cross-frame spacing on the reliability of the flexural limit states for construction using a Monte Carlo Simulation. This reliability evaluation is carried out for different $L_{b}$ values. As a result of this effort, fragility curves are developed in terms of the unbraced lengths $\left(L_{b}\right)$, which are useful to define maximum distance between the cross frames based on the desired reliability level.

\subsubsection{MCS for each cross-frame spacing}

The following procedure is followed for each cross-frame distance to evaluate the reliability of the limit state equations using the Monte Carlo Simulation:

1. Definition of the limit state equations in terms of the random variables:

The limit states described in Section 7.1 are restated in the Resistance - Demand format to define zero $(L S=0)$ as the failure boundary for the reliability analysis, as follows: 


$$
\begin{aligned}
& L S_{1}=\phi_{f} F_{y c}-\left(f_{b u}+f_{l}\right) \\
& L S_{2}=\phi_{f} F_{n c}-\left(f_{b u}+\frac{1}{3} f_{l}\right) \\
& L S_{4}=\phi_{f} F_{y t}-\left(f_{b u}+f_{l}\right) \\
& L S_{6}=0.6 F_{y f}-f_{l}
\end{aligned}
$$

2. Generate $\mathrm{N}$ specific random values for each random variable.

The Monte Carlo Simulation requires the generation of random numbers according to the specific probabilistic distribution of the random variable considered.

Initially, the random numbers are generated distributed uniformly between 0 and 1. Common computer generators use a seed value to produce the required number of random numbers, where a different seed value will produce a different set of random numbers. Next, the normalized and uniform random numbers are transformed to random numbers with the probabilistic characteristics given by the corresponding random variable. This process is known as the Inverse Transformation Technique Method, where the $\mathrm{CDF}$ of the random variable, $F_{x}\left(x_{i}\right)$, is equated to the generated uniformly random number, $u_{i}$, as indicated by Equation 7-30 and Figure 7-5:

$$
F_{X}\left(x_{i}\right)=u_{i} \quad \therefore x_{i}=F_{X}^{-1}\left(u_{i}\right)
$$

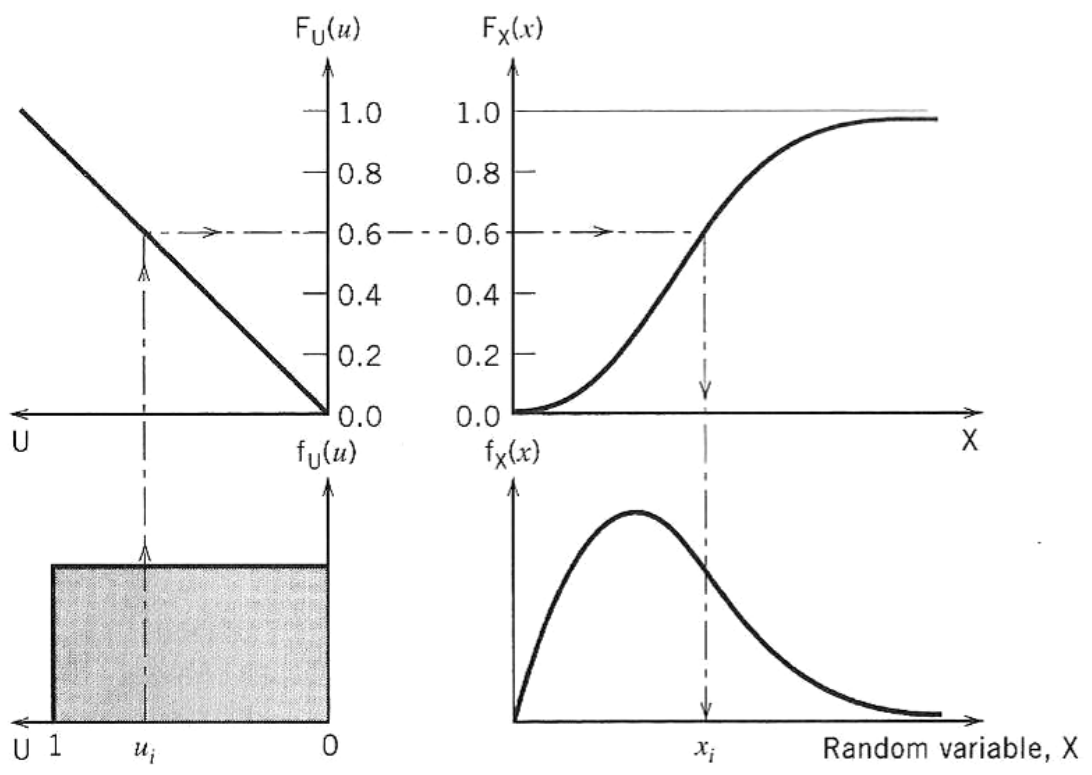

Figure 7-5. Inverse Transformation Technique Method (Haldar \& Mahadevan, 2000) 
Therefore, to generate a set of standard normal random numbers, $z_{i}$, the following transformation is used:

$$
z_{i}=\Phi^{-1}\left(u_{i}\right)
$$

where $\Phi^{-1}$ is the inverse of the standard normal CDF. A standard normal distribution is characterized by having a mean and a variance equal to 0 and 1 , respectively. However, a general normal random variable, $x_{i}$, with mean and standard deviation $\left(\mu_{\mathrm{x}}\right.$ and $\left.\sigma_{\mathrm{x}}\right)$ different than 0 and 1 , is related to $z_{i}$ as follows:

$$
x_{i}=\mu_{x}+z_{i} \sigma_{x}
$$

Therefore, the normal random values for the load variables, $f_{b u}$ and $f_{l}$, are generated using Equation 7-32. For the resistance variables, $R_{n}$, a set of lognormal random values are generated using the corresponding transformation, as follows:

$$
x_{i}=\exp \left(\mu_{\ln x}+z_{i} \sigma_{\ln x}\right)
$$

where $\mu_{\ln x}=\ln \left(\mu_{x}\right)-\frac{1}{2} \sigma_{\ln x}^{2}$ and $\sigma_{\ln x}^{2}=\ln \left(V_{x}^{2}+1\right)$.

In this work, the random numbers of the variables are generated using the design or factored value instead of the mean, since the load and resistance factors have already been calibrated in the Specifications as mentioned previously.

A total of 1,000,000 random values were generated for each random variable $\left(R_{n}\right.$, $\left.f_{b u}, f_{l}\right)$, according to the probabilistic characteristics described previously. For example, Figure 7-6 shows the generated values of $f_{b u}$ with $\mu=13.66 \mathrm{Ksi}$ and $\sigma=1.68 \mathrm{Ksi}$ for the top flange at the positive moment region of the end spans. 


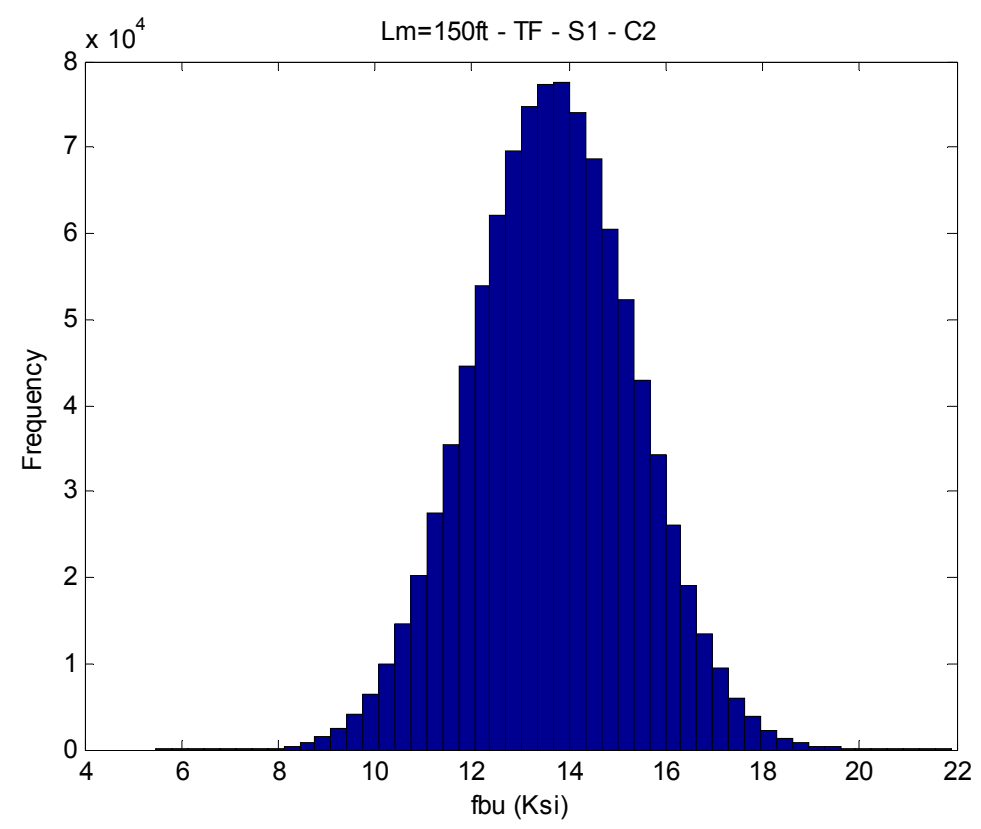

Figure 7-6. Histogram of the simulated values of $f_{b u}$ with $\mu=13.66 \mathrm{Ksi}$ and $\sigma=1.68 \mathrm{Ksi}$

3. Evaluation of the limit state equations for the $\mathrm{N}$ realization sets and determination of their probabilistic characteristics:

The limit state equations are evaluated for 1,000,000 $(\mathrm{N})$ of realization sets of the random variables defined in the previous step, as shown in Figure 7-7 for the ultimate strength limit state of a compression flange. The means and the standard deviations of these limit states were computed based on the evaluated $\mathrm{N}$-set values using the corresponding equations for discrete test data, as follows:

$$
\begin{aligned}
& \bar{x}=\frac{1}{n} \sum_{i=1}^{n} x_{i} \\
& S_{x}=\sqrt{\frac{\left(\sum_{i=1}^{n} x_{i}^{2}\right)-n(\bar{x})^{2}}{n-1}}
\end{aligned}
$$

where $\bar{x}$ is the sample mean, $s_{\mathrm{X}}$ is the sample standard deviation, $n$ is the total number of observations and $x_{i}$ is a single observation. 


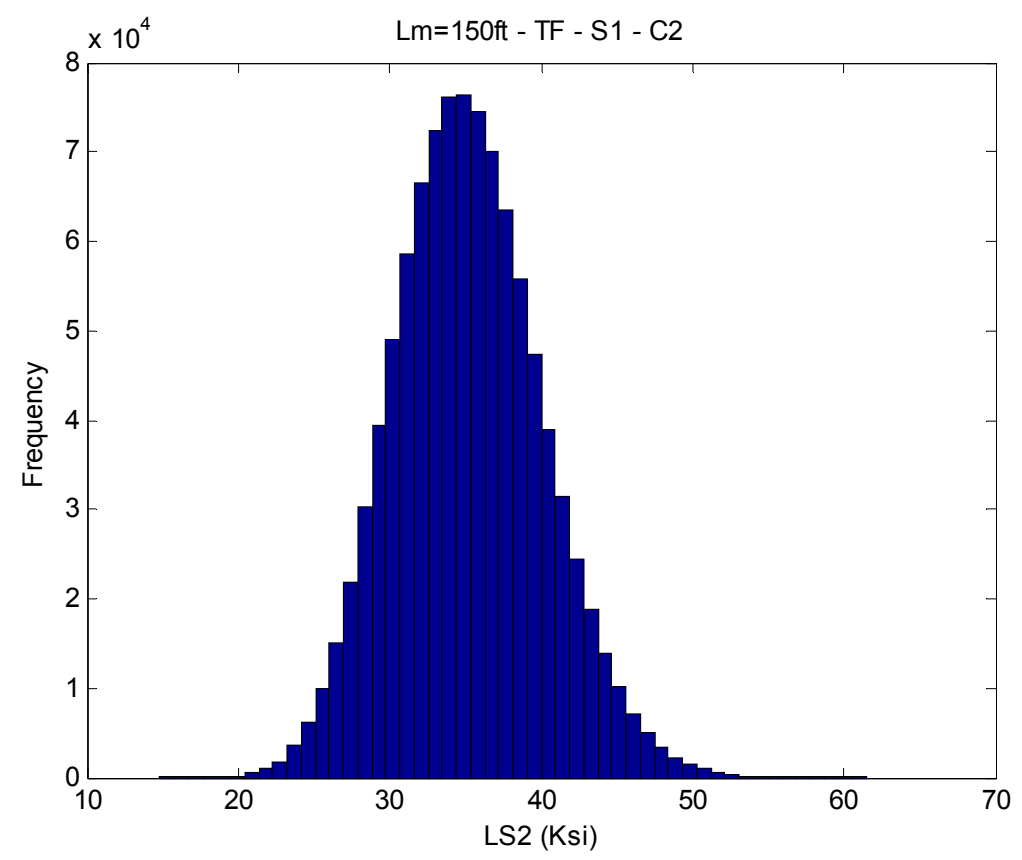

Figure 7-7. Histogram of the simulated limit state LS2 with $\mu=35.18 \mathrm{Ksi}$ and $\sigma=4.88 \mathrm{Ksi}$

4. Probability of failure:

The estimation of the probability of failure for a specific limit state equation improves as $\mathrm{N}$ increases. If the evaluation of the limit state is less than zero, it means a failure state. Therefore, an estimate of the probability of failure is defined as:

$$
P_{f}=\frac{N_{f}}{N}
$$

where $N_{f}$ is the number of simulation cycles that did not satisfy the limit state equation.

5. Evaluation of the accuracy and efficiency of the simulation:

The accuracy of the simulation is estimated assuming a theoretically correct probability of failure close to zero $\left(\mathrm{P}=10^{-4}\right)$ corresponding to a reliability index $(\beta)$ of 3.71 , since a zero probability of failure cannot be achieved. Then, the error is computed based on the $95 \%$ confidence interval of the estimated probability of failure (Haldar \& Mahadevan, 2000), as indicated by Equation 7-36.

$$
\varepsilon(\%)=\sqrt{\frac{1-P}{N P}} \times 200 \%=20 \%
$$


Therefore, it can be affirmed that there is a $95 \%$ probability that the true probability of failure will be in the range of $0.0001 \pm 0.00002(0.01 \% \pm 0.002 \%)$ when the simulation employs $1,000,000$ realizations, for the assumed characteristics of the random variables.

\subsubsection{Fragility curves}

Figure 7-8 shows an example of the CDFs generated for different $L_{b}$ values of the first two limit states for the top flange at the positive moment region of the end spans. It is evident that the CDFs become more critical (i.e., moving toward zero) as $L_{b}$ increases. This effect is more pronounced in the ultimate strength limit state of the compression flanges.
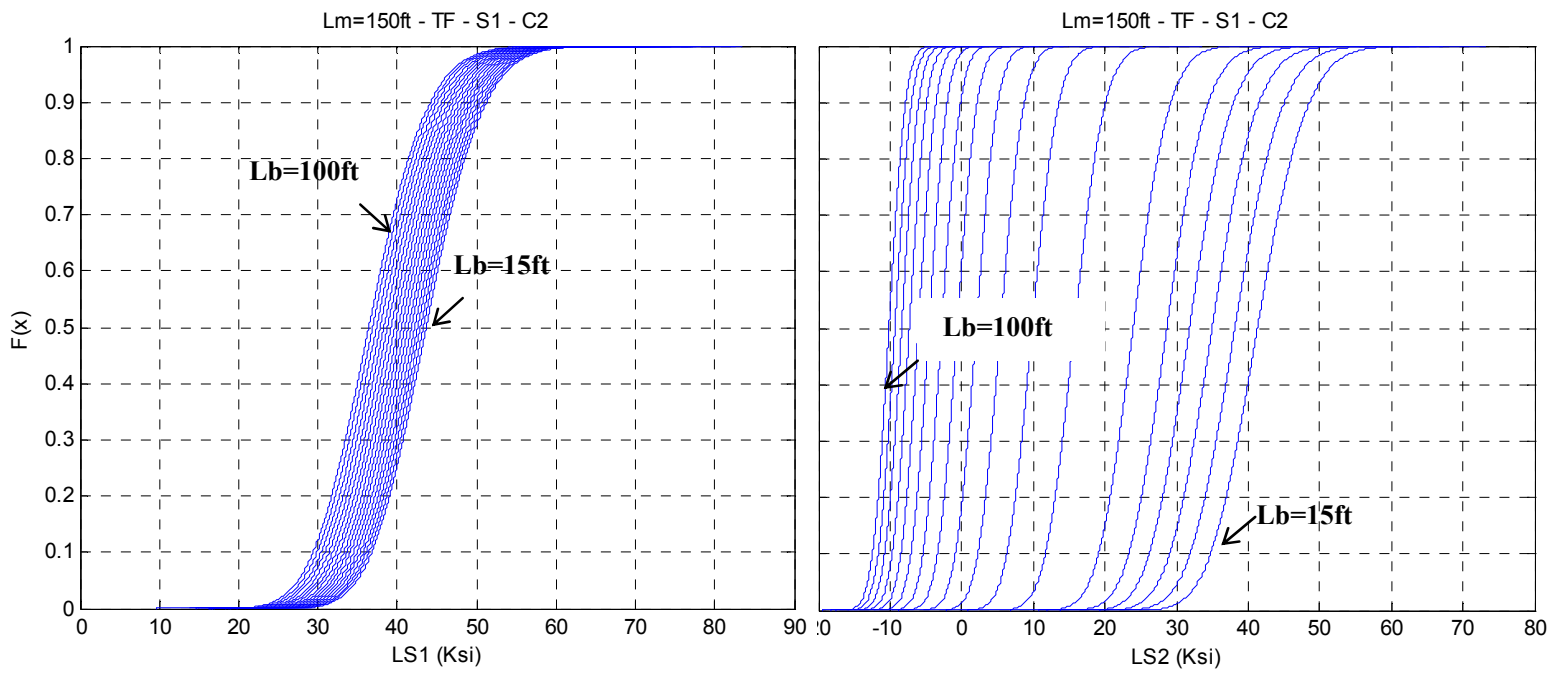

Figure 7-8. Simulated CDFs of LS1 and LS2 for different Lb values in a straight bridge

The simulated probability of failure $\left(P_{f}\right)$ and reliability indices $(\beta)$ of each CDF was plotted against the corresponding $L_{b}$ to obtain the fragility curves for the limit states, as shown in Figure 7-9. These curves represent a practical method to select the maximum cross-frame spacing based on the maximum tolerated probability of failure or reliability index. 

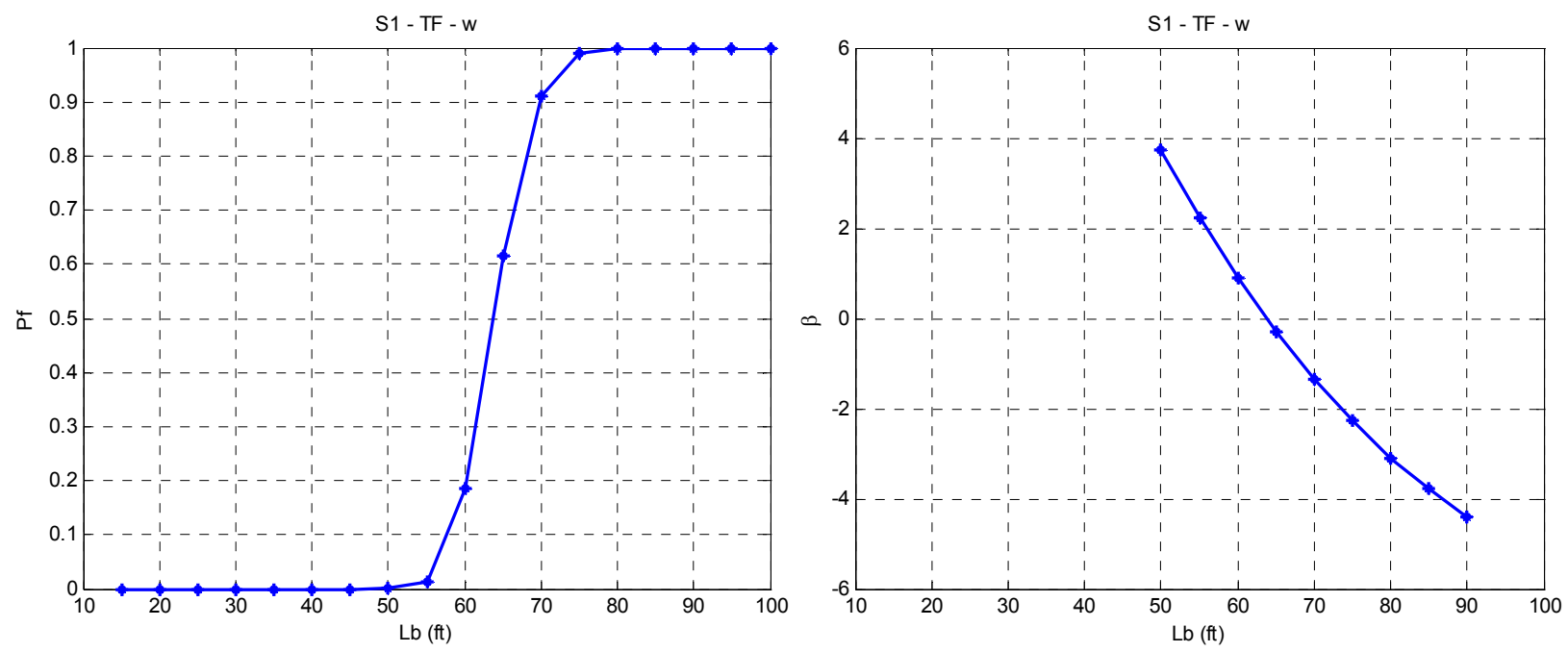

Figure 7-9. Fragility curves of LS2 using Monte Carlo Simulation

\subsubsection{Straight Bridges}

Figure 7-10, Figure 7-11 and Figure 7-12 show that the ultimate strength in the compression flange is the governing limit state in the reliability analysis. It is also observed that the reliability of the system is lower for short span bridges since the LFB effects decrease as the length of the bridge increases. Table 7-5 shows the values of the cross-frame spacing required to meet the code reliability index of 3.5 .

Table 7-5. Maximum $L_{b}(\mathrm{ft})$ corresponding to a code reliability level in straight bridges

\begin{tabular}{cccc}
\hline & End span & Middle span & Pier \\
& S1 & S3 & S2 \\
\hline$L_{m}=150 \mathrm{ft}$ & 51 & 43 & 53 \\
\hline$L_{m}=240 \mathrm{ft}$ & 58 & 54 & 69 \\
\hline$L_{m}=300 \mathrm{ft}$ & 60 & 69 & 75 \\
\hline
\end{tabular}


$\mathrm{S} 1$ - TF - P

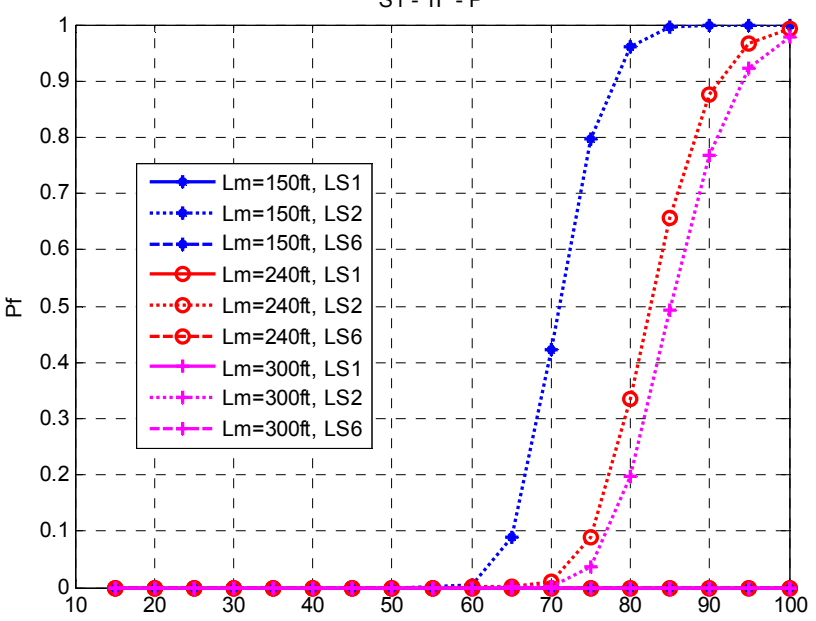

S1 - TF - w

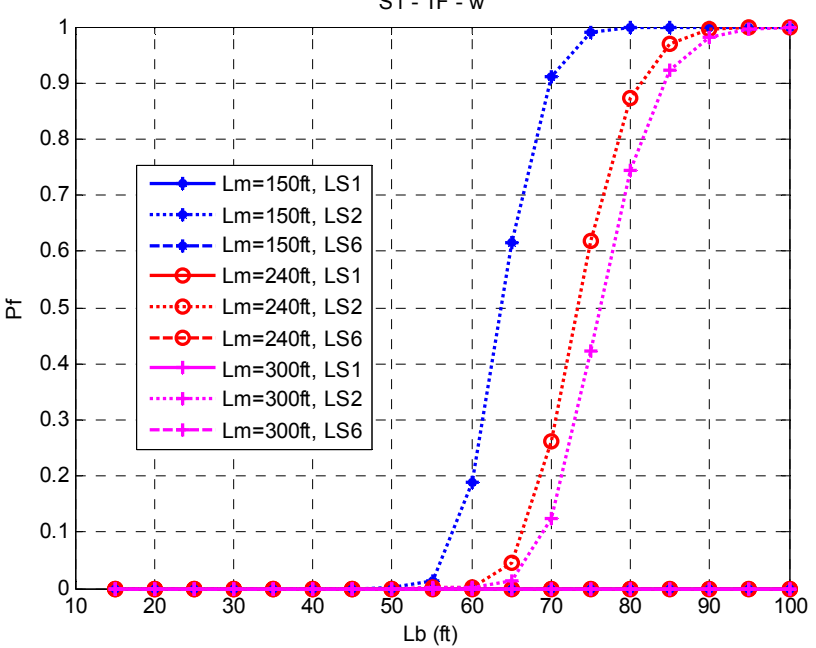

S1 - TF - P

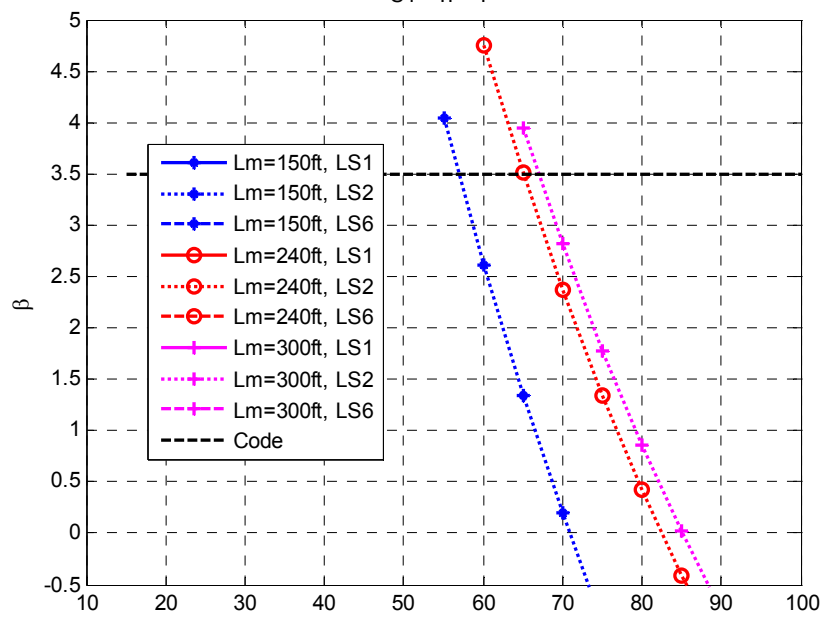

S1 - TF - W

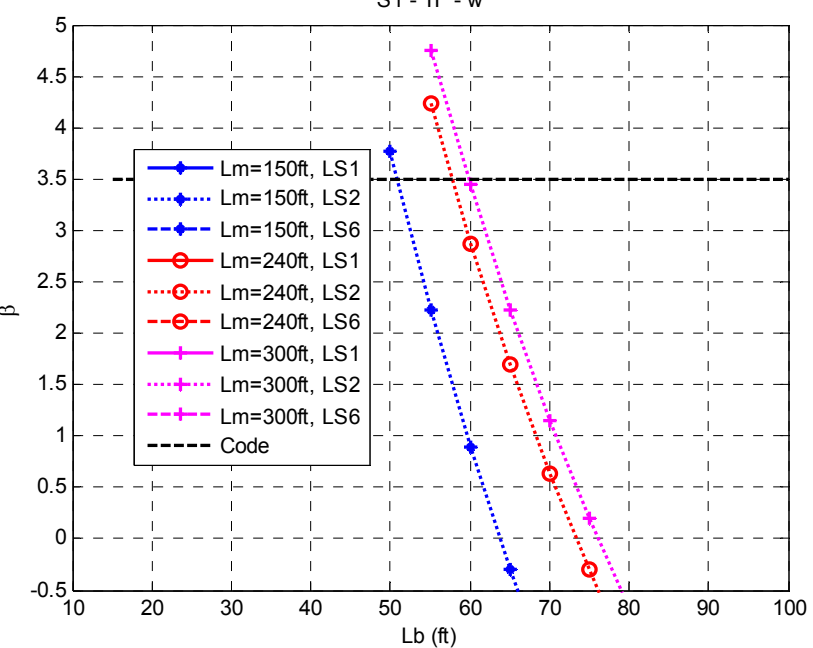

Figure 7-10. Fragility curves for the end-span sections in straight bridges with $L_{m}=150 \mathrm{ft}$
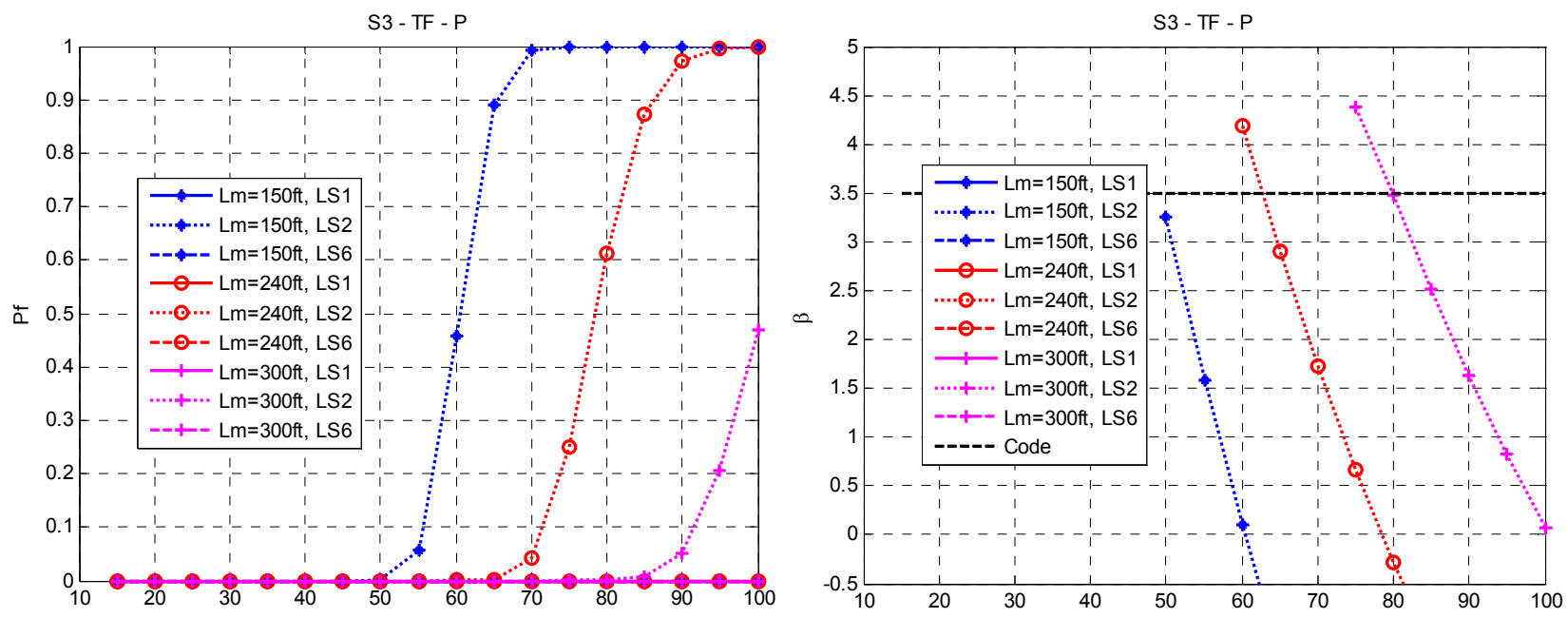

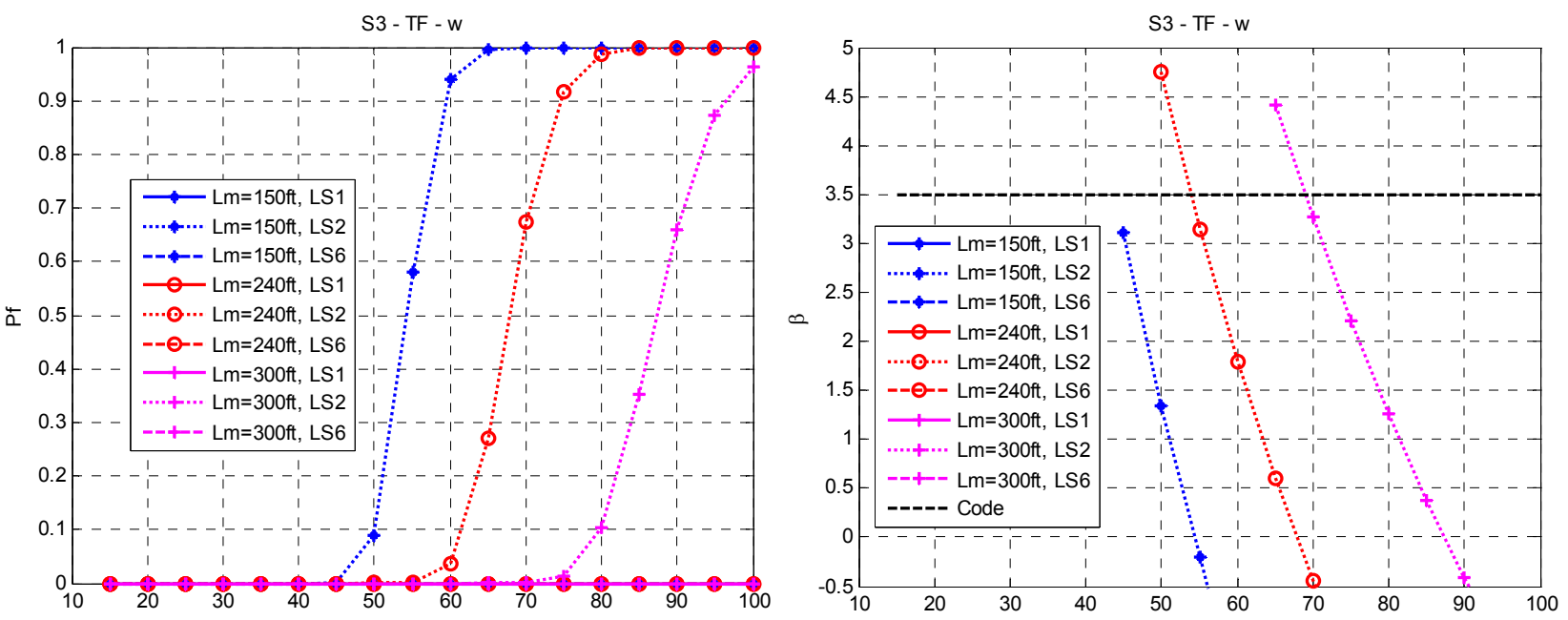

Figure 7-11. Fragility curves for the $L_{m}$ sections in straight bridges with $L_{m}=150 \mathrm{ft}$
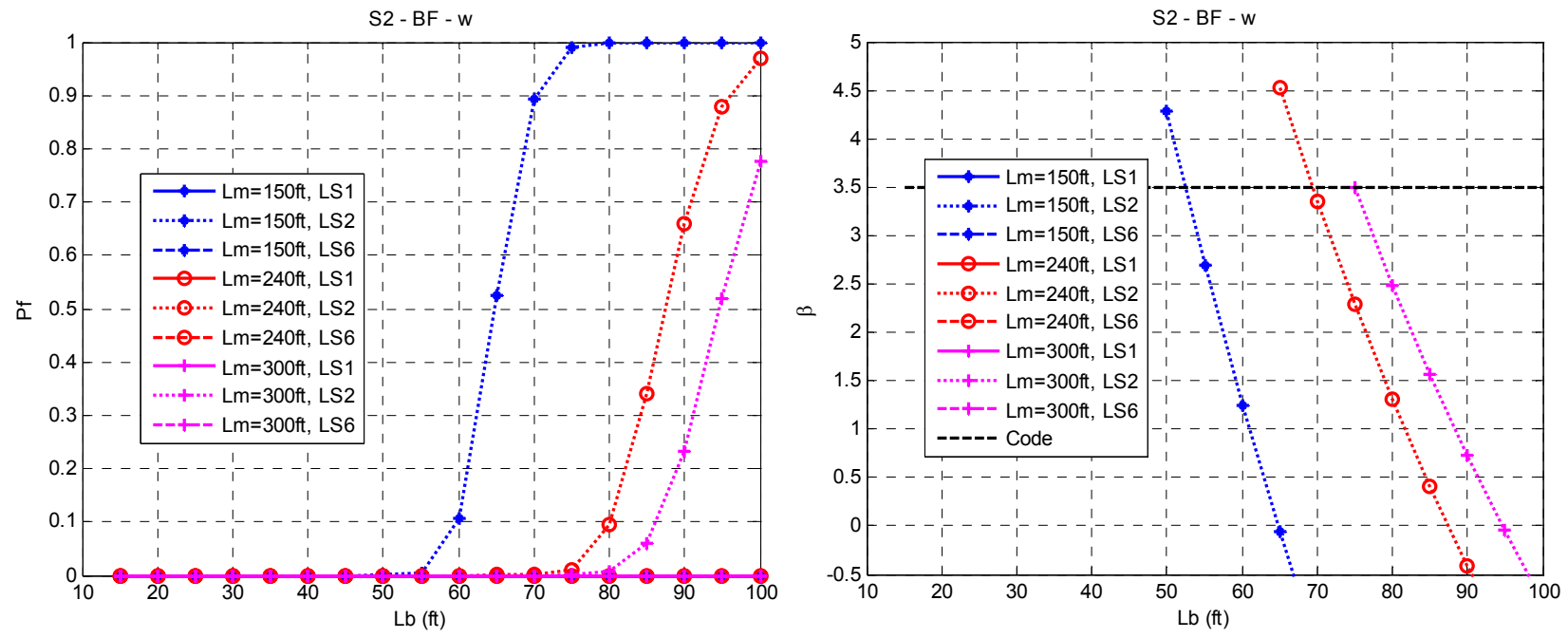

Figure 7-12. Fragility curves for the pier sections in straight bridges with $L_{m}=150 \mathrm{ft}$

\subsubsection{Skewed Bridges}

From Figure 7-13, Figure 7-14, Figure 7-15 and Table 7-6, it is observed that the skew angle does not affect the reliability of the bridges and the same results obtained for straight bridges apply in this case. Appendix E contains the remaining figures corresponding to $\theta=45^{\circ}$ and $60^{\circ}$. 
Table 7-6. Maximum $L_{b}(\mathrm{ft})$ corresponding to a code reliability level in skewed bridges

\begin{tabular}{|c|c|c|c|c|c|c|c|c|c|}
\hline & \multicolumn{3}{|c|}{$\theta=30^{\circ}$} & \multicolumn{3}{|c|}{$\theta=45^{\circ}$} & \multicolumn{3}{|c|}{$\theta=60^{\circ}$} \\
\hline & S1 & S3 & S2 & S1 & S3 & S2 & S1 & S3 & S2 \\
\hline 150ft & 50 & 43 & 54 & 50 & 42 & 54 & 50 & 42 & 53 \\
\hline $240 \mathrm{ft}$ & 58 & 54 & 70 & 58 & 53 & 70 & 57 & 52 & 69 \\
\hline 300ft & 60 & 69 & 76 & 60 & 69 & 75 & 59 & 67 & 74 \\
\hline
\end{tabular}
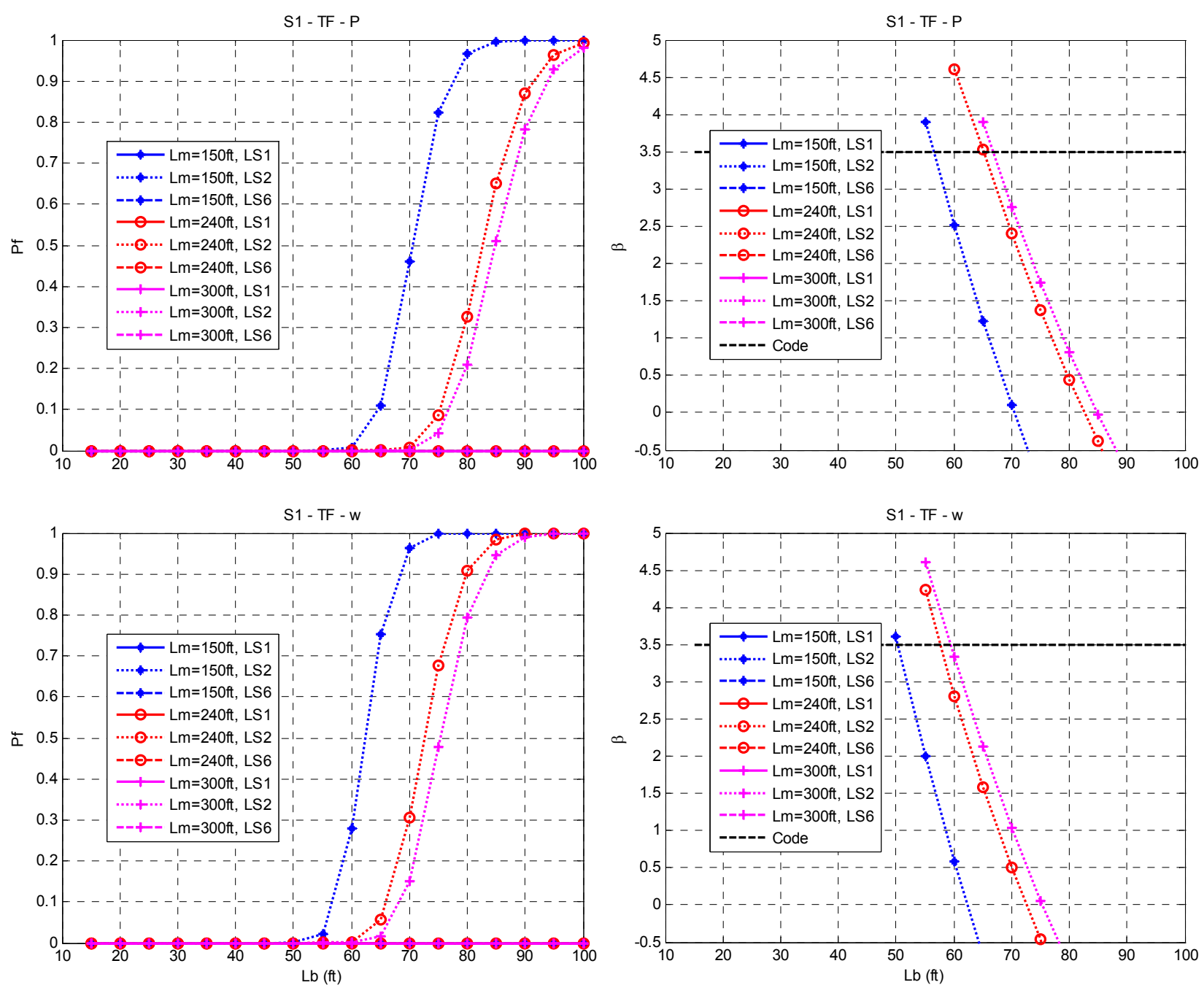

Figure 7-13. Fragility curves for Section S1 in skewed bridges with $L_{m}=150 \mathrm{ft}$ and $\theta=30^{\circ}$ 
S3 - TF - P
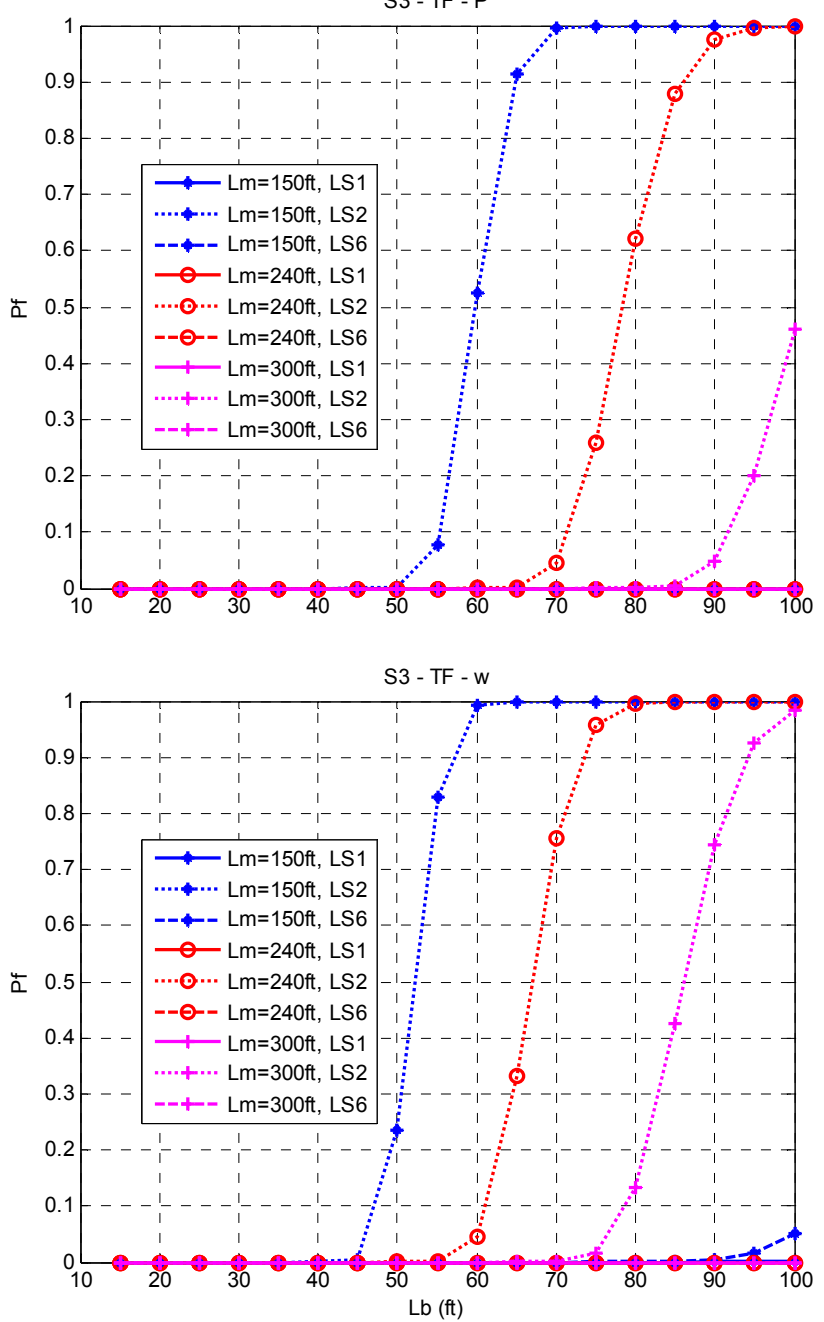

S3 - TF - P

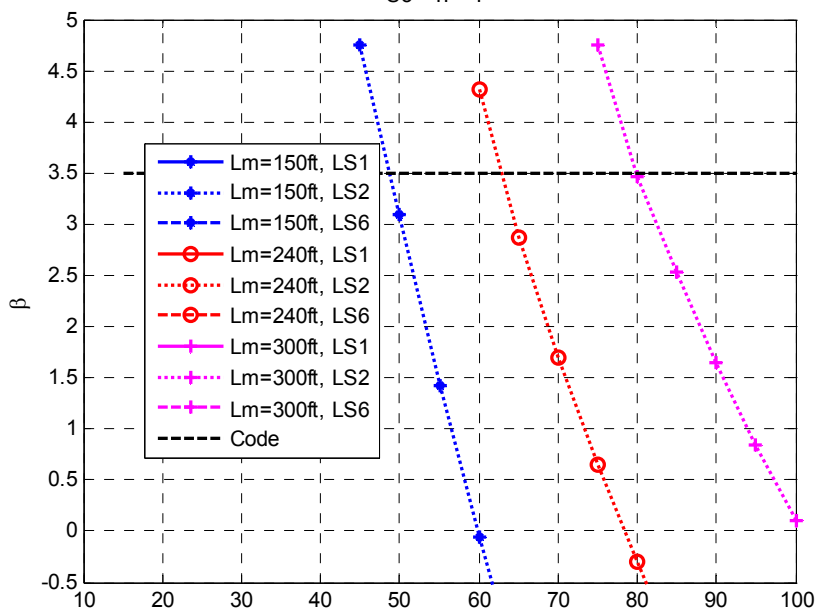

S3 - TF - w

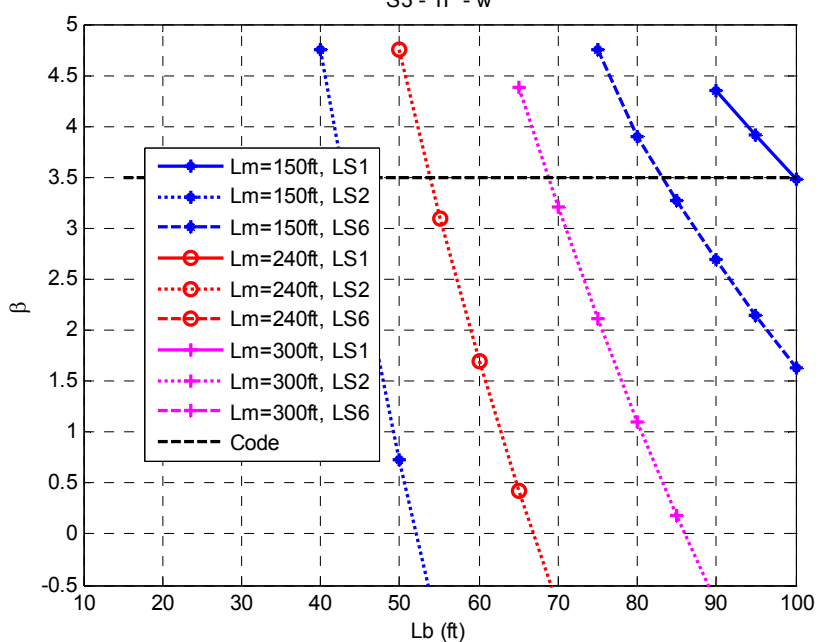

Figure 7-14. Fragility curves for Section S3 in skewed bridges with $L_{m}=150 \mathrm{ft}$ and $\theta=30^{\circ}$
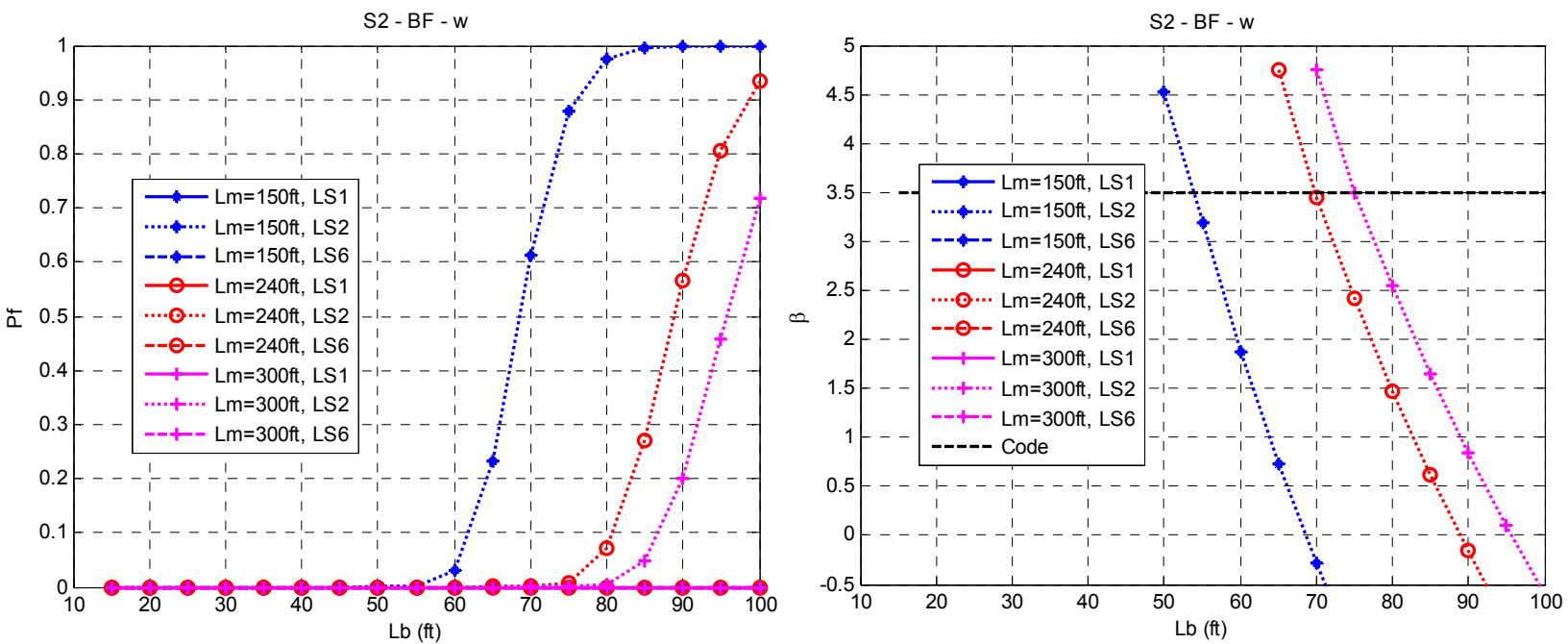

Figure 7-15. Fragility curves for Section $\mathrm{S} 2$ in skewed bridges with $L_{m}=150 \mathrm{ft}$ and $\theta=30^{\circ}$ 


\subsubsection{Curved Bridges}

Figure 7-16 - Figure 7-21, Table 7-7 and Table 7-8 show that the critical crossframe spacing is obtained at the end span of the exterior girder. The ultimate strength of the compression flange is the limit state that governs in most cases. The reliability in the exterior girder decreases for highly curved and long-span bridges. This is principally due to the effect of $f_{b u}$ which is largely increased by the curvature and the span length.

The critical cross-frame distances in the middle span and the pier regions corresponding to a code reliability level are not unfavorably affected by the span length as shown in Table 7-7. However, these distances slightly decrease as the curvature is higher.

In general, it is recommended to use temporary vertical supports at the end spans during construction for highly curved and long bridges that do not meet the minimum levels of tolerated reliability. Appendix $\mathrm{F}$ contains the additional fragility curves corresponding to $L / R=0.45$ and 0.60 .

Table 7-7. Maximum $L_{b}(\mathrm{ft})$ corresponding to a code reliability level in G4

\begin{tabular}{cccccccccc}
\hline & & L/R=0.30 & \multicolumn{3}{c}{ L/R=0.45 } & \multicolumn{3}{c}{ L/R=0.60 } \\
\hline & S1 & S3 & S2 & S1 & S3 & S2 & S1 & S3 & S2 \\
\hline 150ft & 37 & 40 & 46 & 25 & 37 & 42 & $<10$ & 34 & 38 \\
\hline $\mathbf{2 4 0 f t}$ & 15 & 50 & 61 & $<10$ & 48 & 57 & $<10$ & 46 & 54 \\
\hline 300ft & $<10$ & 63 & 65 & $<10$ & 62 & 62 & $<10$ & 68 & 65 \\
\hline
\end{tabular}

Table 7-8. Maximum $L_{b}(\mathrm{ft})$ corresponding to a code reliability level in G1

\begin{tabular}{cccccccccc}
\hline & & $\mathbf{L} / \mathbf{R}=\mathbf{0 . 3 0}$ & \multicolumn{3}{c}{$\mathbf{L} / \mathbf{R}=\mathbf{0 . 4 5}$} & $\mathbf{L} / \mathbf{R}=\mathbf{0 . 6 0}$ \\
\hline & $\mathbf{S 1}$ & $\mathbf{S 3}$ & $\mathbf{S 2}$ & $\mathbf{S 1}$ & $\mathbf{S 3}$ & $\mathbf{S 2}$ & $\mathbf{S 1}$ & $\mathbf{S 3}$ & $\mathbf{S 2}$ \\
\hline $\mathbf{1 5 0 f t}$ & 53 & 43 & 53 & 48 & 42 & 52 & 43 & 41 & 47 \\
\hline $\mathbf{2 4 0 f t}$ & 68 & 51 & 72 & 58 & 49 & 72 & 52 & 47 & 72 \\
\hline $\mathbf{3 0 0 f t}$ & 78 & 64 & 79 & 75 & 62 & 81 & 66 & 59 & 88 \\
\hline
\end{tabular}



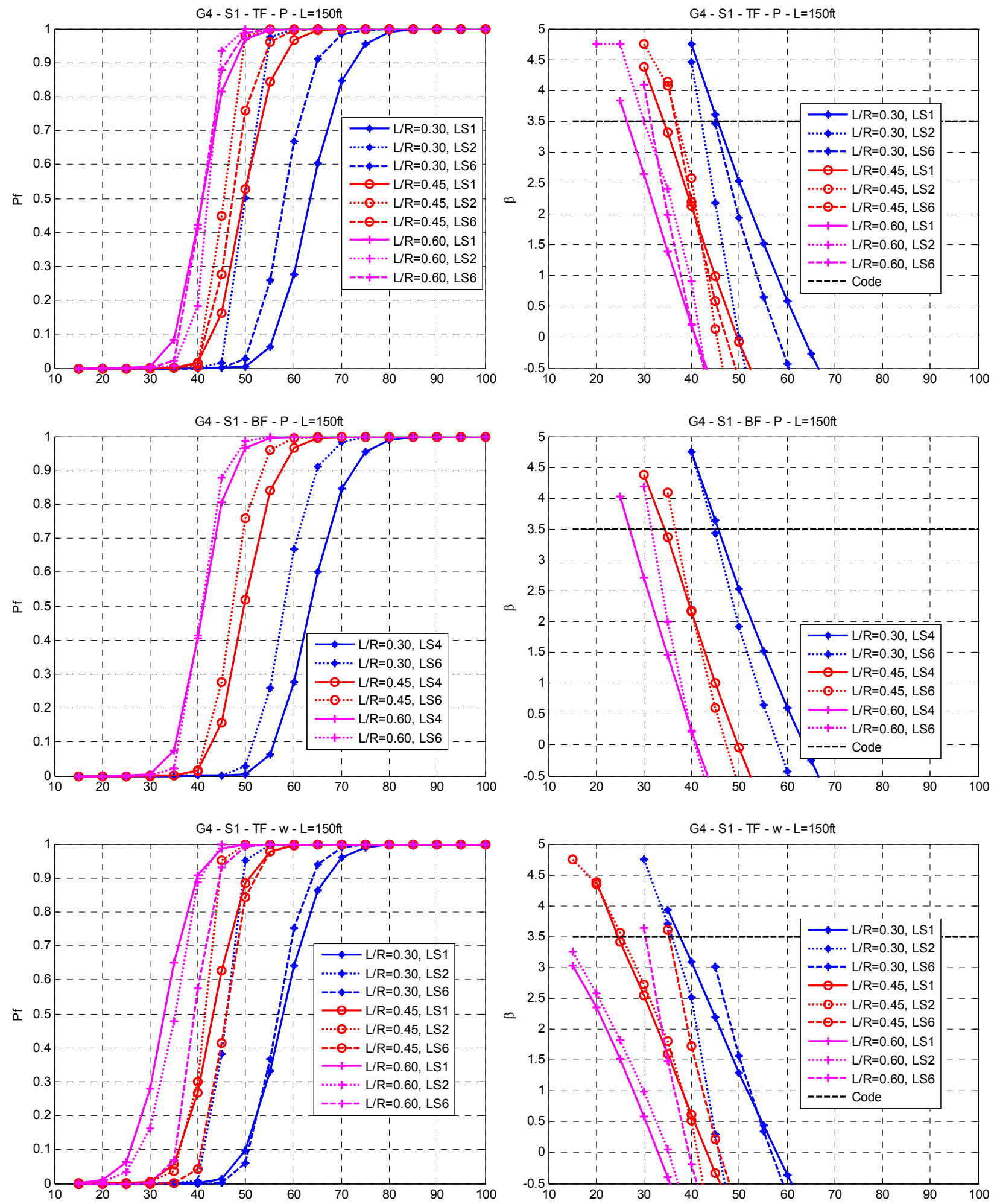

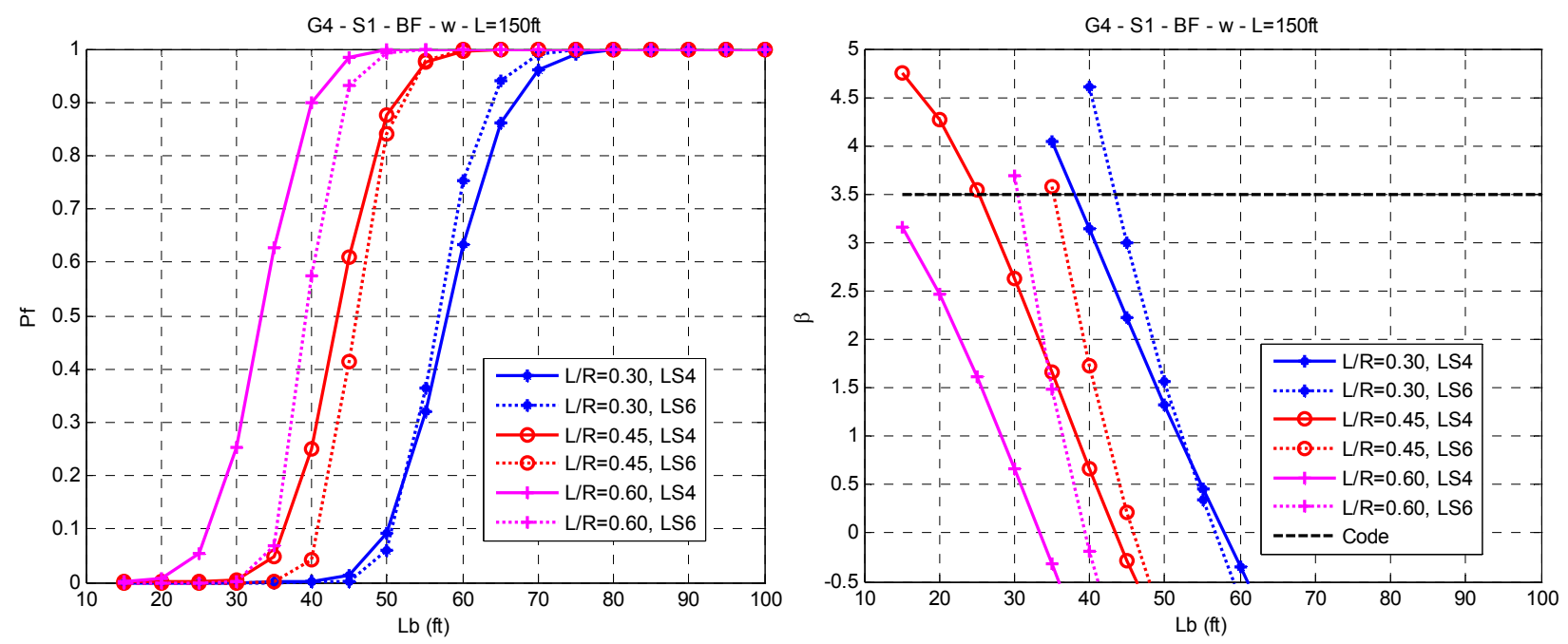

Figure 7-16. Fragility curves for section $\mathrm{S} 1$ in $\mathrm{G} 4$ with $L_{m}=150 \mathrm{ft}-\mathrm{L} / \mathrm{R}=0.30$
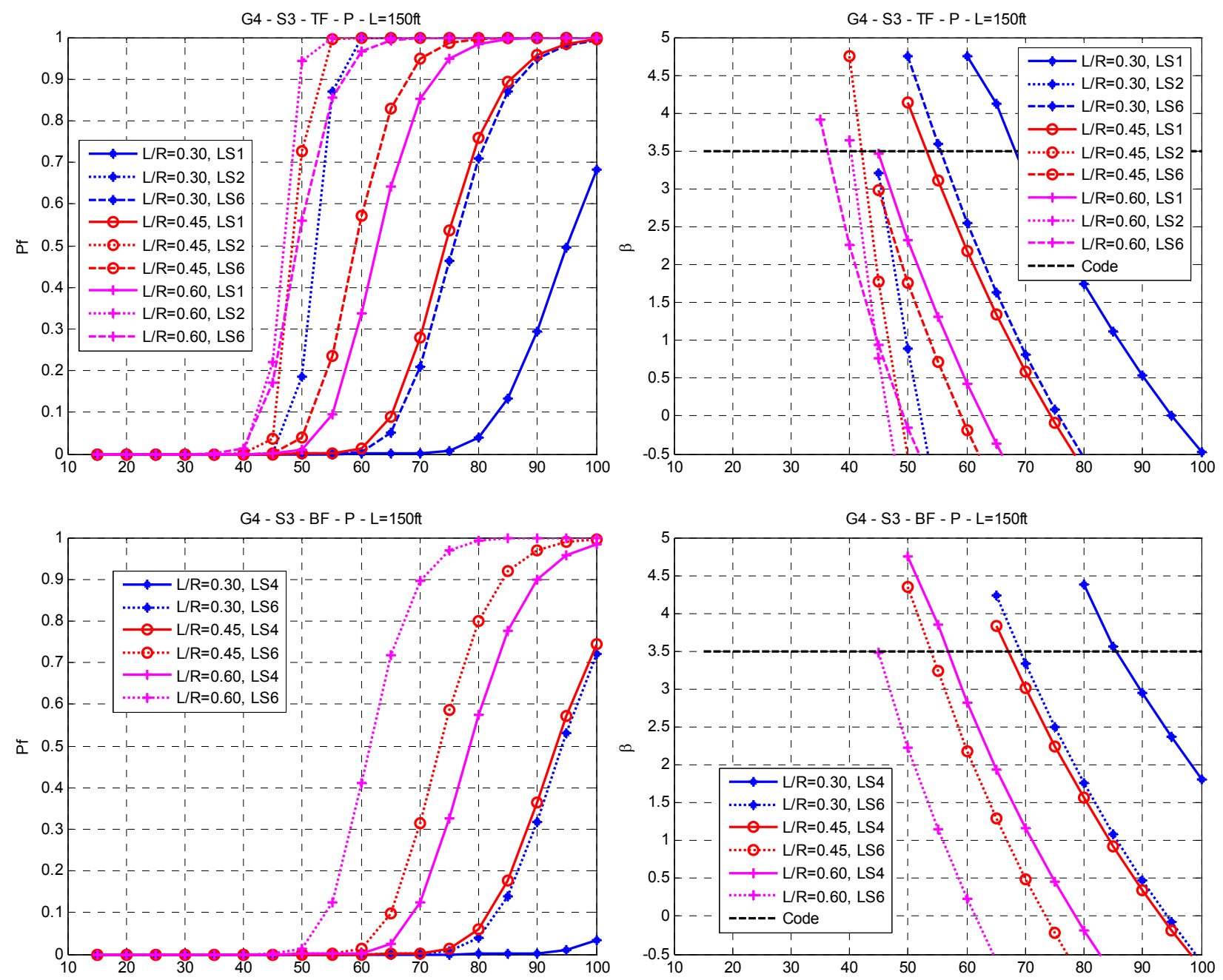

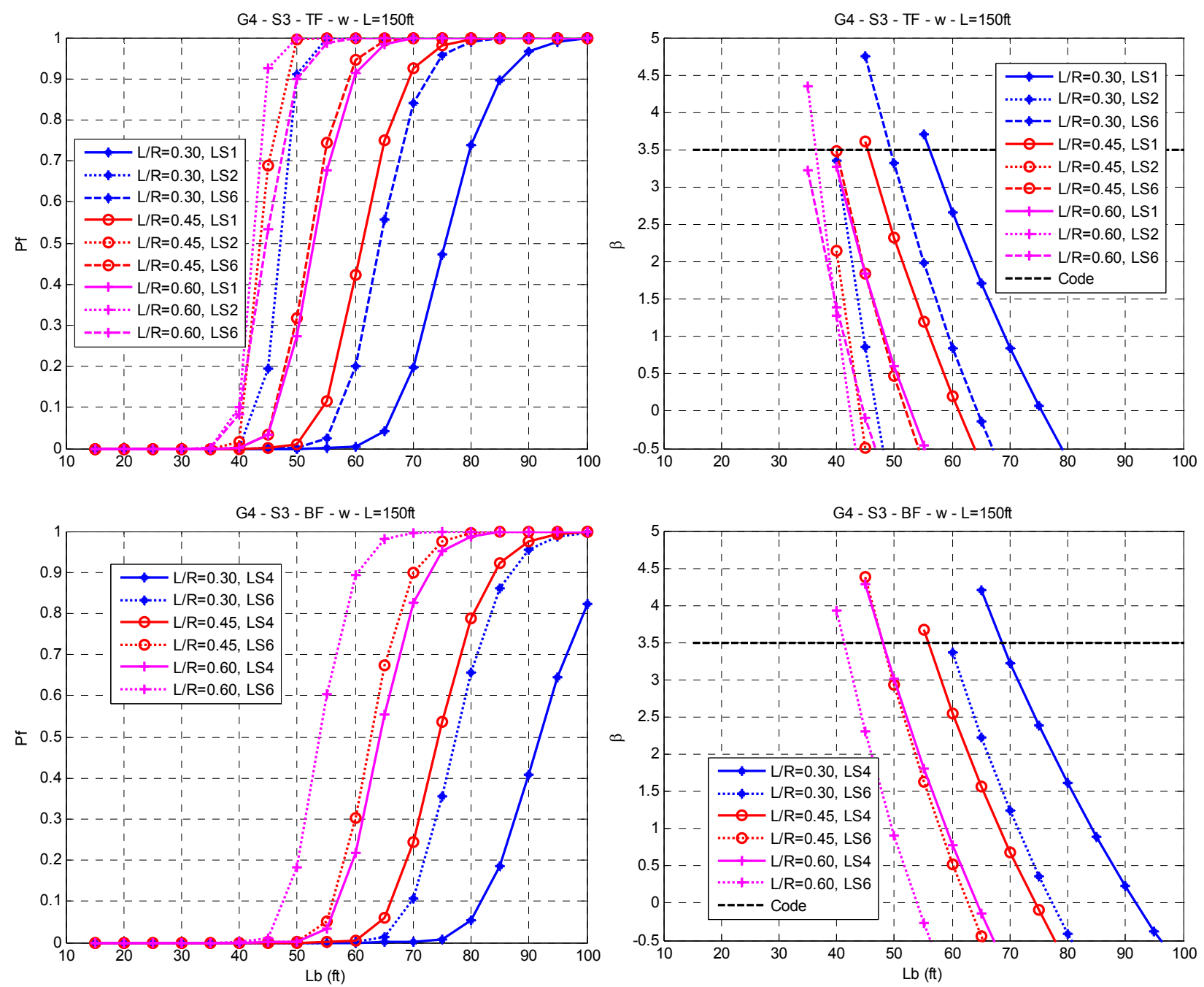

Figure 7-17. Fragility curves for Section S3 G4 with $L_{m}=150 \mathrm{ft}-\mathrm{L} / \mathrm{R}=0.30$
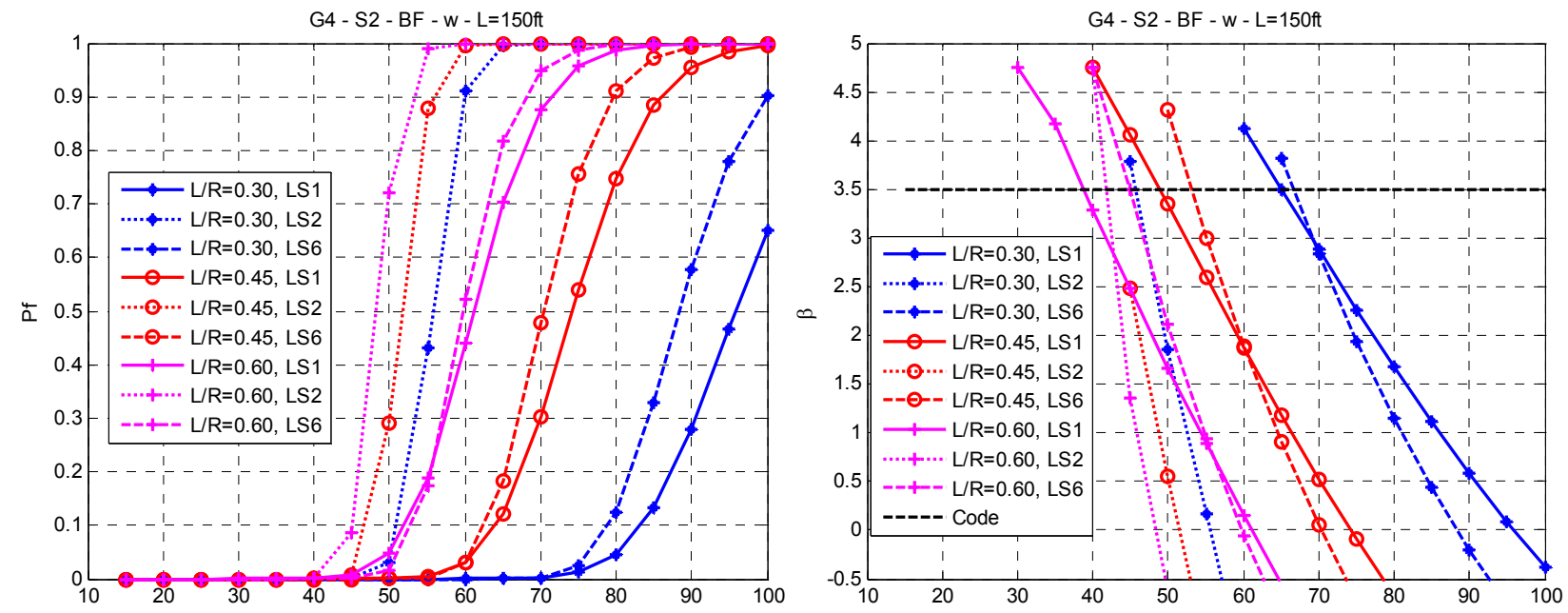

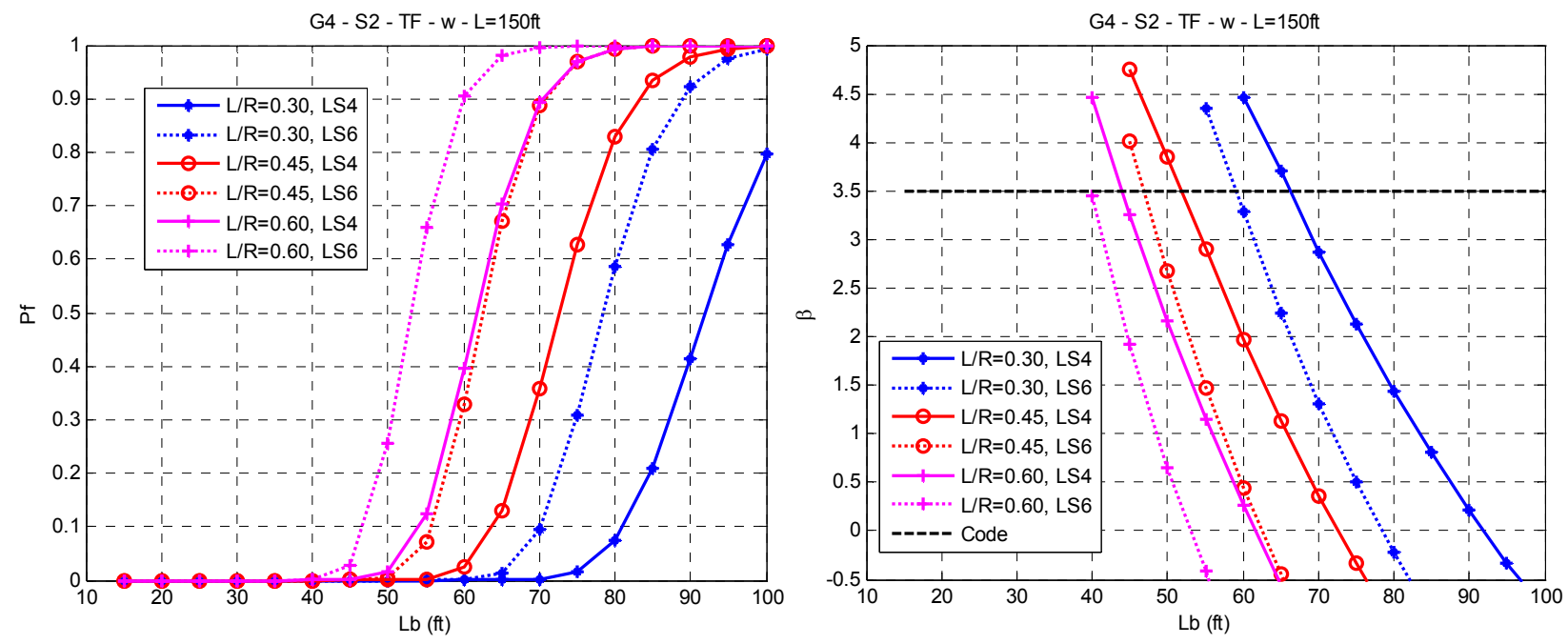

Figure 7-18. Fragility curves for Section $\mathrm{S} 2$ in $\mathrm{G} 4$ with $L_{m}=150 \mathrm{ft}-\mathrm{L} / \mathrm{R}=0.30$
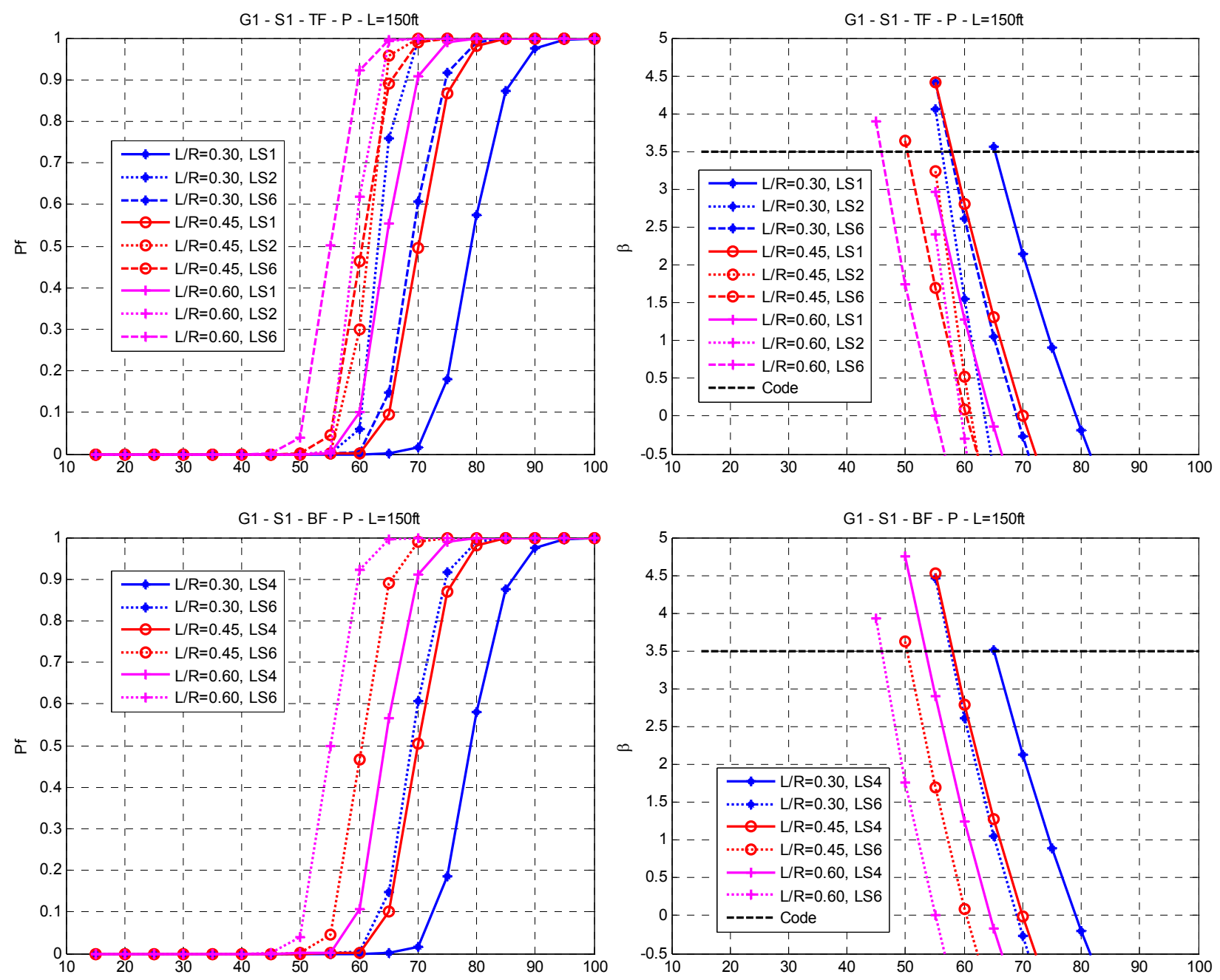

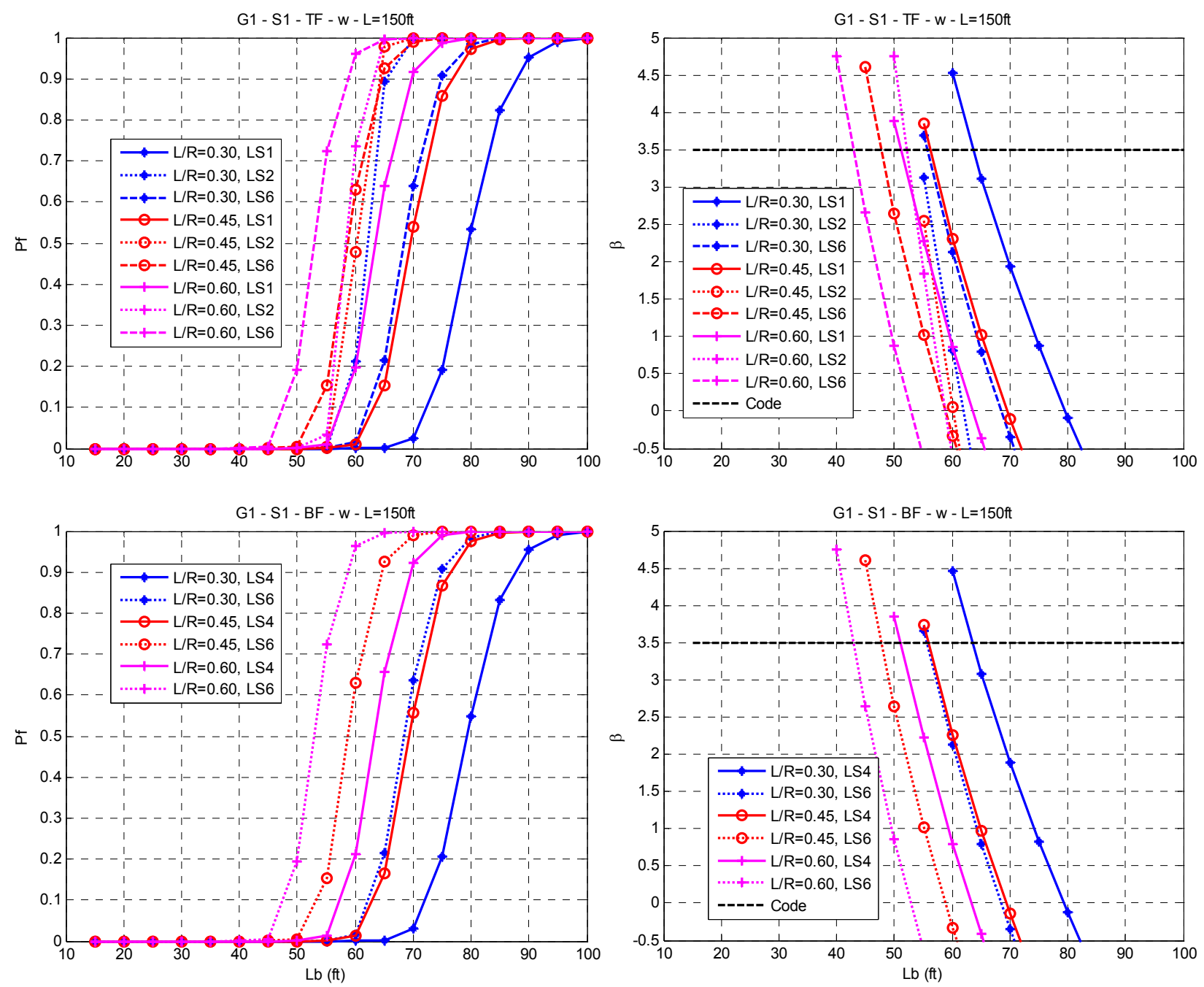

Figure 7-19. Fragility curves for Section $\mathrm{S} 1$ in $\mathrm{G} 1$ with $L_{m}=150 \mathrm{ft}-\mathrm{L} / \mathrm{R}=0.30$
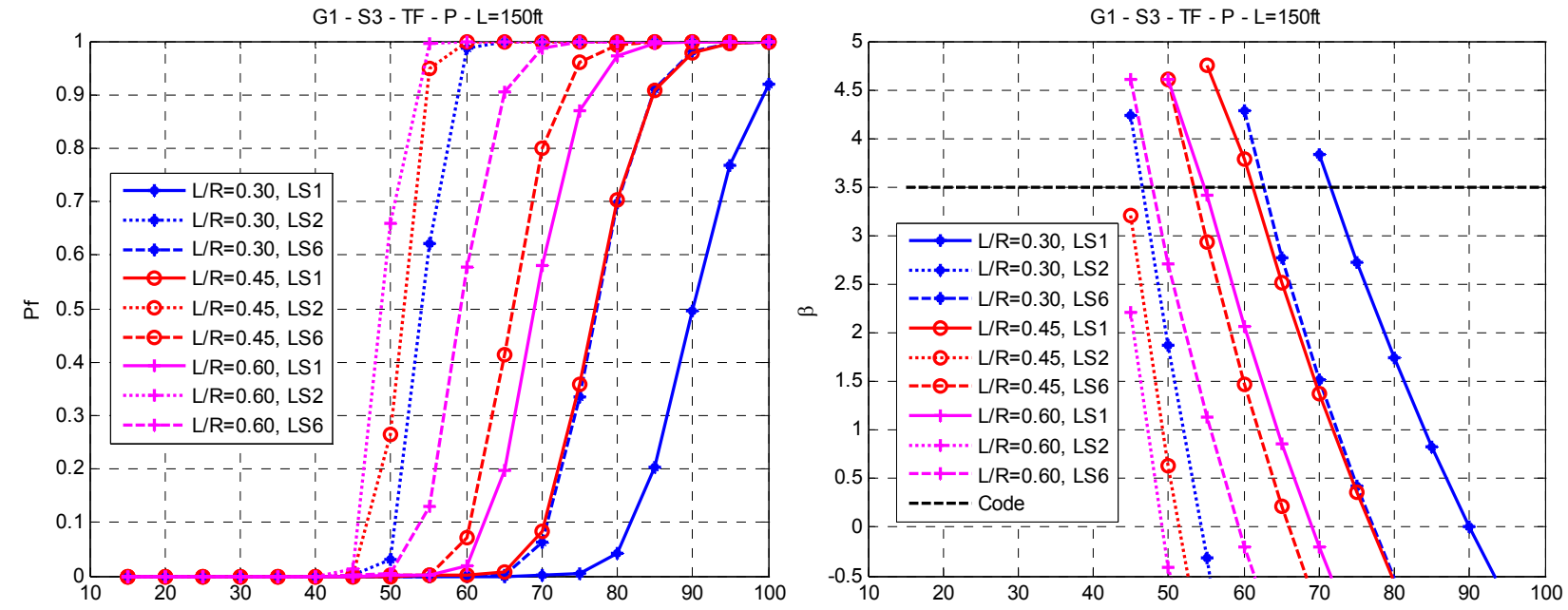
$\mathrm{G} 1-\mathrm{S} 3-\mathrm{BF}-\mathrm{P}-\mathrm{L}=150 \mathrm{ft}$
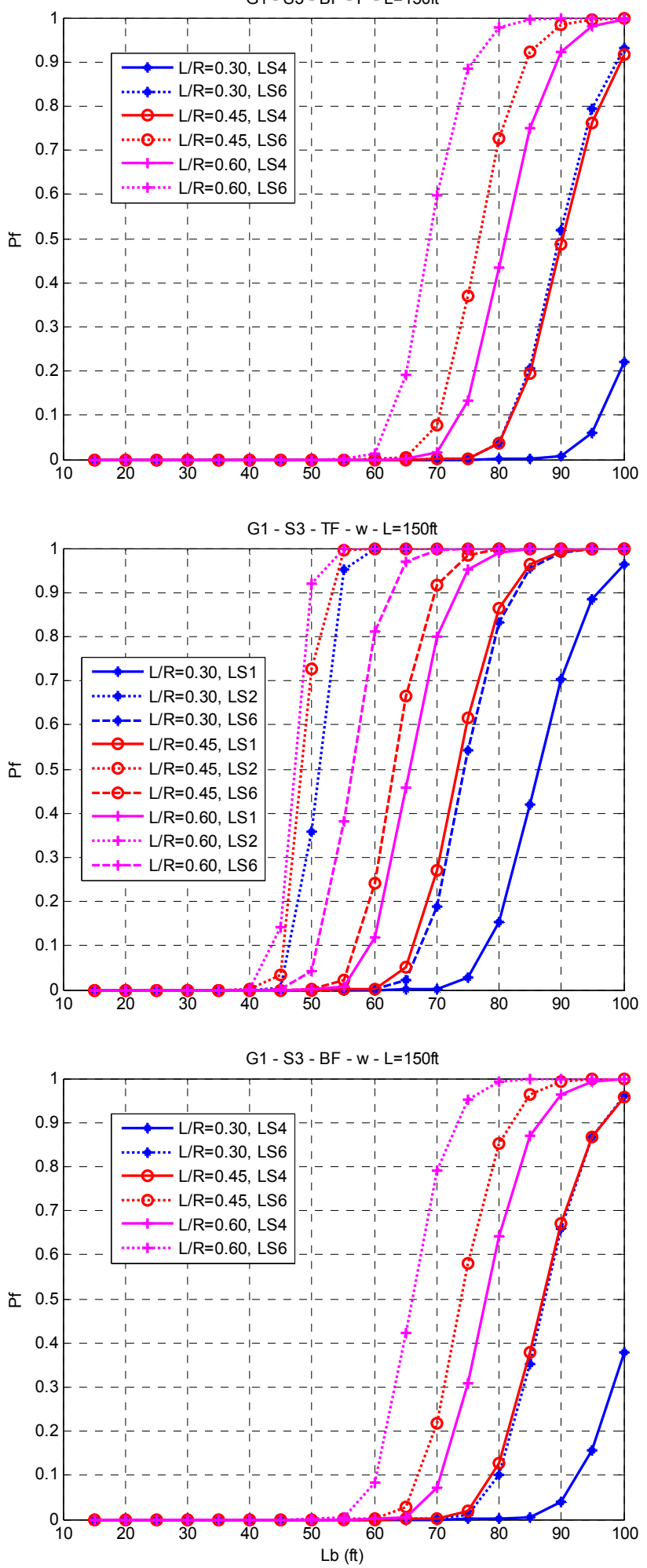

G1 - S3 - BF - P - L=150ft
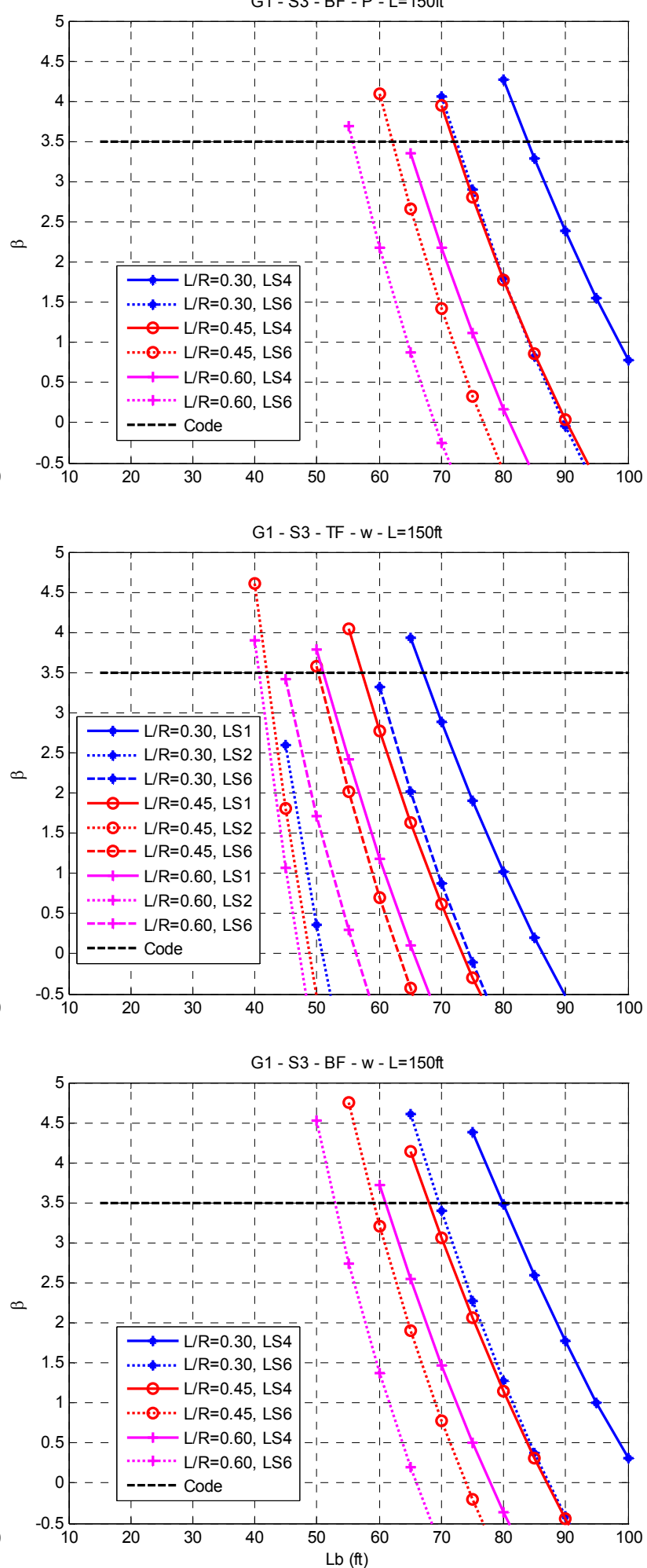

Figure 7-20. Fragility curves for Section $\mathrm{S} 3$ in $\mathrm{G} 1$ with $L_{m}=150 \mathrm{ft}-\mathrm{L} / \mathrm{R}=0.30$ 

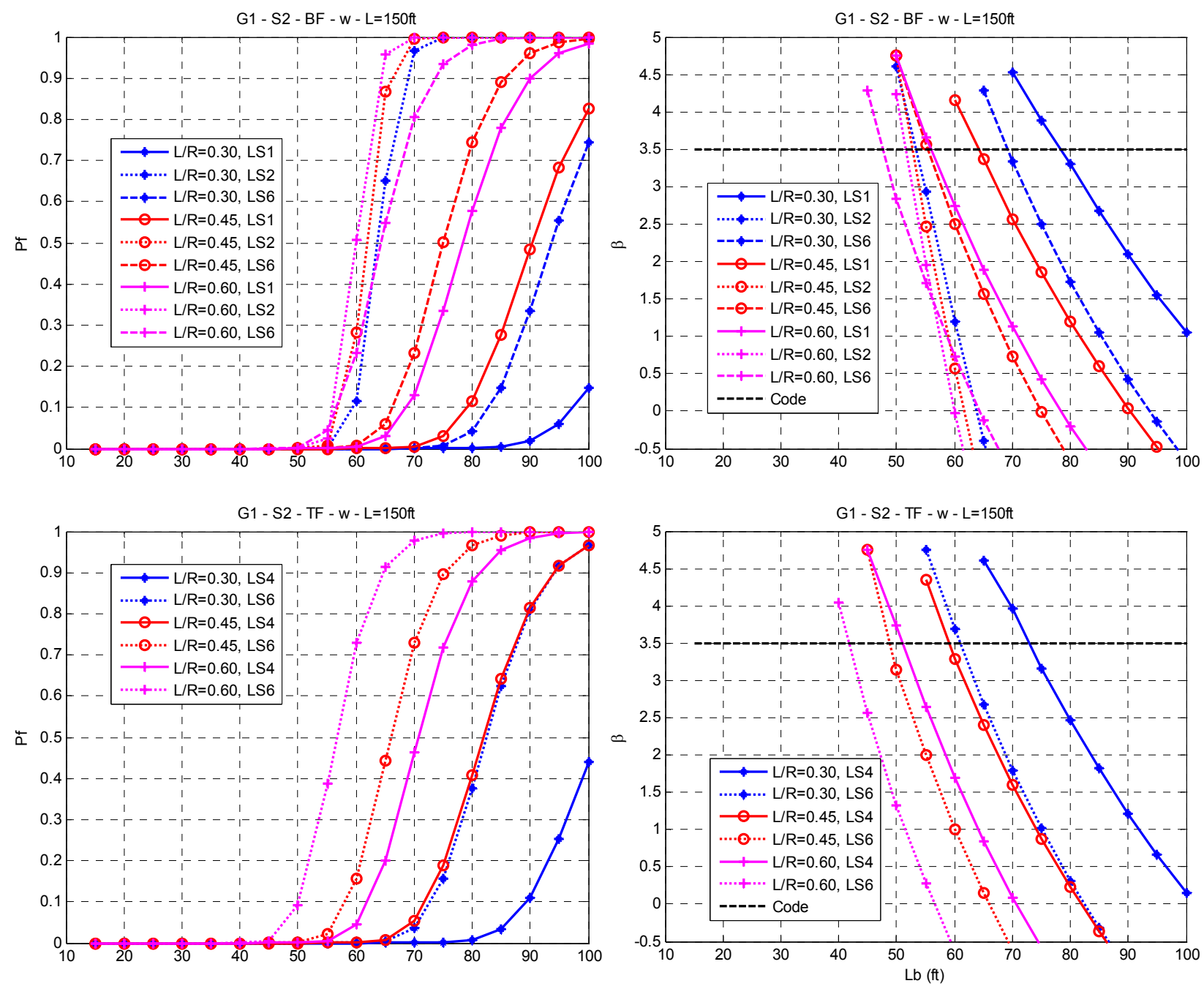

Figure 7-21. Fragility curves for Section $\mathrm{S} 2$ in $\mathrm{G} 1$ with $L_{m}=150 \mathrm{ft}-\mathrm{L} / \mathrm{R}=0.30$

\subsection{Concluding remarks}

A reliability analysis was performed to develop fragility curves that allow the designer to select the cross-frame spacing for deck placement conditions considering the flexural limit states for constructibility that are directly affected by the cross-frame spacing. The adopted structural loading and resistance models were described along with their corresponding probabilistic characteristics. A Monte Carlo Simulation was conducted for straight, skewed and curved steel I-girder bridges to obtain the fragility curves that allow identifying the maximum cross-frame according to the maximum tolerated level of risk. The following observations were established from this effort. 
For all bridge types, the ultimate strength in the compression flange is the governing limit state in the reliability analysis. Therefore, the fragility curves obtained in this work may be valid for service conditions since the compression flange in these cases act as a composite section.

For straight and skewed bridges, it was observed that the reliability of the system is lower for short span bridges since the LFB effects decrease as the length of the bridge increases. The results showed that the skew angle does not affect the reliability of the skewed bridges and the same results obtained for straight bridges apply in this case.

For curved bridges, it was observed that the critical cross-frame spacing is obtained at the end span of the exterior girder. The reliability in the exterior girder decreases for highly curved and long-span bridges due to the effect of $f_{b u}$, which is deeply increased by the curvature and the span length. The critical cross-frame distances in the middle span and the pier regions corresponding to a code reliability level are not unfavorably affected by the span length. However, these distances slightly decrease as the curvature is higher.

In general, it is recommended to use temporary vertical supports at the end spans during construction for highly curved and long bridges that do not meet the minimum levels of tolerated reliability. 


\section{Chapter 8: $\quad$ Summary, conclusions and recommendations}

\subsection{Summary}

This work has resulted in the development of approximated equations that predict the LFB in steel I-girder bridges during concrete deck placement. The effects on the flexural limit state equations for constructibility of variables such as skew, curvature, cross-frame spacing, girder cross section and casting sequence were evaluated as well. In addition, the maximum allowed skews and curvatures were computed for straight bridges based on the limit states for constructibility and the proposed LFB equations. Finally, fragility curves were developed for steel I-girder bridges during construction that allow selecting the maximum cross-frame according to the maximum tolerated level of risk.

Introductory material was presented in Chapter 1 including general information of the research topic, the need for this project, the main research objectives and the research methods. Chapter 2 presented the literature review of topics related to this research project such as LFB, overhang loads, curved and skewed steel I-girder bridges, specifications and structural reliability.

Chapter 3 discussed the principal modeling procedures employed to conduct FEA in this project. A description of the material models, the finite elements and analyses required to model the bridge behavior during deck placement conditions is included. A validation of the modeling procedures was also presented based on the results from different experimental tests.

The parametric study used to investigate the effects of the deck-placement process on the LFB of steel I-girder bridges was described in Chapter 4. The description covered the parametric variables, the loads, the structural design and the analyses for each parametric configuration that properly represent the deck casting sequence.

Chapter 5 presented the results from the parametric study that allow formulating approximate equations to predict the LFB in straight, skewed and curved steel I-girder bridges during deck placement. The major-axis bending stresses were also analyzed, where the principal contribution was made for curved. 
In Chapter 6, the AASHTO flexural limit states for constructibility were evaluated using the results from the parametric study along with the equations proposed in Chapter 5 to estimate the LFB. Initially, the critical sections along the bridge were identified and the effects of the cross-frame spacing and the deck-placement sequence in the governing limit states were evaluated as well. Finally, the maximum allowable skews and curvatures for deck placement conditions were computed for bridges designed originally as straight.

Chapter 7 described the reliability analysis performed to develop the fragility curves. A general description of a structural reliability study is given initially, followed by the definition of the structural loading and resistance models employed in this work. Then, the Monte Carlo Simulations were performed for straight, skewed and curved steel I-girder bridges resulting in the fragility curves that allow identifying the maximum cross-frame spacing for deck-placement conditions according to the maximum tolerated level of risk.

\subsection{Conclusions}

The bending stresses obtained during deck placement conditions in three-span straight, skewed and curved steel I-girder bridges were evaluated in Chapter 5 using the results obtained from a FEA parametric study. A symmetric deck casting sequence was considered where the positive moment regions are poured first. The LFB stresses caused by different sources were estimated by the curves that have the best fit to the numerical critical stresses. The following major conclusions were established from this evaluation.

- Straight structures:

The major-axis bending stresses exhibited by the exterior girders of straight bridges are independent of the cross-frame spacing. Therefore, these stresses may be obtained from the structural analysis of either a simplified model that does not consider explicitly the cross frames or a more detailed model involving any cross-frame distance.

For the positive moment regions, the LFB due to distributed loads was satisfactorily predicted by the equation proposed in this work over the entire range of cross-frame distances, principally at the end spans. Although the LFB obtained in the 
middle span is lower, the same equations were proposed to simplify the approximations. Comparisons with the code estimations showed that the Specifications are highly conservative in all cases, especially for large cross-frame distances.

For concentrated loads, a linear regression considering the most external points from the critical curves was performed to adjust the numerical stresses. These external points correspond to models where the concentrated loads are placed at the middle of a cross-frame spacing which is the critical location. The proposed equation for the bottom flange governs over the code equation for cross-frame distances larger than $26 \mathrm{ft}$. However, both the code and the proposed approximations coincide for the top flange.

The LFB effect on the negative moment regions was approximated considering only the distributed loading case since the contribution from the concentrated loads is negligible. The results showed that the proposed equation satisfactorily predicts the LFB in both flanges.

\section{- Skewed structures}

The results showed that skewed bridges exhibit LFB even when torsional loads are not applied. However, in the presence of torsional effects, the LFB effect is slightly more pronounced when the cross frames are oriented parallel to the supports. Therefore, a perpendicular orientation of the cross frames is recommended for general skewed bridges to increase the lateral stiffness of the structure. This perpendicular configuration was adopted in this work to evaluate the LFB effects.

The results indicated that the major-axis bending in skewed bridges is not only independent of the cross-frame spacing but also of the skew angle. Therefore, these stresses may be taken from structural analyses performed for skewed bridges with any cross-frame spacing and skew angle, including their simplified straight counterpart.

The LFB in the positive moment regions was approximated using curve fitting models similar to the ones used for straight bridges. For distributed loads, a lower limit was introduced in the proposed equation for the bottom flange since this flange exhibits LFB even for closely spaced cross frames. The minimum LFB stress considered in the bottom flange is proportional to the skew angle. The results were compared to the code equation for overhang loads and significant overestimations of the LFB principally for long cross-frame distances were found. For concentrated loads, the results showed that 
the code equation for overhang loads fails to satisfactorily predict the LFB in most of the cases, principally in the bottom flange.

The LFB in the negative moment regions was approximated in this work by a constant term that depends on the skew angle. This approximation was adopted to conservatively estimate the stresses which do not exhibit a consistent trend in these regions. The results also indicated that the code recommendation of using $10 \mathrm{Ksi}$ for the unfactored LFB stresses in skewed bridges is conservative even compared to factored stresses.

\section{- Curved structures}

The evaluation of the bending stresses in curved bridges indicated that the majoraxis bending and the LFB are deeply affected by the curvature and the position of the girder, i.e. outer or inner girder.

The analyses showed that the major-bending stresses in curved bridges are independent of the cross-frame spacing. However, they increase linearly with the curvature at the positive moment regions of the end spans. Conversely, these stresses at the middle span of the outer girder are not significantly affected by the curvature. A linear model was proposed to estimate the major-bending effects in the positive moment regions of both exterior girders. This model computes the major-axis bending in curved bridges based on the major-axis bending stresses exhibited by their straight counterpart. Therefore, the proposed equation allows estimating the major-axis bending in curved bridges from structural analyses of simplified straight bridges. In the negative moment regions, the major-bending stresses also exhibit a linear trend but it is independent of the span length.

The assumption of computing the major-axis bending based on a simplified straight model with the real arc length of the curved girder does not help to represent the curvature effects on these stresses. In fact, the AASHTO recommendation of ignoring the curvature effects for $f_{b u}$ when $L / R$ is lower than 0.06 introduces an error of approximately $10 \%$ in long span bridges.

The LFB in curved girders is caused by both the curvature and the overhang loads. However, it was shown that the participation of the overhang loads in the LFB is low compared to the curvature effects. In fact, the overhang-to-curvature effect ratio 
reduces as the length of the span and the curvature increase. Therefore, the overhang term was dropped from the final expression proposed for the LFB which simplifies to the curvature term only. However, the torsional effects due to overhang loads are implicitly considered since the curve fitting process is applied to the total LFB obtained from the parametric study. A comprehensive formulation to estimate the LFB effects due to curvature was developed for distributed and concentrated loads, respectively. The critical case between the top and bottom flanges was selected to define the equations proposed in this work to estimate the LFB in curved bridges.

The results indicated that the LFB is practically unaffected by the variation of the curvature, a slight effect was only observed in the positive moment regions of the middle span. This observation indicates that these effects need to be considered even in bridges with large curvature radii.

The equations proposed in this project to estimate the LFB in curved bridges work adequately in both exterior girders. Significant reductions were found in most of the cases compared to the estimations given by the AASHTO.

The major-axis bending effects in the AASHTO equation were computed using both the numerical and the estimated major-axis bending stresses, obtaining similar results. The estimated major-axis bending stresses correspond to the stresses computed using the equation proposed in this work to estimate $f_{b u}$ in curved bridges from the results obtained in their straight counterpart. Therefore, the LFB in a curved bridge can be conservatively approximated using the code equation together with the major-axis bending from a corresponding straight bridge. The advantage of the proposed equations over the code approximation is that it is not required to know in advance the majorbending effects to compute the LFB. However, the principal disadvantage is that different expressions are required to define the effects of distributed and concentrated loads, while the code approximation consists of one single equation that applies for all load cases independent of the girder location and flange position.

However, the results from the parametric study indicated that the code equation fails to predict the LFB due to distributed loads at the inner girder of highly curved bridges with long spans. Therefore, it is recommended to use the equation proposed in this work which predicts satisfactorily the LFB in all cases. It was also observed that the 
bottom flange under concentrated loads exhibits the most critical LFB effects compared to the top flange, especially for short span lengths.

The results indicated that the outer girder exhibits the most critical combined bending effects. On the contrary, the curvature decreases the magnitude of the majorbending stresses in the inner girder resulting in a combined bending action much lower than that corresponding to the outer girder. Therefore, the design of both exterior girders shall be based on the evaluation of the outer girder, unless an optimization of the inner girder is pursued. In that case, the effects of the girder stiffness in the behavior of the whole cross section of the deck shall be investigated.

The first part of Chapter 6 was intended to evaluate the behavior of the limit states for constructibility based on the FEA results obtained from the parametric study. The following major results were obtained.

For the skewed bridges analyzed in this work, it was observed that the effect on the limit states of the girder position, the cross-frame orientation and the skew angle is almost negligible. However, the cross-frame spacing has a significant effect on the limit state of discretely braced flanges in compression principally in the positive moment regions, since the LFB depends on the cross-frame distance and the capacity of the flange reduces as this parameter increases.

It was also observed that the cross sections of the end and middle spans are controlled by the casts where the concrete is placed over the complete positive moment region. The compression flanges in both spans are governed by the ultimate strength limit state. The negative moment regions are controlled by the last casting stage. The flanges in compression are governed by the yielding limit state for short span length bridges. However, the web bend-buckling limit state governs the compression flanges for longer spans.

In curved bridges, the results showed that both the curvature and the cross-frame spacing highly affect the limit state ratios in the positive moment regions of the outer girder. However, a stress relief was observed in the inner girder caused by the length adjustments due to curvature. Additionally, the LFB exhibited by the inner girder is relieved since its eccentricity with respect to the supports is smaller. In fact, the LFB 
undergoes a reversing effect in the inner girder compared to its straight counterpart. This is caused from the torsional effects that are controlled by the curvature being counteracted by the overhang load effects in the inner girder.

It was observed that the variables that most affect the limit states are the curvature followed by the cross-frame spacing. The sections of the end and middle spans are controlled by the casts that consider the concrete over the total positive moment regions. The ultimate strength is the limit state that principally governs the compression flanges in these sections. However, the yielding limit state may control in short span lengths with high curvatures.

As for skewed bridges, the negative moment regions are controlled by the last casting stage. The yielding limit state governs the compression flanges of bridges with short spans and the web bend-buckling limit state becomes significant in the pier regions for long span bridges.

None of the critical limit state values resulted from a casting stage where the finishing machine is considered.

In the second part of Chapter 6, the maximum allowed skews and curvatures were computed for straight bridges based on the limit states for constructibility and the LFB equations proposed in Chapter 5. For skewed bridges, it was observed that the maximum cross-frame spacing in skewed bridges is controlled by the ultimate strength limit state in the compression flange. Conversely, the tension flange seems to be unaffected by the skew angle.

On the other hand, the maximum allowed curvature is controlled in most of the cases by the end-span cross section in the outer girder. It was also shown that the ultimate strength limit state of the compression flange controls the maximum allowable curvature for large cross-frame distances. Comparisons with the code recommendation about the maximum cross-frame spacing in curved bridges showed that this specification is adequate in short span bridges when the distance between cross frames is less than approximately $40 \mathrm{ft}$. However, long span bridges present an allowable curvature lower than the one recommended in the code when the cross sections are designed from a straight girder formulation. 
A reliability analysis employing a Monte Carlo Simulation was performed in Chapter 7 to develop fragility curves that allow the designer to select the cross-frame spacing for deck placement conditions. The following observations were established from this effort.

For all bridge types, the ultimate strength in the compression flange is the governing limit state in the reliability analysis. Therefore, the fragility curves obtained may be valid for service conditions since the compression flange in these cases acts as a composite section.

For straight and skewed bridges, it was observed that the reliability of the system is lower for short span bridges, since the LFB effects decrease as the length of the bridge increases. The results showed that the skew angle does not affect the reliability of the skewed bridges and the same results obtained for straight bridges apply in this case.

For curved bridges, it was observed that the critical cross-frame spacing is obtained at the end span of the exterior girder. The reliability in the exterior girder decreases for highly curved and long-span bridges due to the effect of $f_{b u}$, which is deeply increased by the curvature and the span length. The critical cross-frame distances in the middle span and the pier regions corresponding to a code reliability level are not unfavorably affected by the span length. However, these distances slightly decrease as the curvature is higher.

In general, it is recommended to use temporary vertical supports at the end spans during construction for highly curved and long bridges that do not meet the minimum levels of tolerated reliability.

\subsection{Recommendations}

The results presented in this work correspond to steel I-girder bridges with the specific characteristics described in the parametric study. Additional parametric variables such as overhang length, girder spacing, number of girders, number of spans, yielding strength, etc., should be incorporated in future research efforts to complement the existing conclusions. In particular, the parametric study is based on the loads factored according to the Strength I load combination of the Specifications. Additional load 
combinations may be considered where the LFB effects due to wind are analyzed. In addition, in this study, a symmetric deck casting sequence was considered. The evaluation of critical conditions exhibited during unsymmetrical sequences would be a beneficial supplement to the existing work.

The present work is focused on the evaluation of the flexural bending stresses exhibited by the girder flanges. Future work incorporating shear stresses, deflections and distortions will provide the engineer with the criteria required to evaluate the complete structural behavior of steel I-girder bridges during construction. Moreover, the evaluation of the internal forces in the members of the lateral bracing system will contribute to improve the corresponding design specifications.

It is suggested that some of the results obtained in this work be validated by conducting experimental tests. 


\section{References}

AASHTO. (1992). Standard Specifications for Highway Bridges (15th ed.). Washington, DC: Author.

AASHTO. (1994). LRFD Bridge Design Specifications, S.I. units. Washington, DC: Author.

AASHTO. (1998). LRFD Bridge Design Specifications, Customary U.S. units (2nd ed.). Washington, DC: Author.

AASHTO. (2003). Guide Specifications for Horizontally Curved Steel Girder Highway Bridges. Washington, DC: Author.

AASHTO. (2004). LRFD Bridge Design Specifications, Customary U.S. units (3rd ed.). Washington, DC: Author.

AASHTO. (2007). LRFD Bridge Design Specifications, Customary U.S. units ( $4^{\text {th }}$ ed.) 2008 Interim Revisions. Washington, DC: Author.

Abaqus. (2002). Version 6.3 Documentation. Pawtucket, RI: Hibbit, Karlsson \& Sorensen.

American Concrete Institute. (2002). Building code requirements for structural concrete and commentary, ACI 318-02. Farmington Hills, MI: Author.

Applied Technology Council. (1996). Seismic evaluation and retrofit of concrete buildings. Redwood City, CA: Author.

Barker, M., \& Zacher, J. (1997). Reliability of Inelastic Load Capacity Rating limits for Steel bridges. Journal of Bridge Engineering, 2(2), 45-52.

Barth, K., \& Wu, H. (2006). Efficient Nonlinear Finite Element Modeling of Slab on Steel Stringer Bridges. Finite Elements in Analysis and Design, 42(14), 13041313.

Beal, D. (1978). Horizontally Curved Bridges: New York field testing and design studies (Vol. 61). Albany, NY: New York State Department of Transportation.

Beal, D., \& Kissane, R. (1971). Field Testing of Horizontally Curved Steel Girder Bridges. Albany, NY: New York State Department of Transportation.

Beckmann, F., \& Medlock, R. (2005). Skewed Bridges and Girders Movements due to rotations and differential deflections. Paper presented at 2005 World Steel Bridge - Symposium and Workshops. Orlando, FL: National Steel Bridge Alliance. 
Bell, B., \& Linzell, D. (2007). Erection Procedure Effects on Deformations and stresses in a Large-radius, horizontally curved, I-girder Bridge. Journal of Bridge Engineering, 12(4), 467-476.

Brokenbrough, R. (1986). Distribution factors for curved I-girder bridges. Journal of Structural Engineering, 112(10), 2200-2215.

Chang, C., White, D., Beshah, F., \& Wright, W. (2005). Design Analysis of Curved IGirder Bridge Systems - An Assessment of Modeling Strategies. Paper presented at the Annual Stability Conference. Montreal \& Quebec: Structural Stability Research Council.

Chavel, B. W., \& Earls, C. J. (2006a). Construction of a Horizontally Curved Steel IGirder Bridge, Part I: Erection Sequence. Journal of Bridge Engineering, 11(1), $81-90$.

Chavel, B. W., \& Earls, C. J. (2006b). Construction of a Horizontally Curved Steel IGirder Bridge, Part II: Inconsistent Detailing. Journal of Bridge Engineering, 11(1), 91-98.

Cheng, G., Xu, L., \& Jiang, L. (2006). A sequential approximate programming strategy for reliability-based structural optimization. Computers \& Structures, 84(21), 1353-1367.

Choo, T., Linzell, D., Lee, J., \& Swanson, J. (2005). Response of a continuous, skewed, steel bridge during deck placement. Journal of Constructional Steel Research, 61(5), 567-586.

Coletti, D., \& Yadlosky, J. (2005). Behavior and analysis of curved and skewed steel girder bridges. Paper presented at 2005 World Steel Bridge - Symposium and Workshops. Orlando, FL: National Steel Bridge Alliance.

Czarnecki, A., \& Nowak, A. (2006). System Reliability Assessment of Steel Girder Bridges. In M. Pandey, W. Xie and L. Xu (Eds.), Advances in Engineering Structures, Mechanics and Construction, (pp. 699-710). Canada: Springer Netherlands.

Davidson, J., \& Madhavan, M. (2006). Definition of flange compactness for I-beams that are subjected to combined primary bending and torsion or lateral flexure. Paper presented at the 17th Analysis and Computation Specialty Conference. St. Louis, MO: Structural Engineering and Public Safety.

Davidson, J., \& Yoo, C. (2000). Evaluation of Strength Formulations for Horizontally Curved Flexural Members. Journal of Bridge Engineering, 5(3), 200-207. 
Davidson, J., \& Yoo, C. (1996). Local buckling of curved I-girder flanges. Journal of Structural Engineering, 122(8), 936-947.

Davidson, J., Keller, M., \& Yoo, C. (1996). Cross-frame spacing and parametric effects in horizontally curved I-girder bridges. Journal of Structural Engineering, 122(9), 1089-1096.

Davidson, J., \& Yoo, C. (2003). Effects of distortion on strength of curved I-shaped bridge girders. Transportation Research Record, 1845, 48-56.

Davidson, J., Abdalla, R., \& Madhavan, M. (2002). Design and construction of modern curved bridges, Report No. 01223. Huntsville, AL: University Transportation Center for Alabama.

Davidson, J., Ballance, S. \& Yoo, C. (1999). Analytical model of curved I-girder web panels subjected to bending. Journal of Bridge Engineering, 4(3), 204-212.

Davidson, J., Ballance, S. \& Yoo, C. (1999). Finite displacement behavior of curved Igirder webs subjected to bending. Journal of Bridge Engineering, 4(3), 213 -220.

Davidson, J., Ballance, S., \& Yoo, C. (1999). Effects of longitudinal stiffeners on curved I-girder webs. Journal of Bridge Engineering, 5(2), 171 -178.

Davidson, J., Keller, M. \& Yoo, C. (1996). Cross-frame spacing and parametric effects in horizontally curved I-girder bridges. Journal of Structural Engineering, 122(9), 1089-1096.

De Polo, D. \& Linzell, D. (2005). Evaluation of live load lateral flange bending distribution for a horizontally curved I-girder bridge. Paper presented at the Transportation Research Board 84th Annual Meeting. Washington, DC: TRB.

Domalik, D., Shura, J., \& Linzell, D. (2005). Design and Field Monitoring of Horizontally Curved Steel Plate Girder Bridge. Transportation Research Board, $1928,83-91$.

Duwadi, S., Grubb, M., Yoo, C., \& Hartmann, J. (2000). Federal Highway Administration's Horizontally Curved Steel Bridge Research Project - An update. Transportation Research Record, 1696, 152-161.

Eamon, C., Nowak, A., Ritter, M., \& Murphy, J. (2000). Reliability-based criteria for load and resistance factor design code for wood bridges. Transportation Research Record, 1696, 316-322.

Ellingwood, B. (1985). Probability-based load criteria for structural design. Paper published in A Chronicle of Selected of NIST publications 1901-2000. Washington, DC: National Institute of Standards and Technology. 
Ellingwood, B., Galambos, T., MacGregor, J., and Cornell, C. (1980). Development of a Probability Based Load Criteria for American National Standard A58. Washington, D.C.: National Bureau of Standards.

FEMA. (2000a). FEMA 356 - Pre-standard and commentary for the seismic rehabilitation of building. Washington D.C.: Author.

FEMA. (2000b). FEMA 350 - Recommended Seismic Design Criteria for New Steel Moment-Frame Buildings. Washington D.C.: SAC Joint Venture.

FEMA. (2003). NEHRP - Recommended provisions for seismic regulation for new buildings and other structures, Part I: Provisions, Part 2: Commentary, FEMA 450. Washington, D.C.: Building Seismic Safety Council.

Foschi, R., Li, H., \& Zhang, J. (2002). Reliability and performance-based design: a computational approach and applications. Structural Safety, 24(2-4), 205-218.

Galambos, T. (2004). Reliability of the Member Stability Criteria in the 2005 AISC Specification. Steel Structures, 4, 223-230.

Galambos, T., Hajjar, J., Huang, W., Pulver, B., Leon, R. \& Rudie, B. (2000). Comparison of measured and computed stresses in a steel curved girder bridge. Journal of Bridge Engineering, 5(3), 191-199.

Galindez, N., \& Barth, K. (2008). An assessment of the levels of lateral flange bending in skewed and curved girder bridges due to concrete placement. Paper presented at the Annual Stability Conference. Nashville, TN: Structural Stability Research Council.

Gayton, N., Mohamed, G., Sorensen, J., Pendola, M., \& Lemaire, M. (2004). Calibration methods for reliability-based design codes. Structural Safety, 26(1), 91-121.

Grubb, M. (1991). Design for Concrete Deck Overhang Loads. Paper presented at the National Steel Construction Conference. Washington, DC: American Institute of Steel Construction.

Grubb, M., Yadlosky, J., \& Duwadi, S. (1996). Construction issues in steel curved-girder bridges. Transportation Research Record, 1544, 64-70.

Hartmann, J. (2005). An experimental investigation of the flexural resistance of horizontally curved steel I-girder systems. Ph.D. Dissertation, University of Maryland, College Park, MD.

Heins, C., \& Jin, J. (1984). Live load distribution on braced curved I-girders. Journal of Structural Engineering, 110(3), 523-530. 
Herman, R., Helwig, T., Holt, J., Medlock, R., Romage, M., \& Zhou, C. (2005). Lean-on cross-frame bracing for steel girders with skewed supports. Paper presented at 2005 World Steel Bridge - Symposium and Workshops. Orlando, FL: National Steel Bridge Alliance.

Howell, T., \& Earls, C. (2007). Curved Steel I-girder bridge response during construction loading: effects of web plumbness. Journal of Bridge Engineering, 12(4), 485493.

Huang, H., Shenton, H., \& Chajes, M. (2004). Load distribution for a highly skewed bridge: testing and analysis. Journal of Bridge Engineering, 9(6), 558-562.

Issa, M., Yousif, A., \& Issa, M. (2000). Effect of construction loads and vibrations on new concrete bridge decks. Journal of Bridge Engineering, 5(3), 249-258.

Jung, S. (2006). Inelastic strength behavior of horizontally curved composite I-girder bridge structural systems. Ph.D. Dissertation, Georgia Institute of Technology, Atlanta, GA.

Jung, S., White, D., Beshah, F., \& Wright, W. (2005). Ultimate Strength of Horizontally - Curved Composite I-Girder Bridge Structural Systems. Paper presented at the Annual Stability Conference. Montreal \& Quebec: Structural Stability Research Council.

Kansas Department of Transportation. (2005). Design Manual - Ch.5 Falsework design, analysis and inspection. Kansas: Author.

Khaloo, A., \& Mirzabozorg, H. (2003). Load distribution factors in simply supported skew bridges. Journal of Bridge Engineering, 8(4), 241-244.

Kissane, R. (1978). Estimating Lateral Flange Bending in Curved Plate Girders. (Vol. 60). Albany, NY: New York State Department of Transportation.

Kissane, R., \& Beal, D. (1972). Field Testing of Horizontally Curved Steel Girder Bridges (Vol. 8). Albany, NY: New York State Department of Transportation.

Lee, J. \& Fenves, G. (1998). Plastic-damage model for cyclic loading of concrete structures. Journal of Engineering Mechanics, 124(8), 892-900.

Li, J., \& Li, G. (2004). Reliability-based integrated design of steel portal frames with tapered members. Structural Safety, 26(2), 221-239.

Linzell, D., Hall, D., \& White, D. (2004). Historical Perspective on Horizontally Curved I Girder Bridge Design in the United States. Journal of Bridge Engineering, 9(3), 218-229. 
Linzell, D., Leon, R., \& Zureick, A. (2004). Experimental and Analytical Studies of a Horizontally Curved Steel I-Girder Bridge during Erection. Journal of Bridge Engineering, 9(6), 521-530.

Maneetes, H., \& Linzell, D. (2003). Cross-frame and lateral bracing influence on curved steel bridge free vibration response. Journal of Constructional Steel Research, 59(9), 1101-1117.

Mans, P., Yakel, A., \& Azizinamini, A. (2001). Full-scale testing of composite plate girders constructed using 485-Mpa high performance steel. Journal of Bridge Engineering, 6(6), 598-604.

MATLAB. (2004). Documentation - Version 7.0. Natick, MA: The Mathworks.

McElwain, B., \& Laman, J. (2000). Experimental verification of horizontally curved Igirder bridge behavior. Journal of Bridge Engineering, 5(4), 284-292.

NCHRP. (2006a). Synthesis-345: Steel bridge erection practices. Washington, DC: Transportation Research Board.

NCHRP. (2006b). Synthesis-563: Development of LRFD Specifications for Horizontally Curved Steel Girder Bridges. Washington, DC: Transportation Research Board.

Nevling, D., Laman, J., \& D. Linzell. (2006). Examination of Level of Analysis Accuracy for Curved I-Girder Bridges through Comparisons to Field Data. Journal of Bridge Engineering, 11(2), 160-168.

Norton, E., Linzell, D., \& Laman, J. (2003). Examination of Response of a Skewed Steel Bridge Superstructure During Deck Placement. Transportation Research Record, $1845,66-75$.

Nowak, A. (1995). Calibration of LRFD bridge code. Journal of Structural Engineering, $121(8), 1245-1251$.

Nowak, A. (2004). System reliability models for bridge structures. Bulletin of the Polish Academy of Sciences, 52(4), 321-328.

Nowak, A., Szwed, A., Podhorecki, P., Czarnecki, A., Laumet, P., \& Galambos, T. (2006). Appendix C of Synthesis 345: Calibration of LRFD design specifications for Steel Curved Girder Bridges. Washington, D.C.: NCHRP.

NSBA. (2002). Design Example 1: Three-Span Continuous Straight Composite I Girder. Part of The Steel Bridge Design Handbook. Chicago, IL: National Steel Bridge Alliance. 
NSBA. (2007). Steel Bridge Design Handbook: Chapter 13 - Design for constructability. Chicago, IL: National Steel Bridge Alliance.

Ozgur, C., \& White, D. (2007). Behavior and analysis of a curved and skewed I-girder bridge. Paper presented at 2007 World Steel Bridge - Symposium and Workshops. New Orleans, LA: National Steel Bridge Alliance.

Righman, J. (2005). Rotation Compatibility Approach to Moment Redistribution for Design and Rating of Steel I-Girders. Ph.D. Dissertation, West Virginia University, Morgantown, WV.

Roddis, K., Kriesten, M., \& Liu, Z. (1999). Torsional Analysis for Exterior Girders, Report No. K-TRAN: KU-96-3. Lawrence, KS: Department of Transportation of Kansas.

Samaan, M., Sennah, K., \& Kennedy, J. (2005). Distribution factors for curved continuous composite box-girder bridges. Journal of Bridge Engineering, 10(6), 678-692.

Schilling, C. (1996). Yield-Interaction relationships for curved I-girders. Journal of bridge Engineering, 1(1), 26-33.

Schilling, C. and Morcos, S. (1988). Moment-rotation Test of Steel Girders with Ultracompact Flanges. Project 188 - Autostress Design of Highway Bridges: American Iron and Steel Institute.

Seaburg, P., \& Carter, C. (1997). Steel Design Guide No. 9: Torsional Analysis of Structural Members. Chicago, IL: American Institute of Steel Construction.

Shanmugan, N., Thevendran, V., Richard, J., \& Tan, L. (1995). Experimental study on steel beams curved in plan. Journal of Structural Engineering, 121(2), 249-259.

Shura, J., \& Linzell, D. (2006). Behavior of a horizontally curved steel plate girder bridge during steel erection and deck placement. Paper presented at the Annual Meeting of the TRB. Washington, D.C.: Transportation Research Board.

Sun, E. (2006). Shear locking and hourglassing in MSC Nastran, Abaqus and Ansys. Paper presented at VPD Conference 2006. Huntington Beach, CA: MSC Software.

Thevendran, V., Chen, S., Shanmugam, N., \& Liew, R. (1999). Nonlinear Analysis of Steel-Concrete Composite Beams Curved In Plan. Finite Elements in Analysis and Design, 32(3), 125-139.

Thevendran, V., Shanmugam, N., Chen, S. \& Liew, R. (2000). Experimental study on steel-concrete composite beams curved in plan. Engineering Structures, 22(8), 
877-889.

Tiedeman, J., Albrecht, P., \& Cayes, L. (1993). Behavior of two-span continuous bridge under truck axle loading. Journal of Structural Engineering, 119(4), 1234-1250.

Tilley, M., Barton, F., \& Gomez, J. (2006). Dynamic Analysis and Testing of a Curved Girder Bridge. Charlottesville, VA: Virginia Transportation Research Council.

Vrouwenvelder, T. (2001). The fundamentals of structural building codes. Proceedings of the International Conference on Structural Engineering, Mechanics and Computation. Cape Town, South Africa: Elsevier.

West Virginia Department of Transportation. (2004). Bridge design manual - WVDoH Division of Highways. Charleston, WV: Author.

White, D. (2008). Unified Flexural Resistance Equations for Stability Design of Steel Isection Members: Overview. Journal of Structural Engineering, 134(9), 14051424.

White, D., \& Grubb, M. (2005). Unified resistance equations for design of curved and tangent steel bridge I-girders. Transportation Research Board, 121-128.

White, D., \& Grubb, M. (2004). Comprehensive update to AASHTO LRFD Provisions for flexural design of bridge I-girders. Paper presented at Structures Congress 2004. Nashville, TN: ASCE.

White, D., \& Jung, S. (2008). Unified Flexural Resistance Equations for Stability Design of Steel I-section Members: Uniform Bending Tests. Journal of Structural Engineering, 134(9), 1450-1470.

White, D., \& Kim, Y. (2008). Unified Flexural Resistance Equations for Stability Design of Steel I-section Members: Moment Gradient Tests. Journal of Structural Engineering, 134(9), 1471-1486.

White, D., Zureick, A., \& Jung, S. (2001). Development of unified equations for design of curved and straight steel bridge I girders. Atlanta, GA: American Iron and Steel Institute Transportation and Infrastructure Committee.

Yakel, A., \& Azizinamini, A. (2005). Improved moment strength prediction of composite steel plate girders in positive bending. Journal of Bridge Engineering, $10(1), 28-38$.

Yoo, C., \& Davidson, J. (1997). Yield Interaction Equations for Nominal Bending Strength of Curved I-Girders. Journal of Bridge Engineering, 2(2), 37-44.

Zhang, H., Huang, D., \& Wang, T. (2005). Lateral load distribution in curved steel I- 
girder bridges. Journal of Bridge Engineering, 10(3), 281-290.

Zhang, H., Huang, D., \& Wang, T. (2005). Lateral load distribution in curved steel Igirder bridges. Journal of Bridge Engineering, 10(3), 281-290.

Zhao, Y., \& Ono, T. (2001). Moment methods for structural reliability. Structural Safety, 23(1), 47-75.

Zureick, A., \& Naqib, R. (1999). Horizontally curved steel I-girders state-of-the-art analysis methods. Journal of Bridge Engineering, 4(1), 38-47.

Zureick, A., Linzell, D., Leon, R., \& Burrell, J. (2000). Curved Steel I-Girder Bridges: Experimental and Analytical Studies. Engineering Structures, 22(2), 180-190. 


\section{Appendix A: LFB in Skewed Bridges}

- Positive moment region under distributed loads: $\theta=45^{\circ}$
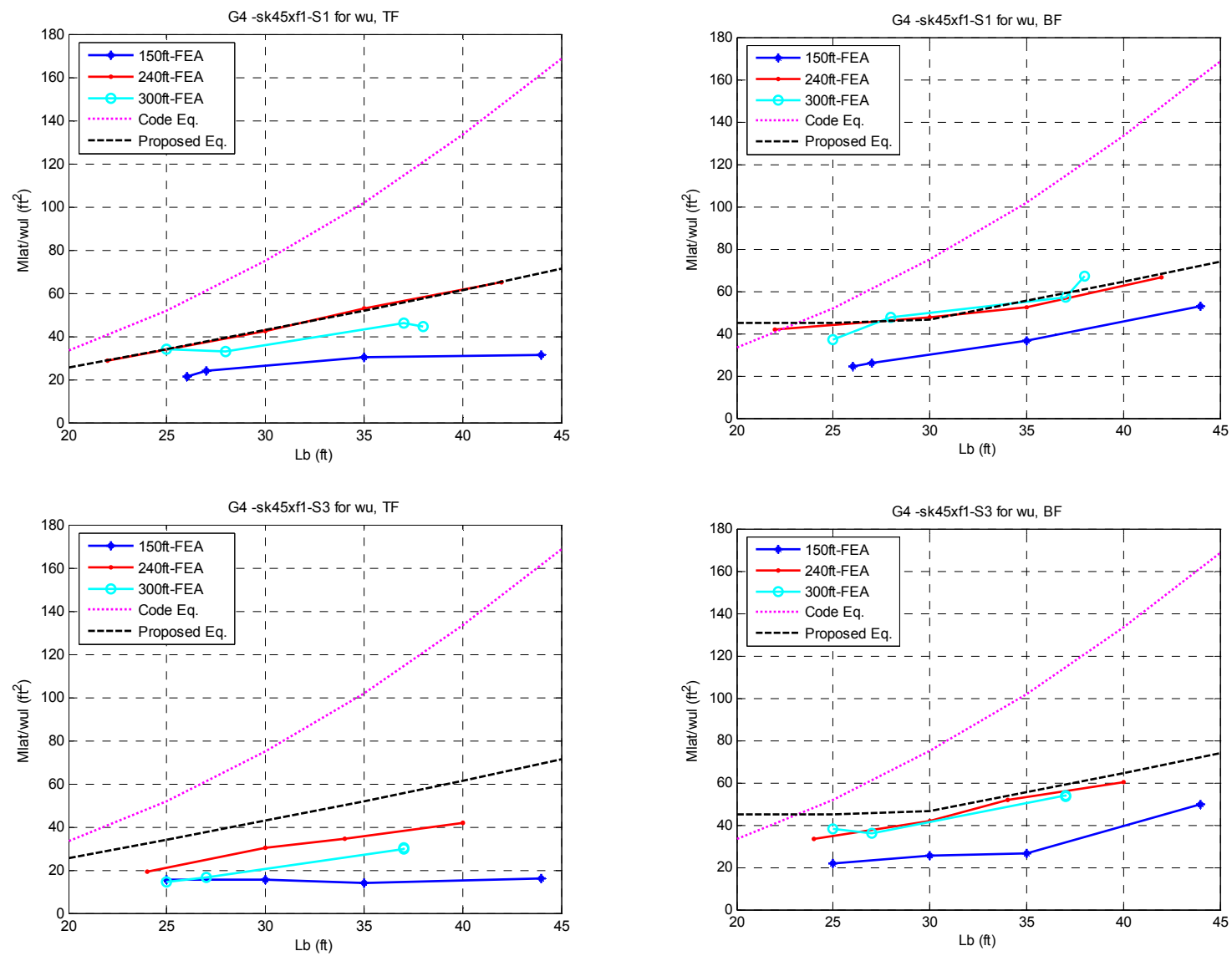
- Positive moment region under distributed loads: $\theta=60^{\circ}$
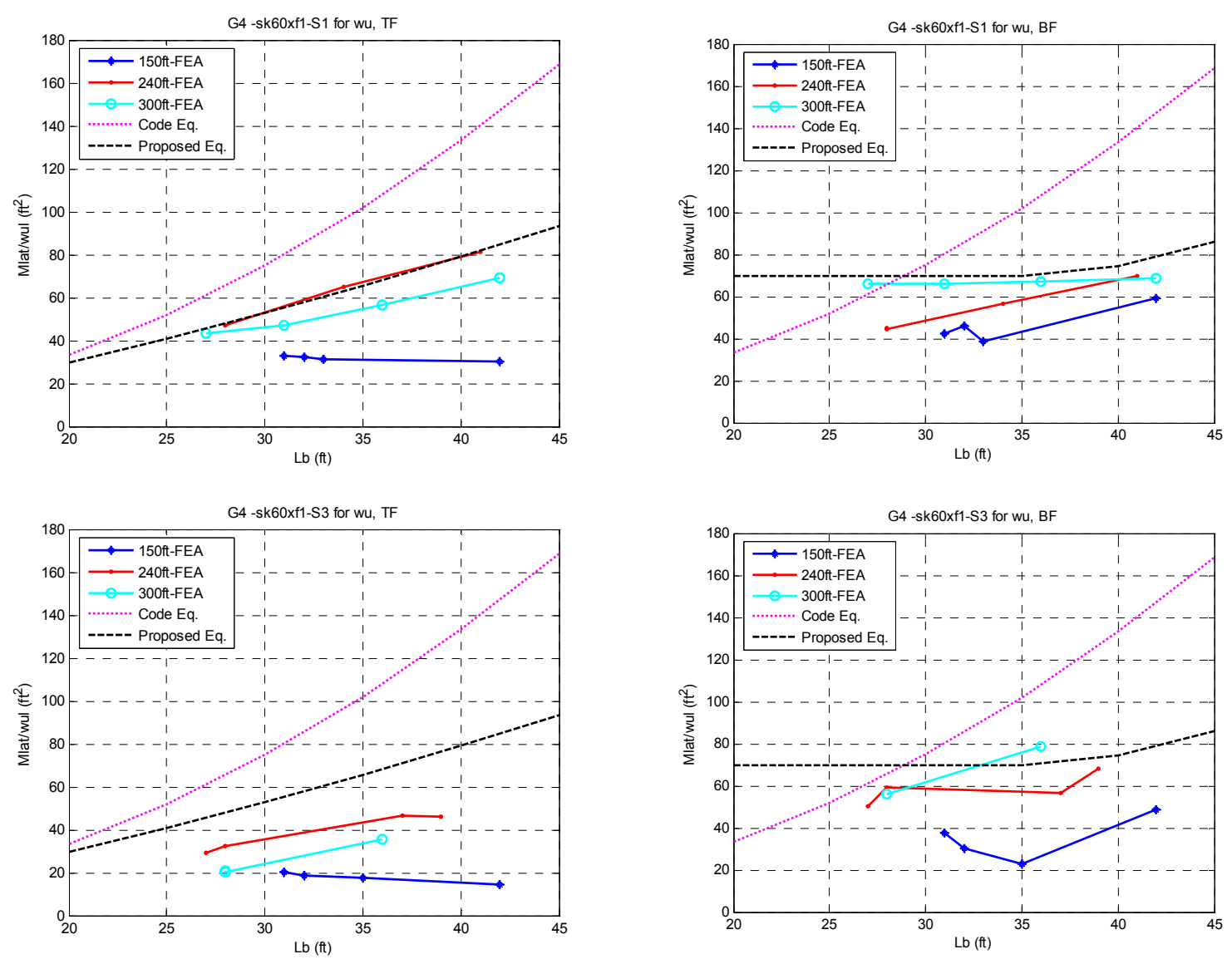
- Positive moment region under concentrated loads: $\theta=45^{\circ}$
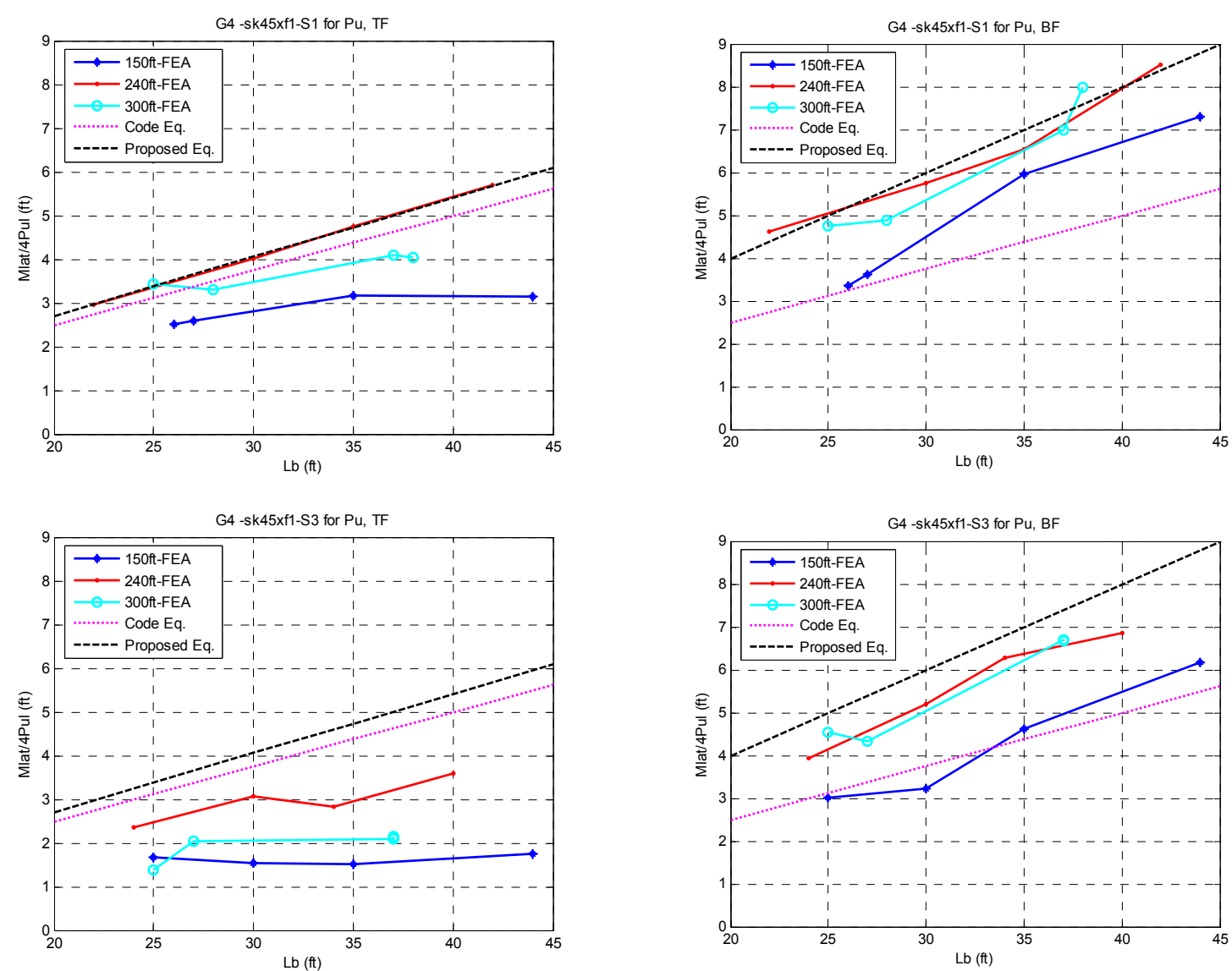
- Positive moment region under concentrated loads: $\theta=60^{\circ}$
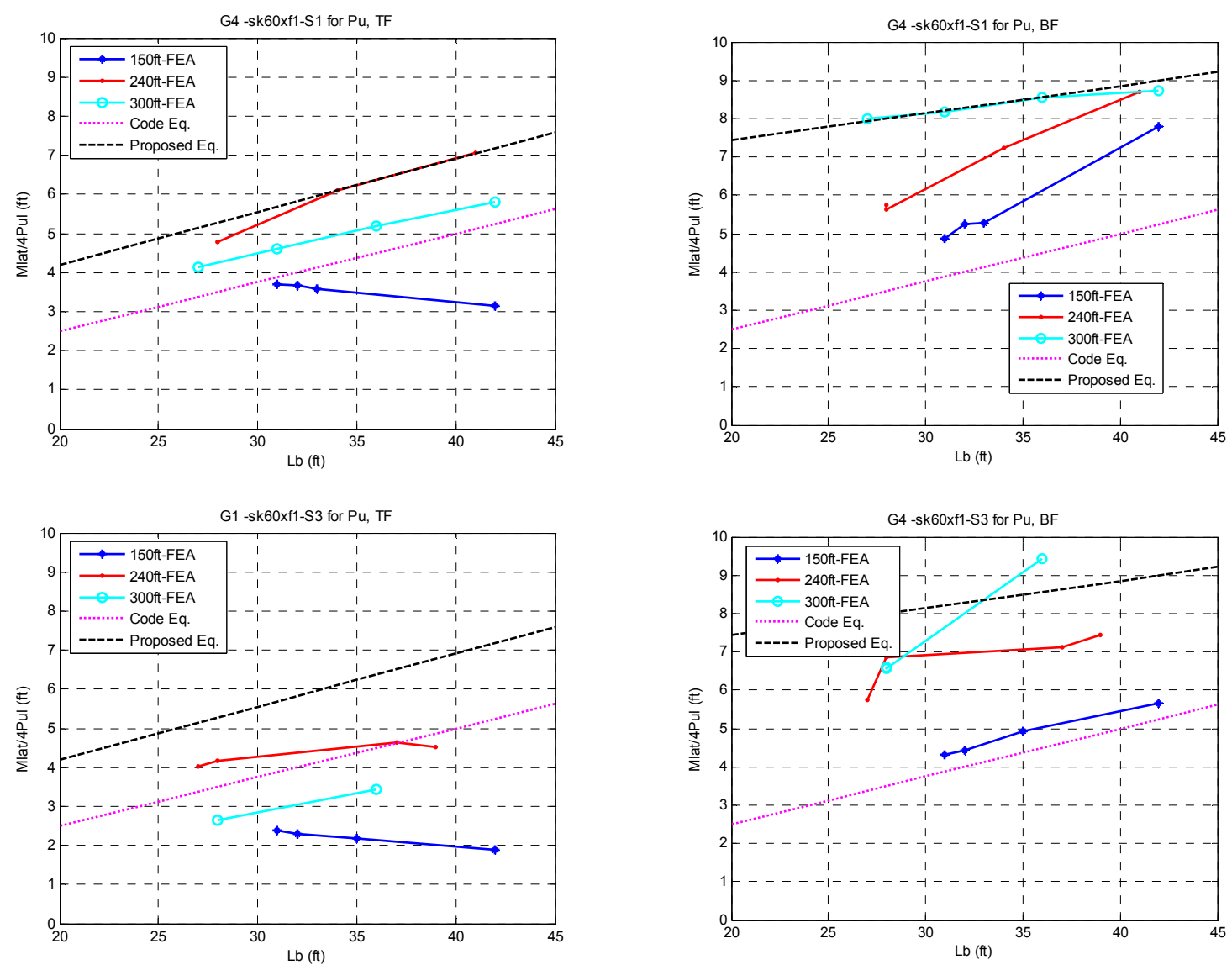
- Negative moment regions:

The figures corresponding to $\theta=45^{\circ}$ and $60^{\circ}$ show some returns on the curves due to the reduction of the cross-frames distances close to the pier zones.
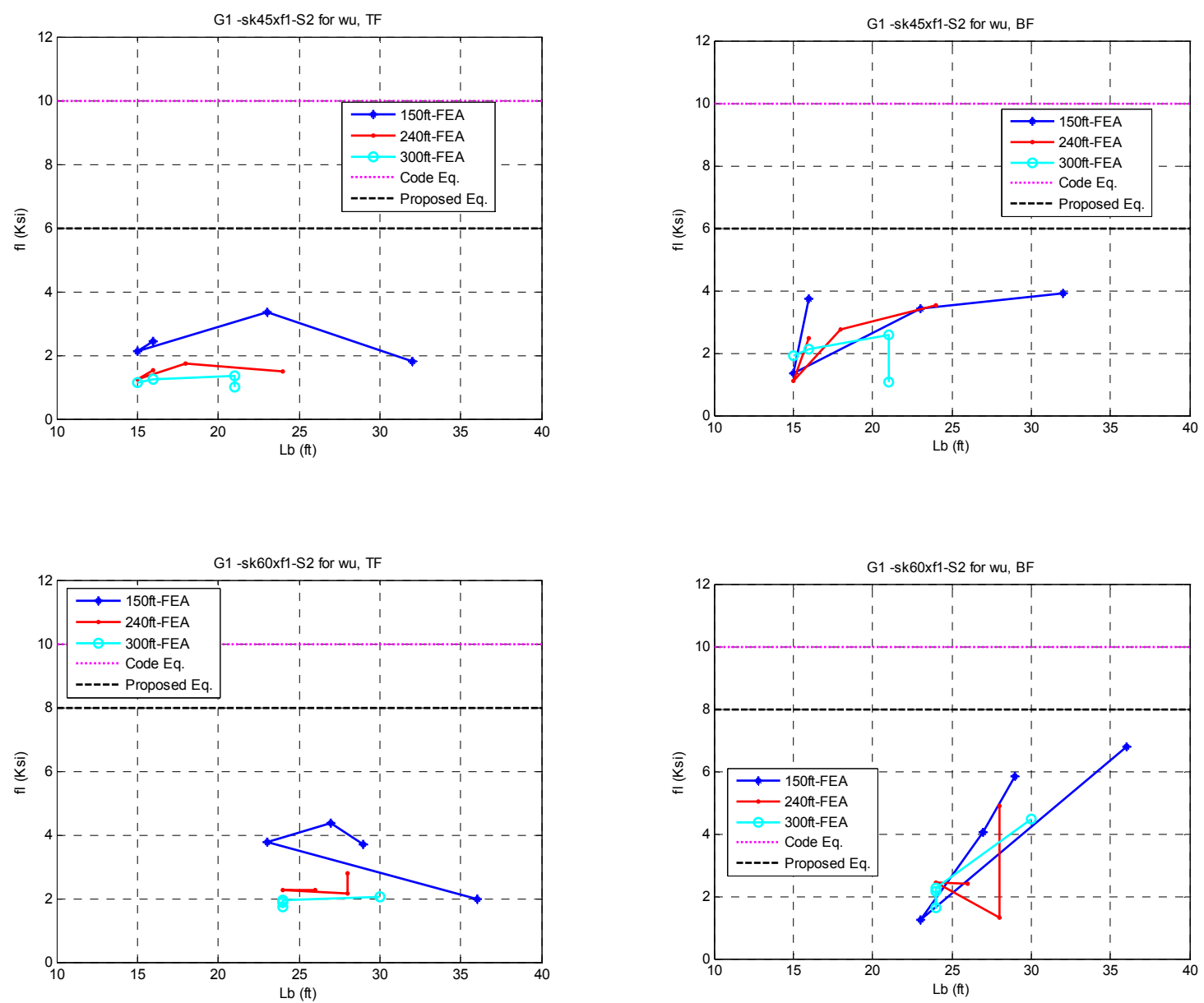


\section{Appendix B: LFB in Curved Bridges}

The figures corresponding to the middle spans $\left(L_{m}\right)$ of $240 \mathrm{ft}$ and $300 \mathrm{ft}$ show some missing points due to computational instabilities that arose during the analyses before the total load was applied.

- Positive moment regions under distributed loads: $L_{m}=240 \mathrm{ft}$ - Outer Girder
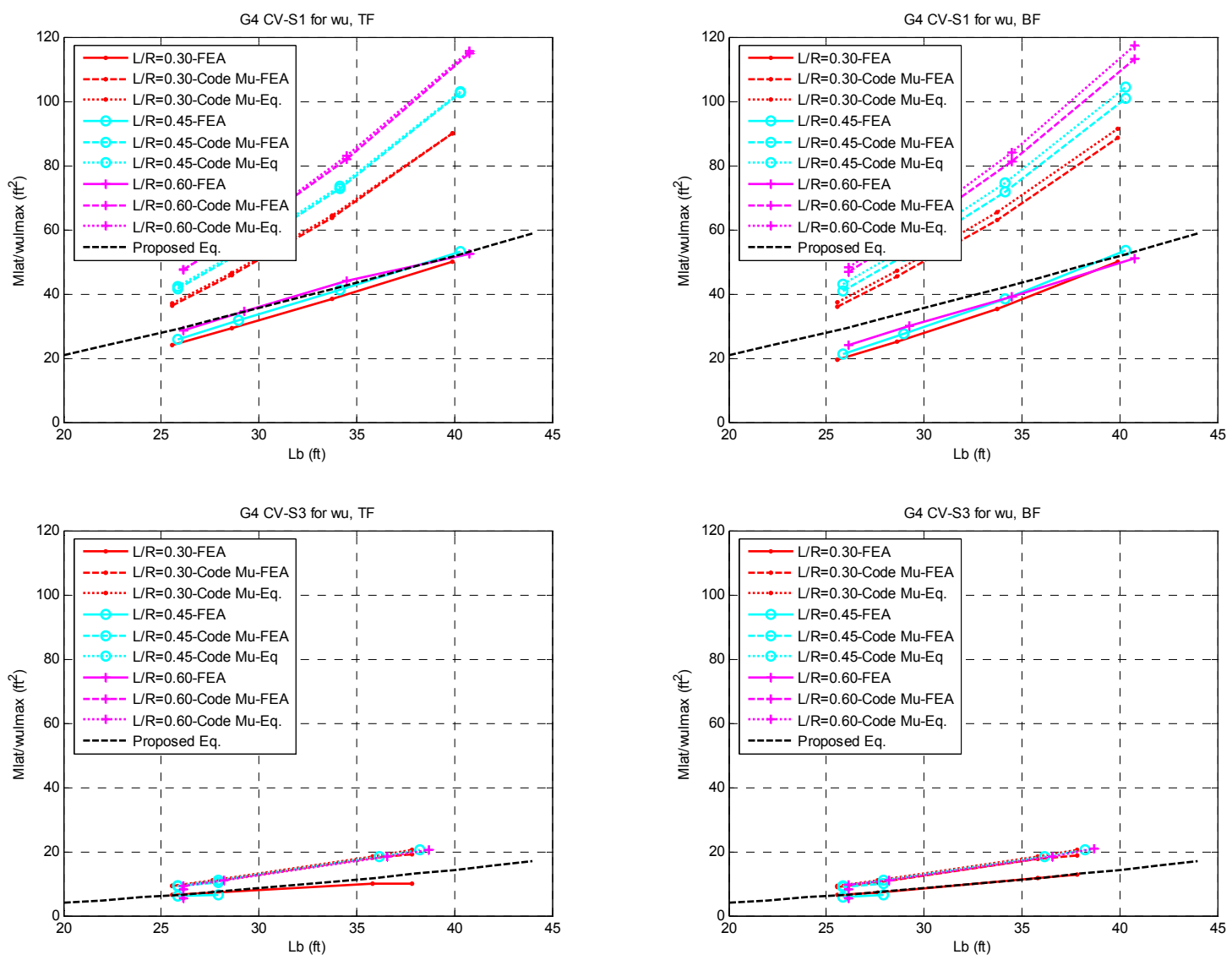
- Positive moment regions under distributed loads: $L_{m}=240 \mathrm{ft}-$ Inner Girder
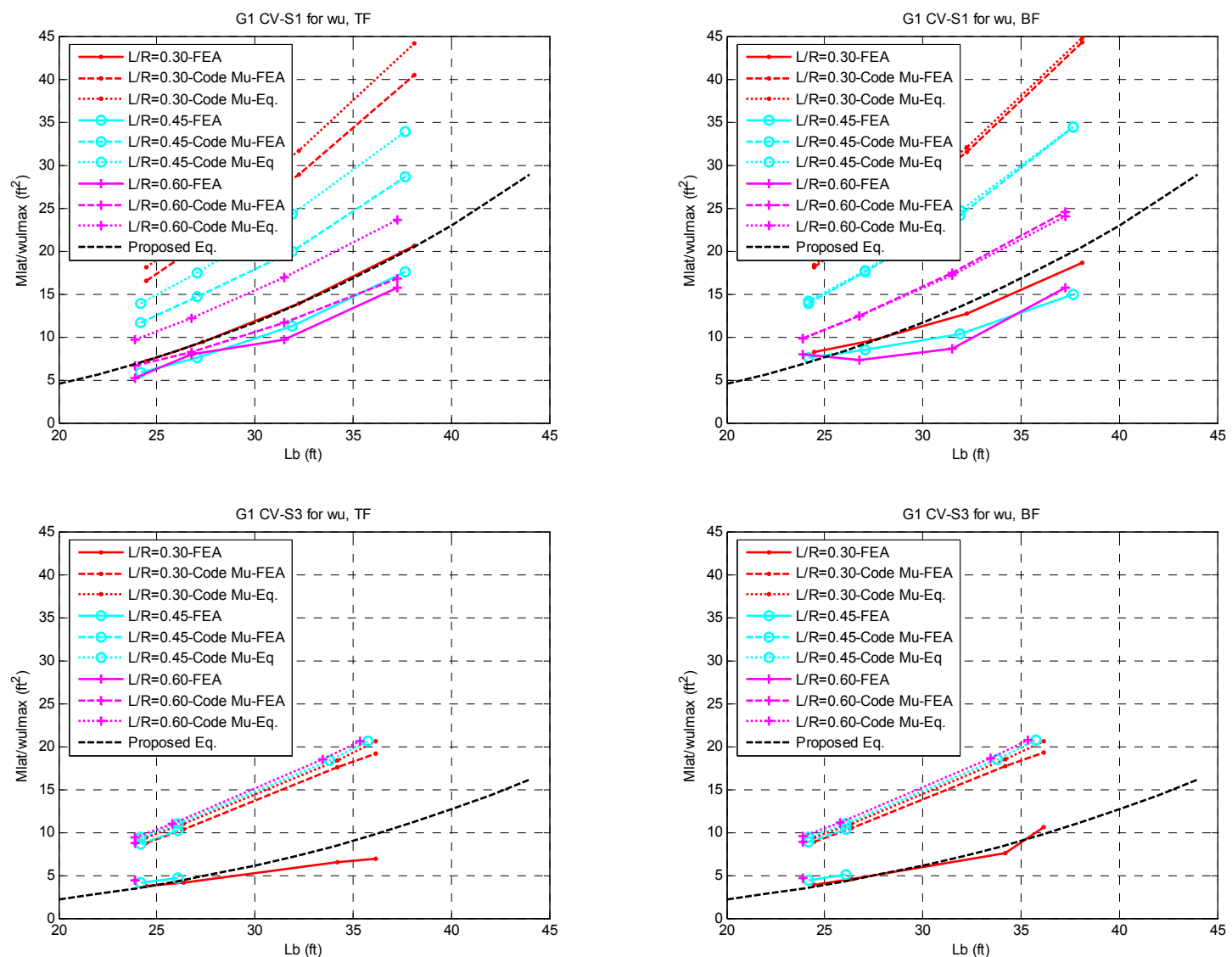
- Positive moment regions under distributed loads: $L_{m}=300 \mathrm{ft}-$ Outer Girder
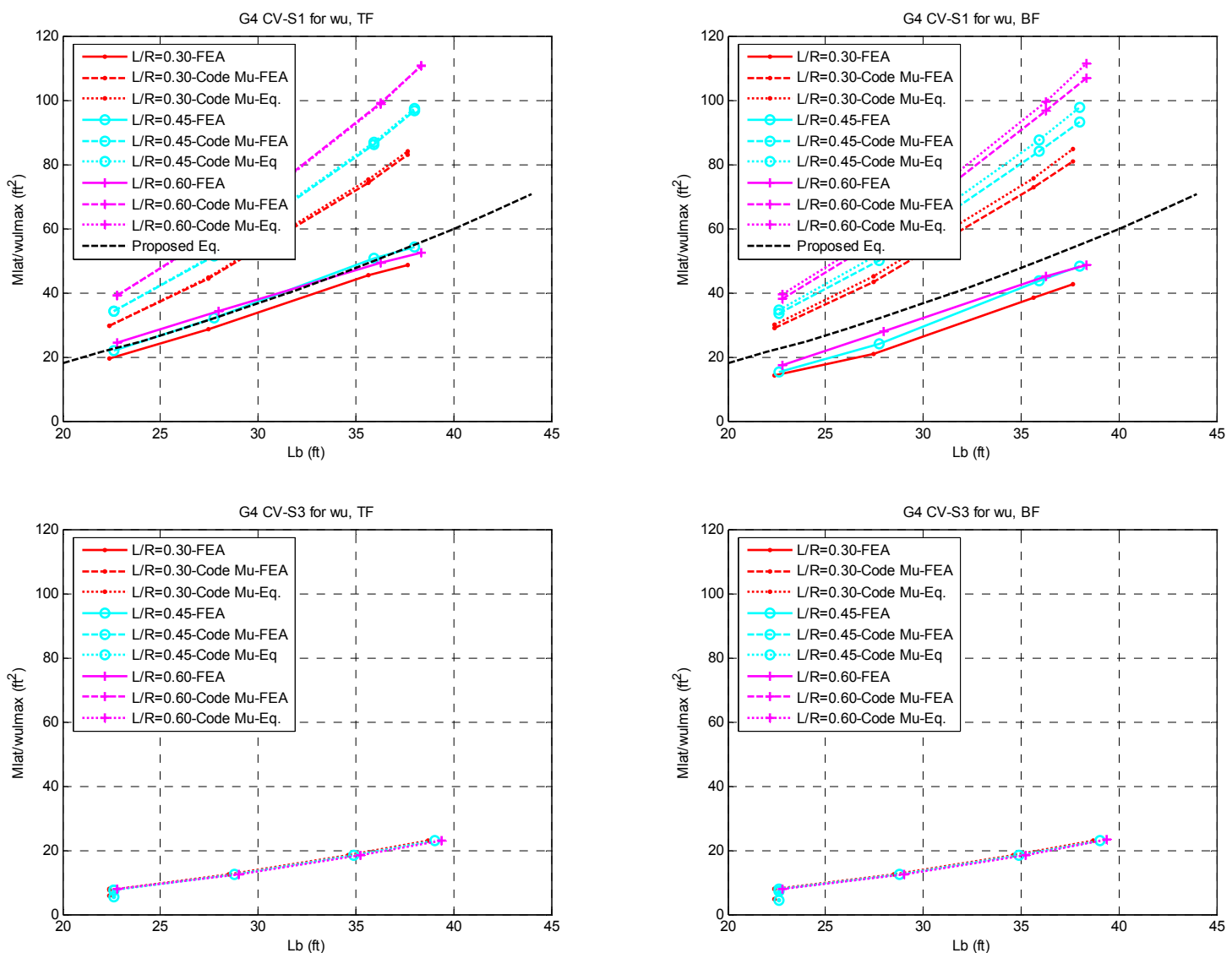
- Positive moment regions under distributed loads: $L_{m}=300 \mathrm{ft}-$ Inner Girder
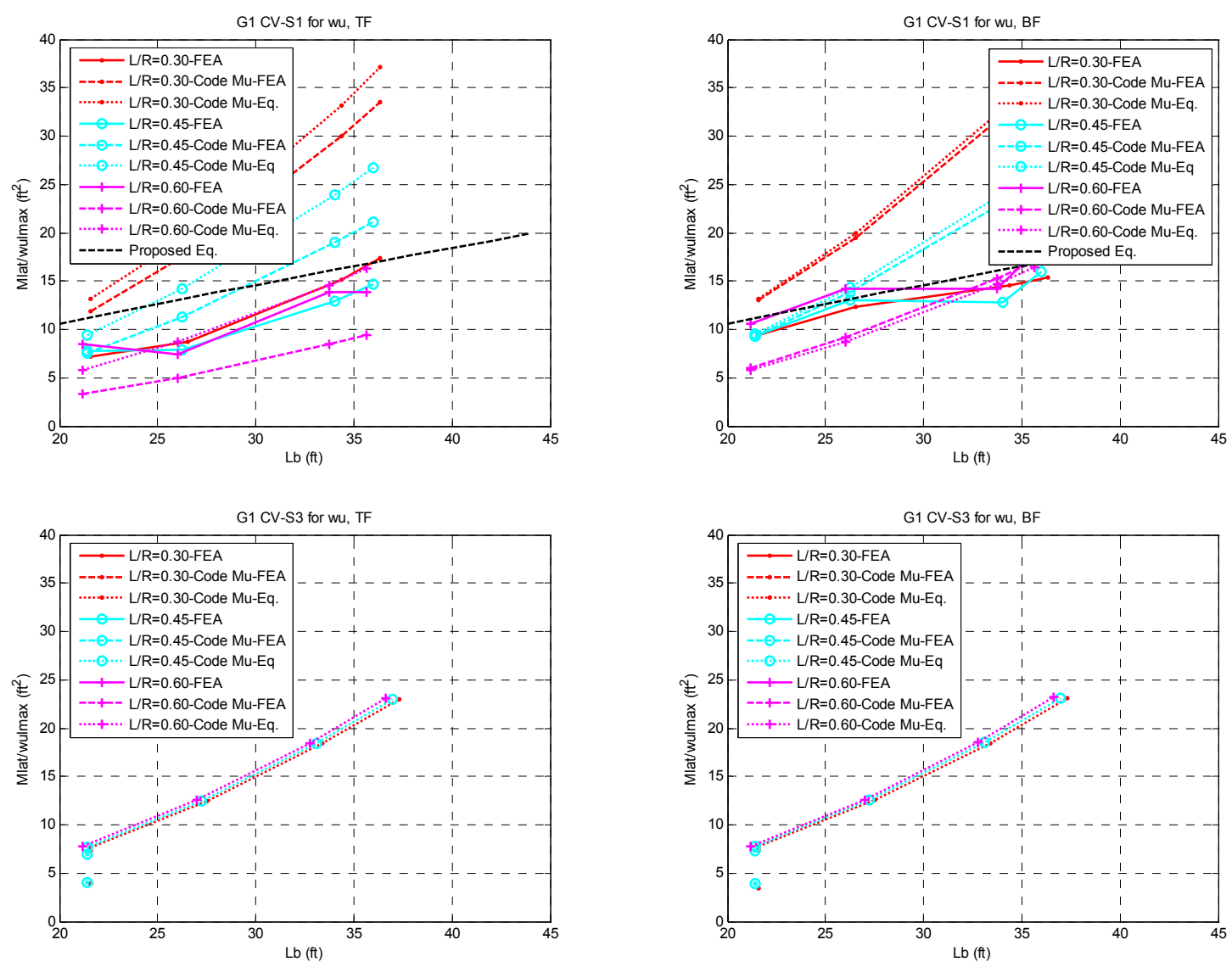
- Positive moment regions under concentrated loads: $L_{m}=240 \mathrm{ft}-$ Outer Girder
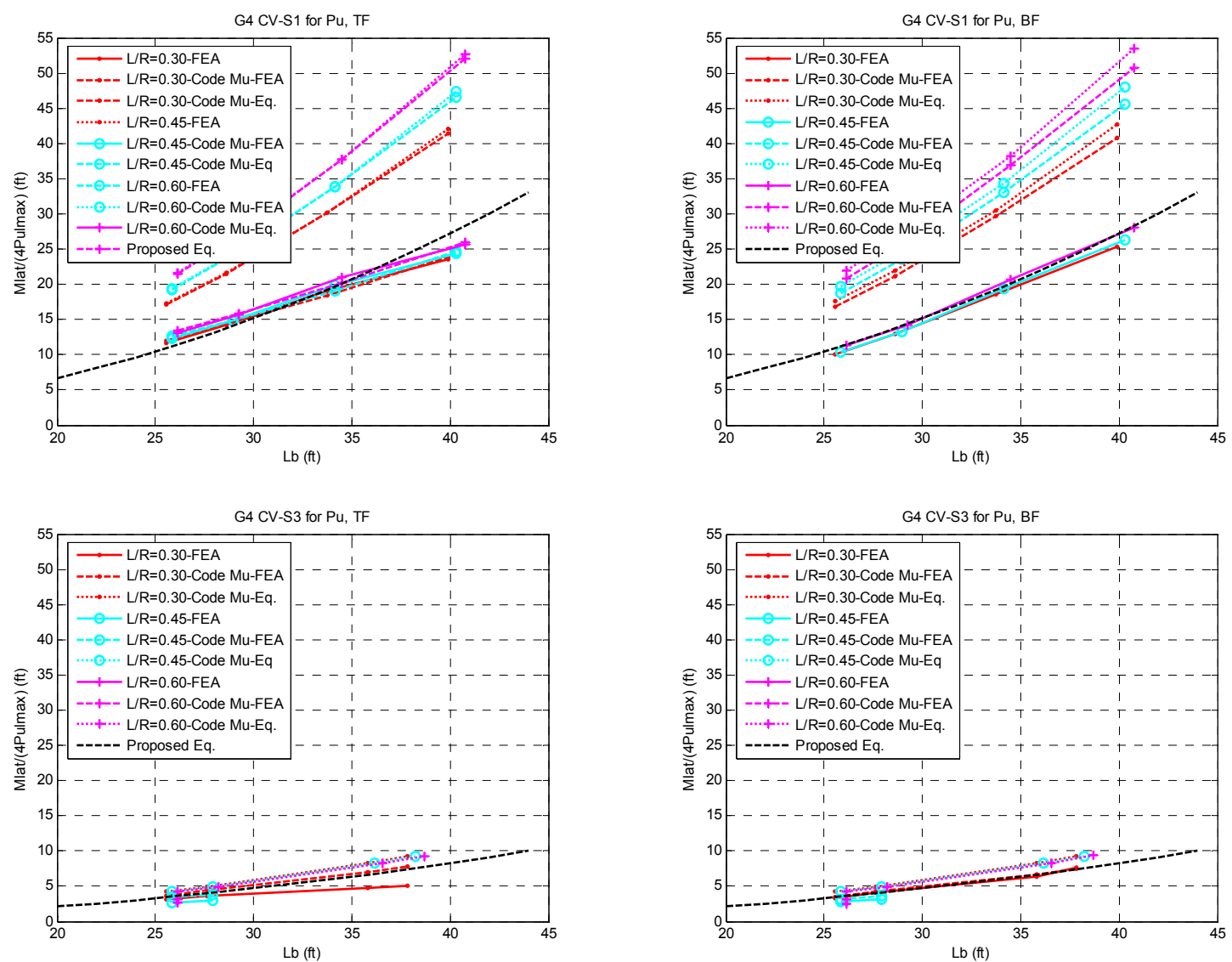
- Positive moment regions under concentrated loads: $L_{m}=240 \mathrm{ft}$ - Inner Girder
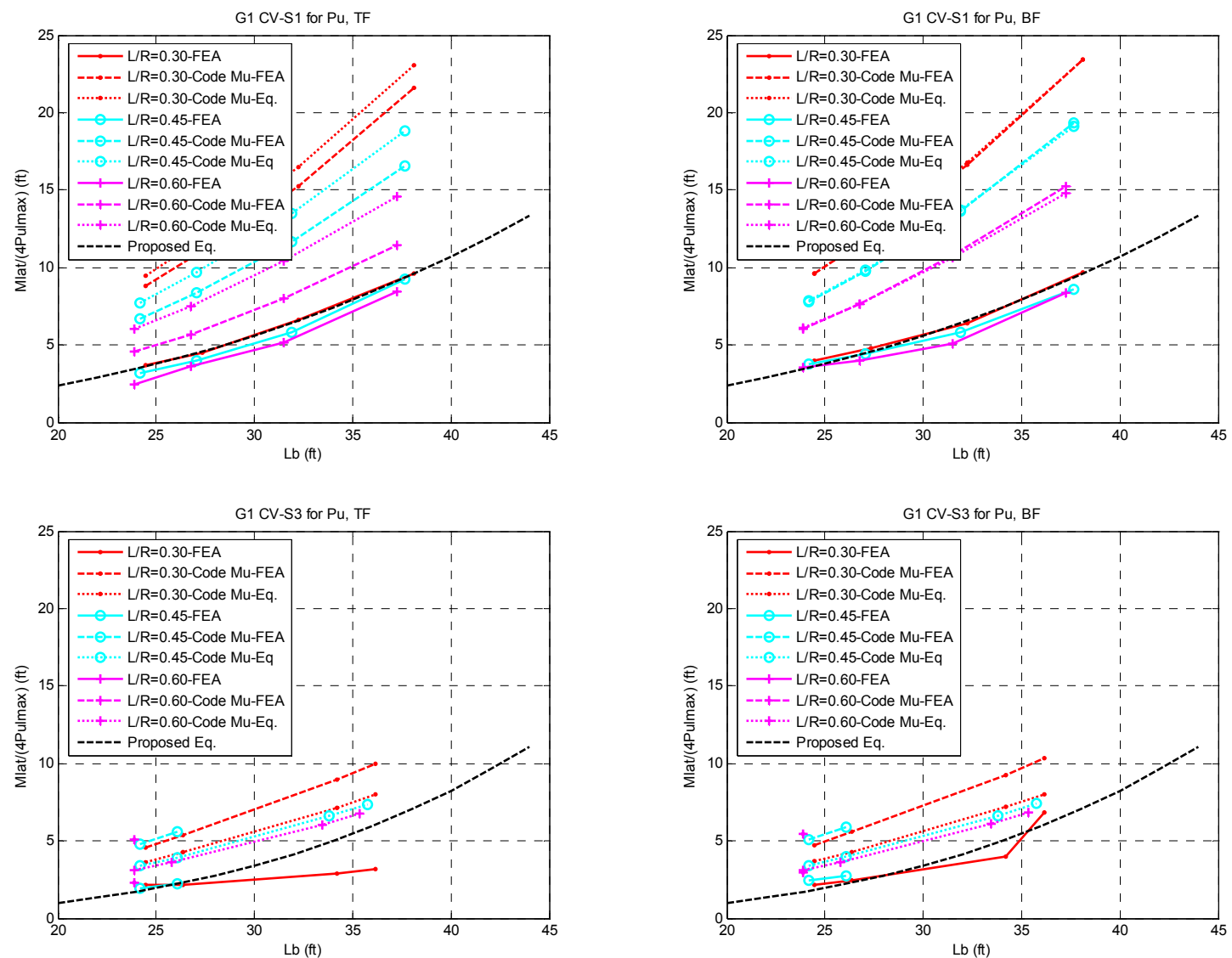
- Positive moment regions under concentrated loads: $L_{m}=300 \mathrm{ft}-$ Outer Girder
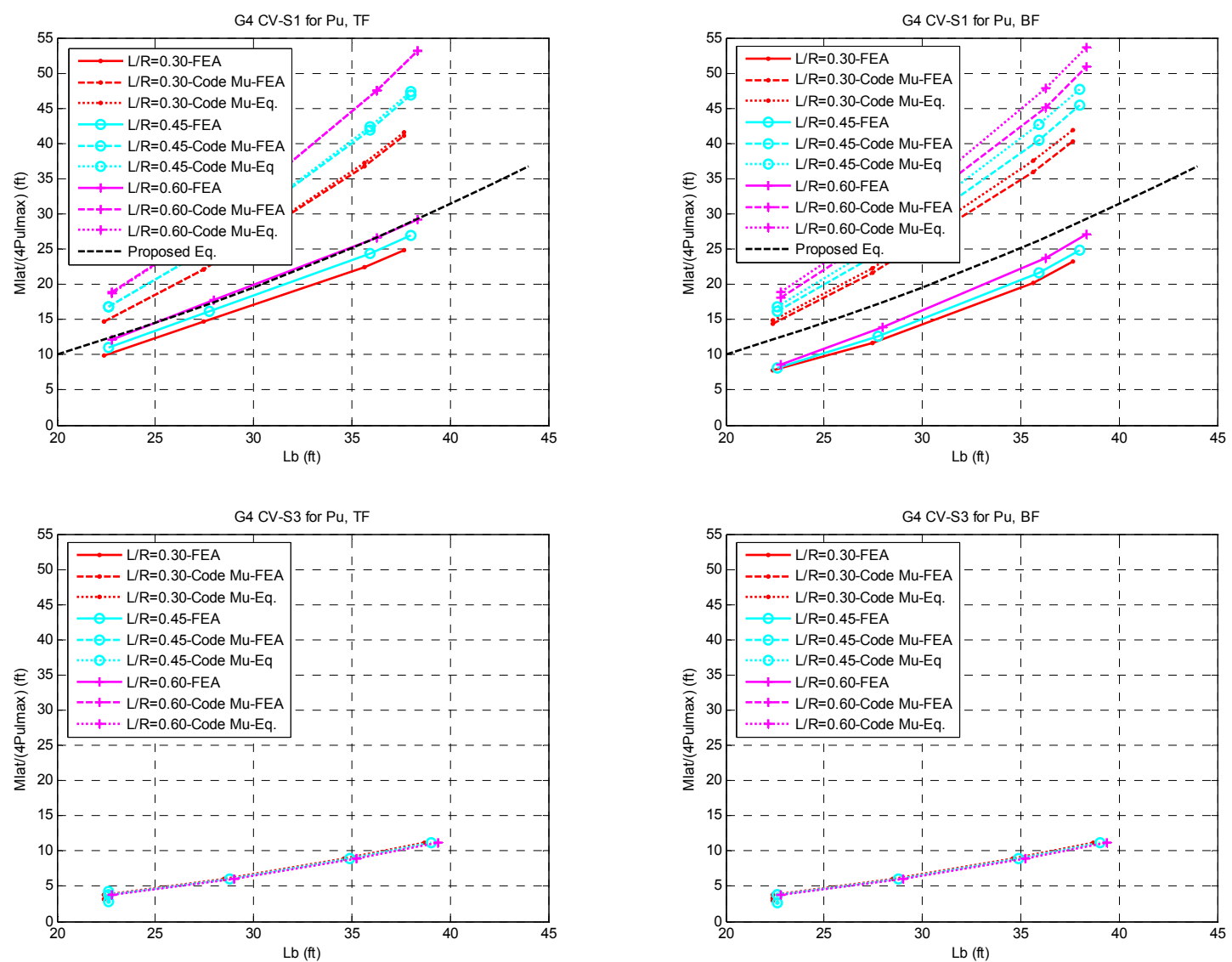
- Positive moment regions under concentrated loads: $L_{m}=300 \mathrm{ft}$ - Inner Girder
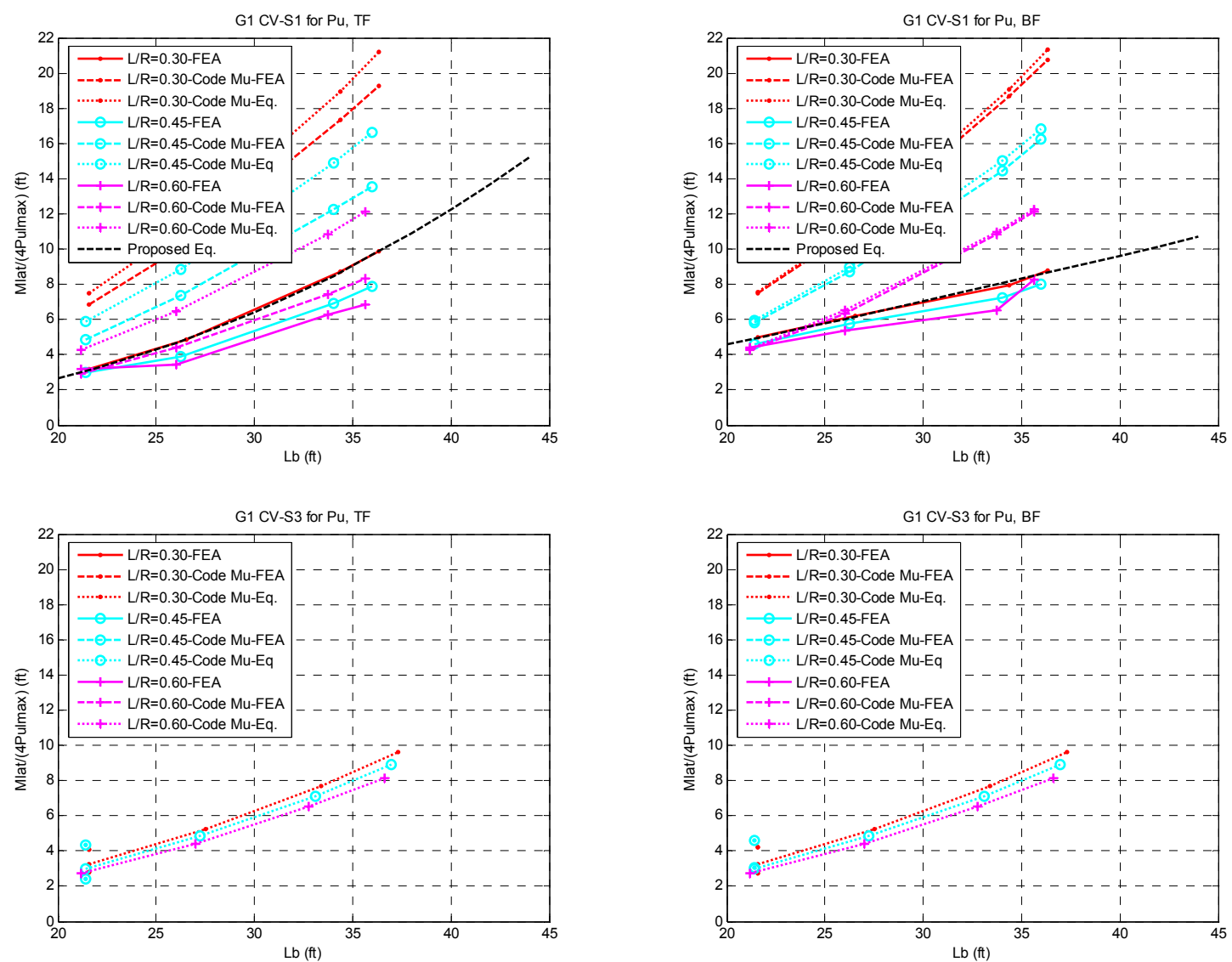
- Negative moment regions: $L_{m}=240 \mathrm{ft}$
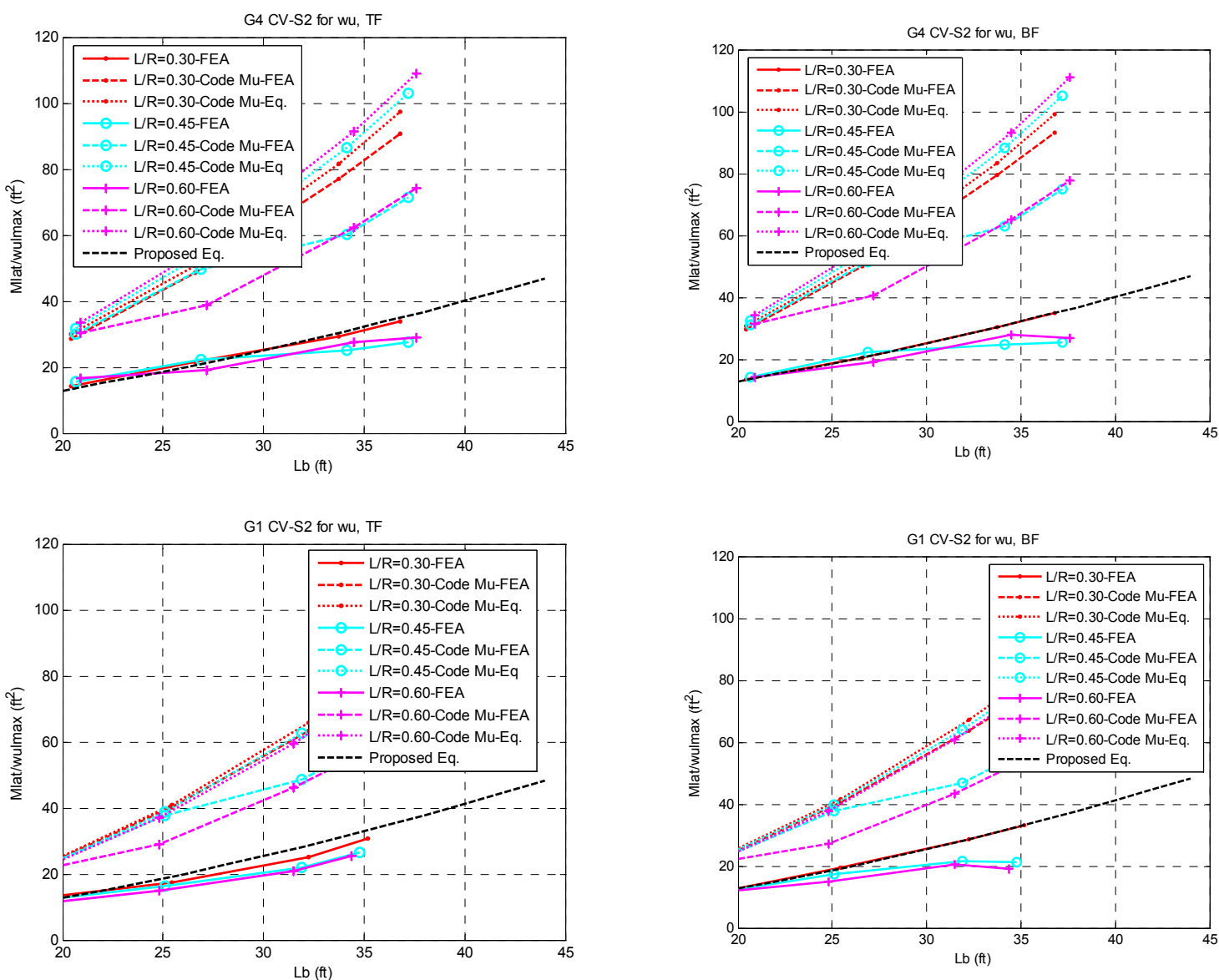
- Negative moment regions: $L_{m}=300 \mathrm{ft}$
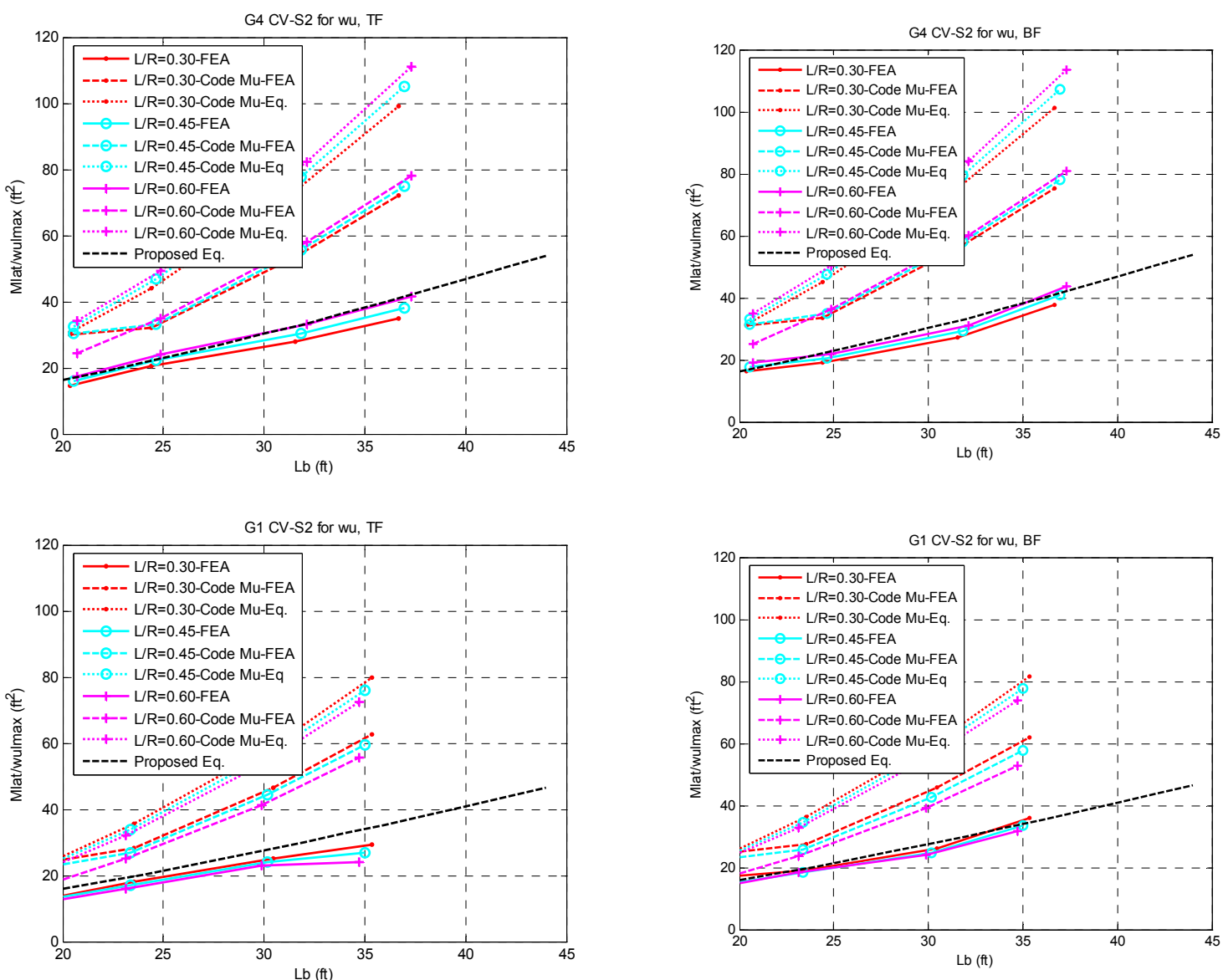


\section{Appendix C: Limit States for constructibility in Skewed}

\section{Bridges}

- Skewed bridges: $L_{b}=25 \mathrm{ft}-$ Cross frames oriented perpendicular to the girders

G4
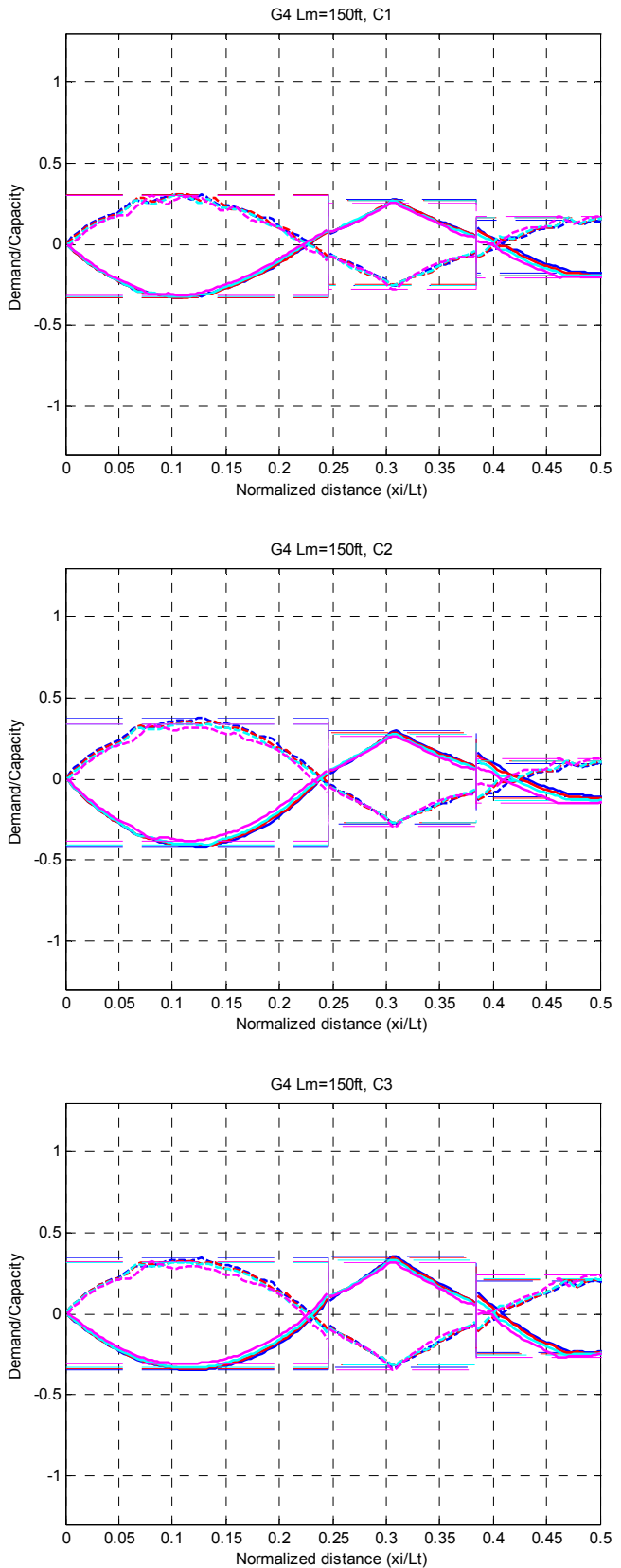

G1
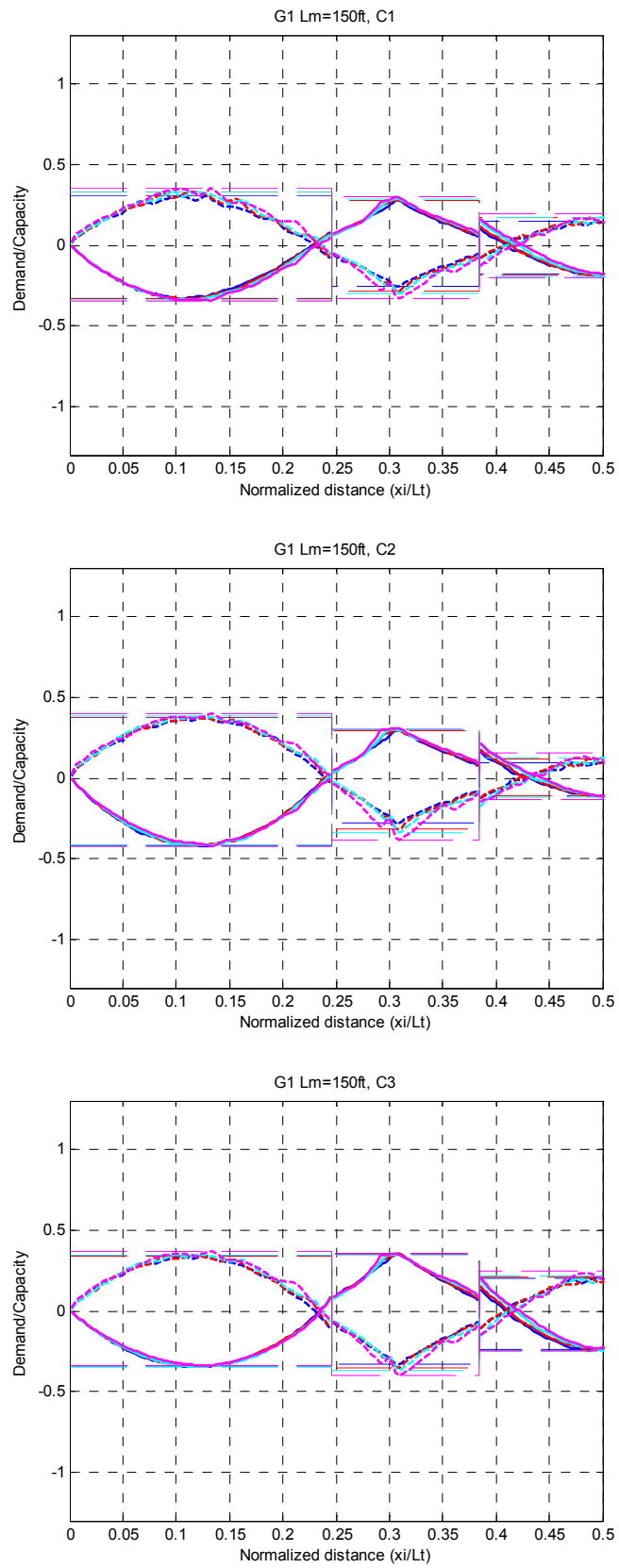

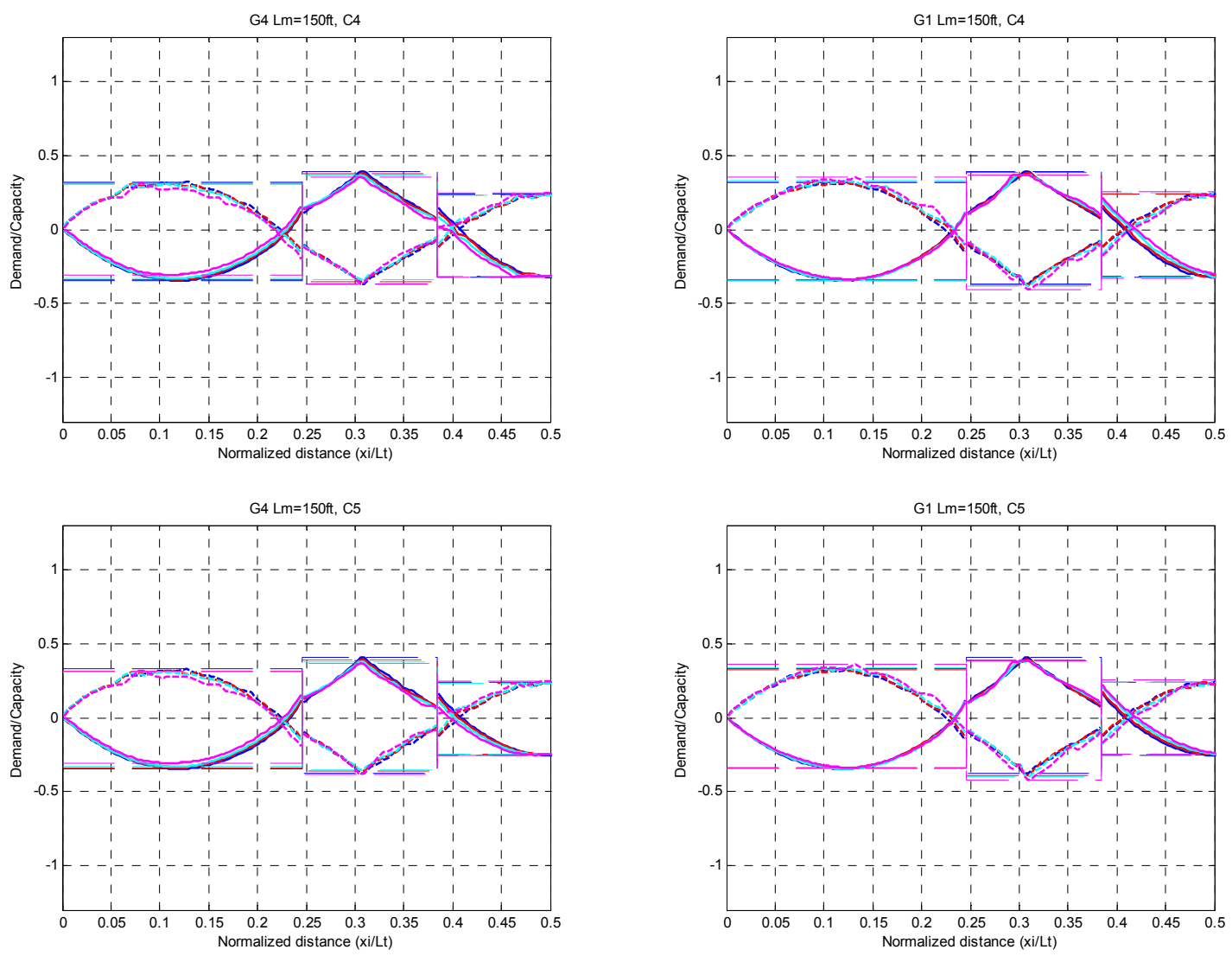

\begin{tabular}{|c|c|c|}
\hline$T F: \longrightarrow \mathrm{ST} \longrightarrow \mathrm{L} / \mathrm{R}=0.30$ & $\mathrm{~L} / \mathrm{R}=0.45$ & $\mathrm{~L} / \mathrm{R}=0.60$ \\
\hline$B F:----\mathrm{ST}----\mathrm{L} / \mathrm{R}=0.30$ & $-----\mathrm{L} / \mathrm{R}=0.45$ & ---- - L/R $=0.60$ \\
\hline
\end{tabular}


- Skewed bridges: $L_{b}=40 \mathrm{ft}-$ Cross frames oriented perpendicular to the girders

G4
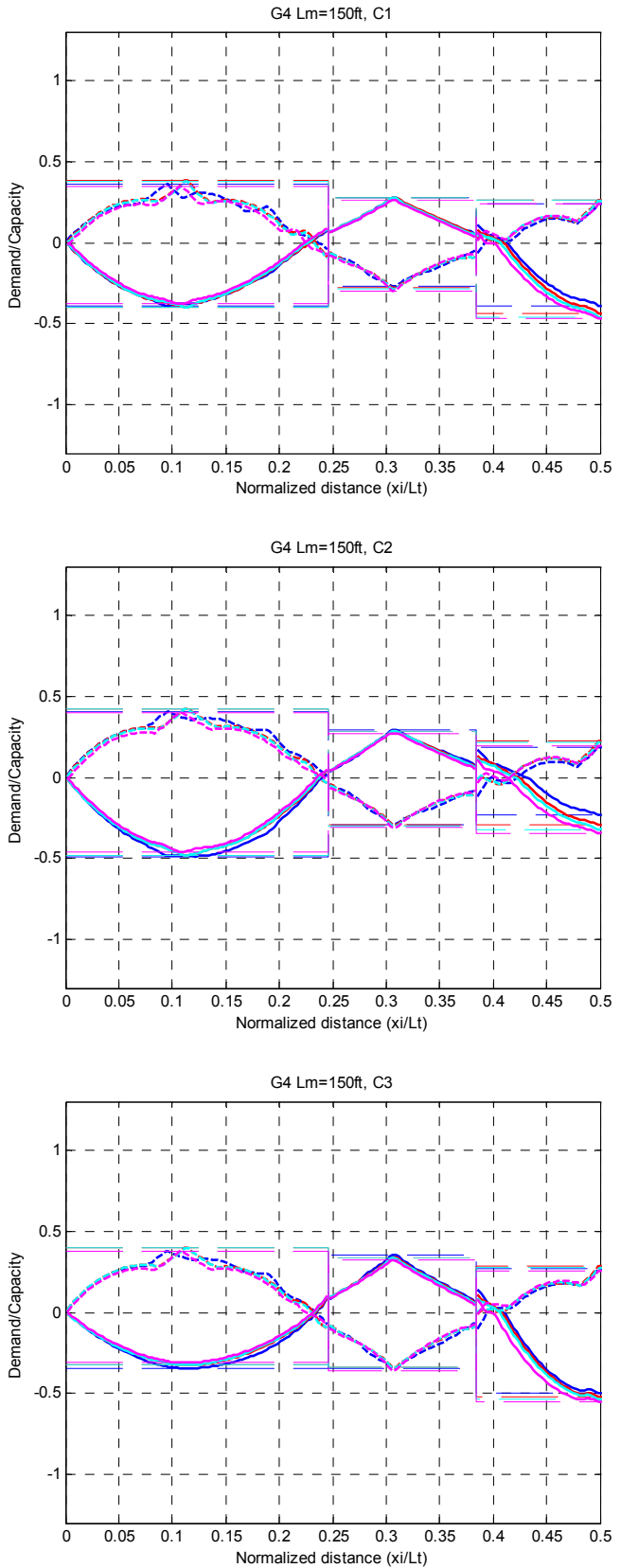

G2
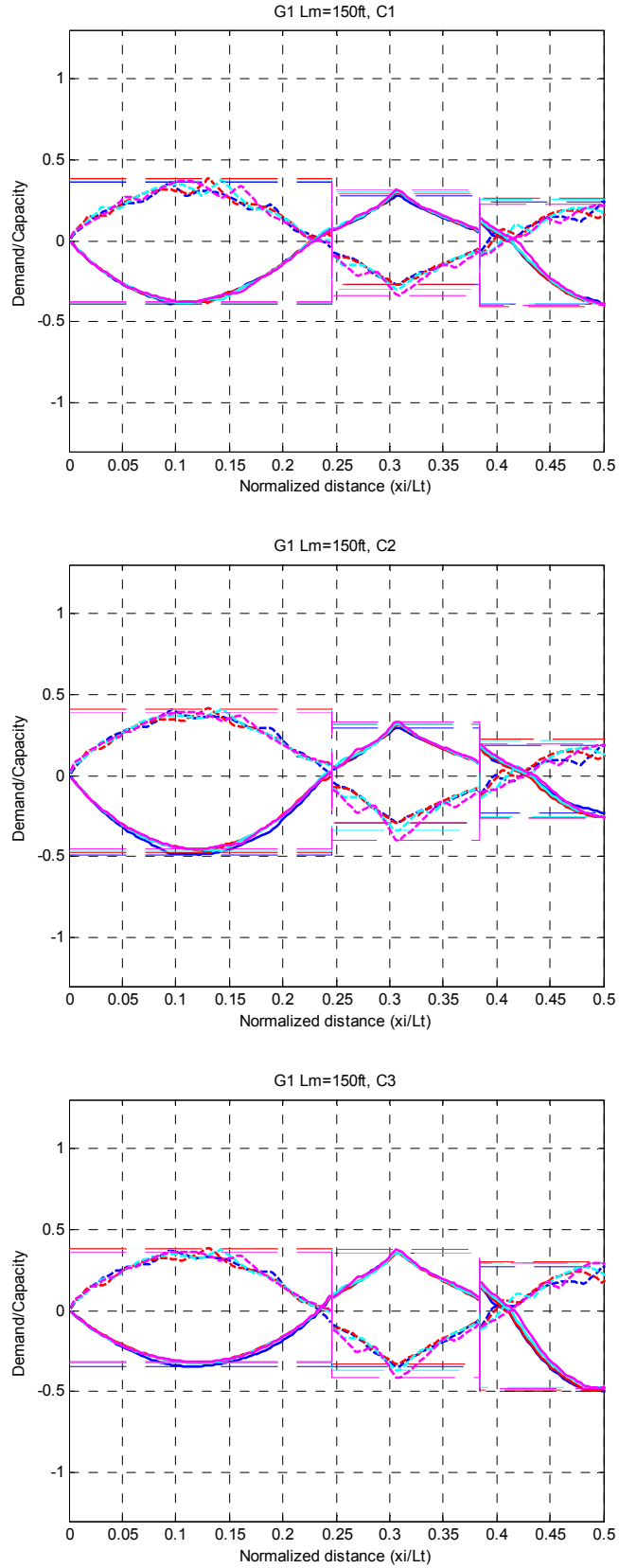

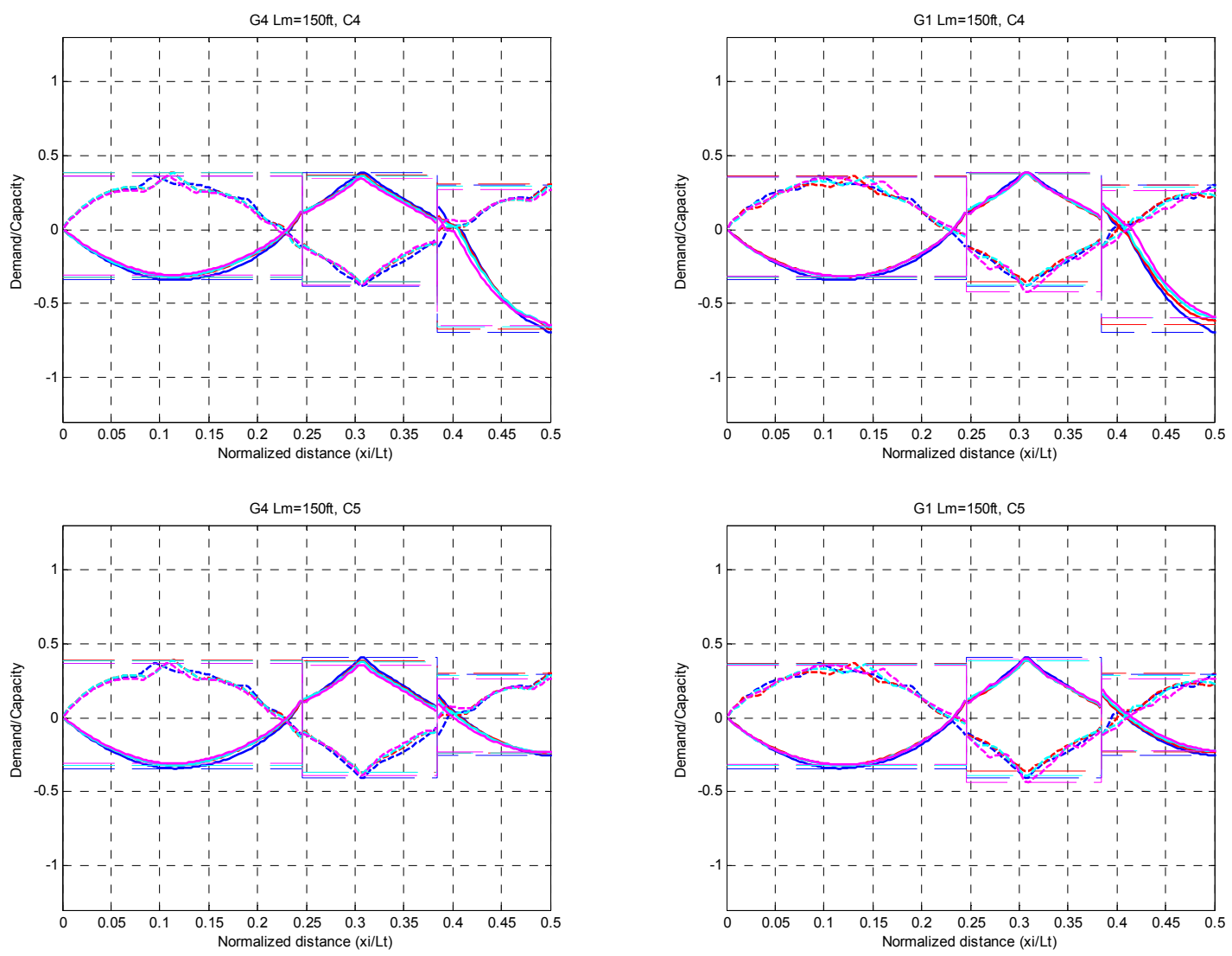

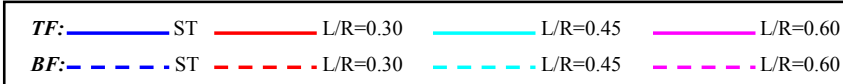


- Skewed bridges: $L_{b}=40 \mathrm{ft}-$ Cross frames oriented parallel to the supports

G4
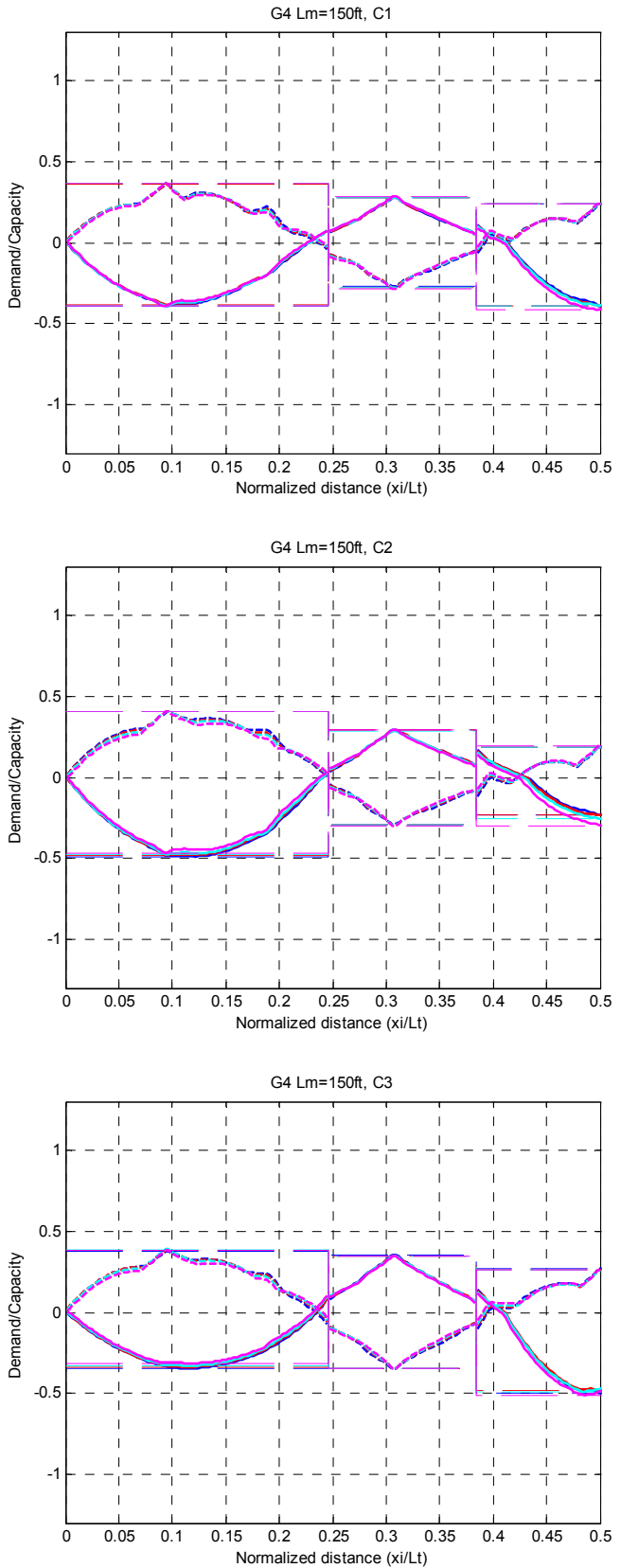

G1
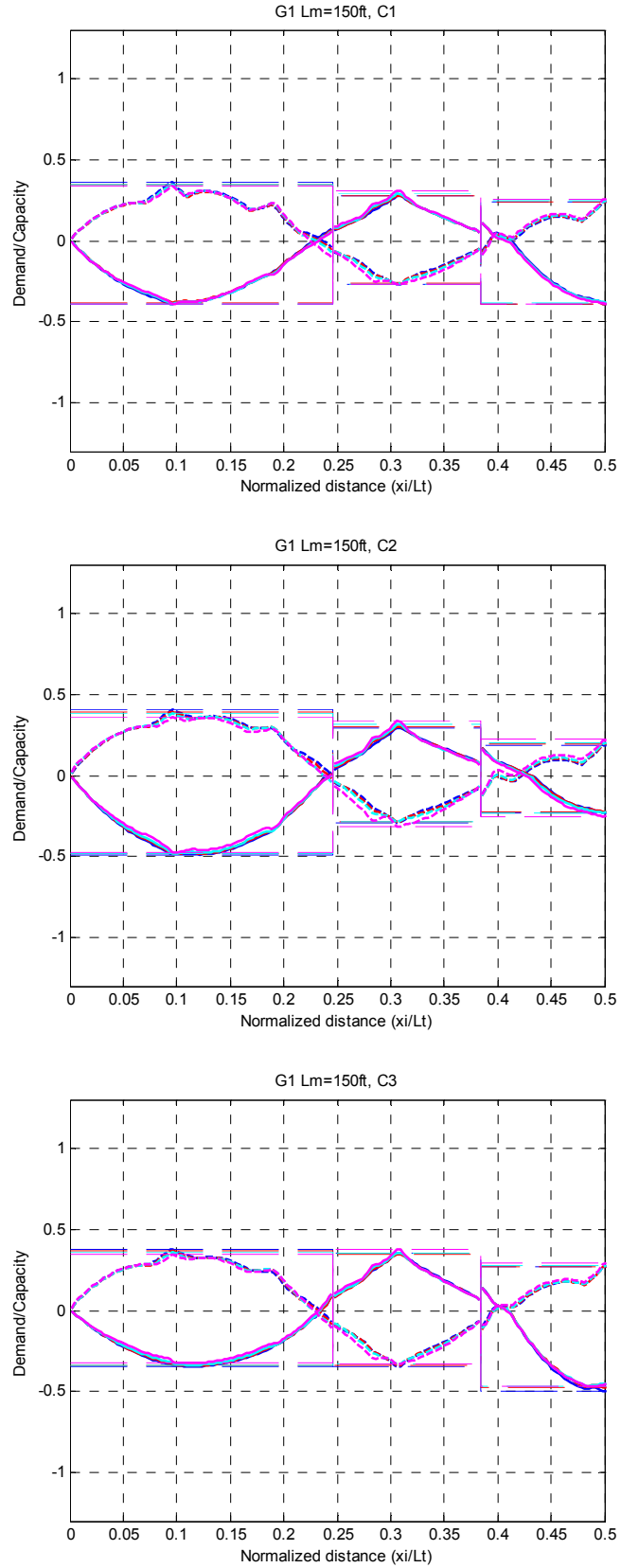

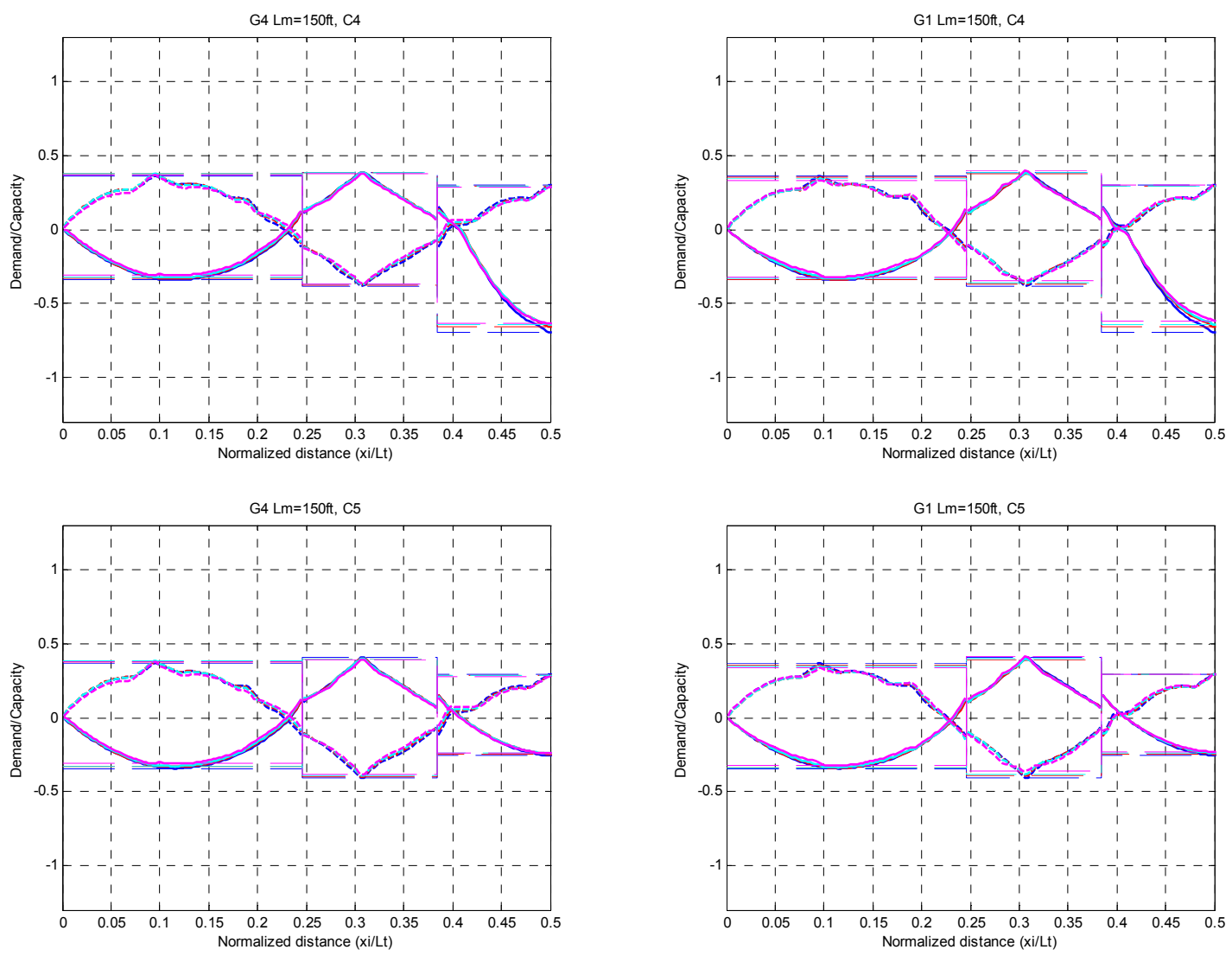

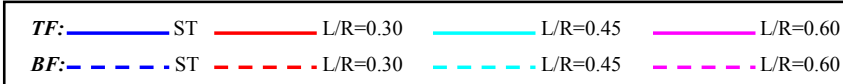


- Skewed bridges: $L_{b}=25 \mathrm{ft}-$ Cross frames oriented perpendicular to the girders
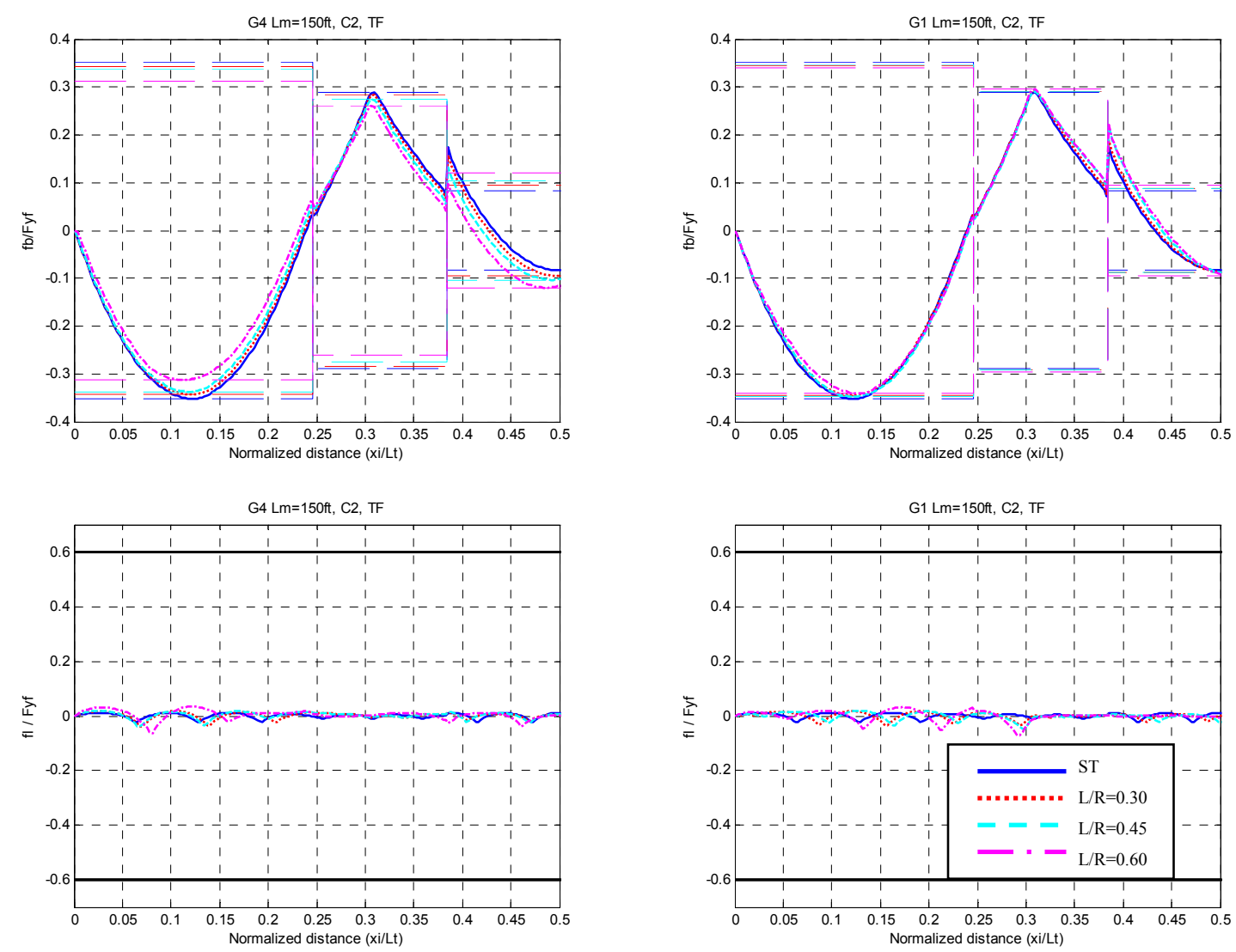
- Skewed bridges: $L_{b}=40 \mathrm{ft}-$ Cross frames oriented perpendicular to the girders
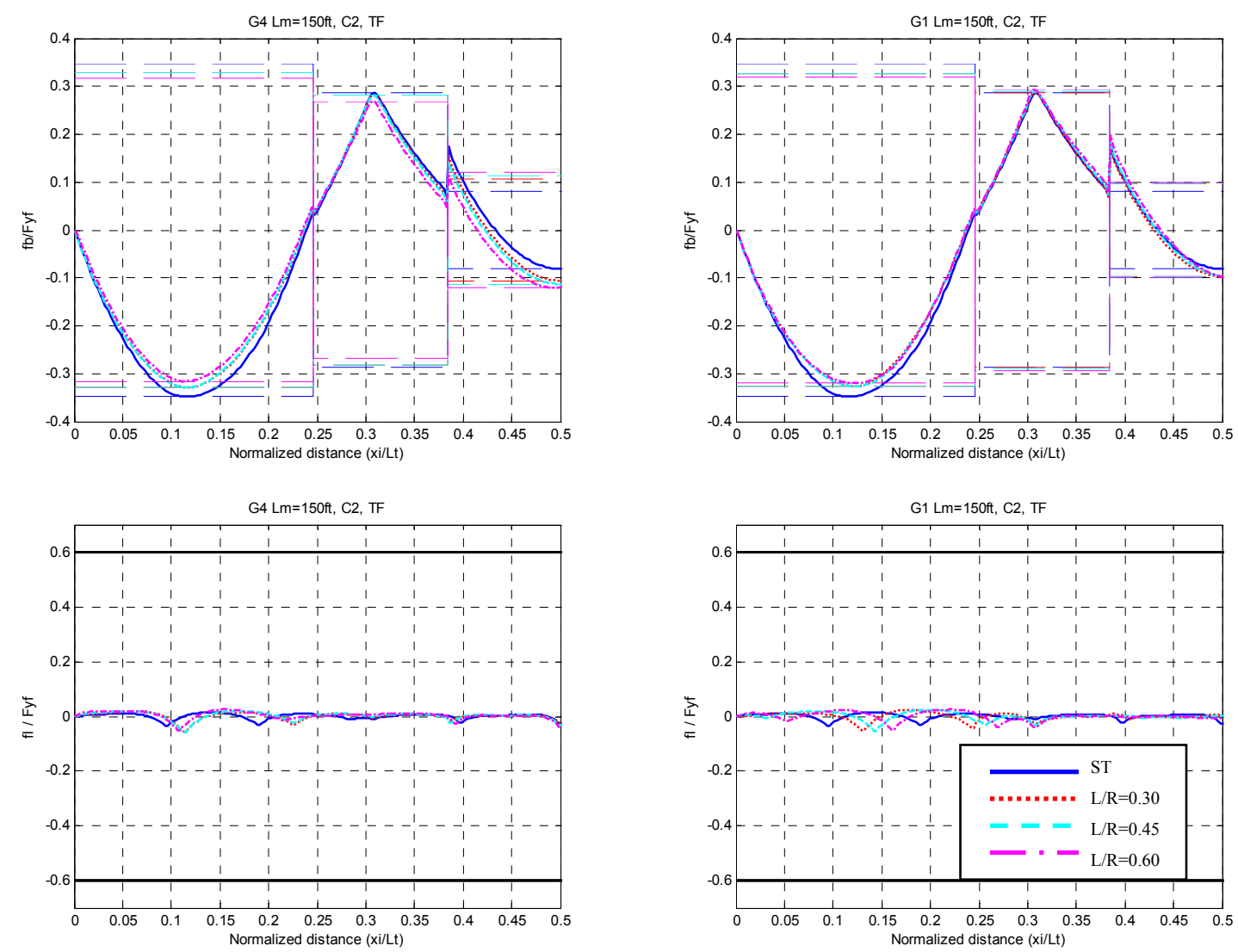
- Skewed bridges: $L_{b}=40 \mathrm{ft}-$ Cross frames oriented parallel to the supports
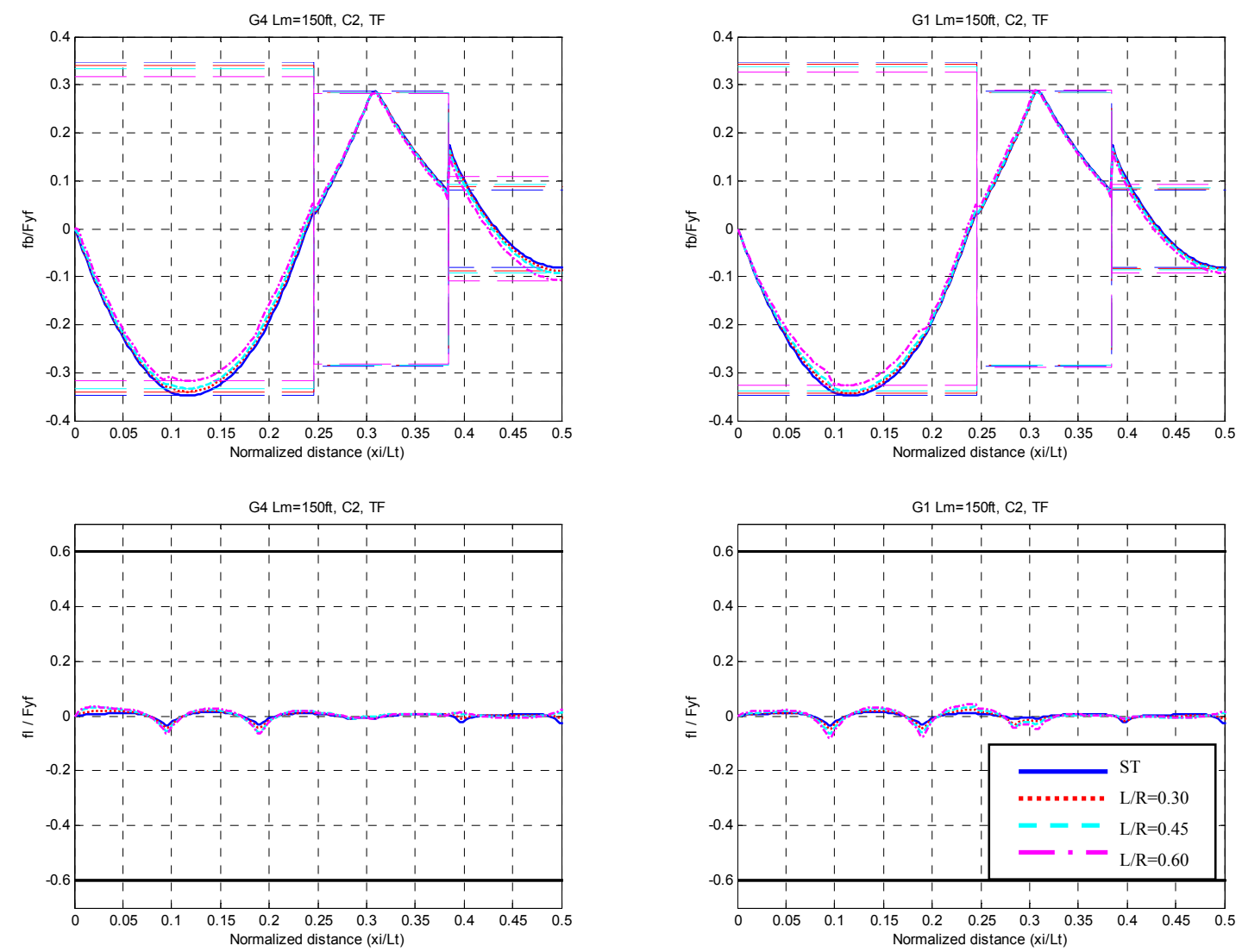
- Skewed bridges: $L_{m}=150 \mathrm{ft}-$ Section 1
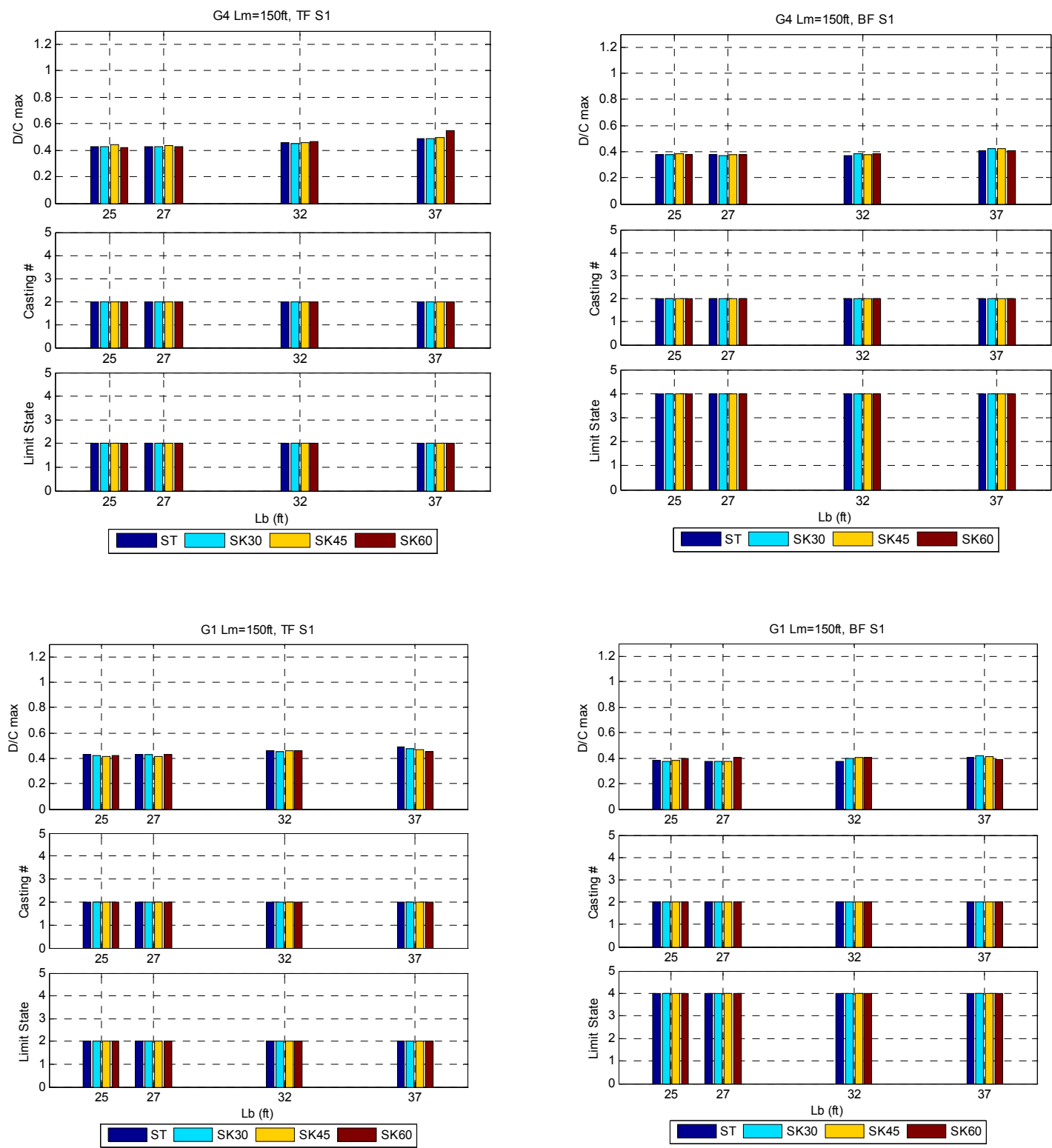
- Skewed bridges: $L_{m}=150 \mathrm{ft}-$ Section 2
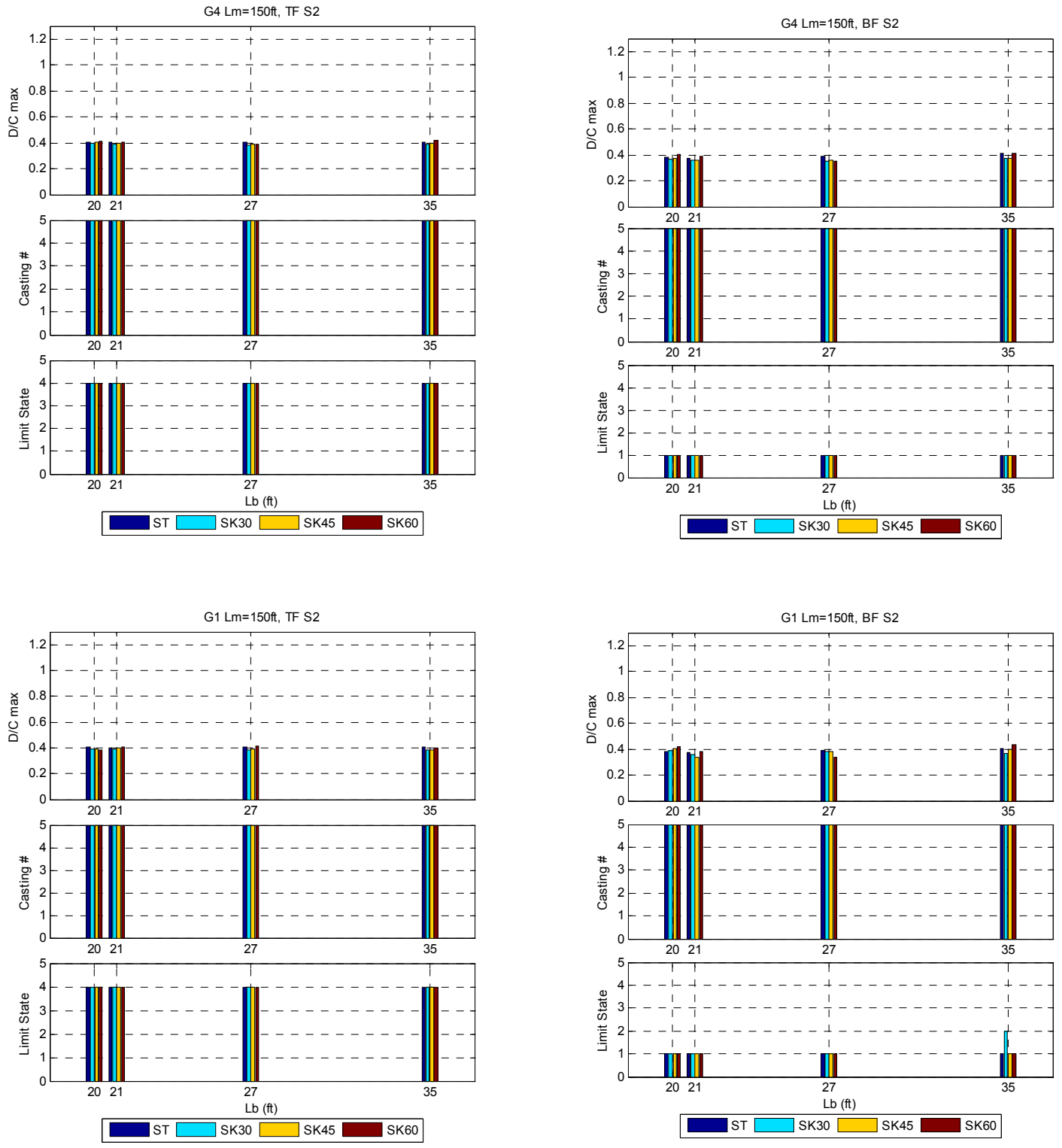
- Skewed bridges: $L_{m}=150 \mathrm{ft}-$ Section 3
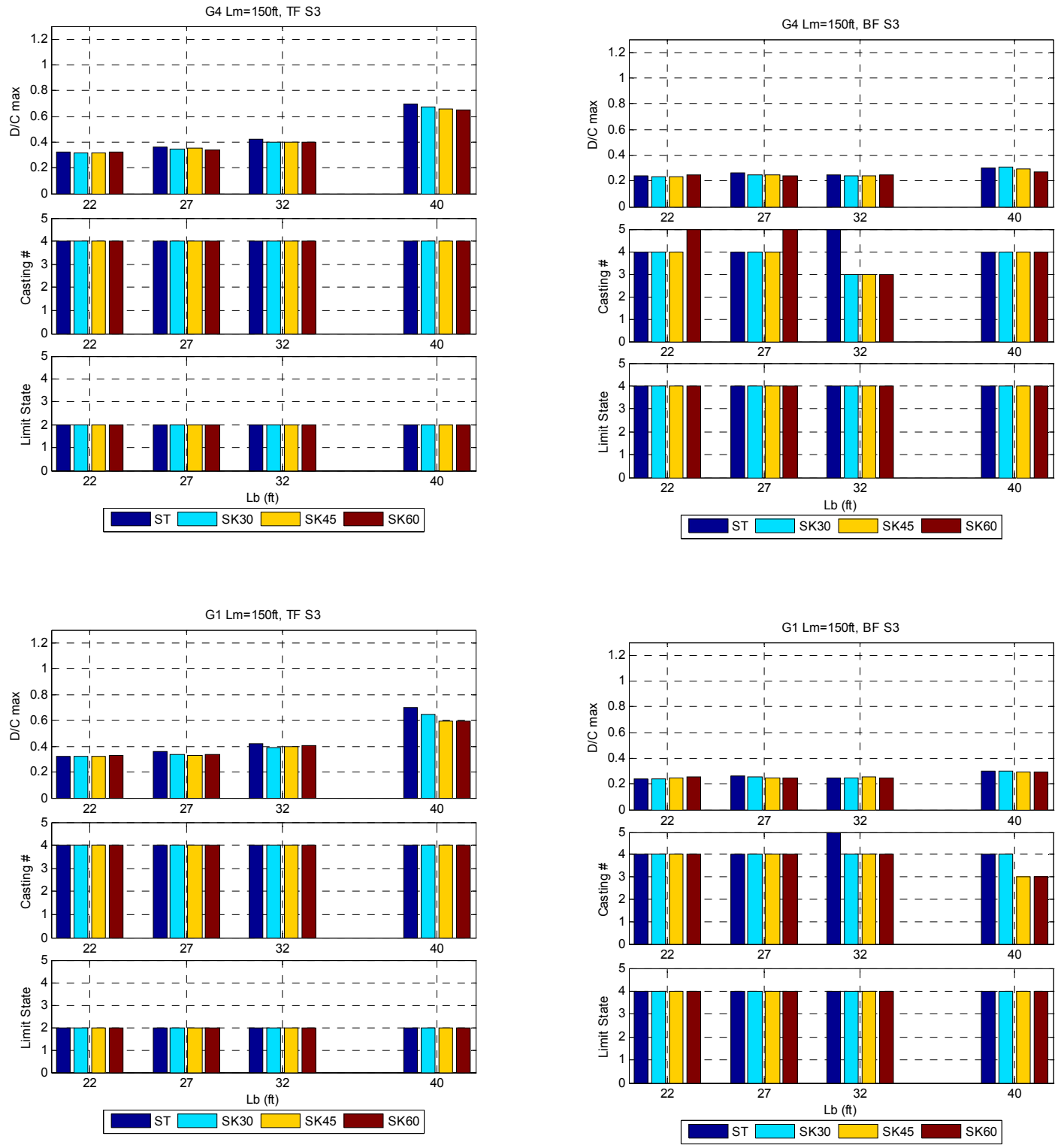


\section{Appendix D: Limit States for constructibility in Curved}

\section{Bridges}

- Curved bridges: $L_{b}=25 \mathrm{ft}$

G4
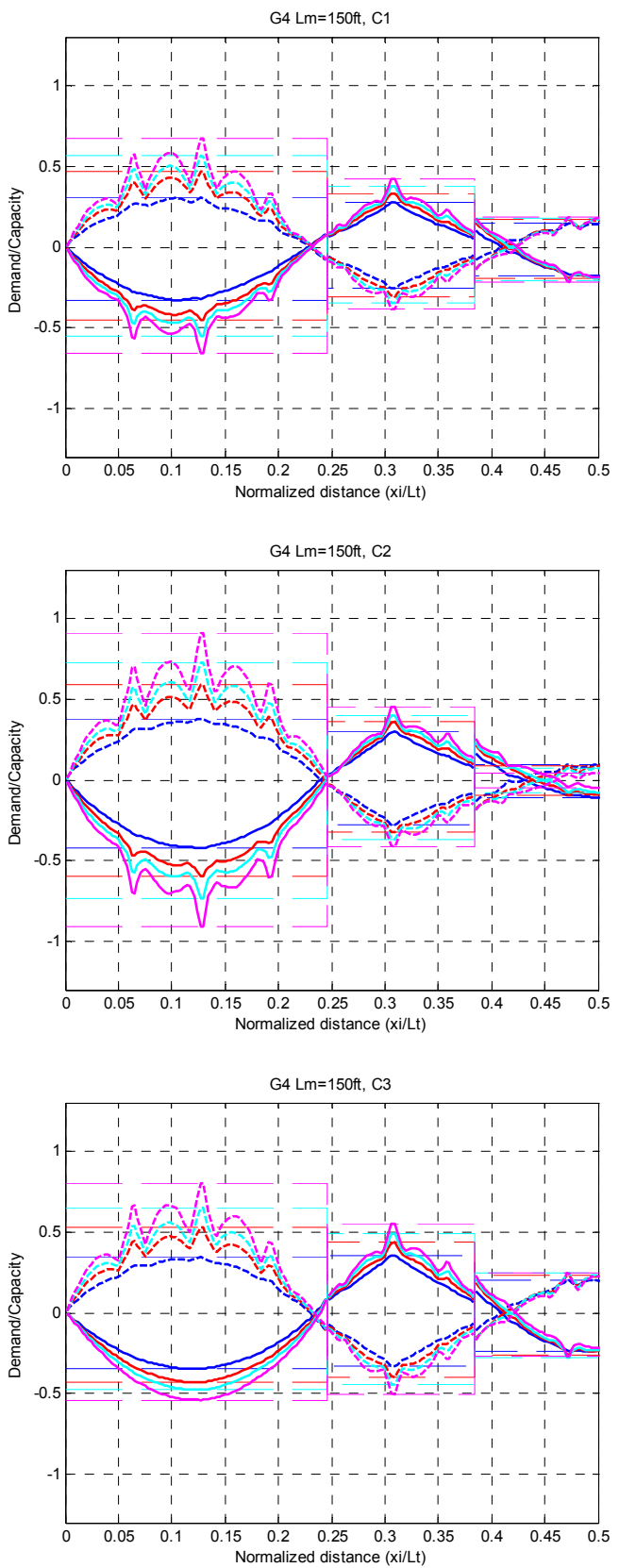
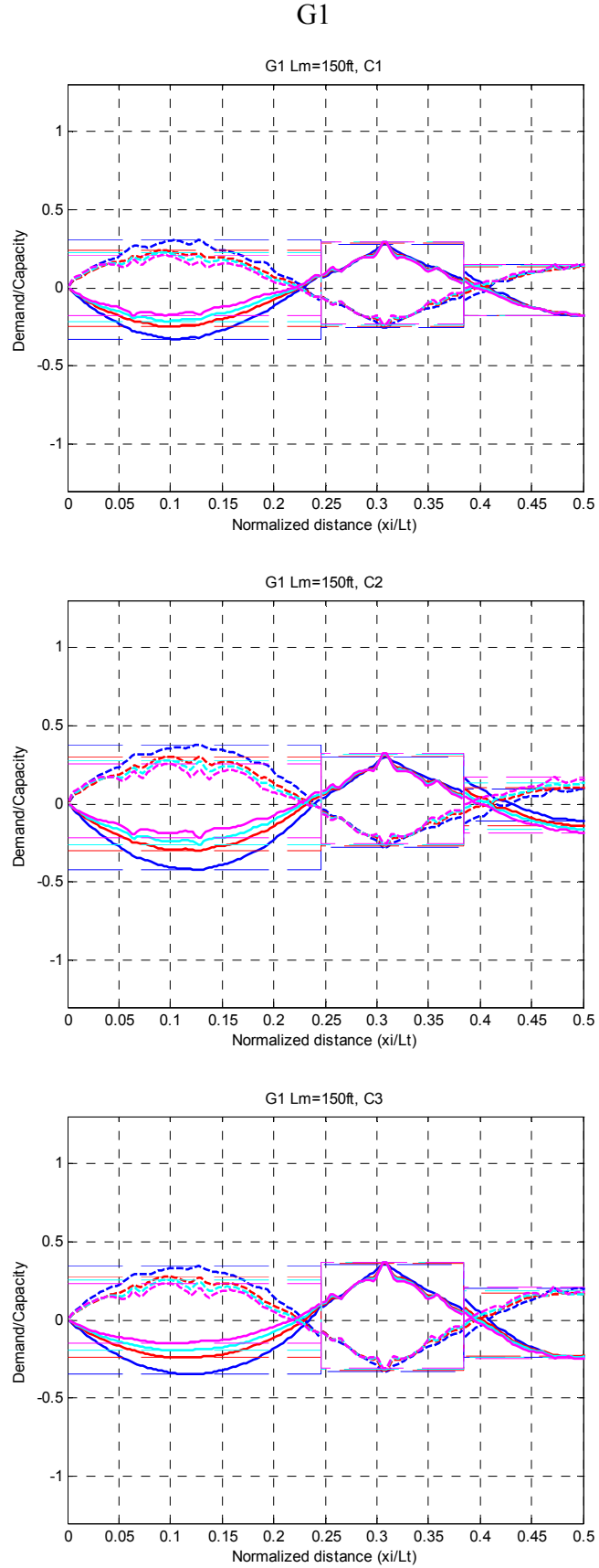

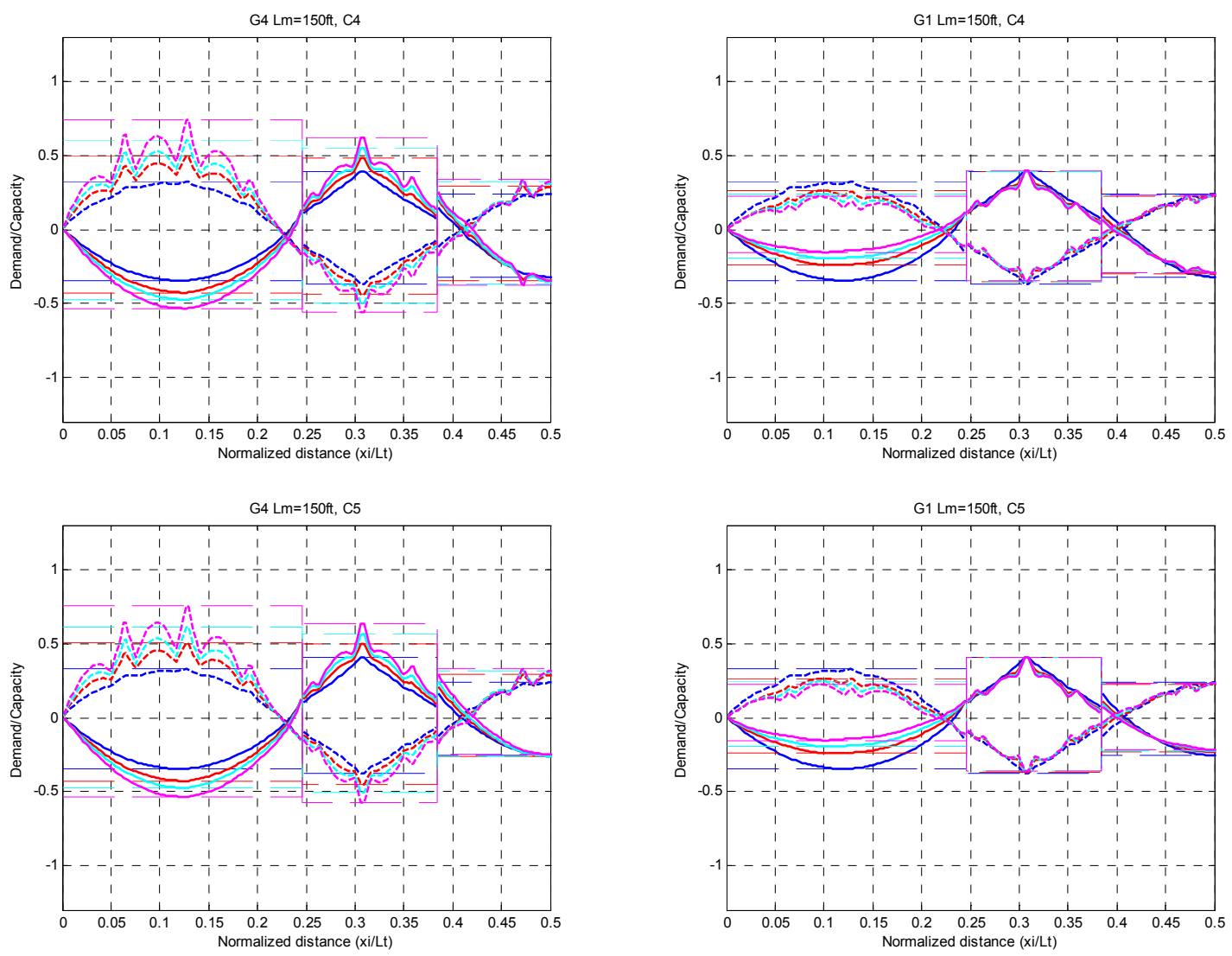

\begin{tabular}{|c|c|c|}
\hline $\boldsymbol{T F}: \mathrm{ST} \mathrm{L} / \mathrm{R}=0.30$ & $\mathrm{~L} / \mathrm{R}=0.45$ & $\mathrm{~L} / \mathrm{R}=0.60$ \\
\hline$B F:----S T$ - - - $\cdot \mathrm{L} / \mathrm{R}=0.30$ & $-----\mathrm{L} / \mathrm{R}=0.45$ & $----. \mathrm{L} / \mathrm{R}=0.60$ \\
\hline
\end{tabular}


- Curved bridges: $L_{b}=25 \mathrm{ft}$
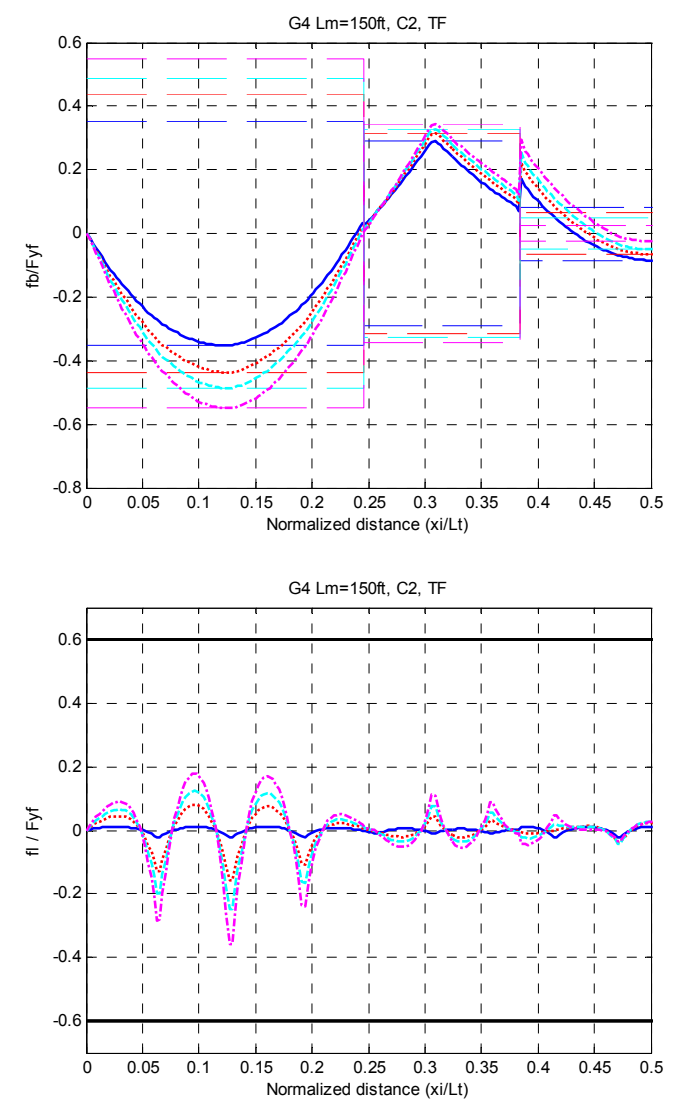
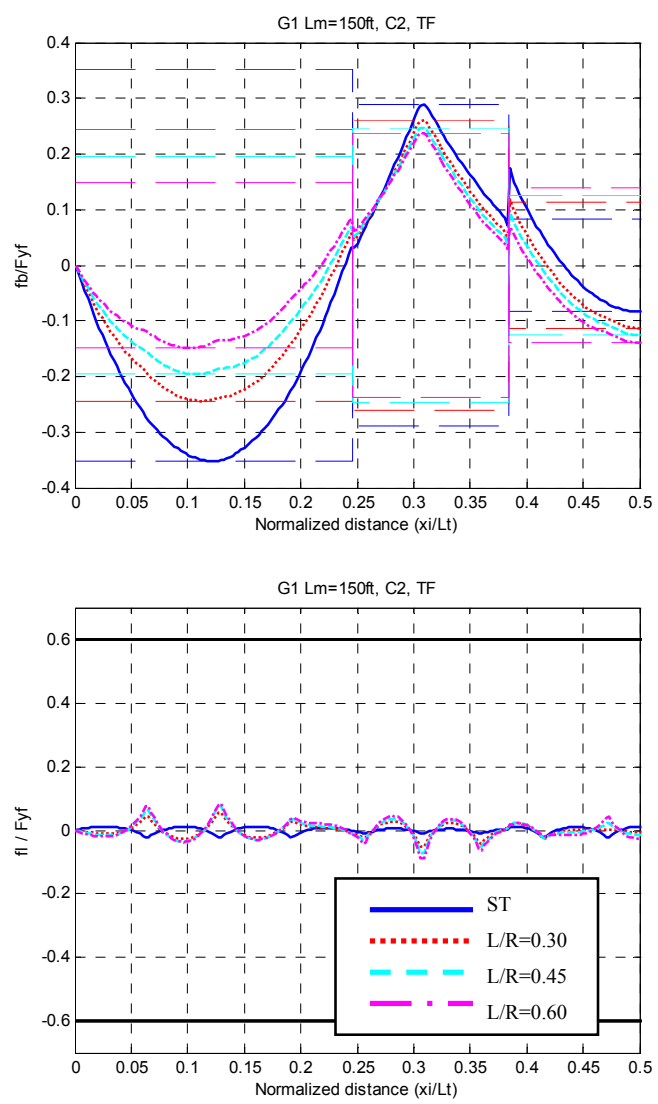
- Curved bridges: $L_{b}=40 \mathrm{ft}$

G4
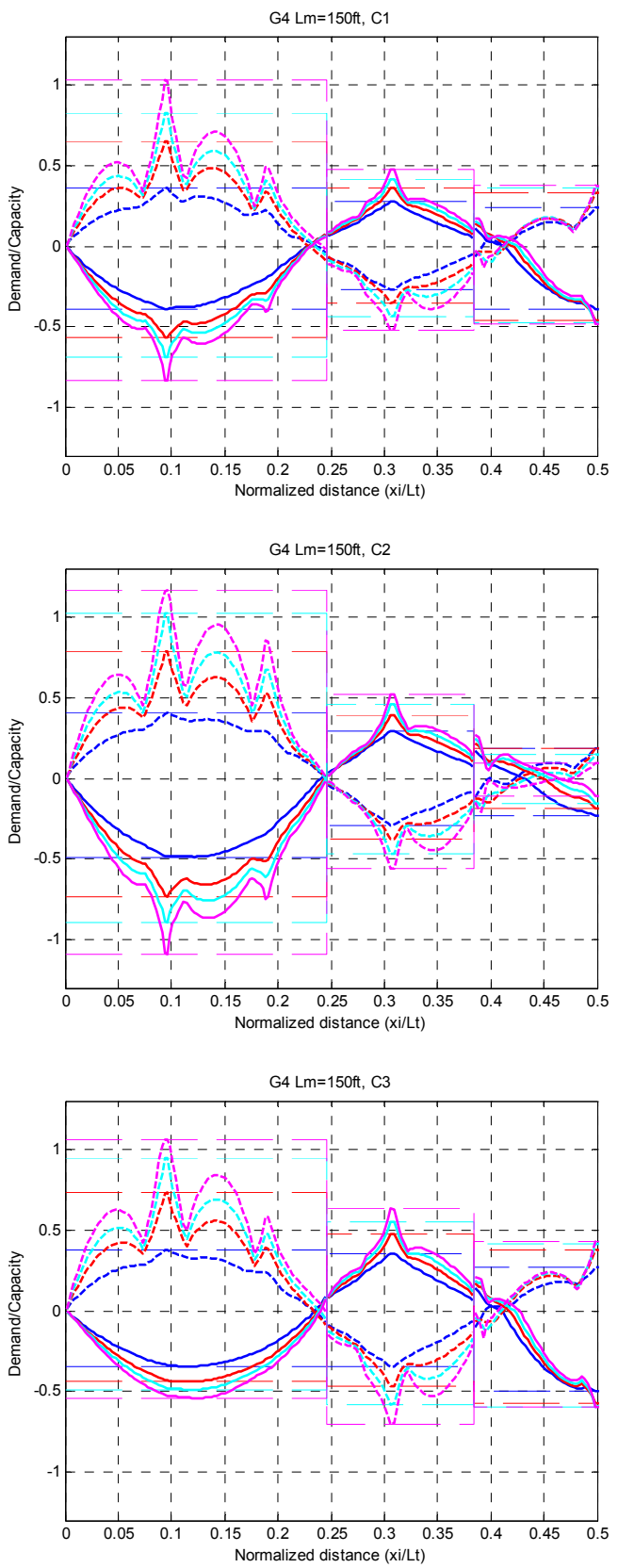

G1
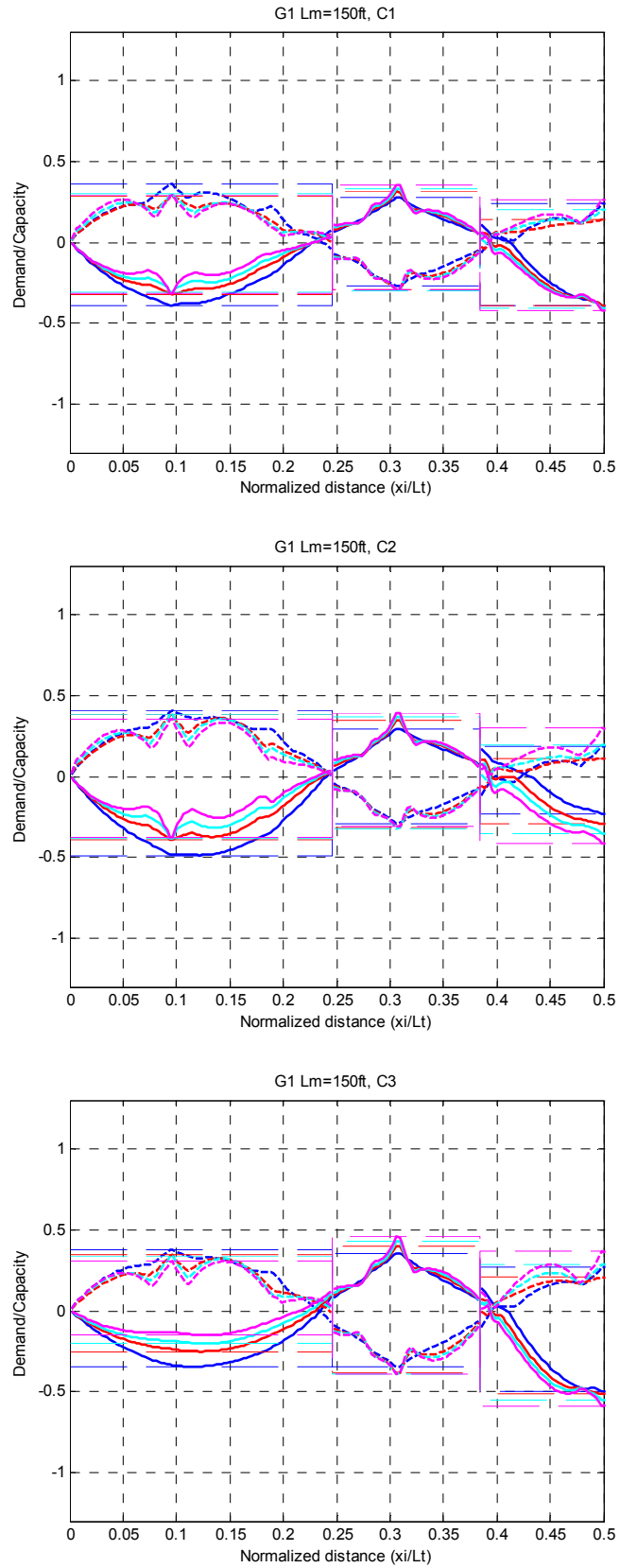

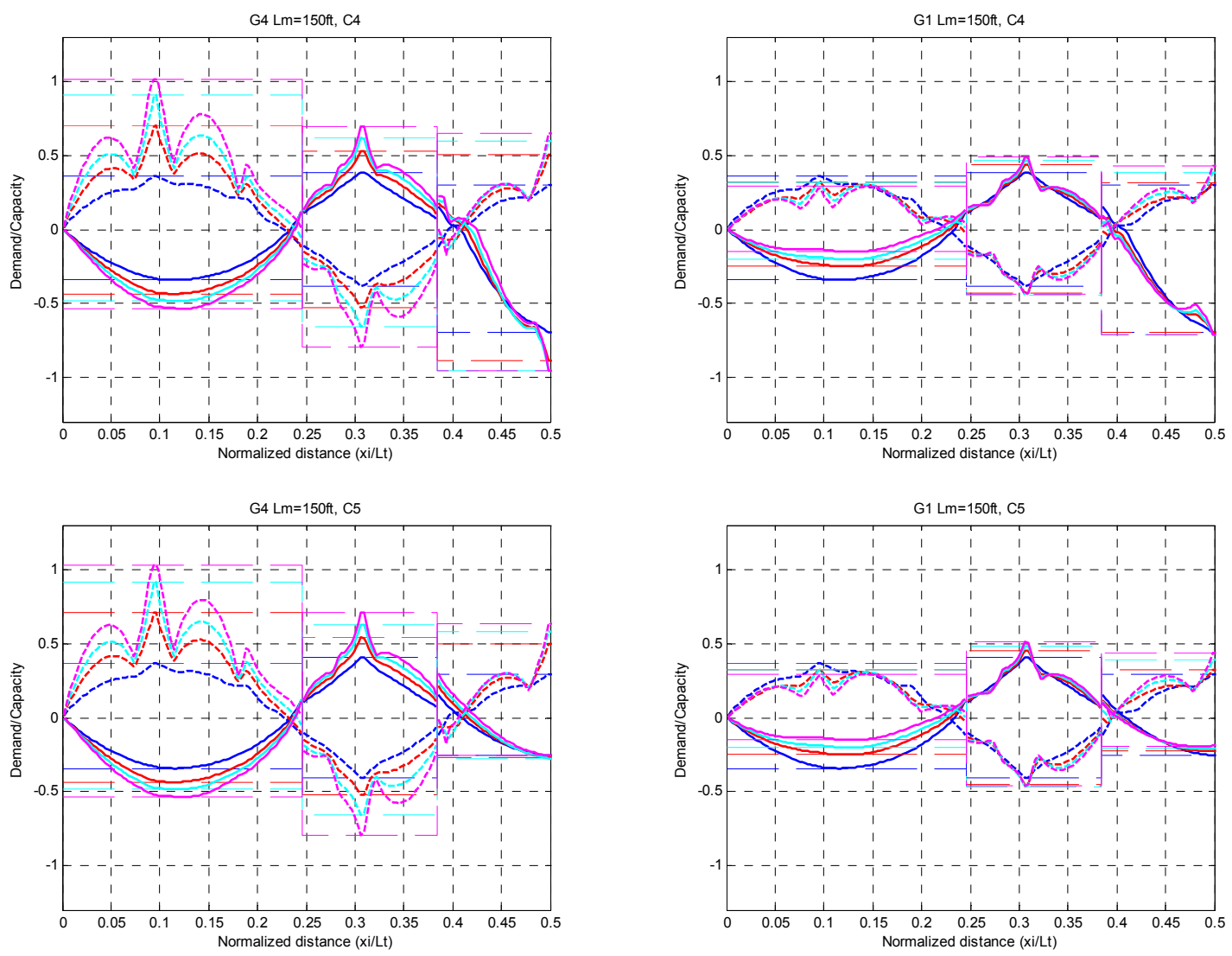

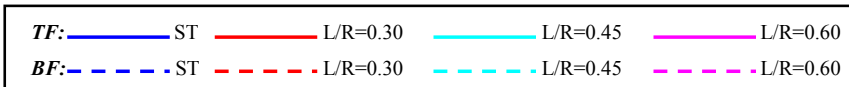


- Curved bridges: $L_{b}=40 \mathrm{ft}$
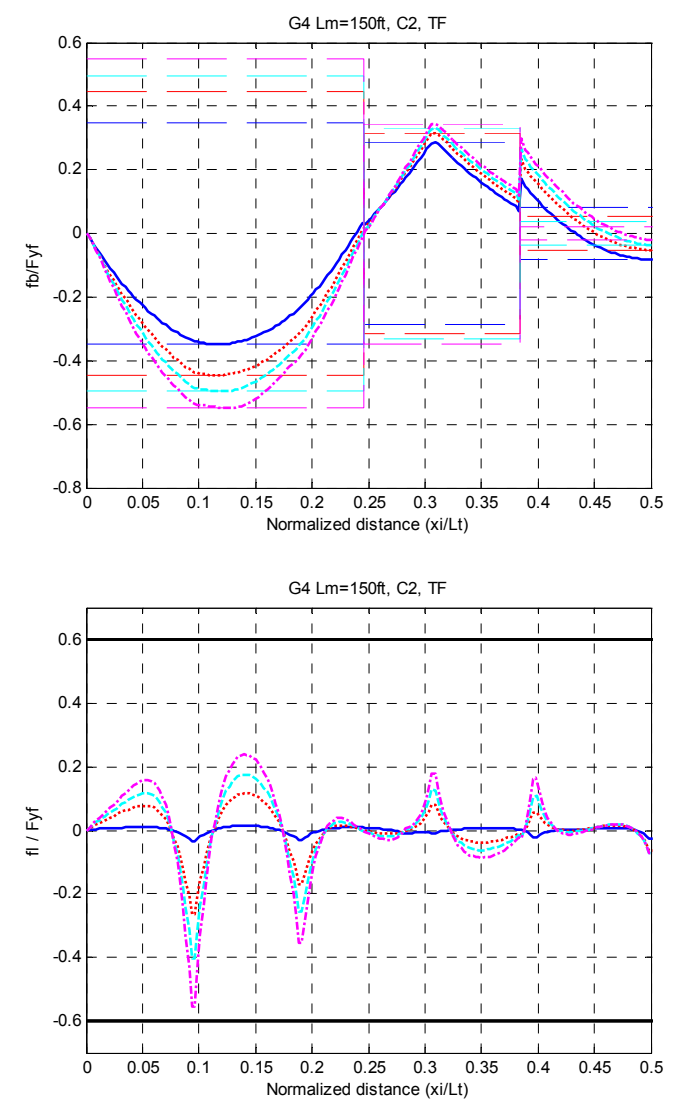
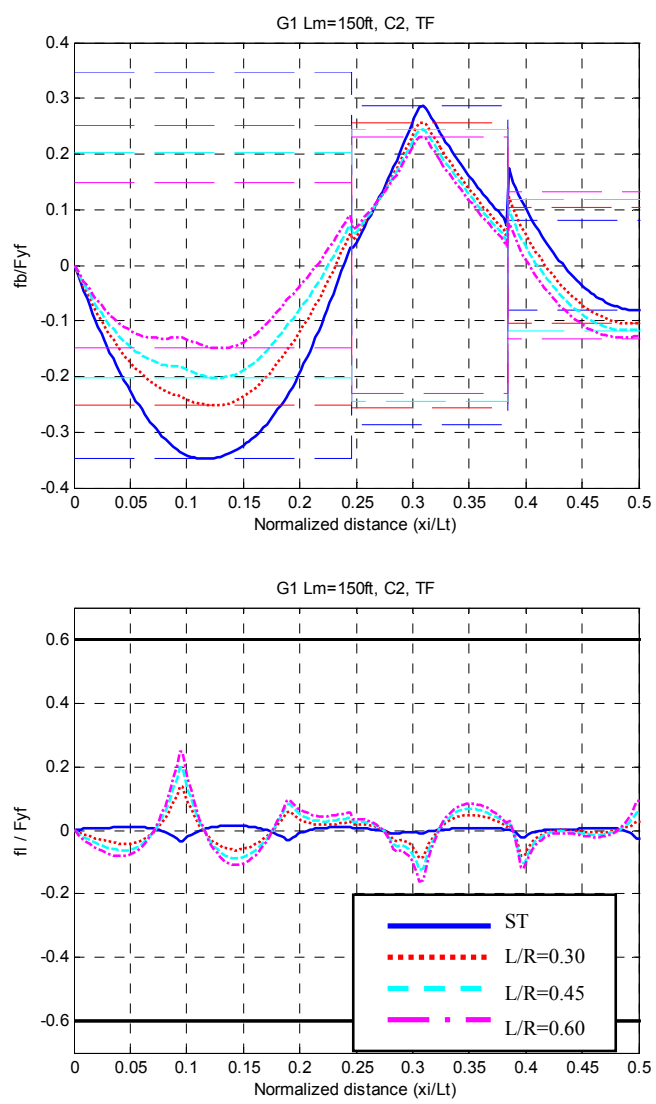
- Curved bridges: $L_{m}=150 \mathrm{ft}-$ Section 1
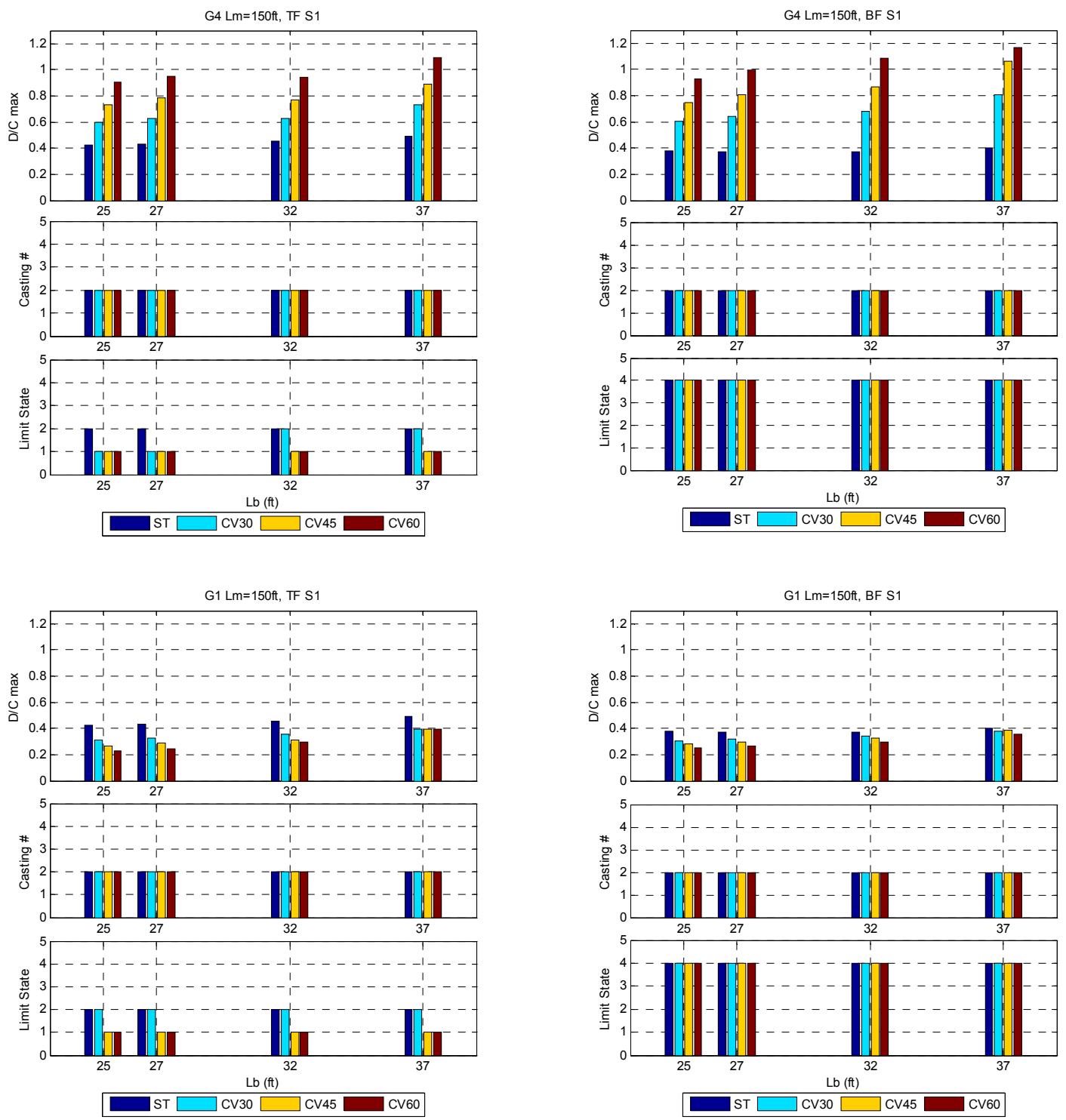
- Curved bridges: $L_{m}=150 \mathrm{ft}-$ Section 2
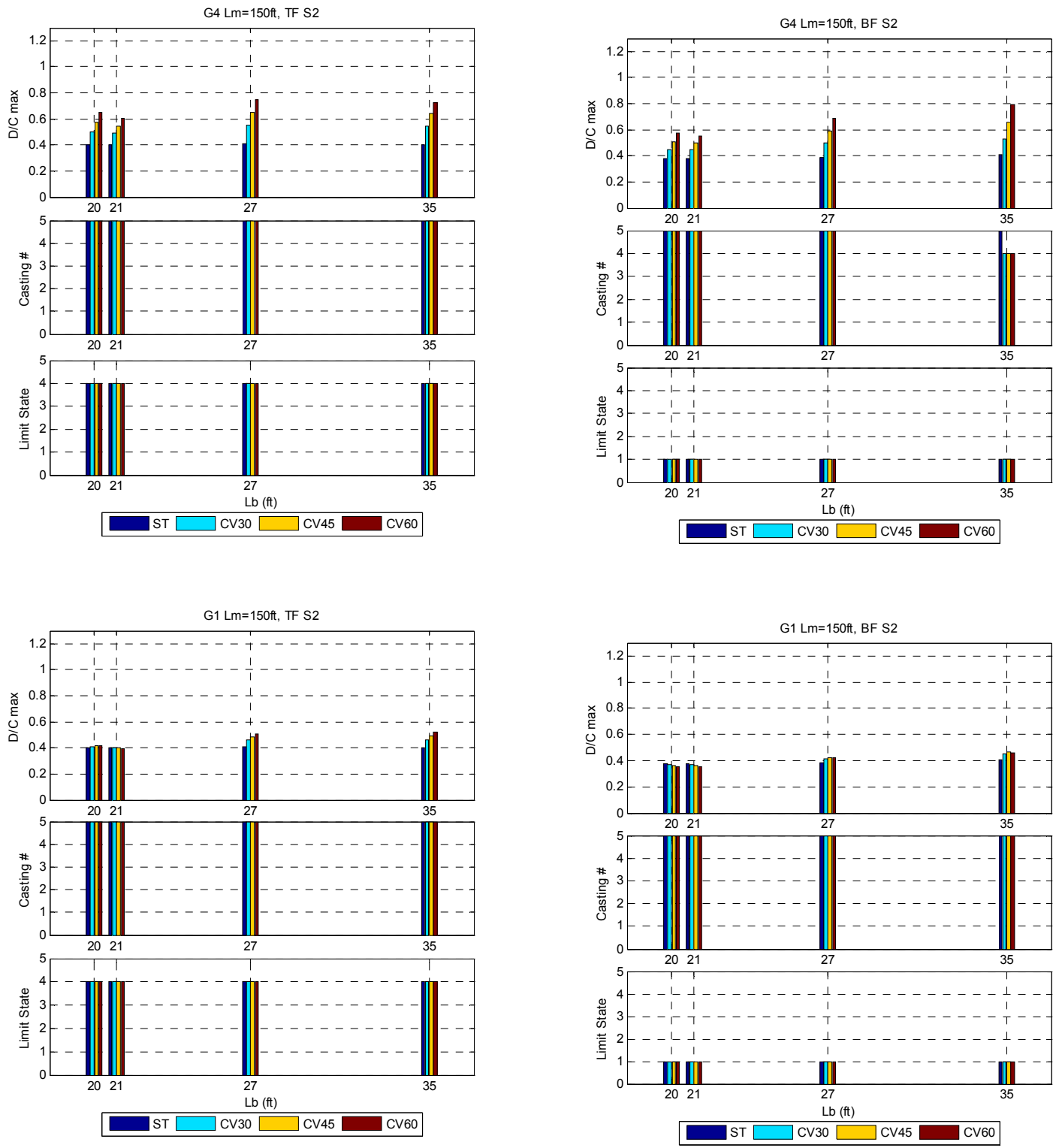
- Curved bridges: $L_{m}=150 \mathrm{ft}-$ Section 3
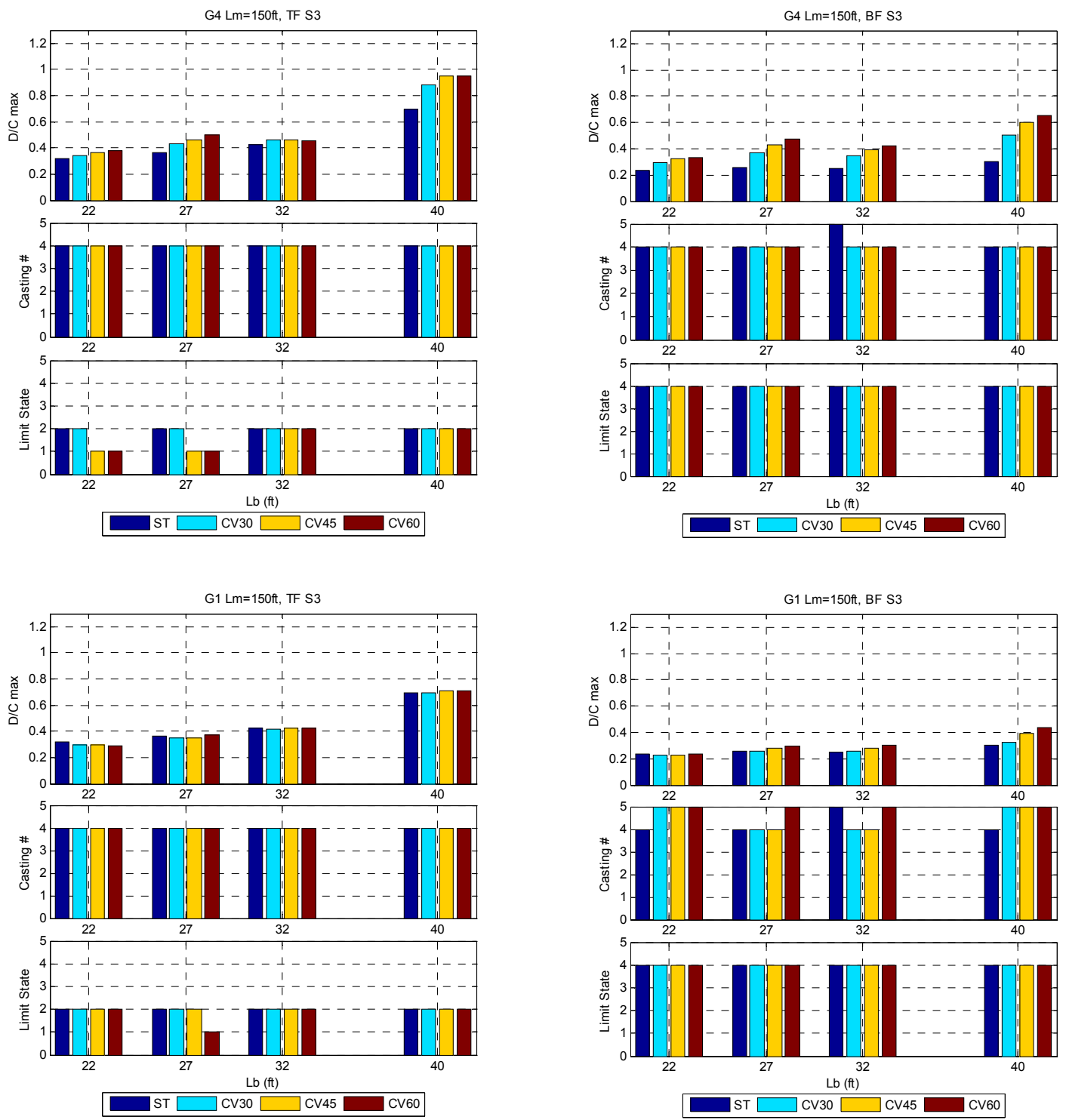


\section{Appendix E: Fragility Curves in Skewed Bridges}

- Skewed bridges: $\theta=45^{\circ}-\mathrm{S} 1$
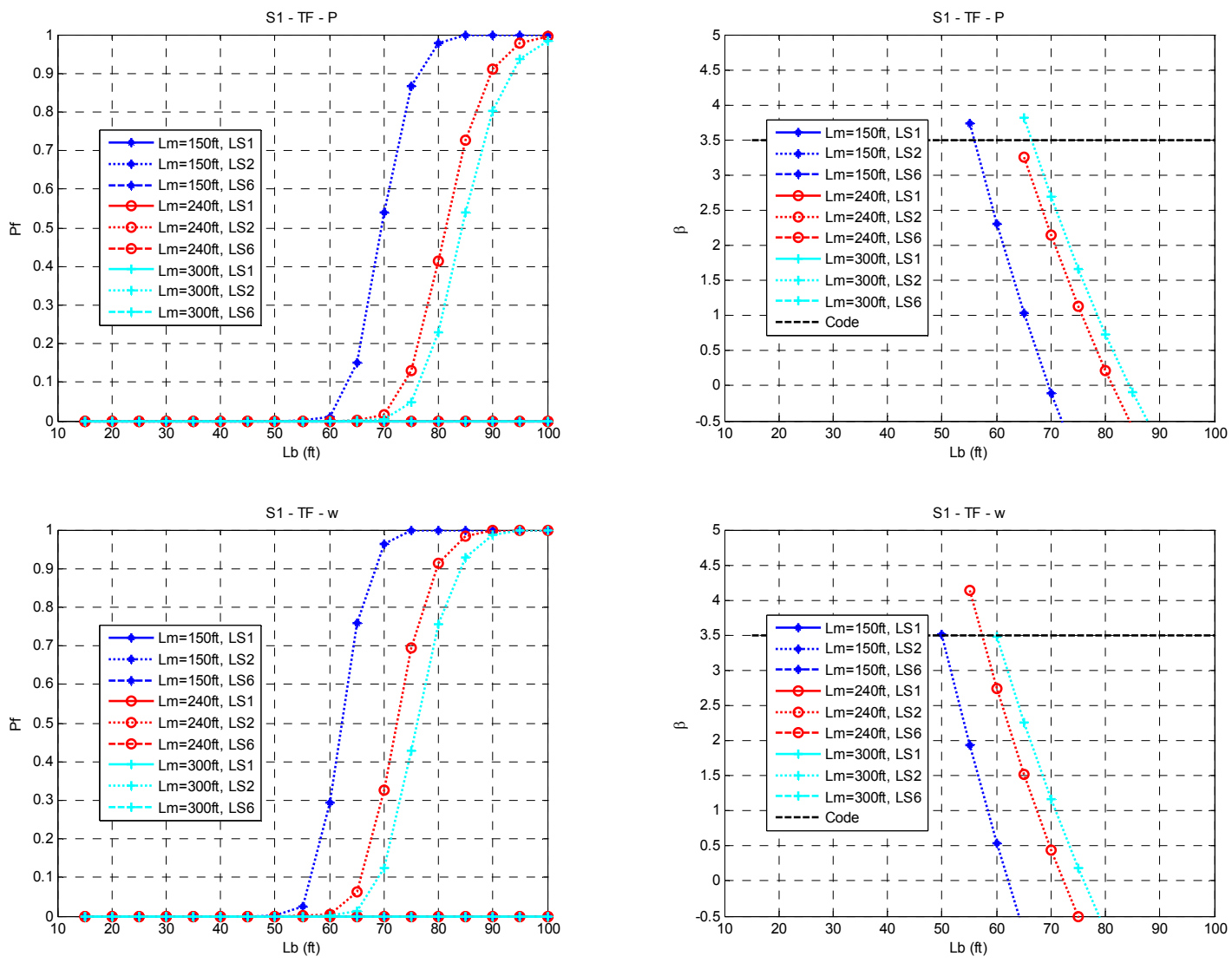
- Skewed bridges: $\theta=45^{\circ}-\mathrm{S} 3$
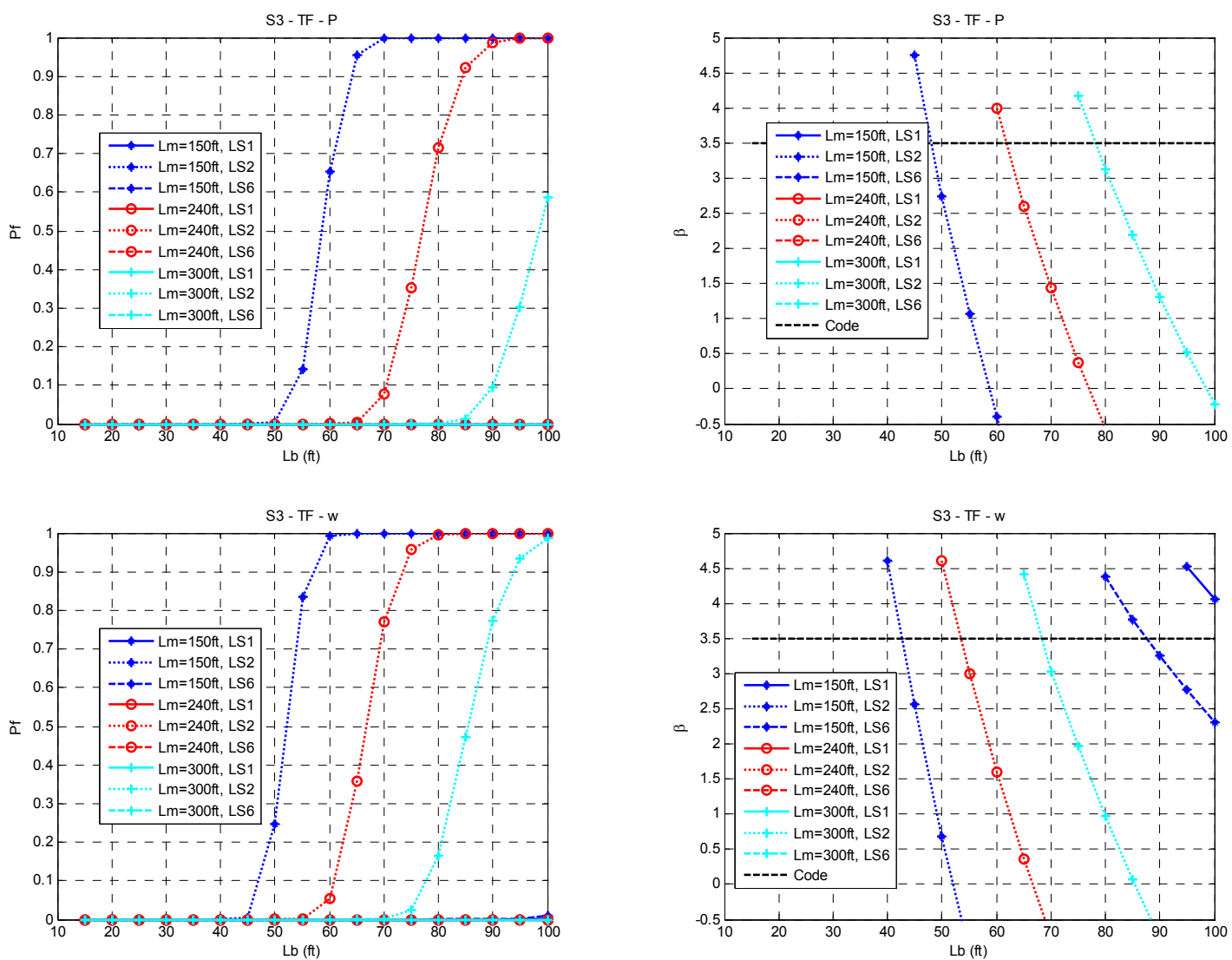

\section{- Skewed bridges: $\theta=45^{\circ}-\mathrm{S} 2$}
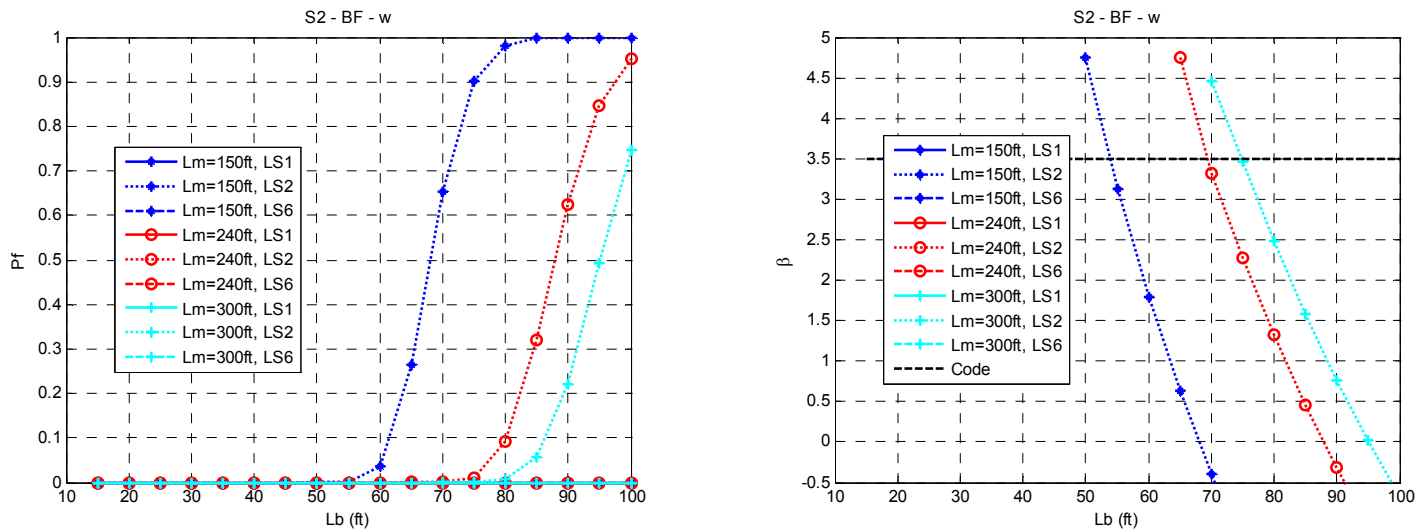
- Skewed bridges: $\theta=60^{\circ}-\mathrm{S} 1$
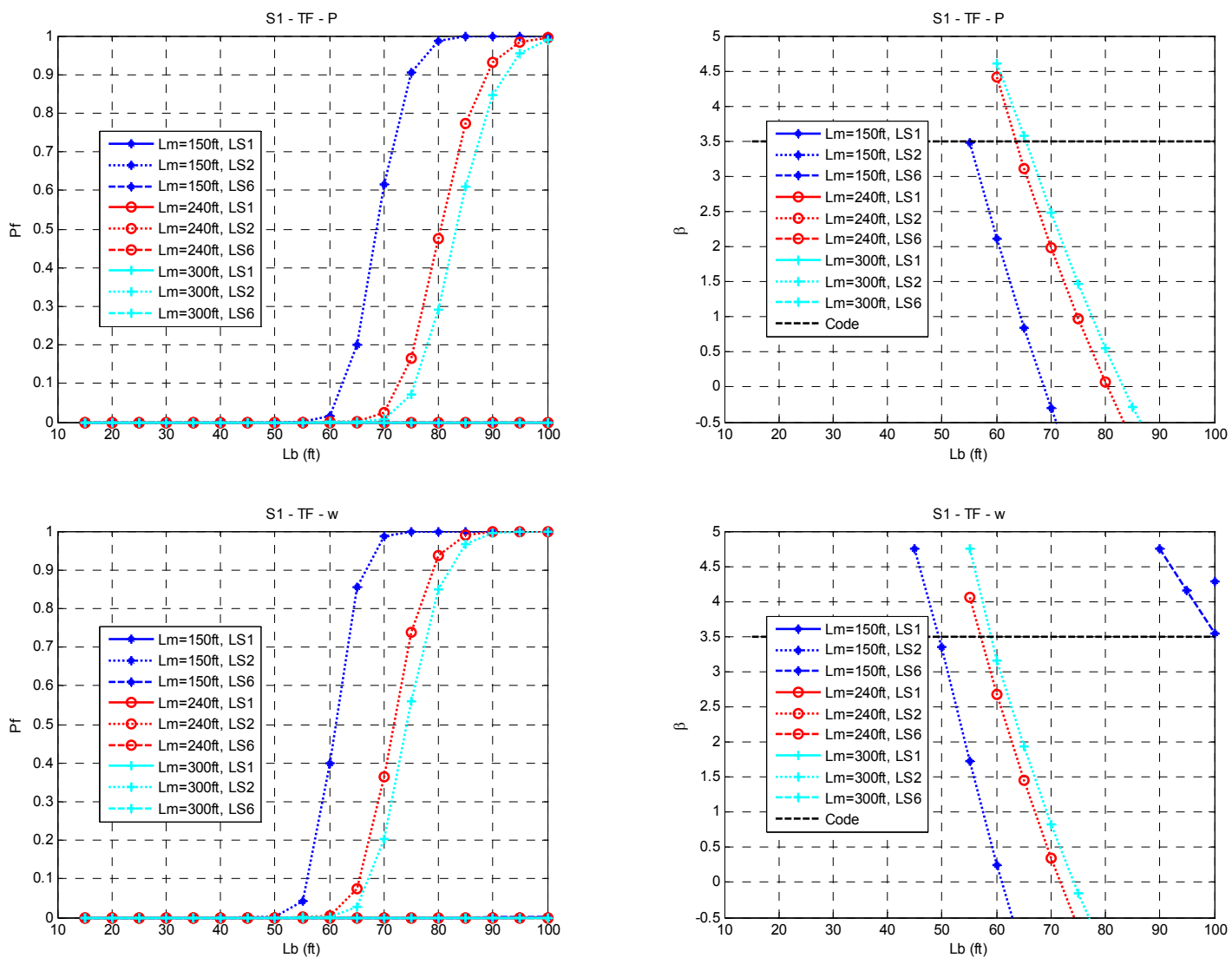
- Skewed bridges: $\theta=45^{\circ}-\mathrm{S} 3$
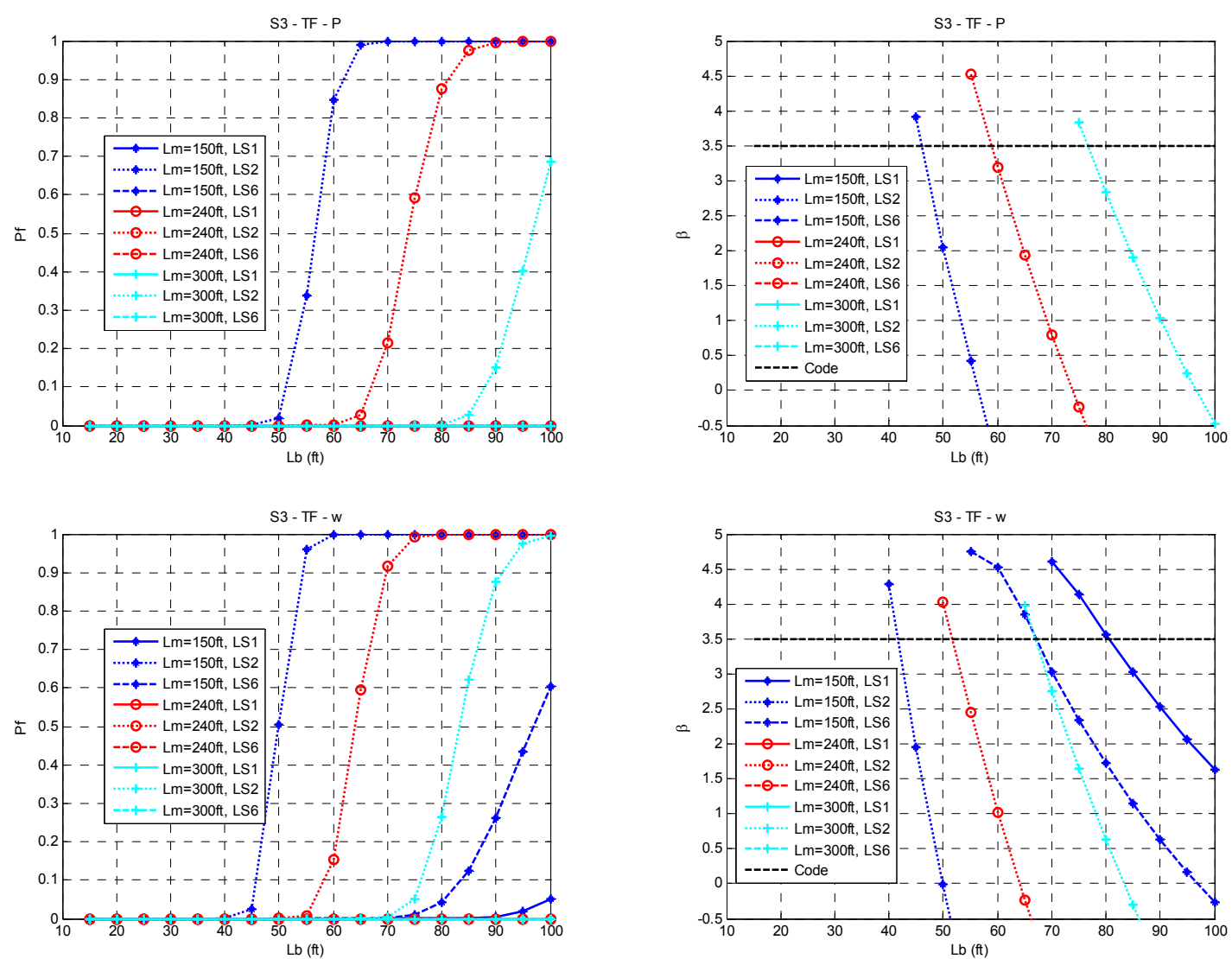

- Skewed bridges: $\theta=45^{\circ}-\mathrm{S} 2$
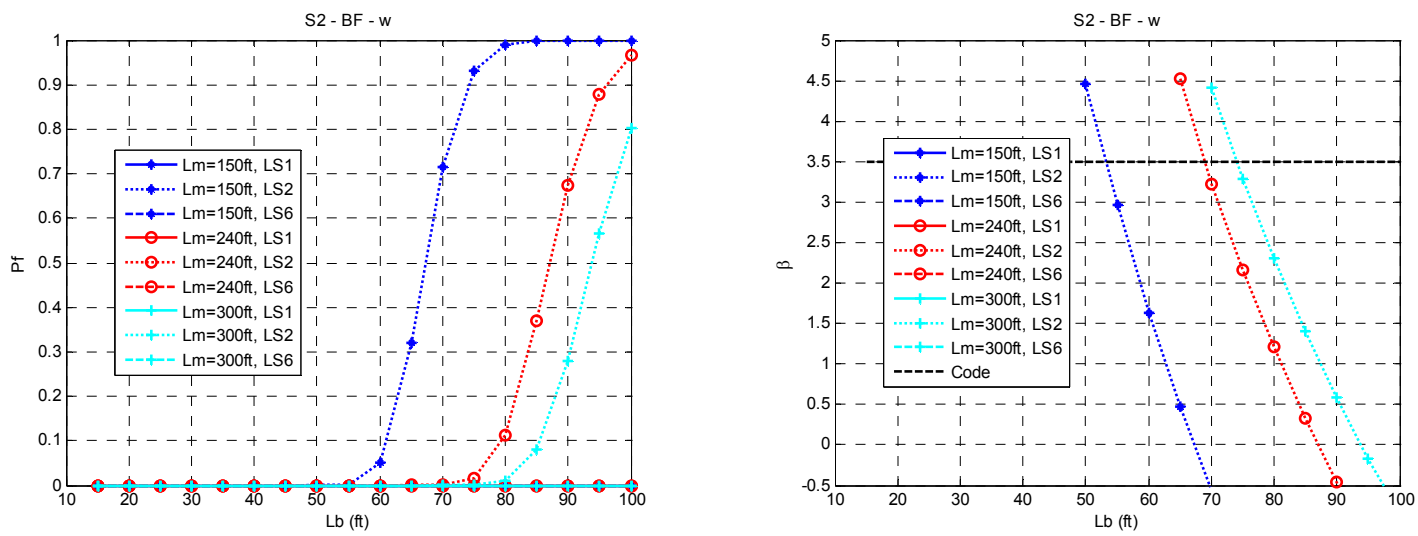


\section{Appendix F: Fragility Curves in Curved Bridges}

- Curved bridges: $L_{m}=150 \mathrm{ft}$ - Outer Girder - S1
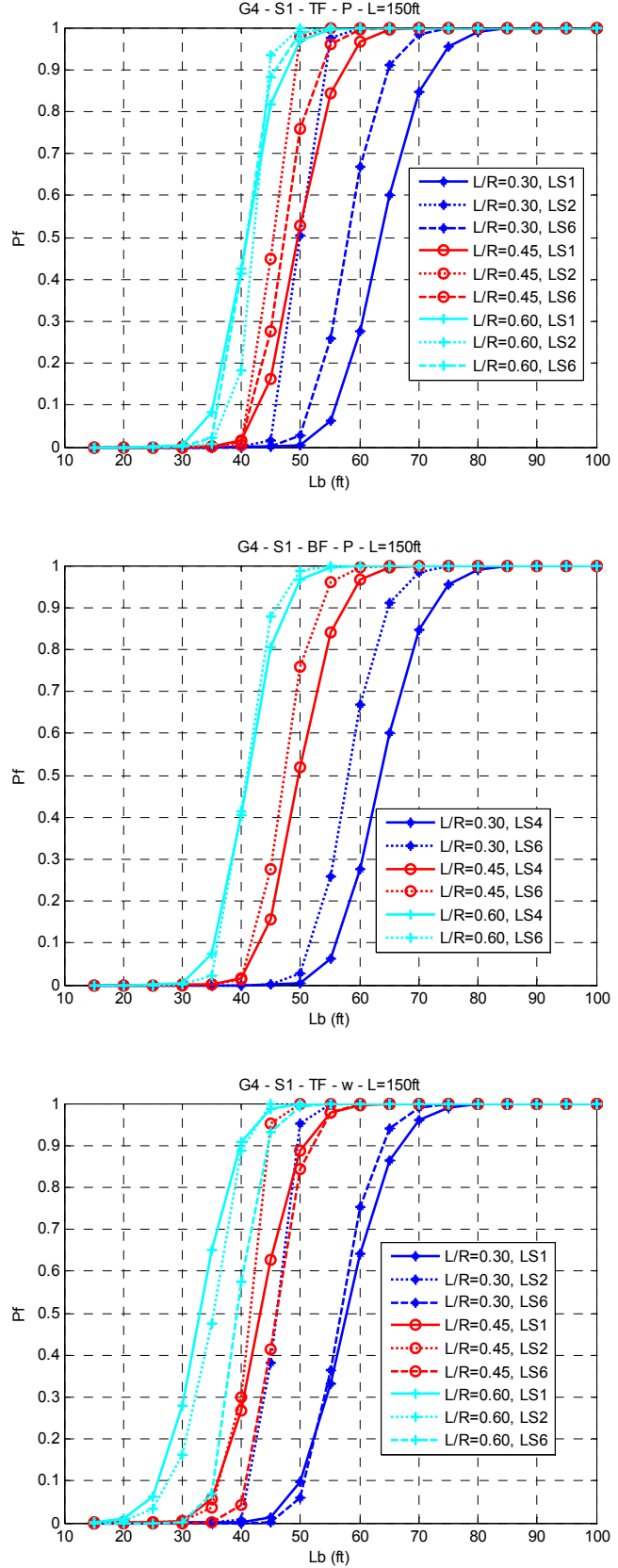
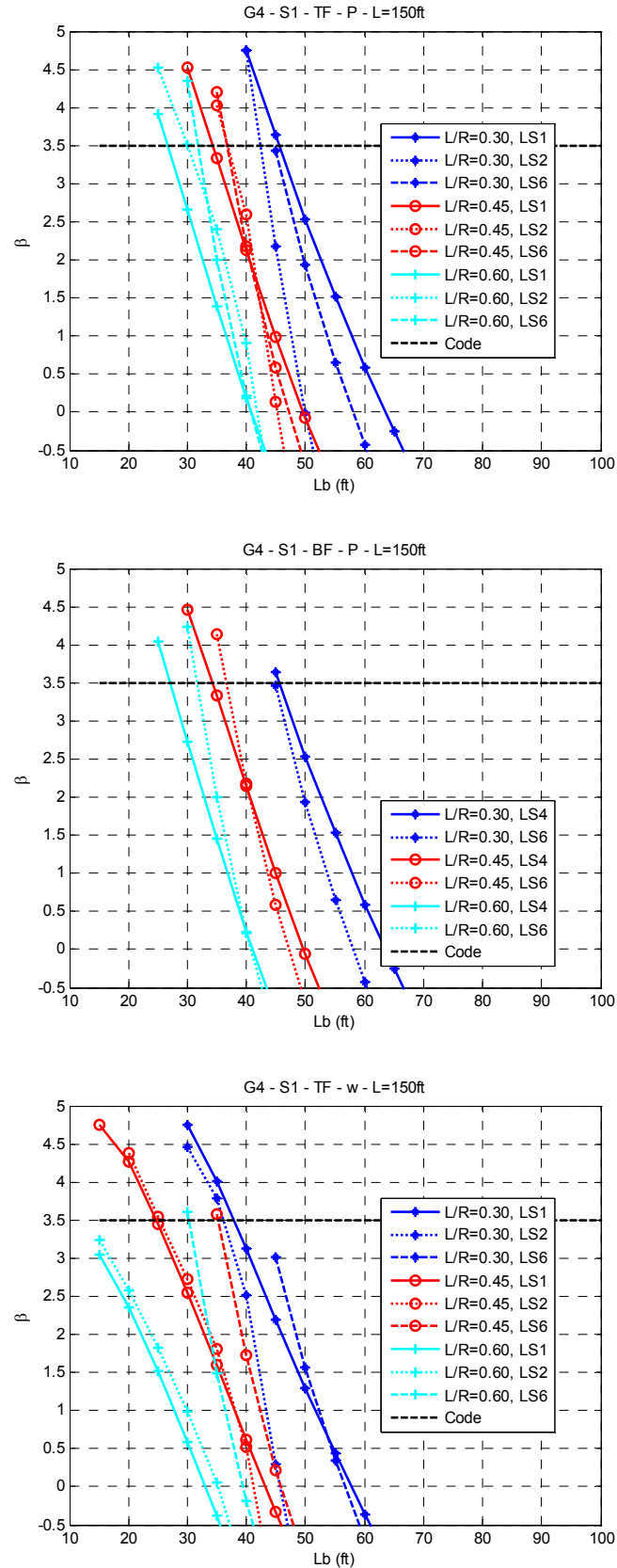

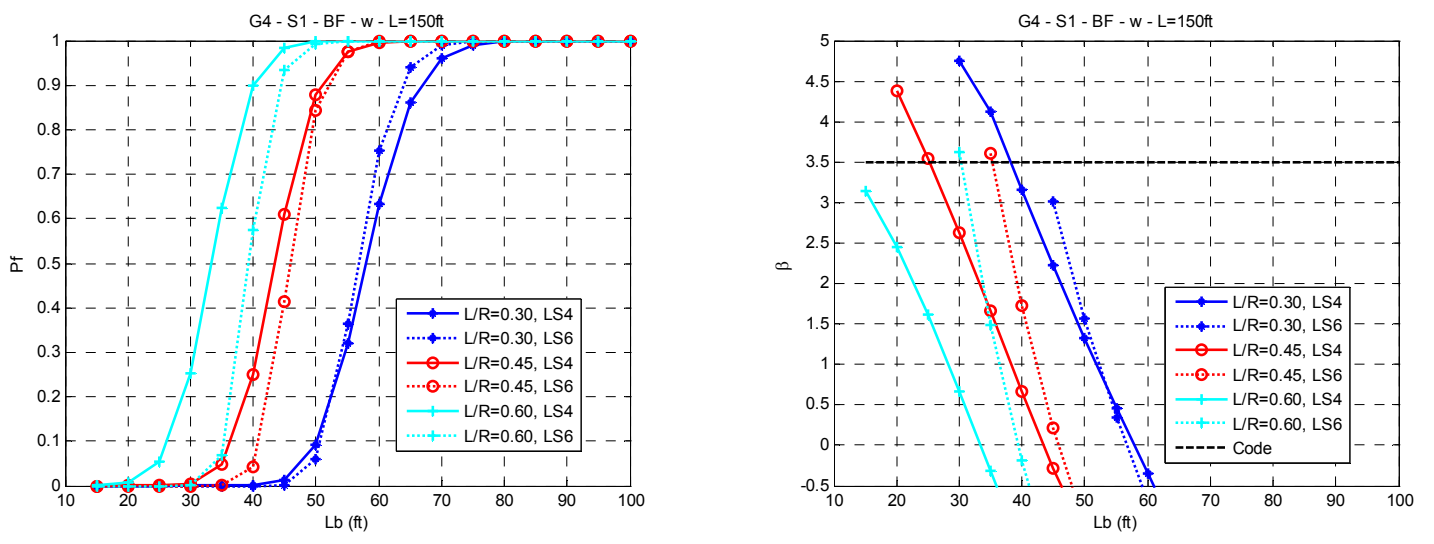
- Curved bridges: $L_{m}=150 \mathrm{ft}-$ Outer Girder - S3
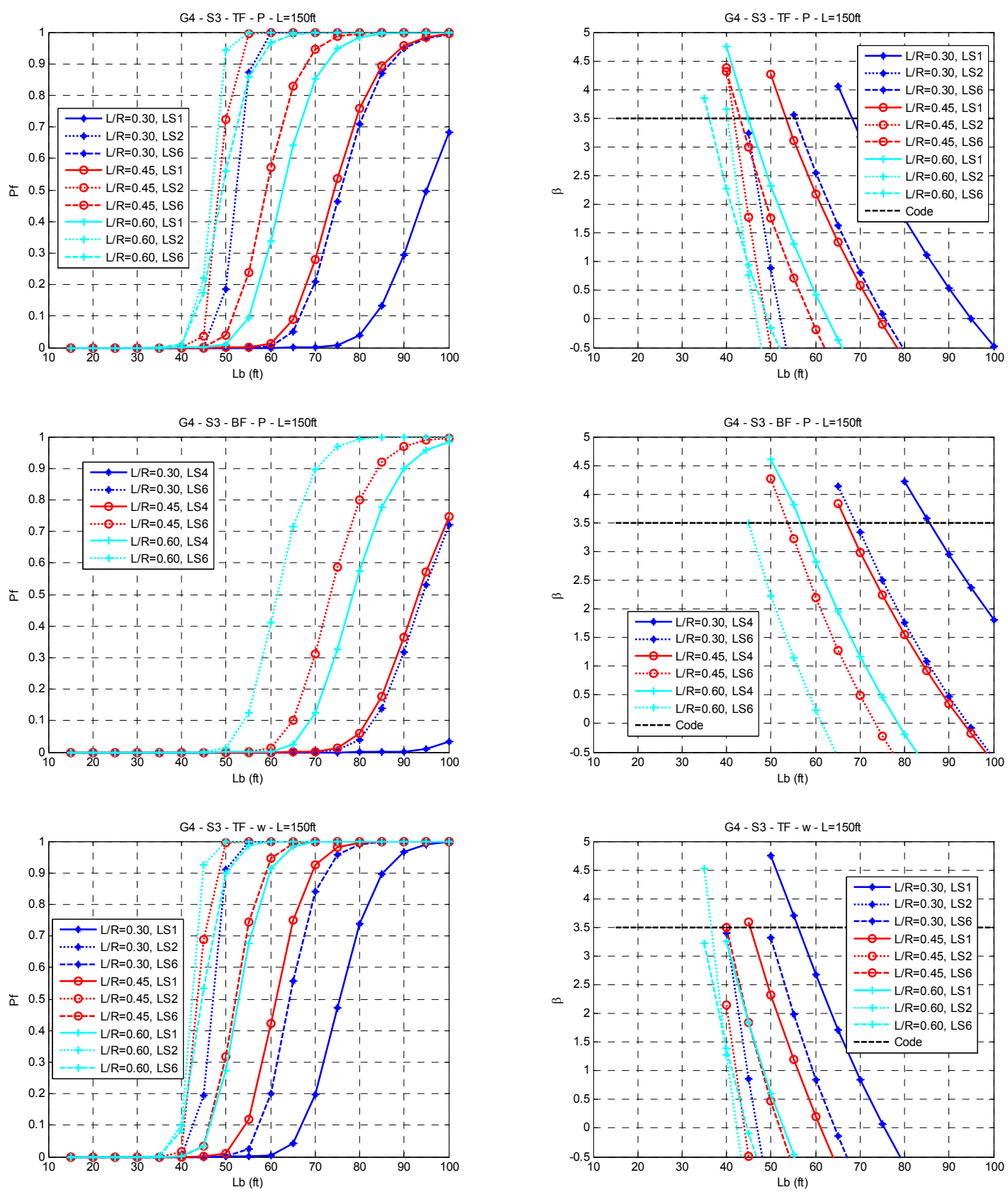

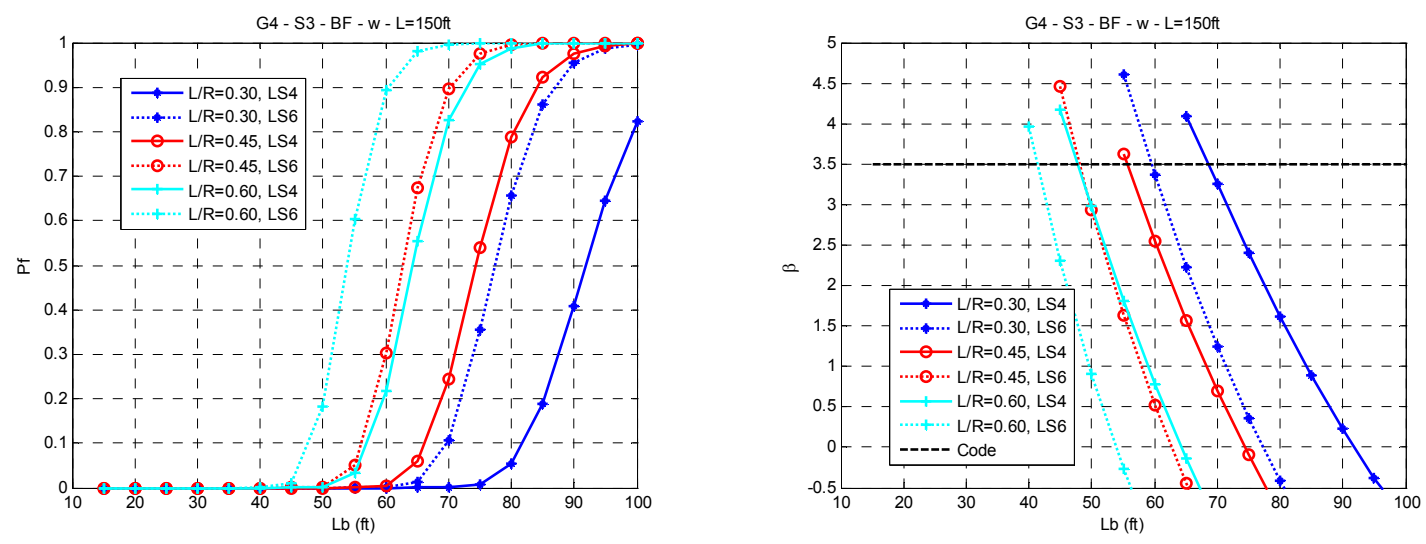

- Curved bridges: $L_{m}=150 \mathrm{ft}-$ Outer Girder - S2
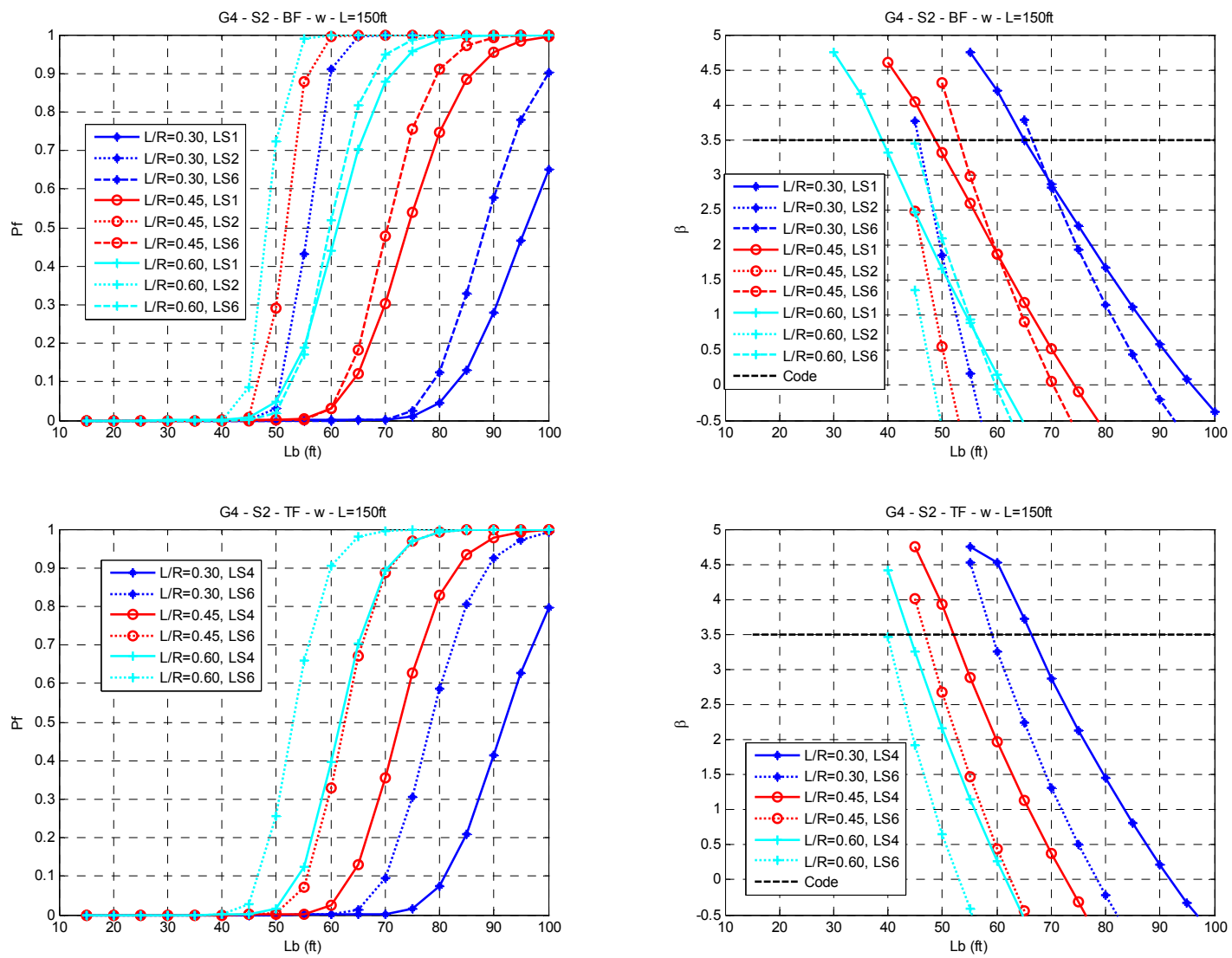
- Curved bridges: $L_{m}=240 \mathrm{ft}-$ Outer Girder $-\mathrm{S} 1$
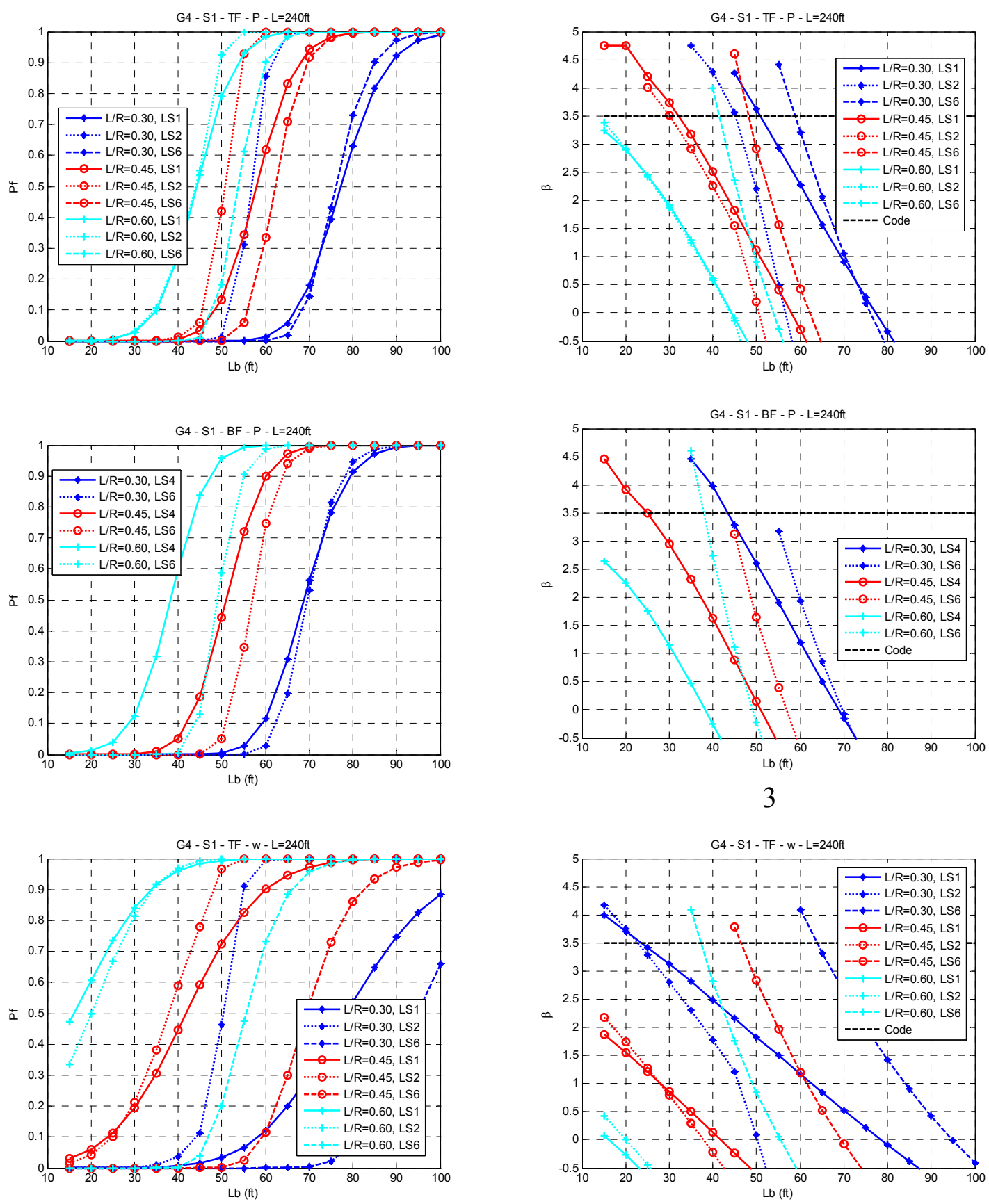
G4 - S1 - BF - w - L=240ft

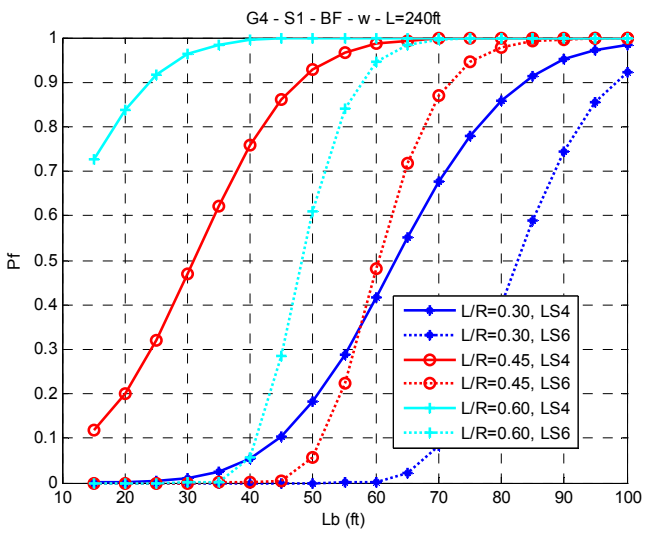

G4 - S1 - BF - w - L=240ft

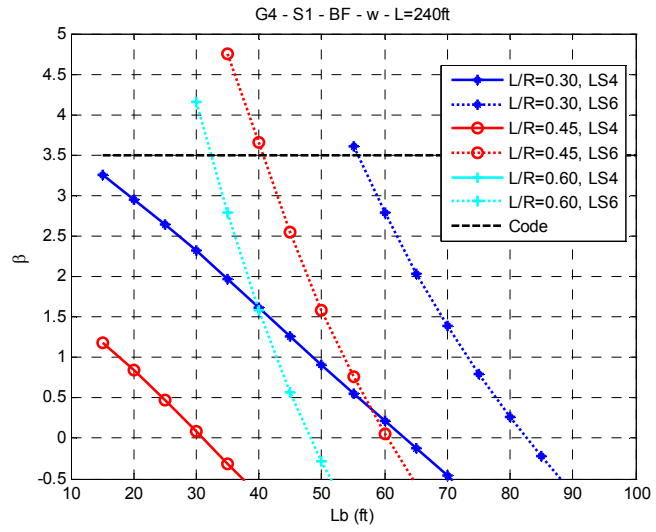


- Curved bridges: $L_{m}=240 \mathrm{ft}-$ Outer Girder $-\mathrm{S} 3$
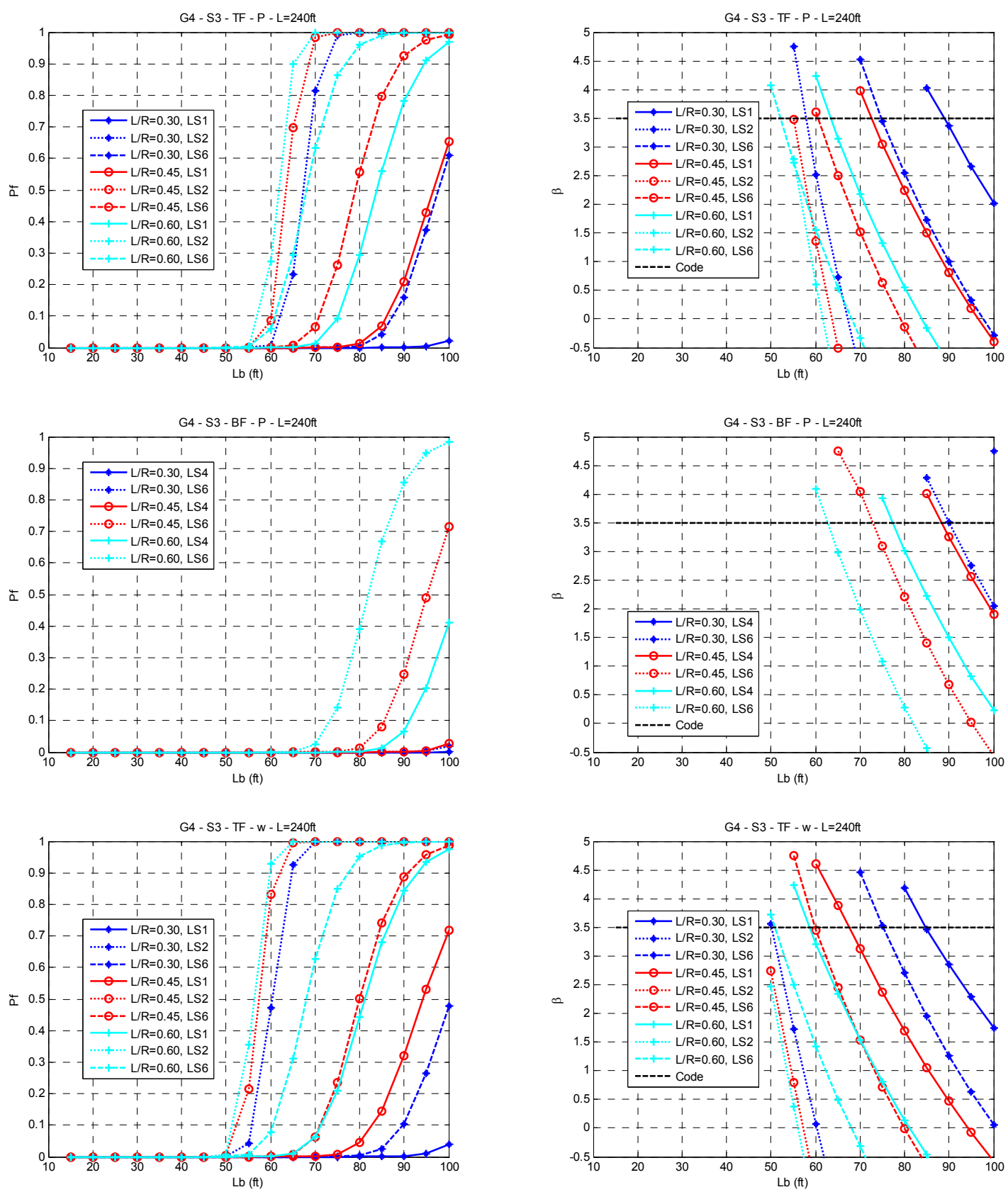

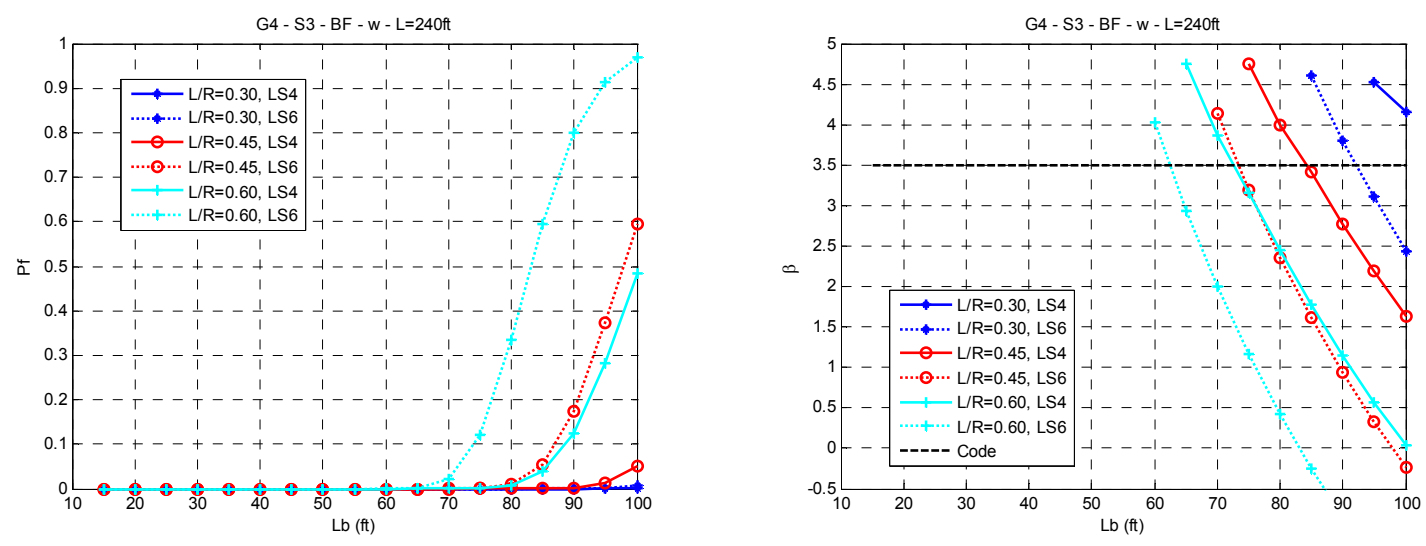

- Curved bridges: $L_{m}=240 \mathrm{ft}-$ Outer Girder - S2
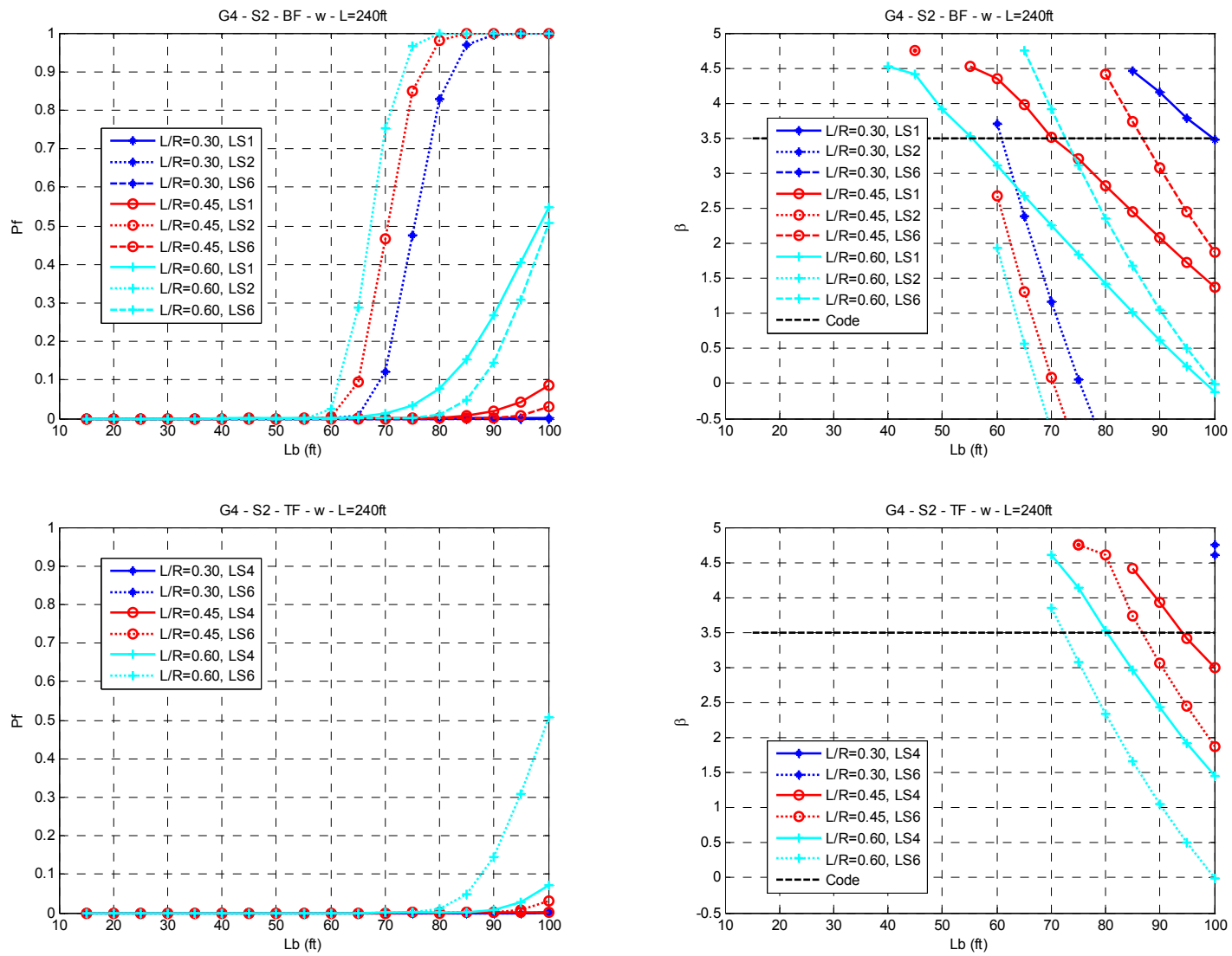
- Curved bridges: $L_{m}=300 \mathrm{ft}-$ Outer Girder $-\mathrm{S} 1$
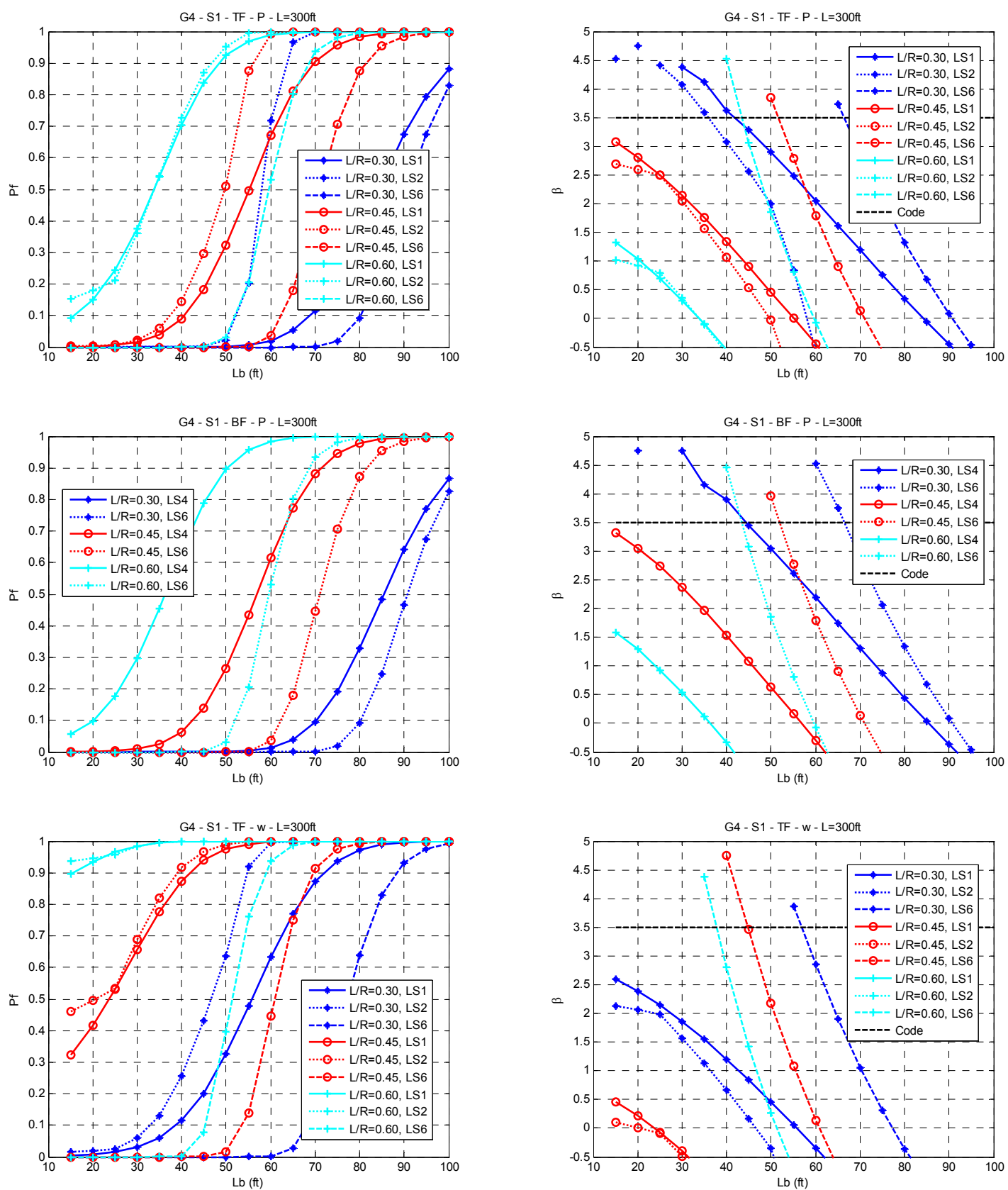
G4 - S1 - BF - w - L=300ft

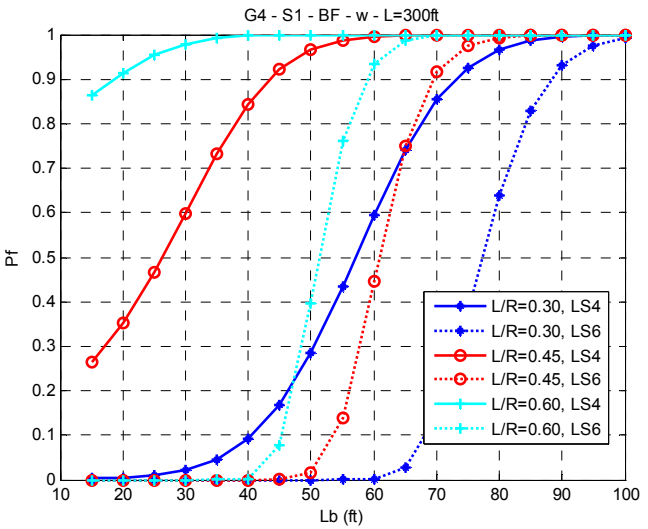

G4 - S1 - BF - w - L=300ft

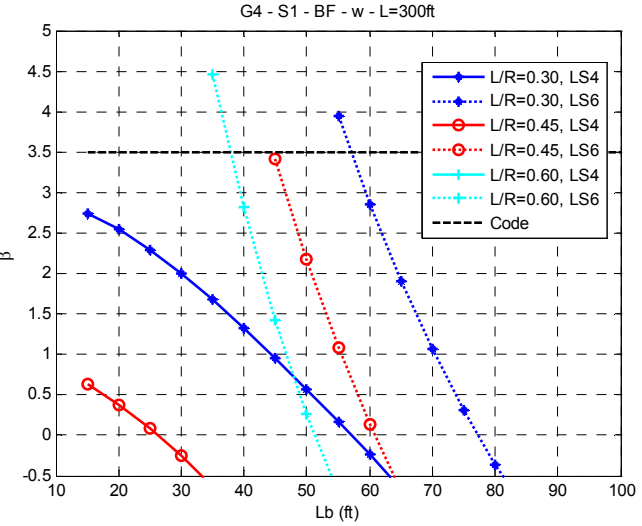


- Curved bridges: $L_{m}=300 \mathrm{ft}-$ Outer Girder $-\mathrm{S} 3$
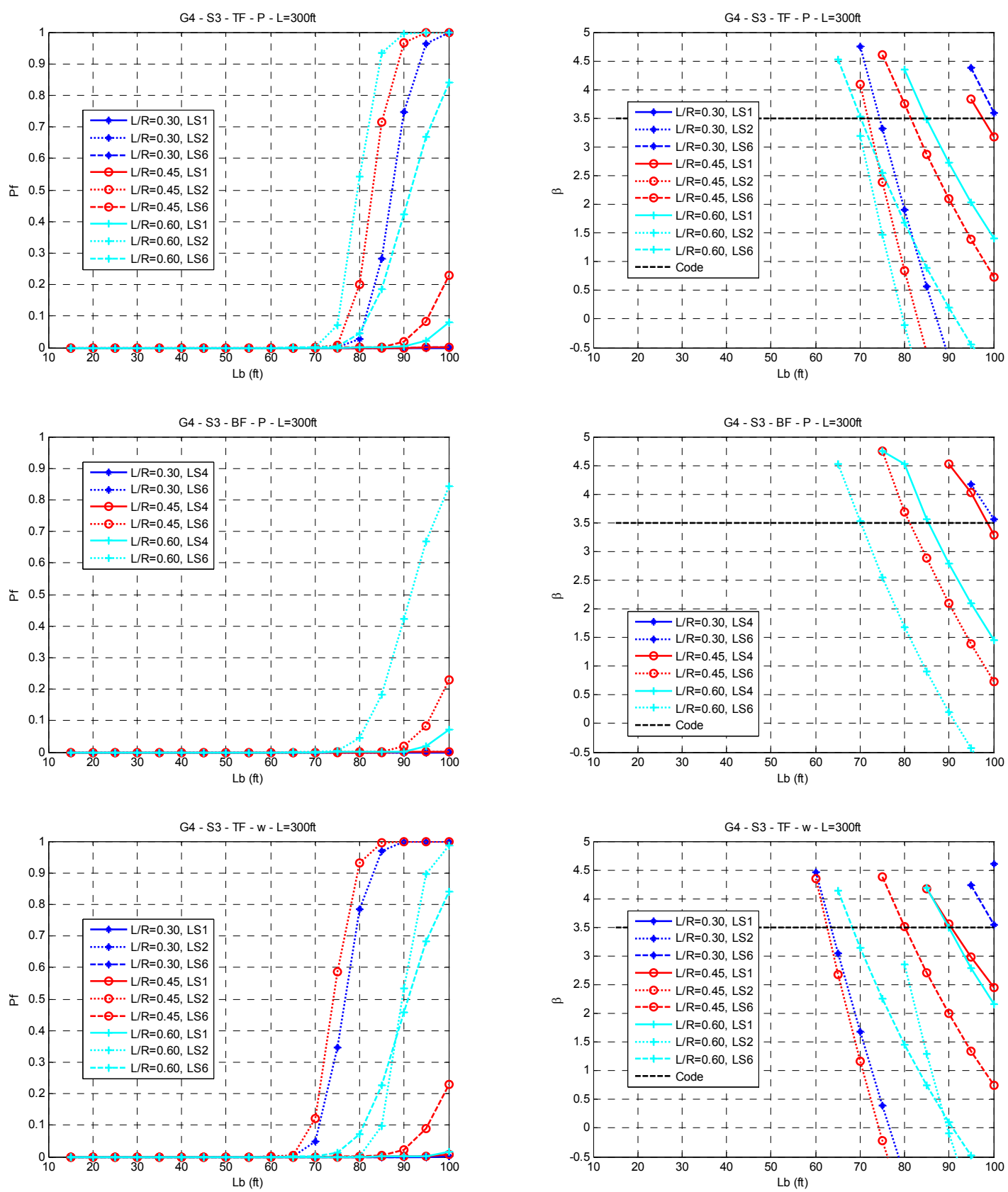

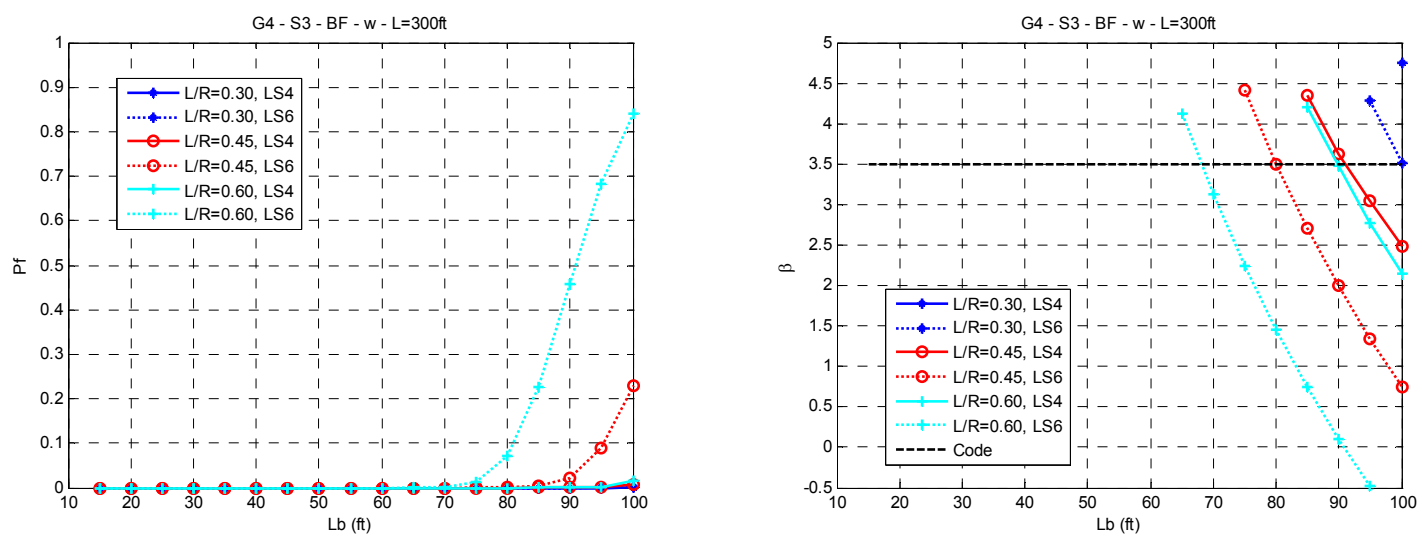

- Curved bridges: $L_{m}=300 \mathrm{ft}-$ Outer Girder - S2
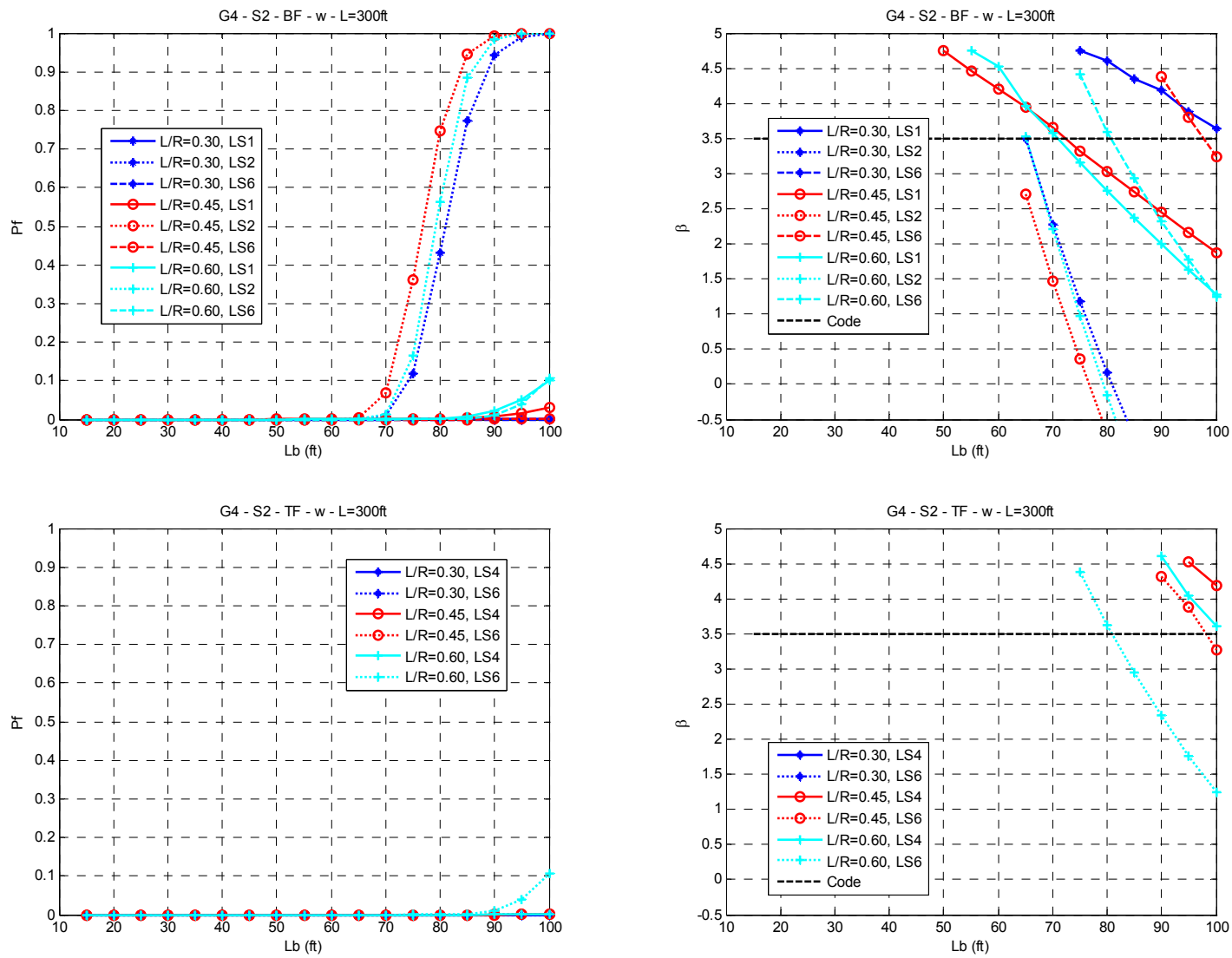
- Curved bridges: $L_{m}=150 \mathrm{ft}-$ Inner Girder $-\mathrm{S} 1$
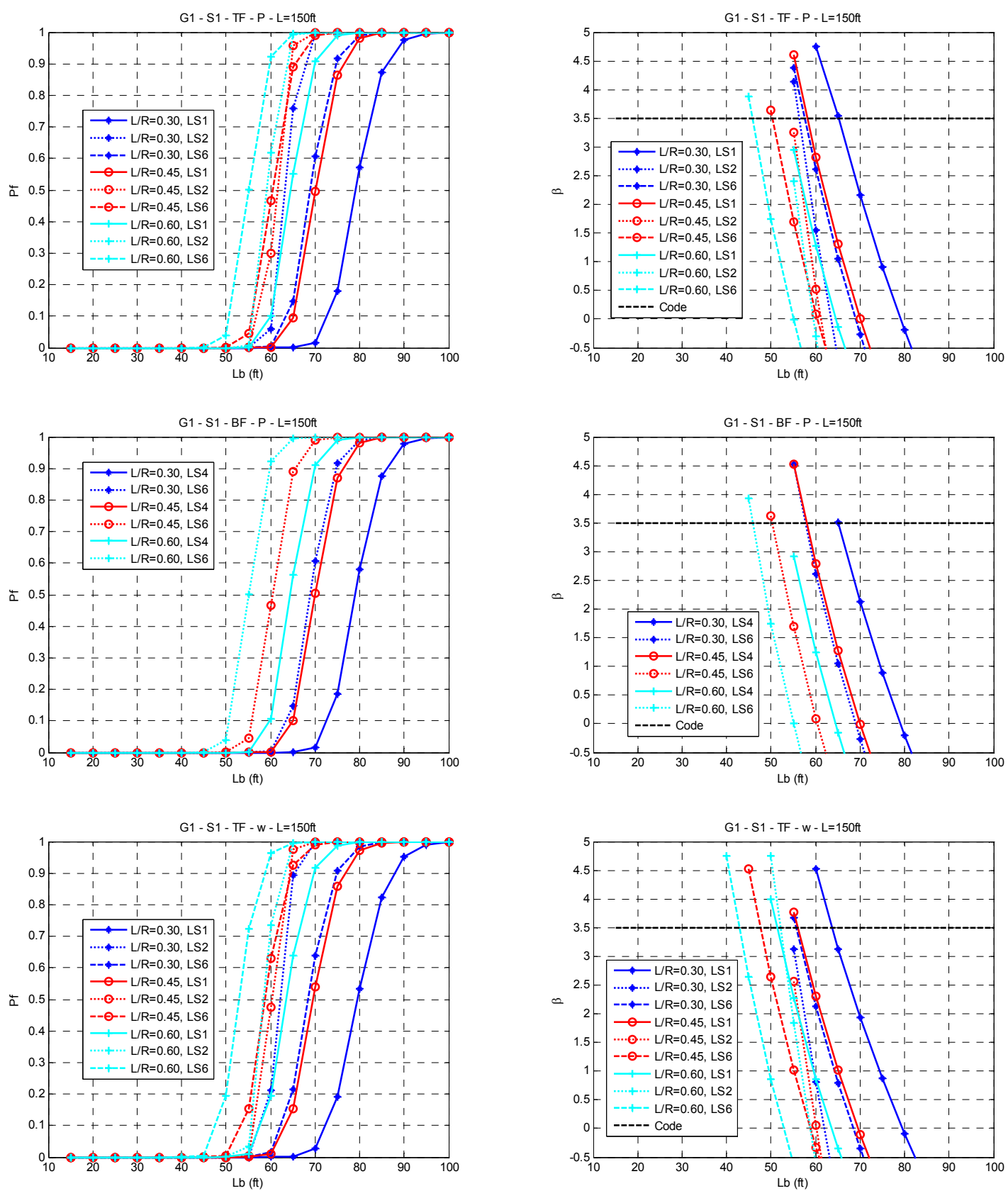
G1 - S1 - BF - w - L=150ft

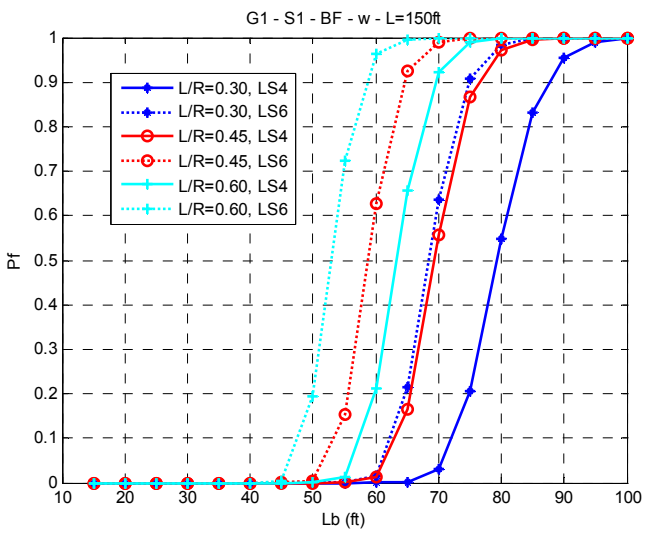

G1 - S1 - BF - w - L=150ft

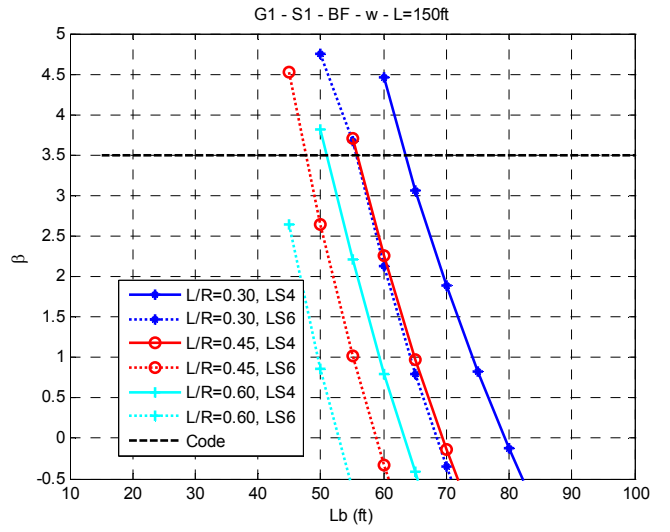


- Curved bridges: $L_{m}=150 \mathrm{ft}-$ Inner Girder $-\mathrm{S} 3$
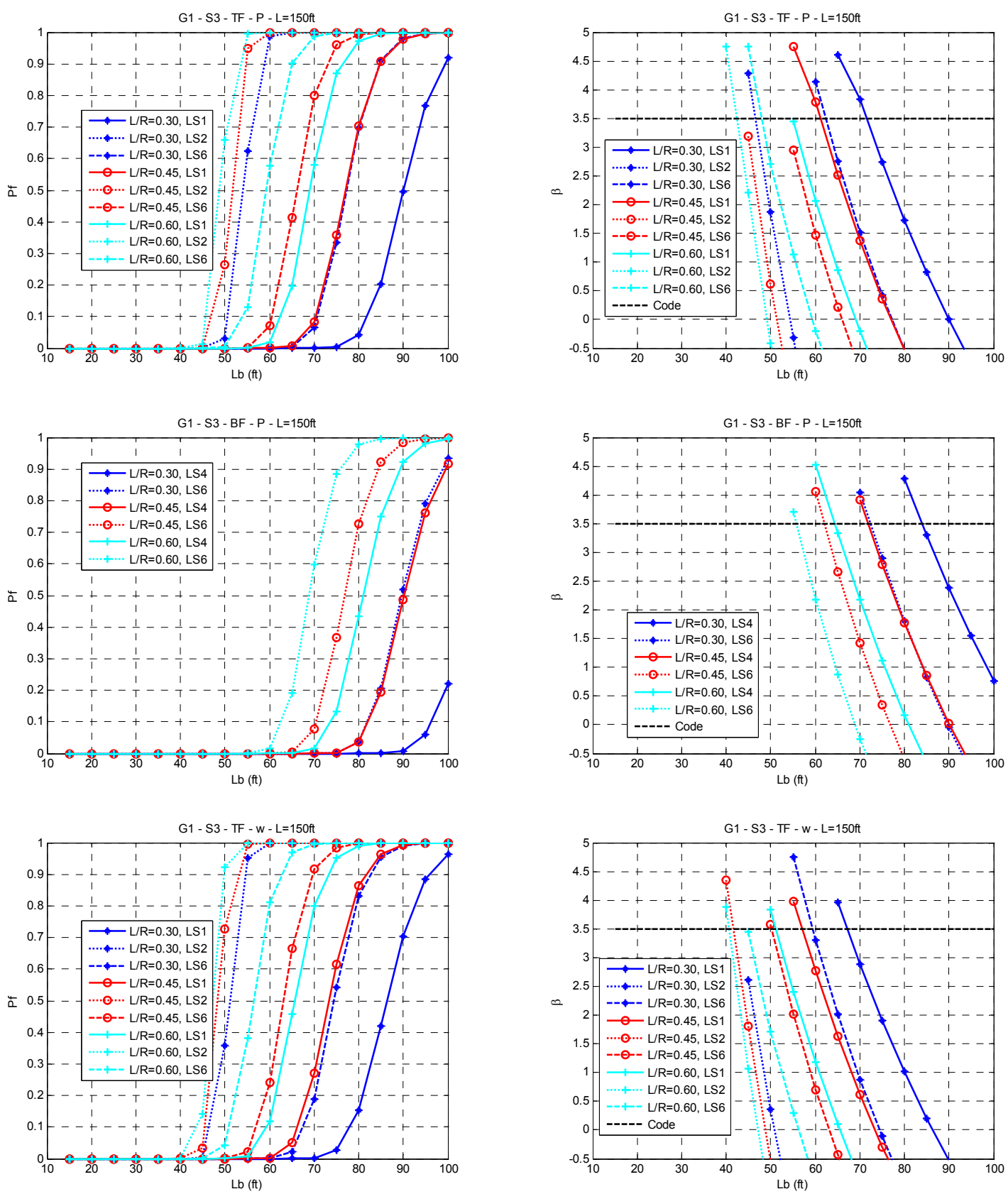

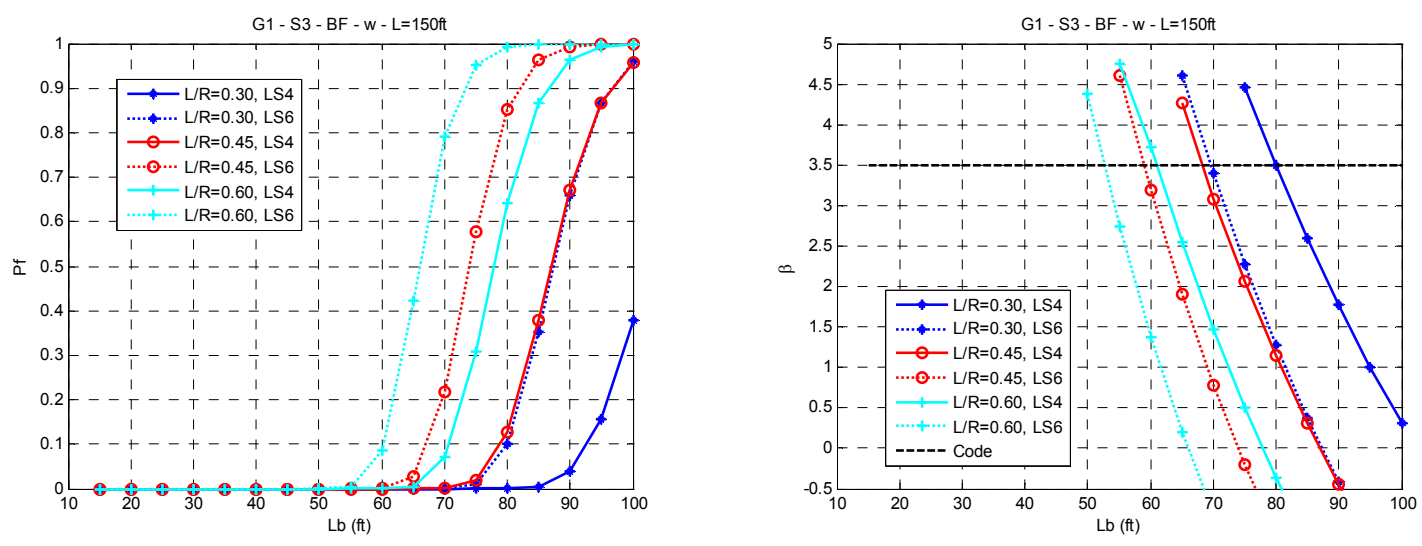

- Curved bridges: $L_{m}=150 \mathrm{ft}$ - Inner Girder - S2
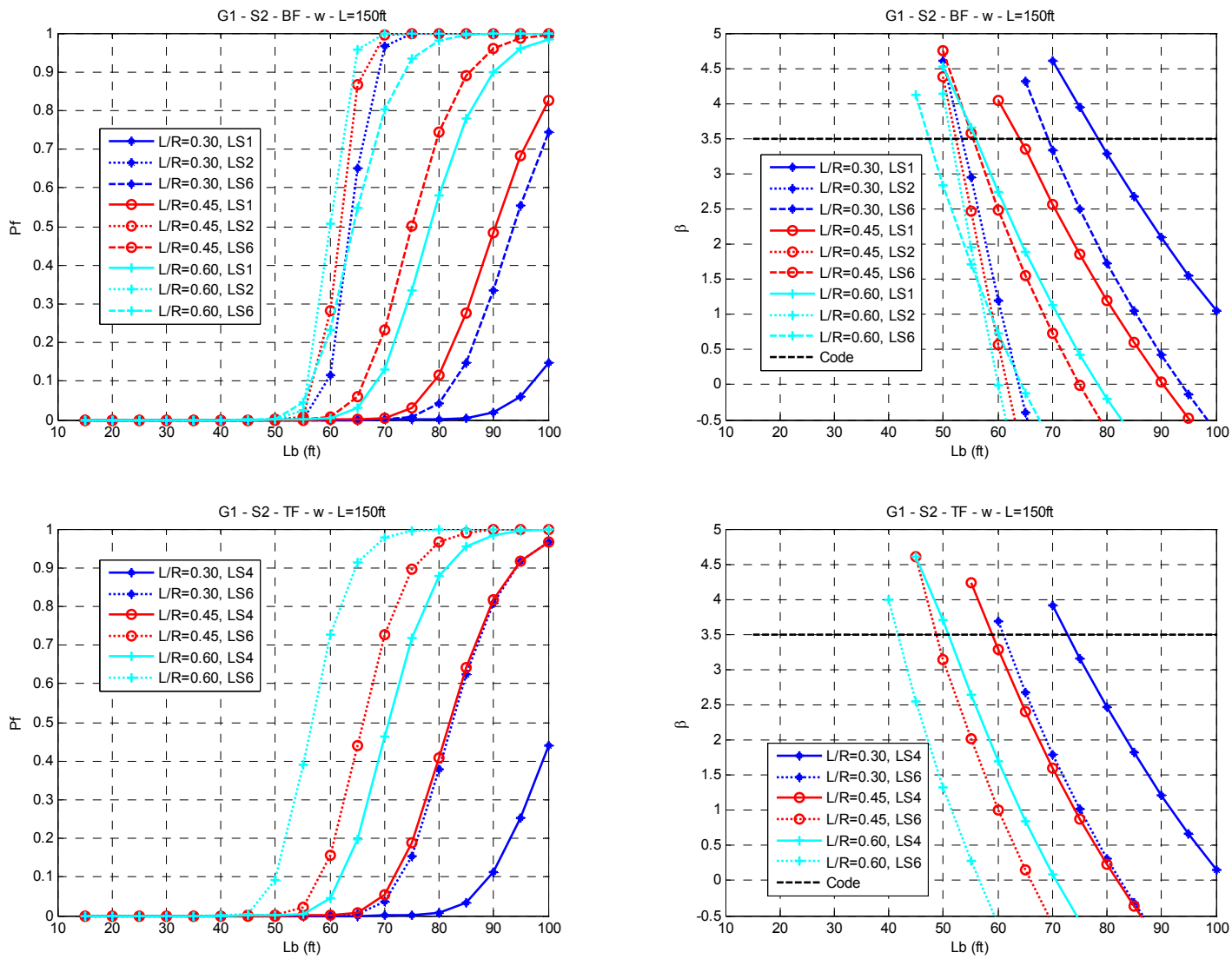
- Curved bridges: $L_{m}=240 \mathrm{ft}-$ Inner Girder $-\mathrm{S} 1$
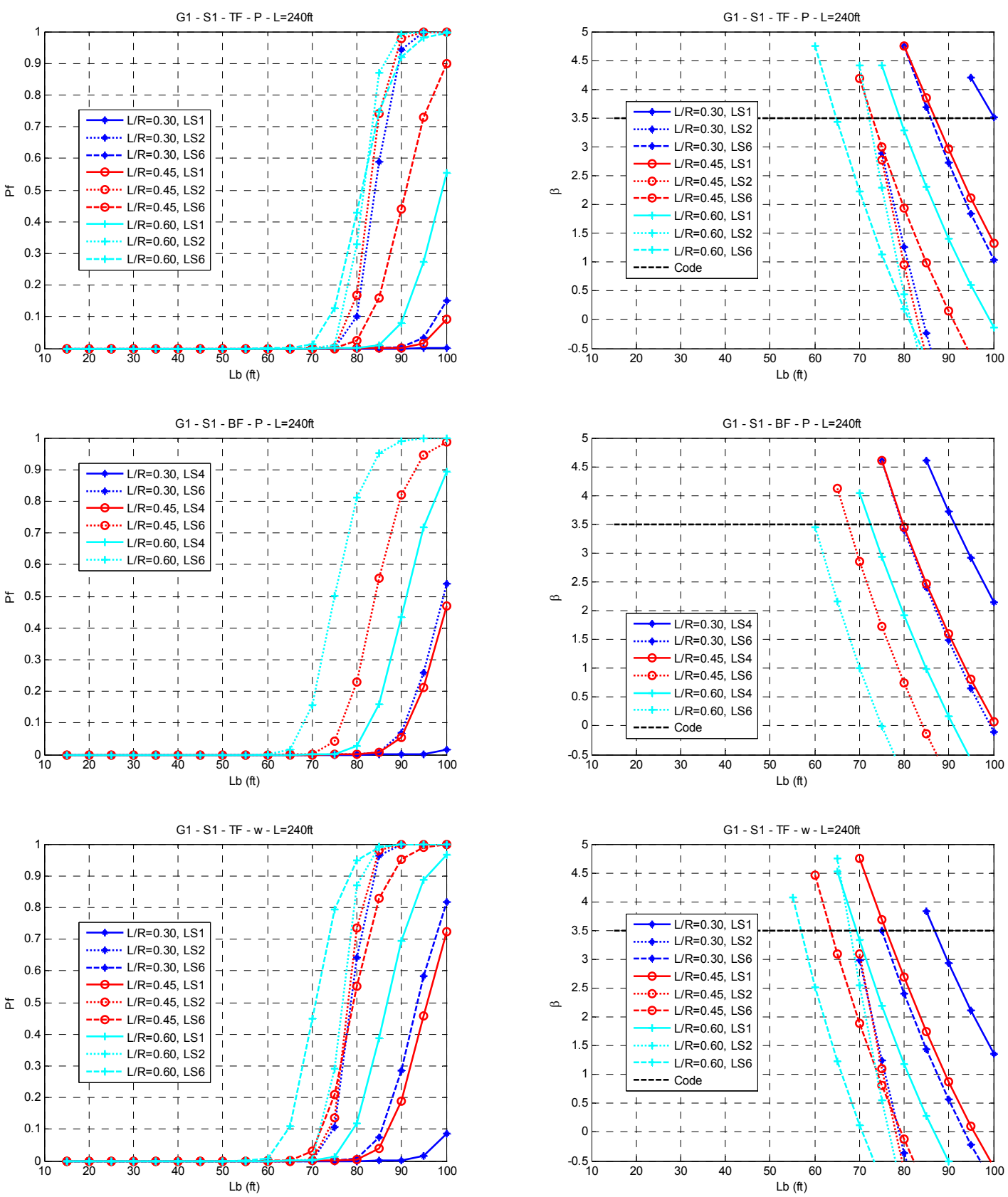

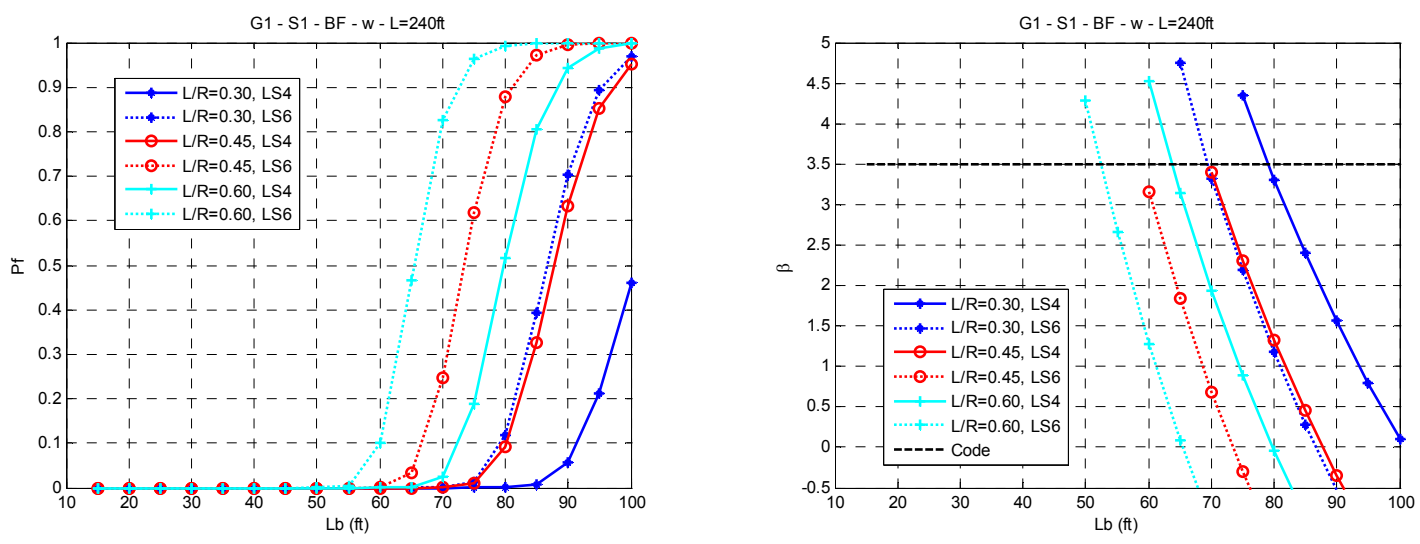
- Curved bridges: $L_{m}=240 \mathrm{ft}-$ Inner Girder $-\mathrm{S} 3$
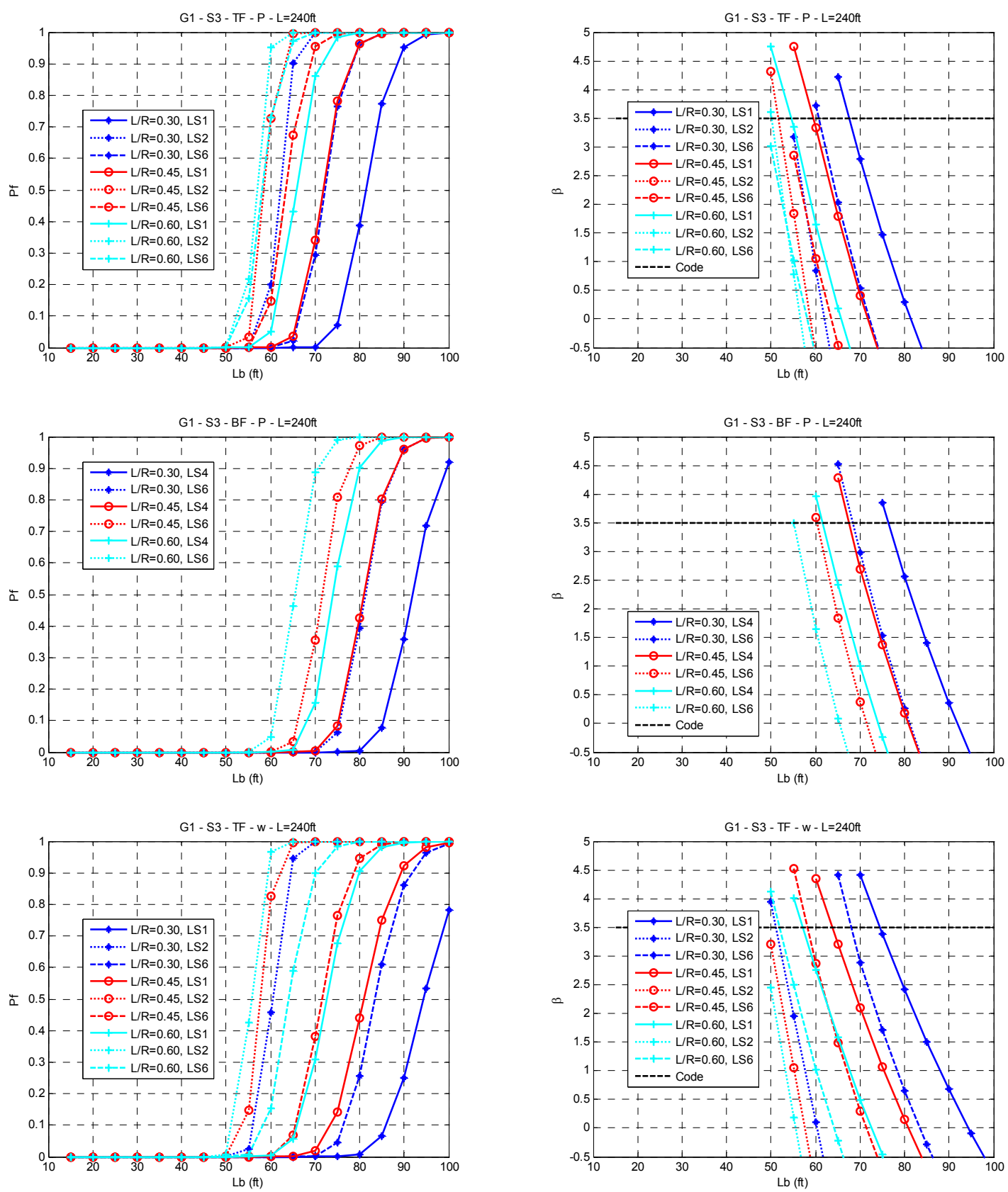

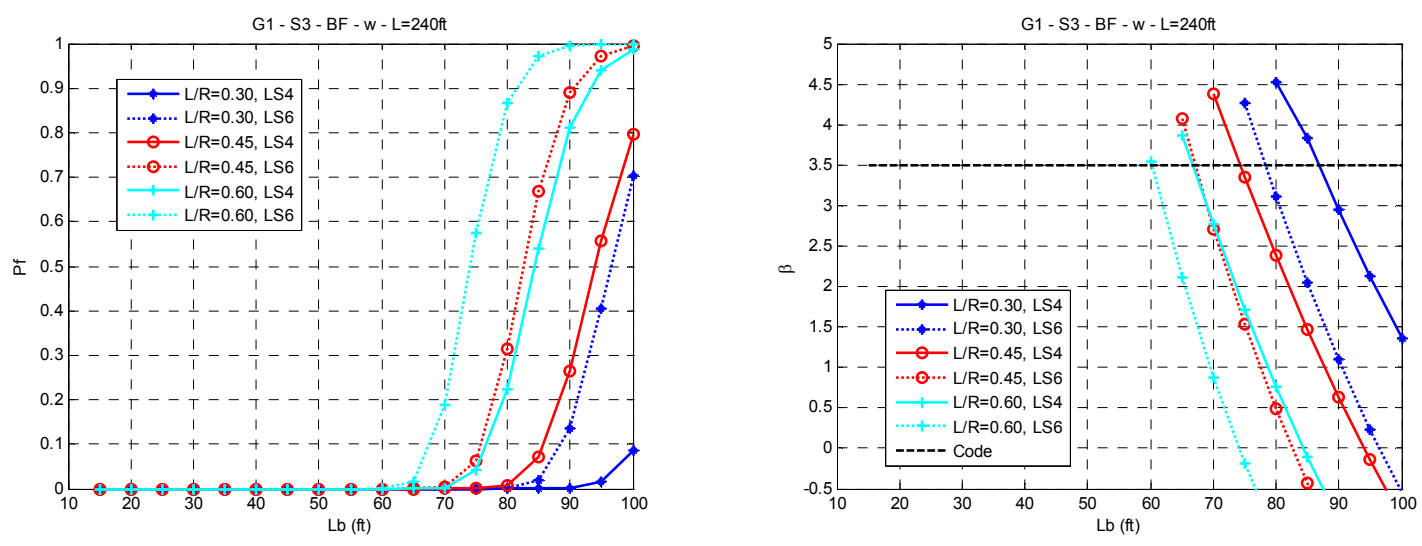

- Curved bridges: $L_{m}=240 \mathrm{ft}-$ Inner Girder - S2
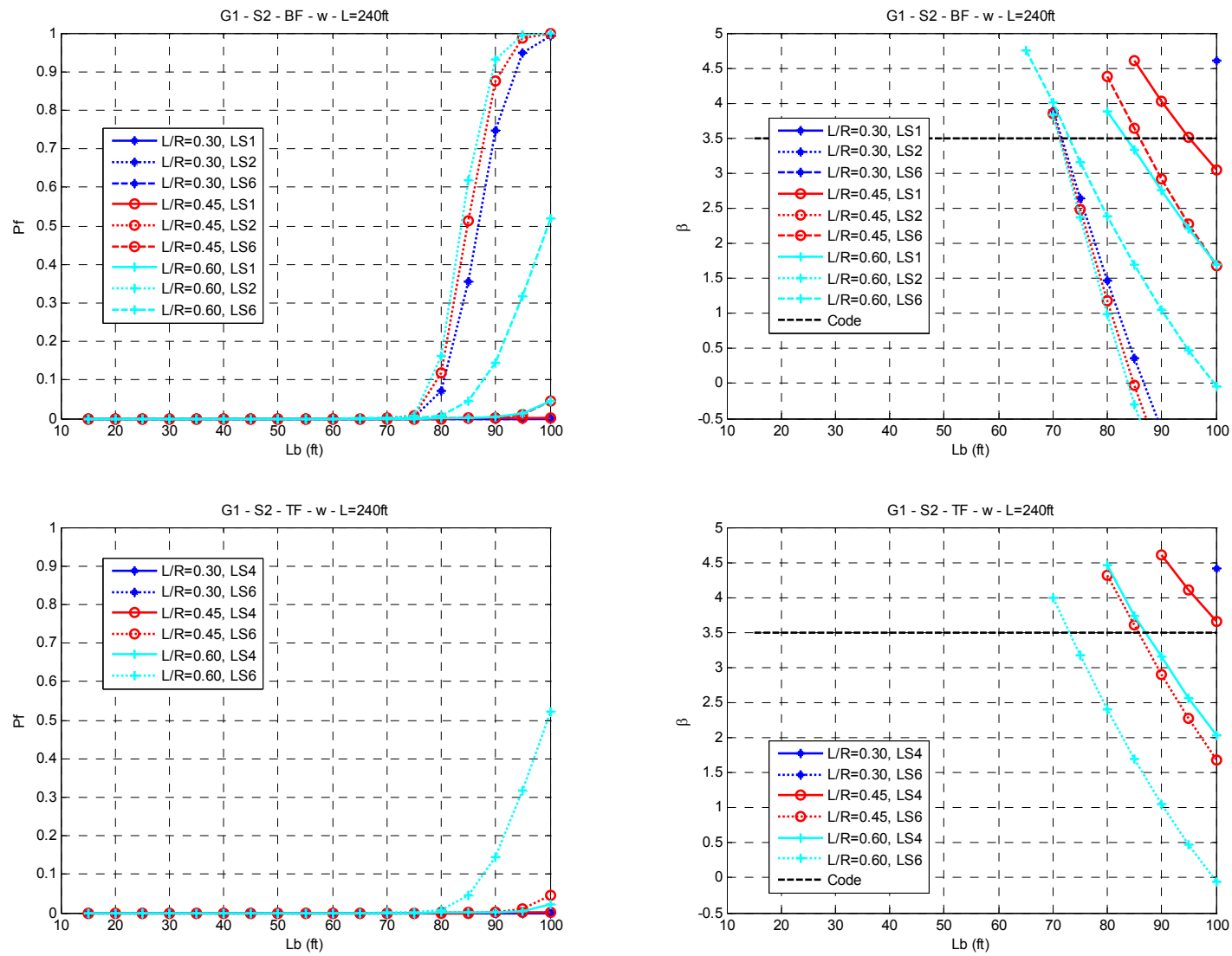
- Curved bridges: $L_{m}=300 \mathrm{ft}-$ Inner Girder $-\mathrm{S} 1$
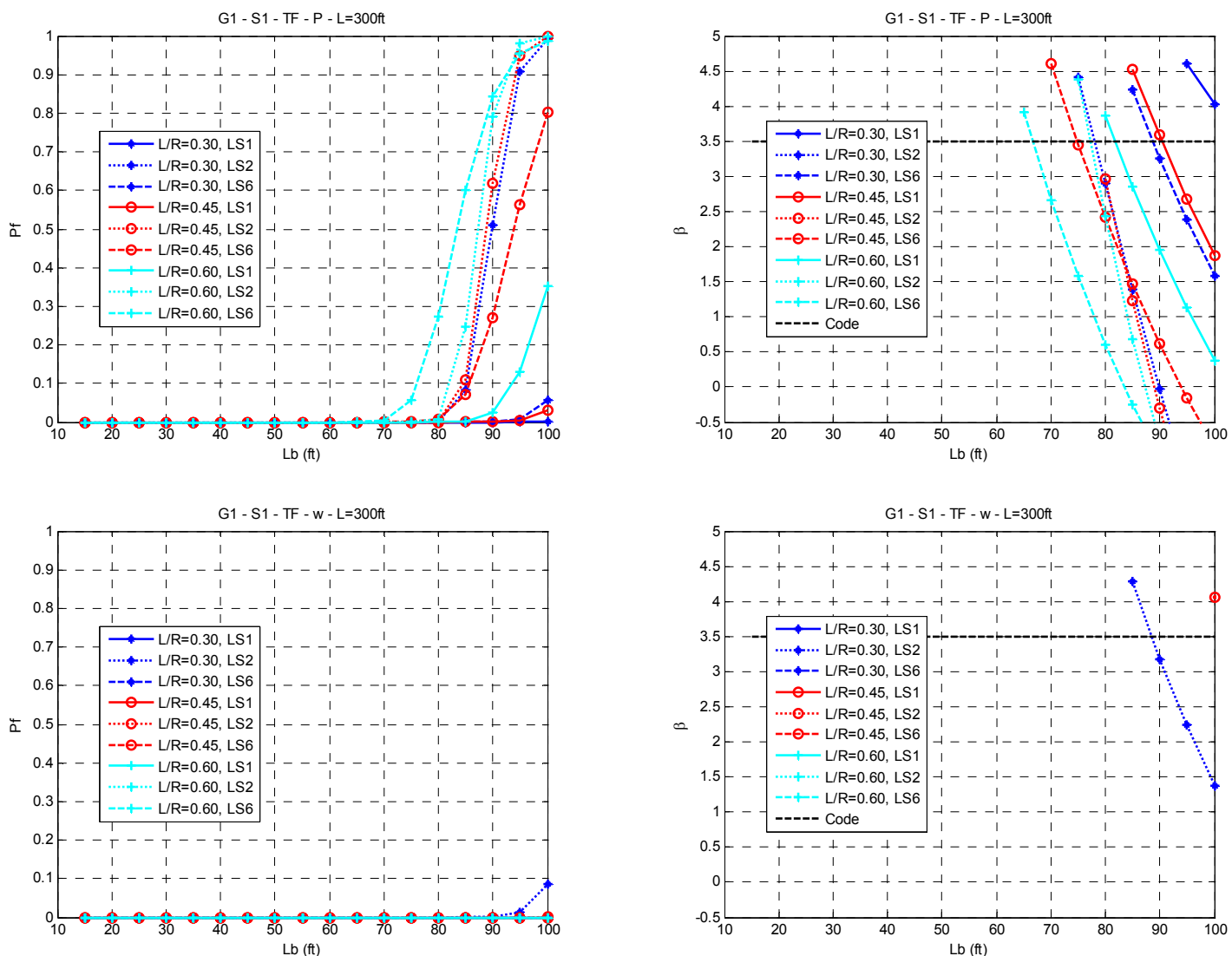
- Curved bridges: $L_{m}=300 \mathrm{ft}-$ Inner Girder $-\mathrm{S} 3$
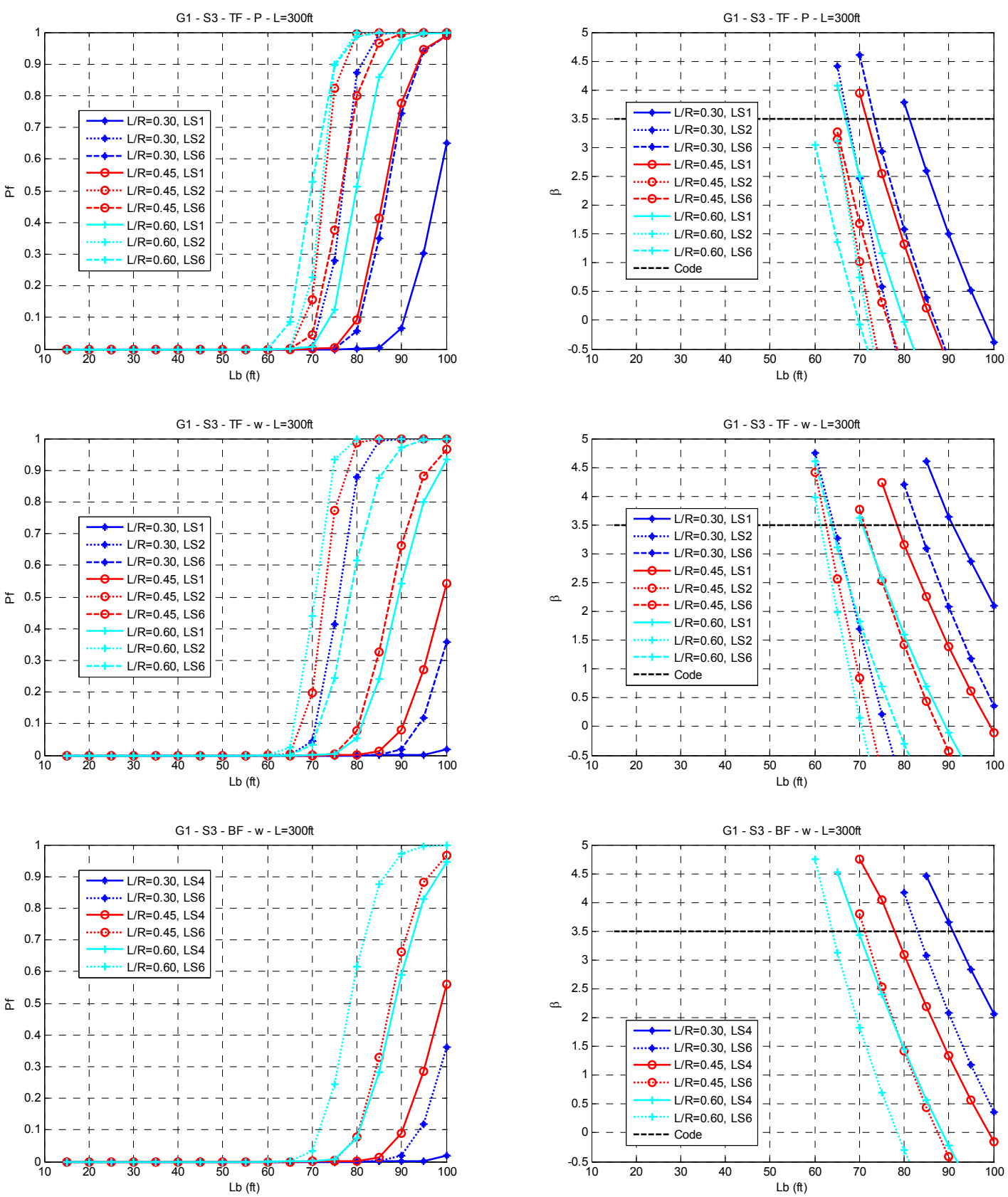
- Curved bridges: $L_{m}=300 \mathrm{ft}-$ Inner Girder $-\mathrm{S} 2$
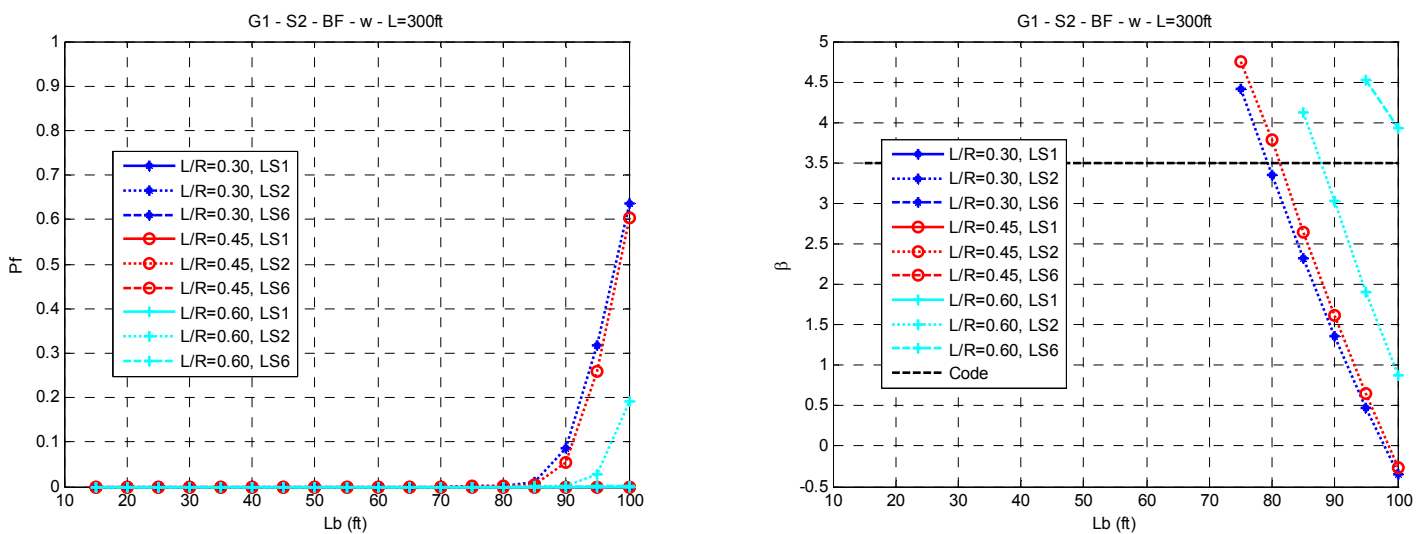


\section{Curriculum Vitae}

Nohemy Y. Galindez received her Bachelor of Science in Civil Engineering in 2002 and a Master's degree in 2005, both with emphasis in structures from Universidad del Valle, Colombia. She worked during her master studies as an adjunct assistant professor in the Department of Civil Engineering at Universidad del Valle, teaching courses related to the mechanics of solids and the structural design of buildings. She joined the School of Civil and Environmental Engineering at West Virginia University in 2006 to pursue a doctoral degree. Her research interests include steel bridge design, structural reliability, nonlinear finite element analysis and earthquake-resistant design of buildings.

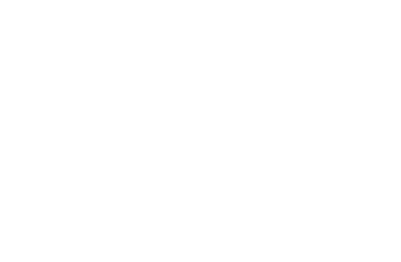

